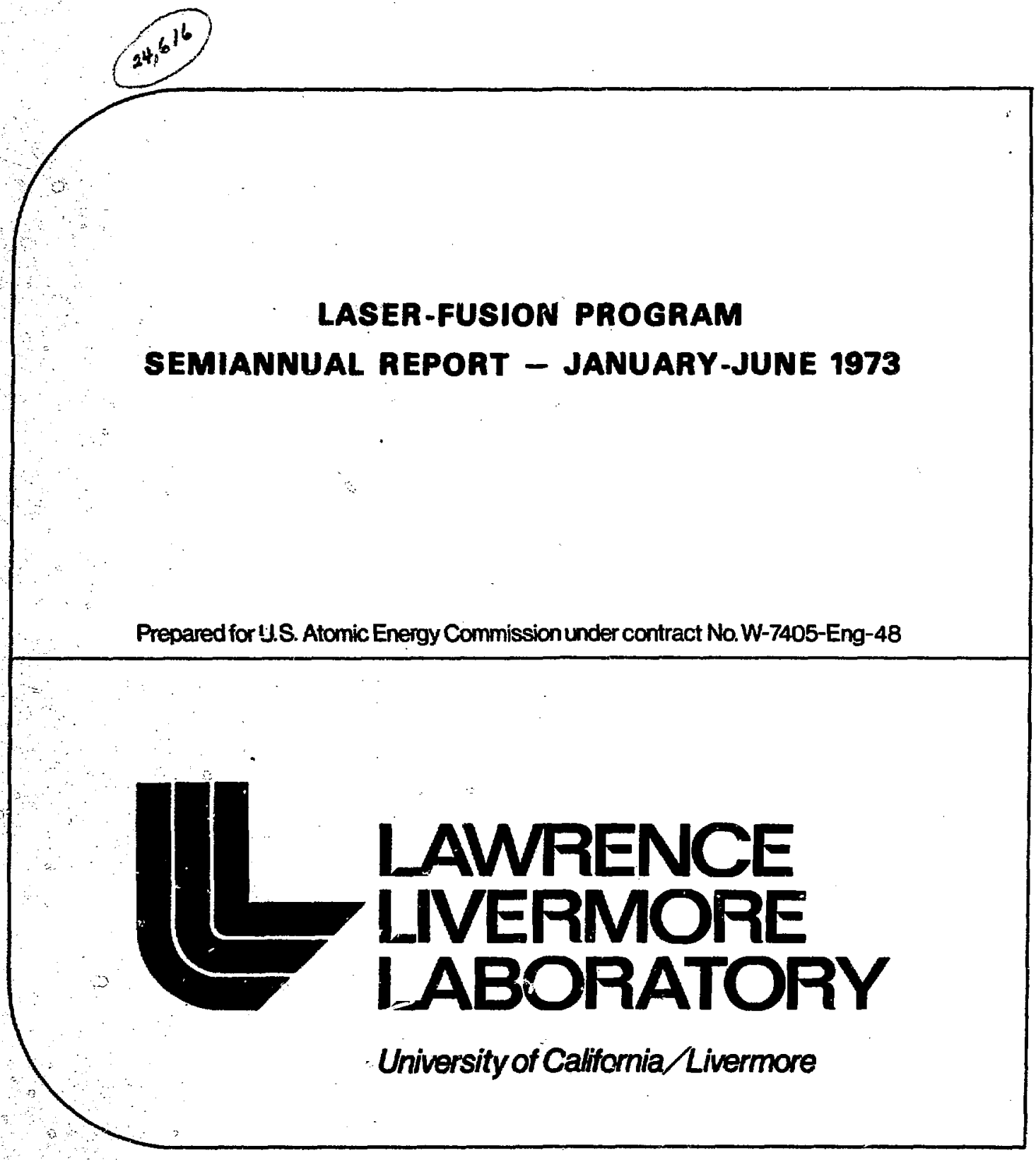


NOTICE

Thin report was jnepend ss an accoun of wook spononed by

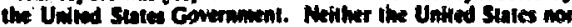

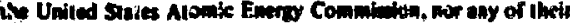

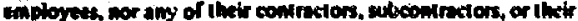

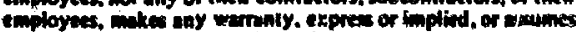

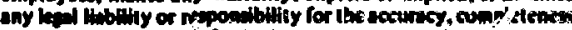
ot unfulase of any informalion. apparalus, product or proceses

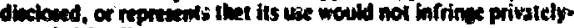
owned riptes."

Printed in the United States of America Available from

National Technical Information Service

U.S. Department of Commerce 5285 Port Royal Road Springfield, Virginia 22151

Price: Printed Copy $\$$ *; Microfiche $\$ 0.95$

\section{* Pages} 1-r;

51

$151-$

326-500

501-1000
NTIS

Selling Price

$\$ 4.00$

$\$ 5.45$

$\$ 7.60$

$\$ 10.60$

$\$ 13.60$ 


\section{世4}

\section{LAMFENCE LNEFMOFE LABOPATOFY}

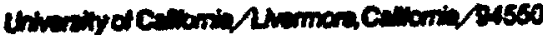

\section{UCRLS0021-73-1 \\ LASER-FUSION PROGRAM \\ SEMIANNUAL REPORT - JANUARY-JUNE 1973}

Stientific Editor: Stanley S. Sussman -

Cerieral Editors: Waitsce Clements

Gary Shaw

Paul Tanasovich

MS, date: August 22, 1973

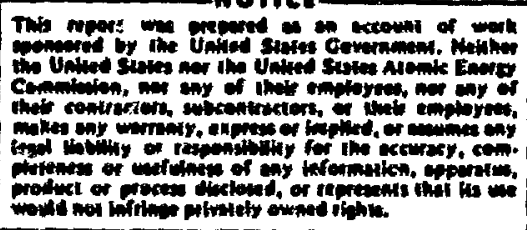

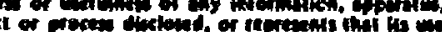

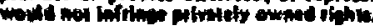




\section{Foroword}

This report covers progress in the laser-fusion program at Lawrence Livennore Laboratory during the period January-June 1973 - the last half of fiscal year 1973. All the work described here is unclassified. Whenever there is classified work to be reported it is covered in a clacsified supplement. No classified supplement is planned for this reporting period.

Some of the articles in this report make reference to company or product names. Such reference does not imply approval or recommendation of the product by the University of Califomia or the U.S. Atomic Energy Commission to the exclusion of others that may be suitable.

Inquiries about the information presented in this report ahould be addressed to the Director's Office, Lawrence Livermore Laboratory, Livermore, California 94550, attention Scientific Editor, Laser-Fusion Program Semiannual Report.

Both printed and microficine copies of this report are arailable from the National Technical Information Bervice, as described on the inside front cover. 


\section{Contents}

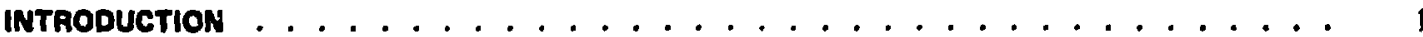

\section{SOLIDSTATE LASER PROGRAM}

Introduction (A. Cabezas) . . . . . . . . . . . . . . . . . . . 4

The primery goal of the solld-state laser program is ro develop a lakj laver fiactitity for lesenfusion expertments. In this facllity, tezet pellett will be untformly spherically inxitlated

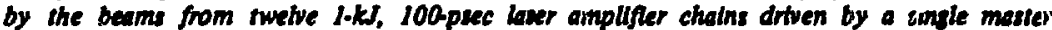

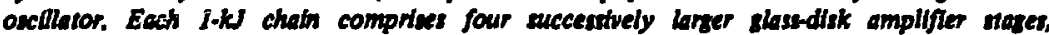
dextmated A, B, C, and D. The development schedule is almed af commencins laser-fuston experiments by mid.FY 1977.

Parasitic Suppression in Large-Aperture Nd:Glass

Disks (J. Gloze, C. Layne) …. . . . . . . . . . . . . . . . . .

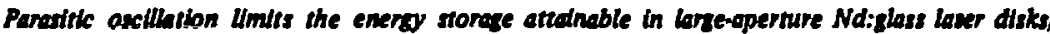
we are restins means of uppreseing if in disks of the stzes planned for the mochules of the 1.kJ enplifier chatin.

Amplifies Development (J. Holertchter) . . . . . . . . . . . . . . . .

To meet the constraints imposed by considerattons of taret interaction, selffocusing, and cost, the deats of the 1-kJ baer chath features full isolation of the ampilften from sarget back-reflection, a conservative limit on the lisht fexx, and modularization and commorallity of chath components. An amplifier-iest device is beins evaluated whtle the destion of the dist amplifier stoges proceeds.

$A$ and $B$ Disk Amplifiers - Prototype Harciware Deaign

(W. Hagen) ...........................

The destisus of the A- and B-nese disk-empllffer modules have been completed

Studies of Material Psoperties for Solid-Siate

Luser Deaign (R. Speck, E. Bliss) . . . . . . . . . . . . . . . . . . .

Material studles supporting the development of the $1 \cdot k J$ laser system have included measurentents of the montinaer index of refraction of ED.2 ghus and Nd:YAG, the rwo-phoson absorpition coeffickent of ED-2, and the intendity at which beem breakup coused by setffocudint instabilities begths 10 occur in a 50-cm.lens ED-2 laser rod

Electrooptic Beamshaping Device (B. Johnson) . . . . . . . . . . . . .

Transverse insensity variations other that Goussim are desirable in laser beams for propazation through loze emplifier systems; we dexcribe an electrooptle device that can, in principle, produce cony desired insusvers beim profile.

Master Oscillator (W. Founsain, P. Johnson, D. Tuft) . . . . . . . . . . . .

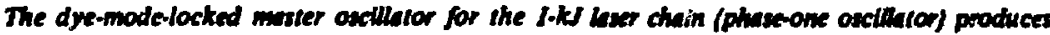

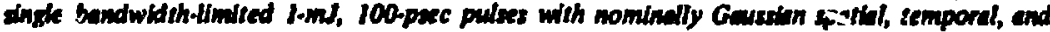

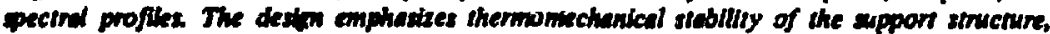
dye solurion, and etolom.

A Synchronoudy Driven Nd:YAG Oxillator for Producing Subnanosecond Bundwidth-Limited Pulses (B. Johneon)

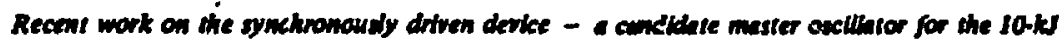

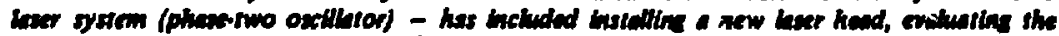

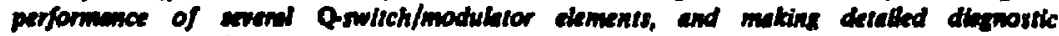
meacurements of the curpus. 
Introduction (J. Trenholme) . . . . . . . . . . . . . . .

The Design Analysis Group has concentrated on support of the solid-state laser program Effort has focused on improving pumping efficiency and unlformity, determining a beam shape that will give stable propagation and efficient energy extraction, and generating detailed amplifier designs

ZAP Compute, Code for Laser Power-Flow Calculations (R. Cooley) . . . . . .

We have continued to improve the $Z A P$ code, and results of $Z A P$ runs have been used to design disk amplifters.

Computer Optimization of Flashlamp Reflectors (E. Goodwin) . . . . . . . . . . $A$ special-purpose code has been written to optimize reflector geometry and lamp posittoning.

The Lambertian-Tube Laser Pumping Approximation (J. Trenholme) . . . . .

An approximate pumping analysis replaces the flashlamps and their reflector by a cylindrical Lambentiar radiating surface to speed up computations.

A User-Oriented Axially Symmetric Diffraction Code

(J. Trenholme) . . . . . . . . . . . . . . . . . .

Efficient calculation of low-angle, far-from-aperture diffraction in a laser beam by means of the Campbell-Deshazer formules is provided in a computer code that is designed for easy use by a broad population of users.

Initial Beam Shaping for a Fusion Laser (J. Weaver) . . . . . . . . . . . .

Detalled studies have been made of methods for generating a beam that will efficiently fill the final aperture of a lange disk laser, taking into account the effects of linear diffraction through the system and the constraints on beam size and intensity in the early stages

Diffractionless Calculation of Aberration and Self-Focusing

Distortion in a Laser Beam (R. Nelson) … . . . . . . . . . . . .

A new ray-tracing code is being used to study the effects of aberration and self-focusing distortion.

Optimizing the Design of a Kilojoule Laser Amplifier Chain

(J. Trenholme) ........................

We have sfudied the overall optimum design of a chain of loser amplifiers, considering the physics constraints on a lange disk laser, the design of a single stage, and the optir a combination of stages.

A Pumping Model for Diak Amplifier Design (J. Trenholme) . . . . . . . .

In this pumping madel the length of the glass path, the number of ailsks, and the capacitor bank size are minimized while satlsfying the constraints of parastic oscillation and lamp life.

BOBG Computer Code for Simulating Laser System Optics

(J. Weaver)

$B O B G$ is a fast and simple geometrical propagation code we have written for use in analysis, desim, and optimization of targe laser systems.

Optimal Selection of Flashlamp and Driving Circuit Parameters

is Laser Design (J. Trenholme) . . . . . . . . . . . . . . . . . .

We have developed a method for selecting the flashlamp size and the driving-circuit parameters for a disk-iaser pumping module.

\title{
COMPONENT DEVELOPAENT
}

\author{
Introduction
}


Faraday Isolators (G. Leppelmeier, W. Simmons) . . . . . . . . . . . . . .

A prototype of the Faraday-rotator isolator for the A module of the $1-k J$ laser chain has been evaluated. Verdet constants have been measured for several rotator glasses. A multilayer dielectric-coating polarizer has been selectew for use with the Faraday isolators.

Development of Calorimeters to Measure the Beam Energy

of High-Power Pulsed Lasers (S. Gunn)

An evaluation of absarbing glasses has led to the selection of Schott BG-18 as the preferred calorimeter absorber. A more compact twin-disk calorimeter and improved entry tubes and enclosures have been developed, and calorimeters are being fabricated for the l-kJ laser chains.

Cyiindrical Ring-Electrode KD*P Pockels Cell

(B. Johnson, L. Steinmetz)

Cylindrical ring-electrode (CRE) Pockels cells offer significant improvements in transmission uniformity and contrast ratio over commersially built cells. Switching times are as short as 200 psec or less.

Electrooptic Modulators for the 1-kJ System (S. Sussman) . . . . . . . . . .

We are fabricating CRE Pockels cells with apertures up to $18 \mathrm{~mm}$ for use in the l-kJ laser system.

\section{DIAGNOSTICS}

Introduction (L. Coleman) …................. .

A Compact Streak Camera (L. Coleman, S. W. Thomas,

G. Tripp) . . . . . . . . . . . . . . . . . . . . .

Three compact 10-psec-resolution streak cameres are operating. Characteristics of the microchannel image intensifiers have been determined.

An U!trafast Streak Camera with the Versatility of a

Standard Camera (R. Carman, H. Spracklen, S. Thomas) . . . . . . . . . . .

$A$ new plug-in unit lends versatility and flexibility to the ultrafast streak camera for a variety of research situations.

A Streak-Camera Tube for Improved Resolution

(R. Kalibjian, C. McConaghy) …...................

A circular-sweep streak-camera tube based on a modified cathode-ray tube has an expected ultimate time resolution of 1 psec.

An X-Ray Streak-Camera Tube (C. McConaghy, R. Kalibjian,

L. Mancebo) . . . . . . . . . . . . . . . . . . . . .

A streak-camera tube sensitive to 1-to-10-keV $x$ rays is vieing developed to temporally resolve $x$-ray events occurring in the time regime of 100 psec or less.

Diagnostics of Wavefront Distortion in Lasing Media

(D. Attwood, H. Chau, L. Coleman) . . . . . . . . . . . . . . .

Holographic interferometers will be used with a 1.A-J double-pulse reby laser for studying pump-induced wavefront distortion in Nd:glass disk amplifiers.

Holographic Interferometry of Laser-Produced Plasmas

(D. Attwood, D. Podesta, L. Coleman)

Because of its limited resolution, scatter-plate holographic interferometry is not particularly well suited for measuring electron-density distributions in laser-produced piasmas; holographic microscopy is a prime candiste for providing the desired higher spatial resolution. 
An Ultrafast 10-\%m Detector Based on the Optical

Kerr Effect in $\mathrm{CS}_{2}(T$, Owen)

A Rosowskl-proffle mode-locked TEA $\mathrm{CO}_{2}$ osclllator with single-pulse switch-out capabillty is now operating in provide the $10 . \mu \mathrm{m}$ modulating pulse for the visible $(0.5-\mu \mathrm{m})$ probe-beam pulse.

\section{GAS LASERS}

Intsoduction (W. Krupke) …....................

The LLL ges laser program is aimed at provlding a technical base for developing efficient, short-wavelength, pulsed gas lasers that can dellver high overage power.

\section{Gas Laser Research}

Demonstration of Temporal and Spatial Coherence and Threshold

Effects in the Molecular Xenon Laser (P. Hoff, J. Swingle,

C. Rhodes) . . . . . . . . . . . . . . . . . . . . . .

We hove observed stimulated emission at $1722 \pm 1$ A in high-pressure xenon gas originating from the bound-free conthuum of the $X e_{2}^{*}$ molecule.

Observations of Stimulated Emission from High.Piessure Krypton and Argon/Xenon Mixtures (P. Hoff, J. Swingle, C. Rhodes) . . . . . . . . . .

Stimulated emission has been observed in high-pressure krypton (1457 1 A, with 8-A bandwidth) and argon/xenon mixtures (which showed a 25-fold enhancement of the xenon loser output at $\sim 1720$ A) when excited by a relativistic electron beam.

Kinetic Model of Ultraviolet Inversions in High-Pressure

Rare Gas Plasmas (V. George," C. Rhodes) . . . . . . . . . . . . . . . . . .

Stimulated vacuum-ultraviolet emlssion at $\sim 1700 \mathrm{~A}$ is posslble in the afterglow of a sultably prepared lonized xenon plasma, as shown theoretically with a model which involves the distociative recomblnatlon of $\mathrm{Xe}_{\mathrm{j}}^{+}$as a key siep in the kinetic scheme leading to the production of exclted $\mathrm{Xe}_{3}^{*}$ dimers.

High-Resolution Infrared Spectroscopy (T. Meyer, C. Rhodes) . . . . . . . . . .

Using a high-resolution infrared spectrometer formed with stable $\mathrm{CO}_{2}$ osclllators, we have measured the pressure dependence of the linewidth and the pressure broadening coefficient for $\mathrm{CO}_{2}$ in collision with is different gases as perturbers.

Gas Laser Technology (L. Bradley, T. Kan) . . . . . . . . . . . . . . . . .

The gas laser technology effort at LLL aims at applying the technology of lasers and excitation sources (primarily electron beams) to develop a new generation of laser systems powerful enough for fusion appllcations. $A$ recent addition to the experimental capability is a $1 \cdot \mathrm{MeV}, 100 \mathrm{kA}$, so-nsec-pulsewidth electron beam source which will be used for pumping a variety of gases.

\section{$\mathrm{CO}_{2}$ Laser System}

Introduction (K. Manes) …....................

The $\mathrm{CO}_{2}$ laser system program at $\mathrm{LLL}$ has two objectives: (1) to establish $\mathrm{CO}_{2}$ system scaling parameters through analytical and computatlonal modeling supported by experimental measurements, and (2) 10 define and construct a small $\mathrm{CO}_{2}$ research laser system incorporating the best present technology to use for testing design concepts and doing plasma physics experiments.

System Design (K, Manes) . . . . . . . . . . . . . . . . . . . . . .

The $\mathrm{CO}_{2}$ laser system will have two types of amplifiers: (I) uv-preiontaed, and (2) cold-cathode, e-beam-sustained. A laser-simulating computer code (BOBG) is being used extensively to calculate the spatial shape of the optlcal pulse as it passes through varlous hyposhetical systems.

\footnotetext{
"Massachusetts Institute of Tochnology, Cambridge, Mass.
} 
Major System Components (K. Manes, w. Lindquist) … . . . . . . . . .

A preliminary design for the $\mathrm{CO}_{2}$ research laser system with an output of $50 \mathrm{~J}$ has been completed. The oscillators and amplifiers lave been specified, and some work has been done on isolator development.

$\mathrm{CO}_{2}$ Modeling . . . . . . . . . . . . . . . . . .

Energy Fluence Limitations for Nanosecond $\mathrm{CO}_{2}$ Pulse

Amplification (Y, Pan) . . . . . . . . . . . . . . . . . . . .

We are studying the energy fluence limitations for nanosecond pulses from $\mathrm{CO}_{2}$ lasers with an improved experimental apparatus which eliminates the need for visual observation of the spark formation.

Rotational Relaxation Measurements (R. Jacojos) . . . . . . . . . . . .

An experimental investigation of rotational relaxation in the upper $\mathrm{CO}_{2}$ laser level and its dependence on the rotational quantum number $J$ is under way.

Five-Temperature Kinetic Model for a $\mathrm{CO}_{2}$ Laser Anplifier

(K. Manes, P. Renard)

A previously reported five-temperature kinetic model for a TEA $\mathrm{CO}_{2}$ osclllator has been extended to include rotational and intramode vibrational relaxation processes.

Four-Temperature Kinetic Model for a $\mathrm{CO}_{2}$ Laser Amplifier

(R. Harrach, T. Einwohner)

................. . .

A four-temperature kinetic model for an e-beam-initiated $\mathrm{CO}_{2}$ laser amplifier is described. Sample calculations are presented, and a comparison of the model predictions with experimental measurements is given.

\section{CHEMICAL LASERS - THE IODINE LASER}

Introduction (D. Gregg) . . . . . . . . . . . . . . . . . . .

We have continued our efforts to better understand and improve the performanre of the lodine photodissociation laser in response to encouraging predictions for energy storage copability at high pressures of rare gas diluent.

Photodissociation Quantum Efficiency (D. Gregg, $E$. Huss)

We have deduced from absorption spectra and relative laser performance that the quantum efficiency for phorolytic production of excited iodine atoms is nearly unity for $\mathrm{n}-C, F, I$ and $\mathrm{i}-C_{2} F_{7} I$, in contrast with the value of 0.68 for $C F_{3} I$.

Kinetic Modeling (C. Turner, L. Rapagnani)

$A$ kinetic model of $C_{1} I$ photolysis, including three-body recombination, has been used to make conservative predictions of inversion lifetimes when the $C F_{3} I$ is diluted with rare gas at greater than atmospheric pressure. Our initial studies indicate that inversions of about 1 Torr can be maintained for $\geq 10 \mu \mathrm{sec}$.

\section{BASIC STUDIES AND ADVANCED CONCEPTS}

Introduction ( $A$. Glass)

Research has progressed in four broad areas of investigation: light propagation calculations, experimental devlce development, frequency conversion techniques, and laser materials studies. 
The method of trajectories has been developed and applied to study self-focusing and light propagation in nonlinear media.

Numerical Calculation of Stimulated Raman Scattering in a

Laser Beam (R Tooper $)$. . . . . . . . . . . . . . . . . . . .

A set of computer programs (called SAGITTARIUS) is under development to solve numerically the equations of quasi-optics for the laser, Stokes, and anti-Stokes beams, using the method of trajectories.

Homogeneity Requirements for Minimizing Self-Focusing Damage

(J. Marburger, ${ }^{*}$ R. Jokipii, ${ }^{\dagger}$ A. Glass, J. Trenholme) . . . . . . . . . . . .

We have generatized the theory of wave propagation in random media to include the effects of self-focusing. The simple solutions obtained show that intensity fluctuations - whether in the input beam or induced by random inhomogeneities in the medium - can grow catastrophically lange. These solutions may enable us to determine the conditions on homogeneity and beam fluctuations necessary to prevent such catastrophic self-focusing.

The Role of Linear Dispersion in Plane-Wave Self-Phase

Modulation (R. Fisher, W. Bischel $\neq$ )

Additional calculations are reported on the role of linear dispersion in self-phase modulation. Propagation of laser light in $\mathrm{CS}_{3}$ has been studied more completely, including propagation in $\mathrm{CS}_{\mathrm{2}}$-filled waveguides, and we have also investigated propagation in pumped and unpumped laser glass.

Improvements in the 20-psec Mode-Locked Ruby Laser System

(R Carman, S. Sutton, J. Kysilka) . . . . . . . . . . . . . . . . .

Further improvements have been made in the mode-locked ruby lacer system by eliminating unpumped ends of the ruby rods and optimizing the rod doping.

Nd:YAG Disk Amplifier (S. Sutton, R. Carman, R. L. Smith) . . . . . . . . .

We are proceeding with the development of an Nd:YAG disk laser. The poor quality of available disks remains a serious problem.

Stimulated Raman Scattering in Gases at High Pressure

(H. Lowdermilk, G. Kachen, R. Carman) . . . . . . . . . . . . . . . . .

We have generated stimulated Raman scattering in $\mathrm{CS}_{2}$ and several gases and examined its time dependence using the 5300-A second-hamonic beam of an Nd:YAG laser system. We are modifying a computer code to aid in interpreting the severe temporal modulation we have observed.

Studies of the ${ }^{4} I_{11 / 2}$ Level in ED-2 Neodymium-Doped Glass

(L. James, R. Fisher)

The nonradiative lifetime of the ${ }^{4} I_{11 / 2}$ level in ED-2 laser glass has been measured, and excited-state absorption from this level has been observed and identified. These preliminary

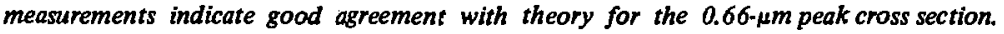

Tunable Laser Spectroscopy $(R$. Jacobs $)$. . . . . . . . . . . . . . . . . .

$A$ tunable $c w$ dye laser pumped by an argon laser has been constructed and characterized, and a pulsed dye laser pumped by the 3371-A line of a pulsed nitrogen laser is nearly completed. These lasers will be used to probe the excited state dynamics of potential lasing medin, and to study the Nd:glass laser medium.

\footnotetext{
*University of Southern California, Los Angeles, Calif.

${ }^{\dagger}$ Callfornia Institute of Technology, Pasadena, Calif.

${ }^{*}$ University of California, Devis - Livermore, Calif.
} 


\section{LASER PROPAGATION}

Two-Dimensional Propagation and Self-Focusing Code

(J. Greene, J. Fleck) ． . . . . . . . . . . . . . . . . . . . . . .

We have developed a time-dependent, twa-transverse-dimenstonat computer code to assess diffraction and self-focusing effects in the ellipticat beam profiles encountered with disk laser amplifiers.

Effect of Nonlinear Absorption on Self-Focusing in Glass

(J. Fleck, M. Feit) .........................

Calculations show that nonlinear absorptton can have a profound effect on the propagation of intense pulses through typical laser amplifier chains, since it tends to flatten the pulse both spatially and temporally and to limit the maximum intensity attainable.

Laser-Pulse-Induced $\mathrm{CO}_{2}$ Breakdown (M. Feit, J. Fleck) . . . . . . . . . . .

$A$ version of the LAMP pulse-propagation code is being developed to model the pulseinduced breakdown of $\mathrm{CO}_{2}$. The code follows in detail how the bulldup of an electron avalanche fed by inverse bremsstrahlung affects the light. Prellminary suns show severe distortion of the pulse shape and refraction of light energy through large angles.

\section{LASER PLASMAS}

Laser-Plasma X-Ray Studies

Introduction (W. Mead)

X-Ray Yields from Laser-Produced Plasmas (C. Violet,

J. Petruzzi, W. Mead, J. Swain, R. Saroyan)

We have evaluated various $x$-ray detectors for measuring $x$-ray ytelds from laser-produced plasmas and used the more promising ones in experiments with the Plasma $X$ and Long Path Laser systems and various target materials.

Preliminary Measurements of the High-Energy X-Ray Spectrum

from Laser-Produced Plasmas (W. Slivinsky, H. Komblum)

Thick K-edge filters and high-gain ssintillation detectors have been used to measure the $x$-ray spectrum in the range of 10 to $140 \mathrm{keV}$ emitred by plasmas produced from $\mathrm{CD}_{2}$ targets irradiated with 50.J, 3-nsec laser pulses.

Hard-X-Ray Evidence for Relativistic Electrons

(J. Shearer, J. Swain)

Analysis of hard-x-ray data from some Long Path Laser experiments done in 1972 indicates that relativistic electrons were produced at the laser focus. This observation is qualitatively consistent with theoretical calculations.

Pair Production by Relativistic Electrons from an Intense

Laser Focus (J. Shearer, J. Garrison, J. Wong) . . . . . . . . . . . . . . .

A consideration of inechanisms of relativisticelectron production by focused laser light has Bed to the conclusion that laser-driven plasma instabilities moy be capable of producing relativistic electrons at lower laser intensities than previously estimated.

LASNEX Calculations of X-Ray Experiments (H. Shay) . . . . . . . .

LASNEX calculations of laser $x$-ray experiments agree reasonably well with measured conversion efficiencies and $x$-ray spectra but predict a much lower laser-light reflection coefficlent. 
The Computer Progrum FOREST (w. Grasberzer) . . . . . . . . . . . . .

POREST is a singlenelement multipteion computer program for onedimensional plene geometry

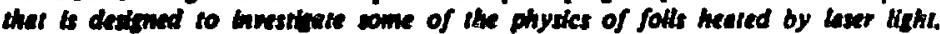

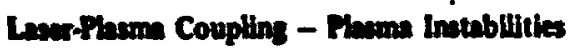

Introduction (W. Kruer) …..................

Mode-Coupling Saturation of the Parametric Instability and

Electron Heating (J. Thomeon, R. Faeht, W. Knuer) . . . . . . . . . . . . .

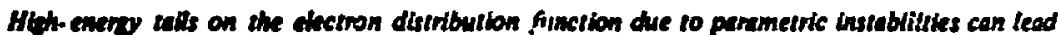
to serious prebent and decoupling probiems in the dextsn of peltets. We present esimple theory of the sumution and hatins mechouism for the parmetric instebility thet occurs at critical denilgy. The theory predicts an upper bound for the supnithermal iall formulton co be expected in pellet epplications.

Improvements in Turbulently Heated Distribution Functions

(J. DeGroot, W. Knuer, J. Tuln

Shere very energetic electrons formed in the barmpellot interection send to degnede pellet performence, we are looktins for mays to minimize their production Two promising reetiniques

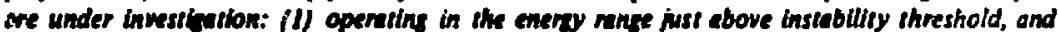
(2) operatins with a hish netis of atectron to ion tempernture.

Plame Heating at One-Fourth the Critical Density

(A. Langdon, B. Lasinsk, W. Kruer)

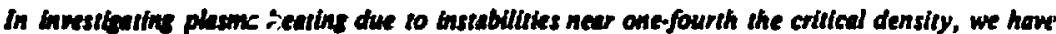

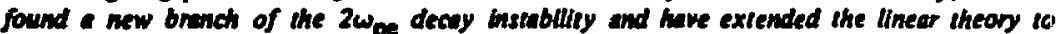

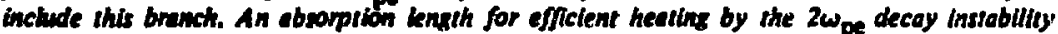
hes been extimuled on the basis of nesulis of computer simulations used to stridy the nontlineal evalution of the plasme.

Instubility-Generated Laser Reflections in Piasmas

(i.. Kruer, K. Estabrook, K. Sinz)

To exemine the imporience of instabilliygenereted reflection of laser titht from a plesme (from: the Romen and Brillouin instablitiks), we hrve calculated the refection of bser lisht propogating through an undendense plasme with a density srodient. The reflection decreases ropidly as the retio of the laser lith pressure to the phame pressure becomes small. Several rechniques are demonsmited which further neduce the instebillty effects.

Self-Focusing and Filamentation in Plasmas

(A. Landon, B. Lasinski)

A beam of iser light in pheme cen self-focus into morrow flaments of higher intensity light, which cen have importens effects on tarer heating of the plasma. We present a linear analysis of selffocusing in planess 10 assess the rate at which fltaments form. A speciffc example of enlffocusing is exemined with an etectromagnesic plasmasimulation code.

Large Plasma-Simulation Codes Using Virtual Memory

(C. Bames)

We have devetoped a scheme for using magnetle disk storage as virtual memory for both particles and felds in the plusm-simulation codes. A iwadimensional electrostatic particle code with - $512 \times 5 / 3$.polnt potenthl meth is being sit up in ints way.

Stanfoed Univeralty, Stanford, Calitornis. 
REFERENCEE. . . . . . . . . . . . . . . . . . . . . 220

GIBLIOCRAPHY OF RECENT PUBLICATIONE . . . . . . . . . . . . . . 228

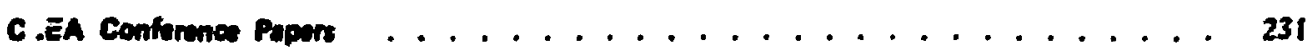


The AEC-zponsored laser proyam has three primary objectives:

1. To demonatrate the scientific fessibility of initiatine thermonuclear bum in a fuel pellet by irradiating it with a high:power, short dupation laper pule.

2. To exploit the knowledse grined in echieving the first objective to develop miliury and commercial applications (the milisary applications fint becuuse of economic consinints, with the commercial applications following later). A broad level of adranced Irer lechnoloy is beine established at $L L$ and other laboralories in the process of attuining these first two objestives.

3. To apply this laser technoloy to vorious nonfusion appliations of interat to she AEC and to other sovimment agencies.

An outline of these objectives is given in Table 1.

\section{Teth 1. Priming obioctives of the AEC}

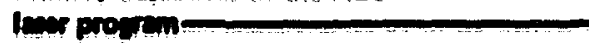

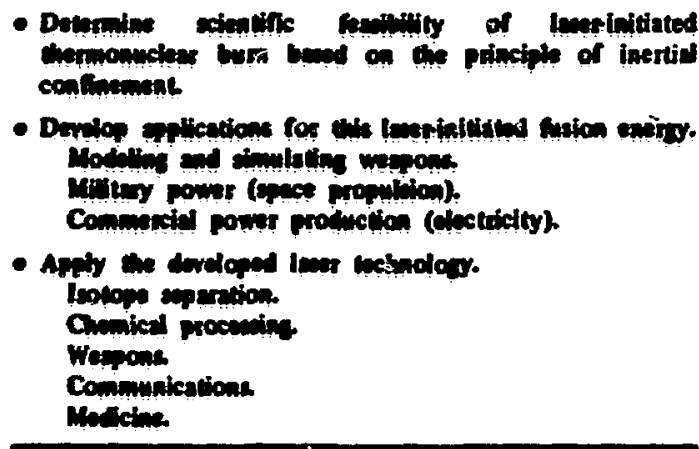

The baric taks in the initial phases of the lawer fusion program are to determine the kind of laser output puke - in terms of energy, duration, wavelength, coherence, and symmetry - that will be required to compress a pellet of thermonuciear fuel to burn conditions, to design and build a laser that will produce this output pulse, and to carry out experiments demonstrating the fexibility of lacer-induced fusion.

Hot electrons play a key role in compression of the pellet. The laser/plama interaction heats the electrons, and energy is transported from the plasma to the denser pellet region by these electrons. Heating of the pellat surface generates the implosion presures. In order to reach very high compreasions, shaping of the laser pules (or multiple pulses) will be required, with the find power level rising by severd orden of maznitude from the initiol power level.

Five mileatones can be set down to meanre our progres:

1. Rexching in adequate undentanding of the physics of the interaction between laser light and plesme.

2. Demonatrating laser-induced implosion in which the fusion enersy produced is greater then $1 \%$ of the light onergy from the lewer, and compresions greater than tenfold are attuined.

3. Achievine significant thermonuclear bum, with comprewsions greater than a hundredfold and fusion energy cutputs greater than $10 \%$ of the laner tight eneryy.

4. Achioving light energy breck-even, by raising the pellet bum efficiency so that the fusion enery produced is of the some monitude $a$ the laner light energy.

5. Achieving net enerzy gin, by raing the system efficiency to the point where the furion enerby output subatuntially exceed the energy input to the laver.

Our present eatimates of the laser technology needed to reach these milestones are given in Table 2.

For the next few years (FY 1974-1976), the Leboratory will continue to perform buic leser/matter experiments using laters of moderate size. Pube energies will range from tens of joules to about a thousand joules, and pulse lengths will range from the low nanowecond region to tens of picoseconds. The main purpose of these experiments will be to provide data for theoretical calculations, so 20 to develop the capability to predict with more confidence the key lawer and target parameters necestary to obtain thermonuclear bum. We hope to attain the first milestone and possibly the second one during this period. The Laboratory will also woik on obtaining sources of laser-produced $x$ rays, which could be used for $x \cdot$ ray simulation experiments.

Neodymium-doped glass lasers are the best understood high-nergy, short-pulse-duration lavers. With Nd:glass lasers we will be able to thoroughly investigate the physics of laser/plama coupling, which is so fundamental to the eventual success of laser fusion. Glass laser offer the short-term potentid of a possible lightenergy break-even. However, the high energy and high average power eventually required for controlled thermonuclear power generation preclude the use of solid-state lasen ase now concejve them.

We are confident that our glas laser effort offers the most reliable path to accomplishing the near-tarm 


\begin{tabular}{|c|c|c|c|}
\hline & & & $n$ \\
\hline 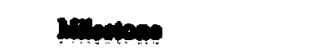 & $E_{\text {putan }} / E_{\text {urith }}$ & Enepro $(0)$ & Rum lonst (n) \\
\hline 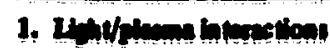 & - & $10+10^{3}$ & 1elice \\
\hline 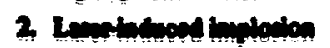 & $10^{-2}$ & $10^{2}-10^{3}$ & sores \\
\hline 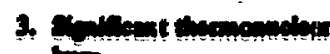 & $10^{-1}$ & $100 \cdot 104$ & 100100 \\
\hline & $10^{\circ}$ & $100 \cdot 10^{\circ}$ & solote \\
\hline 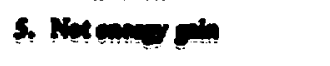 & $\geq 10^{4}$ & $100 \cdot 104$ & 1001000 \\
\hline
\end{tabular}

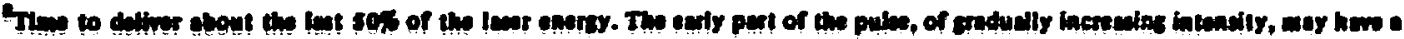

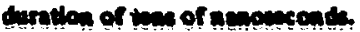

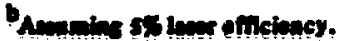

rols of the Imer fucion program. A Imar with Nd:-1es dilo (the Lons Poth Laer) producing $200 \mathrm{~J}$ in 5 neec and $100 \mathrm{~J}$ in 2 nece hes bon dowloped at LL and in in opontion. Uhdor devlopment now is a 1.kJ, 100-pece Nateles leer sytem. It will be constneted in four eucceivive incements producing uproximately 5, 50, 250 , and $1000 \mathrm{~J}$. Severil replices of the loner-unery incremente will be built nd used to study the detail of the lever/pluma interaction. These will be extremoly alexible devices providing use. ful enery output at pule durations from 20 peec to 1 neec.

The IteJ sytem, which will be almat entirely contructed from dick leer umplifien, will form the module beris for the development of the $104 . \mathrm{J}$, 100-pece spherical inndiation facility to be used for the proof-of-principle experiments. Twelve of the 1-kJ laners will be opernted in parellel, driven by a common cocillator, to make the 104.J lewer facility. The program for the $10^{4-J}$ facility will be initiated in FY 1974.

Nd:sims Imers opernte at a wavlength of $1.06 \mu \mathrm{m}$. However, certsin theoretical ccnsidentions indicate that shorter varvelengths would be more uneful for plema heatiag. Thus, the $18+-1$ factity will incorporate the cepabiity of renerating the recond and fourth hamanics 2 well $a$ Stokesthifted radiation in $\mathrm{H}_{2}$, sllowing coveras of the waviength repion from 0.265 to $1.9 \mathrm{~mm}$. At all of these wavelengths, total enertes in exces of $2 \mathrm{~kJ}$ will be wailable. Thus we will hure a flexible facility for puruing the proof-ofprincipie experiments.

Cas lmon appear to provide greater long-term potential for the eneration of enerpies in the 104-to.105.J region at hiph efficiency. The $\mathrm{CO}_{2}$ Iaser operating at $10.6 \mathrm{~mm}$ has demonatrated the atility to conente 1-nece pulsen with an efficiency of $5 \%$.
Development of shortopula $\mathrm{CO}_{2}$ laen is preaently under way at LL, with operation of a S0-to-100J. t-nec $\mathrm{CO}_{2}$ lener expected by the midde of FY 1974. If physice permits, the selling of this devies to lager eneries my be undertaken.

Enery docoupling and tarest proheating effects, during implosion, hive been found which may make it las decirable to use lawer radiation of 20 long a wavelength $210.6 \mathrm{\mu m}$. Both problems, it wapens may be dleviated to some extent by using rediation of shorter wavelength. An extensive program is in progress at LL to develop short-wavelength, highefficiency gat laver media which could thare many of the stvantages of the $\mathrm{CO}_{2}$ system but operate at more desirable wavelengths. We have observed, for example, lacer action (1716 A) from molesulas xenon $\left(\mathrm{Xe}_{2}^{*}\right)$ in xenon as at $200 \mathrm{pri}$, and stso liser ection in molecular krypton ( $\left.K_{2}^{*}\right)$ at 1457 A at 400 pei. Similar experiments with other rareges syatems and other van der Wads type molecules are under way. Short-wavelength laser systems using both resonme and nonresonent stimilated anti-Stokes Reman sattering ane under development.

Pulsed shemical Iasers sppear to offer the greatest potentia for weapon applications ultimately, because they may hrve the ability to store lare amounts of energy in a reasonably snall volume and weight. We are still in the very early research stages with these concepta, however, and plen only a modest level of effort in the near future. The chemical laver propram will be rapidly expanded when ireakthroughs or other technological development permit.

For all of these systems, disprostic capsbility is of paramount importance - especislly for mikauring lifht pulses of aubnanomecond duration. Our dieprostic program addreses these needs. We have developed a strock camen with 10-pece reaclution and are 
extending its capability to 1 peec. An $x$-rety photocathode is unde? development which will allow time-resolved $x$-ray spectroscopy on the laser. produced platmes. In senerch, we are workins to extend the time resolution of all the conventional direnostics for lacers and plesmas to the 1:t0-1000 pace regine.
The extentive lmer tewlopment effort in sepport of the lewrefusion projam will clearly hawe applica. tions in ares other then fusion. A propram directed at optical eparation of a wide nne of ieotopes includ ing urmiun isotopes in particular, has been initiated. Other lmer applicitions will be explored 2 ins needs apput and the tochnolosien become milable. 
The primary goal of the solid-state laser program is to devalop a $10 \mathrm{~kJ}$ neodymium-glass laser facility for lecr-fusion experiments. Nominally, twelve $1 \cdot \mathrm{kJ}$, 100-piec beams will be used to uniformly and spherically irradiate target pellets in cxperiments aimed at demoristrating significant themonuclear bum and possibly schieving the lightenergy break-even point. The latter is defined $\&$ the point at which the thermonuclear output energy is equal to the total energy of the laser ceams.

A scale model of a $10 \mathrm{~kJ}$ array of 12 chains driven by a single master cecillator is shown in Fig. 1 . This configuration is preliminary; it is intended only to clarify the concept of an array of beams uniformly and spherically irradiating a target. It is also shown to draw attention to the basic chain that is replicated 12 times to make up the array; it is this chain that we are presently developing and optimizing in output performance, energy efficiency, and system reliability.
(Details of the program approach were discussed in the previous Semiannual Report. ${ }^{1}$ ) In the model shown, the last arnplifier in each chain is oporated at twice the maximum energy flux in the rest of the chain; note how these last amplifiers are positioned radially around the target so that the turning mirrors are not subjected to this high flux level.

The schedule for the development of the 10-kJ system is shown in Table 3. The schedule is aimed at commencing laser-fusion experiments by the middle of FY 1977. The increments outlined in Table 3 are amplified below.

The developmental and prototype program for the 1-kJ chain will be completed in FY 1974. The prototype chain will be used to thoroughly work out the physics (such as self-focusing and clean beam propagation) and the engineering required to generate approximately $1 \mathrm{~kJ}$ in 100 psec from a single $\mathrm{Nd}$ :glass chain. In FY 1975, the design of the basic 1-kJ chain

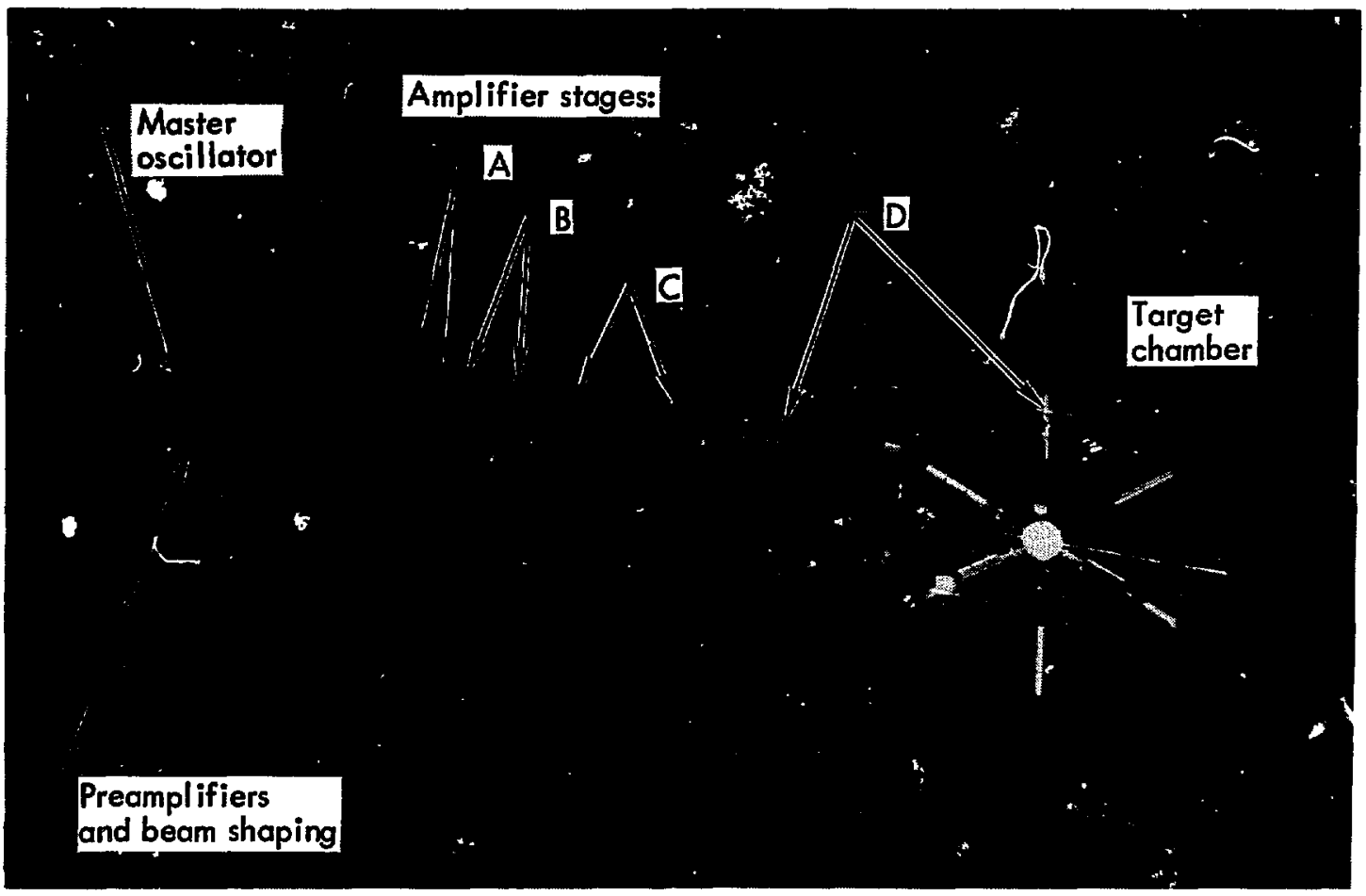

Fig. 1. Scle model of a conceptual design of the 12-chain 10-kJ laser array uniformly irradiating a target. In this model, there are two amplifier modules in each stage of each 1-kJ chrin. 
FY 73

FY 74 FY 75

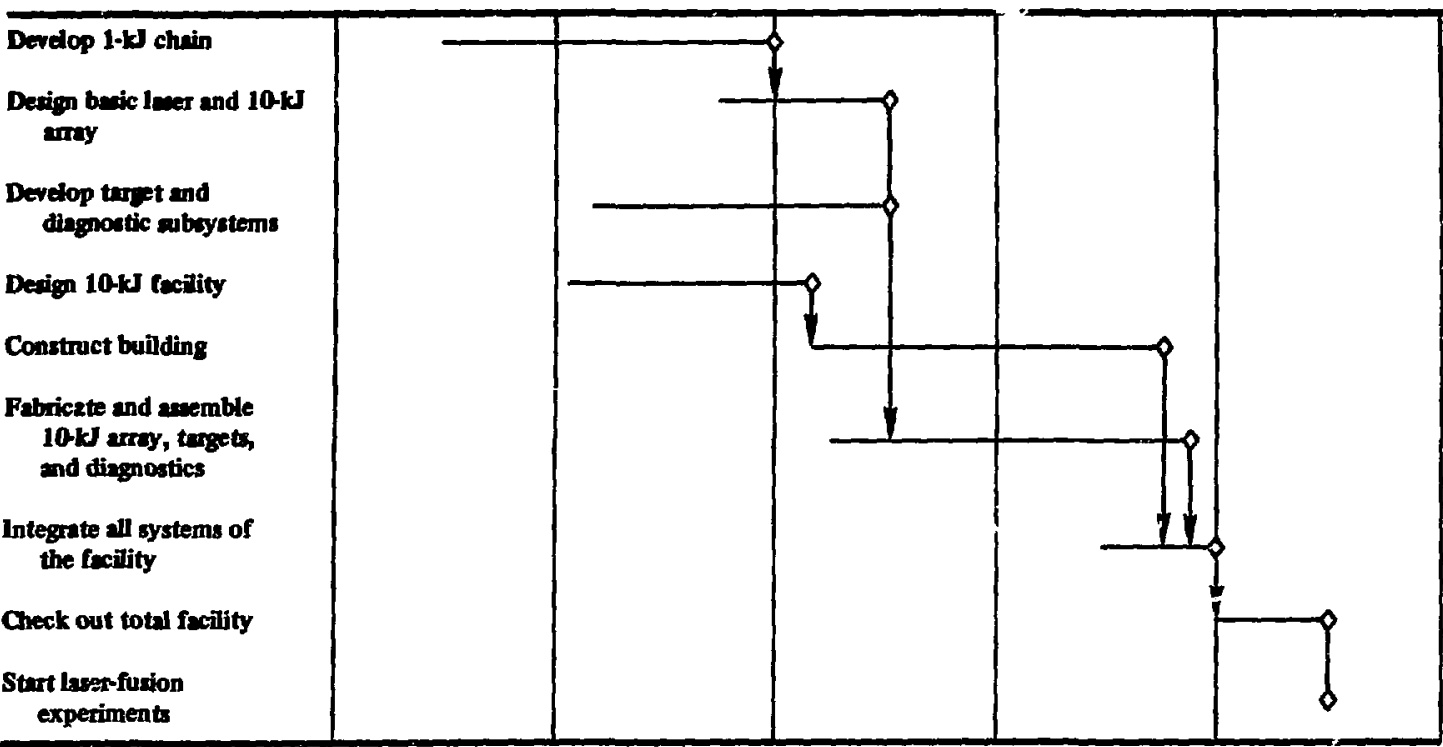

for the 10-kJ laser array will be completed, and fabrication will be started; the array will be assembied by the end of FY 1976.

The building will be designed in FY 1974, and construction will start in FY 1975 and be completed in FY 1976.

The target and diagnoatic subsystems will be developed in FY 1974 and 1975; fabrication and assembly will proceed in FY 1975 and 1976.

The laser array, the target and diagnostic subsystems, and the building will be integrated by the end of FY 1976.

The total system will be thoroughly checked out during the first half of FY 1977.

Barring slips in this schedule, the laser-fusion experiments should start in mid.FY 1977.

We are now in the midst of the $1-\mathrm{kJ}$-chain developmental program. Our major accomplishments during the second half of FY 1973 are summarized here and then described under separate headings below.

- Chain design. The design of the developmental chain was completed. The main features of the design are: consideration of the limit on the operating flux in order to control self-focusing; provision for complete isolation from maximum target returns possible in both polarizations; and modularization and commonality of chain components. A block diagram of the chain design for a 1-kJ, 100-psec output is shown in Fig. 2. The oscillator output of about $1.5 \mathrm{~mJ}$ is preamplified and spatially shaped, and then sent through disk amplifier stages denoted as $A$ through $D$. The clear apertures of stages $A, B, C$, and $D$ are 3.5, 8.5 , $\sim 20$, and $\sim 30 \mathrm{~cm}$, respectively.

Hardware for the prototype chain through stage $B$ is being built. Stages $C$ and $D$ are being designed, and the hardware will be built in the next six months. All of the hardware for the prototype chain should be completed by April 1974. Initial assembly dates for each of the amplifier stages are shown in Table 4.

- Amplifier development. Optimization studies on amplifier gain, pumping efficiency, and engineering details of the design of disk amplifiers were started using the DATUM 1 test unit. DATUM 1 incorporates

Table 4. Initial assembly dates for the prototype hardware of the 1-kJ chain

\begin{tabular}{lrl} 
Stage & $\begin{array}{c}\text { Tots } \\
\text { energy } \\
(J)\end{array}$ & \multicolumn{1}{c}{$\begin{array}{c}\text { Assembly } \\
\text { date }\end{array}$} \\
\hline$A$ & 5 & Sept. 1, 1973 \\
$B$ & 50 & Oct. 1, 1973 \\
$C$ & 250 & Jan. 1, 1974 \\
$D$ & 1000 & Apr. 15, 1974 \\
\hline
\end{tabular}




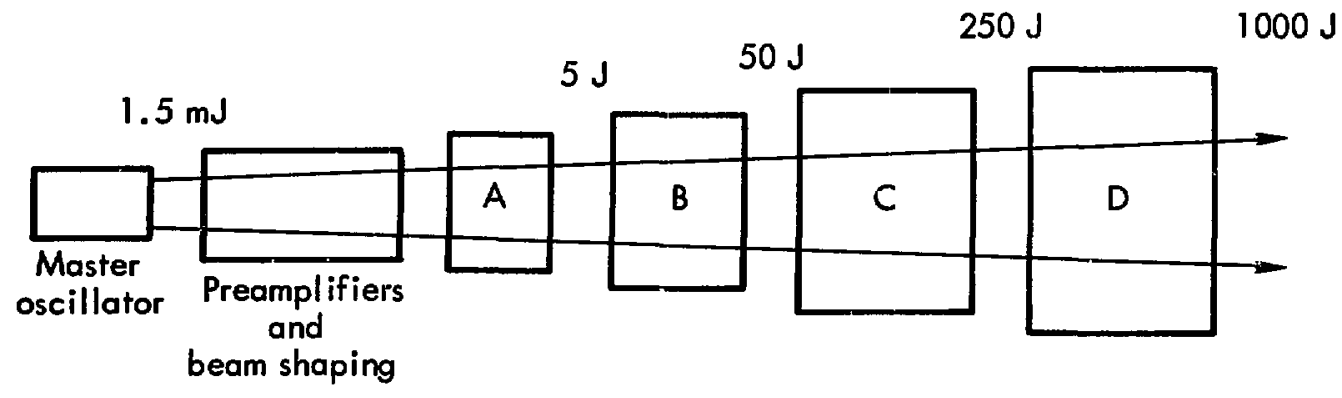

Fig. 2. Block diagram of the Nd:ghass chein for a 1-kJ, 100-psec output. $A, B, C$, and $D$ are disk amplifier stages with clear apertures of $3.5,8.5, \sim 20$, and $\sim 30 \mathrm{~cm}$, respectively.

eight $7-\times 14 \times 2-\mathrm{cm}$ disks and is designed to produce a small-signal gain of about 5 .

Parasitic suppression in large disks was investigated using both DATUM I and DATUM II, a test unit that allows large disks to be pumped to values of $\alpha D$ in excess of 2.5, the design value for the $C$ - and $D$-stage disks. Here, $\alpha$ is the small-signal gain coefficient and $D$ is the maximum physical dimension (the major axis) of the elliptical disks. The energy storage in a large disk is limited by the total-intemal-reflection (TIR) mode propagating along the major axis of the disk and reflecting back from the disk edge. Significantly, a samarium-copper cladding has been developed that will permit an $\alpha D$ value of 2.1 . This is more than adequate for the $A$ - and $B$-stage disks. This particular cladding has also been found to be more thermomechanically stable than black solder-glass cladding under intense flashlamp irradiation of up to $13 \mathrm{~J} / \mathrm{cm}^{2}$. This new Sm-Cu cladding has a higher transmission for the pump light while at the same time exhibiting sufficient selective absorption at $1.06 \mu \mathrm{m}$. Work on developing a cladding for the $C$ and $D$ disks is continuing.

The $A$ and $B$ disk-amplifier designs were completed. Particular attention was given to achieving the required overall gain (3.2) while minimizing the pump energy required and, more significantly, while minimizing the total glass thickness along the propagation direction. The latter is important in considerations of self-focusing.

- Beam-propagation studies. The Intermediate Laser System (ILS), ${ }^{2}$ a high-power Nd:glass laser test bed, now generates an energetic, high-quality, large-diameter beam for studies of beam propagation and material characterization. This laser delivers a $0.7-\mathrm{cm}$-diam (at $1 / e), 5-\mathrm{J} / \mathrm{cm}^{2}, 150$-psec pulse with less than $2 \%$ spatial and temporal ripple. Self-focusing and two-photon absorption effects in Owens-Illinois ED-2 glass have been investigated with this beam.

- Oscillator development. The first-generation master oscillator for the $1-\mathrm{kJ}$ developmental chain has been designed and is now being built. This passively dye-mode-locked oscillator has been designed for maximum output reliability and reproducibility by emphasizing the thermomechanical stability of the resonator support structure and resonator components. Particular attention has been given to precise thermal control of the dye solution and the intracavity etalon. Tests using a brassboard of the design indicate that the output energy will be about $1 \mathrm{~mJ}$, and that bandwidth-limited 100-psec pulses with smooth Gaussian temporal, spectral, and spatial piofiles will be obtained. By varying the thickness of the intracavity etalon, the pulse width can be varied from $30 \mathrm{psec}$ to 1 nsec.

In our investigation of an oscillator design that uses a synchronously driven Pockels cell instead of a dye cell for generating short pulses, the difficulty of reducing the pulse width to $\sim 100$ psec by reducing the response time of the Pockels cell to 100-200 psec remains.

- New facility for the 1-kJ chain. The 1-kJ developmental chain will be housed in the new laboratory facility, Building $174 . .^{3} \mathrm{~A}$ plan view of the part of this building in which the laser will be installed is shown in Fig. 3. This modem facility provides the control of dust, humidity, and temperature that is necessary for high-power laser systems. The laser facility consists of a $30-\mathrm{ft}$-wide by 180 -ft-long main bay; a control room, which will also house the PDP-8 computer control system; and approximately $4 \mathrm{MJ}$ of energy storage, consisting of capacitor banks and power supplies. 
The chain through stage $B$, occupying the first 60-ft-long wing (see Fig. 3), will be completed by September 1973 . The $0.7-\mathrm{MJ}$ energy-storage module is already installed and will be ready when needed, Stage $C$ and the 1.1-MJ module required to pump it will be assembled by December. The 60-ft-long by 70-ft-wide addition indicated by the dashed lines is also scheduled for completion by this December; as shown in Fig. 3, this section will house the last half of stage $D, 2.2 \mathrm{MJ}$ of energy storage for the $D$ modules, and diagnostics for the full-output beam.

Fig. 3. Plan view of the new lawer facility (Building 174) and installation of the $1-k J$ chnin.

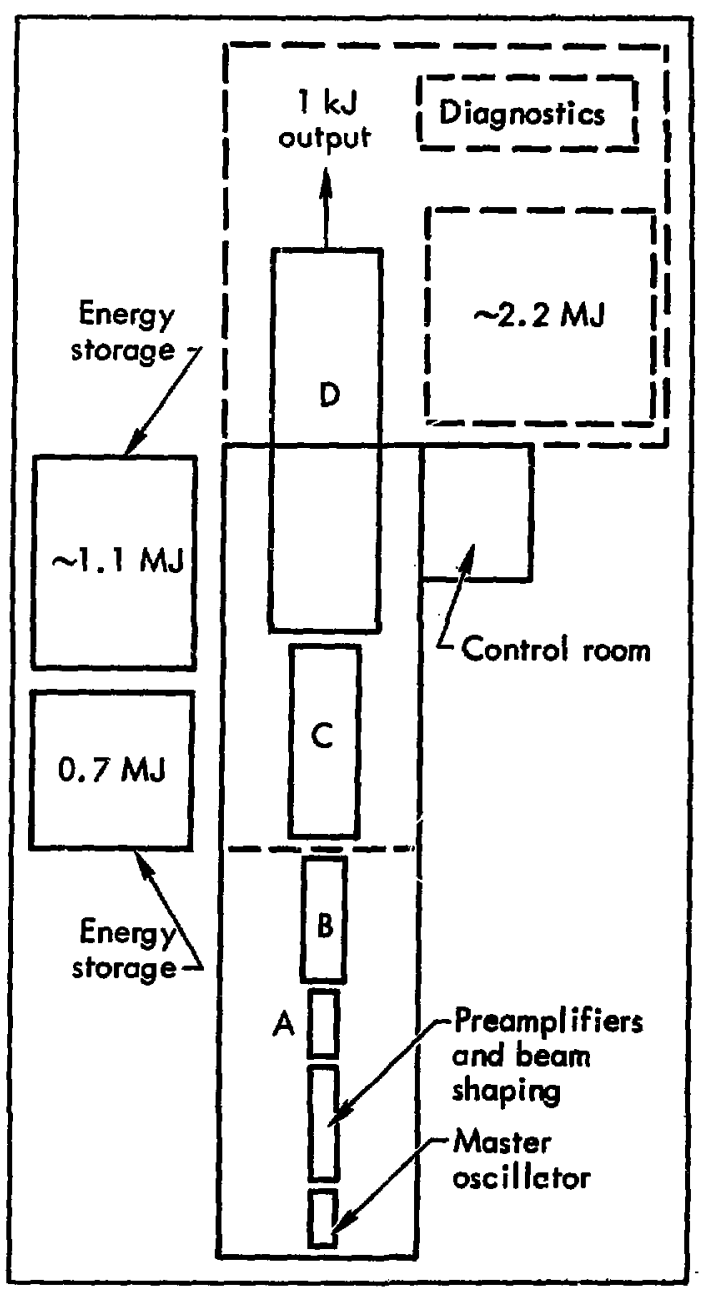

\section{PARASITIC SUPPRESSION IN LARGE-APERTURE Nd:GLASS DISKS}

\section{Introduction}

The threshold for parasitic oscillation sets an upper limit on the energy storage attainable in large-aperture Nd:glass laser disks. In extreme cases, for example, nearly lossless parasitic modes can reduce the population inversion to very small values over a substantial portion of the disk volume. This has been observed in early work on the NRL disk amplifier ${ }^{4}$ and has been substantiated in work described here.

Trenholme ${ }^{5}$ has analyzed the problem of parasitic oscillation for the special case of uniform energy storage throughout the volume of the disk. In this work he has shown that the lowest threshold mode of oscillation is one in which a ray undergoes a number of lossless reflections at the disk surface and, in the case of a cladded disk, a lossy reflection at the disk edge. The threshold condition for this mode, as given by Trenholme, can be simply expressed as

$$
R e^{n \alpha D}=1
$$

where $R$ is the reflection coefficient at the disk edge, $n$ is the ratio of the index of refraction of glass to that of air, $D$ is the major axis, and $\alpha$ is the small-signal gain coefficient.

In practice, the gain is not distributed uniformly throughout the disk but is highest at the face and lowest at the center. As a consequence, if the pumping 


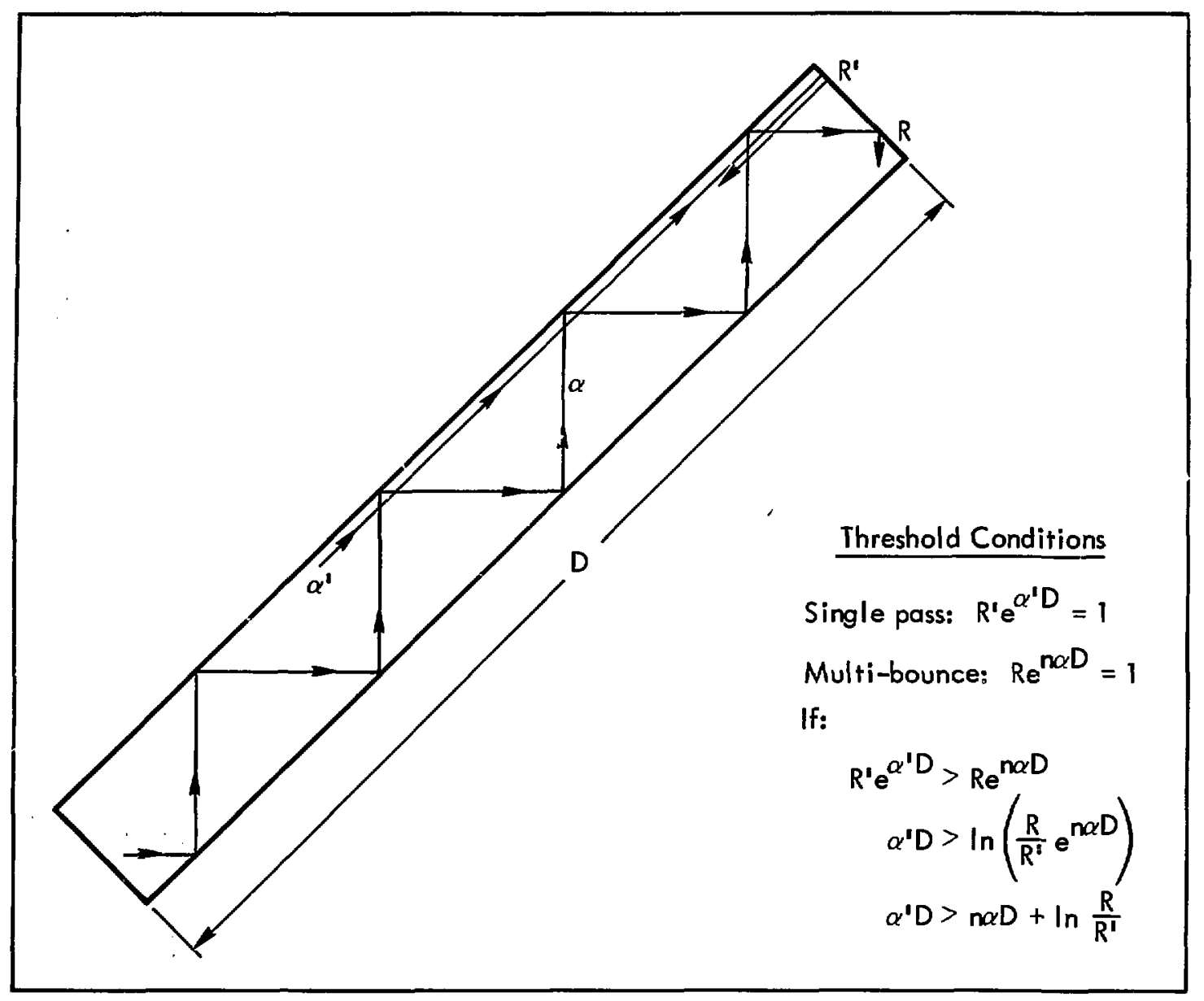

Fig. 4. Two possible modes of parasitic oscillation. See text, Eqs. (1) and (2), for definitions of tems.

is large at the face, the net gain in a single pass across the face may exceed that of the bounce-across path (Fig. 4). This will be the case if the following inequality is satisfied:

$$
\alpha D>n \alpha D+\ln \frac{R}{R^{\prime}}
$$

where $\alpha^{\prime}$ is the gain coefficient at the disk face, $\alpha$ is now interpreted as an average gain coefficient, and $R^{\prime}$ is the reflectivity for normal incidence at the disk edge. For our present amplifier designs, this inequality is not satisfied, and so we will be concerned only with the mode described by Eq. (1).

\section{Glass Cladding}

Black solder glasses such as Hoya BSDL-3 and Owens-Illinois G-9000 are commonly used as disk cladding materials. With such coatings, parasitic thresholds are determined largely by the index mismatch at the interface between the cladding and the substrate. For smaller disks such as those proposed for our $A$ and $B$ modules, the primary objection to the use of such cladding material is the poor thermal-mechanical properties it exhibits under intense flashlamp illumination. In brief, we have observed catastrophic mechanical failure of the G-9000 coating following a single irradiation at $13 \mathrm{~J} / \mathrm{cm}^{2}$. Gradual degradation is anticipated at lower illumination levels.

In an effort to produce a cladding material with a high damage threshold, Owens-Illinois has developed a solder-glass coating which exhibits reduced absorption over the flashlamp spectrum and at $1.06 \mu \mathrm{m}$ as well. This coating consists of the same basic solder glass used for the black coating, but it has been doped with copper and samarium for selective absorption at $1.06 \mu \mathrm{m}$. To date, this coating has 
shown excellent resistance to damage. In particular, it has withstood many irradiations at $13 \mathrm{~J} / \mathrm{cm}^{2}$. We are currently evaluating it for use on our $A$-and $B$-module disks.

In Trenholme's calculations ${ }^{5}$ it is assumed that there is no return of that portion of the optical wave propagating through the substrate/cladding interface. Since this is not the case for a partially transmitting cladding, it becomes necessary to extenc the calculations to account for a finite return from the cladding/air interface.

Figure 5 is a schematic of the ray geometry required for the calzulation. The figure shows that the total return from the disk edge is composed of a partial reflection at the substrate/cladding interface and a total reflection at the cladding/air interface. Denoting this return as $R$, we have

$$
R=R_{1}+R_{2}
$$

where

$$
R_{1}=\left[\frac{\sin \left(\theta_{2}-\theta_{3}\right)}{\sin \left(\theta_{2}+\theta_{3}\right)}\right]^{2} \text {, }
$$

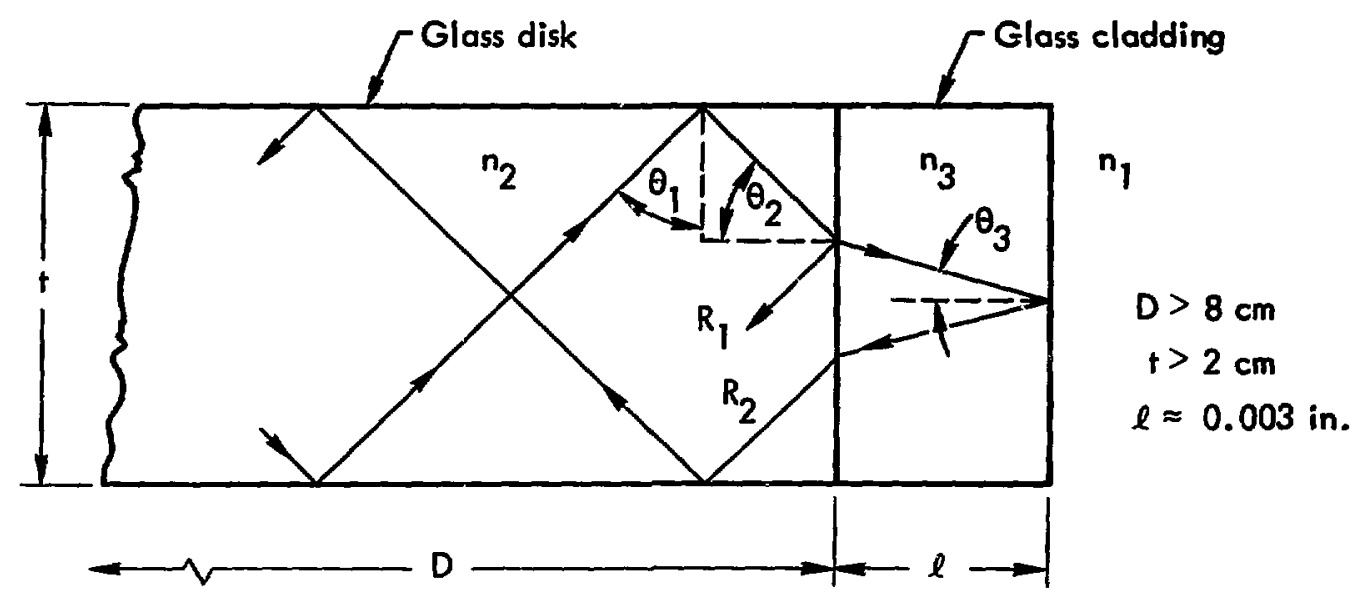

$\theta_{1}=\sin ^{-1} \frac{n_{1}}{n_{2}}$ (TIR angle at substrate/air interface)

$\theta_{2}=90^{\circ}-\theta_{1}$

$\theta_{3}=\sin ^{-1} \frac{n_{2}}{n_{3}}\left[1-\left(\frac{n_{1}}{n_{2}}\right)^{2}\right]^{1 / 2}$
$\theta=\sin ^{-1} \frac{n_{1}}{n_{3}}$ (TIR angle at cladding/air interface)

$\theta_{3} \geq 0$ for $n_{2} \geq \sqrt{2} n_{1}$

Fig. 5. Schematic of the ray geometry used in calculating the parasitic threshold condition for a disk with a partially transmitting edge coating. 
and

$$
R_{2} \approx \exp \left(-2 \beta 2 \sec \theta_{3}\right)
$$

In Eq. (4), $\beta$ is the absorption coefficient of the cladding at $1.06 \mu \mathrm{m}$, and $l$ is the thickness of the cladding. It is convenient to write $R_{2}$ in terms of the single-pass transmission, $T$, of the coating:

$$
R_{2}=\tau^{\left(2 \sec \theta_{3}\right)} \text {. }
$$

With the aid of Fig. 4, the trigonometric functions appearing in the above equations can be expressed in terms of the indices of refraction to obtain

$$
\begin{gathered}
F:=\left[\frac{\left(n_{1}^{2} \cdot n_{2}^{2}+n_{3}^{2}\right)^{1 / 2} \cdot n_{1}}{\left(n_{1}^{2}-n_{2}^{2}+n_{3}^{2}\right)^{1 / 2}+n_{1}}\right]^{2} \\
+T^{2 n_{3} /\left(n_{1}^{2}-n_{2}^{2}+n_{3}^{2}\right)^{1 / 2}},
\end{gathered}
$$

where $n_{1}, n_{2}$, and $n_{3}$ are the indices of refraction for air, glass, and cladding, respectively.

The threshold condition can now be expressed as

$$
\begin{aligned}
(\alpha D)_{c r i t}=-\frac{n_{1}}{n_{2}} \ln \{ & {\left[\frac{\left(n_{1}^{2} \cdot n_{2}^{2}+n_{3}^{2}\right)^{1 / 2}-n_{1}}{\left(n_{1}^{2}-n_{2}^{2}+n_{3}^{2}\right)^{1 / 2}+n_{1}}\right]^{2} } \\
& \left.+T^{2 n_{3} /\left(n_{1}^{2}-n_{2}^{2}+n_{3}^{2}\right)^{1 / 2}}\right\}
\end{aligned}
$$

Figure 6 shows a plot of $(\alpha D)_{\text {crit }}$ versus single-pass transmission $T$. The maximum transmission permitted for the $A, B, C$, and $D$ amplifier modules is also indicated. The present coating obtained from Owens-lllinois has a measured transmission of about $25 \%$. This coating is currently being applied to a set of $A$ disks; we anticipate that it will also be used on the $B$ disks. Future development of glass claddings will be directed toward achieving transmissions compatible with our $C$ and $D$ disk designs.

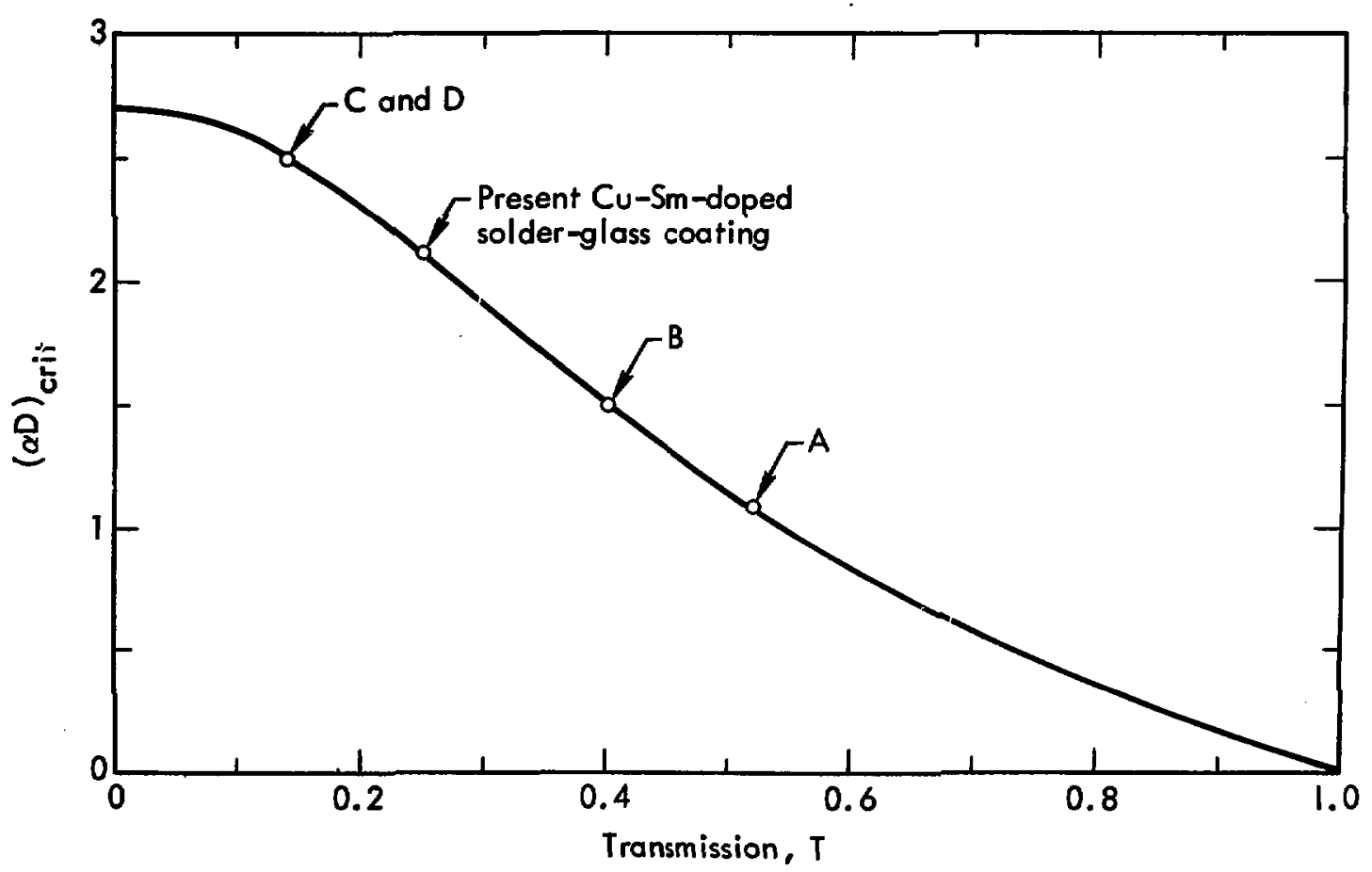

Fig. 6. Critical poin coefficient as a function of single-pass transmission of the edge conting at $1.06 \mu \mathrm{m}$. 


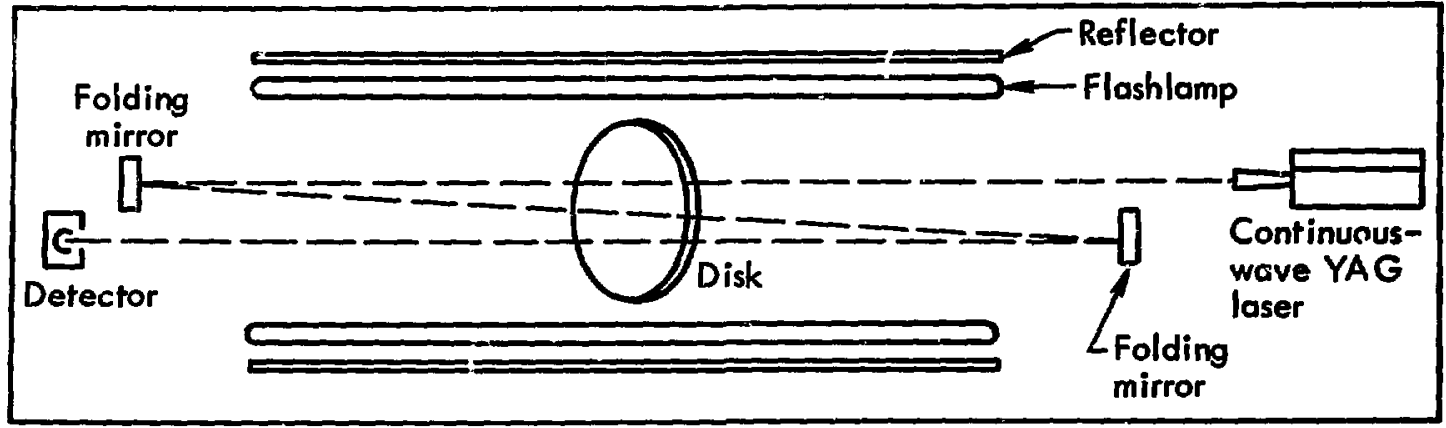

Fig. 7. Apparatus for small-signal gain meusurements using a single disk at the center of the DATUM I amplifier.

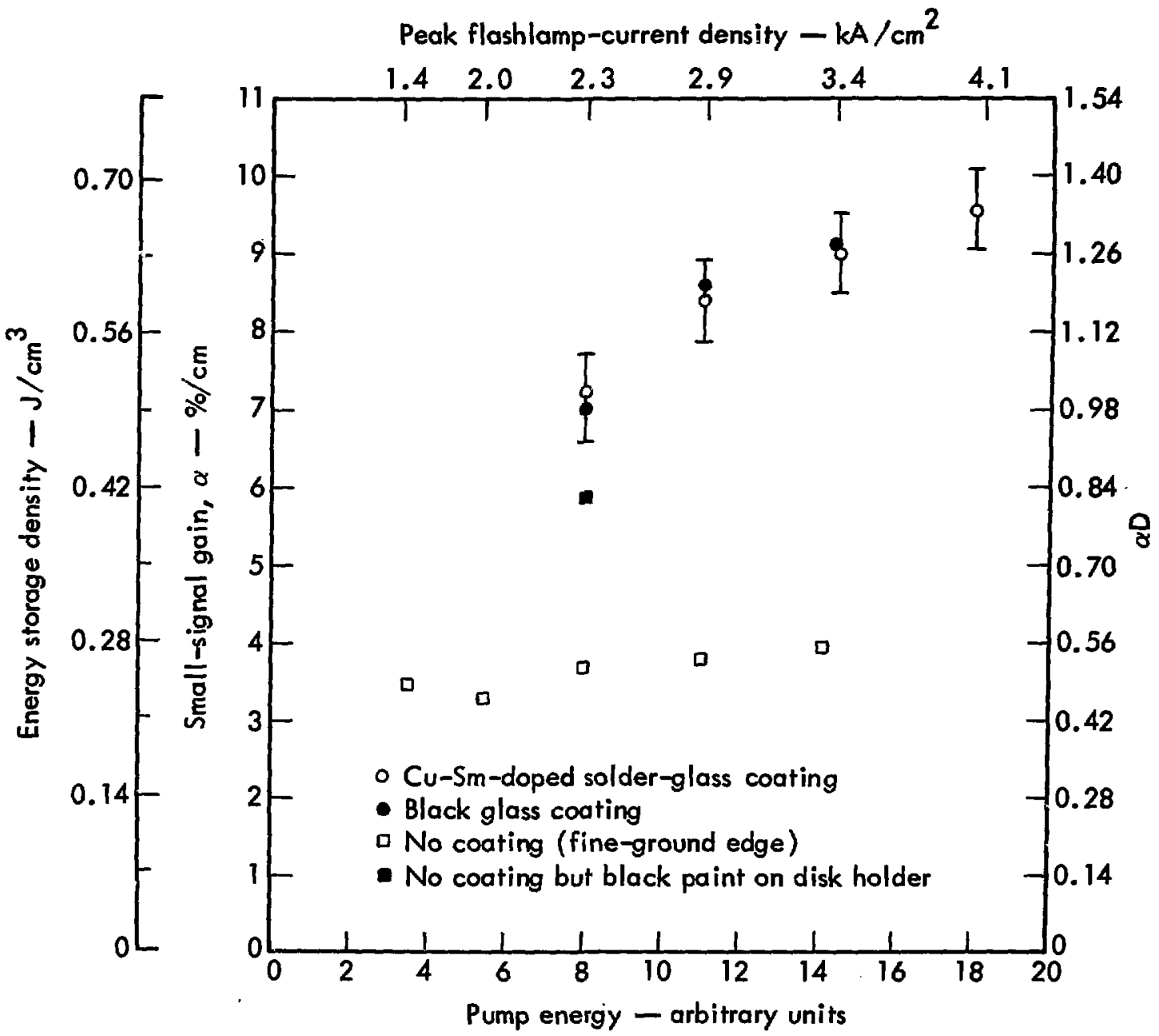

Fig. 8. Measurements of the gin and energy-storago density in gass disks with various coge treatments. 


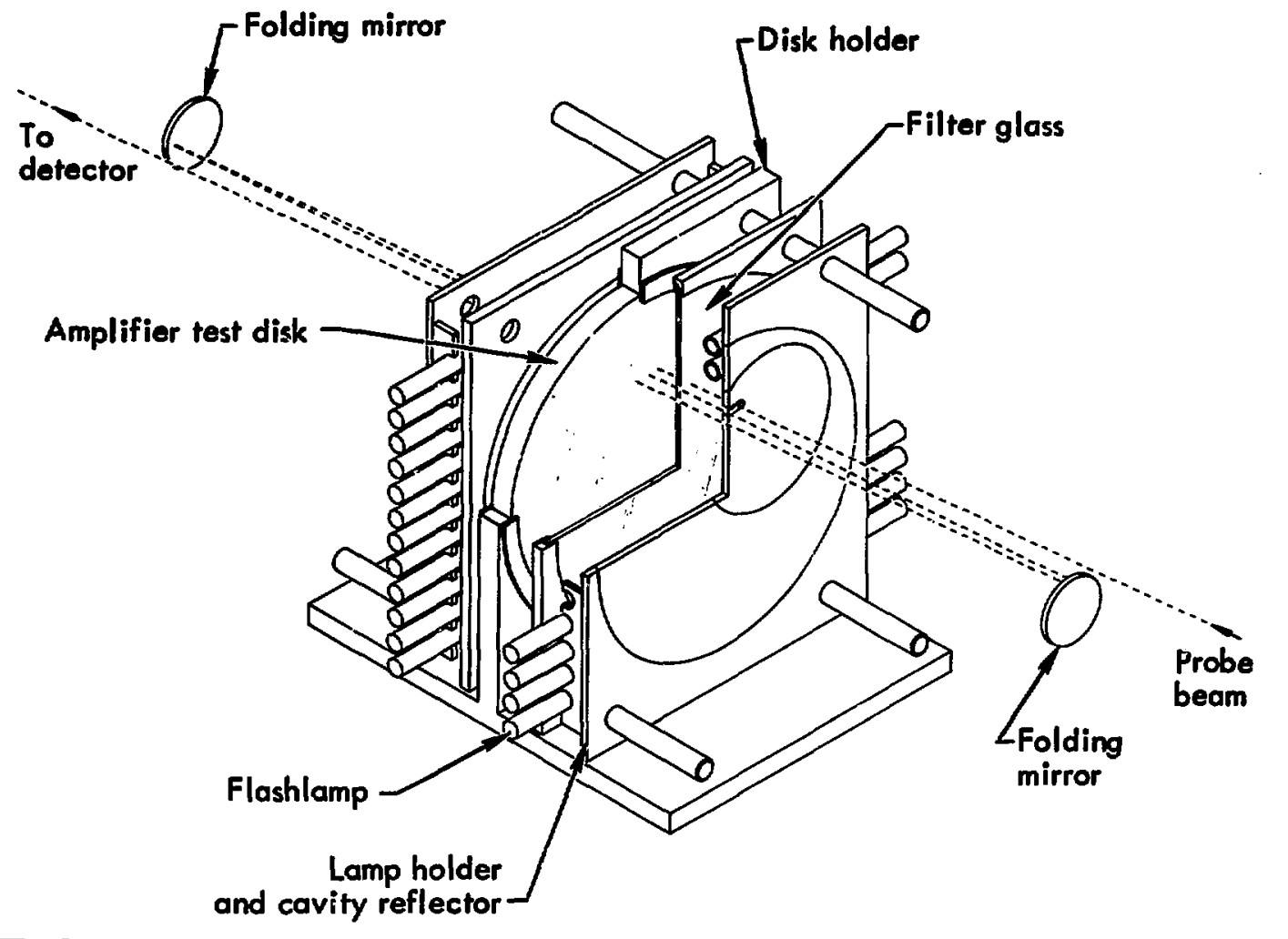

Fig. 9. Schematic of the aetup usad for gein mensurements with DATUM II.

\section{Energy Storage of Glass Disks}

We are currenily evaluating the energy-storage capability of ED-2 laser disks. Two amplifier modules have been used in this evaluation: DATUM I and DATUM II (DATUM is an acronym for disk-amplifier test-unit module).

DATUM I is an eight-disk amplifier similar in design to that used by NRL. (The details of the development of DATUM I appear in the following article.) Figure 7 is a schematic of how this amplifier was used in a multiple-pass small-signal gain measurement. Briefly, a single elliptical glass disk $(7 \mathrm{~cm}$ by $14 \mathrm{~cm})$ was positioned at the center of the module. The ouput beam from a continuous-wave Nd:YAG oscillator operating at $1.06 \mu \mathrm{m}$ was folded twice through the disk and then detected with a photodiode.

Figure 8 shows the results of gain measurements for both cladded and uncladded disks. An energy storage of about $0.7 \mathrm{~J} / \mathrm{cm}^{3}$ at an $\alpha D$ of 1.36 was achieved in the disk coated with Cu-Sm glass. Limitations of our energy-delivery system precluded the extension of the data to larger values. Similar results were, obtained for the disk with the black cladding; we did not, however, pump this disk quite as hard because of recurrent problems with the energy-storage system. It is clear from the data that the Cu-Sm-doped glass cladding is sufficient to prevent self-oscillation of the $A$-size disks $(\alpha D=1.1)$. The data do not allow us to make a definitive judgment concerning the use of $\mathrm{Cu}-\mathrm{Sm}$ glass on the $B$-size disks, which require an $\alpha D$ of 1.5. Optical transmission measurements, as previously noted (Fig. 6), suggest that this coating will work on the $B$ disks.

The flattening of the gain curves at the larger values of peak current density is tentatively attributed to spectral shifting (blue shift) of the flashlamp output. Additional support for this view has been obtained from fluorescenc $:$ measurements currently in progress.

The low energy storage achieved in the uncladded disks results from the very rapid onset of parasitic oscillation. It is also observed that blackening the disk holder increases the storage significantly. 


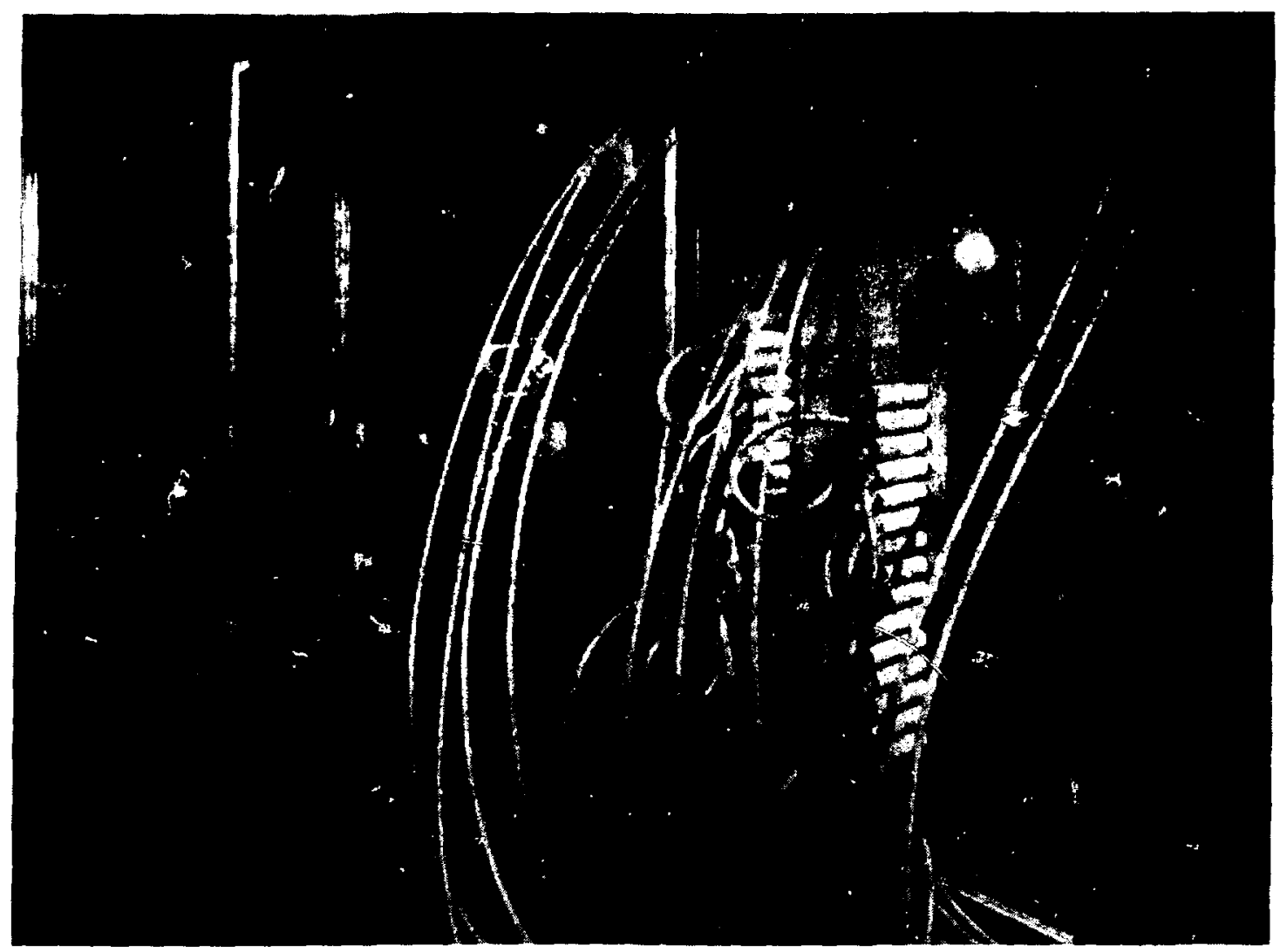

Fig. 10. DATUM II setup.

The second amplifier module, DATUM II, has been designed to accommodate single disks $u_{\Gamma}^{-}$to $12 \mathrm{in}$. in diameter. The purpose of this module is to pump glass disks to values of $\alpha D$ in excess of about 2.5. Our current $C$ and $D$ amplifier designs are predicated upon achieving such values. Figures 9 and 10 show a schematic and a photograph of DATUM il. Basically, it consists of two vertical flashlamp banks whose positions relative to the face of the disk can be adjusted. Each bank contains 16 flashlamps. The lamps are 14 in. long and $1 \mathrm{~cm}$ in i.d. At present, up to $135 \mathrm{~kJ}$ can be delivered to the banks in about $550 \mu \mathrm{sec}$. Gain measurements are made by folding a continuous-wave probe beam several times through the disk.

Preliminary attempts at gain measurements using DATUM II have revealed strong shock waves propagating throughout the module enclosure. These waves apparently arise as a result of uv absorption by residual $\mathrm{O}_{2}$. Our $\mathrm{N}_{2}$ purging system is being improved; however, even at $\mathrm{O}_{2}$ concentrations as low as $1 \%$, some shock waves are formed. The presence of the shock waves is manifested by strong and seemingly random directional modulation of the probe beam. It is hoped that an evacuated window cell positioned between the lamps will reduce this beam deflection.

\section{AMPLIFIER DEVELOPMENT}

\section{Introduction}

The detailed design of the $1-\mathrm{kJ}$ amplifier system is governed by the many constraints imposed by target interaction effects, self-focusing limits, and minimum cost considerations. (These considerations are discussed in detail under "Design Analysis.") The results of a detailed analysis of the main amplifier system lead to the following system description: An overall gain of 
$10^{4}$ is required to amplify $0.1 \mathrm{~J}$ to $1 \mathrm{~kJ}$. Each amplifier stage will have a maximum small-signal gain of $\mathbf{3 . 8 5}$ in order to provide complete isolation from target back-reflection. For a 100-psec pulse width, the maximum flux densit; at the output of each stage is $1 \mathrm{~J} / \mathrm{cm}^{2}$, except for the final stage, which will run at $2 \mathrm{~J} / \mathrm{cm}^{2}$. To provide commonality, a maximum of four aperture sizes will be used $-3.5,8.5,20$, and $30 \mathrm{~cm}$ in diameter.

\section{Experimental Program}

The goal of the experimental program is to accumulate a data base for amplifier design. The data base that is presently available for checking the analytical design tools is not as accurate or extensive as we require. Therefore, the major part of our experimental effort has been directed toward improving this situation. This effort includes studies of flashlamp model verification, laser glass pumping dynamics, and the suppression of parasitic oscillation in large glass disks with edge coatings. We are also evaluating disk-amplifier performance, including measurements of pumping uniformity, pumping efficiency, phase-front distortion, and self-focusing.

Two test devices have been constructed specifically to provide experimental data for amplifier development. They are an experimental disk amplifier, DATUM I, and a large-aperture-disk excitation cavity, DATUM II. (DATUM II is described in the previous article.) DATUM I is designed to test mechanical, electrical, and optical engineering concepts. It is being used to provide detailed measurements of absolute efficiencies, gain uniformity, phase front distortion, etc. The apparatus is shown in Fig. 11.

The amplifier uses elliptical disks of 3\%-doped ED-2 glass, $7 \mathrm{~cm}$ by $14 \mathrm{~cm}$ by $2 \mathrm{~cm}$ thick. (This is the same disk design as that used at NRL. ${ }^{4}$ ) Special mechanical features include the disk-support method, which uses two rails to hold the disks and allows independent removal of the flashlamps and reflector modules. The

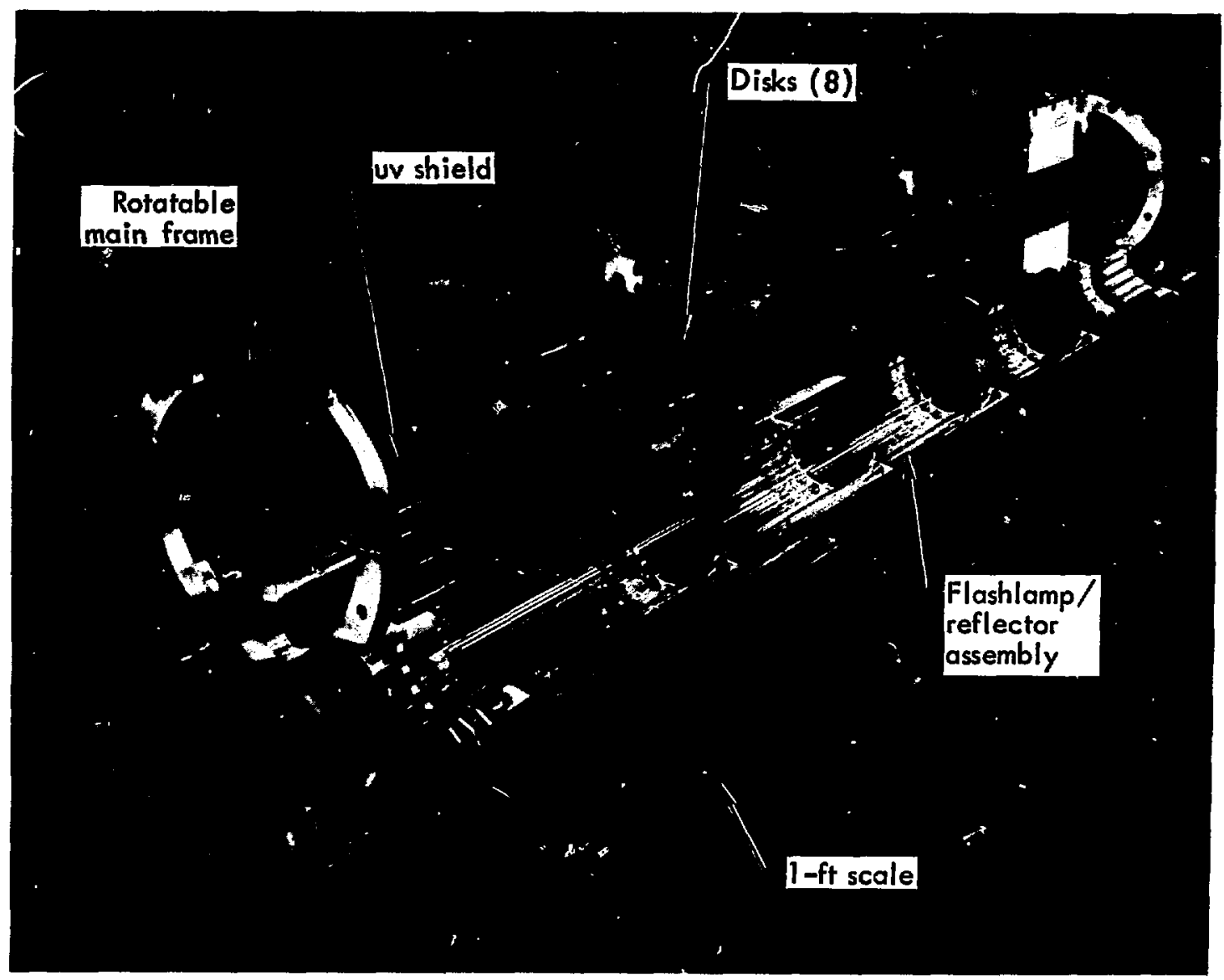

Fig. 11. Side view of the DATUM I prototy pe disk amplifier with flashiamp reflectors in the open position. 
disks are protected from the ultraviolet flashlamp output by filter-glass shields. These shields also protect the disk surfaces from contamination by organic material and dust particles. Contamination of the laser modules by photodegraded organic materials and by dust particles is a recurring problem which can be solved only by eliminating all organic materials from inside the laser head and by sealing the laser disks so that no foreign matter can reach them. Methods of fabricating disk-laser structures are being further investigated with the design of the $A$ and $B$ modules.

The electrical and optical performance of DATUM I is being evaluated. Electrically the amplifier appears to operate satisfactorily. Some electrical storage problems have been identified and are being resolved. Flashlamp reliability has not been a problem in this laser head, although a detailed long-term study of flashlamp reliability remains to be conducted.

The amplifier gain that we have measured confirms the code predictions, which are normalized to the NRL disk-laser data. ${ }^{4}$ We have measured energy storage in the glass of $\sim 0.6 \mathrm{~J} / \mathrm{cm}^{3}$, although at very high flashlamp energies and current densities the energy storage appears to roll off more rapidly than expected. Detailed optical measurements on DATUM I are in progress.

\section{Amplifier Design}

The objective of the design program is to understand the trade-offs involved in selecting competing designs for laser amplifiers. This study requires extensive use of computer-aided design techniques and reliable experimental data. (The analytical techniques that we use for system design are discussed in detail under "Design Analysis.") Codes are available that provide information on flashlamp spectral output as a function of electrical input (GENEFF), light distribution and pumping in arbitrary geometries (ZAP), flashlamp driving-circuit optimization, and laser-beam propagation and amplification. These codes are used to model the performance of contemplated amplifier designs, which allows us to analyze many possibilities without actually constructing the amplifiers.

These design techniques have been applied to the problem of selecting disks for the anıplifier stages. Disk selection is governed primarily by the nunimum cost calculations presented in the Design Analysis section of this report. These calculations define the aperture size and gain of each stage in the $1-\mathrm{kJ}$ laser amplifier chain. Four modules with increasing aperture size $(A$, $B, C$, and $D$ ) have been chosen. Once the aperture size is chosen, the major and minor axes of a disk are approximately fixed by the requirement of setting the disk at Brewster's angle. We then have to select the thickness of the disk and neodymium doping content of the glass. These two parameters are chosen so as to minimize the thickness of glass in the laser beam to reduce self-focusing. Two constraints, energy storage and parasitic oscillation, affect this selection.

To prevent self-focusing, the ideal disk would have a thickness of zero; however, very thin disks could not absorb enough flashlamp radiation to provide the required module gain $(\sim 3.85)$. To estimate the minimum disk thickness and Nd doping, we consider a disk-laser environment. Within the cavity, the disk surfaces receive froti the lamps a fixed total energy flux per unit surface area. The number of $\mathrm{Nd}$ ions in the disk determines the fraction of incident flashlamp energy that is turned into stored laser energy - more Nd ions imply that a larger fraction of flashlamp energy is absorbed. The stored energy, however, is reduced because of $\mathrm{Nd}$ fluorescence. Since the fluorescence lifetime of $\mathrm{Nd}^{3+}$ in the laser-glass host decreases with the Nd density (310 $\mu \mathrm{sec}$ at $3 \%$, $147 \mu \mathrm{sec}$ at $6 \%$ ), a very high Nd concentration will lead to a fast fluorescence decay, reducing the stored energy and gain. As a consequency we cannot raise the Nd concentration to an arbitrarily high level.

The second constraint is imposed by the parasitic-oscillation threshold within the individual disk (see the previous article). The gain $\alpha\left(\mathrm{cm}^{-1}\right)$ in a disk with a given major diameter $d$ is bounded by a maximum value, which we presently believe is given by $\alpha_{\max }=2.5 / d \mathrm{~cm}^{-1}$. In addition, we desire to select the doping so that the pumping nonuniformity through the disk is restricted to a level such that the peak face gain divided by the average through-disk gain is 1.5 . This reduces the possibility of a parasitic face oscillation. Such a pump profile is found to occur when the optical thickness (disk thickness in centimeters times the percent Nd doping) is no greater than $4.2 \mathrm{~cm}-\%$.

The energy flux into the disks is determined by the electrical energy delivered to the flashlamp. However, flashlamp life is related to the rate of energy delivered. ${ }^{6}$ We determine the flashlamp pumpng conditions by specifying the desired flashlamp shot life. We then maximize the energy stored in a disk (and hence the gain) consistent with the selected shot-life constraint by varying the pulse duration (and hence, the lamp energy loading). This yields a curve of gain as a function of pulse duration for a given Nd concentration and disk thickness. We obtain a family of such curves by varying concentration and thickness while keeping the optical thickness constant. An example of these calculations is shown in Fig. 12, for an optical thickness of $4 \mathrm{~cm}-\%$. The highest values of gain are obtained for the thickest disks because of the increased fluorescence lifetime at lower Nd 
Table 5. Possible choices for $A$-module disks ( 4.8 by $8.4 \mathrm{~cm}$ )

Number

of disks

Thickness (cm)

Nd doping (\%)

Optical thickness

(cm-\%)

Total glass path (cm)

1. No edge coating ( $30 \%$ rellectivity)

$\begin{array}{lll}5 & 3.2 & 2.8 \\ 6 & 2.1 & 2.4 \\ 7 & 1.75 & 2.3 \\ 8 & 1.55 & 2.6\end{array}$

2.8

2.4

2.6

$\begin{array}{ll}9 & 19 \\ 5 & 15 \\ 4 & 15 \\ 4 & 15\end{array}$

2. Minimal edge coating ( $10 \%$ reflectivity)

\begin{tabular}{lllll}
$6^{2}$ (selected) & 1.5 & 3.7 & 5.5 & 10.5 \\
7 & 1.3 & 3.1 & 4 & 11 \\
8 & 1.0 & 3.0 & 3 & 9.5 \\
\hline
\end{tabular}

${ }^{3}$ Needs more pumping $\left(2 \times 10^{4}\right.$ shot life $)$.

Table 6. Possible choices for $B$-module disks (10 by $18.4 \mathrm{c}$.n)

Number of disks

Thickness (cm)

Nd doping (\%)

Optical thickness

Totol glass path (cm-\%)

(cri)

1. Minimal edge coating (10\% reflectivity)

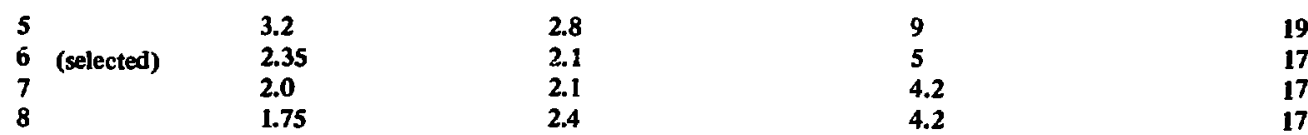

2. Good edg: coating ( $2 \%$ reflectivity)

\begin{tabular}{lllll}
$6^{\mathrm{a}}$ & 1.5 & 2.8 & 4.2 & 10.5 \\
7 & 1.3 & 3.1 & 4 & 11 \\
8 & 1.1 & 3.6 & 4 & 0.5 \\
\hline
\end{tabular}

$a_{\text {Needs more pumping. }}$

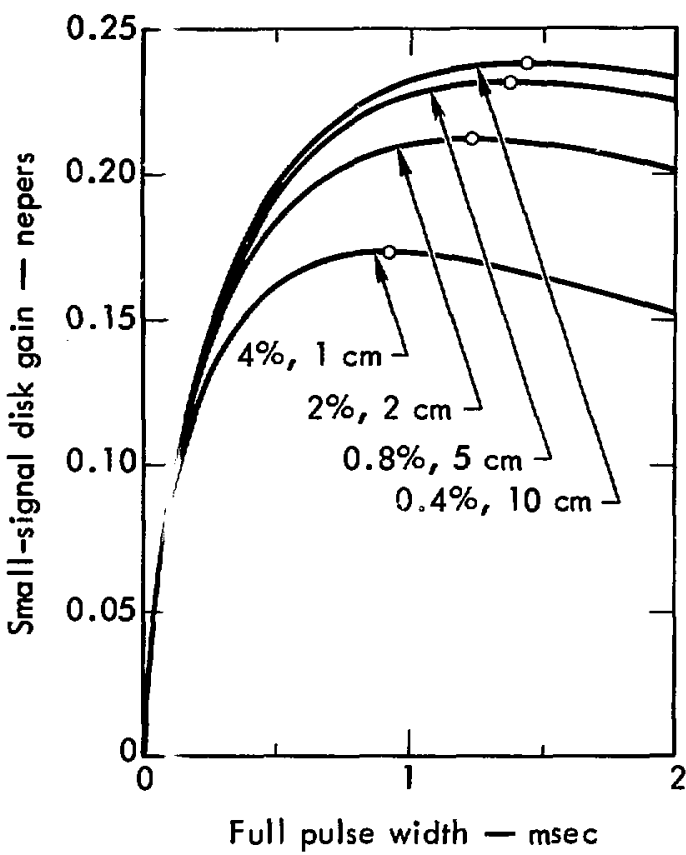

concentrations. For a given thickness and concentration, each curve peaks at a flashlamp discharge time $(=3 \sqrt{L C})$ of about three times the $\mathrm{Nd}$ fluoresc nce decay time for that concentration.

The peak gain value for each constant-opticalthickness curve of Fig. 12 is then plotted as a function of disk thickness. Thus each graph is displayed as a point on a locus of peak-gain points. Examples are shown in Figs. 13 and 14, which show the family of gain curves for various optical-thickness selections, consistent with a flashlamp life of $10^{5}$ shots. Note the upper limiting value on the family of gain-versus-thickness curves for the $10^{5}$-shot life. This is the maximum gain available from a disk laser pumped by a xenon flashlamp operated at the $10^{5}$-shot life expectancy. Also shown are similar

Fig. 12. Calculated small-signal gain as a function of pulse width for a disk amplifier. These calculations are for a flashlamp lifetime of $10^{5}$ shots and various $\mathrm{Nd}$ concentrations and disk thicknesses giving a constant optical thickness of $4 \mathrm{~cm}-\%$. 


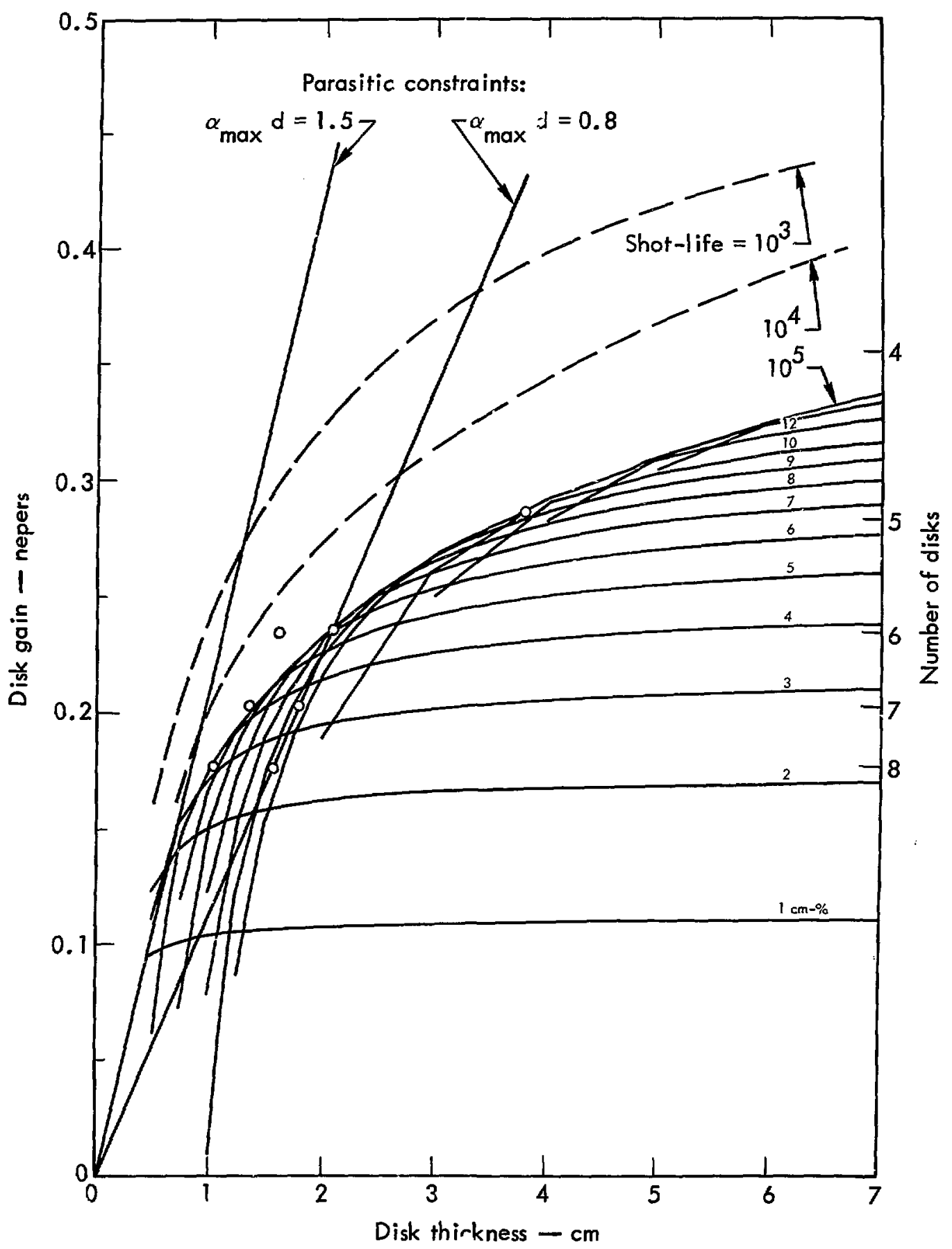

Fig. 13. Using data like those given in Fig. 12, one can draw plots of disk gain as a function of disk thickness. The lashlamplife curves represent the maximum obtainable gain for each life constraint $\left(10^{3}, 10^{4}\right.$, or $10^{5}$ shots $)$. Optical-thickness curves generating the $1 v^{5}$-shot curve are shown. The disk-gain scale on the left, together with the disk-count scale on the right, indicates the gain per disk required for a given number of disks to obtain a fixed module gain of 3.85. This plot is for the $A$ module; the dots indicate the possibilities that have been selected for comparison (see Table 5 ). 


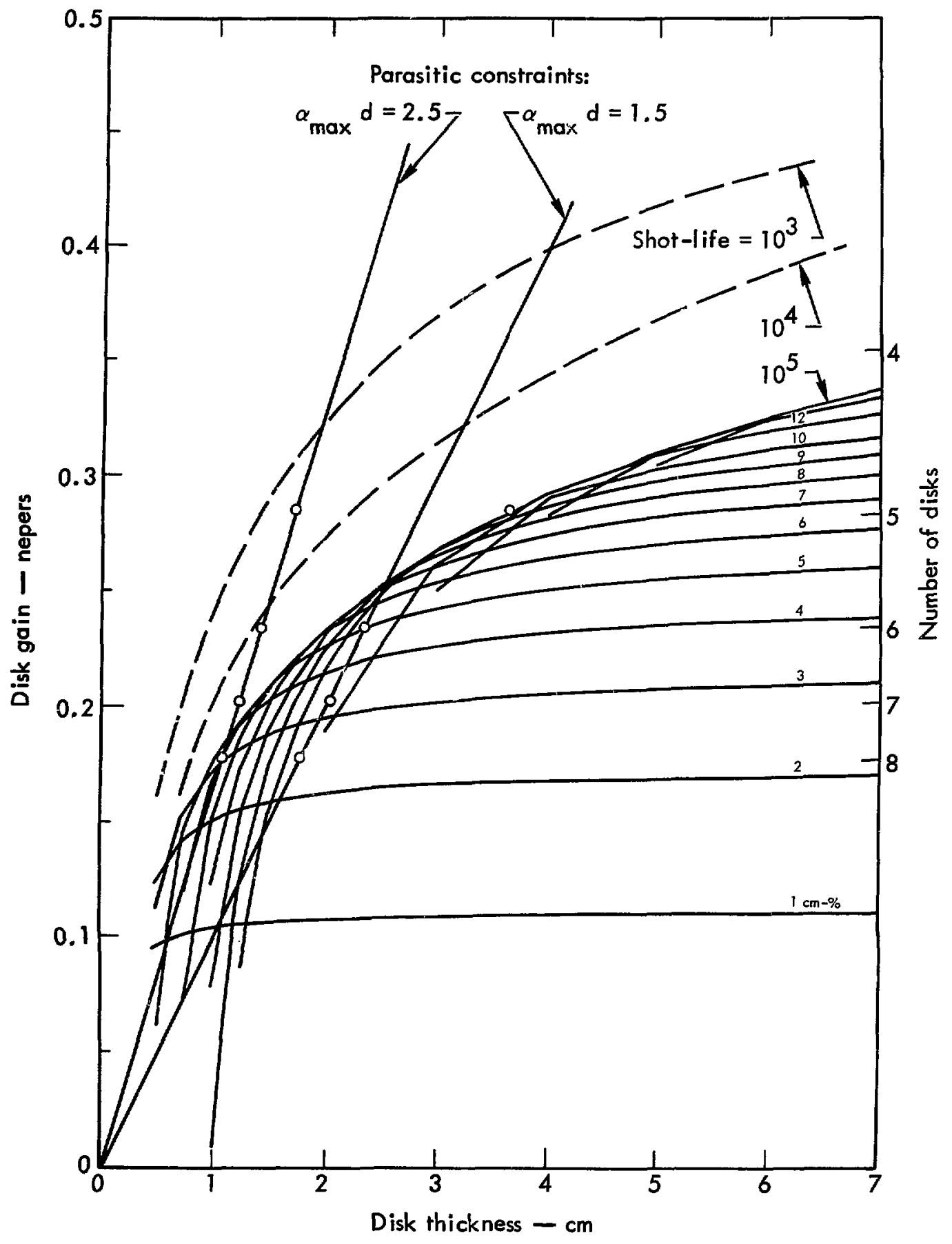

Fig. 14. The selections for the $B$-module disks were based on the data shown in this plot, in the manner described for Fig. 13. 
maximum-gain curves for $10^{3}$. and $10^{4}$-shot life constraints. Operation on or below a maximum-gain curve yields the indicated shot life.

The parasitic-limit lines are plots of maximum disk gain $g$ versus thickness $t\left(g=\alpha_{\max } t\right.$, where $\alpha_{\max }$ is defined above). We must operate on or to the right of a parasitic limit line to satisfy the indicated constraints. For a fixed module gain, usually 3.85 , with a given number of disks, the required gain per disk can be determined by using the number-of-disks scale on the right of Figs. 13 and 14.

Tables 5 and 6 show possible choices of laser disks for the $A$ and $B$ modules; the data are taken from Figs. 13 and 14. We have selected $A$-module disks to have a minimum glass thickness commensurate with a reasonable flashlamp life $\left(\sim 2 \times 10^{4}\right.$ shots $)$.
In the case of the $B$ module, we would have to operate very close to the parasitic limit $\left(\alpha_{\max } d=2.5\right)$ for a minimum-thickness disk (Fig. 14). Since the parasitic limit has not been tested experimentally, we have chosen to build the prototype $B$ module with $2.35-\mathrm{cm}$-thick disks (2\% doping) rather than the desired $1.5 \mathrm{~cm}$ (2.8\% doping). We can conveniently retrofit the $B$ moduie with the thinner disks when improved edge coatings are available.

The energy required to drive the $A$ module is estimated to be $70 \mathrm{~kJ}$, delivered in $800 \mu \mathrm{sec}(3 \sqrt{L C}$ $=800 \mu \mathrm{sec})$. The $B$ module will require $180 \mathrm{~kJ}$, delivered in $800 \mu \mathrm{\mu ec}$. The mechanical design of the $A$ and $B$ modules is described in the following article; the $C$ and $D$ modules are still in the process of being designed.

\section{A AND $B$ DISK AMPLIFIERS - PROTOTYPE HARDWARE DESIGN}

The designs of the hardware for the $A$ and $B$ disk-amplifier modules have been completed. These are the first two of four disk-amplifier stages being considered for the prototype $1-k J$ laser system. The $A$ and $B$ disk amplifiers provide nominal apertures of $3.5 \mathrm{~cm}$ and $8.5 \mathrm{~cm}$, respectively, and are designed for a numerical small-signal gain of $\mathbf{3 . 2}$.

The designs emphasize reliability, ease of maintenance, and simplicity, plus flexibility to allow for future improvements. The disk amplifiers consist basically of a stable rail structure, which sunnorts and aligns the disks, and removable flashlamp modules for optical pumping of the disk array, as shown in Fig. 15. The disk array is surrounded by uv-absorbing filter tubing that protects the laser glass against excessive uv exposure, which could cause solarization and grazing of the disks. This filter tubing also makes it possible to seal and pressurize the disk assembly in order to avoid contamination and damage to the disk surfaces.

For reliable operation of the system it is important to keep the disk assembly and optical components as clean as possible. The optical path will therefore be completely sealed off and purged with nitrogen to maintain a clean environment and to avoid the thermal distortions of the optical beam that are observed in. air.

The laser disks are mounted in holders which provide kinematic support of the disk for minimum deflection and shield the edge coatings from the flashlamp light to avoid deterioration. The disk holders incorporate flow channels which concentrate the nitrogen flow over the disk surfaces for better cooling. For ease of maintenance, the disks and disk holders can be individually removed from the disk array.
To reduce the down time of the laser system, the amplifiers are designed so that they can be serviced in place. The reflectors and flashlamp modules can be individually removed from the disk assembly for inspection, cleaning, or replacement without disturbing the clean environment of the disk assembly. The disk amplifiers are mounted in an adjustable base which allows them to be aligned with the optical axis of the laser systein.

The amplifiers were designed for flashlamps with bore diameters of 10 or $15 \mathrm{~mm}$. The $10 \mathrm{~mm}$ flashlamps were chosen because they have been previously tested and there is an appreciable amount of performance data available. Less performance data is available for $15-\mathrm{mm}$ flashlamps; however, they would better match the existing $20-\mathrm{kV}$ power supplies, would require fewer flashlamp circuits, and would provide a lower current density. A low current density is desirable because it reduces the flashlamp uv output, which could cause solarization, grazing, and deterioration of the exposed materials. In addition, a low current density will improve the pumping efficiency and hence the reliability of the disk amplifier.

A flashlamp design was chosen which employs an insulated flexible lead. This approach requires only a single connector to make the transition from the long, heavy cable from the power supply to the short, flexible lead of the flashlamp. High-voltage terminal boxes are mounted in close proximity to the disk amplifier to allow the flashlamp modules to be quickly disconnected.

The flashlamps are connected in series (at least two in each circuit) to reduce the number of power-supply circuits and to provide opposing current paths through 


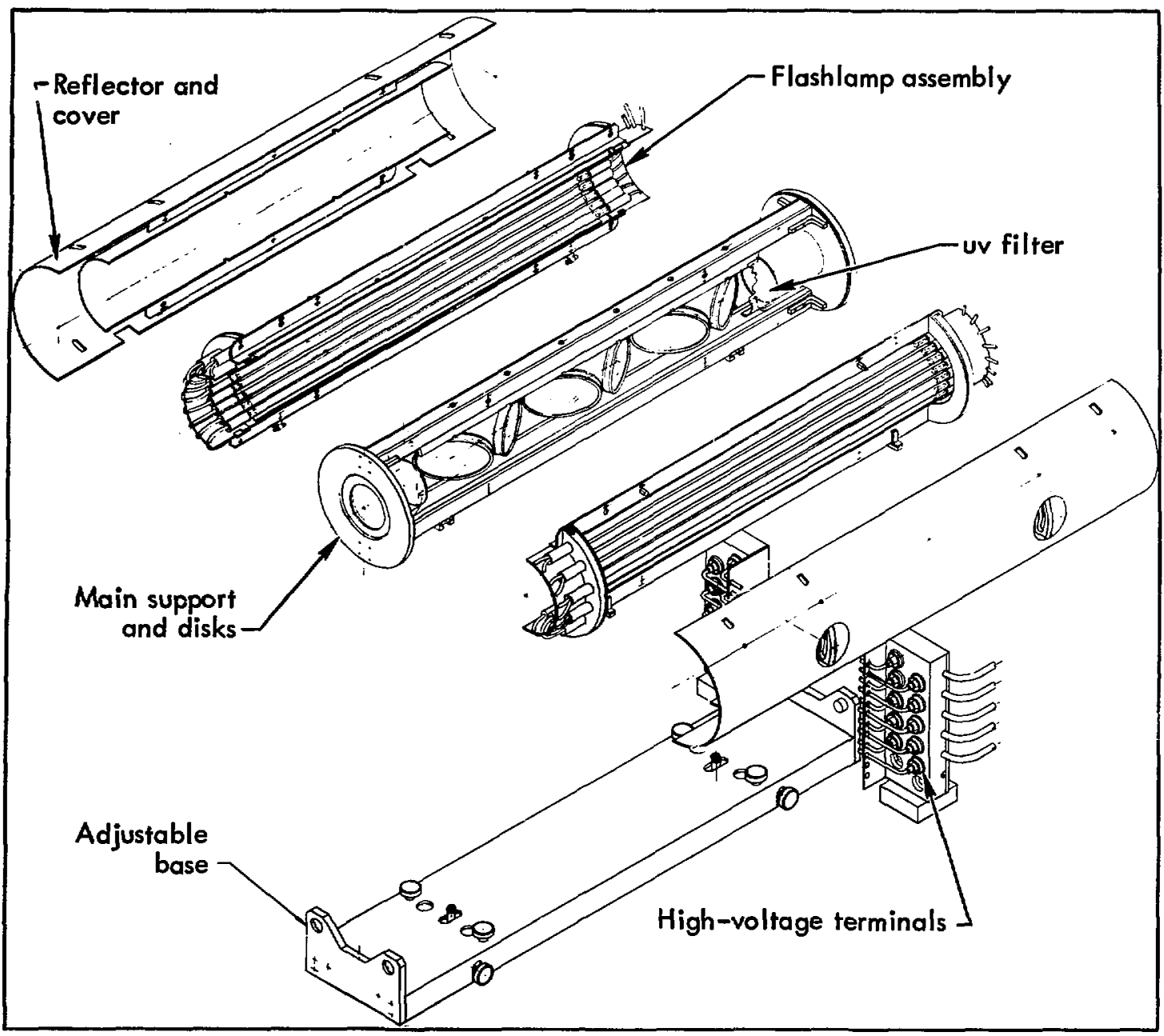

Fig. 15. Major components of the $B$-module assembly.

adjacent flashlamps. This arrangement significantly reduces the magnetic forces exerted on the flashlamps.

The $A$ and $B$ disk amplifiers were designed with machining tolerances as loose as possible to reduce cost but not performance and reliability. To further reduce cost and complexity, we will attempt to use the same kind of flashlamps for all disk amplifiers in order to reduce the number of spares required and to simplify the requirements on the power supply.

\section{STUDIES OF MATERIAL PROPERTIES FOR SOLID-STATE LASER DESIGN}

The design of the LLL 1-kJ laser system requires an understanding of the interaction of high-power laser beams $\left(10^{10} \mathrm{~W} / \mathrm{cm}^{2}\right)$ with the components in the beam path, since absorption, nonlinear propagation effects, and damage will limit system periormance. We have begun to study these problems using the beam from the Intermediate Laser System (ILS) as a source.
Here we report on experiments to determine the nonlinear index of refraction $\left(n_{2}\right)$ of ED-2 laser glass and Nd:YAG, the two-photon absorption coefficient of ED-2, and the intensity at which the beam becomes unstable to small-scale structure when propagated through 50-cm-long unpumped ED-2 laser rods. 


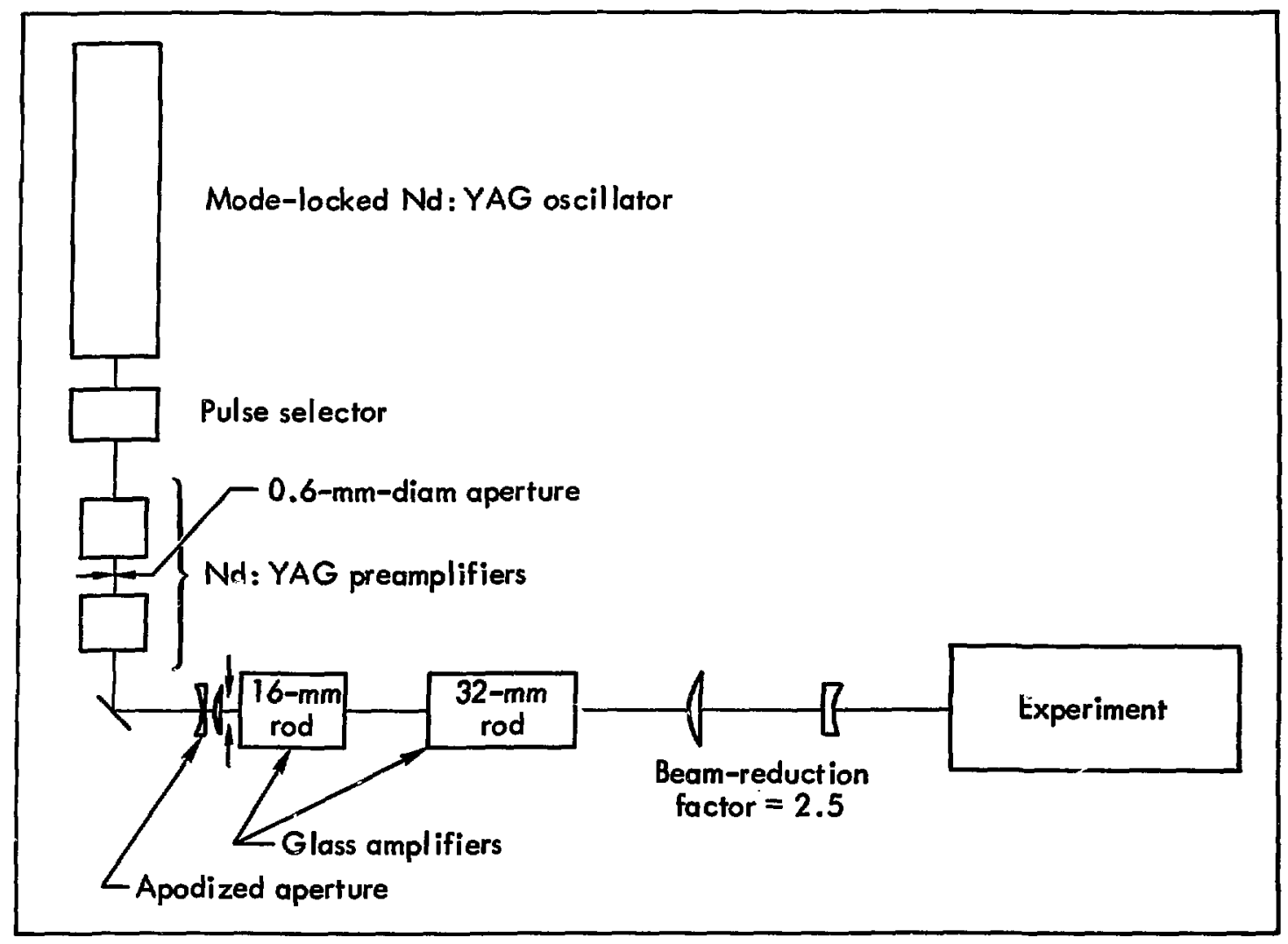

Fig. 16. A simplifed line dingram of the ILS used for material studies

\section{ILS Output Characteristics}

The results of laser-interaction experiments are easy to interpret only if they are performed with a beam that is smooth in both time and space. The ILS provides such a beam with a diameter large enough for detailed beam diagnostics. Figure 16 is a line diagram of the system, and Fig. 17 is a photograph of some of the apparatus. Changes made since the last semiannual report ${ }^{2}$ include modifying the oscillator to produce 150-psec pulses, adding a second Nd:YAG preamplifier, and modifying the aperturing used to shape the beam before it enters the first Nd:glass amplifier. Beam shaping is done with a $0.6-\mathrm{mm}$-diam aperture located between the two YAG amplifiers and a second aperture $2 \mathrm{~m}$ away, immediately ahead of the 16-mm glass rod. The first aperture truncates the beam at $0.85 I_{\max }$ to produce an Airy pattern at the second aperture, which is an apodized aperture similar to that described in the last semiannual report. ${ }^{2}$ It has a 10-mm-diam output window, corresponding to the diameter of the first minimum in the Airy pattern and a small-signal transmission at this diameter of approximately $10^{-2}$. This arrangement produces a smoothly truncated beam with a measured spatial intensity modulation of less than $2 \%$. This smooth beam is preserved to the output of the $32-\mathrm{mm}$ glass-rod amplifier by keeping the diameter of the beam smaller than the diameters of the glass rods. Also, the beam intensity in the $32-\mathrm{mm}$ rod is kept below $5 \mathrm{GW} / \mathrm{cm}^{2}$, the level where the beam quality deteriorates substantially because of small-scale self-focusing; a beam-reduction telescope at the output of the amplifier reduces the beam diameter by a factor of 2.5 and provides a beam of up to $30 \mathrm{GW} / \mathrm{cm}^{2}$ for the experiments.

We routinely monitor the output from the last glass amplifier with the LLL 10-psec-resolution streak camera to determine the temporal characteristics of each shot. A pair of partially reflecting mirrors in front of the streak-camera slit splits the beam into an array of successively less intense beams. Figure 18 shows a densitometer scan of a typical photograph in which four of these beams were stieaked. We obtain a calibration of film density versus laser intensity for 


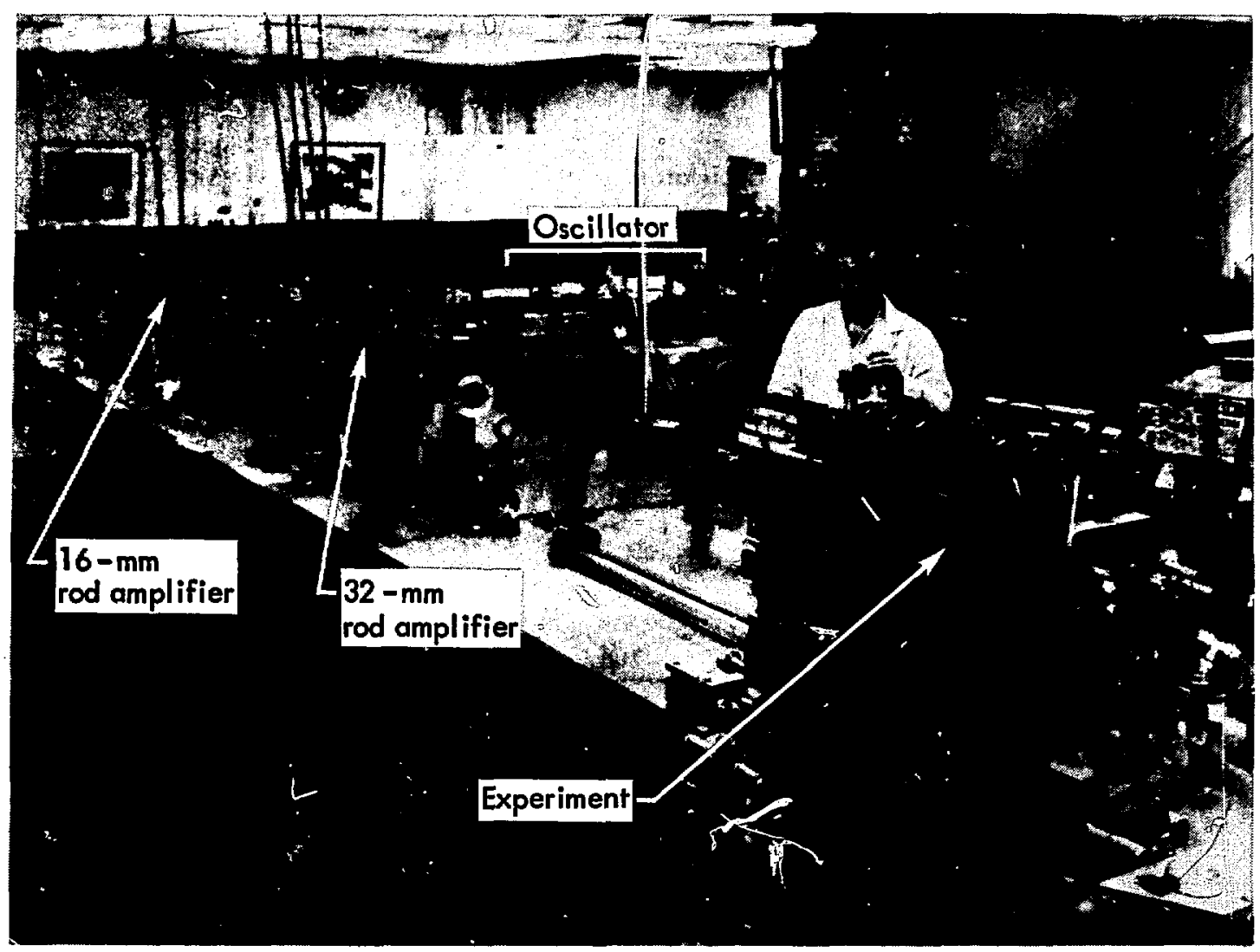

Fig. 17. The ILS retup.

each shot by using the fact that adjacent exposures differ in intensity by the product of the mirror reflectivities. ${ }^{7}$ We believe that the moduiation observed on the densitometer traces is from film and streak-camera noise because it does not reproduce on adjacent exposures. The peak-to-peak amplitude of these density variations is equivalent to a $5 \%$ intensity medulation. We conclude that any temporal modulation on the pulse is less than $2 \%$ of the local intensity.

We also monitor the beam spatial profile at the input to the experiment on each shot by splitting off a portion of the beam, sending it through a mirror pair, and photographing the resulting beams on Polaroid or IZ spectroscopic film. The densitometer trace of a typical photograph, shown in Fig. 19, demonstrates that spatial modulation of the beam is less than $2 \%$ of the local intensity. Beams of this quality were produced with peak intensities after the reduction telescope up to about $20 \mathrm{GW} / \mathrm{cm}^{2}$. Above this intensity, beam quality began to deteriorate from small-scale self-focusing in the glass-rod amplifier train. The diameter of the beam at $e^{-1}$ of maximum intensity is $7 \mathrm{~mm}$.

In summary, the characteristics of the beam input to our experiments are:

$\begin{array}{ll}\text { Average pulse duration } & 150 \mathrm{psec}(\mathrm{FWHM}) \\ \text { Peak intensity } & \text { up to } 30 \mathrm{GW} / \mathrm{cm}^{2} \\ \text { Beam diameter }\left(e^{-1}\right) & \begin{array}{l}7 \mathrm{~mm} \\ \text { modulation }<2 \% \text { in } \\ \text { Beam smoothness }\end{array} \\ \begin{array}{l}\text { both space and time. } \\ \text { meth }\end{array}\end{array}$

\section{Experimental Results}

Nonlinear Refractive Index. We have used two methods to determine the nonlinear refractive index coefficient, $n_{2}$. In the first method, a beam is passed through an unpumped laser rod and photographed several meters beyond the rod output; a part of the beam is split off before it enters the rod and is photographed on the same film. The photograpis record the decrease in the size and the increase in the 


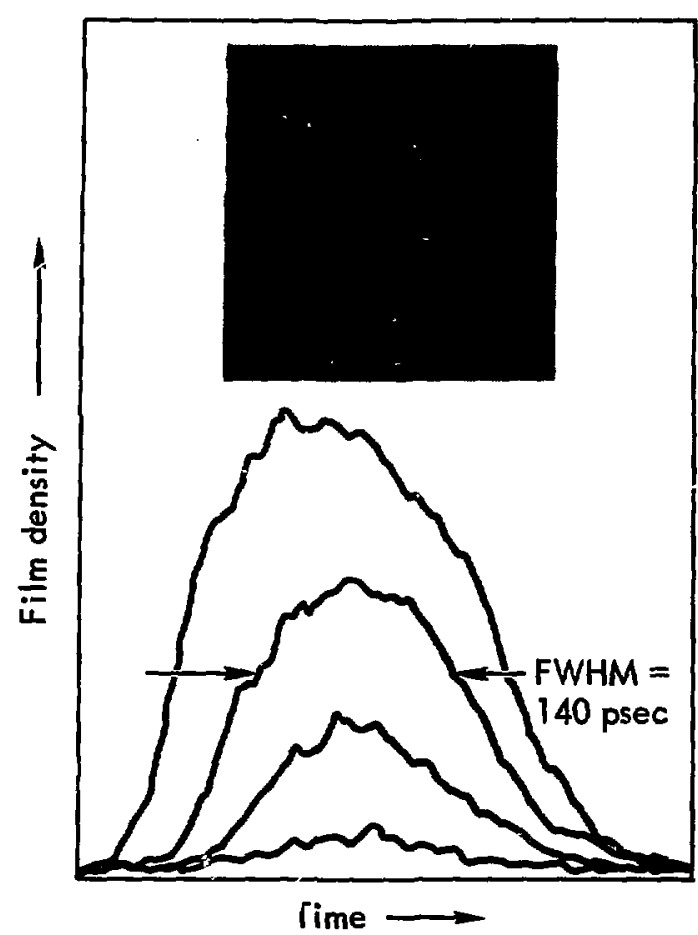

Fig. 18. A densitometer scan of a streak-camera record of a beam at the output of the ILS. The four streaks were generated from a pair of mirrors, and adjacent exposures bear an intensity ratio equal to the product of the minor reflectivities.

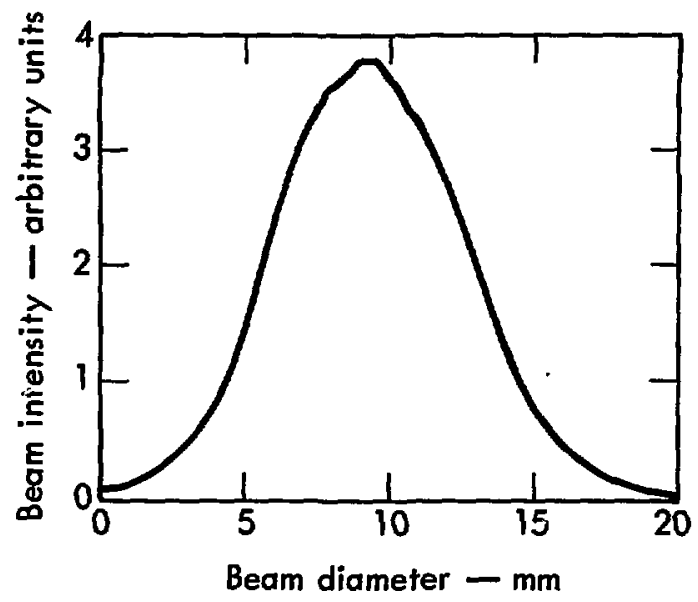

Fia. 19. A densitometer san of the beam eross section of the output of the beam-reduction telescope. This beam quality is preserved up to a peak intensity of sbout $20 \mathrm{GW} / \mathrm{cm}^{2}$. intensity of the beam due to the passage through the rod. The coefficient is determined by assuming that the changes in beam size are produced by whole-beam self-focusing.

In the second method, the phase difference between low- and high-intensity beams that pass through identical rods is measured as a function of time with a Mach-Zehnder interferometer and a streak camera. The coefficient is calculated from the measured peak intensity and maximum phase difference.

Figure 20 shows the setup of the experiment in which whole-beam self-focusing is observed. The smooth beam from the beam-reduction telescope first impinges on a beam splitter, where $4 \%$ of the energy is split off and sent to a pair of mirrors that divide the beam into a series of beams of known energy ratio. These beams are recorded on either Polaroid or IZ spectroscopic film placed at the end of the optical table, $7 \mathrm{~m}$ from the beam splitter. The main beam passes through a 50-cm-long ED-2 rod, where the self-focusing effect in the laser glass produces an intensity-dependent convergence. Beyond the rod another beam splitter splits off $4 \%$ of the beam to another pair of mirrors. The beams from this mirror pair are recorded on the same piece of film as the input beam. In the experiments reported here, the beam intensities were kept below the levels at which beam breakup in the rod begins.

As shown in Fig. 20b, a low-intensity beam will pass through the sample unaffected, since the self-focusing effect will be negligible, and the input and output beams will be of the same diameter. At higher intensities, however, self-focusing will cause the beam to start to converge in the rod. The bearn will continue to converge after leaving the rod, and the output beam will be smaller than the input beam. This is a dynamic process that changes as the intensity of the pulse changes. The focus moves from infinity to a minimum distance from the rod as the pulse intensity rises, and returns to infinity as the pulse intensity retums to zero. Figure 21 shows time-integrated photographs taken at different pulse energies; Fig. 22 is a reproduction of a densitometer scan of the photograph shown in Fig. 21b.

To determine the nonlinear index coefficient, the ratio of the on-axis intensities of the two beams measured from densitometer traces similar to Fig. 22 was compared with the time-integrated self-focusing equations. We used the paraxial ray approximation, fitted the measured spatial-intensity profile with a Gaussian and the measured temporal-intensity profile with a parabola, and assumed an instantaneous nonlinear response in integrating the equations. This procedure yielded a value for $n_{2}$ of $(1.6 \pm 0.3) \times 10^{-13}$ esu for ED-? glass. 


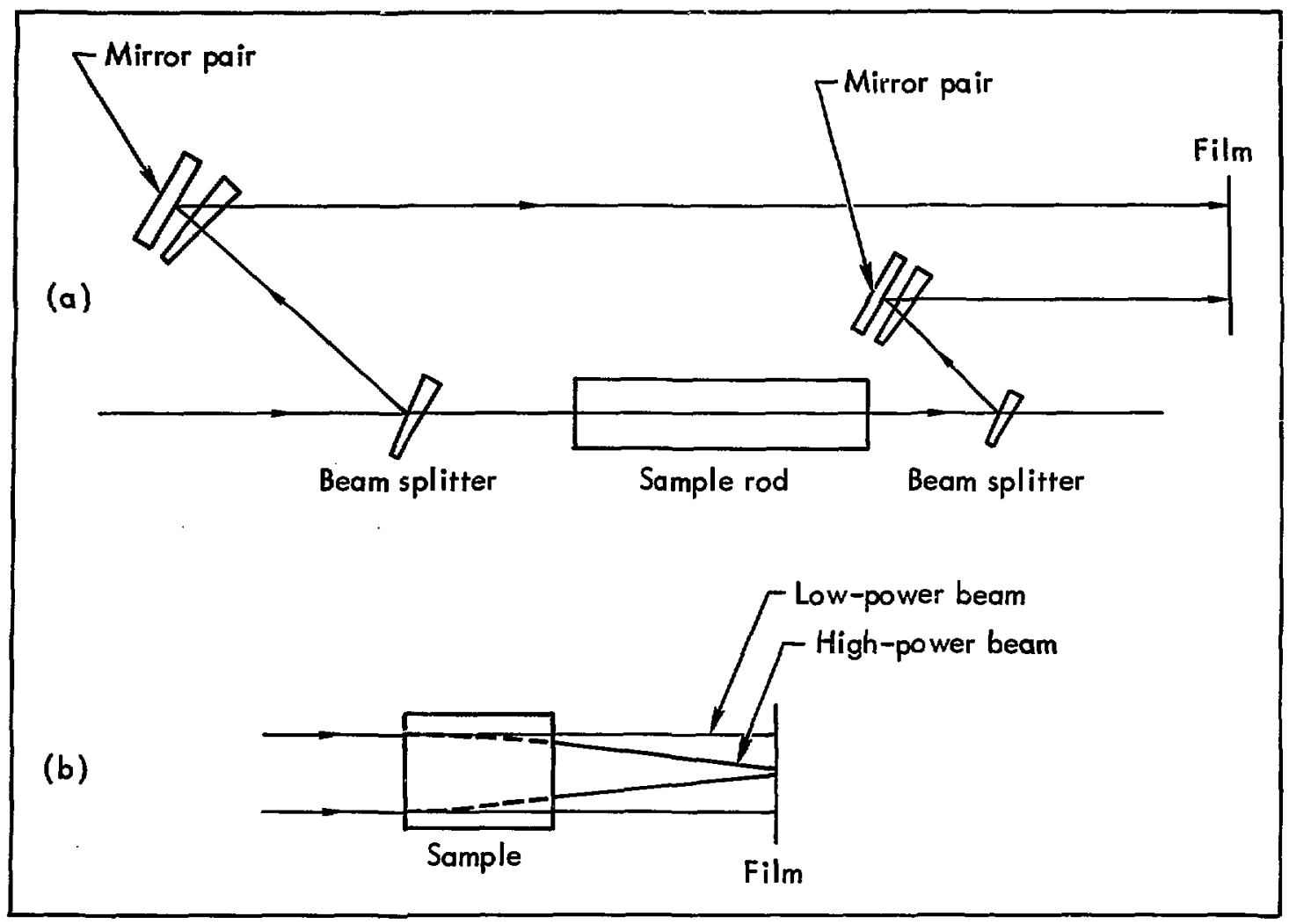

Fig. 20. (a) A simplified dingram of the experiment to measure $n_{2}$ from whole-beam self-focusing. (b) Beam propagation tirough a laser rod at low and high intensity.

The setup of the Mach-Zehnder interferometer experiment to measure $n_{2}$ is shown in Fig. 23. The smooth beam from the beam-reduction telescope is the input beam. The main part of the beam propagates through a sample rod in one arm of the interferometer, while about $2 \%$ of the input beam, which has been split off by a fused-silica beam splitter, is sent through an identical sample rod in the reference arm of the interferometer. A second beam splitter at the output of the rod in the main beam splits off another $2 \%$ of the beam, which is combined with the reference beam to produce a set of interference fringes. The optical-path lengths of the two arms are adjusted so that the pulses are ajpproximately coincident in time. The streak camera records the position of the fringes in time along a beam diameter perpendicular to the fringes. Figure 24 shows a typical record. The value of $n_{2}$ is calculated from the maximum measured phase shift between the two beams and the measured peak intensity. This procedure yielded $n_{2}$ values of (1.5 $\pm 0.5) \times 10^{-13}$ esu for ED-2 glass and $(4 \pm 1)$ $X 10^{-13}$ esu for Nd:YAG.
Two-Photon Absorption Coefficient. In a recent paper, Penzkofer and Kaiser ${ }^{8}$ reported the two-photon absorption coefficient of Nd in Schott LG-630 laser giass to be $4 \times 10^{-12} \mathrm{~cm} / W$. To investigate this effect in ED-2 glass, we measured the transmission at 1.06 $\mu \mathrm{m}$ of $50-\mathrm{cm}$-long 3\%- and 1\%-doped ED-2 laser rods at peak input intensities of 1 to $30 \mathrm{GW} / \mathrm{cm}^{2}$. Figure 25 shows the arrangement for the experiment. A calorimeter measures the energy reflected from a fused silica beam splitter to determine the input beam energy. The main beam passes through the sample and into a second calorimeter. Photographs from mirror pairs monitor the spatial character of the beam before and after it passes through the sample on each shot. Figure 26 summarizes the data from this experiment. All of the points except for the three at the high-intensity end of the scale represent averages of at least four shots. Errors shown are calculated standard deviations. The solid curves are calculations of the transmission averaged over the pulse, using a computer code which included two-photon absorption and self-focusing. Our data for the 3\%-doped rod fit 

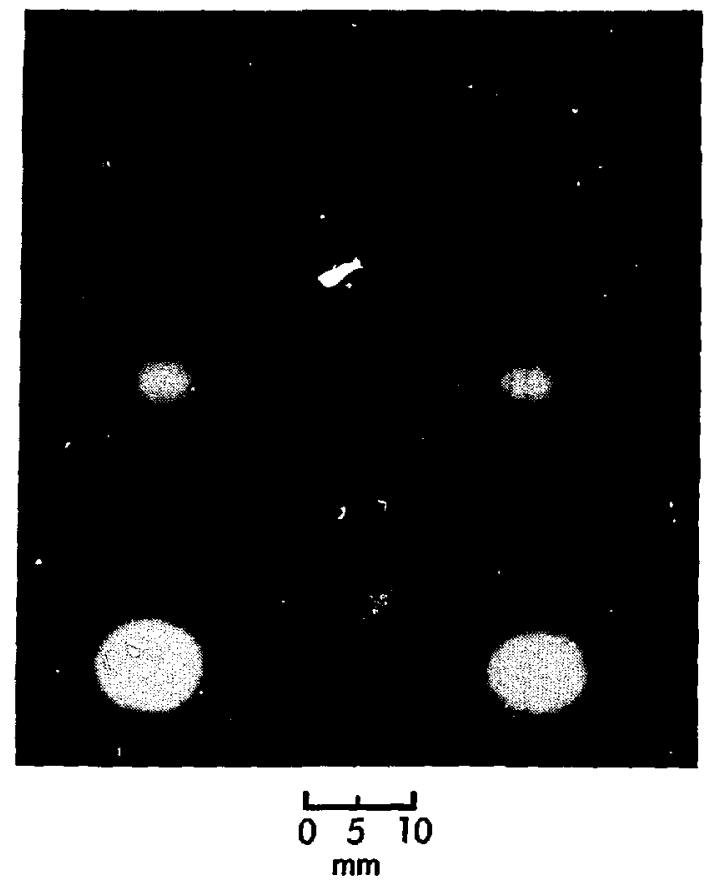

(a) Peak intensity $=1.2 \mathrm{GW} / \mathrm{cm}^{2}$
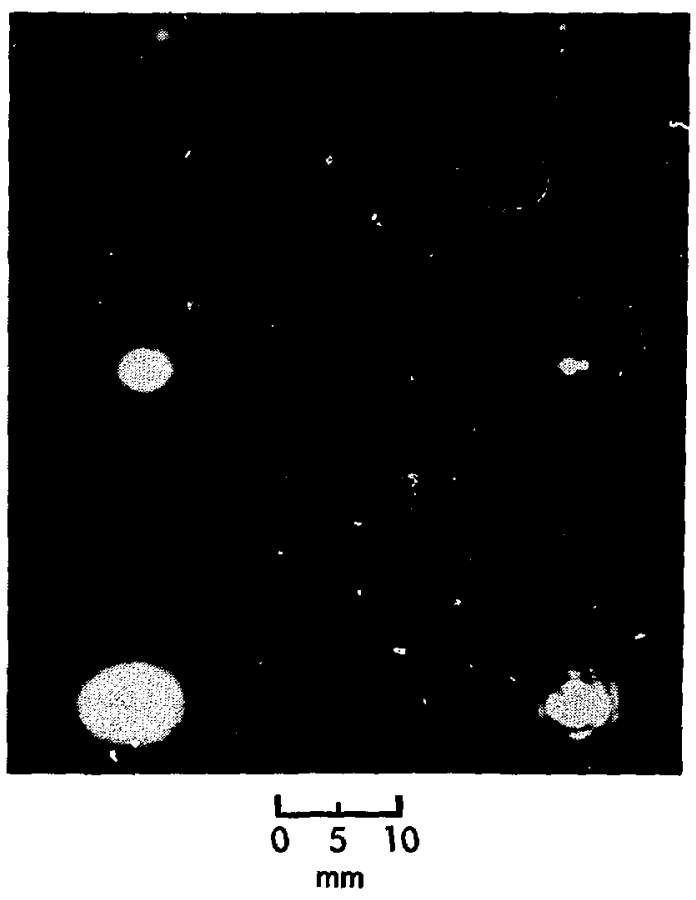

(c) Peak intensity $=7 \mathrm{GW} / \mathrm{cm}^{2}$
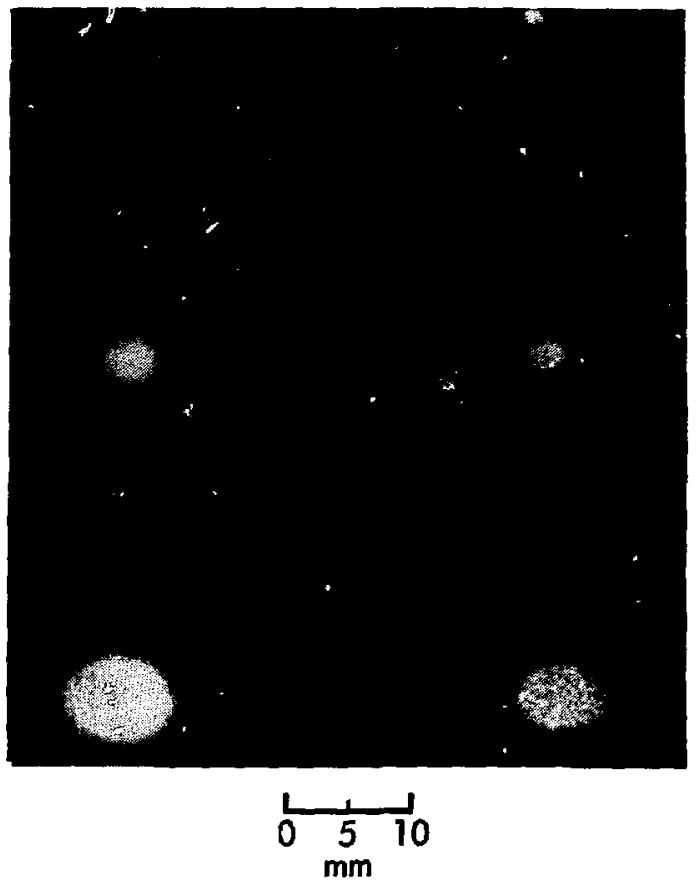

(b) Peak intensity $=2.5 \mathrm{GW} / \mathrm{cm}^{2}$

Fig. 21. Photographs showing wholeseam self-focusing of beams of various peak intensities after passing through a 50-cm-long ED-2 leser rod. The beams split off at the input to the rod are on the left, and the beams split off after passing through the rod are on the right; note the grenter degree of selffocusing at the higher intonsities. The multiple images were generated by a mirror pair. 


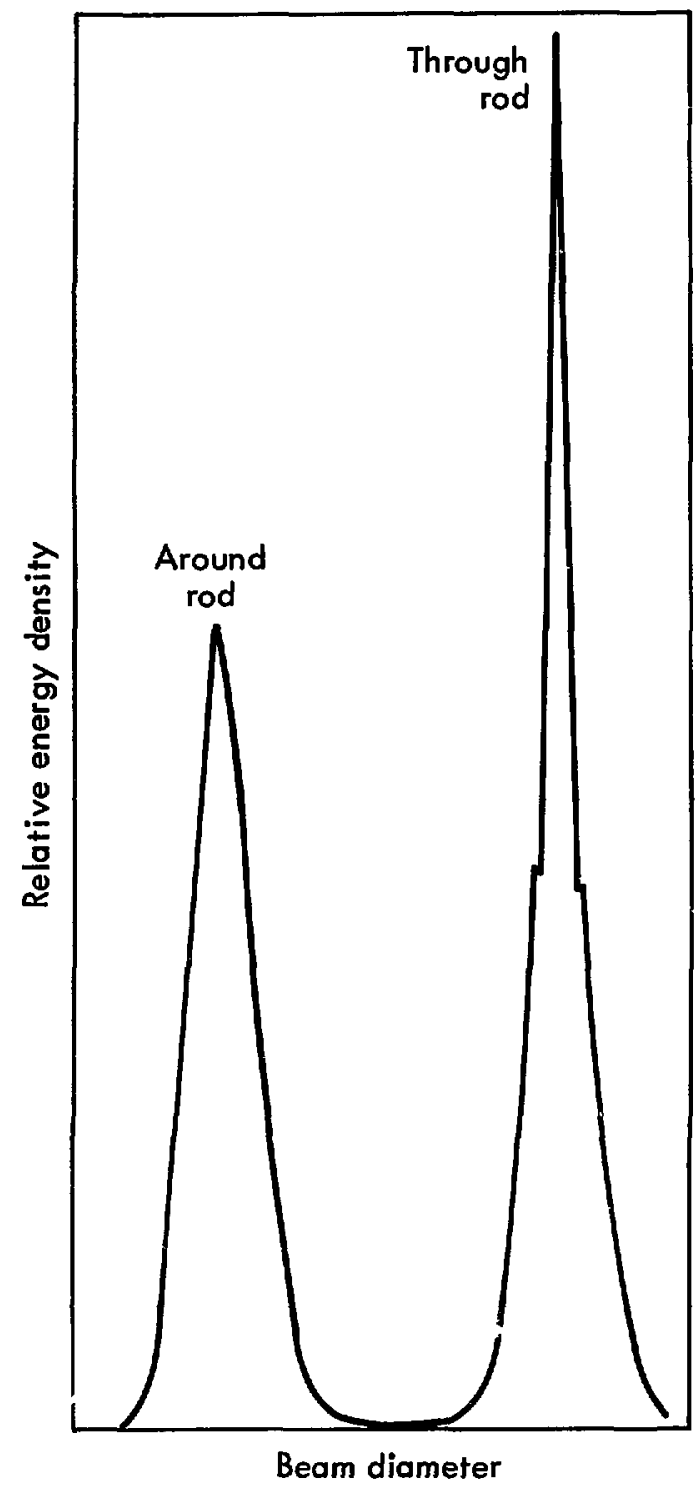

Fig. 22. A densitometer scan of photograph 21b. After correcting for absorption and reflection losses in the two paths, the beam through the rod has 4.2 times the peak energy density of the beam around the rod.

a value for two-photon absorption coefficient $(\gamma)$ of

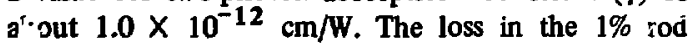
appears to be only slightly smaller than that in the $3 \%$ rod; the difference is smaller than expected if the observed effect is totally from two-photon absorption in Nd. Since there is evidence of beam breakup from self-focusing at high intensities, which may scatter energy out of the beam, we concluded that the two-photon absorption coefficient for 3\%-doped ED-2 is less than $1.0 \times 10^{-12} \mathrm{~cm} / \mathrm{W}$.

Small-Scale Breakup. While doing the two-photon absorption studies, we took near-field photographs at the input and output of the sample rod using the mirror-pair technique. These photographs provide examples of small-scale beam breakup from self-focusing in laser glass. Irregularities in the input 


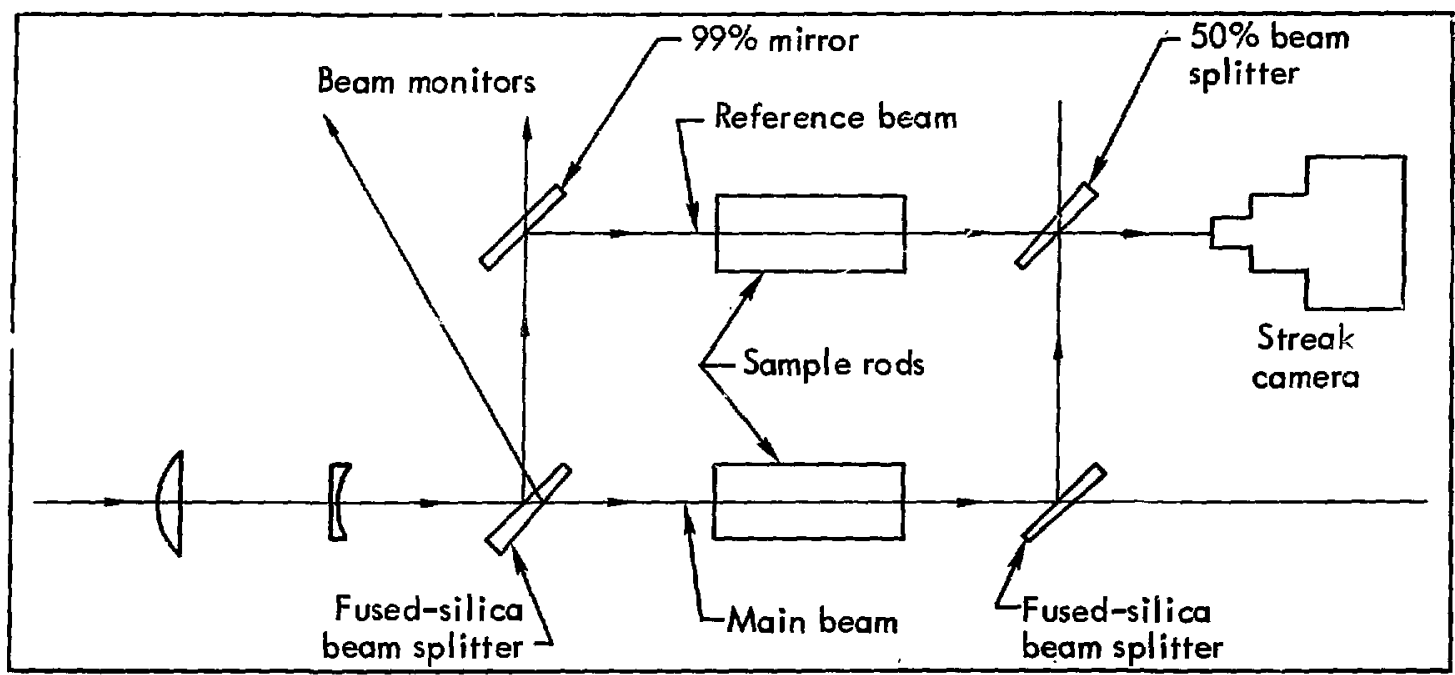

Fig. 23. Diagram of the Mach-Zehnder interferometer experiment used io measure $n_{2}$ for ED-2 and YAG. The high-power beam passes through the lower leg of the interferometer, while a low-power reference beam passes through the upper leg The streak camera is used to photograph the fringe displacement in time.

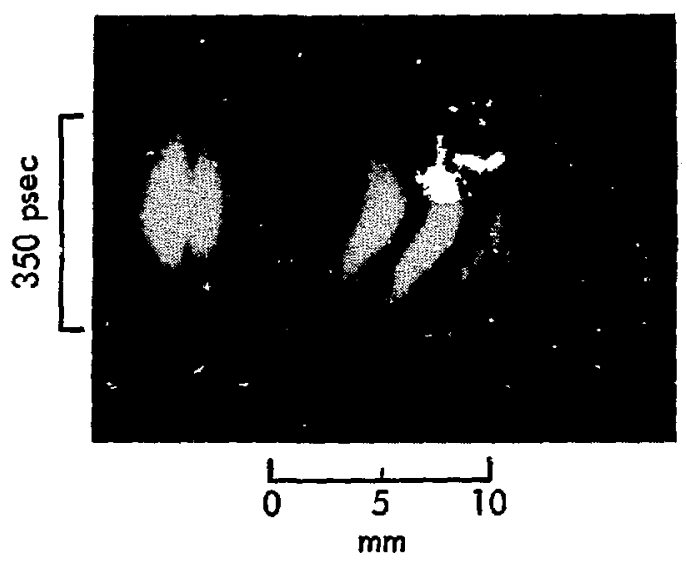

Fig. 24. A typical streak-c.imera photograph of the output from the Mach-Zehnder interferometer. The peak intensity of this pulse was $6.5 \mathrm{GW} / \mathrm{cm}^{2}$. The streak on the far left gives the timing of the putse through the main-beam arm of the interferometer. The pulse through the referencebean arm for this shot arrived at the camera about 1/3 pulse length earlier.

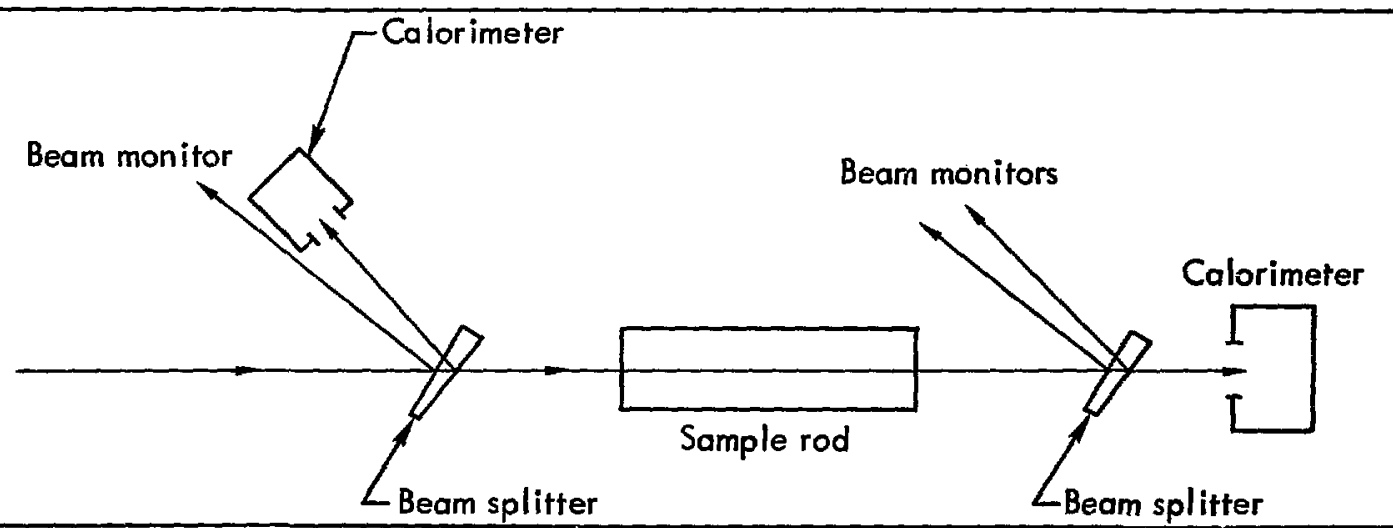

Fig. 25. Simplified dingram of the experiment to measure power-dependent transmission. Calorimeters measure the input and output energy. Beam monitors determine the spatial and temporal characteristicy of the beam. 
beam, that correspond to less than $2 \%$ modulation, increase in contrast in passing through the rod and dominate the beam pattern at the output. We observed this effect to begin at peak int nsities of $4 \% W / \mathrm{cm}^{2}$ and to grow progressively worse with increasing intensity for the passive $50-\mathrm{cm}$ rod in our experiments. The hotspots ranged in size from just resolvable to about $1 \mathrm{~mm}$ in diameter. Figure 27 shows photographs of the beam below and above the intensity threshold for breakup.

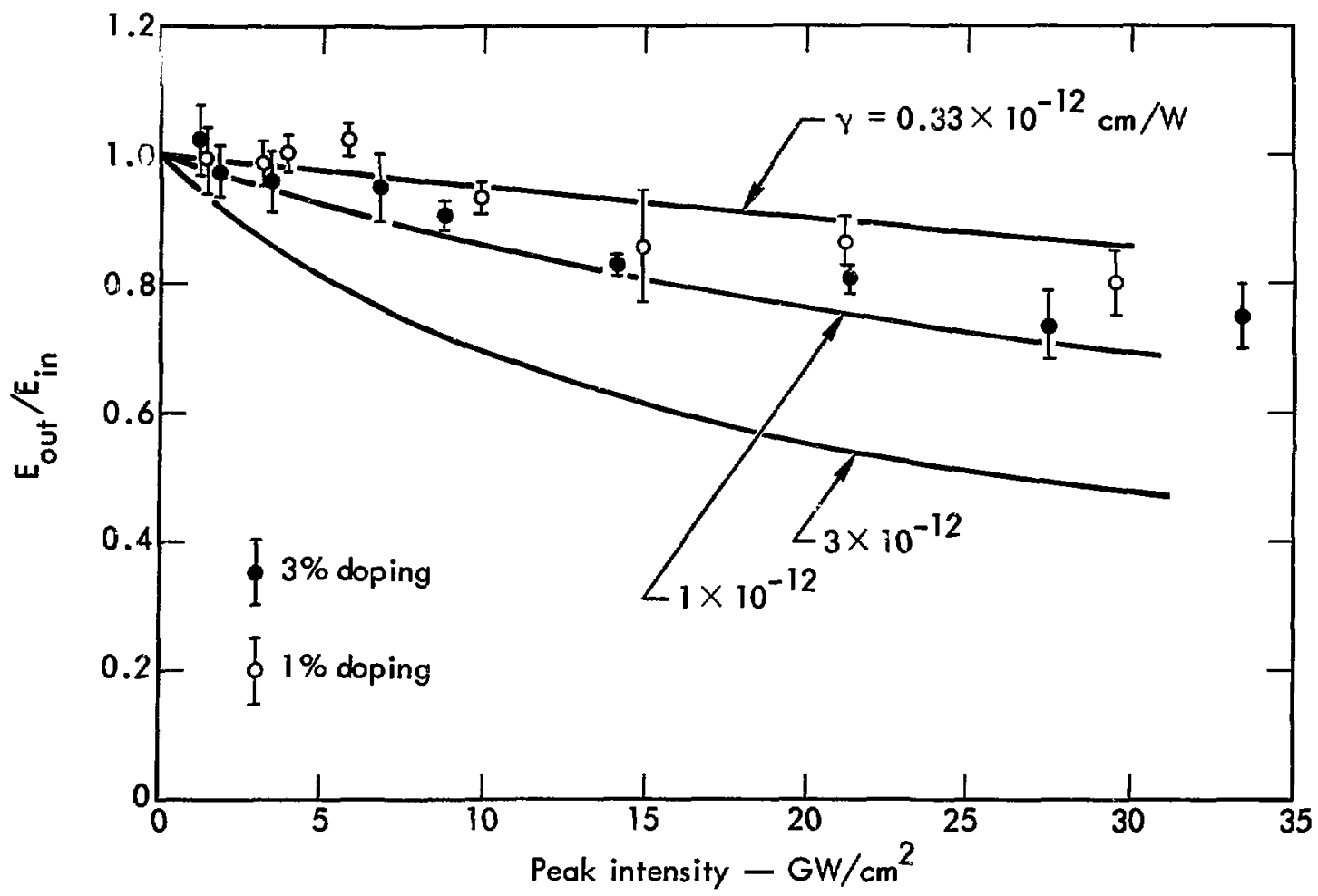

Fig. 26. Ratio of output to input energies measured for 50-cm-long ED-2 laser rods of nominal $3 \%$ and $1 \%$ doping versus the peak input beam intensity. The ratio is nomalized to 1.0 at low intensities. The solid curves are calculated assuming twophoton absorption coefficients of $0.33,1.0$, and $3.0 \times 10^{-12} \mathrm{~cm} / \mathrm{W}$, respectively.

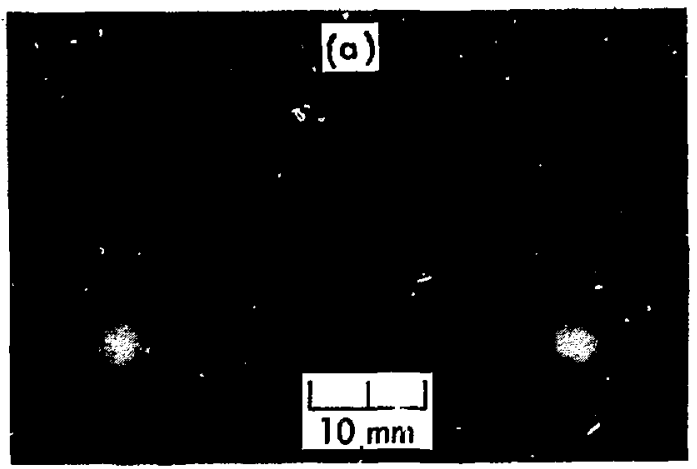

Input
Output

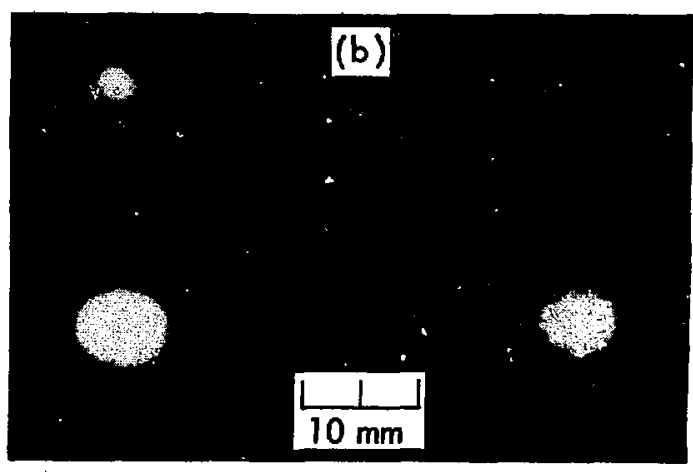

Input
Output

Fig. 27. Near-field photographs of the beam at the input to and output from a $50-\mathrm{cm}-10 \mathrm{~g} g$ ED-2 rod at (a) $2 \mathrm{GW} / \mathrm{cm}^{2}$ and (b) $6 \mathrm{CW} / \mathrm{cm}^{2}$. Breakup of the beam that could soon lead to self-focusing damage is evident in (b). 


\section{Future Plans}

We plan to compare beam-breakup thresholds in a laser rod and an array of laser disks of the same glass length and to measure $n_{2}$ and two-photon absorption effects in candidate Farsday rotator glasses in the near future. Damage testing of rotator glasses, thin-film polarizers, and thin-film mirrors is in progress (see under "Component Development").
We are developing an electrooptic device that is capable of producing a sadially varying transmission density. Devices with smooth, continuous, radially varying transmission characteristics are generally referred to as apodized apertures. ${ }^{9}$ Figure 28 shows the electrooptic (Pockels) effect apodizer. It consists of an electrooptic crystal placed between a polarizer/analyzer cornbination. The device operates by selectively rotating the plane of polarized radiation over its cross-sectional aperture. Utilizing this effect in combination with the polarizer/oralyzer transmission characteristic results in the - ired transmission variation.

Apodized apertures are useful in high-energy lasen systems when it is desirable to have a beam whose transverse intensity variation is other than Gaussian. The requirement for laser beam shaping stems from two main considerations. The first is that a truly Gaussian beam, even though it will theoretically propagate without diffraction, never reaches zero intensity at its edges. Therefore, its aperture will, in general, be determined by other system elements - disk apertures, for example. In a beam that is amplified many times in energy, the small initial diffraction effects resulting from truncating the wings of a Gaussian can result in undersirable amplitude modulation of the beam. The fact that such modulation will inevitably be compounded by amplifier nonlinearities requires that it be eliminated.

The second consideration is energy extraction efficiency. A beam with uniform intensity across the aperture will obviously extract more total energy from the amplifier than will a Gaussian beam of equal peak intensity. In a system where overall efficiency is critical, factors of 3 or more can be gained by using beam shapes other than Gaussian. The development of beam-shaping techniques is therefore an important consideration in the design of lorge laser-amplifier systems.

The present objective for the $1 \cdot \mathrm{kJ}$ solid-state laser system is to obtain a transverse beam pattern that has zero intensity at its edges, fills the effective aperture about 3 times more efficiently than a Gaussian shape, and will propagate through the amplifier chain without suffering appreciable diffraction effects.

The Pockels cell apodizer of Fig. 28 differs from other apodizing schemes ${ }^{9}$ in that the crystal is a lossless element with a transmission characteristic that is independent of input beam intensity. Since the device does not rely on absorption to selectively attenuate the beam, it avoids damage and phase-distortion problems induced by thermal and other nonlinear effects in the absorbing medium.

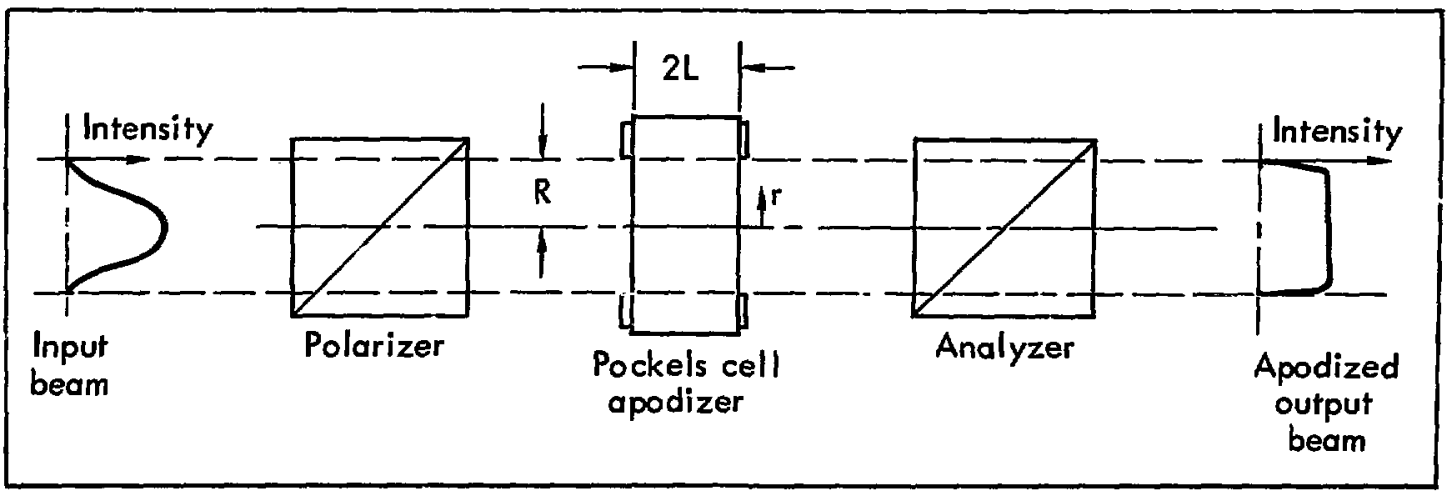

Fig. 28. Electrooptic apodizer configuration. The beam entering from the left is selectively attenuated by the radially varying transmiscion function of the device. The radius $R$ and length $2 L$ of the electrooptic cryatn are indicated on the figure. Polarizer and unalyzer are parallel in this example of the device. 


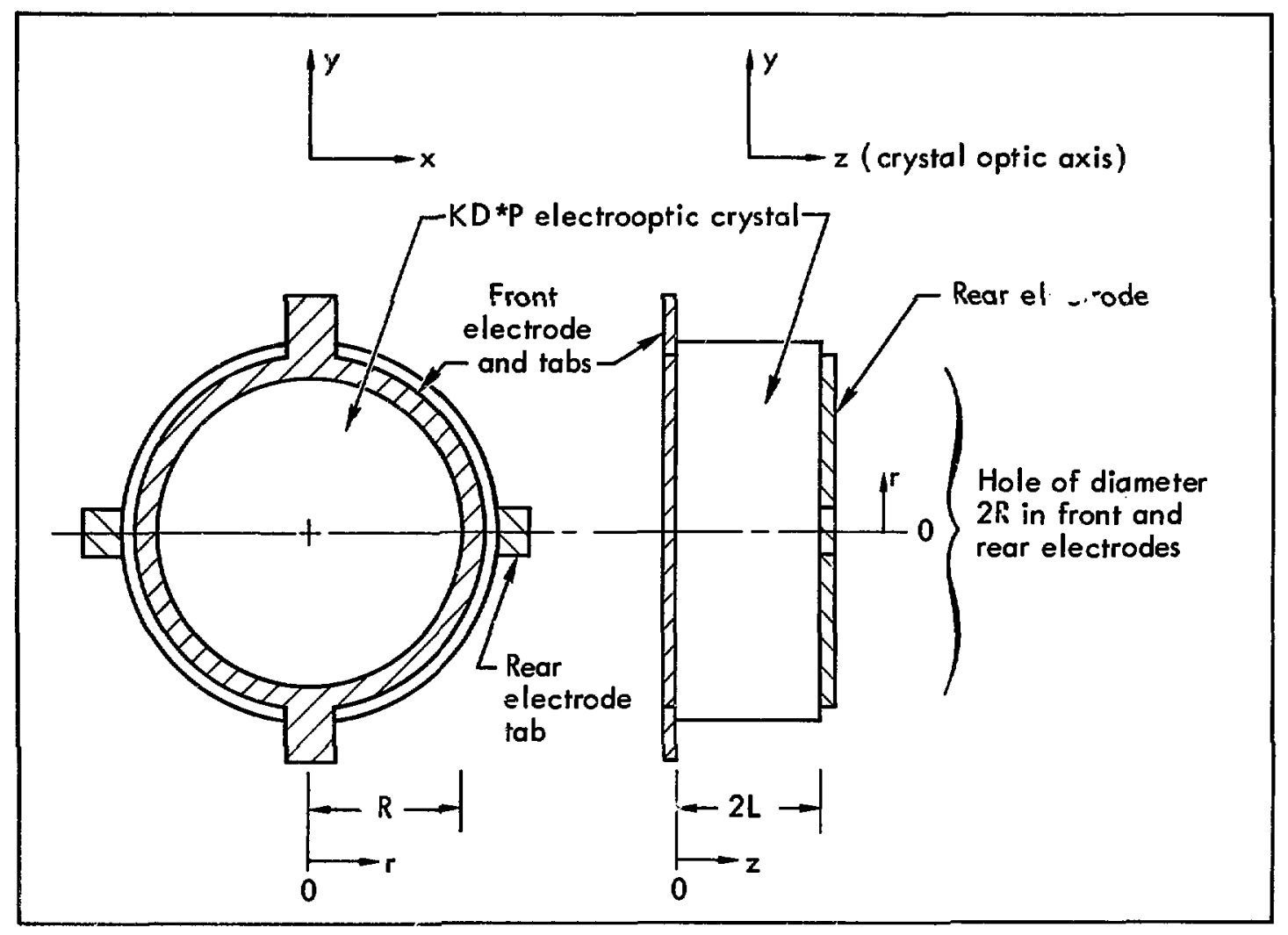

Fin 29. Dotuind stetch of a KD०P rpodining crystal. Voltage is applied across the fwo electrodes.

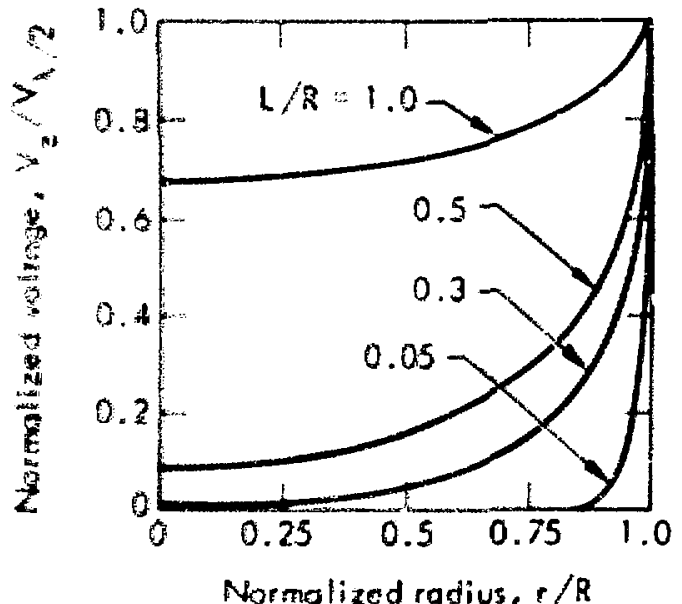

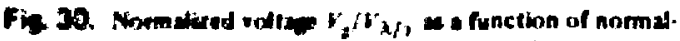

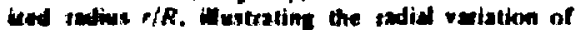

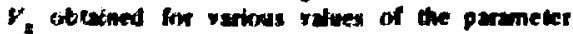
CIR. These theoretices results are obteined from a comprter code colvtion to the Liplace equation for the nin incide the KD*P crystal.

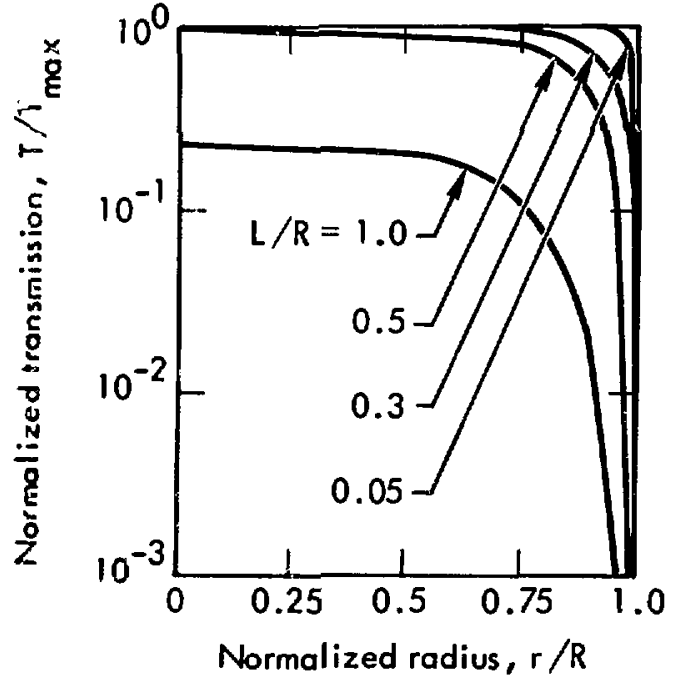

Fig. 31. Nomelized transmiscion $T / T_{\max }$ as a function of normslized radjus $r / R$ for an apodizing cell between paraliel polarixers, culculated from Eq. (1) and the reautis shown in Fit. 30. 
It can be easily demonstrated that the transmission function of a Pockels cell placed between parallel polarizers is givcn by

$$
T(r)=\frac{I_{\text {our }}}{I_{\text {in }}}=T_{\text {max }} \cos ^{2} \phi(r),
$$

where $\phi$ is the effective polarization rotation angle induced by the Pockels cell, and $r$ is the radial distance from the center of the cell. For the longitudinal-field $\mathrm{KD} * \mathrm{P}$ Pockels cell ${ }^{10}$ shown in Fig. 29,

$$
\phi(r)=\frac{2 \pi}{\lambda} n_{0}^{3} r_{63} \quad V_{z}(r)
$$

where $\lambda$ is the optical wavelength, $n_{0}$ is the optical refractive index, $r_{63}$ is the electrooptic coefficient, and $V_{z}(r)$ is the applied voltage. At $V_{z}=V_{\lambda / 2}$ (half-wave voltage), $\phi \equiv \pi / 2$ and $T=0$ [Eq. (1)]. We have indicated that $V_{z}$ and $\phi$ are functions of $r$, the distance from the center of the crystal; that is, we allow $\phi$, and hence the transmission $T$, to vary radially. This radial dependence of $T$ is the basis of the beam-shaping capability of the cell.

The radial variation of $V_{z}$ results from fringing field effects obtained by varying the cell geometry shown in Fig. 29. In the simplest version of the device, we ail w the length/diameter ratio $(L / R)$ of the cell to vary and calculate the total voltage drop, $V_{z}(r)$ $=\left.V_{z}\right|_{z=0}-\left.V_{z}\right|_{z=2 L}$, as a function of $r$ for $0 \leqslant r \leqslant R$. The fields are calculated by solving the linear Laplace equation for the field inside the crystal with a computer code ${ }^{10}$ that takes into account all geometrical and the cell. Resulting plots $\because$ ormalized voltage $V_{z} / V_{\lambda / 2}$ are given in Fig. 30 for several values of the ratio $L / R$. Applying Eqs. (1) and (2) to the results of Fig. 30 gives the results shown in Fig. 31, where we plot normalized transmission ( $\log$ scale) as a function of radius, with $L / R$ as a parameter. In order to obtain the resultant intensity profile for any given beam, we simply multiply the incident intensity at any given point by the transmission function for that point, i.e.,

$$
I_{\text {out }}(r)=I_{\text {in }}(r) T(r)
$$

For an infinite plane wave $[I(r)=$ constant $]$, the curves in Fig. 31 give the intensity profile of the beam transmitted through the apodizer. The actual beam propagated in the LLL 1- and 10-kJ laser systems will be obtained by truncating an inilially Gaussian beam and performing detailed shaping with an apodized aperture. In this case, we perform the operation indicated by Eq. (1) with $I_{i r}(r)$ a truncated Gaussian.

The preceding examples demonstrate the possibility of modifying the transverse spatial dependence of an incident optical beam using an electrooptic cell between polarizers as the apodizing element. By extending these techniques, it would be possible to obtain virtually any desired transmission-versus-radius function with a device of this type. This would involve the use of more cumplex geometries, multiple electrodes, and a variety of potentials applied to these electrodes.

\section{MASTER OSCILLATOR}

The oscillator-development effort has two phases, each of which is to culminate in a hardened, reliable laser oscillator that will be used as a tool rather than as a subject of research. The primary use of the phase-one oscillator will be as the master oscillator for the $1-\mathrm{kJ}$ chain, while that of the phase-two oscillator will be as the master oscillator for the $10-\mathrm{kJ}$ chain. The phase-one oscillator will produce a single bandwidth-limited pulse per firing command, having nominally Gaussian spatial, temporal, and spectral profiles. The phase-two oscillator will approximately produce the temporal shape that is considered optimal $^{11}$ for laser-induced fusion. With both systems the controlling requirements will be reproducibility, reliability, and smooth temporal and spatial profiles.

The phase-one oscillator is a hardened, dye-mode-locked device, which is described in this article. A second device, which is mode-locked by an externally driven Pockels cell, is also being developed and is a candidate for the phase-two oscillator. It is described in the following article.

The dye-mode-locked oscillator is based on the LLL version of the NRL oscillator design, ${ }^{12}$ but with considerable differences in implementation reflecting the decision to "harden" this system. Since this system is to be. a tool, it should be movable from room to room and from building to building without requiring realignment. The operational and environmental goals are shown in Table 7.

The laser chassis is basically an H-section structure, with four cylindrical Invar bars mounted near the four corners of the top half of the $H$. The resonator components (mirrors, etalon) are referenced to the Invar bars, while the oth.er components (e.g., the laser 
Table 7. Operational and environmental design guals for the phaso-one oscillator

Output

"Hend-ofr" fifetime

Operating shock and vibration

Nonoperating shock and vibration

Operating temperature

Nonoperating temperature

Operating relative humidity

Nonopernting relative humidity
$>1 \mathrm{~mJ}$ in a single TEM $_{00}$-mode pulse, nominally 100 psec (FWHM) at

$1.06 \mu \mathrm{m}$; pulse width range: 30 psec - 1 nsec

$>10^{4}$ shots (single-pulse selector);

$>10^{6}$ shots (all other components)

Normal laboratory ambient

MILSTD-810B, Procedure VII, Fig 514-5, Curve V, as modified by Fig. $514-7$. ( $11.3 \mathrm{~g}$ peak, 5 to $50 \mathrm{~Hz})$

$22^{\circ} \mathrm{C} \pm 10^{\circ} \mathrm{C}$ air temperature

$0^{\circ} \mathrm{C}$ to $55^{\circ} \mathrm{C}$ air temperature

$20 \%$ to $50 \%$ (nominal)

$0 \%$ to $99+\%$ head) are mounted to the horizontal plate. The bottom half of the $H$ contains some of the oscillator electronics, also mounted to the horizontal plate. Top, bottom, and end plates provide some degree of EMI suppression, and permit the chassis to be slightly pressurized with dry nitrogen. This structure is innately thermally stable. The laser rod temperature will be controlled to $\pm 1^{\circ} \mathrm{C}$ by the cooling water. The pitch-yaw optical mounts will be constructed totally from stainless steel, specifically including the adjustments, in order to preclude bimetallic thermal misalignment.

A schematic of the oscillator is shown in Fig. 32. The components requiring the greatest degree of thermal control are the dye solution ${ }^{13}$ and the intracavity etalon. 14 The dye cell and dye-pumping system are based on an LLL modification of an NRL design. ${ }^{15}$ The new dye-solution reservoir is thermally insulated, and its capacity has been increased from one-half pint ( 0.28 liter) io 1 liter. The temperatures of the dye solution and the dye celi are being controlled to $\pm 0.1^{\circ} \mathrm{C}$. Temperature control is provided by circulating water controlled to $\pm 0.01^{\circ} \mathrm{C}$ at a flow rate of $1 / 4 \mathrm{gpm}$ through the system. The counterflow water path is through a collar : Jund the dye cell, then to a stainless-steel heat exchanger, and out via several wraps of copper tubing around the dye reservoir. The dye solution flows from the reservoir through a pump, through the heat exchanger, and then into the dye cell. This system insures that the dye and cell will be at the same controlled temperature. The temperature of a breadboard system has been monitored for a period of a week; it exhibited a maximum variation of $\pm 0.1^{\circ} \mathrm{C}$ from the mean temperature.

A new laser head, based on an afocal double-ellipse pumping geometry, is being designed with the aid of the ZAP code, ${ }^{16}$ modified for use with Nd:YAG. ${ }^{17}$ The objective is to obtain better gain uniformity than

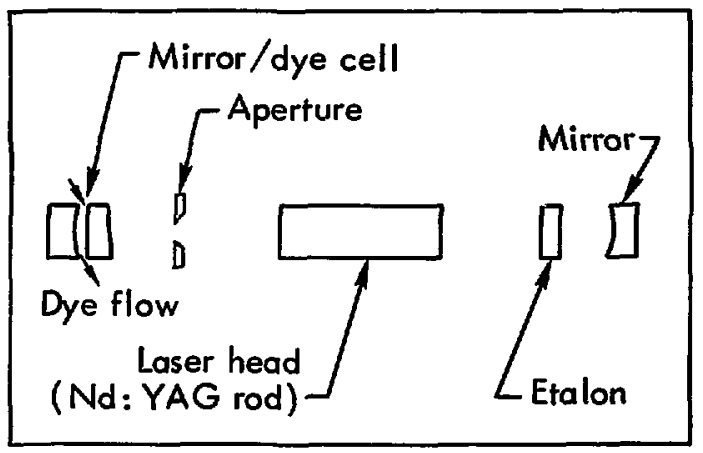

Fig. 32. Schematic of the dye-mode-locked oscillator.

provided by the NRL head (which is a focal single ellipse), with greater efficiency and maintainability than provided by the "four-leaf-clover" head. 18 The lamps will be operated in the simmer-flash mode, ${ }^{19}$ which should increase both pulse-to-pulse and long-term reproducibility. ${ }^{20}$

The resonant cavity will be hemiconfocalequivaient. ${ }^{21}$ This is the most stable configuration available, ${ }^{21,22}$ and the $e^{-2}$-intensity spot radius (for an optical length on the order of $1.5 \mathrm{~m}$ ) varies from 1 to $0.7 \mathrm{~mm}$. The cavity length is chosen to provide a cavity-round-trip time near 10 nsec. The flat mirror will be essentially hard-mounted to the laser chassis in order to eliminate the possibility of angular misalignment with the amplifier chain (which is nore forgiving of slight translational misalignment). We are initiating computer simulation of the oscillator to assist in determining proper component positioning within the resonant cavity.

The weakest part of present passively mode-locked oscillators is the single-pulse-selection technique. Since the time of emission of a pulse train is neither 
accurately predictable nor extemally controllable, the train must be detected and then one of its pulses selected. It is desirable at this point to be able to affirm that the train is not anomalous - or at least to check that one or more pulses preceding the one to be selected are "typical" in some quantitative sense - in order not to spoil a target-interaction experiment or damage the amplifier chain. The present technique utilizes a laser-triggered spark gap, ${ }^{12}$ which performs no logic on the pulse train and which has a threshold that is a monotonic function of the number of triggerings. We are currently examining several approaches to the single-pulse-switchout problem that offer advantages over the present technique because they feature separation of the detection, logic, and switching functions.

\section{A SYNCHRONOUSLY DRIVEN Nd:YAG OSCILLATOR FOR PRODUCING SUBNANOSECOND BANDWIOTH-LIMITED PULSES}

Previous reports ${ }^{18,23-25}$ have described in detail an Nd:YAG laser oscillator that directly produces short-duration, bandwidth-limited output pulses at $1.06 \mu \mathrm{m}$. Single pulses containing 1 to $2 \mathrm{~mJ}$ have been tuned in duration from about $400 \mathrm{psec}$ to $>2.5$ nsec with this oscillator. Our present objective is to extend the operating range of the system down to pulse durations of $\leqslant 100$ psec (FWHM) while maintaining the bandwidth-limited character of the pulse.

Most recent efforts on this oscillator system have involved: (1) fabrication, assembly, and testing of an oscillator system that employs a new laser head with improved pumping uniformity, (2) operation of this new system with several different fast $Q$-switch/ modulator elements in order to evaluate their performance, and (3) an extensive and detailed diagnostic effort designed to determine the time and frequency properties of the oscillator output pulses as accurately as possible.

Figure 33 is a photograph of the new oscillator system. ${ }^{27}$ The mirror mounts are temperaturecontrolled to provide stability of alignment and separation.28 The new laser head 28 is a cloverleaf arrangement containing four linear 3-in.-arc-length xenon flashlamps surrounding a

*A bandwidth-limited pulse is defined as a pulse completely devoid of amplitude or frequency modulation. Its field amplitudes as functions of time and frequency are identical Fourier transform pairs; i.e.,

$E(t)=\int_{-\infty}^{\infty} E(v) e^{-l 2 \pi \nu t} d \nu$, and $E(\nu)=\int_{-\infty}^{\infty} E(t) e^{l 2 \pi \nu t} d t$

where intensity $I=|E|^{2}$. A bandwidth-limited pulse with Gaussian time dependence has a time-frequency product

$$
\Delta v(1 / 2) \quad \Delta t(1 / 2)=\frac{2 \ln 2}{\pi} \sim 0.44
$$

where $\Delta v(1 / 2)$ and $\Delta r(1 / 2)$ are intensity widths measured at full width at half maximum. 26
double-Brewster Nd:YAG rod $1 / 4$ in. in diameter and 3.3/4 in. long. This new laser head has several advantages over the previous unit, ${ }^{24}$ including (1) improved efficiency while maintaining uniform illumination of rod volume and (2) a wider range of available flashlamp input energies, allowing needed. flexibility in adjusting the overall gain of the system. The first advantage reduces unwanted heating effects in the rod and its environment; the second permits us to operate with lower flashlamp input energies (hence lower gain in the Nd:YAG rod) because of the smaller minimum voltage required to operate a linear (3-in. arc length) versus a helical (19-in. arc length) flashlamp.

In Fig. 33, the optical cavity is defined by the two reflecting elements located near the left and right edges of the photograph. The temperature-controlled mirror mounts contain a flat mirror and a two-surface output-coupling etalon. When additional frequency selectivity is required, either or both of the reflecting elements may be replaced by a multi-element etalon. Figure 34 is a photograph of a four-element etalon contained in a newly designed temperature-controlled mirror mount and housing. This design improves the thermal contact to the optical element. Thermal stability of the oscillator components and their environment is required to guarantee alignment and etalon spacing.

One system element that has received considerable attention is the fast-switching $Q$-switch/modulator cell.18,23-25 This particular element is vital to successful operation of the oscillator. Tc obtain output pulses of 100 psec or less from the oscillator, it is necessary to obtain an electrical risetime on the Pockels cell (10 to $90 \%$ ) on the order of 200 psec. This risetime cannot be obtained with current Pockels cells because of their high capacitance. Therefore, we have designed a special high-switching-speed Pockels cell employing a KD*P crystal of the cylindricalring-electrode (CRE) configuration ${ }^{27}$; this cell is described elsewhere in the report (see under "Component Development"). The CRE-configuration 


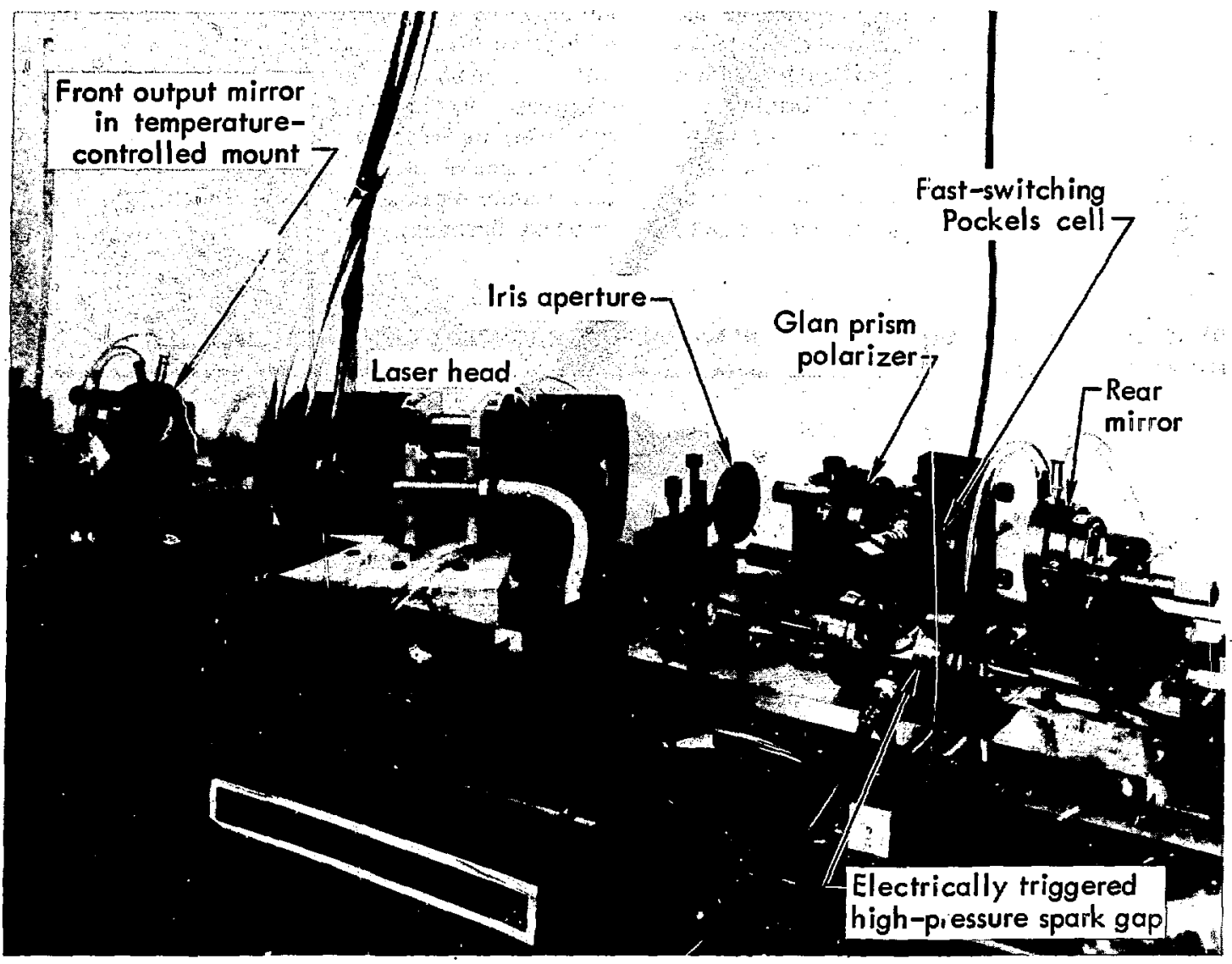

Fig. 33. The synchronously driven Nd:YAG oscillator. All components are rigidly mounted to the optical bench and placed on a vibration-isolated table to enaure mechanical stability.

crystal has a capacitance of $2 \mathrm{pF}$ (about one-fifth that of crystals with conventional electroding) and is contained in a special housing such that the entire cell is impedance-matched to a $50-\Omega$ transmission line. A 2-pF capacitance driven by a $50-\Omega$ transmission line will have a risetime (10 to $90 \%$ ) of about 200 psec," a value consistent with our switching requireme.ts. We are currently dynamically testing the cell and experimentally evaluating its performance in our oscillator.

Most of our other efforts on the oscillator have been devoted to diagnosing the time and frequency properties of the output pulses. For a pulse to be bandwidth-limited, it must be completely devoid of

*The cell as a whol has a characteristic impedance $\sqrt{L / C}$ $=50 \Omega$; however, the voltage risetime arross the crystal is governed by the crystal capacitance.

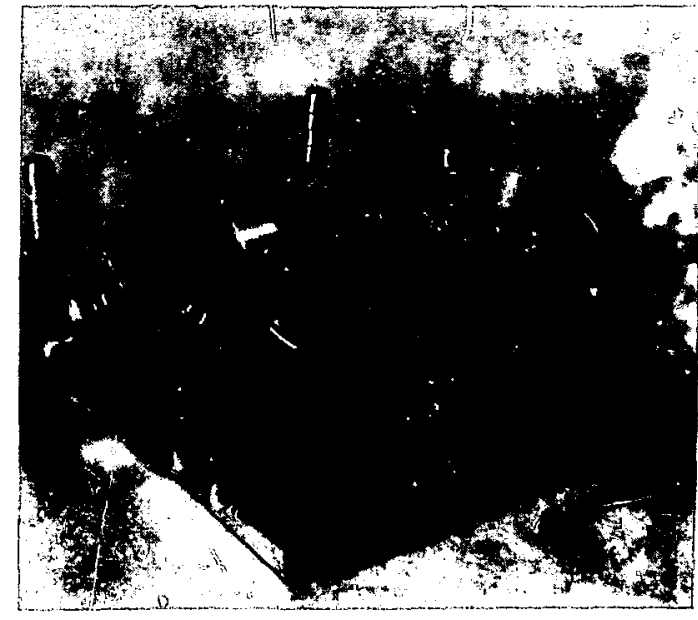

Fig. 34. Temperature-controlled stainless-steel mirror mount containing a resonant reflector (otalon). 


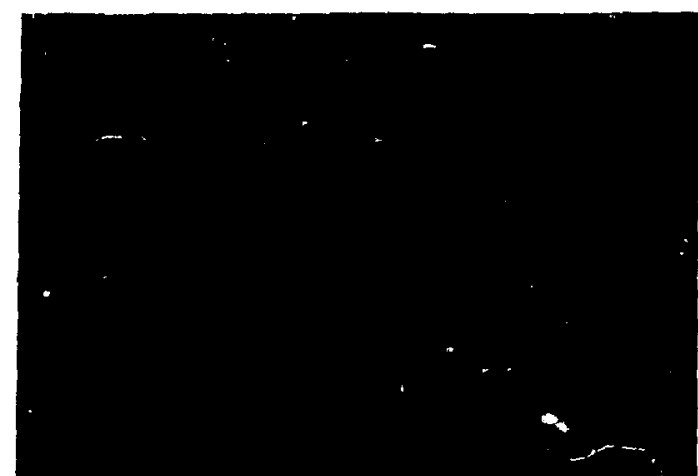

(a)
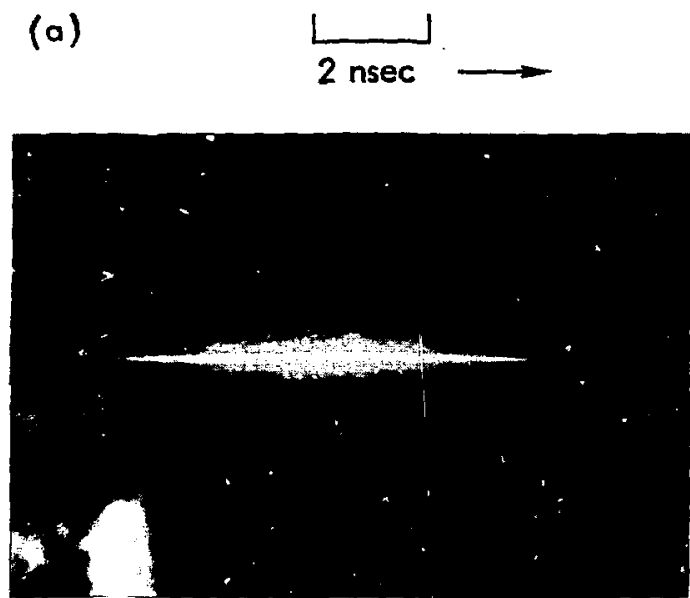

(b)
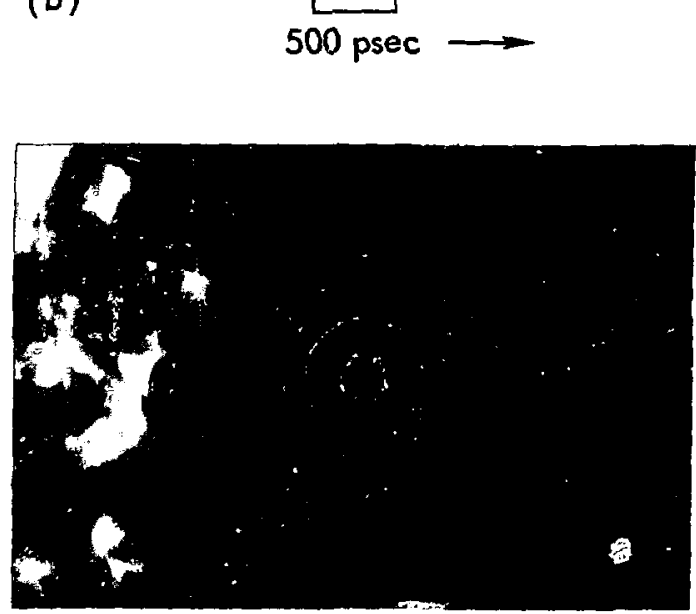

(c)

Fig. 35. Diagnostics perfomed on a bandwidth-limited oscillator output pulse, (a) is en oxcillograph, (b) is a streak-camera trace, and (c) is a Fabry-Perot interferogram.
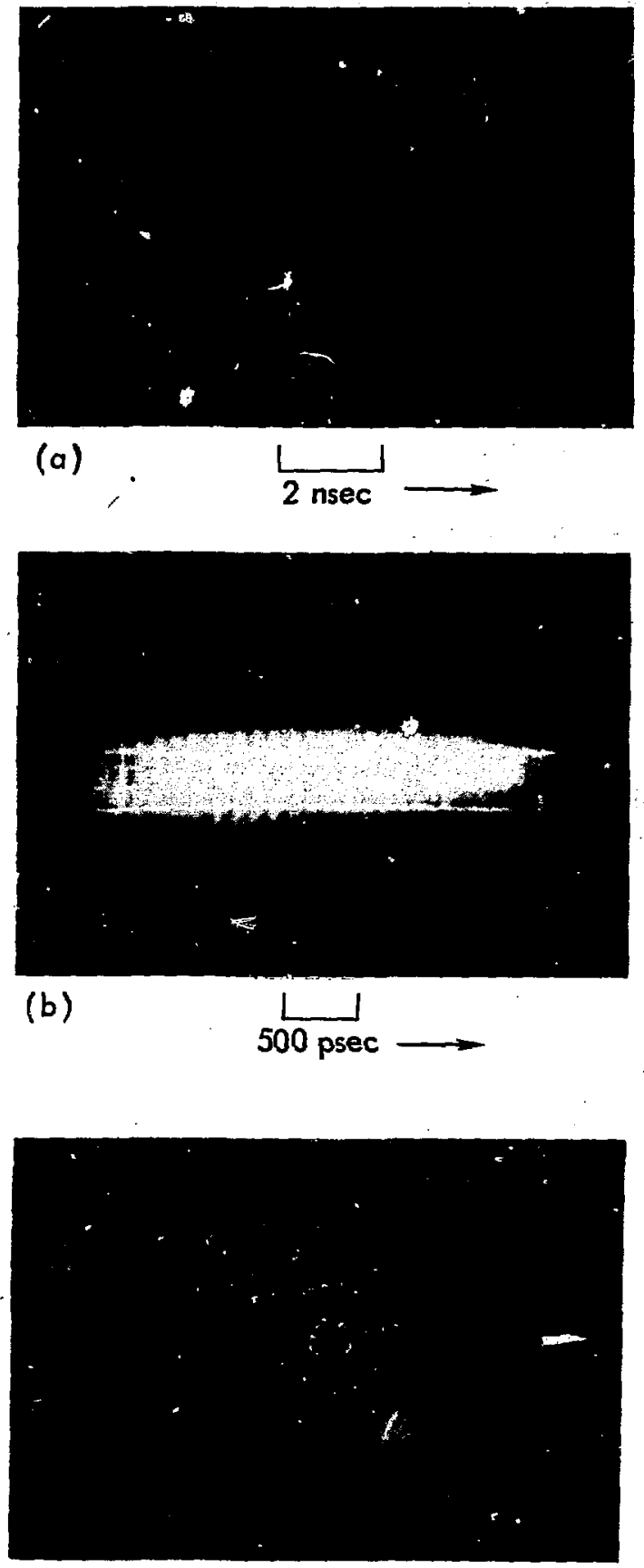

(c)

Fig. 36. Diagnosties performed on non-bandwidth-limited pulso. (a) is an oscillograph, (b) is a streak-camera trace, ard (c) is a Fabry-Perot interferogram. 


\begin{tabular}{c} 
Result obtained \\
for \\
Intrument \\
\hline
\end{tabular}

\section{Oxcilloweope \\ foudwilth $\approx 1 \mathrm{CH}$ )}

High-resolution (10-psec) stroak comere

Grating pectrogreph

( 0.1 A resolution)

Fobry-Perot interferometer (<0.01 A resolntion)
Geussinn time shere for single pulse; smooth, bell-ahaped pulse train

Smooth stredk

Single spectral line at resolution limit

Frequency width (FWHM) of $<0.018$ A for 1-nsec Guvinn pulse
No low-frequency emplitude modulation on single pulse or train (0.3 nsec resolution)

No high-frequency amplitude modulation or short-pulse substructure; microdensitometer trece of film plate confirms Gausian time shepe and provides correct half width, $e^{-2}$ values to $\pm 1 \%$

No multiline outputs or brodband frequency modulation on the pulse

Frequency width of the pulse at the uncertainty principle limit amplitude or irequency modulation. Verification of bandwidth-limited operation requires that very careful and detailed diagnostics be applied to each oscillator pulse in both the time and the frequency domains. There are two basic measurement techniques applied in each domain. The ternporal character of the pulse is determined by (1) a fast photodiode in coi:junction with a 1-GHz oscilloscope and (2) an LLL 10-psec-resolution streak camera. The frequency (or wavelength) properties of the pulse are determined by (1) a grating spectrograph and (2) a high-resolution Fabry-Perot interferometer. The measurement techniques and the expected results for a bandwidth-limited pulse are summarized in Table 8. Note that it is necessary to perform all of the measurements described in the table on the same single pulse.

Figure 35 shows the results of measurennents performed on our oscillator. Figure 35a is an oscillograph trace of a single switched-out bandwidthlimited pulse from the oscillator. The pulse duration (FWHM) in this case is 1.1 nsec, and the pulse exhibits a smooth, time-symmetric temporal dependence. Figure $35 \mathrm{~b}$ is a streak-camera photograph of the same pulse. The streak is smooth and exhibits no amplitude-modulation effects, and a microdensitometer trace of this photograph shows the pulse to be Gaussian in shape to the $e^{-2}$ points with a duration (FWHM) slightly less than 1.1 nsec.
Figure 35c is a Fabry-Perot interferogram of the pulse. The interorder spacing of the interferometer is about $0.2 \mathrm{~cm}^{-1}$ with a resolution of $<0.015 \mathrm{~cm}^{-1}$; the spectral width for the pulse is $\leqslant 0.015 \mathrm{~cm}^{-1}$, a value closely approaching the bandwidth limit for the pulse. Not shown in the figure is the spectrograp. result, which showed the pulse to consist of a single spectral line, and a pulse-train oscillograph, which exhibited a smooth, bell-shaped behavior. We interpret these results as experimental confirmation that the pulse is bandwidth-limited.

In contrast to Fig. 35, we show in Fig. 36 the results of data taken with the oscillator out of proper adjusiment. The oscillograph of Fig. 36a shows distinct asymmetry and time distortion of the pulse, the streak in Fig. 36b shows distinct amplitucle-modulation effects (the modulation period is about $50 \mathrm{psec}$ ), and Fig. 36c shows interorder rings as well as some measured line broadening for the pulse. In addition, the spectrograph data taken on this particular pulse showed the presence of some additional spectral lines with freque $y$ spacing corresponding to the inverse of the 50-psec modulation observed by the streak camera.

We are continuing our efforts to refine these measurements and to improve their accuracy in order to provide a better understanding of oscillator performance as we pursue our objective of a bandwidth-limited outpri $\hat{\imath}$ pulse of 100-psec duration. 


\section{DESIGN ANALYSIS}

\section{INTRODUCTION}

The Design Analysis Group has spent most of its effort on the design of a $1-\mathrm{kJ}$ prototype laser amplifier train. This device, with improvements, will become one arm of the multiple-laser spherical irradiation facility. The principal areas of activity involve:

1. Improving pumping efficiency and uniformity.

2. Producing a beam shape that will propagate stably and extract energy efficiently.

3. Generating detailed amplifier designs for engineering use.

In addition, some help has been given to the $\mathrm{CO}_{2}$, dye, and rare-gas laser efforts.

The most expensive portion of the projected $1-\mathrm{kJ}$ laser will be the components associated with pumping the laser: the power supplies, energy storage capacitors, high voltage switches, cabling, connectors, and flashlamps. Any increase in the efficiency of pumping is reflected in a reduction of these items, which means that increasing pumping efficiency is one of the best ways of cutting system costs. We have therefore followed a number of different paths to this end.

To begin with we have continued to improve ZAP, our large computer code which analyzes optical power flow. We have developed an automatic disk-laser input coder for ZAP, and used it for the analysis of a number of different sizes and configurations of disk lasers. The results of ZAP runs have been used to design disk amplifier parameters such as disk count, disk thickness, doping level, pump pulse duration, and required energy input.

Second, an effort has been mounted to improve the reflector geometry and lamp positioning. ZAP has not been used for this purpose because it is too slow; instead a special-purpose code has been written. This code permits a rapid search through the multidimensional space of lamp and reflector configurations in order to find the optimum,

A third pumping project has led to the creation of an approximate pumping analysis code which replaces the bank of flashlamps and their reflector by a cylindrical Lambertian radiating surface. This method has been used to estimate the effect on total pumping and pumping uniformity of the overlap of lamps beyond the end disks, of zigzag versus chevron disk positioning, and of closer-than-normal positioning of chevron disks.

Another very important area in the design of the large laser is beam propagation. This includes questions of diffraction, amplification, static and dynamic aberrations, and damage. A beam must be generated which will efficiently fill the final amplifier apertures for good energy extraction, propagate through the amplifier chain without serious shape changes, and be spatially smooth enough to avoid self-focusing damage. Four major efforts are under way in this area. First, numerical diffraction codes have been used to define a family of stably propagating, efficiently filling beams. Second, various methods of generating the required beam shapes have been analyzed, and the optimum ones chosen. Third, a code has been written to calculate aberrations of the beam as a whole; these include the effects of distortions due to the tilted amplifier disks, and the self-focusing effect of the ronlinear refractive index. Fourth, the problem of the amplification of small perturbations on a beam has been reviewed with emphasis on the implications for disk amplifier design." In addition, the well-known equations of a saturating laser amplifier have been analyzed to find their implications for spatial and temporal distortion of laser pulses. 29

In the system analysis and synthesis area, parametric studies of the effect of various system variables have been carried out using the design codes briefly described in the last semiannual report. ${ }^{30}$ The methodology behind these codes is described here. A system model code has been written which allows rapid evaluation of the performance of a set of amplifier components arrayed in space, not including diffraction anô self-focusing effects.

Smaller efforts have been carried out in two areas: (1) The Manes-Seguin five-temperature model of the $\mathrm{CO}_{2}$ laser has been extended by the addition of rotatioial and vibrational relaxations. This model will now be incorporated into an amplifier simulation code. $^{\dagger}$ (2) Dye laser cavities have been modeled on ZAP in support of the isotope separation group. Considerable improvement to the initial efficiency was made.

From the above outline of activity, it is clear that a number of powerful and complex tools are now available for lise in the computer-aided laser design area. The major emphasis at present is to integrate these methods inte a whole-problem approach to overall laser design.

\footnotetext{
* See article by Marburger et al, later in this report, in section on Basic Studies and Advanced Concepts (p. 145).

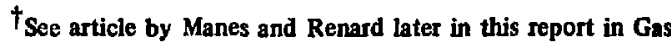
Lasers section, under $\mathrm{CO}_{2}$ Modeling (p. 125).
} 
ZAP is a Monte Carlo ray-tracing computer program that is used to determine power deposition in a laser cavity. " It can model absorptive and reflective properties of the various materials in a laser system. These materials and the boundaries that specify their location for a particular laser are defined by the input file for ZAP. ZAP reads the input file to determine the system characteristics, and then simulates the firing of randomly generated rays out of the lamps, tracing them through the system. This results in a computed distribution of the system energy deposition. The associated error is an inverse function of the square root of the number of rays used, which is directly proportional to the running time. Figure 37 shows a typical energy deposition result from ZAP.

A number of improvements have been made on ZAP since it arrived at LLL. The top-level control routine which had been patched and had evolved to a rather awkward form was restructured to run in a more

* ZAP was developed for the Naval Research Laboratory by Systems, Science and Software, Inc. (See AD-884-920.) logical and straightforward manner. A ray leg stack, which contained up to 40 different legs with up to 200 energies per leg ( 40 deep by 200 wide), was being repacked fairly often at a significant time cost. The stack control was changed so that only pointers to the leg arrays were moved instead of the whole energy arrays. This and several other changes resulted in a $20 \%$ speed improvement.

A large percentage of ZAP's future work will be modeling disk laser systems which include a number of tilted-elliptical-cylinder boundaries. This type of boundary was not available in ZAP and had to be approximated by a combination of several circular-cylinder-type boundaries. Therefore we added the capability to produce elliptical cylinder boundaries with any orientation.

Formerly much care had to be exercised in preparing the input geometry system description to avoid false boundaries with real reflective properties. Changes have been made so that any boundary hit with the same segment on either side is treated as completely transparent. Boundaries with the $\mathrm{s}^{*}$. material on either side may be given any pror desired. If

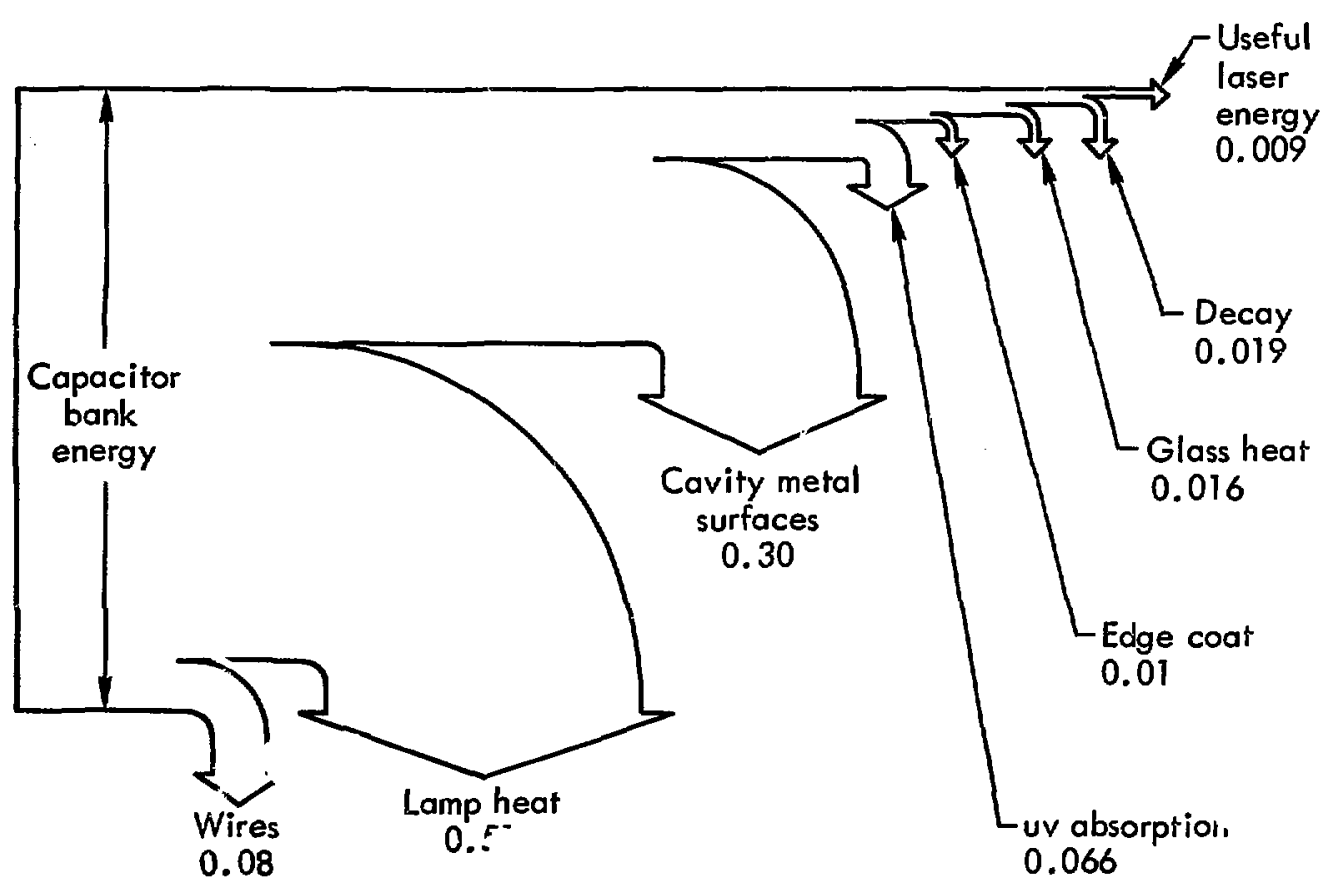

Fig. 37. Power tlow diagram of a typical disk-laser system simulated by ZAP. 

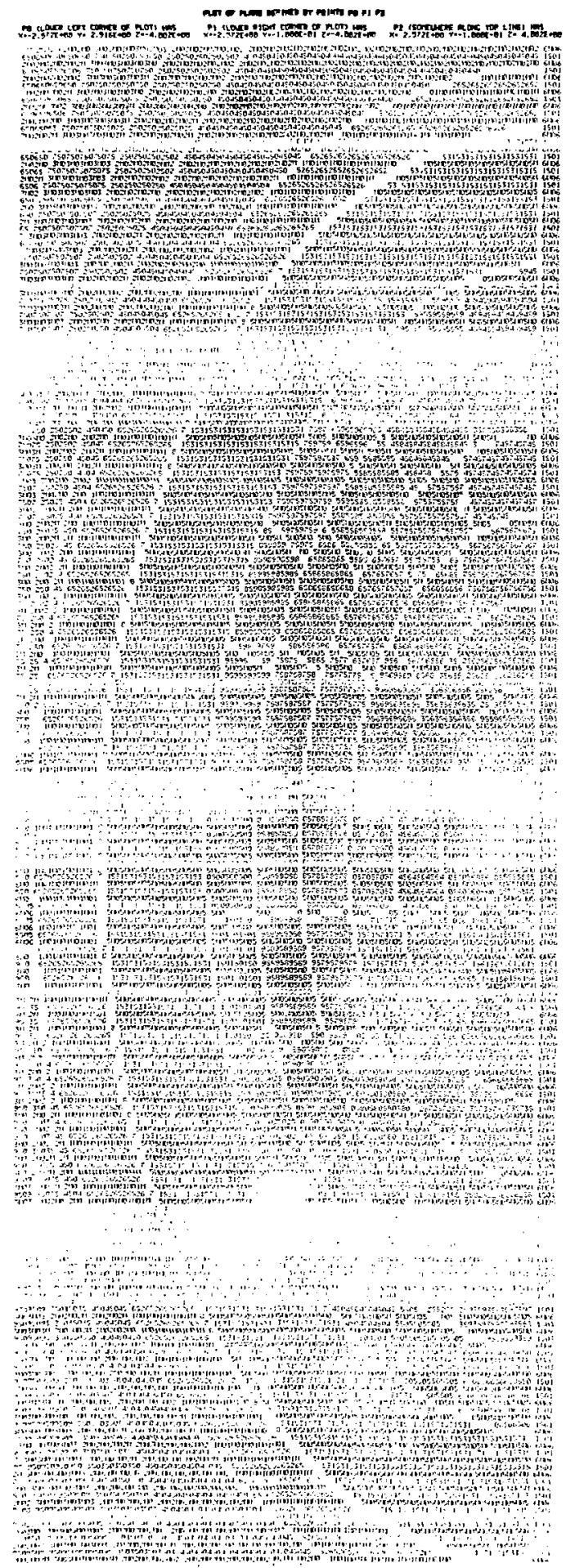

certain materials in a system should never touch, this can now be specified and the program will halt and give an error printout if the materials are found to be touching due to an input error. Problems involving inaccurate computation of the position of certain boundaries are being automatically detected and recorded so they can be studied. Since these problems only occur in about 1 out of 100,000 legs, and since the program has been fixed to correctly racover from most of them, this is not an urgent matter.

To help diagnose ZAP operation, two options have been added. One option prints out the absorption arrays used for each of the materials. The other option prints out the entire deposition spectrum for a particular 'material. With these diagnostic printouts, it is very easy to find mistakes in abs orption or emission input.

Release of ZAP to other groups has revealed many ways in which input mistakes can be made that will cause the program to crash without an explanation of the error. A number of checks have been added to the input routines to catch these errors at their source, thereby making it easier to leam to use ZAP.

The biggest problem remaining, now that computer time allocation has been increased, is to provide fast, correct input file generation. For the disk laser geometries we e..pect to use most in the future, a 1500-line input file generator program has been written. This interactive program operates in the following manner. First the basic geometry type is entered; then the program asks for various physical size parameters, and uses them to calculate and retum resulting overall sizes, gaps between lamps, and apertures. Based on these retumed sizes, the operator can either continue with the design or change a value. When all values have been okayed, a complete and fairly optimal input file is produced for ZAP. Zoning options available include segmenting half the disk into up to 49 equal-volume segments. This segmentation is shown in Fig. 38. The amount of three-dimensional translation and rotation needed to produce this zoning would be practically impossible to do correctly in a finite amount of time without the aid of a computer. Wie generator can now produce an $\boldsymbol{A}$-module input file and is being modified to be able to produce a $B$-, $C$, or $D$-module input file.

\footnotetext{
${ }^{*} A, B, C$, and $D$ are the designations of the four amplifier stages of the $1-k$; laser.

Fig. 38. ZAP plot of the $A$-module disk laser showing half of the disk zoned into 49 equal-volume segments for deposition distribution studies.
} 


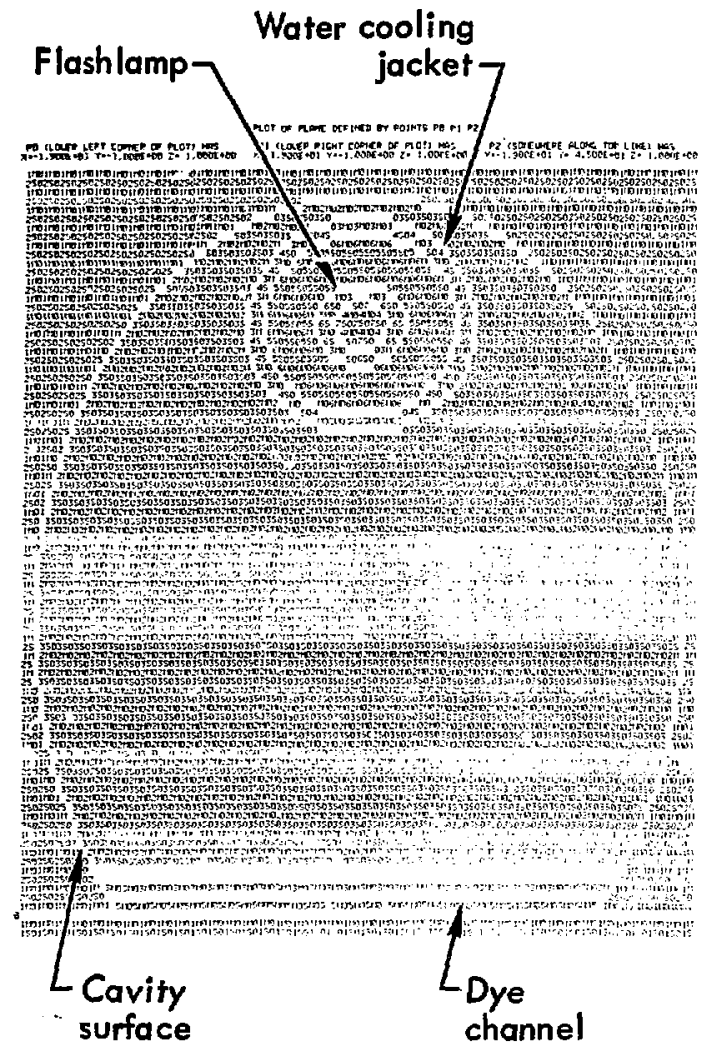

ZAP has been used for various cavity designs. Extensive test runs have been performed on a system intermediaze in size between the $C$ and $D$ modules to determine approximate expected efficiencies for various laser glass dopings and lamp currents. A very detailed model for the $A$ module has been made which effectively divides the laser disk into 100 segments, and a very long run with this model has produced a detailed pumping profile over the disk. A number of runs have been made with the NRL system geometry to determine the effect of the overhang of the lamps bey ond the end disks on the pumping profile. As shown in Fig. 39, a dye laser cavity has been modeled. By varying the position and type of materials in the cavity, it was possible to increase the calculated cavity efficiency from 3.6 to $7.3 \%$.

Most of ZAP's present run time is spent on modeling the larger amplifiers in the $1-\mathrm{kJ}$ chain. The output is converted into design charts which are used for selecting the correct doping and thickness of the disks.

Fig. 39. ZAP plot of an elliptical dye-laser cavity with a cylindrical lamp at the upper focus and a dye channel at the lower focus.

\section{COMPUTER OPTIMIZATION OF FLASHLAMP REFLECTORS}

One problem in building large-scale laser systems is the expense of the huge capacitor banks required to store energy for the flashlamps. The obvious approach in attacking this problem is to increase the pumping efficiency, thereby reducing energy requirements and, accordingly, capacitor requirements. Pumping efficiency may be optimized by reflector changes and by modification of flashlamp count and loading. $A$ computer program was therefore written which computes efficiencies of various laser configurations. The following simplifications of the actual problem were made for tractability and speed of computation:

1. A two-dimensional calculation was used since end effects are small and since ray paths in skew planes are the same as those in nonskew planes except for a change in lamp opacity.

2. The quartz envelope around the flashlamp plasma is omitted since the direction and strength of rays are only slightly affected by the envelope.

3. The cross section of a reflector segment is limited to symmetric portions of ellipses. This gives a wide variety of shapes that are practical because they are easily machinable; more complex shapes than this are íess likely to be used.

4. Only a single ellipse segment per lamp is normally allowed (alsc for ease of machinability).

5. Wavelength variations in flashlamp output and opacity are approximated by varying the lamp opacity and considering only a single wavelength at a time.

6. The Monte Carlo method is used to trace rays from the flashlamp model around the reflector cavity and (hopefully) to the target diameter of the lasing material. In their fight the rays may be lost by reflector absorption or lamp reabsorption.

The program was written to allow input of various parameters describing such a lamp/reflector/laser model. One output from these calculations consists of a computer-drawn pie-slice picture of the model and numbers such as relative pumping and efficiency. An example of this output is shown in Fig. 40.

As a first step, we assume some number and size of flashlamps and then determine the best reflector 


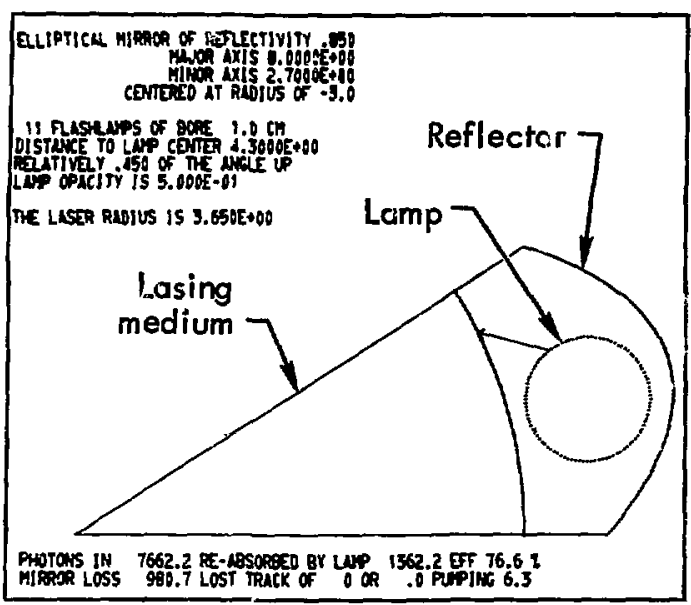

configuration. This involves a five-dimensional optimization:

1. The ellipse's major axis.

2. The ellipse's minor axis (this and the major axis define the shape and size of the reflector segment associated with one lamp).

3. The radial distance from the laser centerline to the reflector.

4. The radial distance from the laser centedine to the lamps.

5. The angle of the $-s$ relative to the reflector.

Fig. 40. Computer-generated picture of a pie-slice from the cross section of a typical leser-crvity configuration.

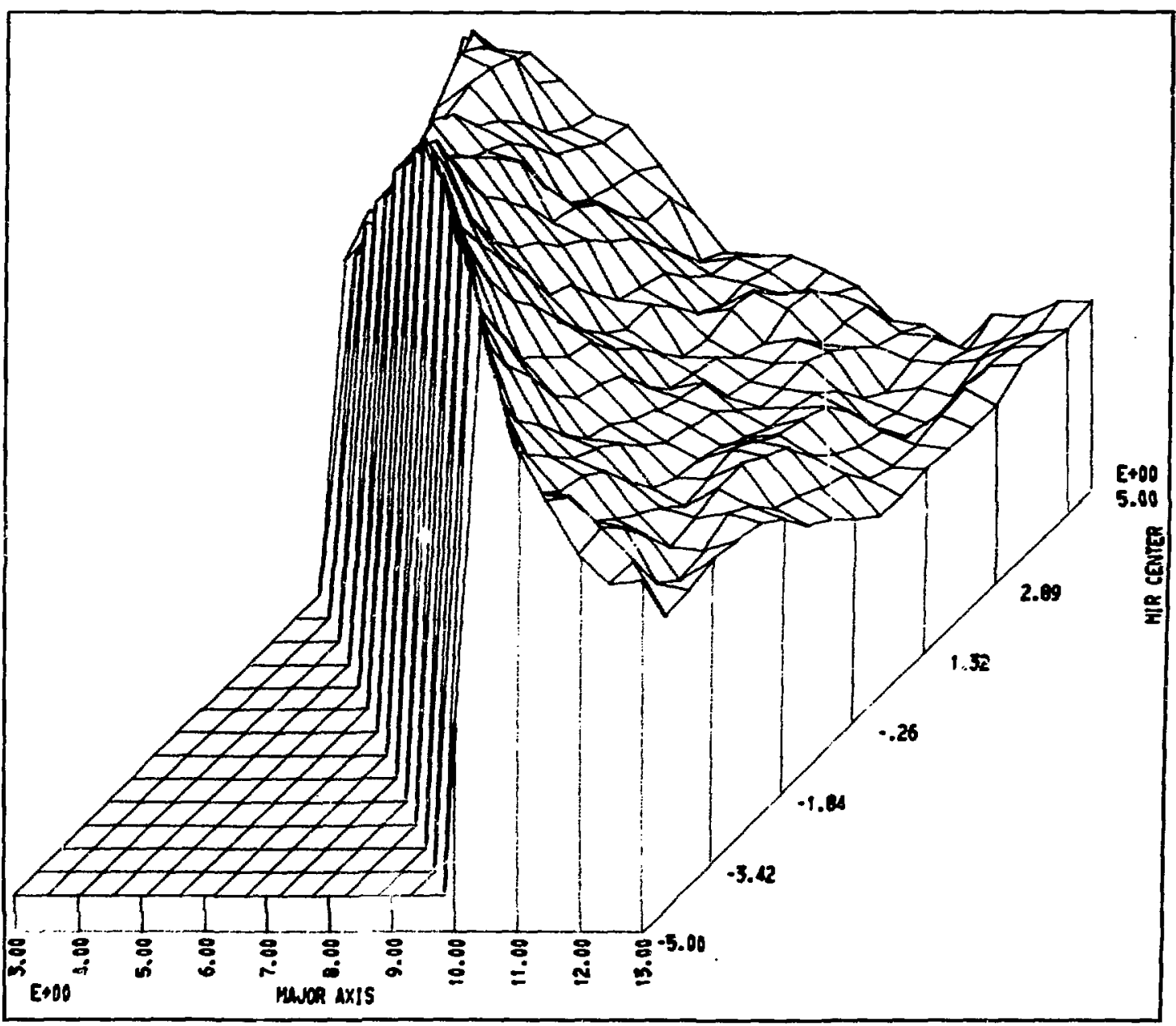

Fig. 41. Computer-generated threedimensional sketch of the efficiency (height) a a function of two of tho variable parametera (ellipe major axis and reflector centerline position in this case). 
An iterative approach is used, varying two of the five pararneters at a time and plotting a threedimensional graph of efficiency as a function of the two parameters (an example is shown in Fig. 41). Then the two parameters for that maximum point are held constant and two others are varied. This is repeated in an automatic way until the fivedimensional optimum is reached. Calculations were made for a typical system with a low flashlamp plasma opacity, and for one with a high opacity. The results in the low-opacity case show an improvement of $29 \%$ over those for a cylindrical reflector. A few other reflector shapes were also calculated, such as parabolas, dual ellipses with lamps at alternate cusps, and ellipses with blinder mirrors between lamps. None of these was better than an elliptical shape.

The second step toward the solution to the problem of optimum pumping is to vary the lamp count and size for best results while holding the input loading fixed. This is done by dividing a constant energy among the lamps with the constraint that the energy per lamp will not explode it, and by varying the lamp opacity appropriately as the diameter of the lamp and the current density change. This lamp count and size optimization will require an extensive parametric study for each change in lamp count and for various lamp sizes, following the methods described in step 1 above. Such a study is currently in progress.

\section{THE LAMBERTIAN-TUBE LASER PUMPING APPROXIMATION}

A method of pumping analysis that is more rapid than a detailed ray-tracing power flow computation is needed for initial evaluation of various disk laser amplifiers. For this purpose, a method which replaces the actual flashlamp geometry with a simple one is being used. The pumping is assumed to come uniformly from the inside of a cylindrical shell or tube surrounding the disks; the pumping is emitted in a Lambertian pattern (i.e., as from an ideally rough surface).
Other simplifying assumptions are usually made to speed the calculation:

1. The disks absorb radiation equally from all incoming angles. Fresnel losses at the surface and varying path lengths in the disks are ignored.

2. The disks absorb all the pump light, so that disks are not pumped from the direction of other disks (this means reflections are alse ignored).

3. There are no geometrical shadowing effects due to the finite thickness of the disks.

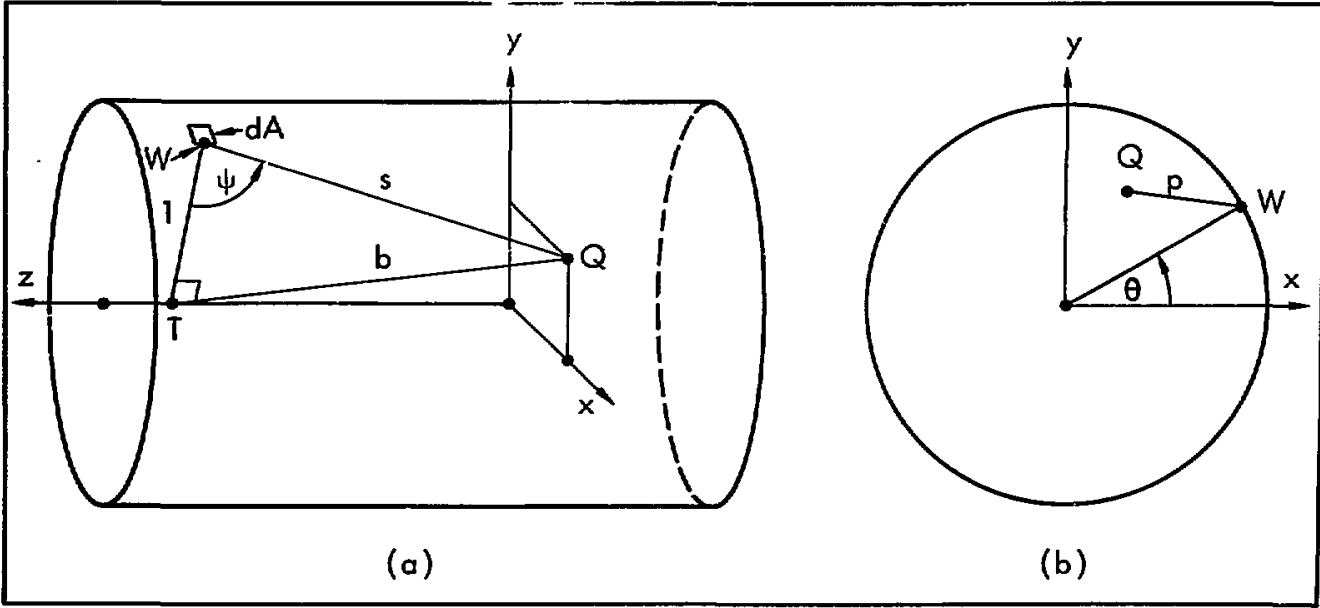

Fig. 42. Geometry of the Lambertian pumping approximation. (a) Radiation is emitted in a Lambertian pattern from the inner surface of a cylinder of unit radius. The observation point $Q$ inside the cylinder lies a distance $s$ from an emitting elf ment $W$ on the surface. The line from $Q$ to $W$ makes an angfe $\psi$ with the normal to the inner surface at $W ; \psi$ ralay be found from the triangle WTQ, with sides unity, $s$, and $b$. (b) View along the cylinder axis. The projection $p$ of the distance $s$ (Fig 42a) is easily calculated. The axial distance $z$ and $p$ form two sides of a right triangle from which $s$ may be found. 
4. For convenience, the disks are inclined at $60^{\circ}$, rather than at Brewster's angle.

With these assumptions, the problem of calculating the pumping reduces to finding the solid angle of pumping surface seen from an arbitrary point on the surface of a disk. This may be done by integrating solid angle elements over the inside of the pumping tube. We now proceed to find the solid angle elements and the appropriate limits of integration.

Consider a Lambertian pumping tube of unity radius (Fig. 42a). Introduce a rectangular coordinate system with the $z$-axis along the tube axis. The illuminated point $Q$ is located at coordinates $(x, y)$ with $z=0$. An element of tube surface of point $W$ of area $d A$ is a distance $s$ from the observation point; the line from $W$ to $Q$ makes an angle $\psi$ with the inner normal to the tube. The element of solid angle is

$$
d \Omega=\frac{d A \cos \psi}{s^{2}} .
$$

The distance $s$ is found as the hypotenuse of a right triangle. The sides are the axial distance $z$ from $W$ to $Q$, and the projection $p$ of $s$ on the $x-y$ plane (Fig. $42 \mathrm{~b}$ ). If the source point $W$ is at an angle $\theta$ from the $x$-aris, then

$$
p^{2}=(\cos \theta-x)^{2}+(\sin \theta \cdot y)^{2}
$$

and so

$$
s^{2}=z^{2}+(\cos \theta-x)^{2}+(\sin \theta-y)^{2} .
$$

The $\cos \psi$ term is found by considering the triangle formed by $W, Q$, and the point $T$ on the $z$-axis at the same distance as $W$ (Fig. 42a). The distance $b$ from $T$ to $Q$ is given by

$$
b^{2}=x^{2}+y^{2}+z^{2},
$$

and we must have

$$
b^{2}=s^{2}+1 \cdot 2 s \cos \psi,
$$

so that

$$
\cos \psi=\frac{1 \pm s^{2} \cdot x^{2} \cdot y^{2} \cdot z^{2}}{2 s} .
$$

Since the element of area is $d A=d \theta d z$, we have

$$
d \Omega=\frac{1}{2}\left\{\frac{1+(\cos \theta-x)^{2}+(\sin \theta-y)^{2}-x^{2}-y^{2}}{\left[z^{2}+(\cos \theta-x)^{2}+(\sin \theta-y)^{2}\right]^{3 / 2}}\right\} d \theta d z .
$$

The fraction of sphere seen is $d f=d \Omega / 4 \pi$, so that

$d f=\frac{1}{4 \pi} \frac{1-x \cos \theta-y \sin \theta}{\left[(\cos \theta-x)^{2}+(\sin \theta-y)^{2}+z^{2}\right]^{3 / 2}} d \theta d z$.

The fraction of the maximum possible pumping which our disk point sees is then

$$
f=\frac{1}{4 \pi} \int_{z_{l o}}^{z_{h i}} d z
$$

$$
\times \int_{0}^{2 \pi} d \theta \frac{1-x \cos \theta \cdot y \sin \theta}{\left[(\cos \theta \cdot x)^{2}+(\sin \theta-y)^{2}+z^{2}\right]^{3 / 2}}
$$

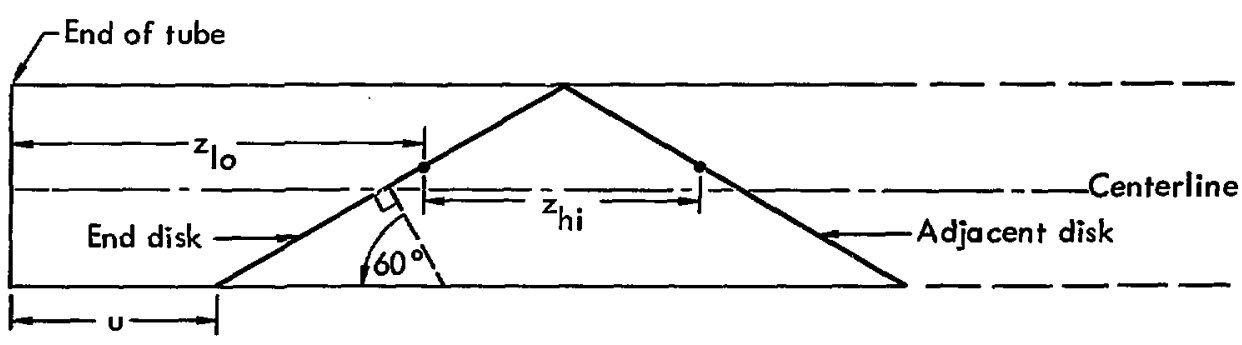

Fig. 43. Calculating the limits of integration in the Lambertien tube. Here the lower limit is to the end of the pumping tube, whie the upper limit is to the adjacent disk. 


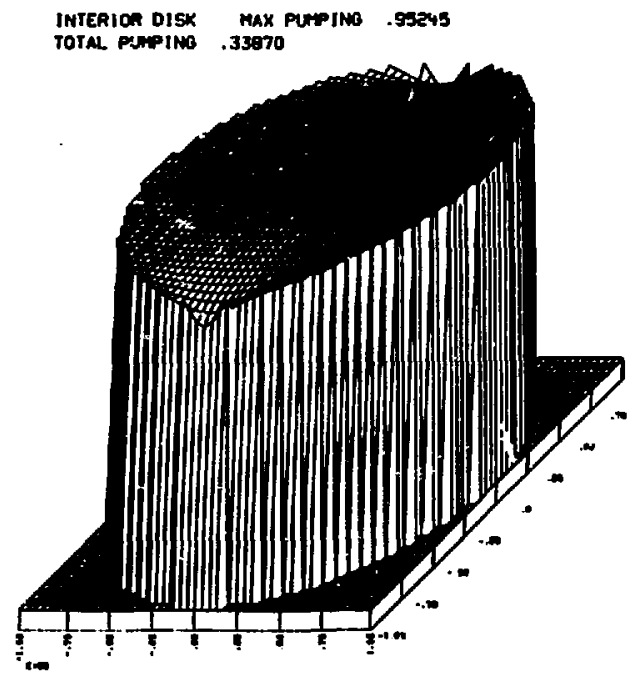

(a)

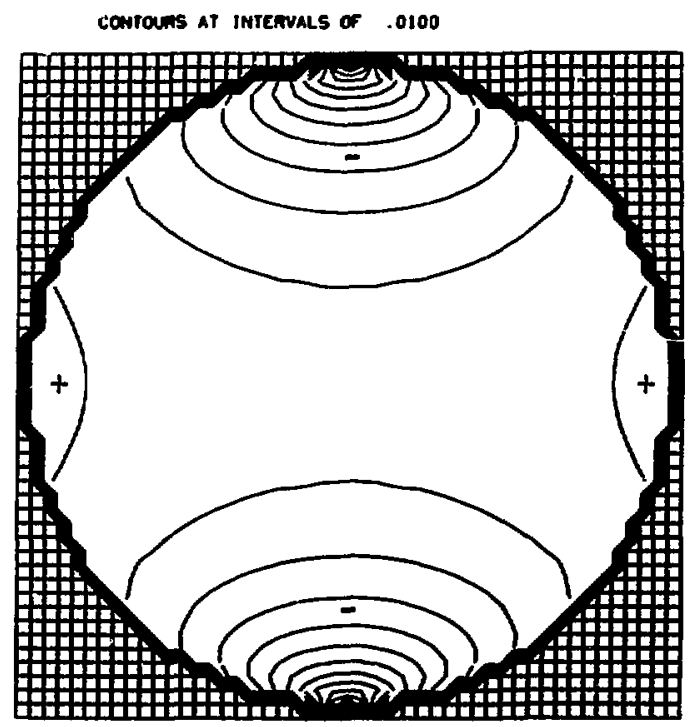

(b)

Fip. 44. Computer plots of pumping for an interior diak, with the disk in a zipag arangement. (a) Three-dinescioncl piot. (b) Conionr plot. Contours are at $1 \%$ intervaly, and the relatively andil number of contou in indicales nea uniformity of pumping. Flus or minus tips indicate regions of relatively hijh or low pumping.

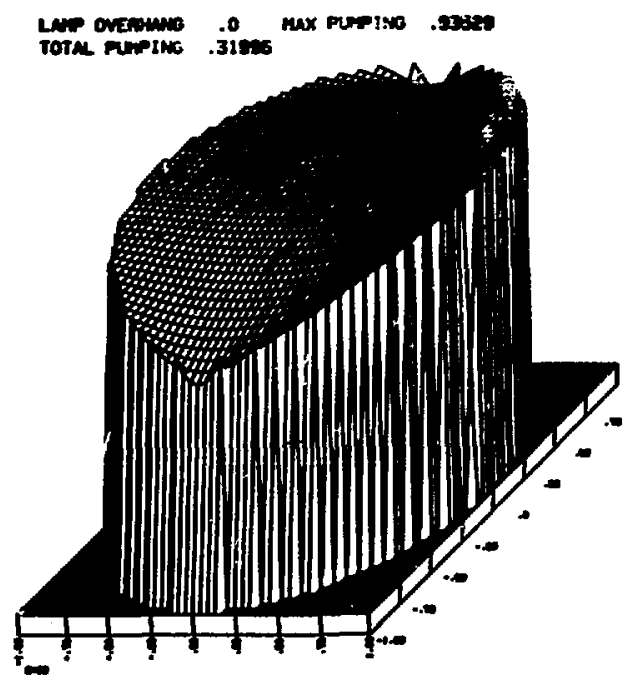

(a)
CONTOUAS AT :WIERYALS Of .0100

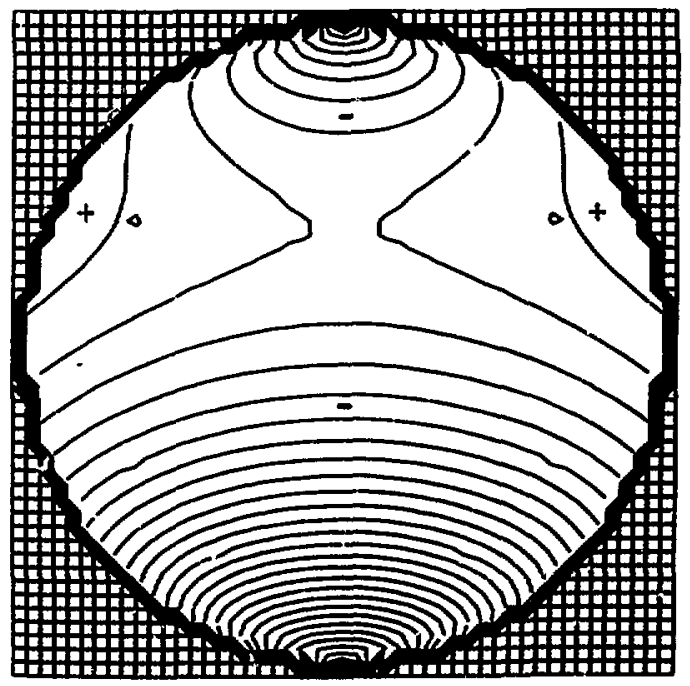

(b)

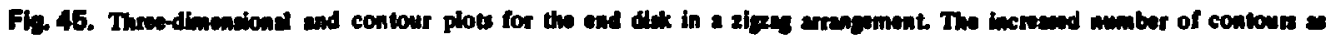
compand with Fie 44 mesas poorer uniformity of pumping in this and ditk es compand with the iatorior disk. 

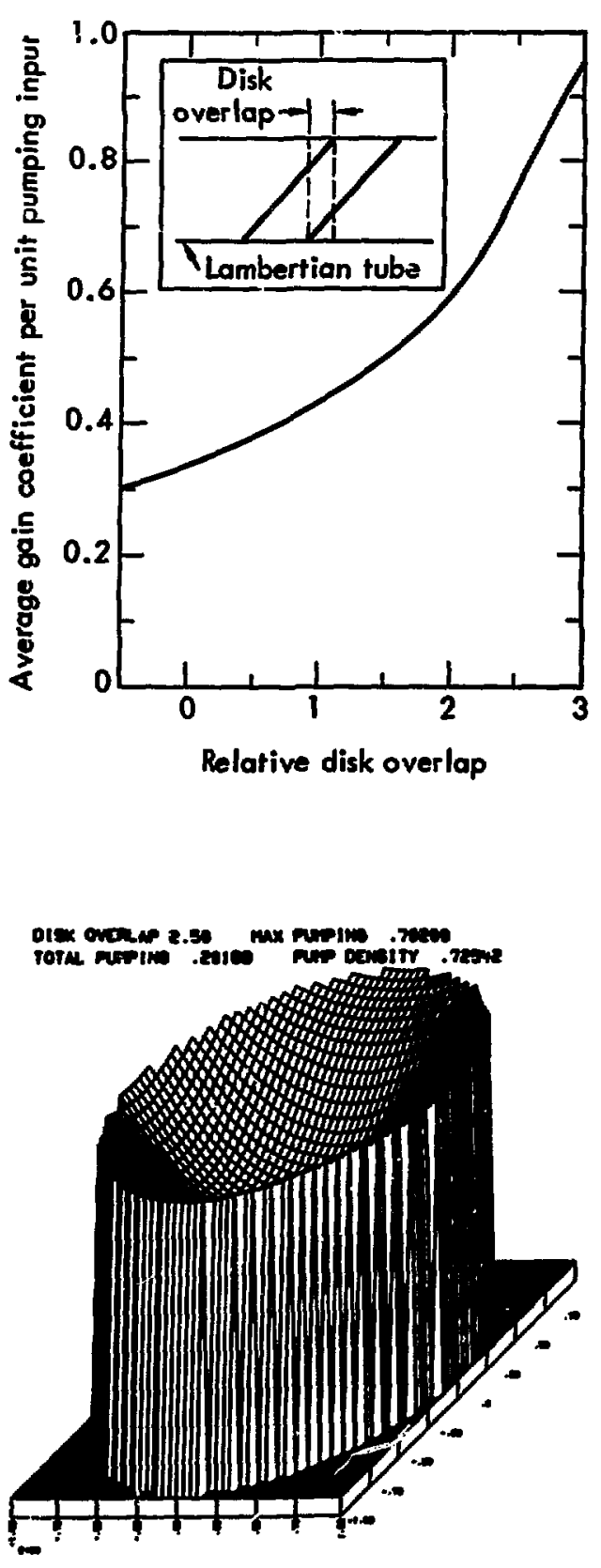

(a)
The integral over $z$ is in atandard tables ${ }^{31}$; we are left with only one integration:

$$
\begin{aligned}
& f=\frac{1}{4 \pi} \int_{0}^{2 \pi} d \theta\left(\frac{1 \cdot x \cos \theta \cdot y \sin \theta}{G}\right) \\
& \times\left(\frac{z_{h l}}{\sqrt{G+z_{h t}^{2}}} \cdot \frac{z_{l o}}{\sqrt{G+2_{l o}^{2}}}\right)
\end{aligned}
$$

where

$$
G=(\cos \theta \cdot x)^{2}+(\sin \theta-y)^{2} .
$$

The integration over $\theta$ is done numerically by adaptive Simpson integration. ${ }^{32}$

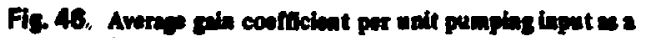

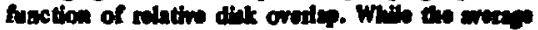

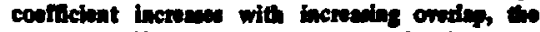

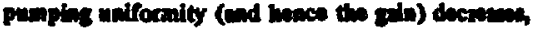
at dhow in the next Igare.

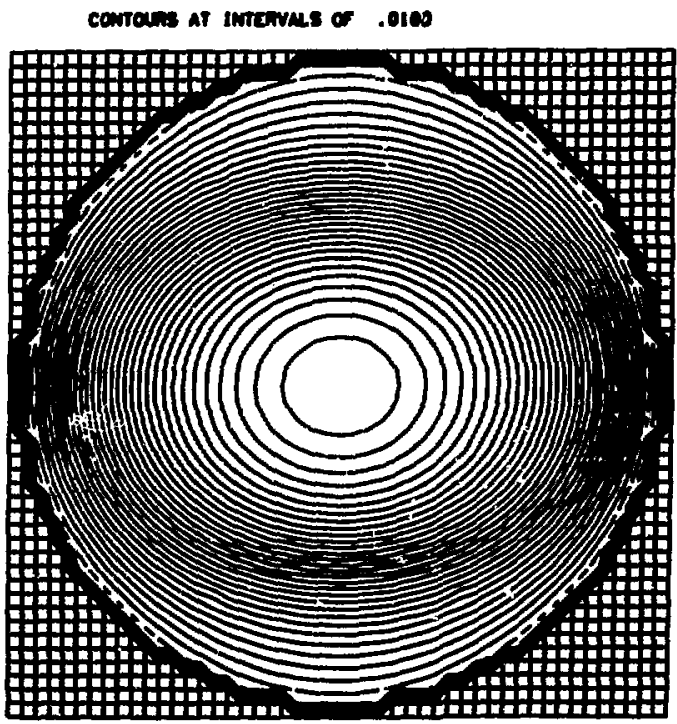

(b)

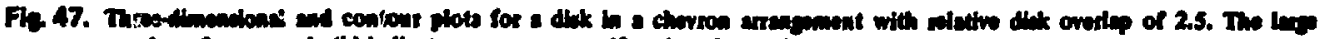

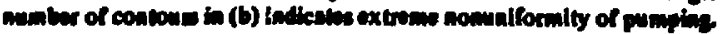


The $z$ limits ( $z_{l o}$ and $z_{h i}$ ) depend on the specific geometry being considered. Suppose, for example, we have a disk at the end of a multidisk laser amplifier (Fig. 43). If the pumping tube overhang the disk by a distance $u$, then

$$
z_{10}=u \cdot \sqrt{3(1+y)}
$$

since the tilt of the disk varies $z_{l o}$ as $y$ varies (the factor of $\sqrt{3}$ comes from using $60^{\circ}$ rather than Brewster's angle). The distance to the next disk depends on $\theta$; it is given by

$$
z_{h i}=\sqrt{3}(2 \cdot \sin \theta \cdot y)
$$

when the disks alternate in angle (zigzag configuration), and by

$$
z_{h i}=\sqrt{3}(2+\sin \theta \cdot y)
$$

when they are all at the same angle (chevron configuration).

Results of the computation outlined above are shown in Fig. $\mathbf{4 4}$ for a disk between two altemating disks, and in Fig. 45 for a disk at the end of the pumping cavity. In order to find the approximate effect of overlapping chevron disks, the pumping was found as a function of overlap (Fig. 46). However, the disadvantage of increasing the overlap is the increasing pump nonuniformity which results, as shown in Fig. 47.

In summary, the Lambertian-tube pumping approximation is a useful way to quickly get approximate pumping distributions. Once the desired result has been achieved, it can be checked in detail by more exact but time-consuming methods, such as ZAP calculations. Efforts are now under way to compare the Lambertian tube approximation with ZAP and with experiments on disk amplifiers in order to determine its accuracy.

\section{A USER-ORIENTED AXIALLY SYMMETRIC DIFFRACTION CODE}

A computer code to calculate axially symmetric diffraction has been written. The code makes the asumptions (discussed by Campbell and DeShazer ${ }^{33}$ ) that the observation point is many wavelengths from the initial plane, and that the angle of observation from the axis and the subtended angle of the initial aperture from the observation point are small. With these limitations, approximations can be made that allow rapid computation of the diffraction pattems.

The code was originally set up to analyze double-aperture beam shaping schemes. It thus starts with a Gaussian beam which is specified by its waist position and size. An aperture is placed in the beam, which is then propagated to another aperture. The amplitude and phase of the diffracted beam from the first aperture are then used as input to the diffraction calculation for the second aperture, including the phase factor $\exp (i k r)$ left out by Campbell and DeShazer. This procedure is not strictly proper, mathematicaily spesking, but it gives results in good agreement with experiments. After the second aperture, snapshots of the beam may be taken at various distances.

The code is written so that the user may enter the specifics of a given problem from the Teletype (Fig. 48). The output is a series of pictures showing the beam at the first aperture, second aperture, and Il requested snapshot points (Fig. 49). The user may view these pictures to see if he has achieved the desired result. If changes are needed, another run is easily made.
Another version of the code has been written which automatically produces a series of runs, with a parameter varied between runs. The parameter may be used to set any variable desired (for example, aperture size or spacing) to a series of specified values. This version allows rapid selection of specific values to achieve a desired result. In addition, the code has been changed by various users to allow the input of beam profiles other than Gaussian, and to modify the layout of elements. It has turned out to be a useful and popular code.

Fig. 48. User-computer interaction to et up a diffaction problem. The computer requests for input are completely selfexplanatory.

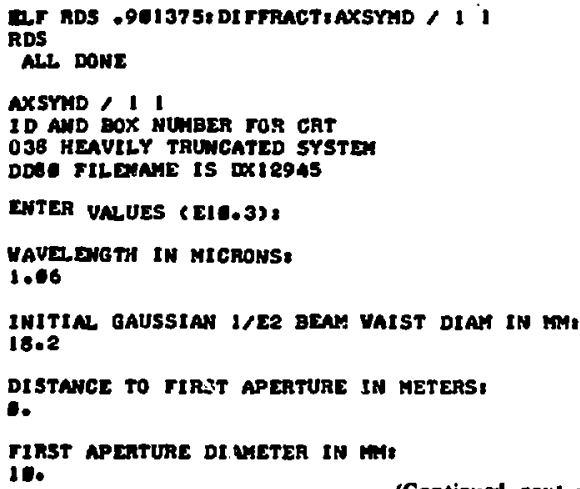

ENTER UNUES (EIC.3):

MAVELDUTH IN MTCRONS:

1.06

INITIN GAUSSIAN I/E2 BCAY UAIST DIAH IN ma 18.2

DISTANCE TO TIRST APERTURE IN METERS,

๑.

FIRST APERTURE DI METER IN MA 10.

(Continued nexl page) 


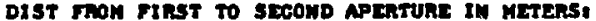

Jocs

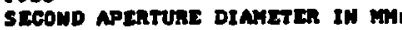

19.7

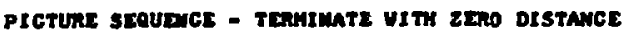

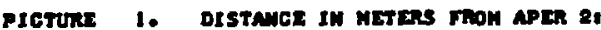

10399

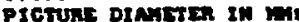

13.

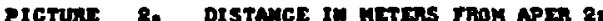

C.

at. Don:

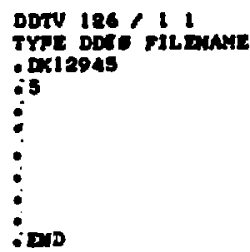

ate bons
Beam at first aperture
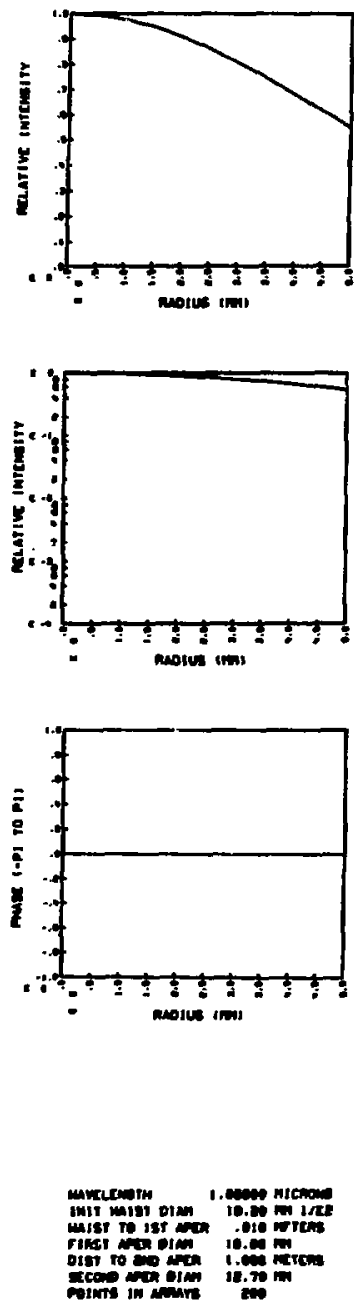

Beam at second aperture
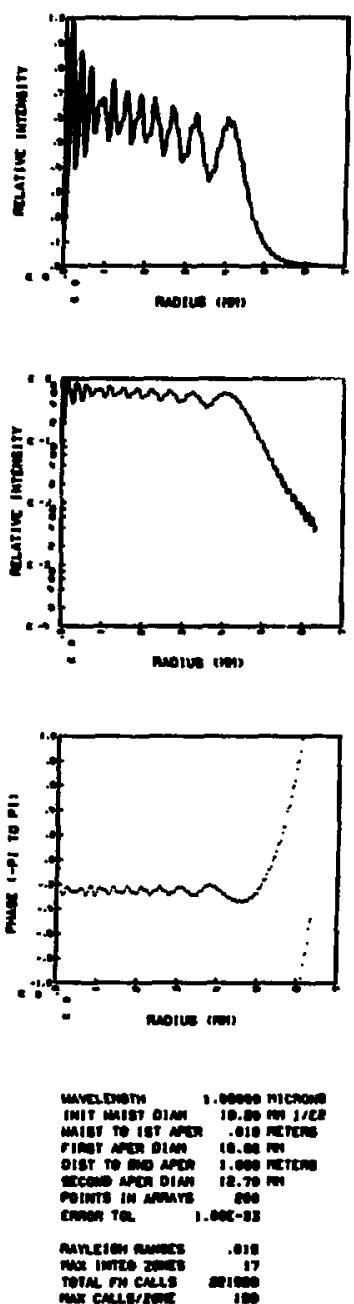

Beam $1.329 \mathrm{~m}$ from second aperture
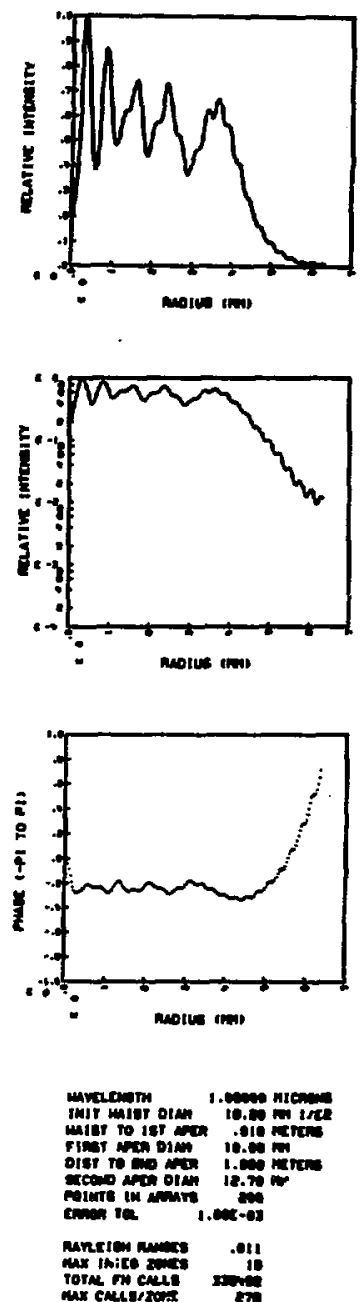

Fis. 48. Cuculated difraction for the computer gun shown in Fig. 48. From top to bottom, the plots are of linear bean intendity, log besm intersity, and phase. System description and diapnostic information is printed out sloss with the plots. 
The laser beam to be injected into the $1 \cdot \mathrm{kJ}$ glass Jeer system must have a radial energy-density profile (i.e., energy density profile through the beam's cross section) that minimizes diffraction effects, to avoid self-focusing, while at the same time allowing maximum extraction of energy. These two requirements are interrelated, but we will discuss them separately at first for the sake of clarity.

Diffrection effects fall into two categories. The first involves the general evolution of the beam's radial profile into its far-field diffraction pattern or Fourier transform; since the Fourier transform is unique, each beam profile will evolve into its own unique diffraction pattern if allowed to propagate unaltered. The second category is the cutoff effect which results from passing the beam through an aperture to remove the lower-intensity edge portions. The effect of the aperture is to convolve the unaltered diffraction pattem with high frequency fluctuations across the entive beam

The two diffraction effects are illustrated in Fig. 50. We have empirically demonstrated that if the beam is

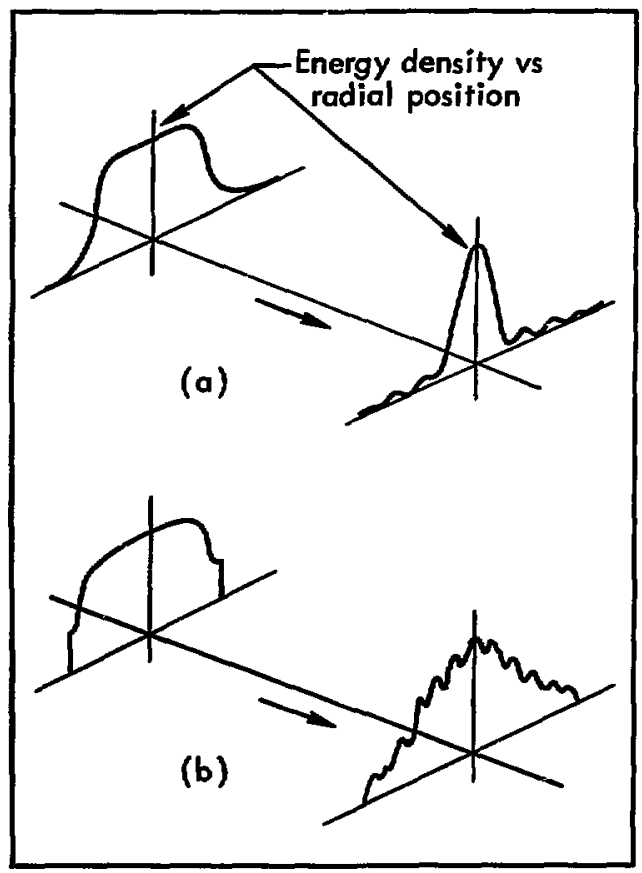

Fly. 50. (a) Cenerd evolution of beem dification (uncperwind. (b) Difinction effects produced by apertar he the beam.

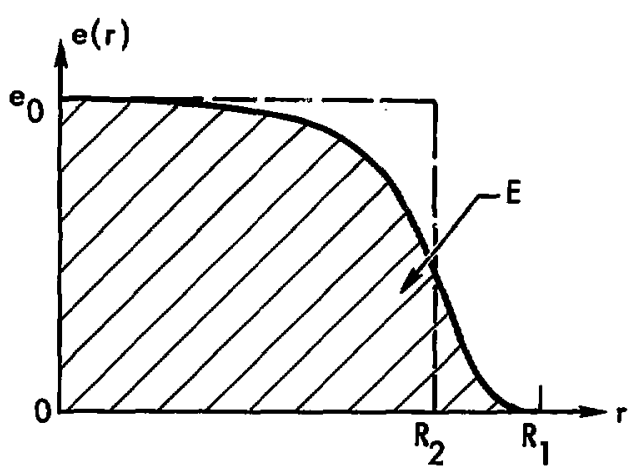

Fig. 51. Fill factor is defined $x\left(R_{1} / R_{2}\right)^{2}$.

apertured so as to cut off portions whose intensity is below $K$, which is a specified fraction of the beam's maximum intensity (for example, $K$ might be 0.1 of the maximum intensity), then the peak-to-peak magnitude of the diffraction ripple or fluctuations will be on the order of $40 \sqrt{K} \%$ for the propagation distance required in the $1 \mathrm{~kJ}$ glass system.

As for maximizing extraction of energy from the beam, we show in Fig. 51 a general spatial profile of a laser beam. The energy density on axis is denoted by $e_{0}$ and the total energy of the pulse, integrated to some radius $R_{1}$, is denoted by $E$. The fill factor of this beam is defined as

$$
\text { fill factor }=\left(\frac{R_{1}}{R_{2}}\right)^{2} \text {, }
$$

where $R_{2}$ is the radius value for which

$$
E=\pi R_{2}^{2} e_{0}
$$

Note that $R_{1}$ is chosen such that the corresponding intensity level $K\left(K=e\left(R_{1}\right)\right)$ produces cutoff diffraction effects that are negligible.

The fill factor is a measure of the extra cross-sectional area of a general laser beam compared to a square-wave beam of the same on-axis energy density. A smaller fill factor permits smaller-aperture laser components, thus lowering the cost substantially. The 1-kJ glass laser system has been designed with a fill factor of 1.5. 
The Ideal Beum Profile

For the $1 \cdot k J$ laser the beam through the disk amplifier system must have a fill factor of 1.5 (as noted above) and must have a radial profile such that it can propagate $40-60 \mathrm{~m}$ with diffraction ripple kept to less than $2 \%$ to avoid selffocusing difficulties. ${ }^{34}$ Our previous remarks on diffraction cut off effects indicate that if the beam is apertured so as to truncate the radial intensity profile at $K=0.0 .01$, then the diffraction ripple will indeed be less th:an $2 \%$. Thus, we will require an ideal beam profile to be such that a value of $R_{1}$ can be found for which

$$
e(r)<0.001 \text { for all } r>R_{1} \text {. }
$$

In addition to the aperture effects the beam must have a profile which when allowed to propagate unapertured will retain its shape and not experience diffraction fluctuations that exceed this $2 \%$. As will be discussed later, lenses and lens systems can be used to control the diffraction evolution of a beam. Here we only point out this fact and thus do not set any minimum propagation distance sequirements.

It is well known that an unapertured Gaussian beam will propagate indefinitely while retaining its radial profile, since the Fourier transform of a Gaussian is still a Gaussian. A Gaussian beam can be described mathematically as

$$
e(r)=\exp \left[-\left(r / r_{e}\right)^{2}\right]
$$

As can be seen

$$
\operatorname{limit}_{r \rightarrow \infty} e(r)=0 \text {, }
$$

and

$$
e(r)<0.001 \text { for all } r>R_{1}=2.628 r_{e} \text {. }
$$

However, the fill factor of this Gaussian to $R_{1}$ equals 6.4, which is much too large.

A square-wave or flat-top pulse, on the other hand, hes the ideal fill factor of unity but suffers from disatrous diffraction breakup effects. A flat-top pulse can be described mathematically as

$$
e(r)=\operatorname{limit}_{p \rightarrow \infty} \exp \left[-\left(r / r_{e}\right)^{p}\right]
$$

The previous remarks on Gaussian and square beam profiles indicate that a beam profile described by

$$
e(r)=\exp \left[-\left(r / r_{a}\right)^{N}\right]
$$

will, for some value of $\boldsymbol{N}$ between 2 and infinity, result in a radial beam profile which will be a reascanble compromise between fill factor (extraction effects) and diffraction effects. This type of beam profile we denote as a WT profile of power $N$.

The propagation of this WT profile was studied for various powers of $N$ using our diffraction computer codes. ${ }^{35^{*}}$ The beam was propagated various distances and the diffraction patterns noted. A safe propagation distance $d_{s}$ was determined to be the distance at which the natural diffraction fluctuations were less than approximately $2 \%$ (in intensity). A tvpical code output is shown in Fig. S2. In the diffraction equation the Fresnel kernel, $\exp \left(\pi r^{2} / \lambda d\right)$, multiplies the optical disturbance function. ${ }^{36}$ Thus, we use $d_{g} / R_{a}^{2}$ to normalize our results and obtain a safe propagation factor $d_{f}$. This factor $d_{f}$ is plotted vs profile power $N$ in Fig. 53. Also plotted in Fig. 53 is the fill factor to $R_{1}$, where $R_{1}$ is such that $e\left(R_{1}\right)=0.001$. As can be seen in this figure, $N$ must be greater than or equal to 10 for fill factor of 1.5 or smaller. On the other hand, as the value of $N$ increases, the safe propagation factor $d_{f}$ decreases. Since we desire $d_{f}$ to be as large as possible and still maintain a fill factor of 1.5, the optimal value of $N$ is 10 . Thus, based on a fill factor of 1.5 and a maximum propagation distance before diffraction breakup, we determine the ideal beam profile to be a 10-power WT aperture described by

$$
e(r)=\exp \left[-\left(r / r_{a}\right)^{10}\right]
$$

For this profile a value of $R_{1}=1.22 r_{a}$ guarantees that $e(r)<0.001$ for all $r>R_{1}$. Also, the safe propagation factor $d_{f}$ is equal to $1500 \mathrm{~cm}^{-1}$.

\section{Effect of a Nepative Lem on Proparation}

From a geometrical-optics point of view, lenses are used to control the size and divergence of a

\footnotetext{
"See also the article immediately precedins this one.
} 


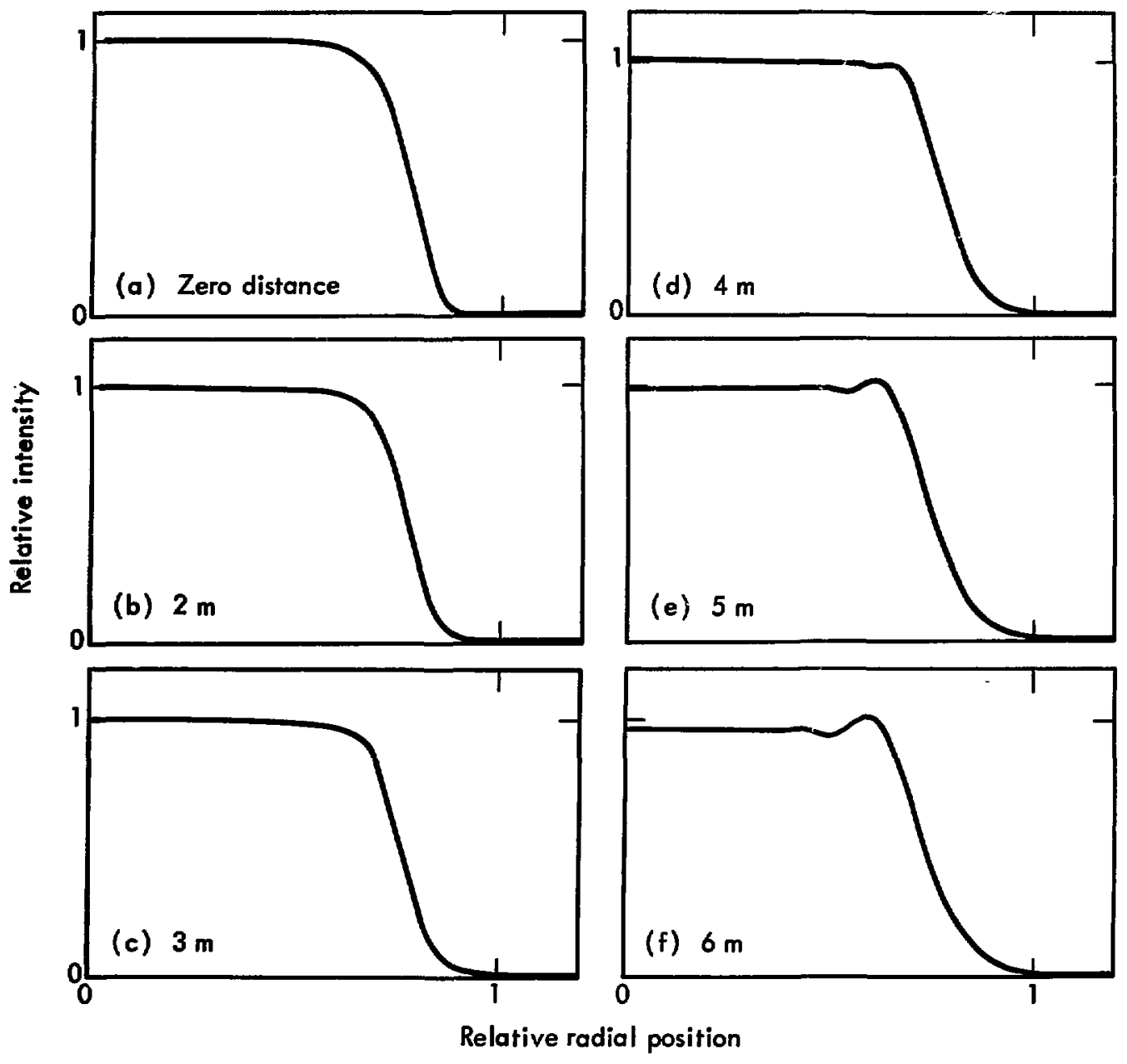

Fig. 52 Typical results of affe propsgation distance calculations.

propagating light beam. However, when lenses are considered from a wave or Fourier-optics point of view, another interesting effect becomes evident. ${ }^{37} \mathrm{~A}$ lens, when placed in a coherent propagating beam, has the effect of retarding (negative lens) or advancing (poxitive lens) the evolution of the diffraction pattern. If we designate by $z$ the distance a light pulse must travel after passing through a lens to reach a certain stage of diffraction development, and by $\hat{z}$ the distance to reach the same stage of development if it does not pass through the lens (i.e., if the lens were removed), then the relation between $z$ and $z$ depends on whether the lens is negative or positive:

$$
\hat{z}=z f /(z+f) \leq f \text { (negative lens) }
$$

or

$$
\hat{z}=z f /(f \cdot z) \text { (positive lens), }
$$

where $f$ is the focal length of the lens. Equation (2a) indicates that a negative lens has the effect of slowing down the development of the diffraction pattem. In fact in the limit as $z$ approaches infinity, $z$ equals $f$. 


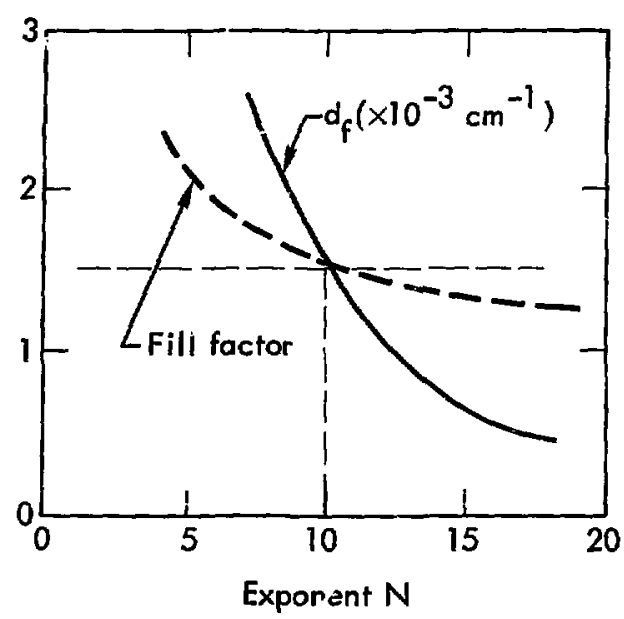

Fig. 53. Safe propagation factor $d_{\text {fand fill factor } a \text { functions }}$ of $N$, where $N$ is from the beam profile description $e(r)=\exp \left[-\left(r / r_{a}\right)^{N}\right]$.

Thus, a negative lens will never permit the diffraction pattern of a beam to develop further than the diffraction pattern of that same beam propagated a distance $f$ without a lens. This fact can be used to prevent diffraction breakup of a beam with a 10-power WT-type profile in the following way. We have previously determined that this beam will propagate a distance $d_{s}=1500 r_{a}^{2}$ (in $\mathrm{cm}$ ) without serious diffraction fluctuations. Therefore if we place a negative lens with focal length $f<d_{s}$ in the beam, the diffraction pattern will never evolve into the serious diffraction breakup region.

\section{Physical Realization of the Desired}

\section{Beam Profile}

A 10-power WT-type beam profile described by Eq. (1) must be obtained, in some fashion, from an oscillator output whose radial bearn profile can be described as an approximate Gaussian. In other words, we must change the radial beam profile from a Gaussian to a 10-power WT-type profile.

If a Gaussian beam is passed altemately through several saturated amplifiers and intensity attenuators, the beam profile theoretically can be altered to very closely resemble that described by Eq. (1). However, recent results from the Intermediate Laser System (see article by Speck and Bliss earlier in this report, in section on Solid State Laser Program, p. 20) indicate that the energy fluxes required to perform this type of beam shaping would lead to self-phase modulation and self-focusing in the preamplifier system. Therefore this approach has been abandoned. $f$ more promising method is to expand the Gaussian beam to a reasonable size and then retain the on-axis flat portion of the beam by aperturing the beam with a transmission filter that has an intensity transmission profile described by Eq. (1). Such an aperture is a particular form of an apodized aperture. The physical realization of a 10-power WT apodized aperture is well within the state of the art. It is a simple matter to generate a photographic transparency or metal film attenuator which has the proper transmission profile. Since such passive elements require absorpition phenomena for their operation, however, care must be used not to expose them to high energy densities which may cause damige. Also, to eliminate emulsion phase distortion; the photographic aperture would have to be placed in a liquid gate. Recent work with Pockels cells and Faraday rotators with nonunifomi electric and magnetic fields respectively indicates that a 10-power WT aperture can also be obtained using these elements in combination with appropriate polarizers." These active elements can withstand higher energy fluxes but are more complicated and costly than the previously mentioned passive elements. Clearly, the choice of aperture element must be determined by more experimental research as to damage limits and performance.

The extent to which the Gaussian beam must be expanded and apertured was studied by forming the product of a 10-power WT aperture, with 1/e transmission radius $R_{a}$, and a Gaussian beam profile with $1 / e$ intensity radius $R_{e} . R_{e}$ was chosen such that

"See article by B. Johnson earlier in this report, in Solid State Laser Program section (p. 29), and article by Leppelmeier and Simmons later in this report in Component Development section (p. 78).

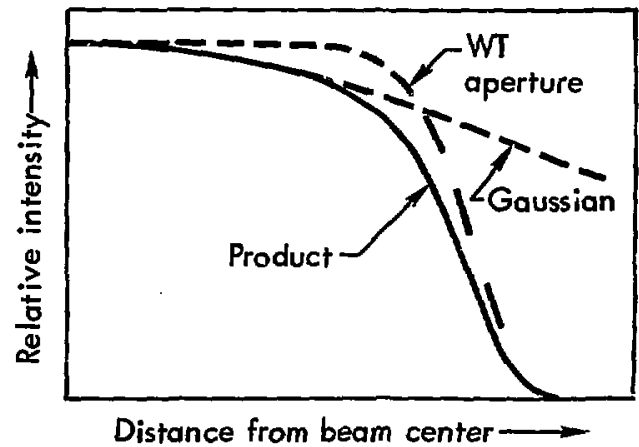

Fig. 54. Froduct of Gaussien beam and 10-power WT aperture. 


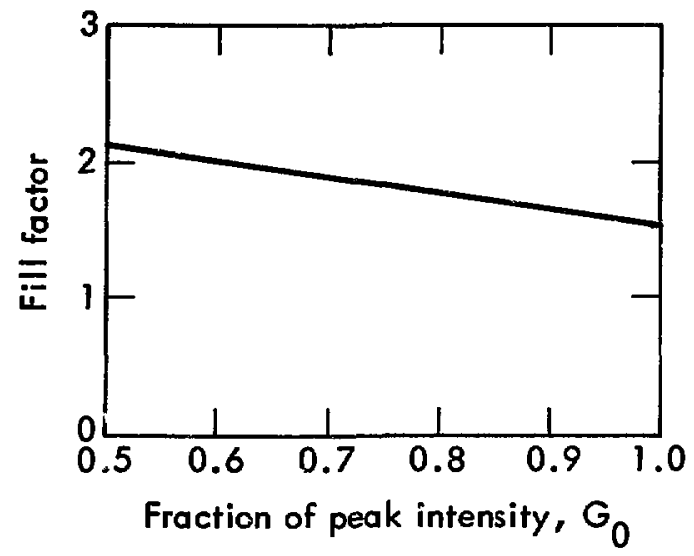

Fig. 55. Fill factor to $R_{a} / R_{a}=1.22$ as a function of $G_{0}$.

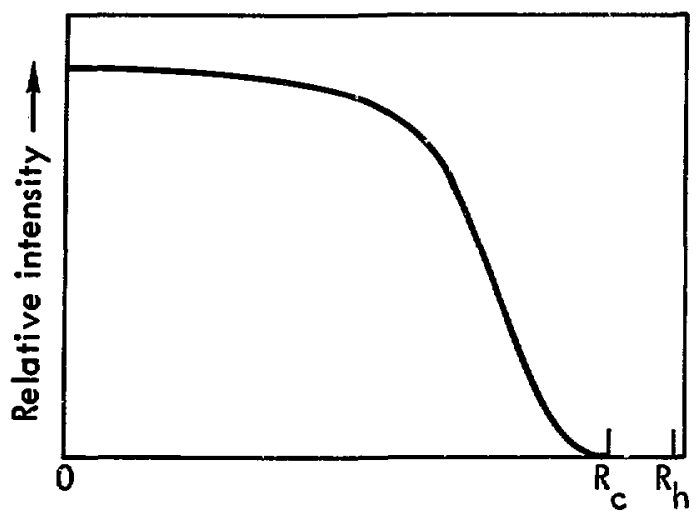

Distance from beam center

Fig. 56. Beam profila for case where clear aperture $R_{c}$ is less than hand sperture $R_{h}$ (edge of disk).

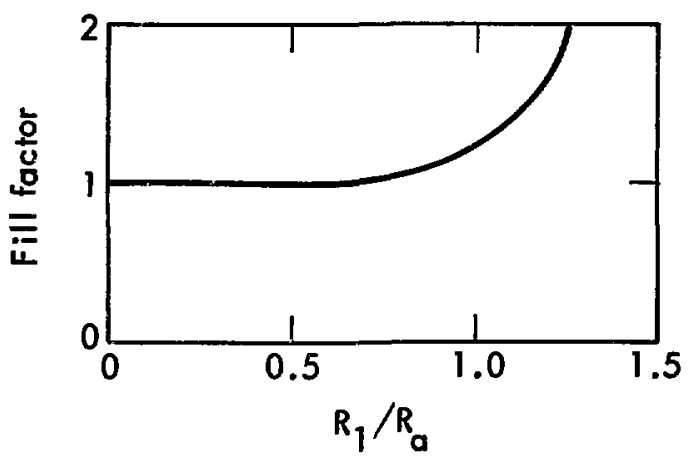

Fig. 57. Fill factor for $G_{0}=0.85$ as a function of $R_{1} / R_{a}$.

$$
\exp \left[-\left(R_{a} / R_{e}\right)^{2}\right]=G_{0}
$$

where $G_{0}$ is a specified fraction of the peak intensity. This is illustrated in Fig. 54. The fill factor to $R_{1}$ (equal to $1.22 R_{a}$ ) was calculated for various values of $G_{0}$. A plot of fill factor vs $G_{0}$ is given in Fig. 55 . Since lower values of $G_{0}$ result in a smoother radial profile, diffraction effects are smaller for decreasing values of $G_{0}$. Therefore the safe propagation factor $d_{f}$ is still valid. (This has been verified by diffraction code runs.)

The disk amplifiers of the $1-\mathrm{kJ}$ laser system are designed with a clear aperture that is less than the physical hard aperture of the disk edge because of disk-holder shadowing effects. ${ }^{38}$ The value of $R_{1} / R_{a}=1.22$ was chosen so that diffraction cutoff effects would be less than approximately $2 \%$. However, if we define the fill factor to the clear aperture $\left(R_{c}\right)$ of the disk while at the same time we define the hard cutoff radius to the physical hard aperture, $R_{h}$, the fill factor is decreased. This is illustrated in Fig. 56. As an example, for the $A$ disk module $R_{c}=1.75 \mathrm{~cm}$ while $R_{h}=2.4 \mathrm{~cm}$. Thus, if we choose $R_{a}$ such that $R_{h} / R_{a}=1.22$, then $R_{c} / R_{a}=0.89$, which results in a fill factor of 1.1. A plot of fill factor vs $R_{1} / R_{a}\left(G_{0}=0.85\right)$ is given in Fig. 57. In actual practice, we usually design conservatively by defining both the fill factor and cutoff radius value to be the clear aperture of the amplifiers and allowing the shaded portion of the radius for tolerance in system alignment. Thus, the real purpose of the "extra" aperture is to reduce diffraction cutoff effects even further than the design value and to ensure that the gain profile is relatively flat over the aperture.

\section{Design Analysis of Preamplifier System}

The disk amplifier system in the $1-\mathrm{kJ}$ laser has been designed so that the energy densities remain well below the saturation flux of the laser gliss. Thus our previous linear diffraction analysis is valid and can be used to describe the propagation of a laser beam through the disk system. Also, since the energy flux throughout the beam shaping and preamplification syst-m (BSP system) will be far below both the saturation and self-modulation flux of the laser materials, this linear analysis is also appropriate for the design of the BSP system. Shown in Fig. 58 is a schematic drawing of the disk amplifier section. Each tube contains the disk amplifiers and required isolation components for each of the $A, B, C$, and $D$ amplifier stages and is characterized by a clear aperture and an overall length. The maximum allowed divergence through each of the tubes is calculated from these two values. As can be seen in Fig. 58, the divergence through the $B, C$, and 


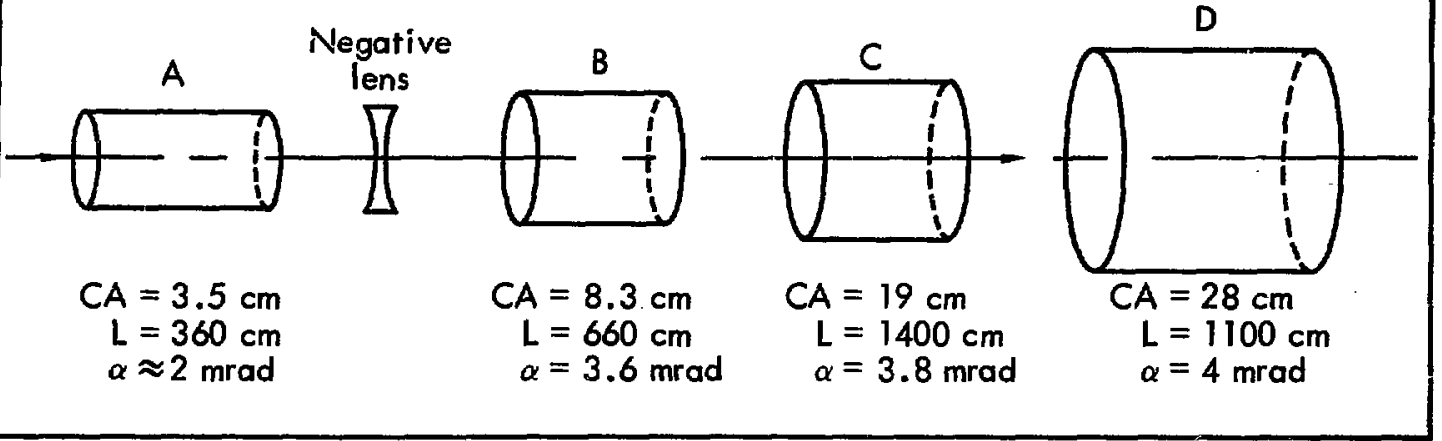

Fig. 58. Schematic diagrum of disk amplifier section of the $1-\mathrm{kJ}$ laser system. $C A=$ clear aperture, $L=$ overall length, and $\alpha=$ half-angle divergence.

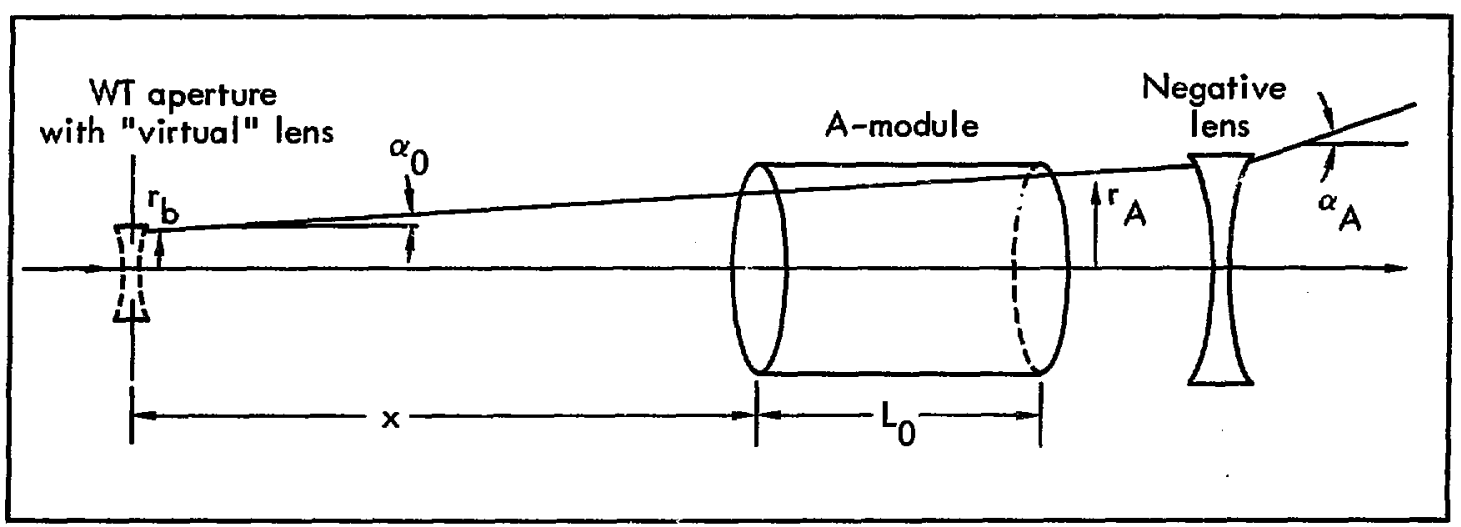

Fig. 59. Basic model for the beam shaping and preamplification (BSP) system.

$D$ tubes is approximately the same and is nominally set equal to $3.8 \mathrm{mrad}$ (milliradians). The slight differences in divergence can easily be compensated for by the proper spacing of the components. Since the divergence of the $A$ tube is considerably less than that of the others, a negative lens is placed after the last $A$ module to increase the divergence to that of the rest of the system.

The function of the BSP system is to take the oscillator output and convert it to a beam that is suitable for injection into the disk system. The Gaussian oscillator pulse is converted to the 10-power WT profile by the apodized aperturing techniques previously discussed. The BSP system must also amplify the beam energy from $1.5 \times 10^{-3} \mathrm{~J}$ to $0.5 \mathrm{~J}$ before it is injected into the $A$ module. If we assume (conservatively) that about $80 \%$ of the energy is lost at the aperture, then the BSP system must supply a small-signal gain of about 1700 . Taking into account the isolator capability, total number of amplifiers, and overall length of the amplifiers, we arrive at four rod amplifier units (each unit has its own isolation) each having a small-signal gain of about 7 . These values indicate a nominal length of about $1 \mathrm{~m}$ per amplifier unit. ${ }^{39}$ We briefly point out here that the isolation requirements are not as severe in the BSP system as they are in the disk system since the feed-forward energy flux in the BSP system is much lower than that in the disk portion of the system. For ease in design, analysis, and fabrication, as well as for keeping the cost as low as possible, we want to use the smallest aperture components consistent with maintaining the energy flux below the nominal $0.3-\mathrm{J} / \mathrm{cm}^{2}$ value in the BSP system.

Figure 59 shows the basic model for our BSP system analysis. The distance $x$ is from the aperture to the $A$-module input aperture. The "virtual" negative lens at the aperture is used to simulate the effect of the beam 
divergence from the aperture. The value $r_{b}$ is the $1 / e$ radius of the profile at the aperture and $r_{A}$ is the $1 / e$ radius at the $A$ tube output. $\alpha_{A}$ is the divergence to $1.22 r_{A}$ of the beam out of the $A$-module negative lens, and $L_{0}$ is the overall length of the $A$ tube. Physical constraints determine the following values:

$$
\begin{aligned}
& L_{0}=360 \mathrm{~cm}, \\
& r_{A}=1.43 \mathrm{~cm}, \\
& \alpha_{A}=3.8 \times 10^{-3} \mathrm{rad} .
\end{aligned}
$$

We now wish to determine a set of values of $x, r_{b}$, and $\alpha_{0}$ (beam divergence to $1.22 r_{e}$ through the BSP system) which will result in a reasonable design. The following three equations can be written which describe the physical constraints relating $x, r_{b}$, and $\alpha_{0}$.

1. The beam radius $r_{b}$ and BSP system diveigence $\alpha_{0}$ must be stich that the beam will fit through the $A$ tube. This condition is given in equation form by

$$
r_{b}<\frac{r_{A}}{1.22}-\alpha_{0} \cdot \frac{\left(x+L_{0}\right)}{1.22} .
$$

2. Due to the divergence $\alpha_{0}$ there is a "virtual" negative lens in the aperture of focal length equal to $1.22 r_{b} / \alpha_{0}$. Because of this virtual lens it is possible to prevent diffraction breakup of the beam if $f<d_{s}=1500 r_{e}^{2}$. Thus the second constraint in equation form is

$$
r_{b} \geqslant \frac{1.22}{1500 a_{0}}
$$

3. There is a second chance to prevent the diffraction breakup of the beam by adding additional divergence from the second negative lens. This constraint is given as

$$
r_{b} \geqslant \frac{1}{2} \frac{1.22}{1500\left(\alpha_{A}-\alpha_{0}\right)}
$$

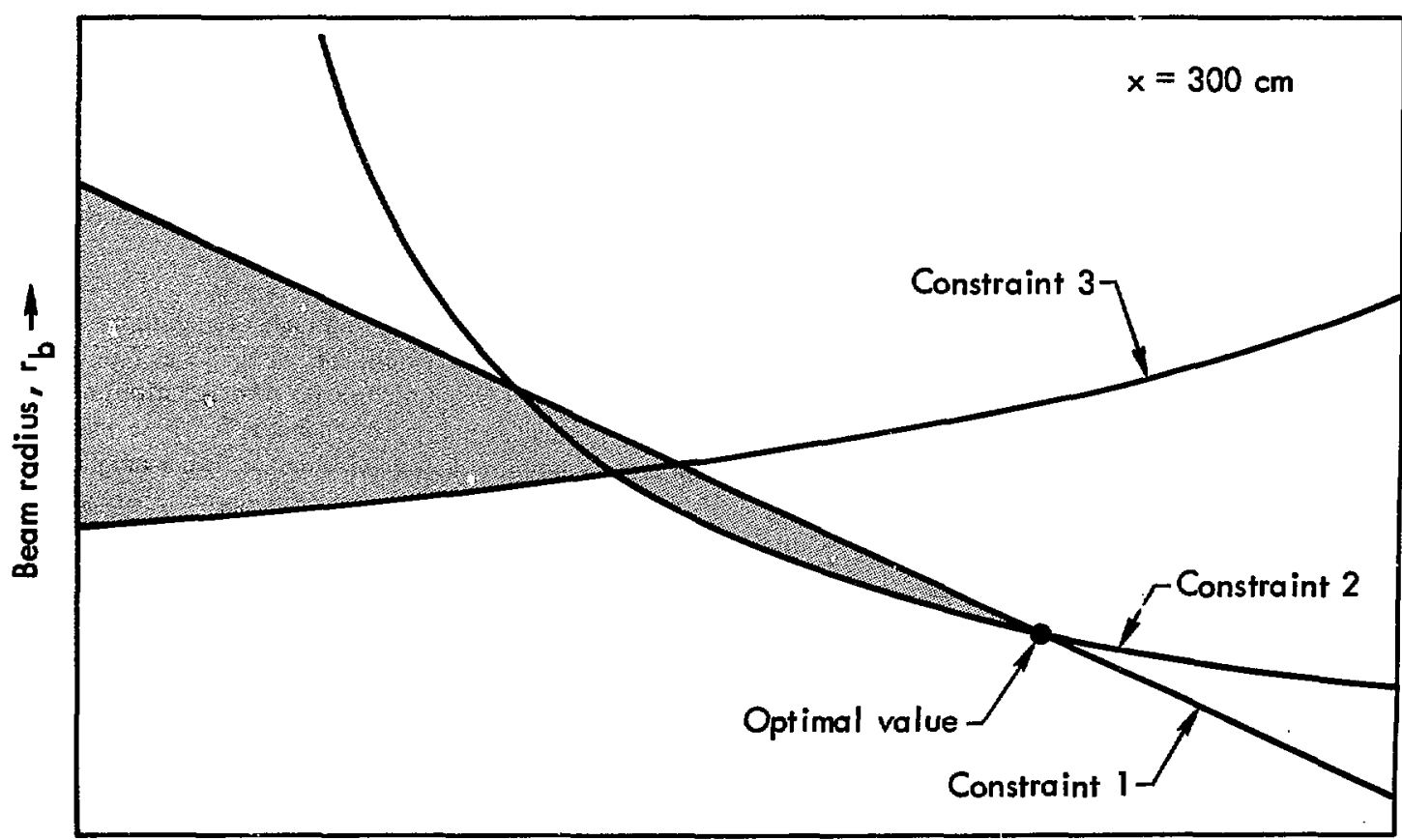

Beam divergence, $\alpha_{0} \rightarrow$

Fig. 60. Flot of bean radius vo beam divergence for constraints 1,2 , and 3 . Values used are $\dot{L}_{0}=360 \mathrm{~cm}, r_{A}=1.43 \mathrm{~cm}$, and $\alpha_{A}=3.8 \times 10^{-3}$ rad. 


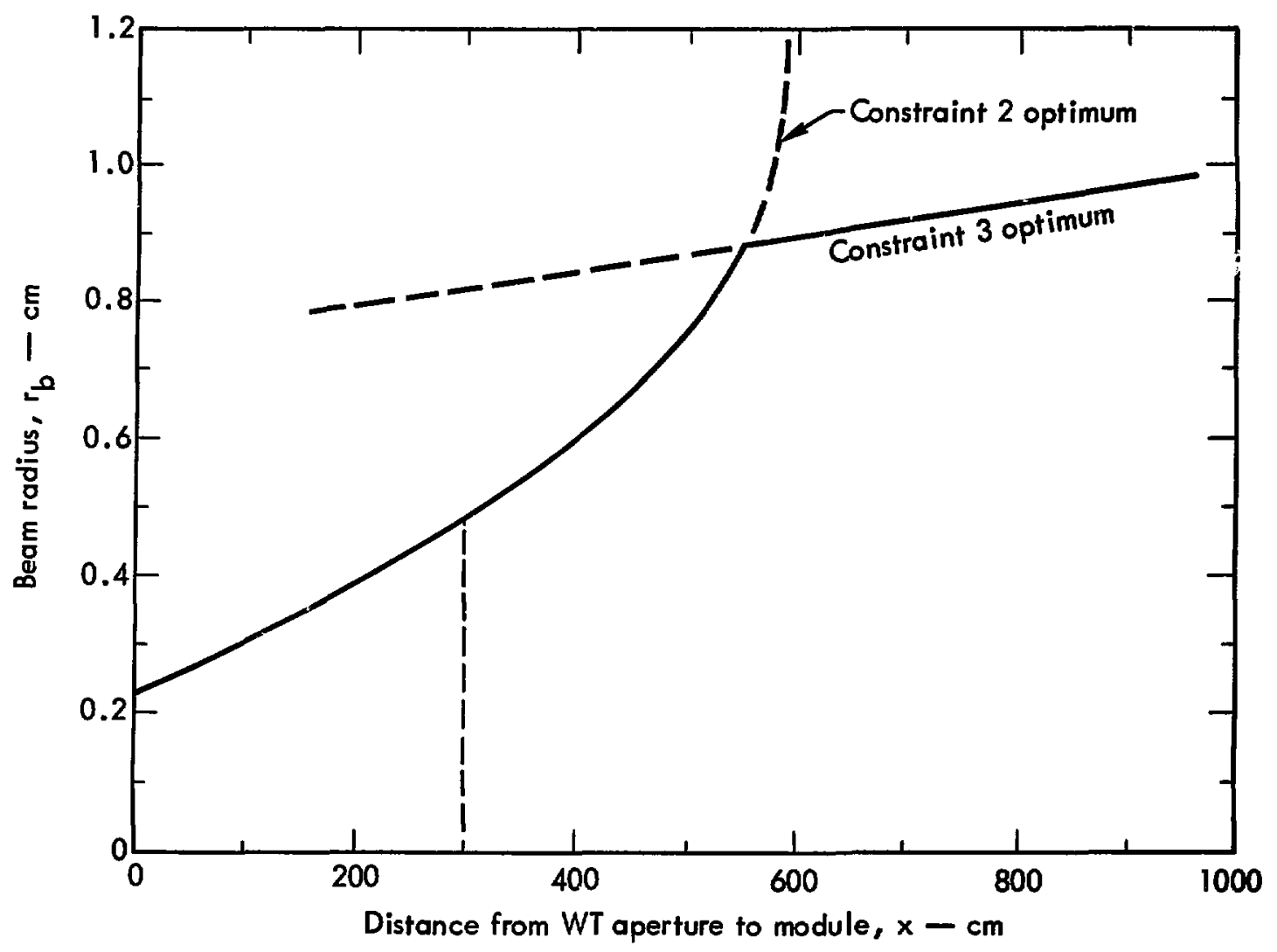

Fig. 61. Optimum beam radius vs distance from WT aperture to module for constraints 2 and 3.

If we require that the maximum input energy density of the $A$-tube i.jput beam should not exceed $0.3 \mathrm{~J} / \mathrm{cm}^{2}$, then we can write a fourth constraint that limits the maximum value of $\alpha_{0}$ so that this energy flux is not exceeded. This results in a maximum value of $\alpha_{0}$ equal to 2.4 inrad.

Shown in Fig. 60 is a typical plot of the three constraints ( $r_{b}$ vs $\alpha_{0}$ for a fixed value of $x$ ) with the shaded area representing the permitted solutions of divergence and heam size. Since we want the smallest diameter components that will fill the clear aperture of the $A$ tube, the optimum solution is the minimum value of $r_{b}$ that lies along the "constraint 1 " line. This is indicated in the figure. If we plot the optimum value of $r_{b}$ vs the distance $x$ as in Fig. 61, we can see that at $x \approx 300 \mathrm{~cm}$, the value of $r_{b}$ increases rather rapidly. Thus, $x=300 \mathrm{~cm}$ is a reasonable choice.

Based on a nominal length of $1 \mathrm{~m}$ per amplifier unit and the fact that we require fou" amplifier units, we see that three of the units should be placed between the aperture and the $A$-tube input aperture while the remaining amplifier unit should be placed before the aperture, as will be discussed shortly. For $x=300 \mathrm{~cm}$, we have $r_{b}=0.452 \mathrm{~cm}$ and $\alpha_{0}=1.8$ mrad.

We now consider the portion of the BSP system which takes the oscillator output and converts it to a beam that is compatible with the WT aperture input. The oscillator output beam will have an energy of $1.5 \mathrm{~mJ}$ in a $1 / e$ diameter of $1 \mathrm{~mm}$, with a divergence (a) of $0.5 \mathrm{mrad}$. We wish to expand the oscillator output beam by a factor $M$ such that the relative intensity value $G_{0}$ at the $1 / e$ radius of the WT aperture is 0.85 . Mathematically this is written as

$$
\exp \left[-\left(r_{a} / M r_{e}\right)^{2}\right]=G_{0}
$$

or

$$
M=\frac{r_{a}}{r_{e} \sqrt{\ln G_{0}}}
$$




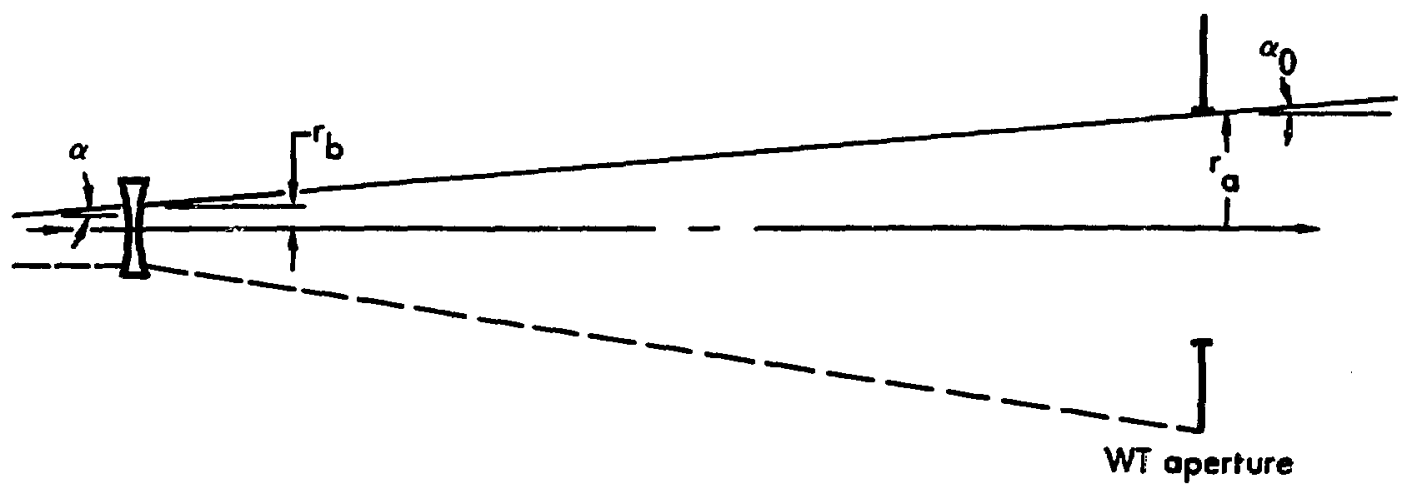

Fig. 62. Expansion syatem with single negattre bas.

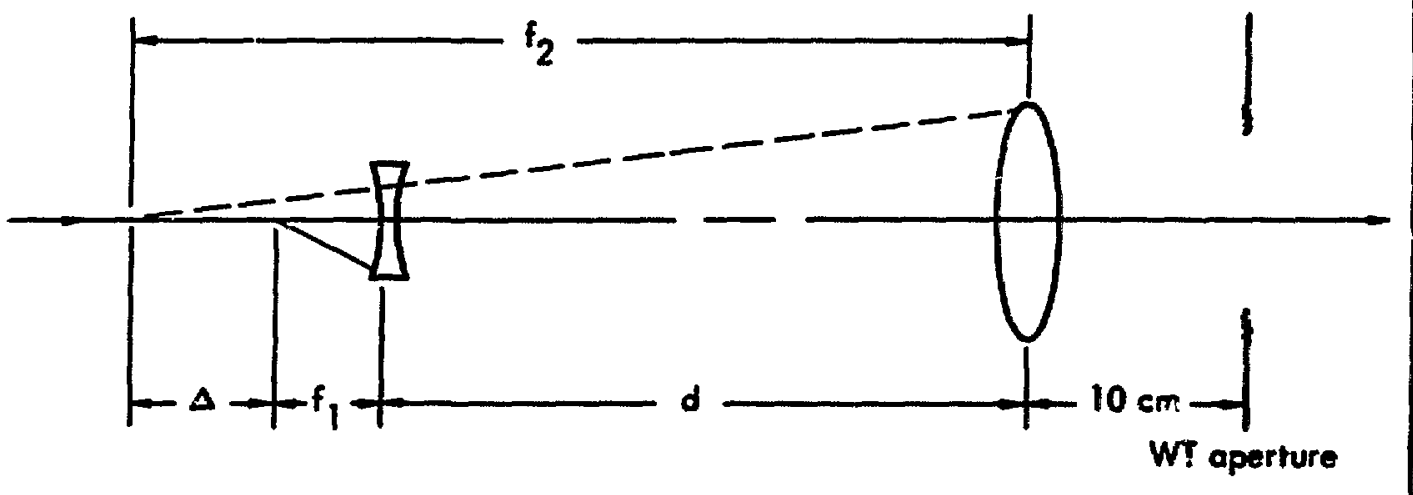

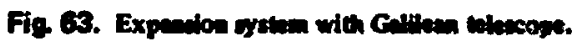

For the BSP system,

$r_{a}=1 / e$ radius of $W T$ aperture $=0.452 \mathrm{~cm}$,

$r_{e}=1 / e$ radius of cacillator beun $=0.05 \mathrm{~cm}$, $G_{0}=0.85$.

These values result in a magnification $\boldsymbol{M} \approx 22$.

This portion of the system must also allow enough distance between the occillatos output and the WT aperture to scsommodace the fourth preamplifier unit. As before we will allow the nominal $1 \mathrm{~m}$.

We have considered two possible beam expansion systems. Shown in Fig. 62 is a singlenegativelens expansion system. The focal length of the lens required to match the aperture divergence is

$$
f=\frac{1.22 r_{0}}{H\left(a_{0} \cdot a\right)}=19.28 \mathrm{~cm}
$$

The distance from the lens to the apefture required for the $22 x$ expansion is eiven by

$$
d=\frac{r_{e} \cdot r_{c}}{r_{0} \cdot a}=808 \mathrm{~cm} .
$$

This distance is cerianly large enough is accoumodale the fourth amplifier unit and in fact is actually wasteful of space. 


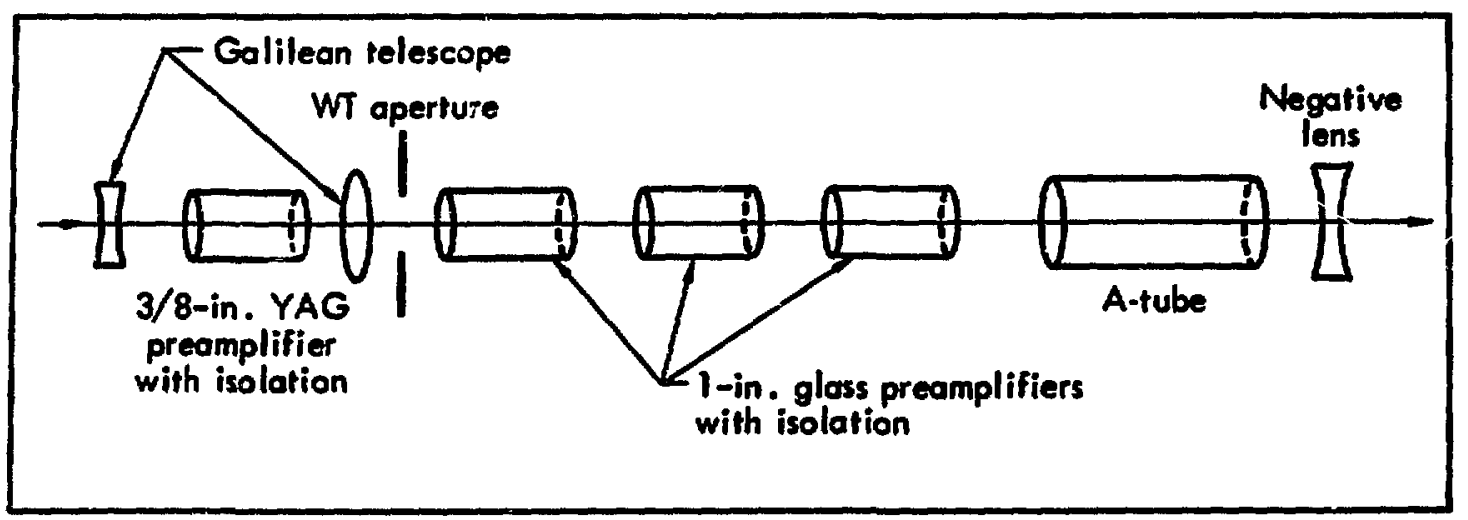

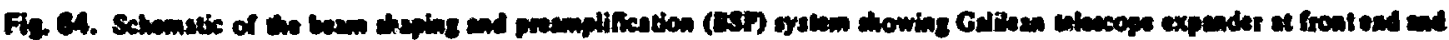
A-molinis of andilint.

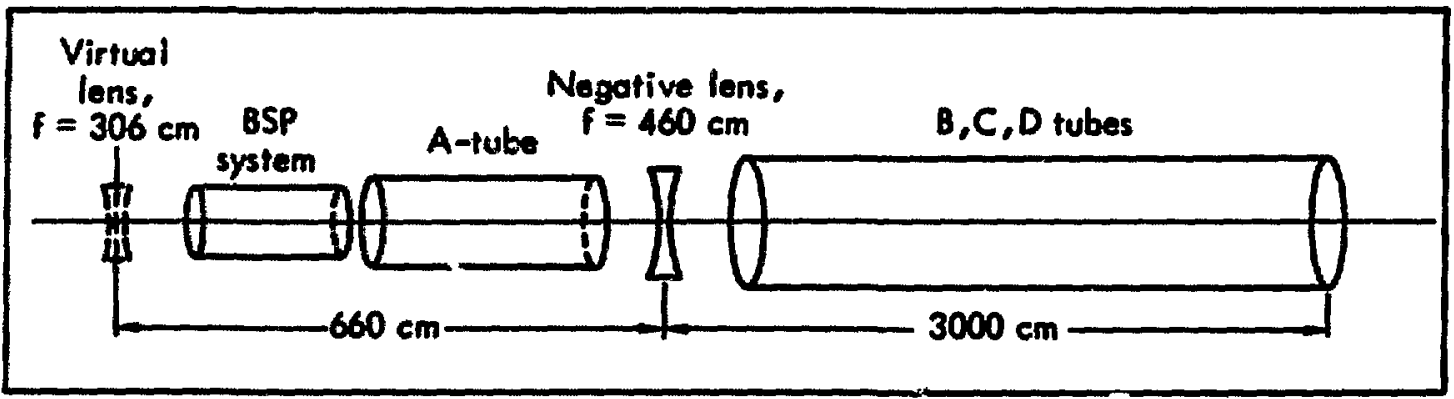

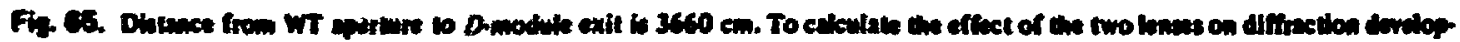

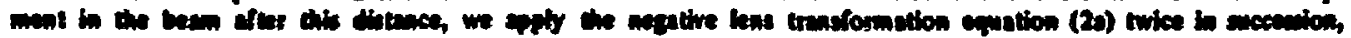

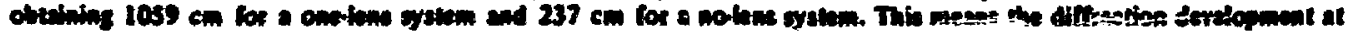

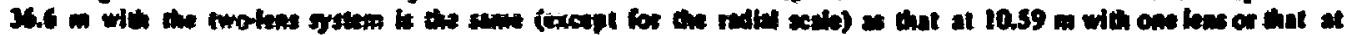

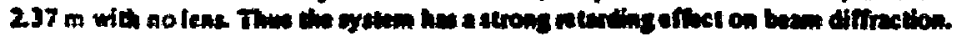

Shown in Fig. 63 is Galilean-telescope magification system. Since the magnification will be sbout 22X.

$$
f_{2}=22 f_{1}
$$

If we choose $f_{1}=6 \mathrm{~cm}$, then $f_{2}=132 \mathrm{~cm}$. The required specing $\Delta$ between the lens focal lengths is iven by

$$
\Delta=\left(\frac{f_{2} a_{0}-f_{1} a}{f_{1} a+r_{c}}\right) f_{1} .
$$

For our particulat system, this results in a value of $\Delta=26.6 \mathrm{~cm}$. The distunce tatwaen the two lenses is

$$
d=f_{2} \cdot f_{1} \cdot \Delta=99.4 \mathrm{~cm}
$$

This distance is adequate 10 secommodite the fourth amplifier and is isolation components.

The Galikan-telescope magnification system is much more compact and veratile (the divergence $\mathrm{cm}$ be finesuned) than the singledens system. Shown in Fis. 64 is a schematic drawing of the BSP system using the Gsileun-telescope mugnification system.

\section{Cem Profie at Exit of D Modve}

We now examine the radid beam proffle at the exit eperture of the $D$ module. Figure 65 shows a schematic diagram of the propagation distance from the WT aperture to the D-module exul aperture. If the negative lens transformation of Eq. (20) is applied wice we obluin an isomorphic propagntion distence of $237 \mathrm{~cm}$. Figure 66 show a computer-generated difrraction pattem of the beem profile at the exit pupil of the $D$ module (using the isomorphic propagation 


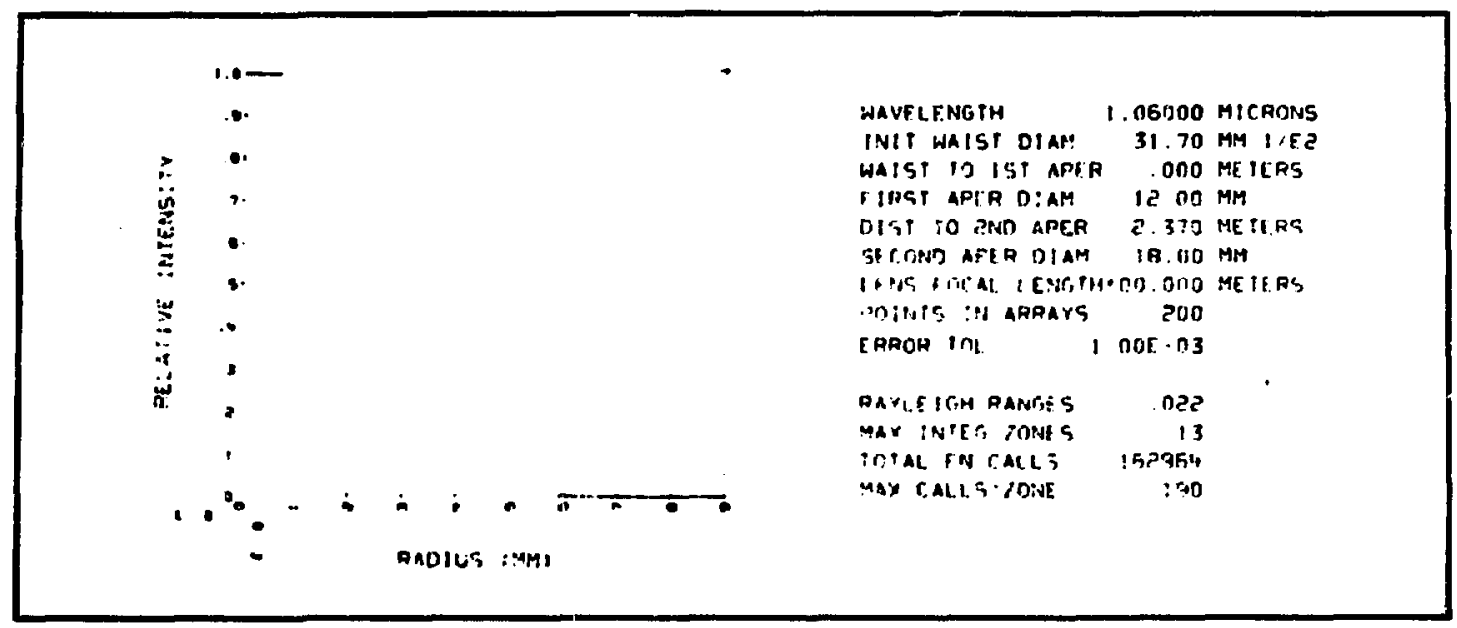

Fi, 63. Companncelentabed ben profit at D.mudule exit eparture (from microfictu).

distence). As can be seen the beam has held its profile ressonably well. The intensity value at the clear aperture ndius, obtained by locating the 1/e radius point and multiplying it by 1.22 , tums out to be 0.01 . We would like to reduce the intersity at the clear aperture radius to 0.001 of the maximum value, but to do 20 we would have to go out to 1.44 times the 1/e radius. This expansion would be very costly, because of the large size of the $D$ modules. Fortunately, the $D$ moduse is the last amplifier aperture the beam passes through before being focused onto the target. Thus diffraction ripple is not as great a concem at this point, and we can allow Inucation at 0.01 .

The preceding linear diffraction andysis indicates that a 10-power WT beam profile will propagate satisfactorily through the disk system. Ho:vever, due to the rather sharp corner on the profile, whole-beam self-focusing may still be a problemin. This possibility will be studied both experimentally and analytically.

\section{DHFFRACTIONLE: CALCULATION OF ABEARATION AND SELF.FOCUSING DISTORTION IN A LASER BEAM}

When a diverging leser beam psses through an asembly of tilted disks, a distortion occurs which may significently affect the focusing of energy or a target pellet. Self-focusing effects in the disks may also cause lare variations in the silstribution of energy at a focus. A computer code to antlyze wholebeam distortions incurred in the traversd of optical systems is therefore being written. The code uses a three-dimensional ray-tracing technique (without diffraction) 10 simulate the behwior of the beam.

Several thousand initid ays are gentrated from a cource point on the optic axis and pased through a polar goid at the entrance aperture (Fig. 67). These rays are given initial intersities corresponding to an abitrary bean profile (currently Gausian). Their pathe are then followed in three dimensions through an anembly of tilted disks and lenses, refracting at each aurfece and amplifying through each diak. To find the effect of seff-focusing, an estimute of the intensity gradient is made for each ray in each disk, and an approximate bend is applied to the ray path according to the equation

$$
\frac{d}{d s}\left(n \frac{d r}{d s}\right)=\nabla n \approx a(\overline{\nabla n},
$$

where $s$ is the disiance along the ray path, $n$ is the index of refraction, $a$ is the nonlinear index coefficient, and $(\bar{\nabla})$ is the average intensity gradient along the ray path. Disks are specified by their position along the optic axis, thickness, axid and azimuthal orientation ingles, index of refraction, small-signal gain coeficient, and nonlinear index coefficient a. Lenses are specified by radii of curvature of two faces, positions along the oplic axis, and index of refraction.

After emerging from the last element of the system, the rays are traced from (or toward) their convergence 
to find the best energy focus. The intensity distribution at this focus is then displayed, both as a three-dimensional surface and as a contour plot as shown in Fips. 68 and 69 respectively. A display of mesh distortions at the focus is also provided (Fig. 70). Displays of intensity distribution and phase surface are optionally available at any number of positions along the beam path. We plan to calculate

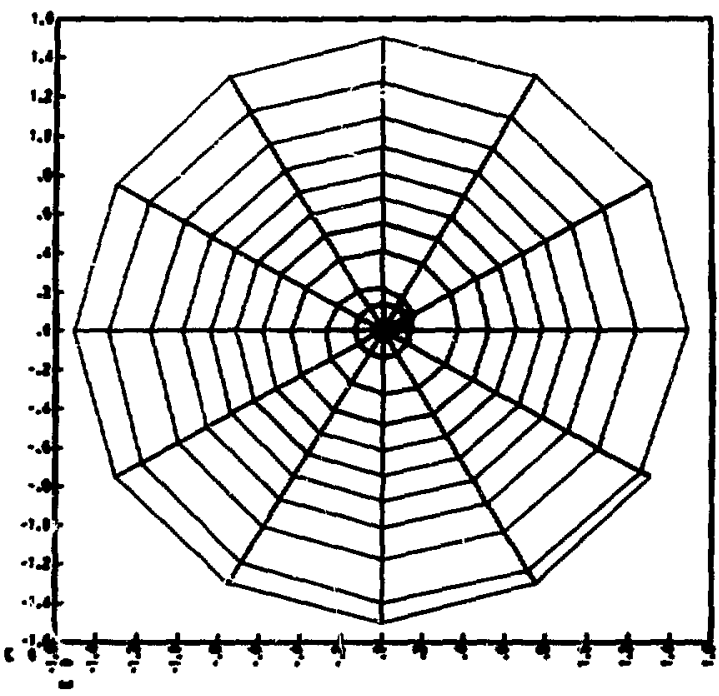

Fin 67. Tyicel rit for a wholeben my-treing cods A

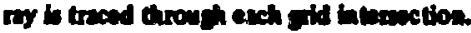

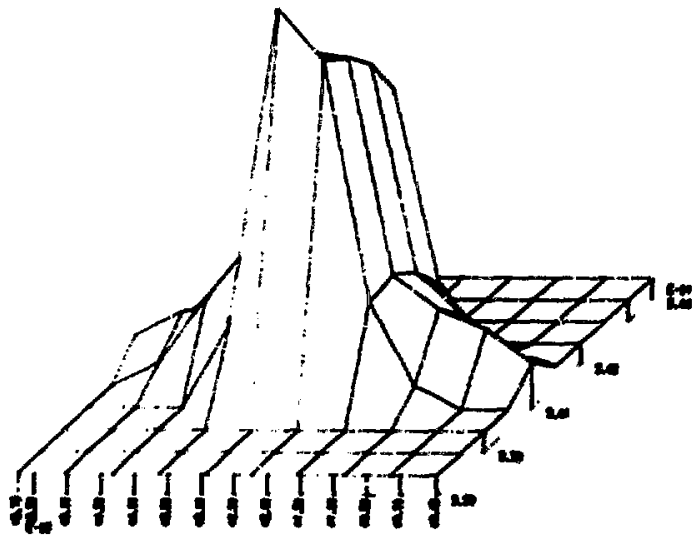

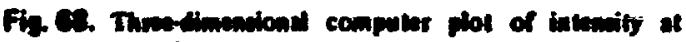
weit tacen. the diffraction pattern at the focus from the phase and amplitude information at the output plane.

The code is currently in a development state and no attempt has yet been made to compare the output with observations.

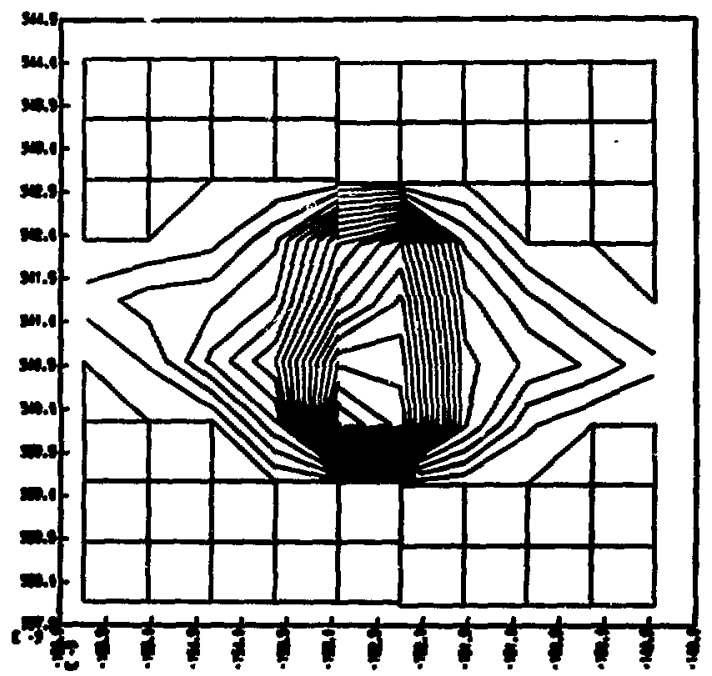

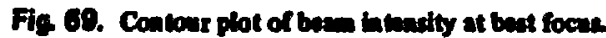

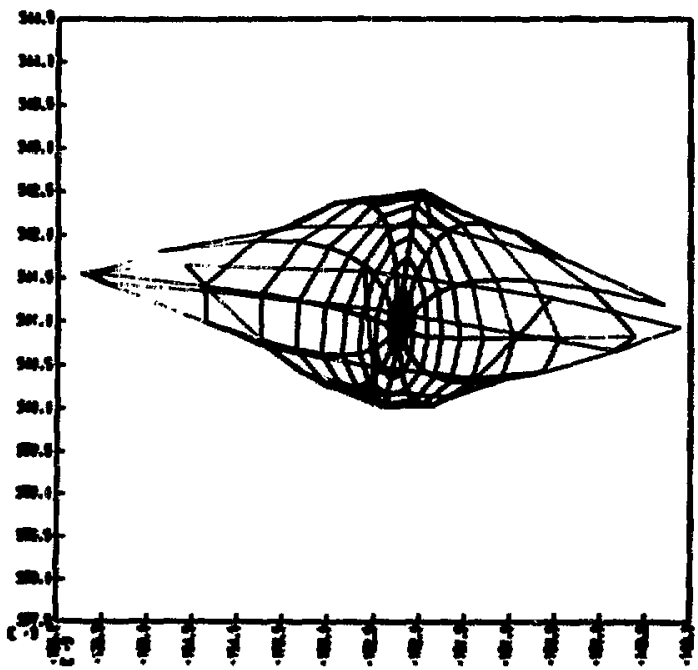

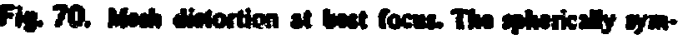

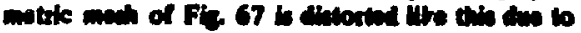
chrotions. 
This article covers the overall optimum design of a chain of laser amplifiers. We first discuss the physics constraints on the design of a large disk laser, then the design of a single stage, and finally the optimal combination of stages to produce $1 \mathrm{~kJ}$. Detailed design of an individual amplifier is covered in other articles in this report.

\section{Physics Constraints}

1. Target isolation. To avoid damage from back-amplified target reflection, the laser must be segmented into stages of gain combined with Farao :y rotator isolators and auxiliary polarizers. The maximum gain per stage is limited to 4 (5 at the most) by the polarizer quality, rotation uniformity, and birefringence of the optical components.

2. Optical damare. For short pulses, the major cause of optical damage is self-focus tracking. This does not arise from collapse of the beam as a whole; rather mall irregularities in amplitude or phase grow and cause local damage. This damage is statistical, with a ratr which rises to dangerous levels at about 10 G. $; \mathrm{cm}^{2}$. Meticulous cleaning of optical components and avoidance of diffraction ripples reduce the danger.

3. Cther nonlisear effects. Self-phase modulation can chirp (i.e., shift the frequency of) the pulse out of the glass gain band. The fractional frequency change is 10

$$
\frac{\delta f}{f} \approx \frac{n}{c \tau_{R}} \int \delta n d x,
$$

where $n$ is the index, $\tau_{R}$ is the pulse rise-time, and $\delta n$ is the intensity-induced index change. The $\delta n$ term is approximated for glass by

$$
\delta n \approx\left(10^{-15} \mathrm{~cm}^{2} / W\right) \times l .
$$

where $J$ is the intensity in $W / \mathrm{cm}^{2}$. Thus a 100 -peec pulse at $10 \mathrm{GW} / \mathrm{cm}^{2}$ will chirp about $10^{-3}$ or $10 \mathrm{~A}$ in $1 \mathrm{~m}$ of gas.

Twophoton absorption may also prevent propagation at high intensity. An intensity-dependent beorption of $\left(4 \times 10^{-12} \mathrm{~cm} / \mathrm{W}\right) \times 1$, which implies $4 \% / \mathrm{cm}$ absorption at $10 \mathrm{GW} / \mathrm{cm}^{2}$, has been reported in one cless."

4. Paswitic cocillation. The avulable inversion in larg disks is limited by paraitic occillations. The maximum gain coefficient is

$$
\alpha \approx \frac{\ln (R)}{n \ell},
$$

where $n$ is the index in the disk, $l$ is the disk's long axis, and $R$ is the edge reflectivity of the disk at the complement of the total intemal reflection angle (i.e., at $\left.\sim 40^{\circ}\right) .5$

5. Pumping limitation. For small disk amplifiers the parasitic limitation may be quite large (several $\mathrm{J} / \mathrm{cm}^{3}$ ). However, a combination of the explosion limitations of flashlamps and the fluorescent decay of the inversion leads to a maximum attainable stored energy density. The exact maximum depends on how clever we can be in our cavity design.

\section{Single-Stage Dexign}

Suppose a stage is to have an output $O$ (measured in joules). We assume that the stage has fixed losses which cause its passive transmission to be $T$. In the worst case where the losses arc lumped at the outpat (due, for example, to the presence of a Faraday rotator), the amplifier must generate an energy of $O / T$ joules.

We now introduce a tlux limitation. To avoid damage, we restrict the peak flux in the glass to $F \mathrm{~J} / \mathrm{cm}^{2}$. Since we know the flux and the total output, we can find the minimum beam area in the glass. This area is $O / T F$. However, we must increase this area by some factor $Q$ to allow for the fact that the beam does not have the peak flux everywhere, but instead falls off near the edges. The required area thus becomes OQ/TF.

The beam area in a disk inclined at Brewster's angle is the refractive index $n$ times the area in the air, ${ }^{41}$ so the beam area in air is

$$
A=\frac{Q Q}{n F T} \text {. }
$$

The beam diameter, and hence the short axis of the disk, is therefore

$$
d=2 \sqrt{\frac{A}{\pi}}=2 \sqrt{\frac{C Q}{\pi n F T}} .
$$

and the diak's long axis (l) is

$$
l=d \sqrt{1+n^{2}}
$$

if the disk is very thin. To allow for finite thickness and for simplicity, we take $Q=2 d$ and have

$$
\ell \approx 4 \sqrt{\frac{O Q}{\operatorname{sn} F T}} .
$$


Now that we have determined the long axis, we can calculate the parasitic limit on the stored energy density. The gain coefficient at the line peak $\alpha$ times the long axis is limited by

$$
a d<P \text {, }
$$

where $P$ will typically lie between 2 and 3 . Then the parasitic oscillation limit on $\alpha$ is

$$
\alpha_{0}=\frac{P}{4} \sqrt{\frac{m F T}{O Q}}
$$

and if $\alpha$ is related to the stored energy density $e$ by

$$
a=k e
$$

(where $k=\sigma / h v$, or about $0.124 \mathrm{~cm}^{2} / J$ for ED-2 glass), we find

$$
c_{0}=\frac{p}{4 k} \sqrt{\frac{m F T}{O Q}}
$$

In the design of the stage, we must use the minimum of the cacillation and pumping limits on $e$, where the pumping limit is due to the fact that there is an upper limit to the amount of energy which can be put into the fanhlarips in a laser. Thus,

$$
e=\min \left(e_{0}, e_{p}\right)
$$

and the gain coefficient to be used is

$$
m=k \min \left(e_{0}, \epsilon_{p}\right)
$$

where we ignore the fact that the operating wavelength may not be at the fluorescent line pesk.

If we specify the stage gain $G$, we may find the path length of glas required to amplify the beam by using the equations for a lostless saturating amplifier in the rate-equation iimit. If the path is s, we find

$$
s=\frac{1}{a} \ln \left(\frac{e^{F / E} \cdot 1}{e^{F T / G E} \cdot 1}\right)
$$

where $E$ is the saturation flux (which will usully depend $c$ the pulse duration). We have asumed that the entire beam has the maximum flus $F$ at the output and thus underestimated the eccurd gin at the edses.

The thicknem of elan normal to the disk feces is lea then s becence the beam is skexsell. The conrection factor is $\sqrt{1+1 / n^{2}}$, so that the thickness is

$$
t=\frac{s}{\sqrt{1+1 / n^{2}}} .
$$

The total volume of glass required is thus the disk area $\pi \ell d / 4$ times the thickness, or

$$
\begin{aligned}
& V_{p}=\frac{2}{\alpha_{p} \sqrt{n^{2}+1}}\left(\frac{O Q}{F T}\right) \ln \left(\frac{e^{F / E} \cdot 1}{e^{F T / G E} \cdot 1}\right) \\
& \text { (pump limited), } \\
& V_{0}=\frac{8}{P \sqrt{\pi n\left(n^{2}+1\right)}}\left(\frac{O Q}{F T}\right)^{3 / 2} \ln \left(\frac{e^{F / E} \cdot 1}{e^{F T / G E} \cdot 1}\right)
\end{aligned}
$$

(oscillation limited).

The stored energy in the glass is

$$
w=e V=\frac{\alpha V}{k},
$$

so that

$$
W=\frac{2}{k \sqrt{n^{2}+1}}\left(\frac{O Q}{F T}\right) \ln \left(\frac{e^{F / E} \cdot 1}{e^{F T / G E}-1}\right) .
$$

If we assume an overall transfer efficiency $f$ from capacitor basiks to peak energy, the bank size required is

$$
B=\frac{2}{k f \sqrt{n^{2}+1}}\left(\frac{O Q}{F T}\right) \ln \left(\frac{e^{F / E} \cdot 1}{e^{F T / G E} \cdot 1}\right) .
$$

Now that we know the bank size and glase rolume required, we can estimate the total cost of the stage since these are the principal cost elements. If costs proportional to the bank size are $\boldsymbol{C}$ dollara/joules, and costs proportional to the glase volume are $D$ dollirs/cn: ${ }^{3}$, then the total cost is

$$
s=C B+D V+Z,
$$

where $Z$ is wn added fixed cost.

For example, let us consider the following case:

$\begin{array}{ll}\text { stage output } & O=1000 \mathrm{~J}, \\ \text { passive transmission } & T=0.85, \\ \text { maximum flux } & F=3 \mathrm{~J} / \mathrm{cm}^{2}, \\ \text { ahape factor } & Q=1.5, \\ \text { dams index } & r=1.56, \\ \text { paraitic limit } & P=2.5, \\ \text { cnergy-pain constent } & K=0.124 \mathrm{~cm}^{2} / \mathrm{J}, \\ \text { pumping limit } & C_{p}=0.6 \mathrm{~J} / \mathrm{cm}^{3} . \\ \text { stage vin } & G=2, \\ \text { saturation fux } & E=3 \mathrm{~J} / \mathrm{cm}^{2} .\end{array}$


These requirements lead to disks about $44 \times 22 \mathrm{~cm}$, with a total thickness of $17.4 \mathrm{~cm}$ (six disks $2.9 \mathrm{~cm}$ thick, for example). The design is parasitic-limited at $0.45 \mathrm{~J} / \mathrm{cm}^{3}$. The glass volume is 13 liters, while the stored energy is $6 \mathrm{~kJ}$.

If we further assume the values

$\begin{array}{ll}\text { bank inversion efficiency } & f=0.008, \\ \text { bank-variable costs } & C=0.35 \$ / \mathrm{J}, \\ \text { glass-variable costs } & D=10 \$ / \mathrm{cm}^{3}, \\ \text { fixed costs } & Z=\$ 100,000,\end{array}$

then the bank-variable cost is about $\$ 260,000$, the glas-variable cost is $\$ 130,000$, and the total cost is $\$ 490,000$.

\section{Multintere Dexinn}

Now that we are able to design a single stage and determine its approximate cost, it is easy to lay out and optimize a chain of laser amplifiers. Each stage his output equal to the input of the succeeding stage and input equal to its output divided by its gain. Thus, by specifying the output of the final stage and the gains of the stages, the entire chain is determined. In general, each stage can have different values of maximum flux, parasitic limit, element costs, and so forth. Once these values are given, the approximate cost of the whole chain is known. The problem is then to adjust the stage gains so that the overall cost is minimized.

The cost optinization of laser amplifier chains was carried out by means of a dynamic programming method. 42 This is a sequential method which is really just a fency form of mathematical induction. We first find the cost of the output stage $a s$ a function of its grin for gains from unity to the maximum gain $G_{M}$ set by isclation considerations. We then find the optimal combination of two stages for all two-stage gins from unity to $G_{M}^{2}$, a follows: For each two-stage gin, we split the gain into output and driver gains, and find the cost (the output cost comes from the previous calculation; the driver gain is calculated). The split point is then varied, and the split which gives the minimum cost is found. We now have a table of optimal two-stage cout as a function of two-stage gain. A third staye is added by splitting the three-stage gain into the added atage part and a twostage part; the twostere cost comes from the previously calculated table. Once more, the optimum split is found by varying the spiit point. This is repeated for various threestage gins to yield a table of optimum threestay cost. This stage-bystuce calculation may be contimued indefinitely. At each addition of a stage, we need only refer to the previous table of minimum cost vernus gain in order to find the beat way to add the stage. The dynamic programming method is illustrated in Fig. 71.

\section{An Optimum Amplifier Chain}

Let us find a minimum-cost amplifier chain to amplify $1 \mathrm{~mJ}$ to $1 \mathrm{~kJ}$. We will assume a maximum flux of $2 \mathrm{~J} / \mathrm{cm}^{2}$ in the output stage, and $1 \mathrm{~J} / \mathrm{cm}^{2}$ in all other stages, so that the system stresses will be concentrated in the iost stage rather than being distributed throughout the chain. Tre beam shape will be taken square enough spatially that only $65 \%$ more area of glass will be needed than in the case of a spatial square wave beam. Faraday rotators at the end of each stage will be uncoated (because of the high energy flux) and so will have a transmission of only $85 \%$. The edge coatings on the disks will absorb parasitics very well, so that we may operate at $\alpha D=2.5$. The maximum pump level will be $0.6 \mathrm{~J} / \mathrm{cm}^{3}$. Cavity design will be assumed to give 0.008 transfer efficiency from stored energy in the capacitor bank to peak stored energy in the laser material. The laser material will be described by an energy-specific gain coefficient of $0.124 \mathrm{~cm}^{2} / \mathrm{J}$ and a saturation flux of $3.3 \mathrm{~J} / \mathrm{cm}^{2}$. Bank-specific costs will be $40 \mathrm{k} / \mathrm{J}$, glass-specific costs will be $\$ 10 / \mathrm{cm}^{3}$, and fixed costs will be $\$ 10,000$ per laser. The is olation requirement will be chosen to give a conservative maximum gain of 3.2 per stage.

With these assumptions, the optimum number of stages is 14. Details of the staging are illustrated in Table 9. The gains of the large, highenergy stages are fixed by capacitor and glass minimization, while the seven smallest stages run at the isolation gain maximum. The output stage has a large gain because it has a higher flux limit; the optimum way to utilize this difference is to concentrate gain in the stage which can be physically smaller. Most of the cost is in the last few stages, so changes in the low-energy portion of the system will have little effect on the cost.

It is interesting to vary one of the assumed design parameters and sae the effect on the optimal system. For example, let us vary the - timulated-emission cross section ( $(0)$ of the laser material. This changes the energy-specifir gain coefficient, the saturation flux, and the bank-inversion transfer efficiency (by changing the absorption spectrum). Because the absorption change is hard te pretict, we show in Fig. 72 a set of curves of glass volume only and ignore any changes in capacitor bank size. We see that over a broad range of flux densities the value of the cross section 0 that gives minimum glass volume is between $2 \times 10^{-20}$ and $3 \times 10^{-20} \mathrm{~cm}^{2}$. This is the $a$ range in which many common laser glasses fall. The value of $\sigma$ thus ha small effect on glass volume, and we must tum to bank size changes to eet cost improvements. We should concentrate on finding glasses which produce 
high products of cross section $\sigma$ times bank-inversion transfer efficiency $f$ (recall that the bank size for one stage goes inversely as $k f$, and $k=\sigma / h \nu$ ). Experimentally, $f$ does not vary by large amounts from glass to glass (it is increased by greater absorption and longer lifetime, but the effects are not large). Thus, cost improvements due to increasing fo will most likely come from increases in $\sigma$. We cannot go too far in this direction, however, because the required glass volume will begin to rise.

\section{Practical Improvements to the} Optimum Design

We have designed a laser chain whic. at the minimum cost in terrins of total capacitor bank size and glass volume. However, this system has a hugh-gain

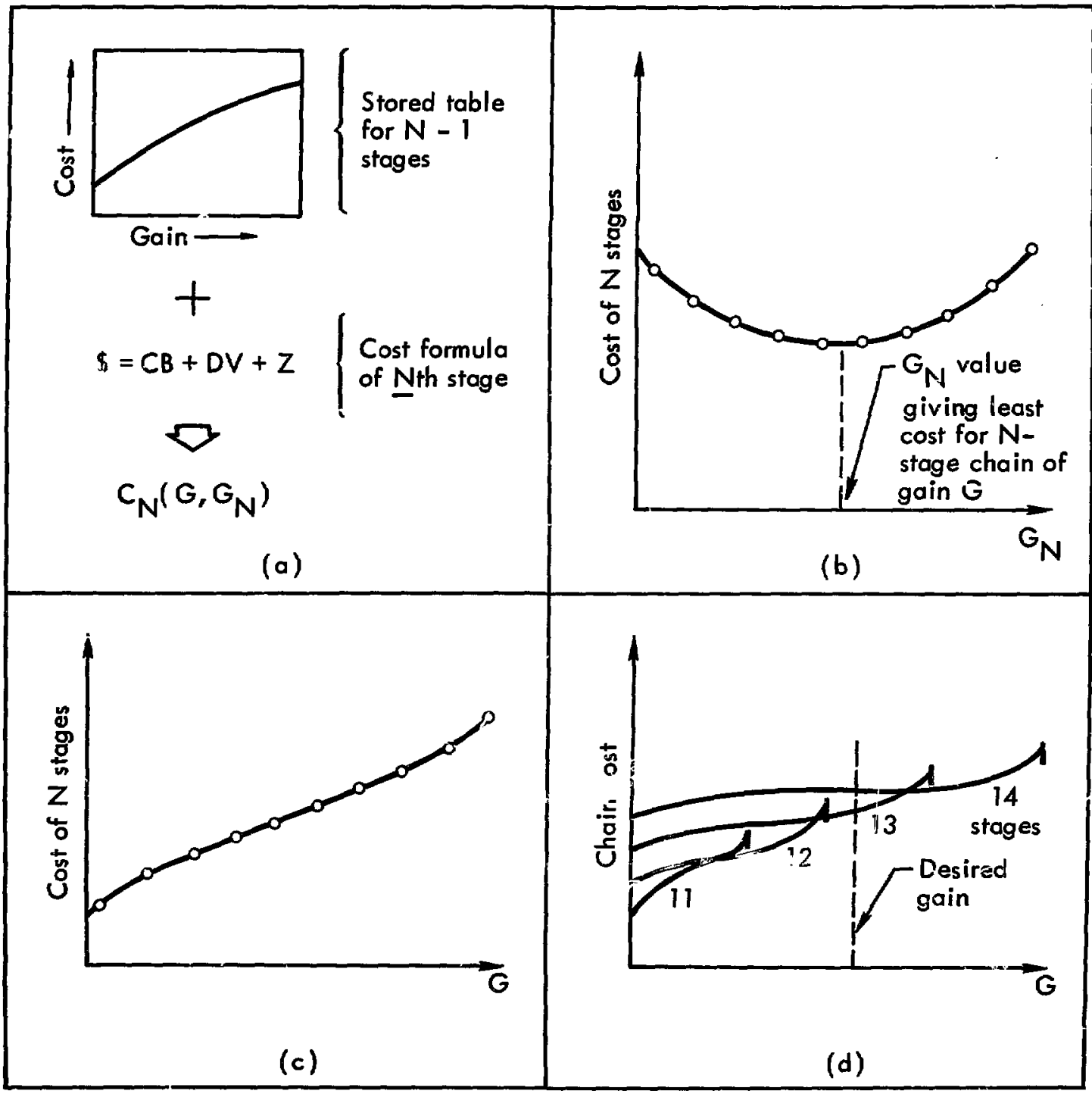

Fig. 71. Dynamic programming optimization of a tece amplifer chain. (a) The goin of $N$ stages is fixed at $G$. The $N$ th stage, of gin $G_{N}$, is added to the remsining $N-1$ stases of gain $G / G_{N}$, and the cott is found by adding the previous computed cost of the $N$ - I stages to the formula value for the $N$ th stage. (b) $G_{N}$ is varied, and the minimum cost is found for the $\kappa^{\prime}-s t a c$ chain of gein $G$. (c) This proces is caried out for various values of $G$, and a table of $\operatorname{cost}$ of $n N_{\text {-r }}$ age chain $v G$ is produced. (d) The coats of achieving the desired chnin gin with various number of stages $(N)$ are compared, and the number of ateres is chowen which gives the dexired gin at minimum cost (in this exemple, 13 stace). 
Table 9. Minimum-cost stagisug for a 1-kJ laser amplifier

\begin{tabular}{|c|c|c|c|c|c|c|c|}
\hline Stage & $\begin{array}{l}\text { Output } \\
\text { (J) }\end{array}$ & Grin & $\begin{array}{c}\text { Beam } \\
\text { aperture } \\
\text { (cm) }\end{array}$ & $\begin{array}{l}\text { Glass } \\
\text { thickness } \\
\text { (cm) }\end{array}$ & $\begin{array}{l}\text { Stored } \\
\text { energy } \\
\text { density } \\
\left(\mathrm{J} / \mathrm{cm}^{3}\right)\end{array}$ & $\begin{array}{c}\text { Capacitor } \\
\text { storage } \\
\text { energy } \\
(\mathrm{MJ})\end{array}$ & $\begin{array}{c}\text { Glass } \\
\text { volume } \\
\text { (liters) }\end{array}$ \\
\hline 1 & 1000 & 3.20 & 28.2 & 30.9 & 0.343 & 1.66 & 38.7 \\
\hline 2 & 313 & 1.81 & 22.3 & 13.2 & .433 & 0.557 & 10.3 \\
\hline 3 & 173 & 1.83 & 16.6 & 9.88 & .583 & .311 & 4.26 \\
\hline 4 & 94.4 & 1.90 & 12.3 & 10.1 & .600 & .179 & 2.38 \\
\hline 5 & 49.6 & 1.99 & 8.89 & 10.6 & .600 & .0987 & 1.32 \\
\hline 6 & 25.0 & 2.51 & 6.31 & 13.4 & .600 & .0629 & 0.839 \\
\hline 7 & 9.96 & 2.91 & 3.98 & 15.2 & .600 & .0283 & .378 \\
\hline 8 & 3.43 & 3.19 & 2.34 & 16.3 & .600 & .0104 & .139 \\
\hline 9 & 1.07 & 3.20 & 1.31 & 16.3 & .6nO & .0033 & .044 \\
\hline 10 & 0.336 & 3.20 & 0.73 & 16.3 & .600 & .0010 & .014 \\
\hline 11 & .105 & 3.20 & .41 & 16.3 & .600 & .0003 & .0044 \\
\hline$\$ 2$ & .033 & 3.20 & .23 & 16.3 & .600 & 0.0001 & .0014 \\
\hline 13 & .0102 & 3.20 & .13 & 16.3 & .600 & $3 \times 10^{-5}$ & .0004 \\
\hline 14 & 0.0032 & 3.20 & 0.07 & 16.3 & 0.600 & $1 \times 10^{-5}$ & 0.0001 \\
\hline
\end{tabular}

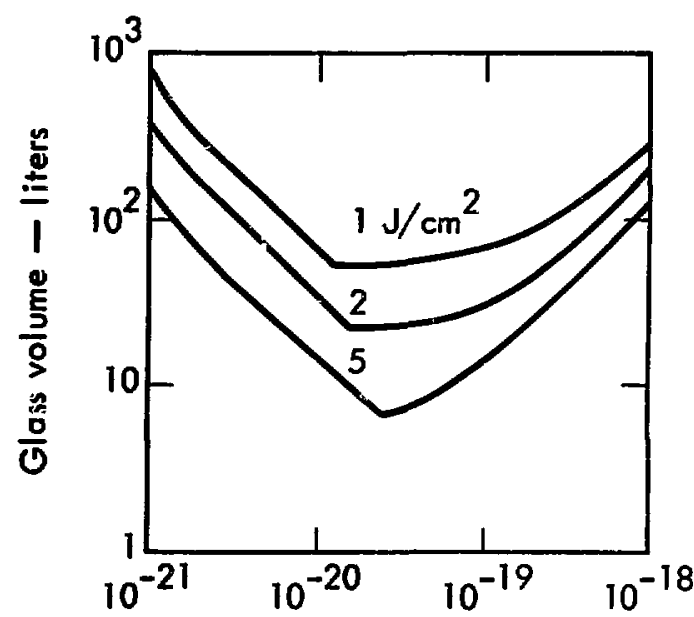

Stimulated-emission cross section,

$$
\sigma-\mathrm{cm}^{2}
$$

Fig. 72. Required glass volume as a function of stimulatedemission cross section ( $\sigma$ ) for a range of flux densities. Note that the cross section value for minimum gass volume is nearly insensitive to flux density.

output stage and 14 different sizes of amplifiers. Both these results are undesirable from the viewpoint of system ownership cost.

The output stage is the most difficult to construct and the most expensive part of the system, and it is not a good idea to have its gain (and thus its size) large. Instead, we should reduce the final stage gain and increase the gain of the other stages somewhat to make up. Since the original design is optimal, the cost increase goes quadratically as we move away from the minimum cost point. This means that the initial cost increase vill be small as we reduce the final stage gain, and so we may proceed without fear of drastic cost penalties.

The large variety of stages is undesirable because of the many different parts which will have to be stocked and because of the separate design efforts required for each stage. In order to maximize system "up-time," it is better to reduce the different kinds of disks and lamps used, so that spares will be more available in the event of failure. The best way to do this is to duplicate stages. Instead of a large stage preceded by a small stage, we should use two large stages. This takes more capacitors and glass than the small-large combination, but it is cheaper in the long run because of the initial design cost reduction and the up-time increase made possible by the smaller number of components. Once more, the cost increase over an optimal system can be small because of the quadratic cost behavior near an optimum.

Let us now modify our optimal design to incorporate paired stages and lower output gain. We will first consider the tradeoffs involved in changes in the output gain, and then the effects of stage pairing.

The effects of modifying the gains in the final three stages of the chain are illustrated in Fig. 73. This diagram shows the combined cost of these stages as the final and driver stage gains are varied. The pre-driver gain is changed at each point to keep the total three-stage gain constant. We see that if we reduce the output stage gain the cost indeed increases, but that we may reduce the gain to 2 for only an added $15 \%$ cost. At this final gain, the driver and final stages have the same aperture, since the final is running at 
twice the flux of the driver. They may thus be made identical, since a gain of 2 is near optimal for the drit us (the driver gain will in fact be slightly larger than the final due to saturation). Thus, for a slight cost increase we have achieved both reduced output-stage gain and pairing of output and driver stages. We deduced this for fixed gain in the last three stages; if we had varied this gain we could have lowered the cost increase somewhat.

Given the paired output-driver stages, we may likewise pair up the remaining stages. Rather than calculate new optimal staging, the gains of the optimal

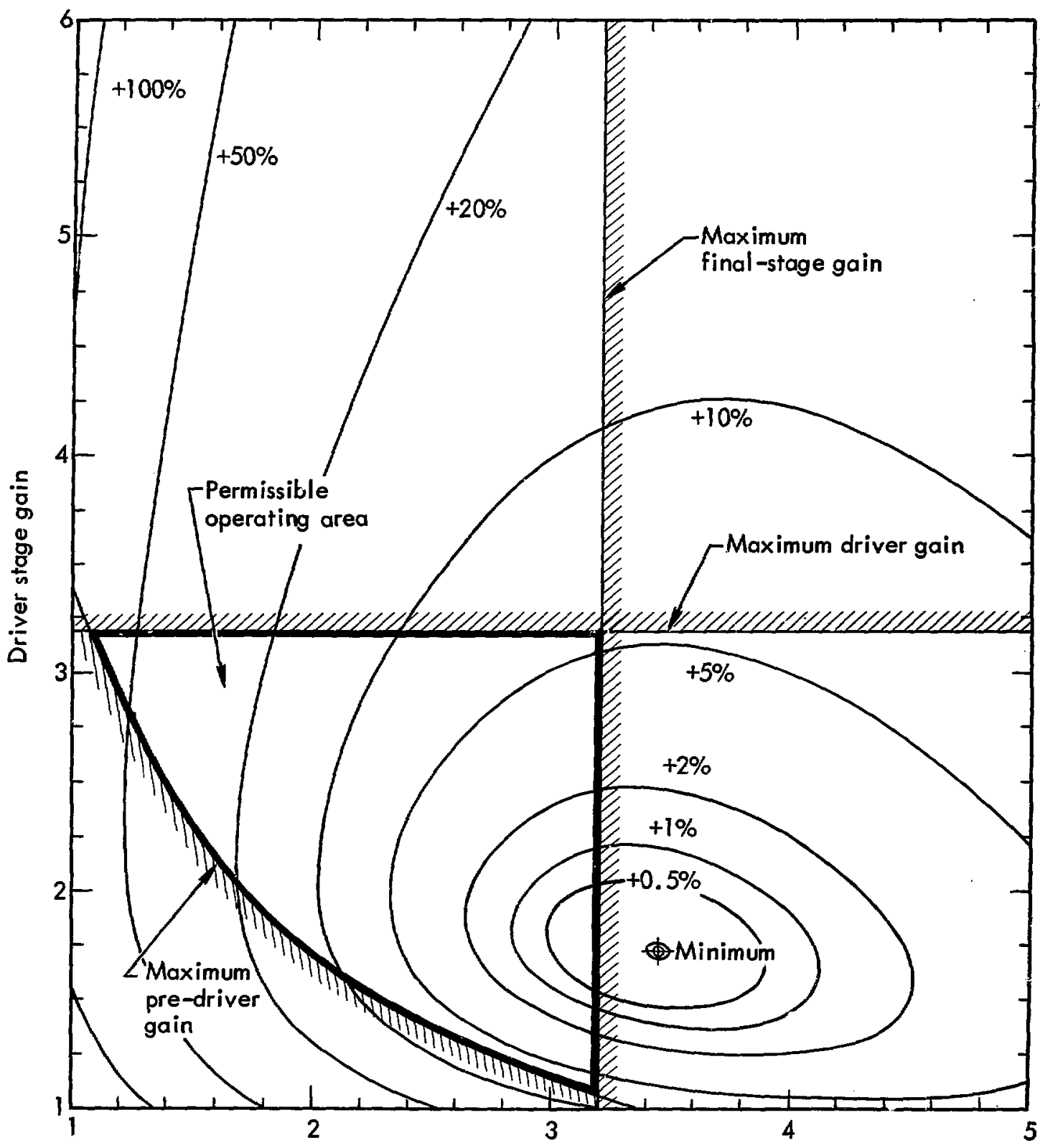

Final stage gain

Fig. 73. Cost analysis of three-stage disk laser amplifier. The constant-cost contours are for the indicated percentuges above the minimum cost shown. The permitted operating area is determined by limits on the maximum gain of the predriver, driver, and final stage amplifiers. 
Table 10. Improved steging for a 1-kJ laser amplifier

\begin{tabular}{|c|c|c|c|c|c|c|c|}
\hline Stage & $\begin{array}{l}\text { Output } \\
\text { (J) }\end{array}$ & Gain & $\begin{array}{c}\text { Beam } \\
\text { aperture } \\
\text { (cm) }\end{array}$ & $\begin{array}{c}\text { Glass } \\
\text { thickness } \\
(\mathrm{cm})\end{array}$ & $\begin{array}{c}\text { Beam } \\
\text { output } \\
\text { energy } \\
\text { density } \\
\left(\mathbf{J} / \mathrm{cm}^{2}\right)\end{array}$ & $\begin{array}{c}\text { Copacitor } \\
\text { storage } \\
\text { energy } \\
\text { (MJ) }\end{array}$ & $\begin{array}{l}\text { Glass } \\
\text { volume } \\
\text { (liters) }\end{array}$ \\
\hline $1(D)$ & 1000 & 2.00 & 30 & 20.3 & 2.0 & 1.10 & 26.0 \\
\hline $2(D)$ & 500 & 2.10 & 30 & 20.3 & 1.0 & 1.10 & 26.0 \\
\hline $3(C)$ & 240 & 2.20 & 20 & 14.2 & 1.0 & 0.45 & 8.6 \\
\hline 4(C) & 108 & 2.26 & 20 & 14.2 & 0.45 & .45 & 8.6 \\
\hline $5(B)$ & 48 & 3.00 & 10 & 15.3 & 1.0 & .24 & 2.0 \\
\hline $6(B)$ & 16 & 3.10 & 10 & 15.3 & 0.33 & .24 & 2.0 \\
\hline $7(A)$ & 5.1 & 3.10 & 3 & 15.8 & 1.0 & .06 & 0.29 \\
\hline $8(A)$ & 1.6 & 3.20 & 3 & 15.8 & 0.32 & .06 & .29 \\
\hline 9 & 0.50 & 3.10 & 1 & 15.8 & 1.0 & .01 & .12 \\
\hline 10 & 0.16 & 3.20 & 1 & 15.8 & 0.32 & 0.01 & 0.12 \\
\hline
\end{tabular}

unpaired chain were used. The difference from the actual optimum is minimal. The results of this pairing, and of the output stage gain reduction, are illustrated in Table 10. This chain (as characterized in Table 10) forms the basis of the prototype fusion laser. Not all of the small stages will be built; instead the last four sizes (called $A, B, C$, and $D$ ) will be fed by a preamplifier section incorporating YAG and glass rods, since these have higher gain per unit length of material.

\section{A PUMPING MODEL FOR DISK AMPLIFIER DESIGN}

Once the aperture and gain of a disk amplifier are specified, we must select the number of disks, their doping, and the flashlamp drive pulse width in such a way that the design is optimal. We want to minimize the glass path, surface count, and capacitor bank size while at the same time satisfying parasitic-oscillation and lamp-life constraints. Our method is based on a mathematical model of the pumping in a disk laser.

Disk laser pumping may be approximated by a series of steps:

1. The relative pumping rate in the disks as a function of lamp current density is found by the ZAP computer program for optical power flow. The results are approximated by an analytic form and then averaged over a critically damped current pulse. The lamp self-loading is also found from the ZAP results.

2. The peak gain during a pulse is found by taking the fluorescent decay of the ions into account.

3. The computed peak gains are normalized to experimental results.

4. The effect of changing optical thickness is found by use of the one-dimensional computer code GENEFF. The face-mode parasitic problem is also analyzed using this information.

Plots of disk gain versus pulse width of the lamp drive pulse can be produced from the model, and optimum performance of the disk amplifier can be determined. We will go through the model in detail, and then show how it is used for design.

\section{Optical Power Flow Calculations}

The ZAP optical power flow program was run on a disk laser georietry with 22 lamps, $10 \times 14 \mathrm{~mm}$ in size, surrounding a $7 \times 14 \times 2-\mathrm{cm}$ elliptical disk of 3\% doped ED-2 glass. The instantaneous conversion efficiency and lamp leading were found for current densities from 440 to $4400 \mathrm{~A} / \mathrm{cm}^{2}$. These calculations were done in a self-consistent manner to include self-loading of the lamps. Figure 74 shows the results of the ZAP calculations and the fit to an analytic expression of the form

$$
\eta_{i}=A\left[1 \cdot \exp \left(-J / J_{0}\right)\right] J^{P},
$$

where $\eta$ is the efficiency, $J$ is the current density, and $J_{0}$ and $P$ are adjustable parameters. This approximation was numerically averaged over a critically damped current pulse to produce the pulse-averaged efficiencies shown in Fig. 75. These points were once more approximated by the same kind of form used for the efficiencies.

In addition to the efficiencies, the power flow calculation also yields the self-loading of the flashlamps due to power reflected back by the cavity. We may use this information to relate the input current density to the larger current density necessary to produce a power equal to the original input power plus the cavity-returned loading, as shown in Fig. 76. This 


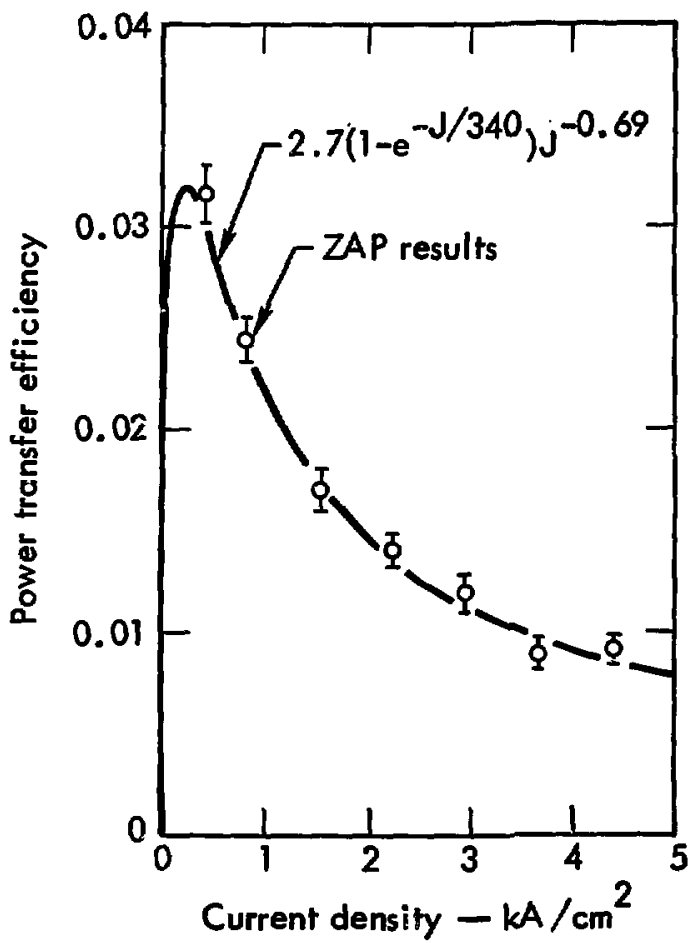

Fig 74. Modeling of a disk lser pumped by closely preiked 10-mm-id. lamps arrounding the dirke. The points with error bass are derived from the Moate Cado power-flow program ZAP; they are epproximately fitted by the anclytic expresion thown.

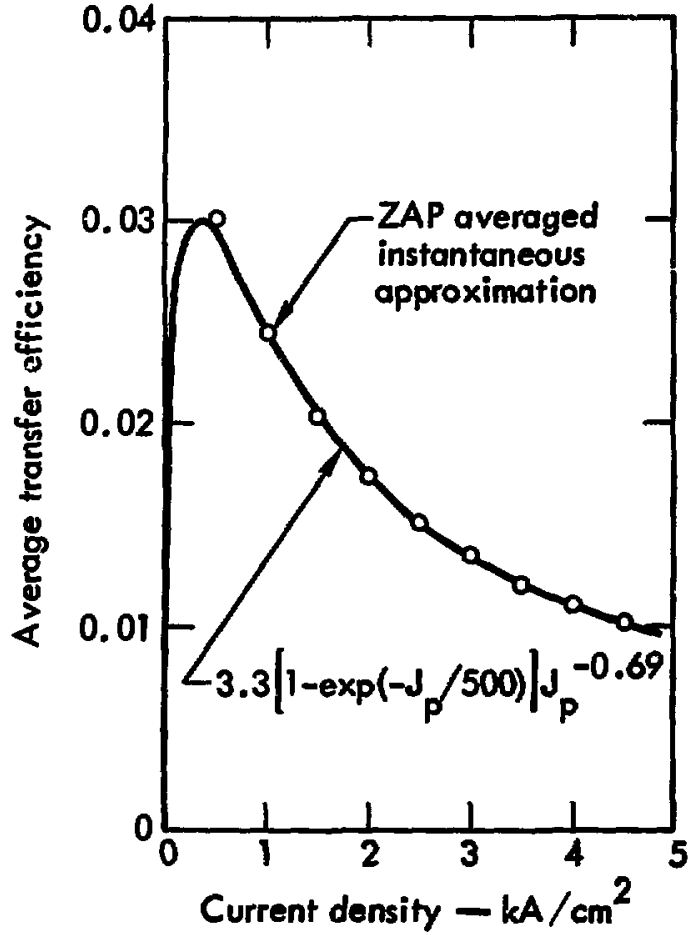

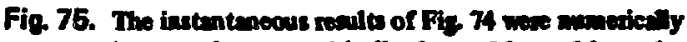
intograted over a critically dumped lapp-drive prime to give the pabe-avercyod points plothd here, wils are anslyticslly approxinated as elom. relation is necessary to determine lamp lifetime as a function of drive conditions.

\section{Fluorescent Decay Loss}

To calculate the effects of variations in the lamp-drive pulse width, we must know the effects of the fluorescent decay of the ions. This decay was modeled by assuming a half-sine drive pulse of base width $\tau$ and $a$ simple exponential decay with time constant $\tau_{F}$. The ratio of peak stored energy to drive energy was found as a function of the ratio of drive pulse width to fluorescent lifetime, and this relation was approximated by

$$
R=\frac{1+\left(u e^{-u / 4}\right) / 6}{1+2 u / 3},
$$

where

$$
u=\tau / \tau_{F} .
$$

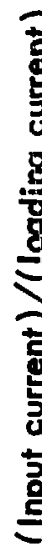

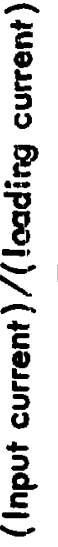

Fig. 76. Self-concibtent reduction of the orifindly mamed dive curreat due to lint retuming firom the cerity to the lampe. The ZAP revilts (plotiod pointo) are agin anslyticelly epproximatod. 
Since we with to optimize the doping we need to know the relation between doping and Auorescent lifotime. This rolation has boen determined experi. mentally and approximated anclytically; the results are shown in Fig. 77.

\section{Norndization to Experiment}

The celculation from electrical power to gein coefficient is uncertain becaus of possible errors in the lemp model in ZAP, the absorption quantum efficiency, the fluorescent docay (a nonexponentis component ha been noted by $R$. Fisher of $\mathrm{LL}$ ), and the stimulatedemision cross section (which relates stored enery to gin coefficient). We therefore use - multiplicative constent (sometimes inelegantly referred to as a fudee factor) to edjunt the celculated resuls to experiment. The gain of a baer identical to that modeled wa measured $s$ a function of bank energy, and the celculated gin was acaled to fit the experiments. The resulting fit is shown in Fig. 78.

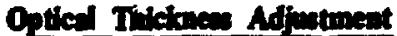

The ciculation so far has been for an optical thickness of $6 \mathrm{~cm}-\%$ ( $3 \%$ doping and $2 \mathrm{~cm}$ thick). We want to be able to adjut these values. We hrve detemined experimentally (experiments by $\boldsymbol{R}$. Fisher of LLI) thet the optical abeorption is proportional to the tots number of ions in the path, and does not depend on the ion concentration (unlike the fluorescent lifetime). We use the one-dimenasiond deposition code GENEFF to find the deposition dentity $a$ a function of optical thickness in a sab of mer glas illuminated from one side. The relative deposition density at optical thickness $\nu$ (in $\mathrm{cm}-\%)$ is well approximated by

$$
f(v)=\frac{1}{(1+0.771 v)^{1.25}}
$$

a can be seen from Fig. 79. The shape of this curve was found to be invensitive to changes in the lamp size or cument density exumed in GENEFF. The deposition predicted by CENEFF wa checksd against the rerult of a ZAP run; agreement was satisfactory.

The deposition profile in a diak is approximated by adding together two curves of the type shown in Fig. 79 to give a saddleshaped curve. To find the gain in one disk, we integrate the deposition across the disk to find the werage gain coefficient, and then multiply

\footnotetext{
By J. Meyen, R. Fither, and G. Leppelmeler.
}

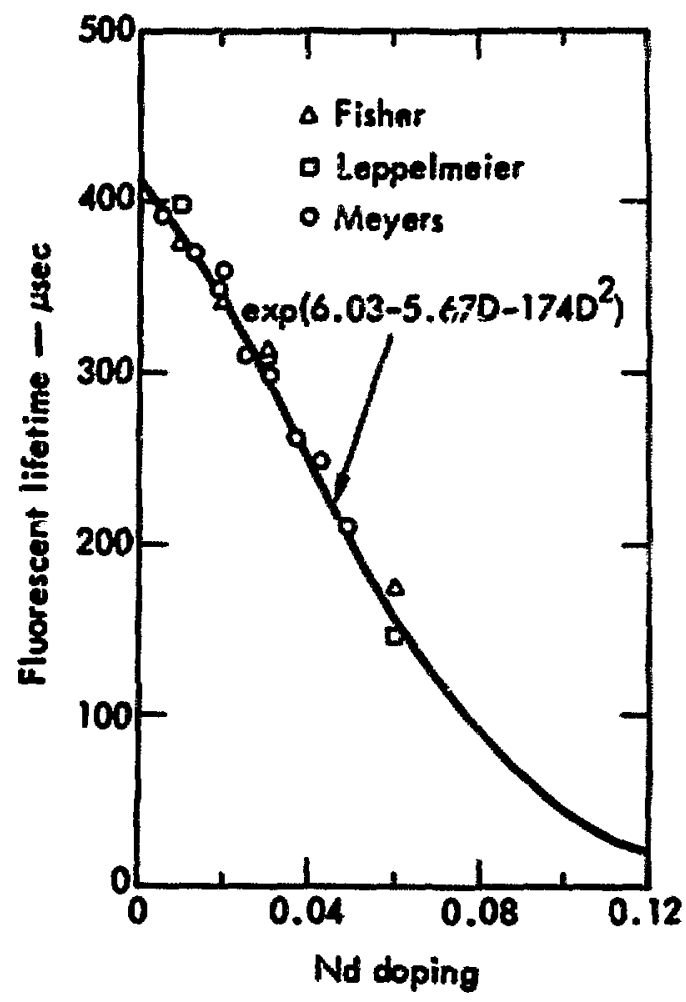

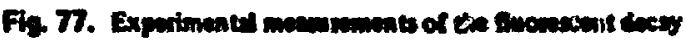

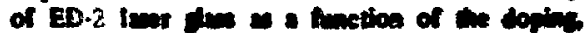

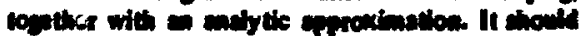
be nowd bat s monexponentill component of ency

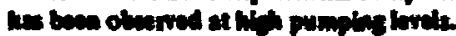

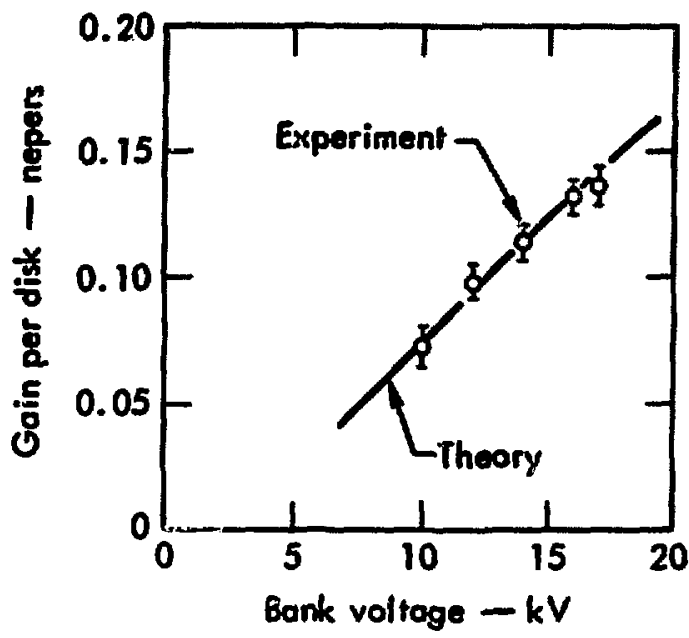

Fi. 78. A singeparameter fit to the meanrenente on a Iner ideatice to the one modvied in ZAP b wood to normelize the theomition nemils. 


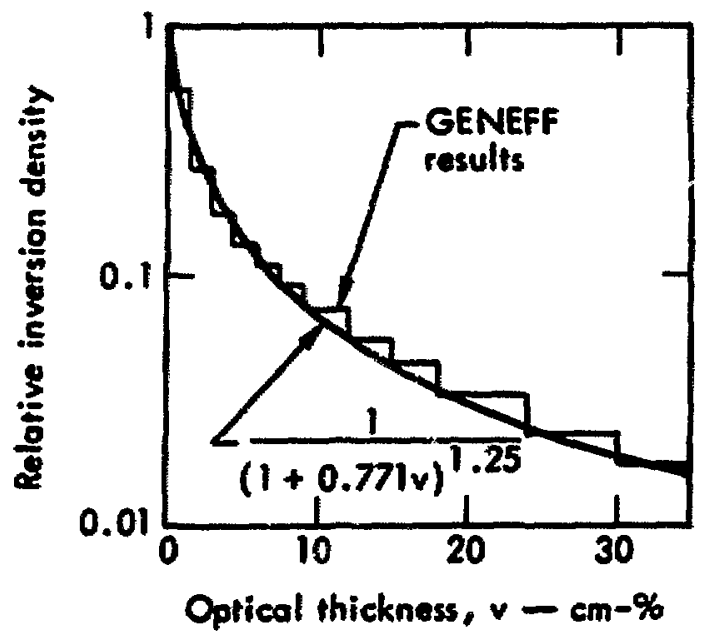

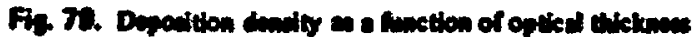

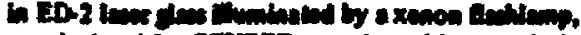

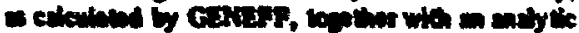

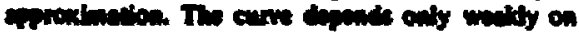

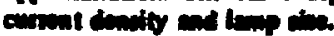

by the path length of the lever beam in the disk. The pin in sepen of then

$$
\delta=A\left[1 \cdot \frac{1}{(1+0.771 D Q)^{0.25}}\right] \text {, }
$$

where $D$ is the doping and 2 is the disk thicknes. This formals dllows to toale to difierent optical depths without doing aparaie ZAP nuts for each one.

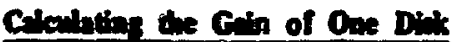

We now have everything we need to find the gain oi one disk. The input information is:

1. The energy loading or lifetime of the lumps.

$\therefore$ The Inmpodrive pule width.

3. The disk thickness.

4. The disk doping.

If the lamp loading is specified, we find the pesk current in a critically damped pule from the equations for a sinde-mesh lamp circuit, ${ }^{43}$ which yield

$$
J_{p}=1.515\left(\frac{W}{s d^{2}}\right)^{2 / 3} \mathrm{~A} / \mathrm{cm}^{2}
$$

where $W$ is the loading in $J / \mathrm{cm}, \tau$ is the full pulse width in sec, and $d$ is the lamp's inner diameter in $\mathrm{cm}$.
If the lamp lifetime ts specified, we fint find the peak current of a single pulse which would tive that lifetime in free air, and then roduce this 10 a value which gives the same lifetime in the cxity. The freedir peak current is given by

$$
J_{p}=\frac{772}{\left(\tau d^{2} N^{1 / 4.3}\right)^{1 / 3}} \mathrm{~A} / \mathrm{cm}^{2} .
$$

where $\boldsymbol{N}$ is the number of shots the lamp will lat. The relation between the free-sir and in-savity peak currents for $N$ chots comes from $2 A P$ code calculations (Fig. 76).

Since the pumpins rate is proportional to $J^{3 / 2} \eta$, the cotal pumping goes $\approx \beta / 2 \mathrm{~m}$. If we recall the reauls for the efficiency averased over the pulve and the optical depth correction, we obtuin the total grin per disk (in nepen) milable from the pumping $S_{0}$ :

$$
\begin{aligned}
s_{0}=3.35 & {\left[1 \cdot \frac{1}{(1+0.77102)^{0.25}}\right] } \\
& \times\left[1-\exp \left(U_{p} / 500\right)\right] \mathrm{J}^{0.01 \mathrm{~g},}
\end{aligned}
$$

when normalized to experimental date. This amount is reduced due to fuorescent loss by the previouly given factor $R$ :

$$
s=s_{0} R=s_{0}\left(\frac{1+\left(u e^{-u / 4}\right) / 6}{1+2 u / 3}\right) \text {. }
$$

\section{Deila Clart}

We may use our pumping model for a variety of taks. One valuable use is the production of laser desion charts, which sllow wa to select the best drive pulse width for a laer, and to find the pump energy required and lamp lifetime resulting from our choice.

A desien chart (Fig. 80) plots disk gain 28 a function of lamp-drive pulse width. There are two families of curves on the plot. One family is a set of curves along each of which the lamp's input energy is kept constsnt. The other set is for constent lamp lifetime. By examination of the chart, we can see that the pulie width which gives maximum lifetime at the detired gain is about 1 meec, while the minimum required input energy is nearer 0.4 mec. An actual system would operate somewhere between these values, depending on the relative importence of energy storage and reliability.

The applicution of this pumping model to the choice of disk thickness and doping is covered in another section of this emiannual report (see p. 13). 


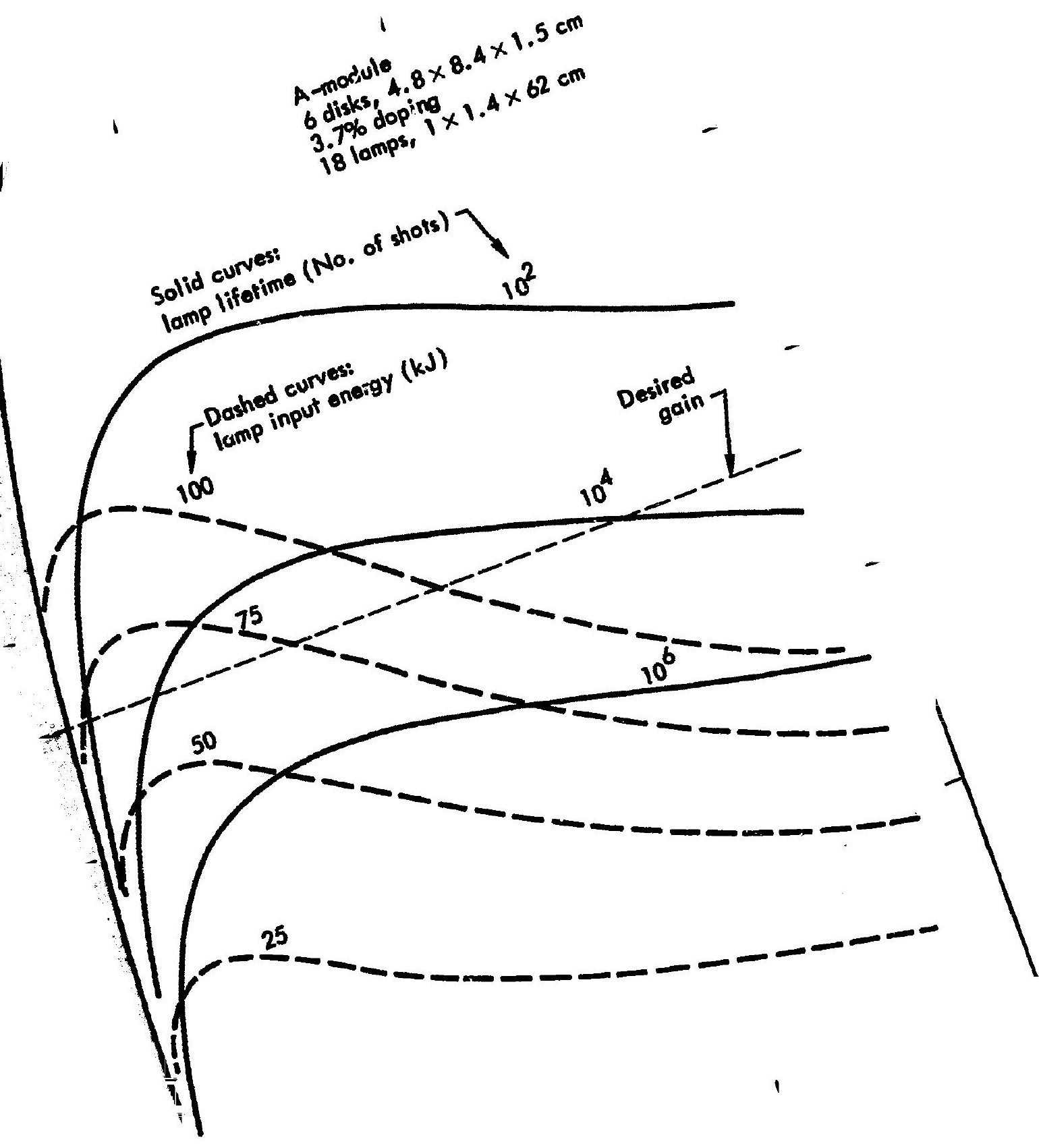


BOBG is a fonctrical-propagation computer code. used $\approx$ an aid in the design and optimization of optical and lawer system. We were motivated to develop BOBC by the success of the LASL code GLASS. The BOBG code is used to simulate systems consisting of a combination of optical and leaer elements. It ures my optios to deccribe the propesation of the light beam from one plane to another. The benm radius (circulur symmetry is aumed) is divided into 100 rays. The five baje properties of the beam that BOBG operates on are:
- Energy density
- Tolal energy
- Polarization ande
- Divergence
- Diameter

BOBG wa designed a a uer's code, and all input is explained in the Fortnn listing. All output is in the form of graphs and tables of important input and output parametern. To date the code will model the following 11 elements:

\section{Attenuator \\ Rod amplifier \\ Polsrizer \\ Optical rotator \\ Pockels cell \\ Tolescope \\ Aperture stop \\ Lens \\ Disk amsplifier \\ Gas amplifier \\ Apodized aperture}

The BOBG code will also simulate target backreflections, which are propagated backward through the system. This is very unful in deaigning the inoletion properties of a lacer system.

As input to the code the uaer applies a list of eloments in the order in which they appear in the system. Each olement is dexcribed by a spocified number of parameten. For example sot amplifier requives the following input:

1. Divtance from previous element in chain.

2. Angte of rod endi.

3. Index of refruction.

4. Angle of plane of incidence.

5. Length of rod.

6. Diumeter of rod.

7. Smalteigon gin coefficient.

8. Soturation flux.

9. Radial invertion ratio.

The code then considen one element at a time and operates on the previoully mentioned five beam

$$
\text { SYSTEM LAYOUT MAP }
$$

No.

ELEMENT

DIST

ROD AMPL IFIER

180.00

TELESCOPE

APODIZED APERTURE

197.60

297.60

Fize 82. BOBG system lyout map (from microfiche).

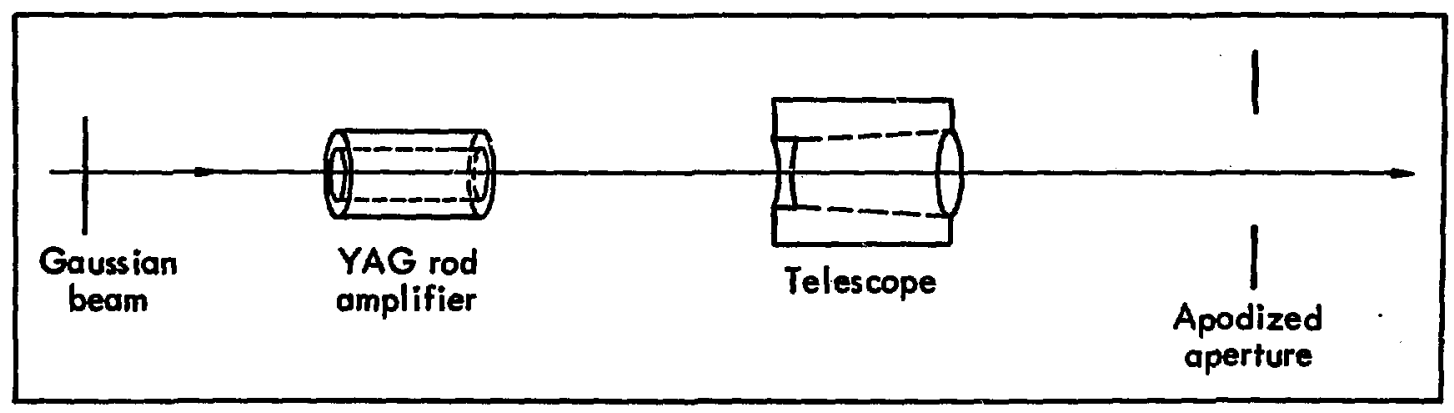

Fig. 81. Exanple system modeled by BOBG code. 
Mria cunstan CEM

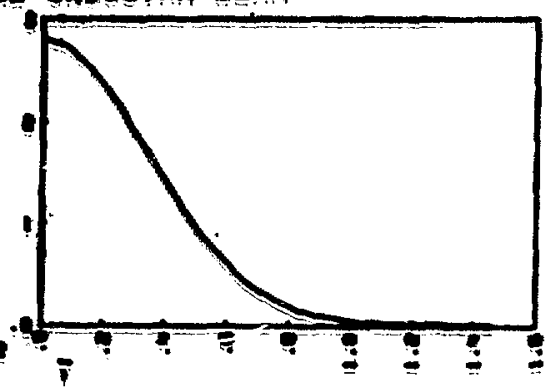

mom atrien

$-1$

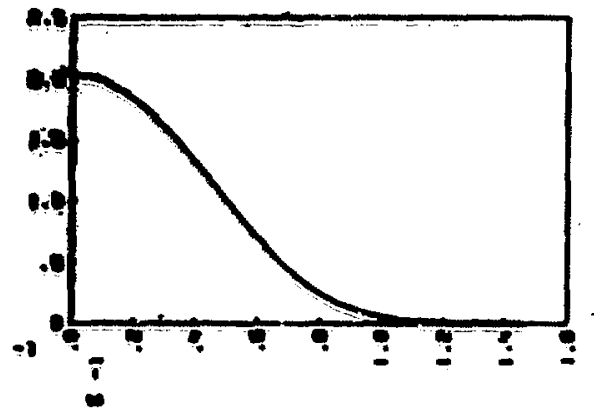

$\therefore$

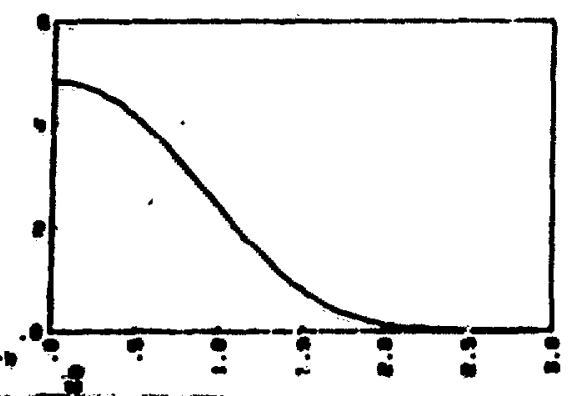

\section{ercusere to 148}

centoderion cines orringere is of

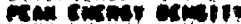
cinesy

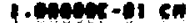

etop exancs -cipe cienr:

P.engerecte $6 \mathrm{cn}-\mathrm{z}$ i. rioct-on sucs
Otstant

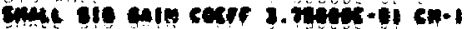

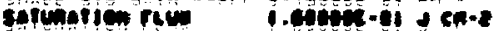

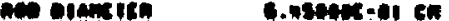

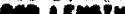

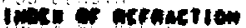
citis of mo tre

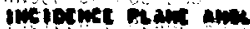

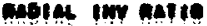
cerantarien smist

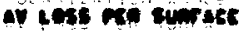
vicimite rasericm crutermet in titGiancish te iac

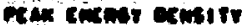
ciment

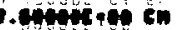

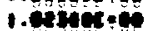

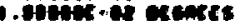

of the ciancs 1. chering

- cers erentes

0.010 .40 - tret cepte ctemes

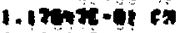

- entri-ei + en-2 coprin.08 vince

\section{enstanct}

artiniun bianter

cess unders

nagifle icatien

1. Cutanten $6 \mathrm{n}$

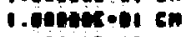

s. 964ty-te

- crencis o 1

odremence is in arcintra te ifs

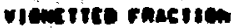

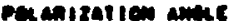

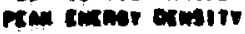
comer exes etches

2. Intrisen of - Crofer

croce exence

- cran-to +6n-e t. cunctos whes

\begin{abstract}
erstanes

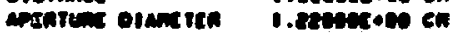

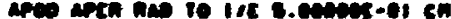

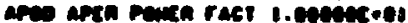

orringusere tes gratiten to se vicutrece rinstien contratien amer ran Eminer exmit cinimer coen cremes r. corengen of a.covrin-1: cisce eremes -.crina-en i ch-s

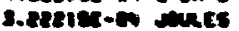

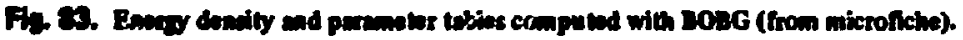




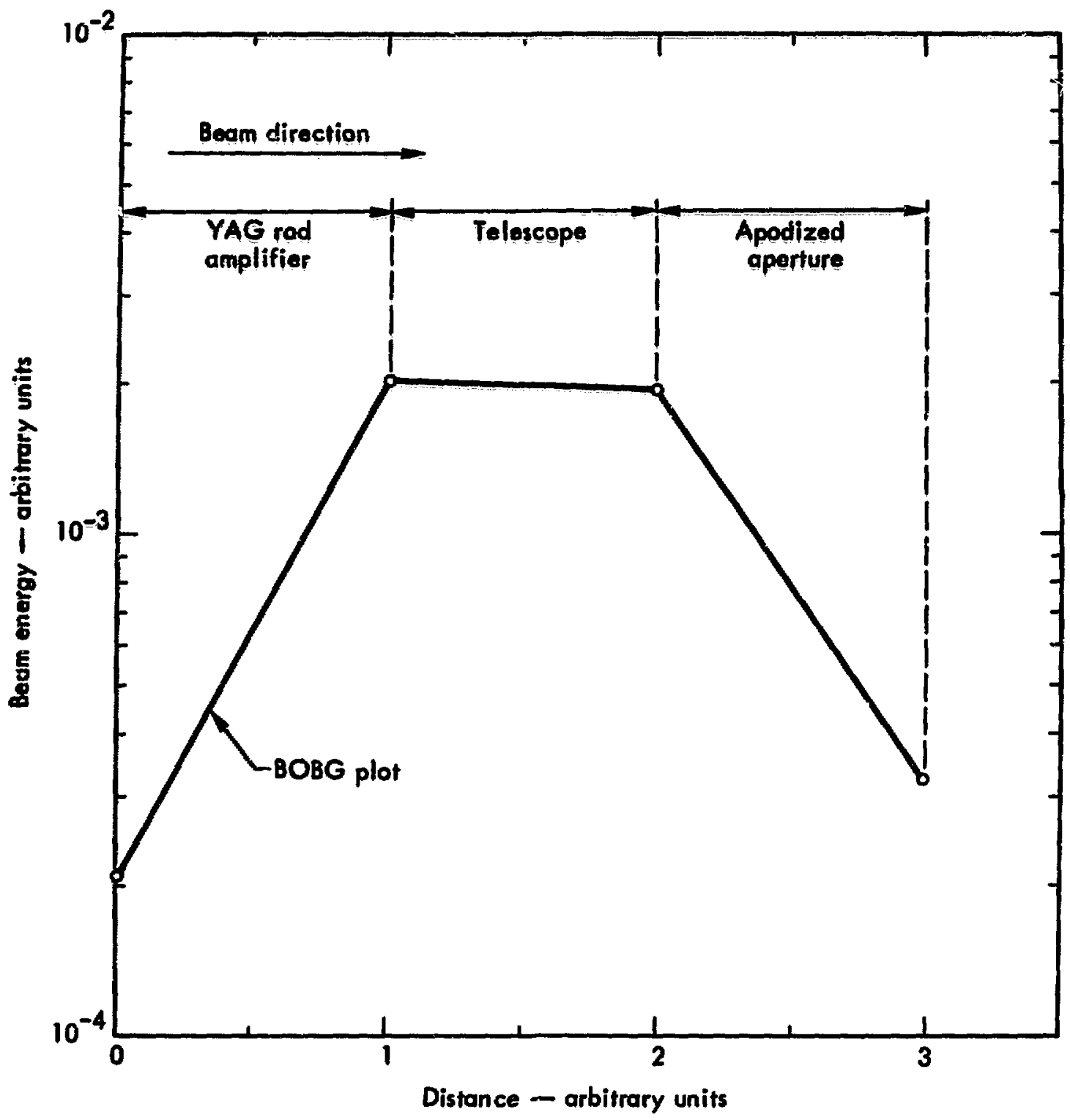

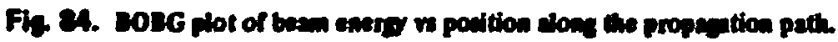

parameters in a manner characteriatic of that element. The operation of BOBG is best illustrated by an example. For a rod amplifier the following sequence of calculations is performed:

1. Calculate radius and energy density change due to beam divergence from previous element.

2. Calculate angle of incidence for beam to propagate along rod axis.
3. Reduce beam energy due to reflection loses at fint surface.

4. Calculate rotation of beam polarization due to difference between parallel and perpendicular polarization reflections.

5. Calculate energy density and radius change due to surface spread of beam going from one medium to enother. 
6. Amplify energy density using saturating gain equation (taking into account quadratic radial invorion profile if any).

7. Reduce enerby due to second surface reflection.

8. Calculate rotation of polarization at second surface.

9. Change enerey density and radius due to second surfuce spread.

10. Calculate portion of beam vignetted by rod aperture.

11. Iniegrate energy density to detennine total beam energy.

Each element has its own series of calculations which best model its effect on the beam properties.
BOBG is constantly being updated as new elements are introduced or as a better model equation is derived.

A simple example will illustrate how the code uses the properties of individual elements to model a system.

Shown in Fig. 81 is a simple system consisting of a rod amplifier (Nd:YAG), a telescupe, and an apodized aperture. This system was modeled by BOBC and the code's output appears in Figs. 82-84.

BOBG has been used in the design of the $1 \cdot \mathrm{kJ}$ solid-state laser system to study the effects of a rotator failure, sensitivity of the output energy to surface losses, and sensitivity of output energy to gain per stage.

\section{CPTIMAL SELECTION OF FLASHLAMP AND DRIVING-CIRCUIT PARAMETERS FOR DISK LASERS}

We have developed a method of selecting the llashlamp size and circuit parameters $(C, L$, and $V)$ for a disk laser module or submodule. The design criteria are:

- An integral number of lamps must fit around the disks, with appropriate allowance for wall thickness and lamp spacing.

- The circuit damping parameter $\alpha$ must lie between specified limits (i.e., $0.7 \leqslant \alpha \leqslant 1.1$ ).

- The charge voltage $V$ must lie between specified limits.

- The capacitance $C$ must be an integral multiple of a basic capacitor value $C_{0}$.

No attention is paid to possible variations in pumping efficiency due to changes in $\alpha$ or the lamp size; these changes should be relatively small.

The input information we assume is the following:

1. The lamps are placed around an exclusion circle of diameter $A$.

2. A space of width $F$ is left between lamps.

3. The ratio of wall thickness to lamp diameter has the consiant value $B$.

4. There are $N$ lamps.

5. The lamp length is $G$.

6. The total energy to all the lamps is $H$.

7. The full pulse width is given $(W=3 \sqrt{L C})$.

First we must find the lamp inner diameter $D$ which satisfies the geometrical constraints. Figure 85 shows that $1 / 2[F+D(1+2 B)]=1 / 2[A+D(1+2 B)] \times \sin$ $(\pi / N)$, or that

$$
D=\frac{A \sin (\pi / N) \cdot F}{(1+2 B)[1-\sin (\pi / N)]} .
$$

Now that we know the lamp diameter, we may find the circuit parameters for a given $\alpha$. We know that the lamp impedance parameter is

$$
K=1.3 \frac{G}{D},
$$

that the lamp input energy is

$$
E=\frac{H}{N},
$$

and that the characteristic time $(\sqrt{L} \bar{C})$ is

$$
T=\frac{W}{3} \text {. }
$$

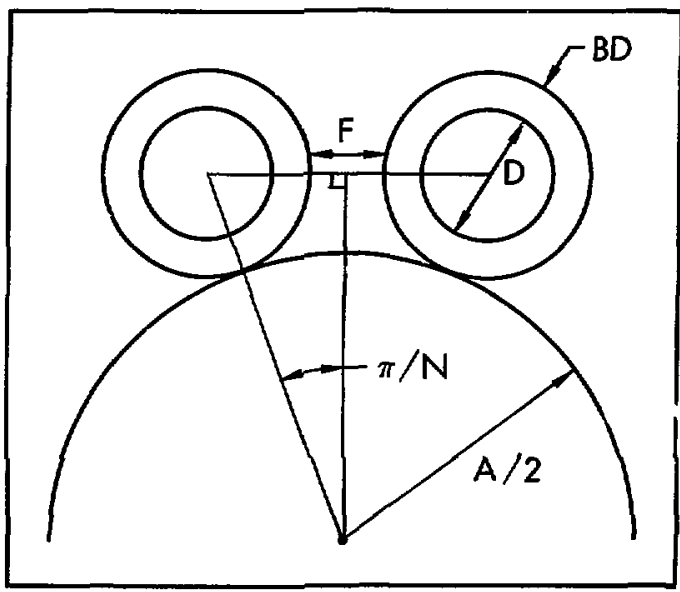

Fig. 85. Lamp geometry assumed in optimization of flashlamp and driving-circuit parameters. 


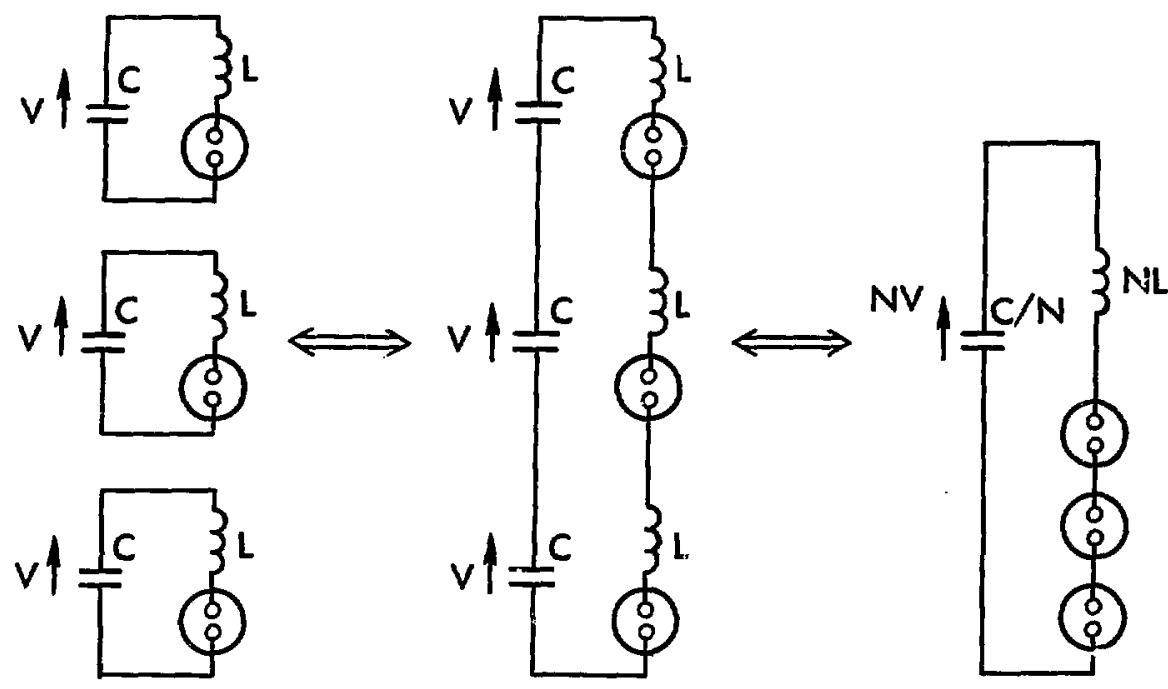

Fio, 86. Equivalent iamp circuits.

We then find the capacitance from the standard equation

$$
C=\left(\frac{2 E \alpha^{4} T^{2}}{K^{4}}\right)^{1 / 3}
$$

The voltage is given by

$$
V=\sqrt{\frac{2 E}{C}}
$$

the inductance is

$$
L=\frac{T^{2}}{C},
$$

and the characteristic impedance is

$$
Z=\sqrt{\frac{L}{C}} .
$$

The peak current density during the pulse is well approximated by

$$
J=\frac{2 V C}{\pi D^{2} T}\left(2 e^{-0.87 \alpha}\right),
$$

where the term in parentheses is a correction for noncritical damping.

We have found circuit values corresponding to one capacitor-inductor mesh driving one flashlamp. For added flexibility, we may wish to drive several lamps in seriez. If there are $M$ lamps in series, they may be driven by a circuit with

$$
\begin{aligned}
& C^{1}=\frac{C}{M}, \\
& L^{1}=M L, \\
& V^{1}=M V,
\end{aligned}
$$

as may be seen by inspection of Fig. 86 .

We can now select possible circuit values. We first set lower and upper limits $D_{\min }$ and $D_{\max }$ which we are willing to accept on the flastiamp inner diameter. These determine lower and upper limits on the number of lamps that can be placed around the disk laser cavity by the formula

$$
N=\frac{\pi}{\sin ^{-1}\left[\frac{F+D(1+2 B)}{A+D(1+2 B)}\right]} .
$$

For each number of lamps between these limits, we investigate all possible combinations of series lamps and parallel capacitors. We use all values $M$ of lamps in series for which $M$ is an integral divisor of $N$ (including, of course, 1 and $N$ ). This allows only situations in which the lamps are grouped in identical series strings; the added flexibility of nonidentical strings is assumed not worth the added complication. We also consider all possible values $X$ of capacitors in parallel from 1 to some maximum value $X_{\max }$ (this maximum is set by the danger of explosion if all the capacitors in a group dump into a fault in one of the 


\section{INPUT PARAIIETERS}

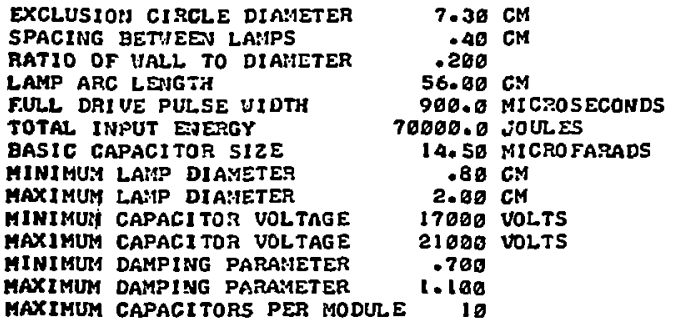

$\begin{array}{lr}\text { LAMP COUNT } & 12 \\ \text { LAMP I. } & 1.435 \mathrm{CM} \\ \text { LANP O.D. } & 2.0 B 9 \mathrm{CN} \\ \text { ENERGY PER LAHP } & 5333.3 \text { JOULES }\end{array}$

EKPLOSION FRACTION - 210

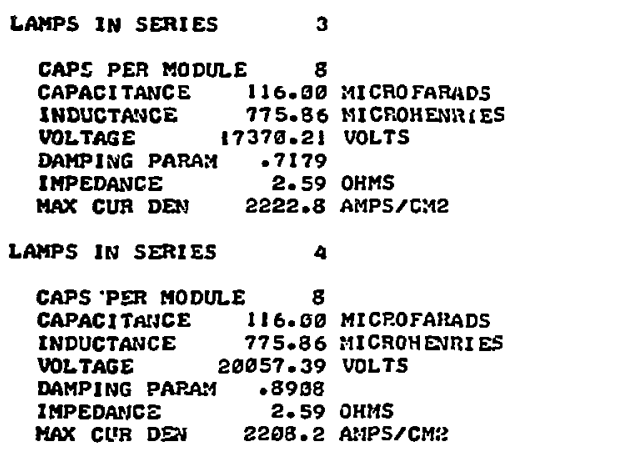

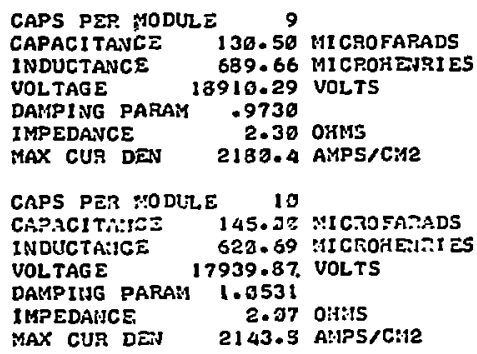

Fig. 87. Output of computer program for calculating lamp-circuit possibilities.

capacitors). Recalling that the groups are made up of capacitors of value $C_{0}$, we have

$$
C=X C_{0}
$$

The energy per lamp is the total input $H$ divided by the lamp count $N$, so the energy in a string of $M$ lamps is

$$
E=\frac{M H}{N} .
$$

The capacitor voltage is then

$$
V=\sqrt{\frac{2 E}{C}}=\sqrt{\frac{2 M H}{N X C_{0}}} .
$$

We reject values of $M$ and $X$ which give voltages above the maximum capacitor rating $V_{\max }$, or below a minimum value $V_{\min }$ (low voltage means inefficient use of bank energy storage ability).
Next, we calculate the circuit damping parameter $\alpha$, which is given by

$$
\alpha=K\left(\frac{c^{3}}{2 E T^{2}}\right)^{1 / 4}
$$

The impedance parameter $K$ we use is the value for $M$ lamps in series:

$$
K=1.3 \frac{M G}{D} .
$$

If we recall that $C=X C_{0}, E=M i / N$, and $T=W / 3$, we get

$$
\alpha=\frac{1}{3} \frac{M G}{D}\left(\frac{9 X^{3} C_{0} N}{2 M H W^{2}}\right)^{1 / 4} .
$$

We then test this value against the limits $\alpha_{\min }$ (underdamped) and $\alpha_{\max }$ (overdamped). Values 
outside these limits are rejected. For each lamp count $N$, there may be values of lamps in series, $M$, and capacitors in parallel, $X$, which lead to integral-number groupings of series strings, voltages between the allowed limits, and permissible damping. If such values exist, they define an acceptable solution to the lamp-circuit problem. The set of all such solutions for various allowed values of $N$ is then examined to find the one which is best in the intended application.

A computer program which automatically carries out the search outlined above has been written. An example of its output for values characteristic of the $A$ module of the $1 \mathrm{~kJ}$ laser system is shown in Fig. 87. The program can be used to systematically study the lamp and circuit constraints on modules of various sizes. 


\section{INTRODUCTION}

The developmental and prototype-hardware effort on components has been concemed mainly with Faraday rotators, Pockels cells, and polarizers. Faraday rotators or Pockels cells will be used as isolators, depending on the beam aperture. For beam apertures up to $1.8 \mathrm{~cm}$, Pockels cells will be used because $K D^{* P}$ of good optical quality is available up to this size. For apertures greater than $1.8 \mathrm{~cm}$, only Faraday rotators can be used.
The Verdet constant and nonlinear index of refraction of candidate rotator glasses such as Owens-Illinois EY-1 and Hoya FR-4 have been measured. Various single-layer and multilayer polarizer coatings have been investigated with regard to trade-offs among rejection level, transmission at the laser wavelength, and resistance to op ical damage. The results obtained have been quite encouraging.

\section{FARADAY ISOLATORS}

Faraday isolators act as optical diodes, perrnitting light to pass in one direction but not in the other. They are used to prevent back-reflected light from reentering the source from which the light originally came. This is accomplished by the Faraday effect, wherein the plane of polarization of the light is rotated by an amount proportional to the strength of a magnetic field applied along the direction of propagation, and the sense of the isolation depends on the direction of the field with respect to the direction of propagation (input or left-hand). Forward-propagating, linearly polarized light can be rotated $45^{\circ}$ in a right-hand sense, whereas back-reflected light will be rotated $45^{\circ}$ in a left-hand sense. Thus the plane of polarization of the back-reflected light will be normal to that of the initial beam, and therefore it can be rejected by a polarizer. The Faraday effect occurs in any magnetic material, particularly in paramagnetic or diamagnetic glasses.

One can calculate the degree of isolation required in a laser chain for a given gain per stage, $G$. If the reflected light returns along the same path as the original forward beam, and the intensity $\left(W / \mathrm{cm}^{2}\right)$ or flux $\left(\mathrm{J} / \mathrm{cm}^{2}\right)$ nowhere exceeds that in the outgoing beam, then it can be shown that the extinction ratio required for polarizers is $G^{-4}$ per stage and the uniformity of rotation required for the rotators is $G^{-2}$. Thus a system consisting of successive amplifier stages with gains of 3.2 requires polarization extinction of 100:1 $(-20 \mathrm{~dB})$ for each stage. In addition, the rotator magnetic field uniformity must be such that after one pass less than $1 \%$ of the light is of the "wrong" polarization at any (transverse) position of the beam. This translates to a magnetic field uniformity of about $10 \%$. In reality we are designing cur magnets to be much more uniform, partly because it is easy to do so, and because it may be possible to remove many of the rotators if depolarization (due to birefringence) turns out not to be a serious problem.

The system's requirements, then, are $-20 \mathrm{~dB}$ extinction ratio per stage for polarizers, and $-10 \mathrm{~dB}$ uniformity for rotators. In practice, $-8 \mathrm{~dB}$ polarization is provided by the glass disks, and $-24 \mathrm{~dB}$ rotation unisormity has been chosen as the goal for the magnets.

Glass for Faraday rotators must have a short optical-path length in order to minimize nonlinear effects, absorption, and the effects of residual birefringence. Hence, a high Verdet constant $V$ is desirable; this has led to the development of terbium glasses.

We have measured Verdet constants of a variety of glasses in pulsed magnetic fields up to $80 \mathrm{kOe}$ (Table 11). The most promising glasses are the Hoya FR-4 and Owens-Illinois EY-1. The EY-1 sample had numerous platinum inclusions (about $10,000 / \mathrm{cm}^{-3}$ ), which led to material damage at about $1.5-2 \mathrm{~J} / \mathrm{cm}^{2}$ for 200-psec pulses. New samples of EY-1 have been obtained with far fewer inclusions, and we are now investigating the damage threshold, absorption, and nonlinear index of refraction of EY-1 and FR-4, using the ILS.

The EWD544A sample listed in Table 11 is a small piece having rather poor optical quality and, therefore, EWD544A is not a serious candidate for large-aperture rotators at present. The Soveril sample is the rod in the CILAS isolator discussed below (p. 79). The LCG-91 and the ED-2 varieties are laser glasses, whose Verdet constants were measured in order to calculate the effect of stray magnetic fields.

The $1-\mathrm{kJ}$ amplifier chain will require rotators and polarizers with apertures up to $30 \mathrm{~cm}$ in diameter. The 
Table 11. Measureá Verdet constants

\begin{tabular}{|c|c|c|}
\hline \multirow{3}{*}{$\frac{\text { Cles }}{\text { FR-4 }}$} & \multicolumn{2}{|c|}{ Verdet constent, $V(\mathrm{~min} / \mathrm{G}-\mathrm{cm})$} \\
\hline & At $0.63 \mu \mathrm{m}$ & A1 $1.95 \mu \mathrm{m}^{2}$ \\
\hline & $-0.137 \pm 0.003$ & $-0.034 \pm 0.0008$ \\
\hline EWDS44A & $-0.17 \pm .02$ & $.0051 \pm .005$ \\
\hline EY-1 & $-0.144 \pm .005$ & $.0041 \pm .001$ \\
\hline Soreril & $0.018 \pm .0005$ & $0.0070 \pm .0004$ \\
\hline LOG-91 & $.015 \pm .002$ & $.0046 \pm .0012$ \\
\hline ED-4 & $.017 \pm .001$ & $.0060 \pm .0008$ \\
\hline ED-2. $1^{b}$ & $.017 \pm .003$ & $.0057 \pm .002$ \\
\hline ED-2.2 & $.016 \pm .003$ & $.0047 \pm .0016$ \\
\hline ED-2.3 & $.014 \pm .003$ & $.0039 \pm .0015$ \\
\hline ED-2.6 ${ }^{b}$ & $0.009 \pm 0.003$ & $0.0034 \pm 0.0017$ \\
\hline
\end{tabular}

${ }^{\mathrm{a}}$ Extrapolated from $1.08 \mu \mathrm{m}$.

b The number after the decimal point is the approximate Nd doping (\%).

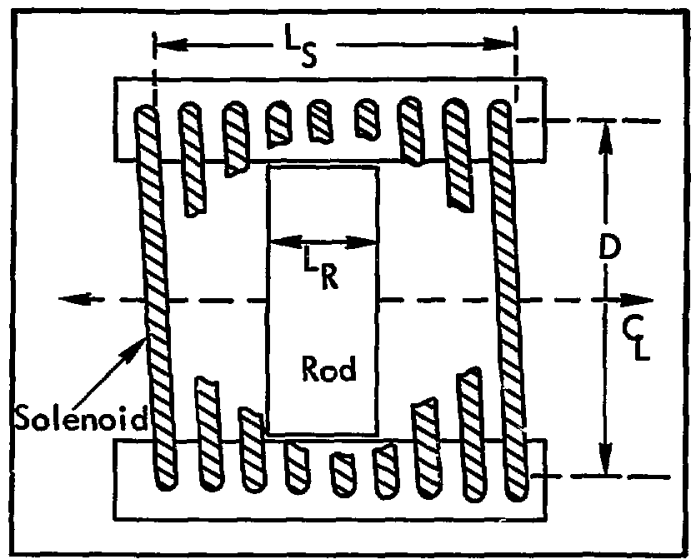

Fig. 88. Sketch of the Faraday rotator. The optical path is slong the center line.

system will employ amplifier modules $A$ through $D$ of increasing aperture. $A$ and $B$ rotator modules are now being constructed; $C$ and $D$ rotator modules have been designed, but it has not been decided whether to use magnetic (superconducting solenoids) or electric (capacitor bank) energy storage.

The essential features of a Faraday rotator are shown schematically in Fig. 88. Rotator design has been predicated upon minimum optical path length through the material, consistent with mechanical and budget constraints. In Fig. 89 we show some of the considerations entering into the design of these components. The magnetic energy required to produce a polarization vector rotation of $\pi / 4$ radians for a light ray traversing a glass rod along a coaxial solenoid center line is plotted for a fixed solenoid inner diameter $D$ as a function of the solenoid jength $L_{S}$. Each solid curve corresponds to a particular Faraday rod length $L_{R}$. For minimum erergy consumption, $L_{S}$ should be chosen such that the appropriate $L_{R}$ curve is near its minimum. Figure 89 has been cosstinucted as a numerical illustration of a $B$ module, with $D=12 \mathrm{~cm}$ (Fig. 88) and $V=0.040 \mathrm{~min} / \mathrm{G}-\mathrm{cm}$. The curves shown ane universal, however, since the energy scales as the cuioe of $D$ and the inverse square of $\boldsymbol{V}$.

Since the magnetic feld of a solenoid of finite length increases with radial distance from the center line, the Faraday rotation angle will be greater than $\pi / 4$ for off-axis light. ronsequently, isolation of forward and backward beam will be less than perfect (even for perfect polarizers), and will be worst near the rim of the rotator element.

We quantify this departire from perfection by defining a rejection ratio $R$ as that fraction of the "wrong way" incidunt intensity which emerges from an isolator unit with perfect polarizers, at a radial distance of nine-tenths of the inner solenoid radius. $\boldsymbol{R}$ was computed, with the help of the computer code COILS, 44 for a variety of $\left(L_{R} / D, L_{S} / D\right)$ combinations. The results are conveniently shown as dashed lines (lines of constant $10 \log R$ ) in Fig. 89. It may now be seen from this figure that specification of required rejection $\boldsymbol{R}$ and rod dimensions (aperture and length) serves to determine the solenoid geometry for minimum energy expenditure. The $B$-module operating point indicated in Fig. 89 corresponds to an energy expenditure of $6.5 \mathrm{~kJ}$.

The effect of strain birefringence in the rod, either residual or thermally induced, must also be taken into account. For design purposes, it is adequate to assume that this birefringence has radial symmetry. In this case, isolator transmission in the reverse direction exhibits the familiar Maltese-cross pattern, having fourfold rotation symmetry about the propagation axis. Choosing a worst-case (maximum reverse transmission) ray, we can use the same definition of $\boldsymbol{R}$ as that above to determine the effect of birefringence upon isolator performance. The result is illustrated in Fig 90. It is seen that severe birefringence, if present, completely outweighs magnetic field inhomogeneity as a degrading factor upon isolation.

One of the first experimental tasks was to test and characterize a CILAS RF70 Faraday isolator consisting of a $64-\mathrm{mm}$-diam by $135-\mathrm{mm}$-long glass rod (Soveril B-1664) in a solenoid magnet. The magnet is driven by a self-contained power supply and capacitor bank. The associated polarizers consist of eight glass plates. The optical properties as measured with a 1-mm-diam beam at $1.08 \mu \mathrm{m}$ are summarized in Table 12 . 


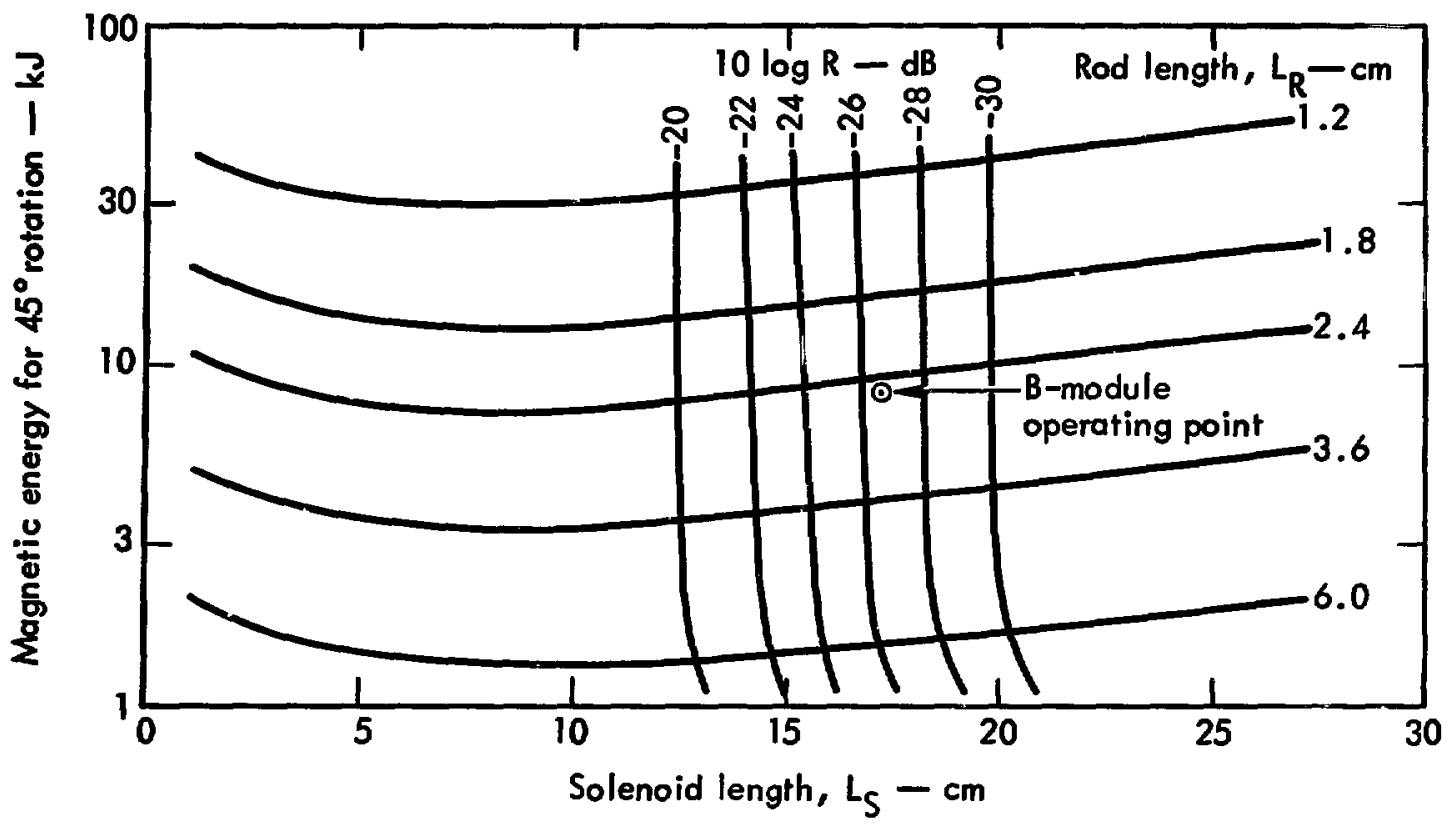

Fig. 89. Dexign curves for the $B$-module Furaday rotator. For minimum energy consumption, the solenoid length $L_{S}$ shonld be chosen 20 that the appropriate $L_{R}$ curve is near its minimum. The rejection $R$ is shown by the family of near-vertical curver. The operating point selected for the B-module rotator is shown.

The CILAS isolator meets or exceeds manufacturer's specifications, and is presently incorporated in the LLL. Long Path Laser.

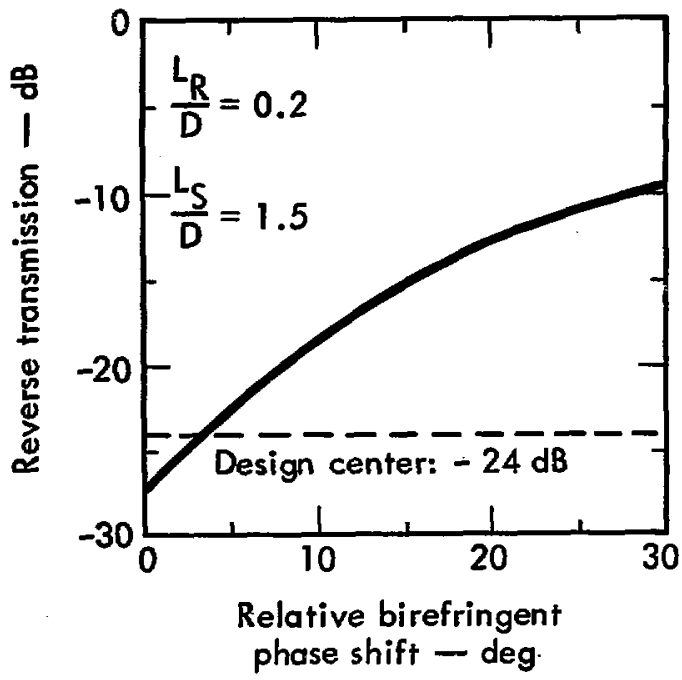

Fig 80. Dependence of reverse isolator transmission upon rotator gass birefringence. The $-27 \mathrm{~dB}$ shown for zero birefringent phase shift is the transmission due to magnetiorield inhomogeneity alone for the parameters thown. The dakhed line shows the required performance limit.
The RF70 glass rotator rod exhibits radial birefringence, which limits its useful aperture to a diameter of $60 \mathrm{~mm}$ for an extinction ratio of $15 \mathrm{~dB}$. It is apparent from such tests that excess strain birefringence must be guarded against in rotator design.

Prototype $A$-module rotators have been fabricated and tested. The solenoid shown in Fig. 91 has operated repeatedly at peak magnetic fields exceeding $100 \mathrm{kG}$ (approximately twice the design center requirement for this unit). The prototype rod, made of Owens Illinois EY-1 glass, exhibited a Verdet constant of $0.041 \pm 0.002 \mathrm{~min} / \mathrm{G}-\mathrm{cm}$ and performed satisfactorily in all respects, except for having a high density of platinum inclusions. This material exhibited slight damage, apparently caused by platinum absorption, when subjected to a 30-psec, 1.06- $\mu \mathrm{m}$ pulse having an energy density of $3 \mathrm{~J} / \mathrm{cm}^{2}$. Static bisefringence was negligible, but thermally induced birefringence was observed. Under ambient conditions, the time constant for the disappearance of thermally induced birefringence was approximately $2 \mathrm{~min}$. Further damage characterization, using the spatially smooth ILS beam, will be undertaken shortly.

Faraday rotation may find a second application in connection with smooth truncation of the radial intensity profile of a high-intensity beam. The usefulness of such "apodized apertures" in suppressing secondary diffraction maxima (which otherwise contribute to self-focusing) has been demonstrated by 
Table 12. Optical properties of a CILAS RF70 Faradry leolator

\begin{tabular}{|c|c|}
\hline $\begin{array}{l}\text { Static incertion los } \\
\text { Faraday sod } \\
\text { Analyzer } \\
\text { Polnizer } \\
\text { Totsl unit }\end{array}$ & $\begin{array}{r}14 \%(-0.65 \mathrm{~dB}) \\
5 \%(-0.22 \mathrm{~dB}) \\
5 \%(-0.22 \mathrm{~dB}) \\
22 \%(-1.1 \mathrm{~dB})\end{array}$ \\
\hline $\begin{array}{l}\text { Polarizer/analyzer extinction } \\
\text { ratio (crosed) }\end{array}$ & $60: 1(-18 \mathrm{~dB})$ \\
\hline $\begin{array}{l}\text { Dynamic insertion low (operating a } \\
\text { isolntor, } 1-\mathrm{mm} \text { beam on sxis) }\end{array}$ & $2 \%(-1.25 \mathrm{~d} G)$ \\
\hline $\begin{array}{l}\text { Dynamic extinetion (operating as } \\
\text { isolator, 1-mm besm on axis) }\end{array}$ & $50: 1(-17 d B)$ \\
\hline Extinction homogeneity & $\begin{array}{l}\text { Limited by readual } \\
\text { strin birefingence }\end{array}$ \\
\hline $\begin{array}{l}\text { Ercinction factor at }+32 \mathrm{~mm} \\
\text { from center line (wort case) }\end{array}$ & $25: 1(-14 \mathrm{~dB})$ \\
\hline
\end{tabular}

recent tests with dye cells using the ILS. ${ }^{45}$ The use of rotator glass and polarizers in this application would essentially eliminate the need for refractive-index matching.

Basically, the idea is to cause the beam polarization vector to rotate through an angle which is a monotonically increasing function of radial distance from the beam center ray. Ideally, this rotation angle will be $90^{\circ}$ at the nominal beam edge (for example, at the first null in a far-field Airy diffraction pattem) and $0^{\circ}$ at the center. When such an element is placed between parallel polarizers, the transmitted beam will exhibit a smooth intensity-profile roll-off with radius.

The inhomogeneous magnetic field required for this application can be obtained, for example, by two dissimilar but coaxial solenoids wound in opposition. An example of the transmission to be expected from this arrangement is shown in Fig. 92. Design work using the computer code COILS is presently under way in an attempt to establish a minimum-energy solenoid configuration for this application.

It should be mentioned that the same concept can be implemented using the Pockels effect as well (see "Electrooptic Beam-Shaping Device," p. 29). Pockels and Faraday apertures complement each other, since Pockels-effect devices are presently limited to apertures of less than about $2 \mathrm{~cm}$ because of crystal growth problems, while Faraday rotators operate efficiently with much larger apertures.

As noted earlier, the nominal gain-per-stage of 3.2 for the $1-\mathrm{kJ}$ chain implies the need for $-20 \mathrm{~dB}(100: 1)$ polarization per stage if Faraday rotators are used. A single plate at Brewster's angle ,rovides about $-1.3 \mathrm{~dB}$, and thus the disks in one amplifier module provide about $-8 \mathrm{~dB}$ polarization, leaving $-12 \mathrm{~dB}$ to be provided by additional polarizers. We have investigated the following possibilities:

1. Ten stacked plates of BK-7 glass at an angle of incidence of $60^{\circ}$.

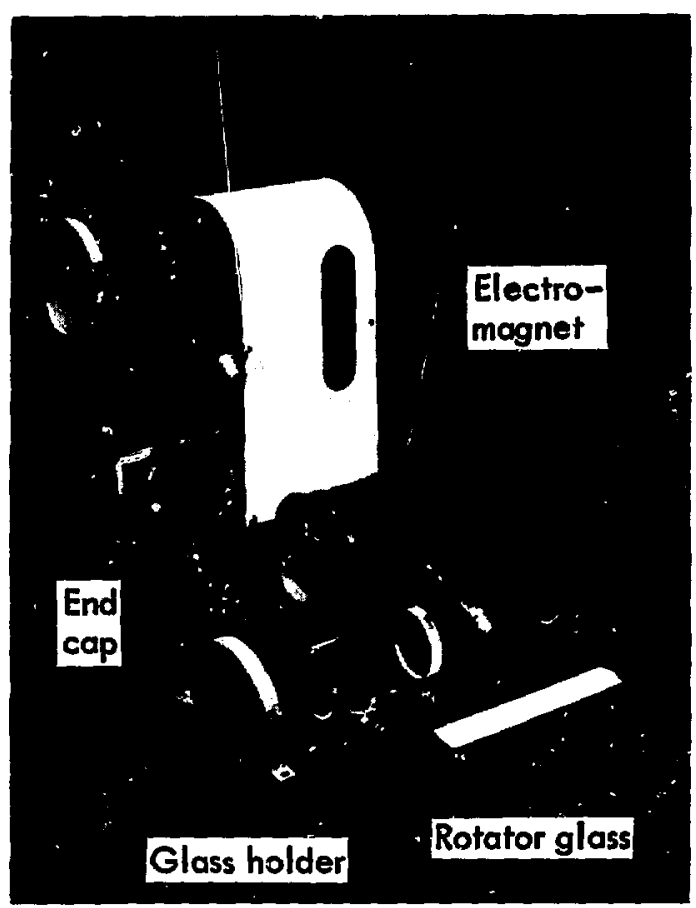

Fig. 91. Faraday rotator for the $A$ amplifier module The complete rotator in its housing is shown in the background. A disassembled unit is in the foreground.

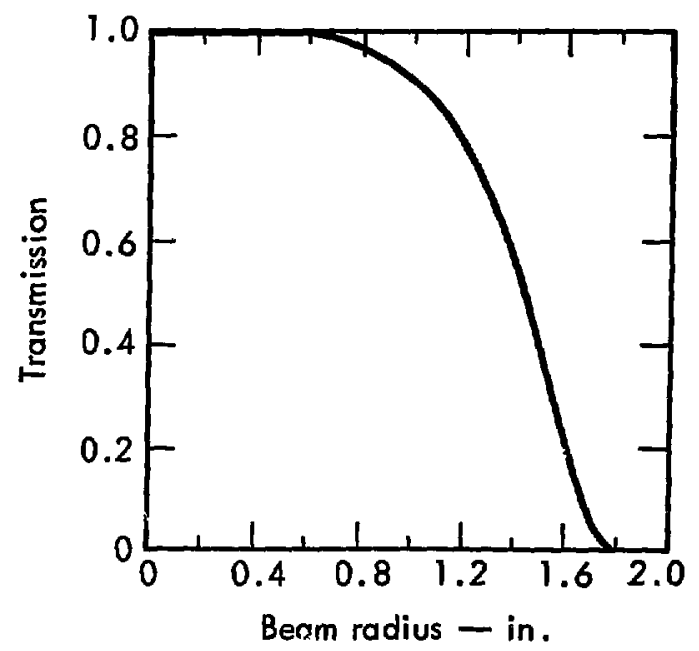

Fig. 92. Calculated transmission for an ideal apodized aperture based on Faraday rotation. 
2. Four pellicles at $60^{\circ}$.

3. A multilayer dielectric coating on one surface of one substrate, at $60^{\circ}$.

4. A single-layer dielectric coating on each surface of two substrates, at $68^{\circ}$.

All of the above altematives tneet the polarization requirement and have an ineertion loss of 5\% or less. The singledayer conting polarizer has to be placed at $68^{\circ}$ because this is the lowest angle of incidence for which high reflection of the $S$ polarizationit is obtained.

Each of the alternatives has its disadvantages. Ten stucked plates will introduce greater wave-front distortions and nonlinear effects than one substrate; in addition, the cost is ten times as great. The pellicles are fragile and of questionable uniformity and damage resiatance in large apertures, although we have mesured samples with a flatness better than $1 / 10$ wave (at $1.06 \mu \mathrm{m}$ ) over $50 \mathrm{~mm}$, and a damage threshold of $2 \mathrm{~J} / \mathrm{cm}^{2}$ (at $60^{\circ}$ angle of incidence for 200-psec pulses). The multilayer, single-substrate polarizer does introduce offset in the beam, whereas the single-layer, double-substrate polarizer has a longer path length in glass because of the higher angle of incidence, a higher substrate cost, and a higher coating cost, since the cost of coating is dominated by the setup time and not the number of layers. Preliminary results indicate that electron-beam-deposited coatings of both the multilayer and single-layer type will withstand the intensities involved as well as provide the necessary polarization.

On the basis of these considerations, we have selected a multilayer dielectric-coating polarizer for the $1-k J$ system - it has a high damage threshold and minimum cost.

\section{DEVELOPMENT OF CALORIMETERS TO MEASURE THE BEAM ENERGY}

\section{OF HIGH-POWER PULSED LASERS}

\section{Aberber Studizas}

The transmittances of several Schott glasses were messured at $1.06 \mu \mathrm{m}$, first with continuous-wave beams and then with short pulses (Table 13). Pulse transmittance measurements were performed in a split-beam setup, with a converging lens in one beam to focus most of the energy through a 3.4-mm aperture located about $20 \mathrm{~mm}$ in front of the absorber of a calorimeter. A sheet of the glass under investigation was placed over the aperture. The average flux density through the aperture was typically $5 \mathrm{~J} / \mathrm{cm}^{2}$, and there were certainly hot spots of substantially greater energy. Since the pulses were of approximately 25-psec duration, the results, while rather rough, do show that there is no gross increase in the transmittance at power densities up to $\sim 2 \times 10^{11} \mathrm{~W} / \mathrm{cm}^{2}$.

The reflectance calorimeter ${ }^{46}$ was used to measure the reflectance of these glasses. For continuous-wave beams, the values ranged from 5.0 to $5.5 \%$, as expected for a single surface of a glass of normal reflective index. For pulses of $\sim 1 \mathrm{~J} / \mathrm{cm}^{2}, \sim 25$ psec wide, preliminary values were essentially the same. Reflectances of $\mathrm{LC} \cdot 1$ cups ${ }^{47}$ containing $\mathrm{Ni}(\mathrm{II}), \mathrm{Co}$ (II), and $\mathrm{Cu}(\mathrm{II})$ solution and fitted with quartz windows having antireflestion coatings on the front surface were found to be about $2 \%$ for continuous-wave beams.

In some of the pulse transmittance tests described above there was damage to the surface of the absorbing giass. Further damage studies were done in a split-beam setup by placing specimens of the various glasses near the focus of a converging beam. The damage thresholds of BG-18, VG-14, BG-7, and NG-1 were all
Table 13. Extomal trenemittances of severd Schott dewes $(1 \mathrm{~mm}$ thick) moasured at $1.06 \mu \mathrm{m}$ with continuous-wave and pulsed beams-

\begin{tabular}{lcc} 
Type of & \multicolumn{2}{c}{ Transmittunce (\%) } \\
\cline { 2 - 3 } dise & CW & Pulsed \\
\hline BG-18 & $<0.006$ & $<0.1$ \\
VG-14 & 2.2 & $\sim 1.5$ \\
BG-7 & 2.8 & $\sim 2$ \\
NG-1 & 2.9 & $\sim 2.5$ \\
UG-1 & 5.0 & -- \\
BG-28 & 5.2 & -- \\
\hline
\end{tabular}

approximately the same; if anything, BG-18 was pitted less than some of the others. This is rather surprising, since the higher absorbance of BG-18 must result in a higher energy dissipation per unit volume near the surface. Absolute values of the damage threshold could not be accurately determined because of the nonuniform bearn cross section.

While further work remains to be done, the above observations suggest with considerable confidence that the Schott glasses are satisfactory calorimeter absorbers, and BG-18 of 1-mm thickness is now considered to be the preferred material for $1.06-\mu \mathrm{m}$ calorimeters.

\section{Design Work}

Aluminum jackets for calorimeters of the LC-3 type ${ }^{47}$ have been built in two sizes: $102 \mathrm{~mm}$ wide 


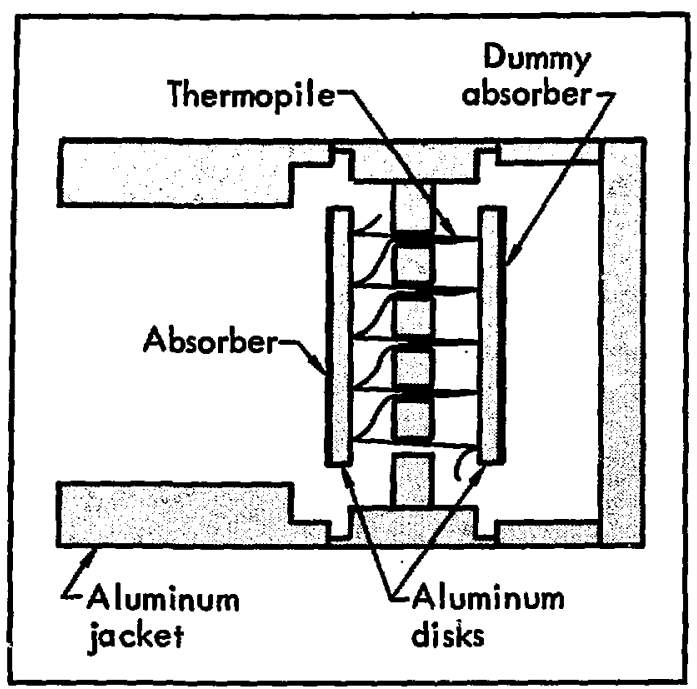

Fig. 93. Cross section of the LC-10 calorimeter.

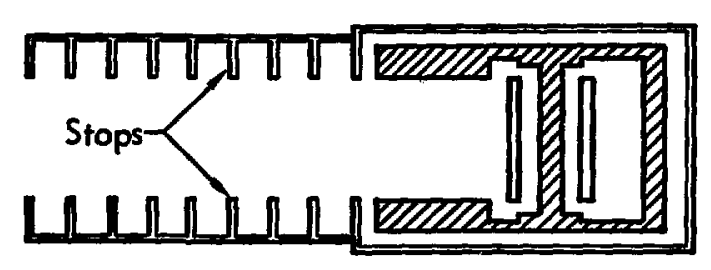

Fig. 94. Calorimeter entry tube with stops to improve absorption of stray rediation.

by $165 \mathrm{~mm}$ high by $152 \mathrm{~mm}$ deep for absorbers from 13 to $51 \mathrm{~mm}$ in diameter, and $165 \mathrm{~mm}$ vide by $292 \mathrm{~mm}$ high by $127 \mathrm{~mm}$ deep for absorbers from 76 to $114 \mathrm{~mm}$ in diameter. For larger diameters, the blocks would be inordinately bulky. Accordingly, a new type, designated LC-10, has been designed. This has a more compact back-to-back configuration, as shown in Fig. 93, compared with the side-by-side configuration of the LC-3. Although this design suffers from having a somewhat less symmetrical environment for the two disks than the LC-3 type, it will generally be preferred for absorber diameters of $38 \mathrm{~mm}$ and up.
It can readily be built with absorber diameters up to the largest for which eventual need is now foreseen (250 to $300 \mathrm{~mm}$ ). A 21-pair thermopile is used for all sizes, and the thermopile resistance is less than for calorimeters of the LC-3 type because the thermopile wire lengths are shorter. The jacket dimensions in millimeters are $(D+38)$ by $(D+38)$ by 178 , where $D$ is the absorber diameter.

A smaller version of the LC-3 type with 25-mm absorbers, designated LC-11, has been designed. This has an aluminum jacket measuring 102 by 127 by $51 \mathrm{~mm}$, and is intended for direct insertion in the laser chain where space is not available for larger jackets.

The calorimeters have heretofore been insulated with 51-imm-thick Styrofoam boxes around the aluminum jackets. A more compact outer jacket of Bakelite and aluminum sheet has now been designed; a of 13 or $19 \mathrm{~min}$ will be left between the aluminum jacket and outer jacket, and this gap can be either filled with insulating material or left empty.

Long entry tubes, of metal painted black on the inside and insulated on the outside, are generally used with the calorimeters to reduce the effects of air currents and to absorb stray visible and infrared radiation from the laboratory. For the latter purpose, their efficiency is degraded by the fact that the absorptivity of the black paint is lower for grazing than for perpendicularly incident radiation. To improve matters in this regard, tubes with a number of black-painted stops have been designed (Fig. 94). Windows (30-min wedges of BK-7 glass) are also on order for use with all of the $1.06-\mu \mathrm{m}$ calorimeters up to $38 \mathrm{~mm}$ in diameter in situations where there is not enough room to use entry tubes.

\section{Calorimeter Fabrication}

Six more calorimeters of the LC-3 type, with diameters from 13 to $114 \mathrm{~mm}$, and one of the LC-10 type, $38 \mathrm{~mm}$ in diameter, have been constructed. Some of these have been put in use at the Intermediate and Short-Path Laser Systems. One was fitted with black-painted absorbers and is being used for flashlamp studies.

For the 1-kJ chains and for oscillator development, parts for 29 calorimeters of diameters from 13 to $127 \mathrm{~mm}$ have been ordered and are being received and assembled.
In the previous Semiannual Report ${ }^{48}$ we discussed in detail the results of our work on a new type of electrooptic (Pockels) cell that achieves a considerable performance gain over other types of cells. 27,49 In this report, we will concentrate on some new results and performance data taken on these Pockels cells. 


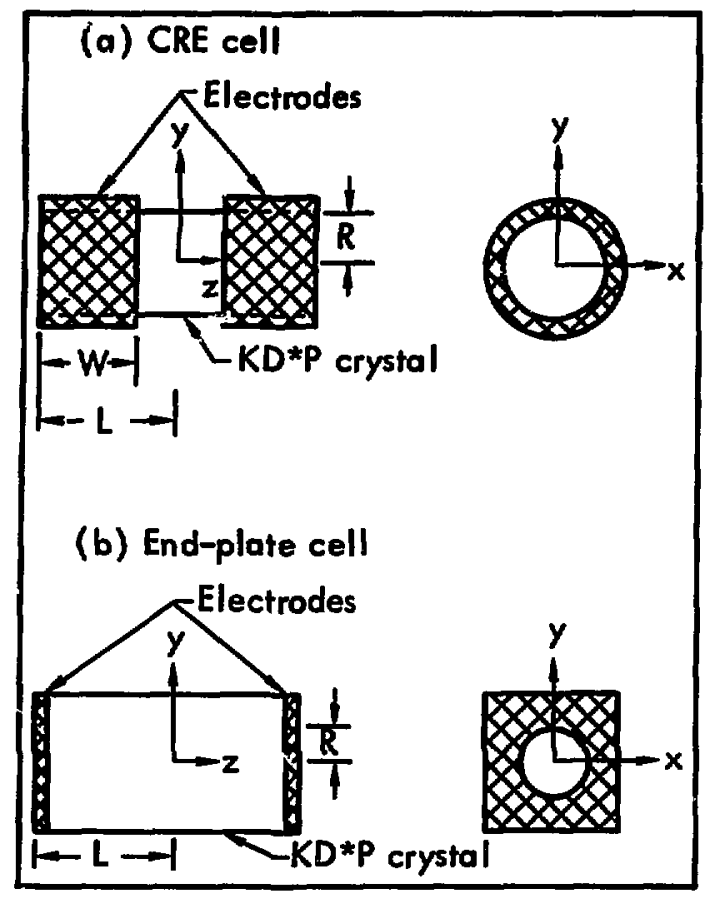

Fig. 85. Schemntics of (a) the cylindrical dingelectrode (CPE) Pockels cell and (b) a stendind end-plate cell. Typical cell dimentions are: cell length $2 L=25 \mathrm{~mm}$, radius $R=6 \mathrm{~mm}$, and electrode width $W=8 \mathrm{~mm}$ (CRE cell only).

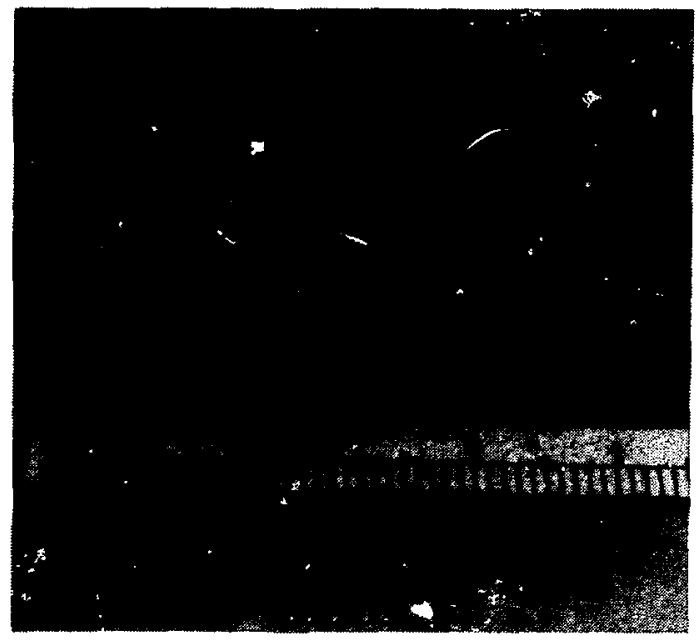

Fip 88. KD*P cryati blanks with gold band electrodes. The crystel on the left is $12.7 \mathrm{~mm}$ in diumeter by $25.4 \mathrm{~mm}$ long; the one on the right is $4 \mathrm{~mm}$ in diumeter by $8 \mathrm{~mm}$ long.

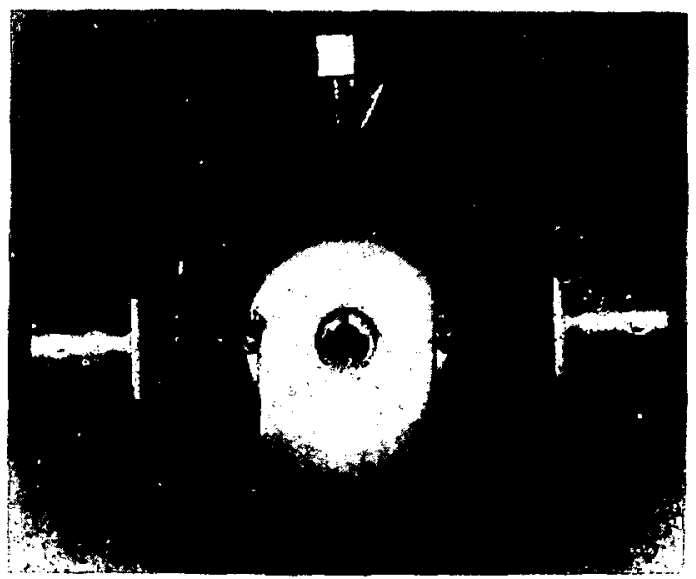

Fig 97. An usembled CRE Pockels cell unit containing a $4 \mathrm{~mm}$ by $8 \mathrm{~mm}$ KD*P cryatel.

The new Pockels cell employs a cylindrical crystal of $\mathrm{KD} * \mathrm{P}$ (potassium dideuterium phosphate, $\mathrm{KD}_{2} \mathrm{PO}_{4}$ ), with the cylinder axis coincident with the optic (or z) axis of the crystal. Voltage is applied to the cell with a pair of ring electrodes fitted over the barrel of the cylinder. Figure 95a shows a cylindrical ring-electrode (CRE) electrooptic cell and defines the cell length $2 L$, cell radius $R$, and electrode width $W$.

An example of a typical commercial cell is shown in Fig. 95b. These cells consist of a rectangular block of electrooptic material with electrodes bonded to the $z$-face ends; a hole in the electrode material determines the aperture. Crystal length $\boldsymbol{Z L}$ and aperture radius $R$ are indicated on the figure. As in the past, all theoretical and experinental comparisons are between cells of this end-plate design and our new CRE design.

Figure 96 is a photograph of two CRE crystal blanks. Gold-alloy electrodes have been evaporated to fomi bands on the outer surface of both blanks. Figure 97 is a photograph of a complete, packaged CRE Pockels cell unit containing a $4-\mathrm{mm}$ by $8-\mathrm{mm}$ KD*P crystal blank.

The performance of the CRE cell is improved over that of the standard cell primarily in two areas: (1) lower voltage nonuniformity across the effective aperture, and (2) lower capacitance. The voltage nonuniformity $\Delta V$ is given by

$$
\Delta V=\left|1 \cdot \frac{V_{z}}{V_{\lambda / 2}}\right|,
$$

where $V_{2}$ is the applied voltage, and $V_{\lambda / 2}$ is the half-wave voltage required to rotate the incoming polarization vector by $90^{\circ}$. In general, $V_{z}$ will be a 
function of radial displacement from the electrode due to fringing effects; the further toward the center of the crystal $(r=0)$, the smaller the value of $V_{z}$ and the higher the value of $\Delta V$. Figure 98 shows computer-calculated curves of $\Delta V$ as a function of normalized radius $r / R$ for a $C R E$ cell and a conventional end-plate cell of identical $L / R$ ratio. For these calculations we have assumed that a voltage $V_{z}=V_{\lambda / 2}$ is applied at $r=R$. Experimental points are indicated with representative error bars; the agreement between calculation and experiment is quite good, both in magnitude and radial dependence.

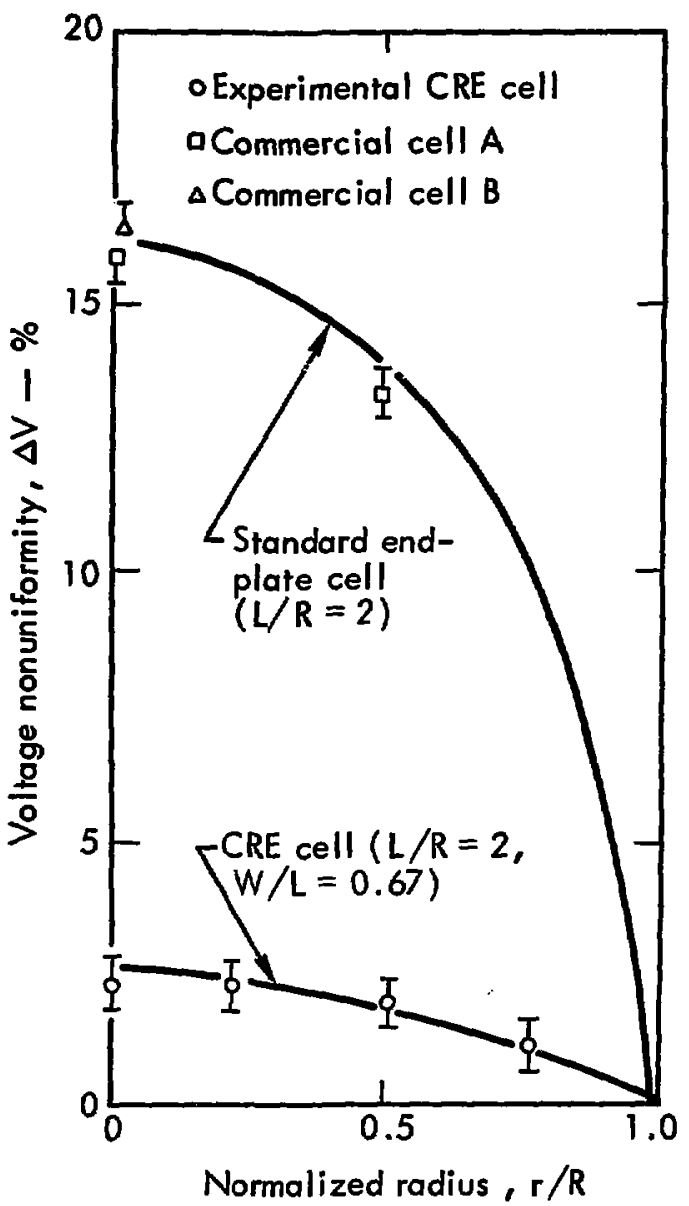

Fig. 98. Voltage nonuniformity $\Delta V$ versus nomalized radius $r / R$. The upper curve is for a standard end-plate cell, and the lower curve is for a CRE cell. Both curves are plotted for full-aperture illumination of the cells. The magnitude and radial dependence of the experimental points are in good agreement with the theoretical curves. Cell contrast ratio is proportional to $\left(1 / \Delta V_{\text {max }}\right)^{2}$.

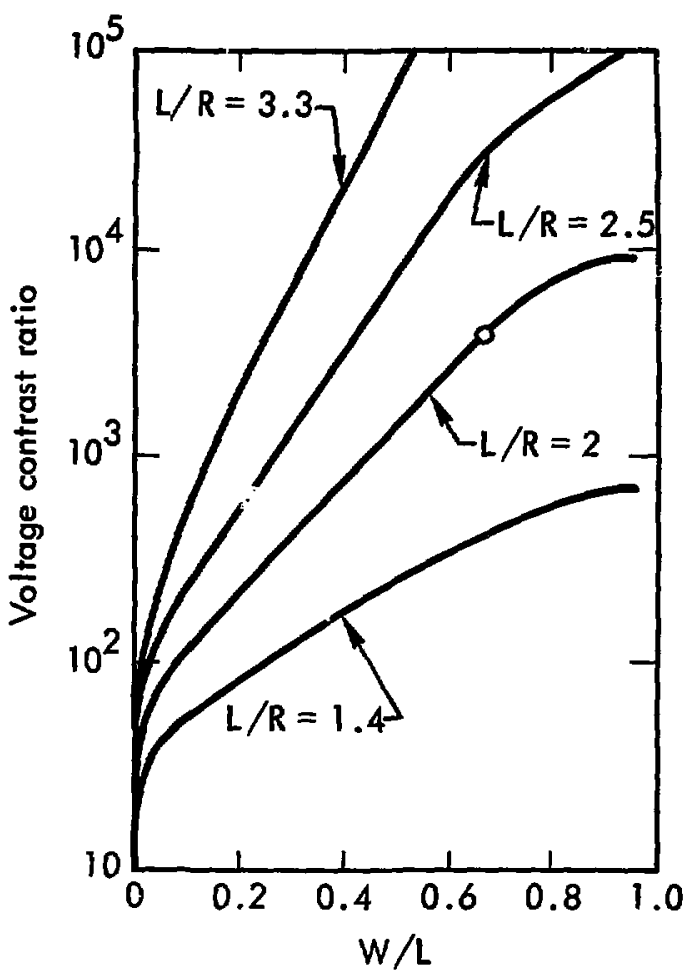

Fig. 99. Pockels-cell voltage contrast matio (VCR) versus normalized electrode width $w / L$, with $L / R \approx$ a parameter. The operating point corresponding to the experimental cell is indicated on the $L / R=2$ curve. VCR's of $>10^{4}$ are generally not attrinable becuse of material imperfections.

The voltage contrast ratio (VCR) for a Pockels cell placed between ideal crossed polarizers is defined as

$$
\frac{I_{\text {out }}\left[V_{z}(R)=V_{\lambda / 2}\right]}{I_{\text {out }}\left[V_{z}(R)=0\right]},
$$

where $I$ is the transmitted intensity, measured across the full aperture of the cell. For small values of $\Delta V$,

$$
\text { VCR } \approx \frac{2.4}{\left(\Delta V_{\max }\right)^{2}}
$$

Using the data of Fig. 98, we computed and experimentally verified a VCR for the end plate cell of $\sim 200: 1$, compared with a value $>3000: 1$ for the CRE cell; this is an improvement ol inore than an order of magnitude.

Another design parameter for the CRE cells is the electrode width $W$ (see Fig. 95a). In general, $\Delta V$ 
becomes smaller and VCR increases as $W \rightarrow L$. This is illustrated schematically in Fig. 99, where we show VCR as a function of $W / L$ for a variety of $L / R$ values. (In practice, values of VCR $>10^{4}$ are generally not attain.ble, particularly in large-aperture cells, because of i'sperfections in the material optical quality.) We see from Fig. 99 that increasing $L / R$ for a fixed $W / L$ leads to an increased VCR. The experimental CRE cell represented in Fig. 98 has $L / R=2, W / L=0.67$; this point is indicated on Fig. 99 and gives a predicted VCR of $\sim 3000: 1$. Conversely, increasing $W / L$ for a given $L / R$ will also tend to increase the VCR. However, other considerations enter the problem as $W / L \rightarrow 1$. The first is voltage holdoff capability; i.e., the electrodes must be sufficiently far apart to prevent breakdown under the operating conditions for the cell. Secondly, the capacitance of the cell, given by the expression

$$
C=\frac{\pi R^{2} \epsilon_{z} \epsilon_{0}}{2(L \cdot W)}
$$

where $\epsilon_{2} \epsilon_{0}$ is the electrical permittivity of the cell in the $z$-directic $n$, will increase as $W \rightarrow L$. (A 4-mm-diam by 8 -mm-long cell with $W / L=0.67$ has a capacitance of $\sim 2.1 \mathrm{pF}$ and will respond with a risetime of $<200$ psec on a $50-\Omega$ line.) These results imply that we must trade off voltage nonuniformity for capacitance, or contrast ratio for speed of response.

Figure 100 is a plot of the product of voltage nonuniformi $y$ squared and capacitance as a function of the $W / L$ ratio for cells of various $L / R$ ratios. Minimizing this product is equivalent to maximizing the product of the contrast ratio and the speed of response (or bandwidth) of the cell. This particular "figure of merit" for the CRE cells shows an optimum range of $W / L$. from about 0.6 to 0.9 . The point for our experimental cell $(L / R=2, W / L=0.67)$ shows that we are operating near an optimum design point.

In summary, we have designed and constructed Pockels cells of a new geometry. The order-ofmagnitude it pprovement in transmission uniformity obtained wit in these cells (as compared with commercially huilt cells) has resulted in significantly higher contrest ratios. The reduction in capacitance obtained witt. the design has resulted in a fourfold improvement in switchirig speed, permitting us to switch our cells in times as short as 200 pser. Experimental measurements have confirmed in detail our design predictions for the CRE Pockels celis.

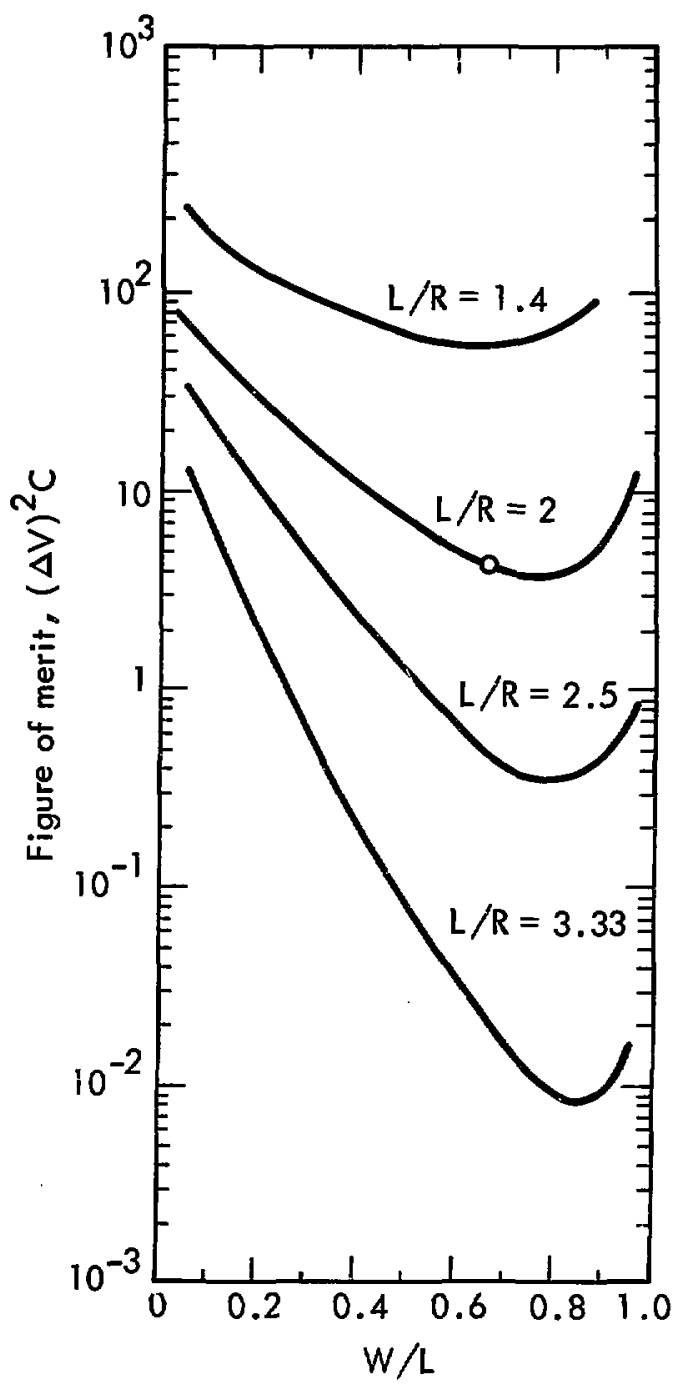

Fig. 100. "Figure of merit" for CRE Pockels cells as a function of normalized electrode width $W / L$, with $L / R$ as a parameter. The ordinate, voltage nonuniformity squared times capacitance, is inversely proportional to contrast ratio times bandwidth for the cell. $A$ minimum on the curve represents an optimum design point. The operating point corresponding to the experimental cell is indicated on the $L / R=2$ curve. 
The effort in the area of electrooptic modulation during the past period has concentrated on the component needs of the oscillator section of the $1-\mathrm{kJ}$ glass laser system. As the needs have been defined, we have procured or fabricated the required components; these include Pockels cells, polarizers, power supplies, spark gaps, etc.

Development of the CRE Pockels cells has continued. We have evaluated three versions of this design, with clear apertures of 4,13 , and $18 \mathrm{~mm}$ diameter, respectively. Typical test results are summarized in Table 14. The high contrast ratio measured with the 18-mris-diam aperture confirms the validity of the scaling of the design, as previously suggested. 48

Procedures for fabricating these cells in small quantities are ready; when the housing parts are completed, we will begin fabricating the 16 Pockels cells required in the $1-\mathrm{kJ}$ oscillator section. A photcgraph of the 18-mm-diam cell is shown in Fig. 101.

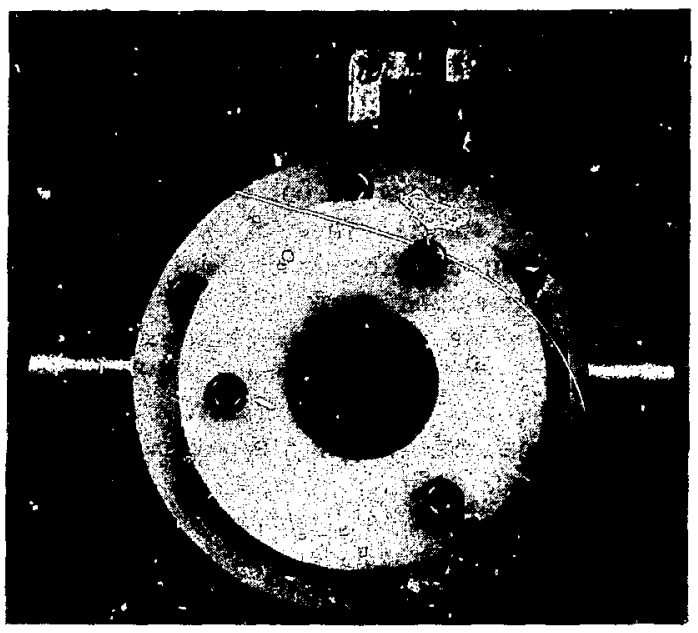

Fig. 101. An 18-mm-diam CRE Pockels cell.

Table 14. Performance of cylindrical ring-electrode (CRE) Pockels cells employing KD*P crystals

\begin{tabular}{|c|c|c|c|c|c|}
\hline \multirow{2}{*}{$\begin{array}{c}\text { Cell clez } \\
\text { aperture } \\
\text { (mm) }\end{array}$} & \multirow{2}{*}{$\begin{array}{c}\text { Test-beam } \\
\text { eperture } \\
\text { (mm) }\end{array}$} & \multicolumn{2}{|c|}{$\begin{array}{l}\text { Voltage-contrast } \\
\text { ratio }^{\mathrm{a}}\end{array}$} & \multirow{2}{*}{$\begin{array}{c}V_{\lambda / 2} \\
\text { at } 0.63_{\mu \mathrm{m}} \\
(\mathrm{kV})\end{array}$} & \multirow{2}{*}{$\begin{array}{l}V_{\lambda / 2}^{b} \\
\text { at } 1.06 \mathrm{\mu m} \\
\text { (kV) }\end{array}$} \\
\hline & & Mensured & Calculated & & \\
\hline 4 & 3 & 2500 & 3000 & 3.5 & 5.9 \\
\hline 13 & 11 & 1500 & 3000 & 3.5 & 5.9 \\
\hline 18 & $13^{\mathrm{c}}$ & 1000 & 600 & 4.0 & 6.7 \\
\hline
\end{tabular}

'The calculated values" ${ }^{4}$ of voltage-contrast fatio are based upon full aperture illumination, which if clearly not the case with the 18-mm-diam cell. This explains why the measured value exceeds the calculated value for this cell.

bCalculated based upon measured values at $0.63 \mu \mathrm{m}$.

Limited by polarizer aperture; will be evaluated with larger-aperture beam when new polarizers are tvailable. 


\section{DIAGNOSTICS}

\section{INTRODUCTION}

We have continued effort on the development, construction, and testing of our compact 10-psecresolution streak cameras. Three of the cameras are operating and in use. The development of a prototype 1-psec-resolution streak tube has proceeded to the point where some streak photographs have been obtained. Development of an $\mathrm{x}$-ray-sensitive streak tube has also progressed; we have made some preliminary measurements with a photodiode using a proposed cathode design.

The specification and design of beam diagnostics for the 1-kJ glass laser system are receiving increasing effort. The diagnostics stations being designed consist of a beam pipe coupling an amplifier module to an isolator module, beam pickoff and steering, and appropriate distribution of the beam to diagnostics detectors. The photodetectors, analog-to-digital conversion, and the computer interface are being defined and the components ordered. The requirements and specifications of the total complement of beam diagnostics are being addressed. We are also closely following device and technique developments in this area.

Considerable experimentation has led to the evolution of the holographic interferometric techniques intended for measuring the density of laser-produced plasmas. In addition, a new holographic effort, directed toward the study of phase distortion in amplifying media (e.g., disk amplifier modules) has been started. The procurement of a laser source and the design of the "holocamera" are well under way.

Diagnostic devices for ion spectroscopy of laser-produced plasmas have been built. We have constructed curved-plate electrostatic and Thomsonparabola charged-particle spectrometers from designs provided by Sandia Laboratories, Albuquerque. These devices are in the final stages of assembly testing. In the near future, we expect to begin operation and calibration of these devices on plasma sources. This will broaden our diagnostic capability for laserproduced plasmas. Work on x-ray streak-camera tubes has direct application to the diagnostics of laserproduced plasmas.

Work has continued on the problem of subnanosecond-resolution diagnostic techniques at $10.6 \mu \mathrm{m}$. Extension of the $\mathrm{CS}_{2}$ Kerr-cell techniques to shorter times has been pursued.

An Nd:YAG - Nd:glass laser-amplifier system has been built up as a diagnostics test and development facility. This system has produced $1 \mathrm{~J}$ in an approximately 150-psec pulse from a 1-in.-diam rod amplifier. A target chamber is being assembled and will be set up at the laser output. This facility will serve as a source for the development, testing, and calibration of both laser and plasma diagnostics.

\section{A COMPACT STREAK CAMERA}

The basic concept and design of streak cameras has been described previously. ${ }^{50}$ A photograph of one of the recent cameras is shown in Fig. 102. We currently have three of the compact 10-psec streak cameras operating; the remaining two are $80 \%$ complete. We have continued to experience difficulty in obtaining reliable delivery of microchannel image intensifiers. A new purchase order was sent to Bendix Corporation for five additional tubes; since the Bendix intensifiers are somewhat different in geometry from those supplied by Litton, we have had to make some mechanical modifications so that the camera tubes and housings will be adaptable to both types of intensifier tube.

We have measured the $P(11)$ spectrum radiant power gain of the Litton microchannel intensifiers used in the operating cameras and find it to be in the range

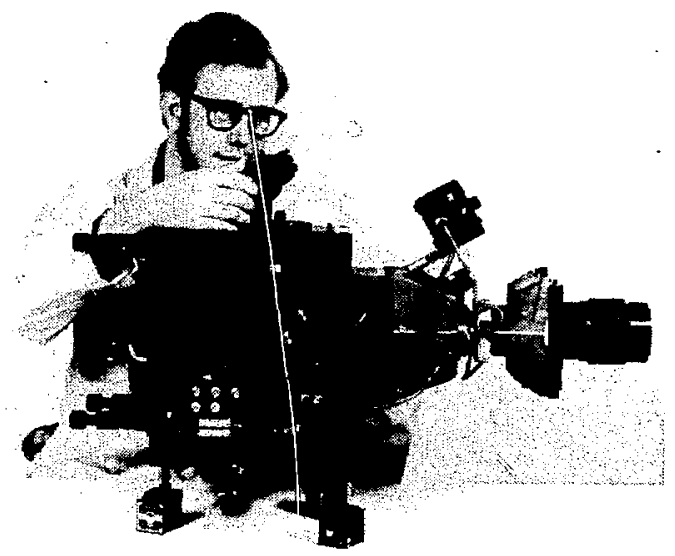

Fig. 102. The LLL compact 10-psec-resolution streak camera. 


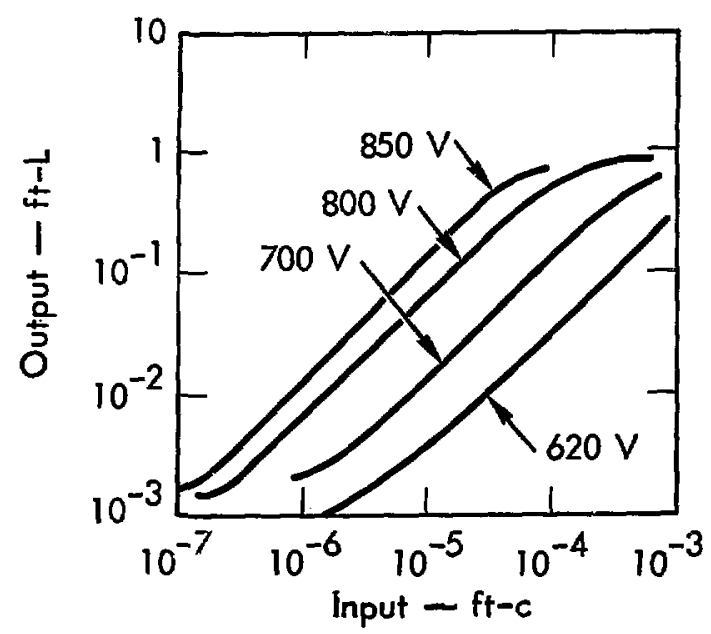

Fig. 103. Gain charecteristics of the microchannel plate image intensifier used on the compact streak cameras. The different curves are for various values of plate voltage.

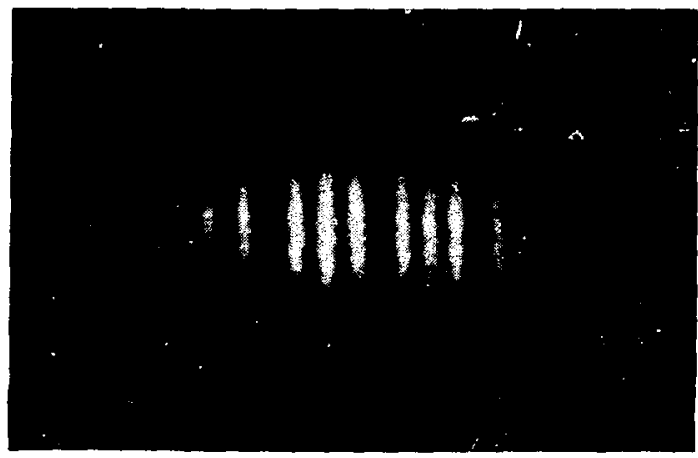

Fig. 104. Streak photograph of a resolution chart at the streak-camen slit. The illuminating source is a 30-psec Nd:YAG laser pulse. Element size varies from four line pairs per millimeter on the left (barely visible) to seven line pairs per millimeter on the right.

of 2-to-6 $\times 10^{4}$. In addition, we have measured the gain characteristics of the tubes, as shown in Fig. 103. We typically operate with a microchannel plate voltage in the range of 650 to $800 \mathrm{~V}$. The dynamic spatial resolution of the camera lias been measured by streaking the elements of a resolution chart that is placed at the camera input slit and illuminated by a laser pulse. Seven line pairs per millimeter are easily. resolved; Fig. 104 is a streak photograph of the resolution chart illuminated by a 30-psec pulse from a mode-locked Nd:YAG oscillator. Finally, the streak speed of the camera has been measured by photographing a train of light pulses of known time separation produced by multiple reflections between a pair of partially transmitting mirrors; the result is shown in Fig. 105. These data are from only one photograph, but we have accumulated many pieces of data that display the same result. The sweep is linear over its central portion, with a streak rate of $34 \mathrm{psec} / \mathrm{mm}$. The electron transit-time dispersion in the streak tube is no greater than 6 psec, as determined by the electric field of about $3 \mathrm{kV} / \mathrm{cm}$ provided at the photocathode. On the basis of these experimentally determined parameters, we predict an overall time resolution for the camera of slightly better than 10 psec.

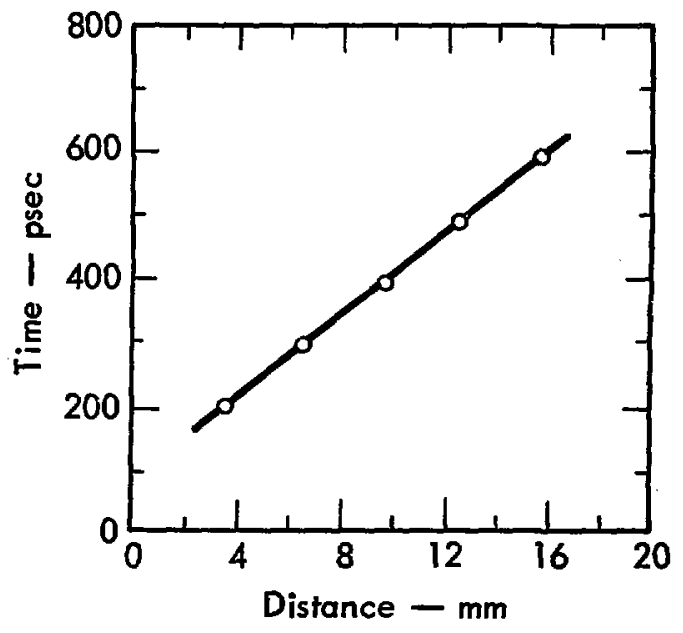

Fig. 105. Streak speed calibration for the compact streak camera.

Mope $=(33.7 \pm 1.0) \mathrm{psec} / \mathrm{mm}$

$=(3.0 \pm 0.1) \times 10^{\circ} \mathrm{cm} / \mathrm{sec}$.

\section{AN ULTRAFAST STREAK CAMERA WITH THE VERSATILITY}

\section{OF A STANDARD CAMERA}

Although the new compact 10-psec-resolution camera is highly satisfactory as a portable, ultrafast streak camera, research situations often require both flexibility and versatility. A 10-psec-resolution streak camera may be required at one point, while an intensified 10-nsec-resolution streak camera or a 


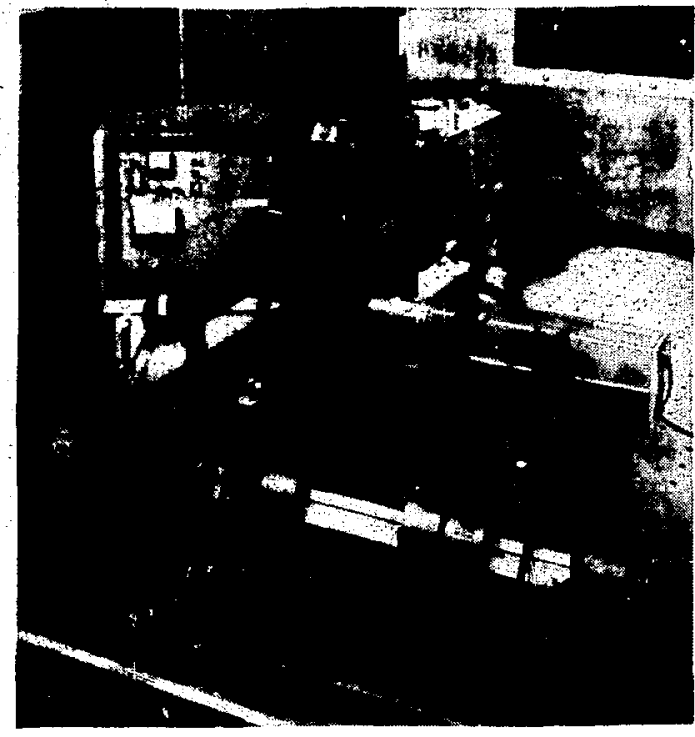

Fig. 106. A 10-pec-resolution streak camera that ha been made from a atandard TRW camera via the plug in. The inage intensification is achieved with a magretically focused tube of gain $10^{5}$.

conventional high-speed framing camera may be required at another point. These needs could be met by using different cameras, but it is also possible to meet them with only one type of unit. To this end, we have developed a new plug-in unit for standard STL-TRW cameras that employs the same avalanche transistor circuits used in the other LLL cameras. 51 It is totally self-contained and is powered by the standard plug-in units. The circuits have been packaged in an empty high-speed streak unit purchased from TRW. The only adjustment which must be made to the camera proper involves the focusing; this is due to an increase in the pulsed grid bias.

We have employed two different techniques to provide for light intensification of the streak-tube output before the photographic record is made. The first involves a rubber diaphragm which allows for removing the standard Polaroid camera and relay lens and replacing it with a commercially available image-intensifier camera. The camera we have used has a magnetically focused tube with a gain of $10^{5}$ and f/1.4 optics at buth ends.

In the second technique, an ITT microchannel plate intensifier is coupled to the film with fiber optics. The unit has a gain of 5000 and uses the standard TRW camera and relay lens where the microchannel plate unit replaces the usual Polaroid roll-film pack.

The two cameras are shown in Figs. 106 and 107. Both intensifiers are $40 \mathrm{~mm}$ in diameter. Streak speeds, linearity of streak, and temporal and spatial resolution are all similar to the other LLLbuilt cameras described previously.

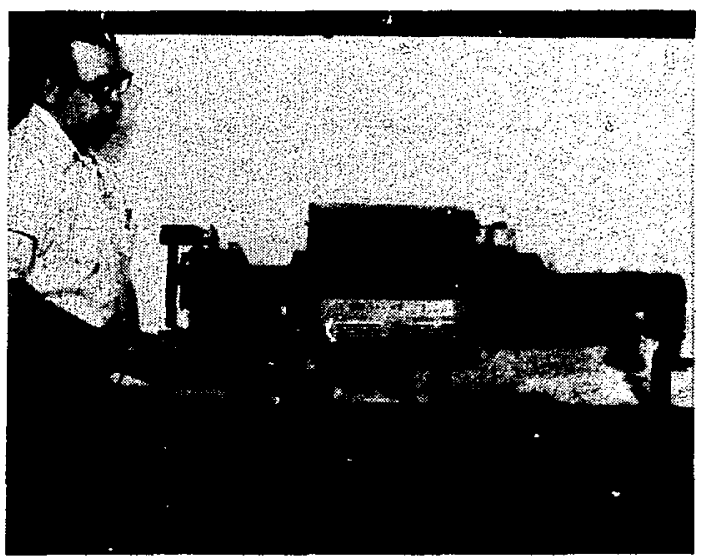

Fig. 107. A second camera that has been converted into a 10-psec-resolution streak camers. Here the image intensification is schieved with a microchennel plate intensifer with a gain of 5004, which is coupled to the film with fiber optick. Note that the size of the intensifier is comparable to that of the conventiond film pack in the foreground.

\section{A STREAK-CAMERA TUBE FOR IMPROVED RESOLUTION}

The development of a circular streak-camera tube (SCT), based upon a modified design of the EG\&G type KR-23 cathode ray tube, has been reported previously. 52 The major advantages of this LLL streak-camera tube as compared, for example, with the RCA C73435 tube, are the following:

- There is no synchronization problem since a trigger pulse is not required for the circular-sweep SCT. This SCT has two sets of wide-bandwidth $(3.6 \mathrm{GHz})$, traveling-wave deflectors, which allow a circular scan of $1.27 \mathrm{~cm}$ maximum diameter on the phosphor screen. The maximum resolution for a $0.01-\mathrm{cm}$ trace-width (cathode field, $10^{4} \mathrm{~V} / \mathrm{cm}$ ), or about 400 resolvable points in the scan, is 0.7 psec per trace-width for a circular sweep frequency of $3.6 \mathrm{GHz}$.

- The superior quality of the electron lens minimizes blooming effects. 
- The electric field at the cathode can be made as high as $3.5 \times 10^{4} \mathrm{~V} / \mathrm{cm}$. The SCT can provide a time resolution of less than 2 psec for $10^{4} \mathrm{~V} / \mathrm{cm}$ at the cathode (zero grid voltage with respect to the cathode) with a $0.01-\mathrm{cm}$ trace-width at the phosphor screen..$^{53}$
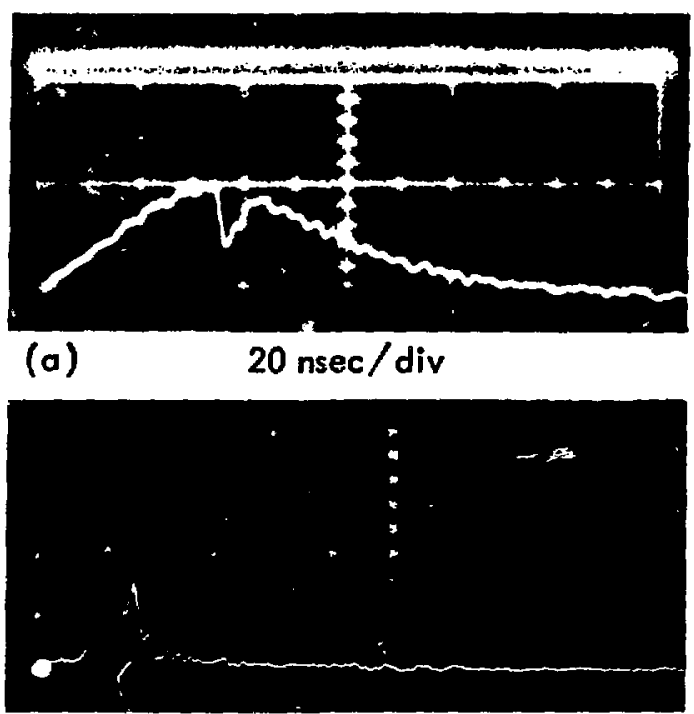

(b)

\section{$10 \mathrm{nsec} / \mathrm{div}$}

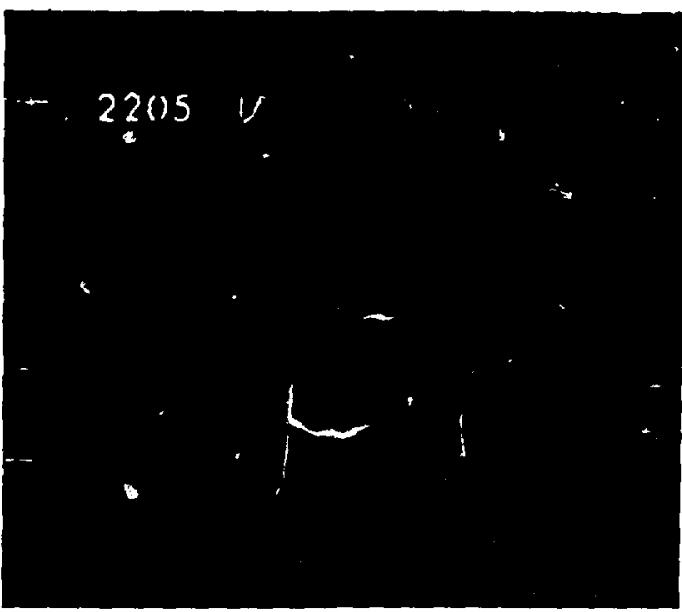

(c)

Fig. 108. (a) Photodiode oscillogram of a pulse from a $Q$ switched nuby laser. (b) Switched-out segment of the pulse in (a). (The portions of the puise between the peaks have been retouched for better visibility.) (c) Circular streak photograph of the pulse shown in (b). The sweep frequency is $0.15 \mathrm{GHz}$, for a total sweep length of 6.65 nsec. The nonunifomity of the trace is due to electrical noise coupling in to the deflectors.

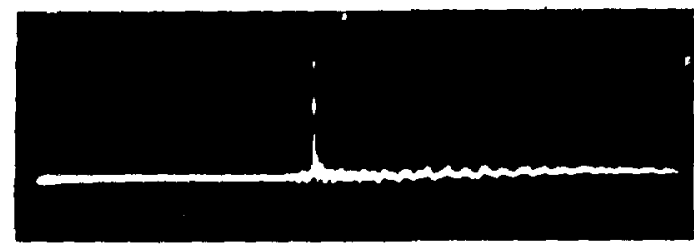

(a)
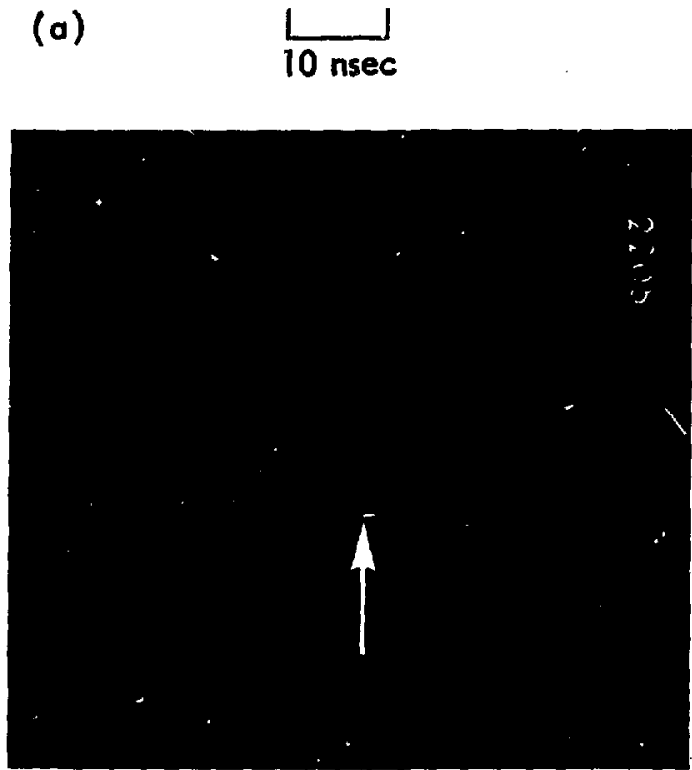

(b)

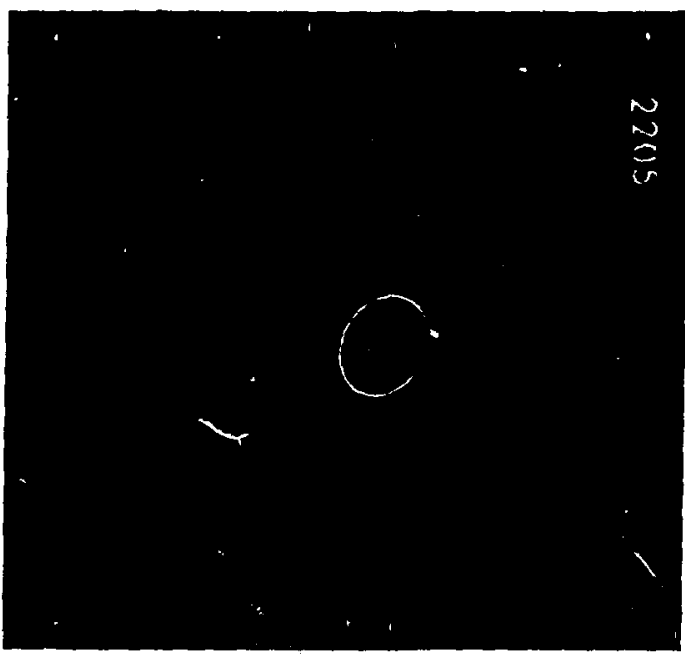

(c)

Fig. 109. (a) Photodiode oscillogram of a pulse from a mode-locked Nd:glass Iaser. (b) Circular streak photograph of the pulse shown in (a). The sweep frequency is $2.75 \mathrm{GHz}$, for a total swoep length c. 364 psec. (c) Continuour-input calibration stroak at $2.75 \mathrm{GHz}$. 
- No optical defining aperture is required at the photocathode. For an electric field of less than $10^{4} \mathrm{~V} / \mathrm{cm}$ at the cathode, the region at the cathode contributing to the beam current is less than $0.01 \mathrm{~cm}$ in diameter when the entire area of the photocathode is irradiated with the laser beam.

The initial applicition of the prototype model of the SCT was with a $Q$-switched ruby laser having a pulse width of about 5 nsec. The photodiode cocillogram and the streak trace for a circula: sweep frequency of $0.15 \mathrm{GHz}$ are shown in Fig. 108. The streak trace reveals greater detail in the valley between the primary and secondary pulses. A second application was with a switched-out pulse from an Nd:glass laser. The photodiode oscillogram, the streak trace for a circular sweep of $2.75 \mathrm{GHz}$ (364 psec total sweep), and the continuous calibration sweep are shown in Fig. 109. The pulse width for Fig. 109 is estimated to be about 60 psec. Pulses of 15 to 20 psec have been identified at $3.75 \mathrm{GHz}$ on the original pictures for the Nd:glass laser. Streak traces were recorded without the use of an image intensifier on the phosphor screen. Some streak traces for the switched-out pulse have shown a long tail of about 100 psec. In some cases a precursor pulse of $10-20 \mathrm{psec}$ would also appear. Whether the low-intensity tail is a part of the real laser pulse is still in question. Hopefully, the future application of the circular SCT with the Nd:YAG lacer will reveal more information on the characteristics of this camera tube.

The following statements can be made concerning the overall performance of the circular SCT and the required modifications for subsequent prototype tubes:

- Very good mechanical alignment of the cathode-grid axis with the lens axis was obtained, and the $0.01-\mathrm{cm}$ spacing between grid and cathode was preserved even after a $425^{\circ} \mathrm{C}$ bakeout of the tube. The spacing was ascertained from the required grid voltage to cut off the beam current and was measured to be $-4 \%$ of the cathode voltage (as specified in the original CRT); furthermore, the alignment of the beam axis and the planarity of the cathode-grid were evidenced by a nonfocused, uniform circular spot on the phosphior screen.

- A sporadic short circuit does occur in the grid-cathode circuit; however, this can generally be eliminated by "burning-out" the short. It is possible that the short circuit is caused by material flakes (due to inadvertent scratching of the grid or cathode structure during the photocathode processing-transfer cycle) which bridge the $0.01-\mathrm{cm}$ gap between the electrodes.

- The cathode was operated at $-8.5 \mathrm{kV}$, not at the specified $-10 \mathrm{kV}$, because of corona-discharge problems on the inner surface of the glass tube at the cathode-grid region. Subsequent SCT models should be deposited with a $\mathrm{Cr}_{2} \mathrm{O}_{3}$ layer in order to allow a leaky path for charges that may accumulate on the glass surface. Additionally, at high negative grid voltages it appears that field emission may occur on the inner edge of the grid aperture; this could be swept into the primary beam. The original thermal-oxide-cathode CRT did not exhibit this phenomenon. It is assumed that redistribution of cesium from the $S-1$ photocathode lowers the work function of the surrounding material (such as the grid and the glass surface) and causes these problems of field emission and corona. The problem does not occur when the grid is operated at zero volts with respect to the cathode $\left(10^{4} \mathrm{~V} / \mathrm{cm}\right)$. Field-emission problems also occur witen the grid is operated at a positive voltage with respect to the cathode $\left(>2 \times 10^{4} \mathrm{~V} / \mathrm{cm}\right)$. Further investigations of the field-emission problems associated with the redistribution of cesium are required.

- With zero volts on the grid, the trace-width in a circular scan was measured to be $0.01 \mathrm{~cm}$. Because of the superior electronic optics in this tube, as compared with the RCA tube, blooming of the spot (or trace-width) has not been a problem.

- Fabrication of a stable S-1 transferrable cathode has been a problem. For the initial SCT prototype, the $1.06-\mu \mathrm{m}$ response was difficult to measure with the 100-mW continuous-wave Nd:YAG laser because of the low sensitivity; however, streak traces from the Nd:glass laser can be identified with a $16-\mathrm{kV}$ postacceleration voltage (applied between the anode and the phosphor screen) and 15-to-20-psec pulses at an intensity of $<1 \mathrm{MW} / \mathrm{cm}^{2}$.

The first prototype model of the circular SCT will receive further tests. The modification of the second KR-23 tube will await the completion of the testing and evaluation of the present tube.

\section{AN X-RAY STREAK-CAMERA TUBE}

The $\mathrm{x}$-ray streak-camera tube is being designed to work over the $x$-ray energy range of 1 to $10 \mathrm{keV}$. The tube will be used to temporally resolve $x$-ray events occurring in the time regime of 100 psec or less. The 


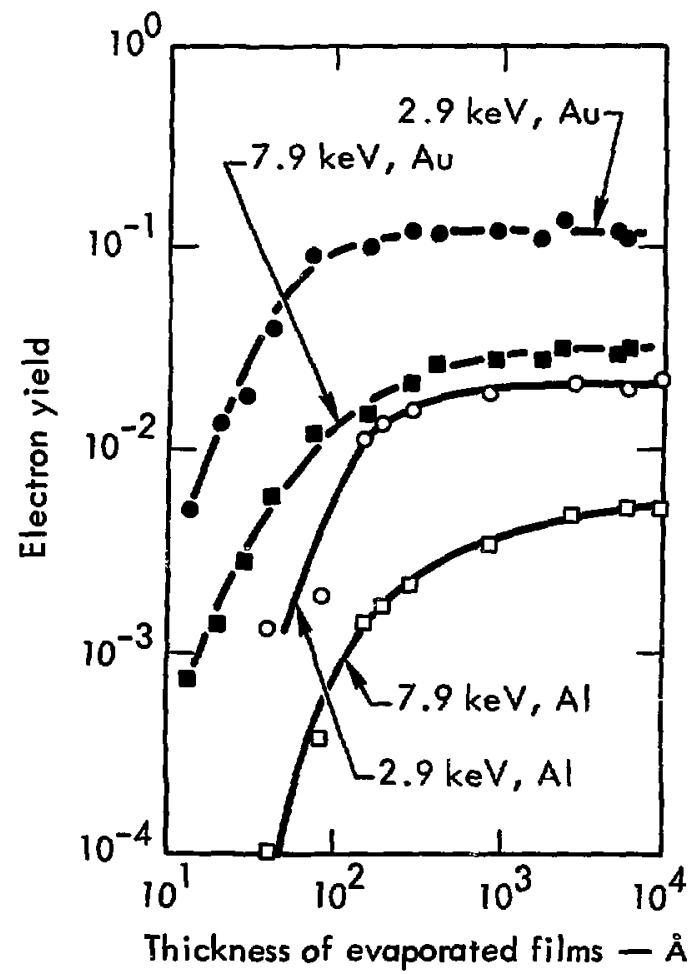

Fig. 110. Quantum efficiency versus thickness for gold and aluminum at two $x$-ray energies.

camera concept is identical to that used in the 10-psec streak camera currently employed for temporally resolving $1.06-\mu \mathrm{m}$ laser pulses; however, the photocathode has been replaced with an $\mathrm{X}$-ray photocathode.

As seen in Fig. 110, $x$ rays are converted to electrons in gold with about a $10 \%$ efficiency for $2.9-\mathrm{keV}$ incident photons. Gold makes a photocathode with about the highest quantum efficiency for this low-energy $x$-ray range. We have experimentally measured the quantum efficiency of gold at $1.5 \mathrm{keV}$ (aluminum $K \alpha$ ) to be as high as $20 \%$ for a 100- $\AA$ thin film. The cathode ir not made thicker than $100 \AA$ since no gain in quantum yield is obtained beyond this thickness, as seen in Fig. 110. Approximately $5 \%$ of the incident $\mathrm{X}$-ray photons (1.5-keV) are absorbed in the first $100 \AA$, and, since the photoelectrons are created within about $10 \AA$ of the vacuum interface, losses in overall quantum yield are suffered with thicker gold films. The gold is presently being evaporated on the thinnest beryllium substrate (0.3-mil) that we have found will support a vacuum. The absorption for $1.5-\mathrm{keV}$ incident $x$ rays in 0.3 -mil-thick beryllium is $22 \%$.
We have two primary questions about the $x$-ray streak-camera tube. First, are the present low-energy $x$-ray soirces capable of delivering enough intensity for streak-camera resoluticn? Seco-id, will the energy distribution of electrous leaving the photocathode be broad enough to cause severe deleterious temporal and spatial dispersions? To help answer these questions, we devised a planar photodiode with our $x$-ray photocathode spaced 0.1 in. from an aluminized P-11 phosphor. The tube is proximity-focused by about $10,000 \mathrm{~V}$ or a field of $40 \mathrm{kV} / \mathrm{cm}$. The tube was used to look at the $\mathrm{X}$ rays emanating from the Plasma $X$ laser-generated plasma. By observing the image at the phosphor, we were able to obtain information about the cross section, and consequently the energy distribution, of the electron beam. (It should be noted ihat our results are tentative, since with this diode there is the possibility of $x$-ray photons penetrating

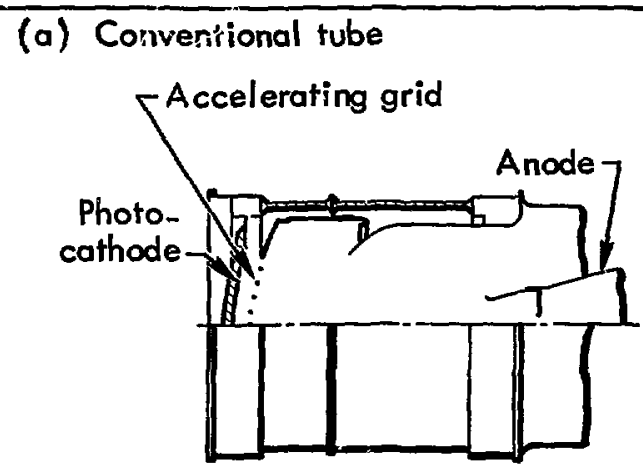

(b) Modified tube

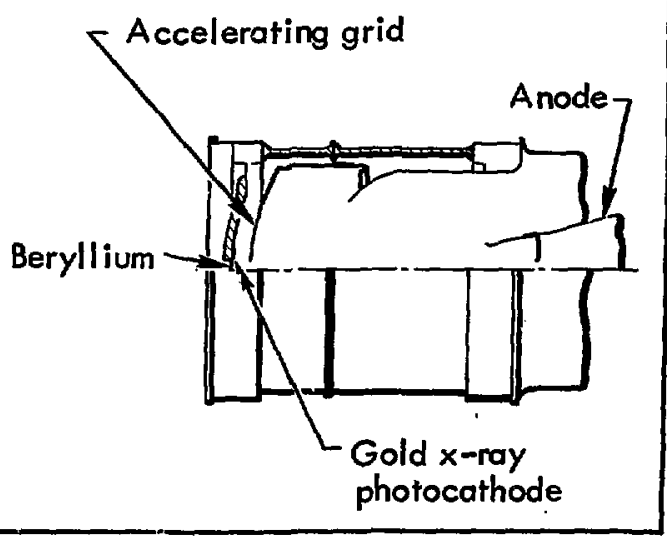

Fig. 111. Cross section of the RCA C73435 tube (a) as conventionally used in streak-camera work and (b) after $x$-ray photocsthode modifications. 

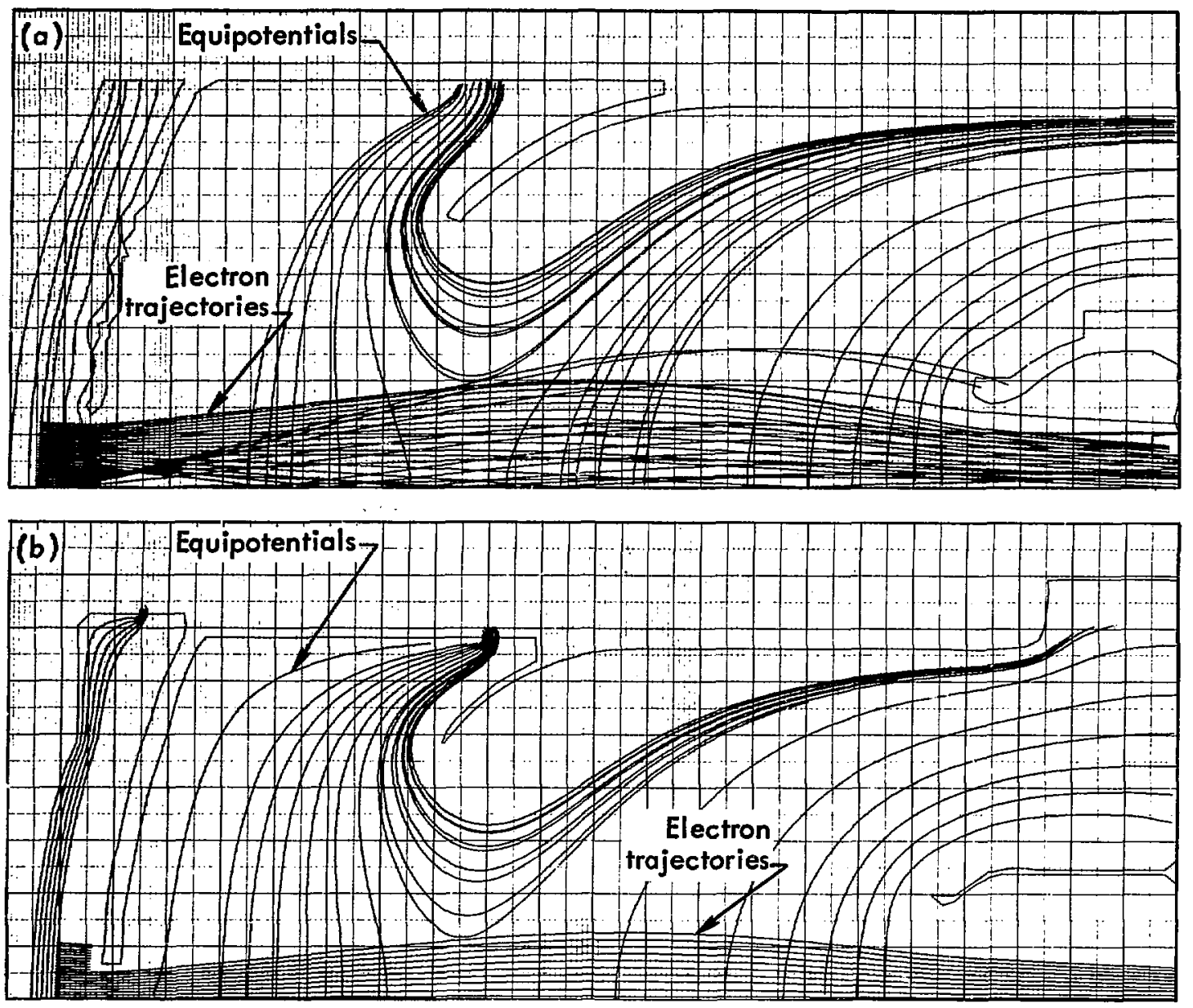

Fig. 112. Computer simulations of electron trajectories and equipotentials. (a) Conventional C73435 tube shown in Fig. 1112 (b) Modified tube shown in Fig 111 b.

through the cathode and directly imparting their energy to the phosphor.) When the diode voltage was removed on some shots, the spot was observed to weaken in intensity or disappear. Assuming that the image at the phosphor was indeed electron-induced, our observations indicated that we obtained an energy intensity of about $1 \mu \mathrm{J} / \mathrm{cm}^{2}$ in the incident $1-\mathrm{keV} \mathrm{x}$ rays. This is the necessary incident $x$-ray intensity for exposing the film by looking at the P-11 phosphor through the image intensifier. We also confirmed that there was little spot size divergence, showing that sine energy distribution of the electrons was no greater than $25 \mathrm{eV}$. A 25-eV energy distribution would result in a cathode transit-time time dispersion of about 17 psec and an optimum spatial resolution of about $11 \mu \mathrm{m}$ using the spherical electron optics in the RCA streak tube. This performance would be encouraging for the present state of the development effort.

The prototype $x$-ray streak tube is a modified RCA streak-camera tube of the type currently in use in standard and ultrafast streak cameras. We have replaced the existing photocathode with a 0.3-mil-thick, 1/8-in.-diam beryllium window on which is deposited the 100- $\AA$-thick gold photocathode. The accelerating grid has been replaced with a disk that has a large aperture in the center. We are utilizing only a small area of the cathode in our first attempts with the tube.

The original tube and the modified tube are shown in Fig. 111; computer-generated electron-trajectory plots for the two tubes are shown in Fig. 112. The computer plot indicates that the electron trajectories 
for the modified tube will be satisfactory. The tube is assembled, and we are now working on the peripheral mounting hardware and power supplies necessary to use the tube in measurements of laser-produced plasmas and for further tests and evaluations of the tube performance.

\section{DIAGNOSTICS OF WAVEFRONT DISTORTION IN LASING MEDIA}

A holographic interferometer is being constructed for the investigation of pump-induced dynamic wavefront distortion in lasing media. The first application will examine distortion due to flashlamp pumping in Nd:glass disk amplifiers. Several disk modules of various sizes will be studied, each of which contains six eliptical disks at Brewster's angle for 1.06- $\mu \mathrm{m}$ radiation. The smallest module $(A)$ has a clear aperture $3.5 \mathrm{~cm}$ in diameter and a total housing length of approximately $1 \mathrm{~m}$. The largest module $(D)$ will have a $30-\mathrm{cm}$ clear aperture and a total length of approximately $6 \mathrm{~m}$. These disk modules are eventually to provide the latter stages of amplification in the 10-kJ glass-laser system.

In large-aperture dynamic systems such as these, holographic interferometry is far more convenient than conventional interferometry; high-quality optics are not required, and precision alignment is not necessary. Because of the significant absorption coefficient (approximately $0.15 \mathrm{~cm}^{-1}$ ) for $6943-\AA$ radiation in Nd:glass (2-3\% doping), and the long optical path (3.5-cm-thick disks in the $D$ module), we require a ruby laser which provides fairly high-energy pulses in afdition to the usual holographic requirements. The nuby laser we have ordered will provide two $700 \mathrm{~mJ}$, 30-nsec pulses and an interpulse separation which is variable from 100 to $1000 \mu$ sec. In addition, the ruby system is specified to have a $\mathrm{TEM}_{00}$ output with a single-pulse coherence length greater than $1 \mathrm{~m}$ and a double-pulse fringe-free region approximately $10 \mathrm{~cm}$ long. The appearance of high-contrast fringes in the double-pulse mode is caused by heating of the ruby rod in its own optical-pump cycle and is thus dependent on interpulse separation time. The variable interpulse separation time of the ruby laser enables us to examine wavefront distortion throughout the optical-pump cycle of an Nd:glass disk module by synchronizing the ruby laser and disk module systems.

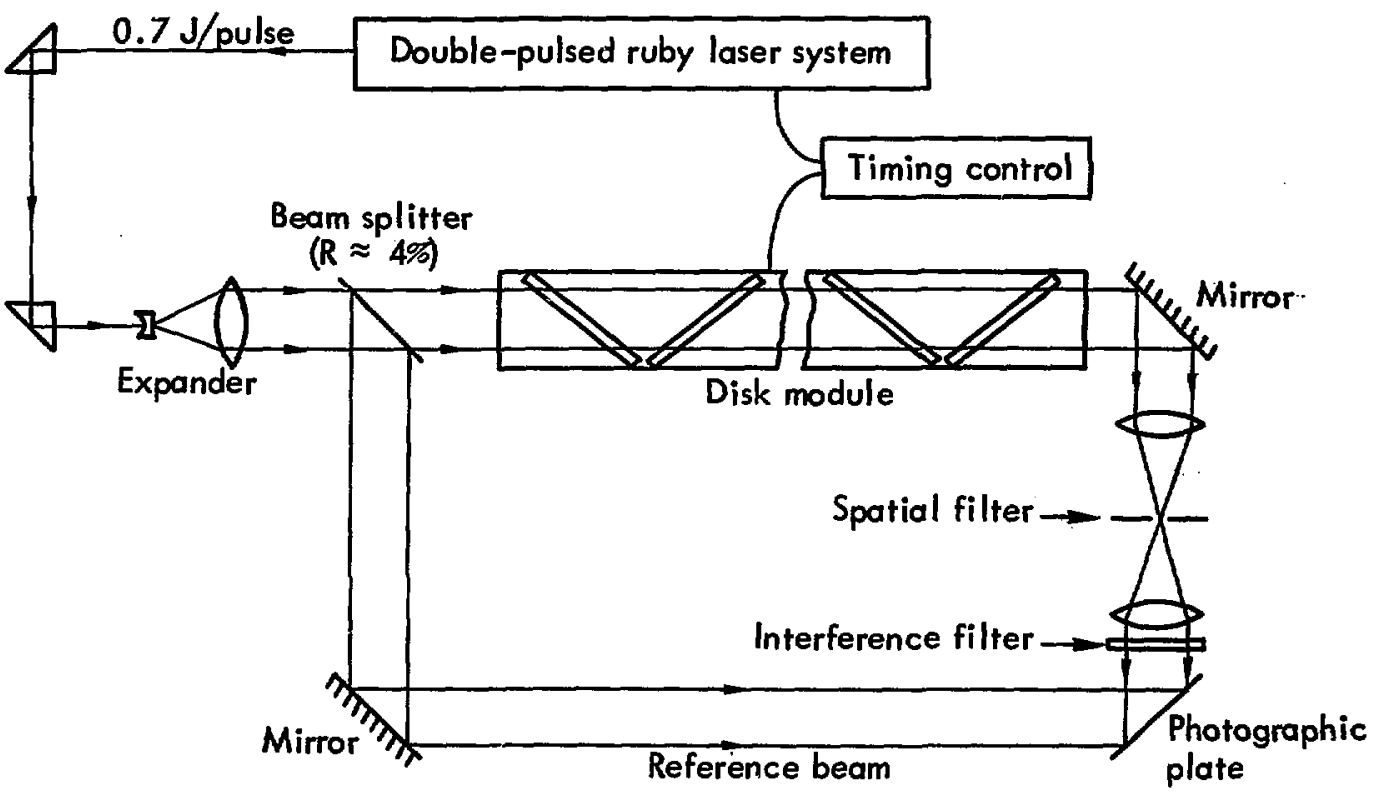

Fig. 113. Dynamic wavefront distortion test of the flass amplifier module by double-exposure holographic interferometry. 
Various holographic interferometers are being evaluated for the above purposes. A simple Mach-Zehnder interferometer, as shown in Fig. 113, can be used in situations which do not require significant longitudinal coherence of the ruby pulses. The limitation set by the Mach-Zehnder arises from the fact that although the interferometer may be path-matched along the central optic axis, it is not path-length-matched for rays transversely displaced from the center, i.e., those corresponding to the outer regions of the transverse profile. With the given coherence limitations of the ruby system, this path mismatch sets predictable limits on the use of a simple interferometer with reasonable angles between "reference" and "subject" beams. With the ruby laser described above, an interferometer such as that shown in Fig. 113 can be used in the double-pulse mode only for the smallest-aperture $(4-\mathrm{cm})$ disk system. Larger systems require either the addition of beam reducers to the setup shown in Fig. 113 or the use of more scphisticated holographic interferometers. ${ }^{54,55}$ By working in the single-pulse mode, with two independent firings of the ruby laser, one could use the simple scheme even for the largest disk modules. This would require one firing of the ruby laser before firing the glass system, several minutes for cooling the flash-heated ruby rod, and finally a second firing of the ruby laser synchronized with a desired time in the flashlamp cycle of the glass disk system. This scheme, however, has the disadvantage that movement and vibration may introduce extraneous fringes in the hologram.
An interferometric scheme which more closely approximates the longitudinal path-match condition, for all transverse positions of the ruby beam, is shown in Fig. 114. This scheme employs a piecewise turning element that provides, to desired accuracy, an effectively tilted phase front. The approximately tilted phase front then strikes the photographic emulsion parallel to the plane wavefront from the second arm of the interferometer with negligible path mismatcin across the surface of the plate. With a path-matched holographic interferometer such as this, one can employ the double-pulse mode of the ruby laser for arbitrarily large disk modules. The piecewise tumi.ig elements have until now been used in conjunction with a nearby ground-glass diffusor and imaging optics in the "scene" beam. 55 We are currently investigating their use in the "reference" beam without the use of ground-glass diffusors or imaging optics.

The various components required for interferometers of the types shown in Figs. 113 and 114 are being procured and evaluated. A 15 -cm-aperture MachZehnder interferometer will be prepared for use before the arrival of the ruby laser. The path-matched interferometer will be available somewhat later, depending on the success of Fresnel turning elements in the reference arm.

\footnotetext{
* The advantages of a reflective turning plate, as compared with a prismatic turning plate, were pointed out by $R$. F. Wuerker of TRW.
}

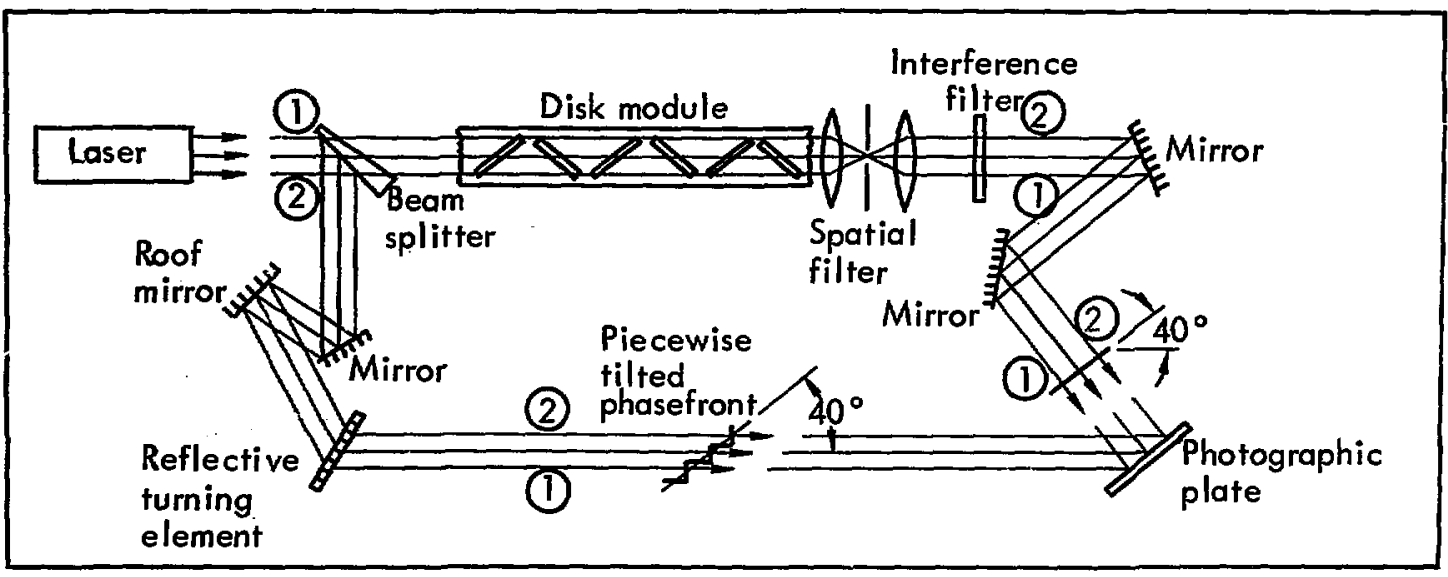

Fig. 114. Path-matched holographic in terferometer. 
In the previous semiannual report ${ }^{56}$ we discussed holographic interferometry as a means of measuring electron-density distributions in laser-produced plasmas. Preliminary results using a scatter-plate interferometer (see Ref. 56, Figs. 74 and 75) have recently been obtained with a 150-psec, mode-locked $\mathrm{Nd}$ :YAG laser. Figute 115 shows the interference pattern obtained $4 \mathrm{nsec}$ after the arrival of a $2-\mathrm{J}$, $1.06-\mu \mathrm{m}$ light pulse on a tilted polyethelene target. Green light $(0.53 \mu \mathrm{m})$ for the interferometer was obtained by frequency-doubling a portion of the incident $1.06-\mu \mathrm{m}$ light pulse with a temperature-tuned CDA crystal. From the photograph in Fig. 115, we estimate a plasma expansion velocity of $3 \times 10^{7} \mathrm{~cm} / \mathrm{sec}$ and electron densities on the ordet of $5 \times 10^{18} \mathrm{~cm}^{-3}$ in the central plasma region.

Although we are continuing to work with scatter-plate holography, we have come to understand that this technique is limited to resolutions on the order of $100 \mu \mathrm{m}$, and is, therefore, not particularly well suited for the investigation of laser-produced plasmas at very early development times. We are examining other holographic techniques which may provide the desired spatial resolution despite the minimal coherence that may be encountered with short-pulse, high-power lasers. A combination of holographic microscopy ${ }^{57}$ and the techniques utilized in so-called "holocameras" 55 is a prime candidate.

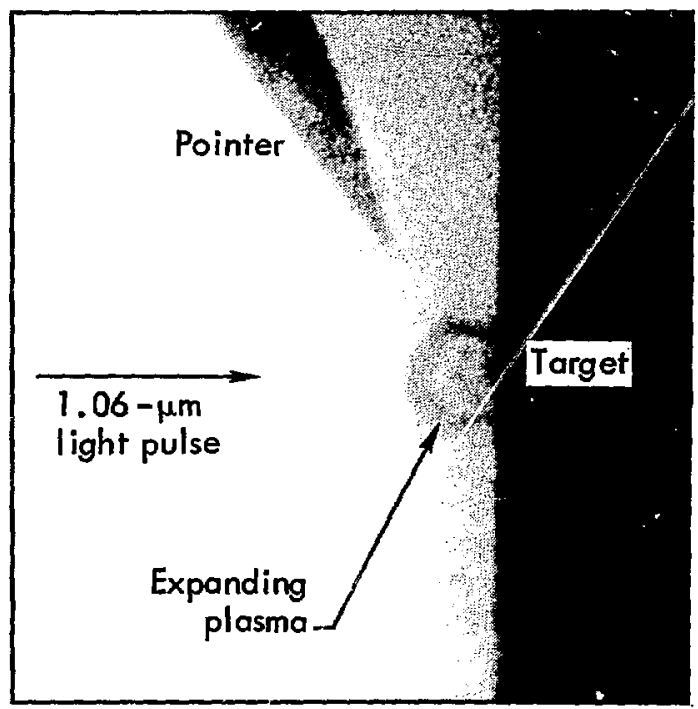

Fig. 115. Double-exposure hologram of a laser-produced plasma. Delay time is $4 \mathrm{nsec}$, expansion velocity is about $3 \times 10^{7} \mathrm{~cm} / \mathrm{sec}$, and electron densities are on the order of $5 \times 10^{18} \mathrm{~cm}^{-3}$.
Using an He-Ne laser and a microscope in one arm of a Mach-Zehnder interferometer, we have made holograms of a standard Air Force test pattern. Reconstructions of the group 6 and groir 7 elements are shown in Fig. 116. The smallest element in group 7, which is clearly resolved, has ine widths of $2 \mu \mathrm{m}$. An interferometer that may extend this technique to 100-psec light pulses is presently being constructed, as shown in Fig. 117. The only unusual element used is a piecewise turning element that effectively matches optical path length across the aperture of the beam. "With high spatial resolution,

*R. F. Wuerker of TRW pointed out the advantages of a reflective turning plate over a prismatic tuming plate.

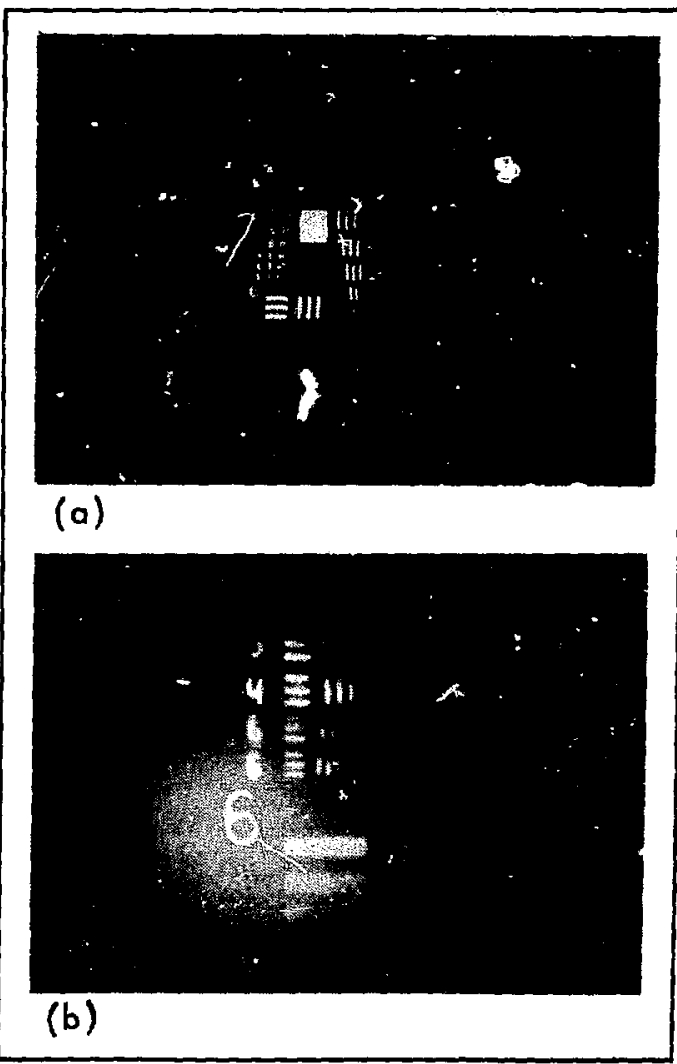

Fig. 116. Holographic reconstructions of a standard Air Force test pattem. (a) Groups 6 and 7. (b) An enlargement of group 7. The smallest pattern in (b) shows a clearly visible $2-\mu \mathrm{m}$ interval. 


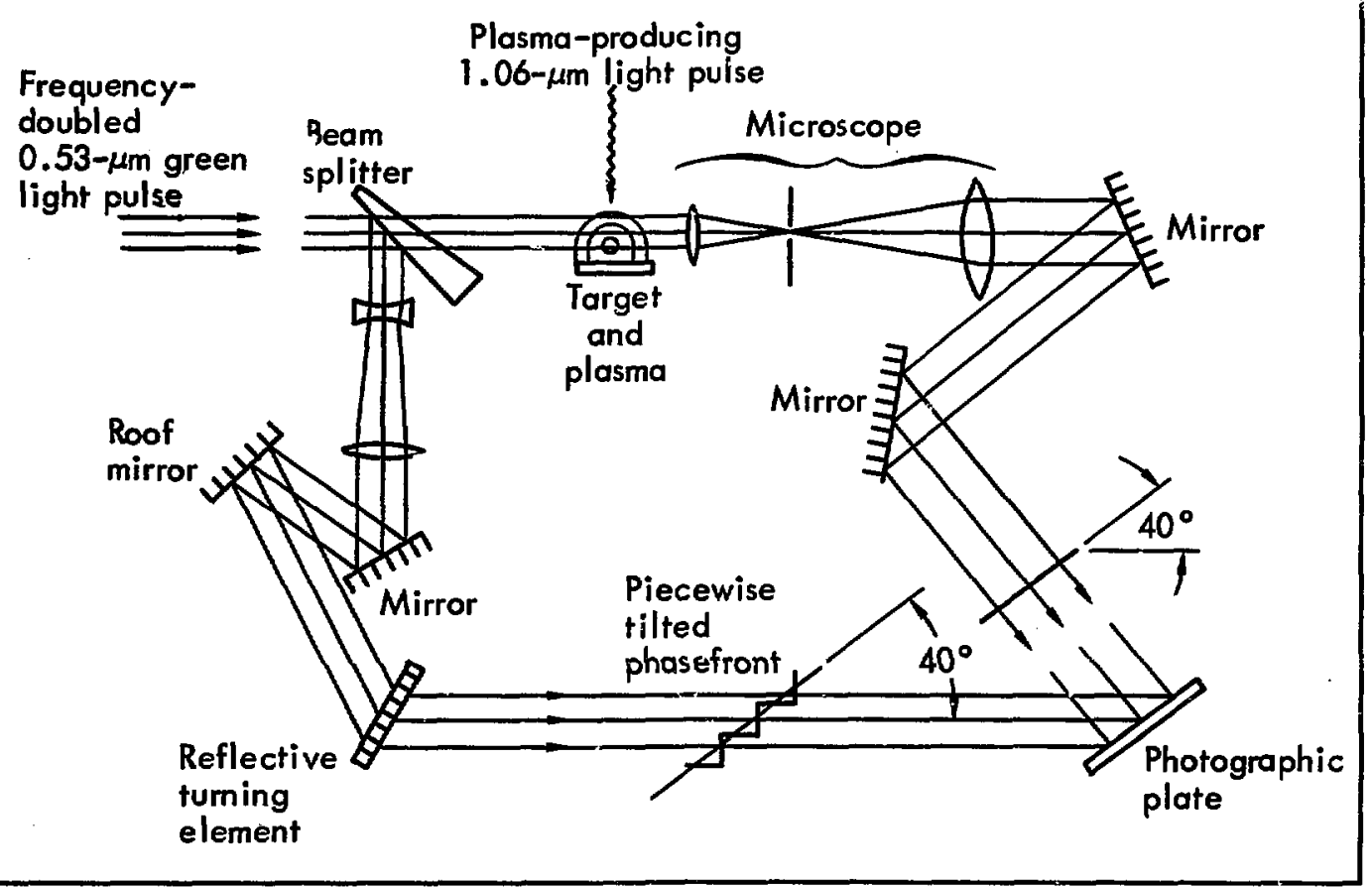

Fig. 117. High-resolution holographic interferometer.

holography of laser-produced plasmas will be limited only by optical-pulse duration and plasma expansion velocity. For instance, an expansion velocity of $3 \times 10^{7} \mathrm{~cm} / \mathrm{sec}$ and a 100-psec light pulse would combine to give a resolution of approximately $30 \mu \mathrm{m}$.
By observing the plasma with near-zero delay time (i.e., immediately after the high-power laser impacts the target), we might then obtain an experimental estimate of the size of the focal spot, in addition to determining electron-density distributions.

\section{AN ULTRAFAST 10- $\mu \mathrm{m}$ DETECTOR BASED ON THE OPTICAL KERR EFFECT IN CS,}

The response times of detection systems at $10 \mu \mathrm{m}$ are currently limited to about 1 nsec. By using the optical Kerr effect in $\mathrm{CS}_{2}$, coupled with a visible probe beam and a streak camera, an ultrafast but nonlinear detection system can be created. The theory of operation of this detection scheme has been described in previous reports 58,59 ; the work reported here is the direct continuation of the development described previously.

A mode-locked Rogowski-profile TEA $\mathrm{CO}_{2}$ oscillator of LLI design has bsen assembled in our new laboratory. This oscillator produces a pulse train which has a higher power and less amplitude jitter and time jitter than did the previous pin-type TEA oscillator. A single-pulse switch-out section ${ }^{60}$ has been added to the system, which allows us to select a single pulse from the pulse train.

A study has been made to compare the structure of the modulating $\mathrm{CO}_{2}$ pulse with the resulting structure of the modulated probe-beam pulse. A single switched-out pulse from the $\mathrm{CO}_{2}$ oscillator is used, to eliminate any possible ambiguity as to which pulse in the pulse train one is observing. The detection system used for simultaneously observing these two pulses consists of a photon drag detector for the $10.6-\mu \mathrm{m}$ radiation, a solid-state pin diode for the visible radiation, and identical 500-MHz oscilloscopes. The net response time of both detector systems is 1 nsec. Figure 118 displays two pieces of data showing the detector outputs overlaid. As can be seen, the two $\mathrm{CO}_{2}$ 


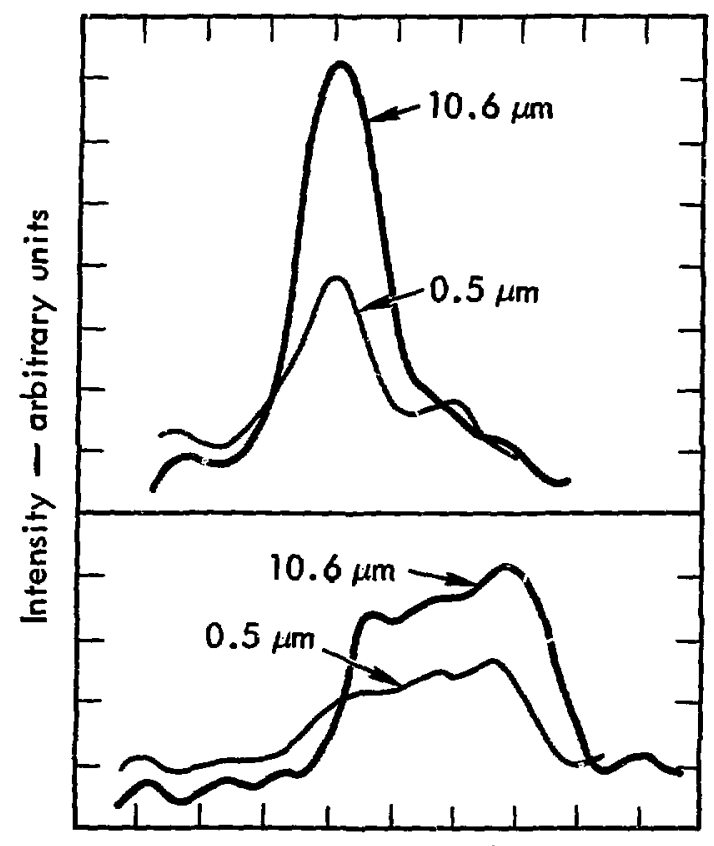

Time -1 nsec/div pulses shown have entirely different structures. These pulses were picked inientionally to show how the modulated visible pulse does indeed take the same shape as the modulating 10.6- $\mu \mathrm{m}$ pulse (to within the time response of the detection system). This is what one exprects, since the response time of the Kerr cell is $\sim 2$ psec.

The experimental apparatus is presently being adapted so that the LLL 10-psec-resolution streak camera may be used as the probe-beam detector. This is expected to increase the time resolution of our system an order of magnitude ( $20 \mathrm{psec})$. The initial investigations with this streak camera showed that the probe beam used in the previous experiments (TRW pulsed argon-ion laser) contains intensity modulation in the 100-psec-period region. This modulation makes this light source unsuitable for a high-time-resolution system. We are looking for a light source that will be acceptable for use in such a systern.

Fig. 118. A pair of pulse traces demonstrating that the a5- $\mu \mathrm{m}$ modulated pulse does follow the shape of the 10.6- $\mu \mathrm{m}$ modulating polse from the $\mathrm{CO}_{2}$ oscillator. 


\section{Introduction}

Laser-driven inertial-confinement fusion reactors for power generation will require the development of efficient, short-wavelength, pulsed gas lasers capable of delivering high average power. A technical base for the developmerit of such lasers is provided in the LLI ptapram by the gas laser activity, consisting of three complementary areas: research, technology, and systems.

There are a great many alomic and molecular gases potutially capable of being driven to provide coherent tmimion in tho vilite and ulluaviotet specteal regions.

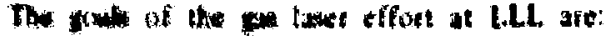

- Pigholify. thesugh computationa and expeci.

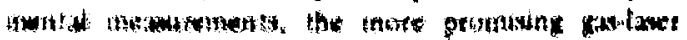

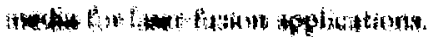

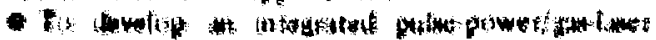

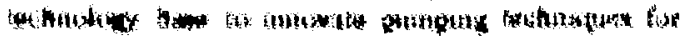

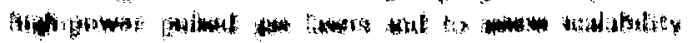

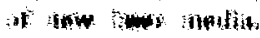

- To develop gas laser systems of appropriate energy, pulsewidth, efficiency, tc., for advanced laser-fusion experiments.

During the past half-year, efforts in new lasers centered on rare-gas excimer lasers. The first articles in this section (under Gas Laser Research) report on conclusive evidence for $1700-\AA$ stimulated emission in $\mathrm{Xe}_{2}^{*}$ together with observations for the first time of $1430-\AA$ stimulated emission in $\mathrm{Kr}_{2}^{*}$. Efforts to deveiop the pulse power technology required to assess sialability of such lasers got under way during the rteporting period and are described next. A $1-\mathrm{MeV}$, $100-k A$. 50 -nsec-pulsewidth electron-beam generator is being installed. Systems activity continues to focus on construction of a $\mathrm{CO}_{2}$ 1-nsec largel-irradiation laser toe lawer plastio coupling experimentation at $10.6 \mathrm{\mu m}$. lixpertrettisl and calculational offorts supporting the Jewer of the $\mathrm{CO}_{2}$ system are described in the

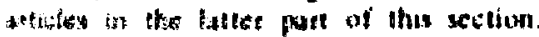

\section{Oan kater novereh}

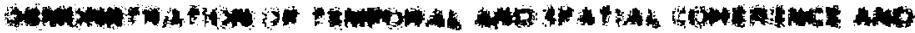

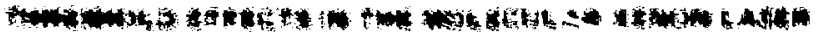

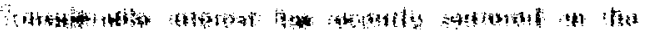

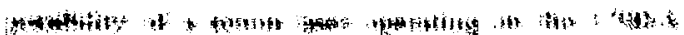

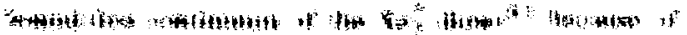

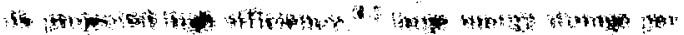

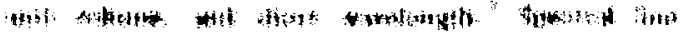

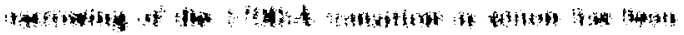

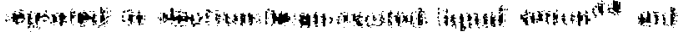

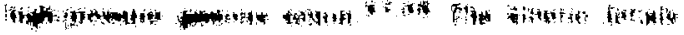

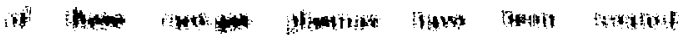
atimitions?

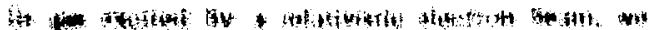

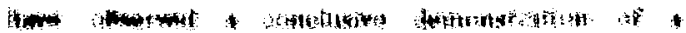

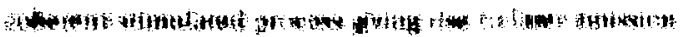

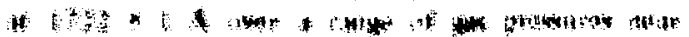

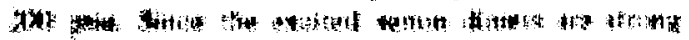

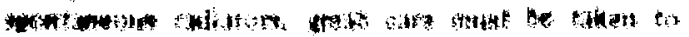

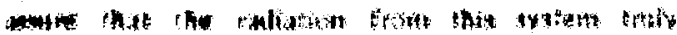

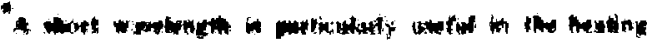

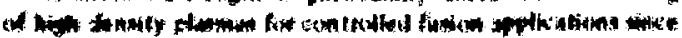

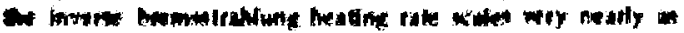
(n) Rat, (3).
}

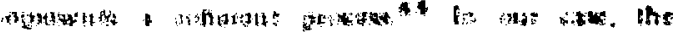

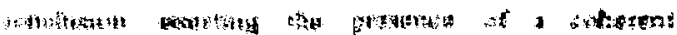

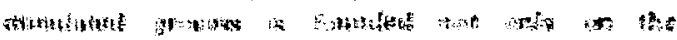

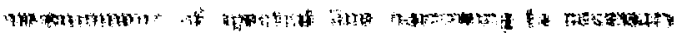

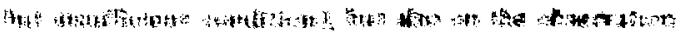

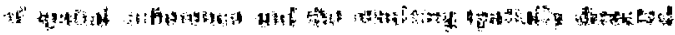

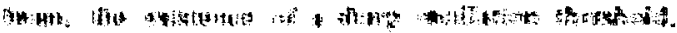

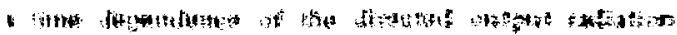

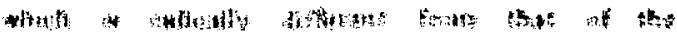
rivan

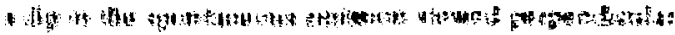

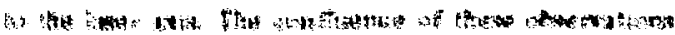

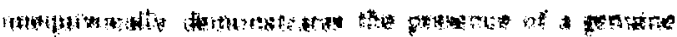

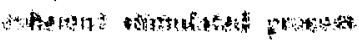

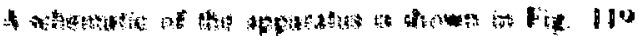

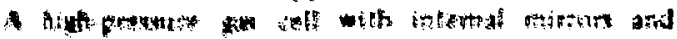

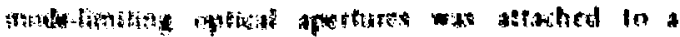

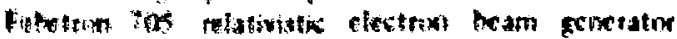

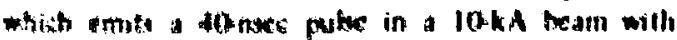
a tomina cherg af 1.5 MeV uret a Letrdiam aptefure, The optical cavity cenststed of two mirtois whth I.tis falius of currature, separeted from each 


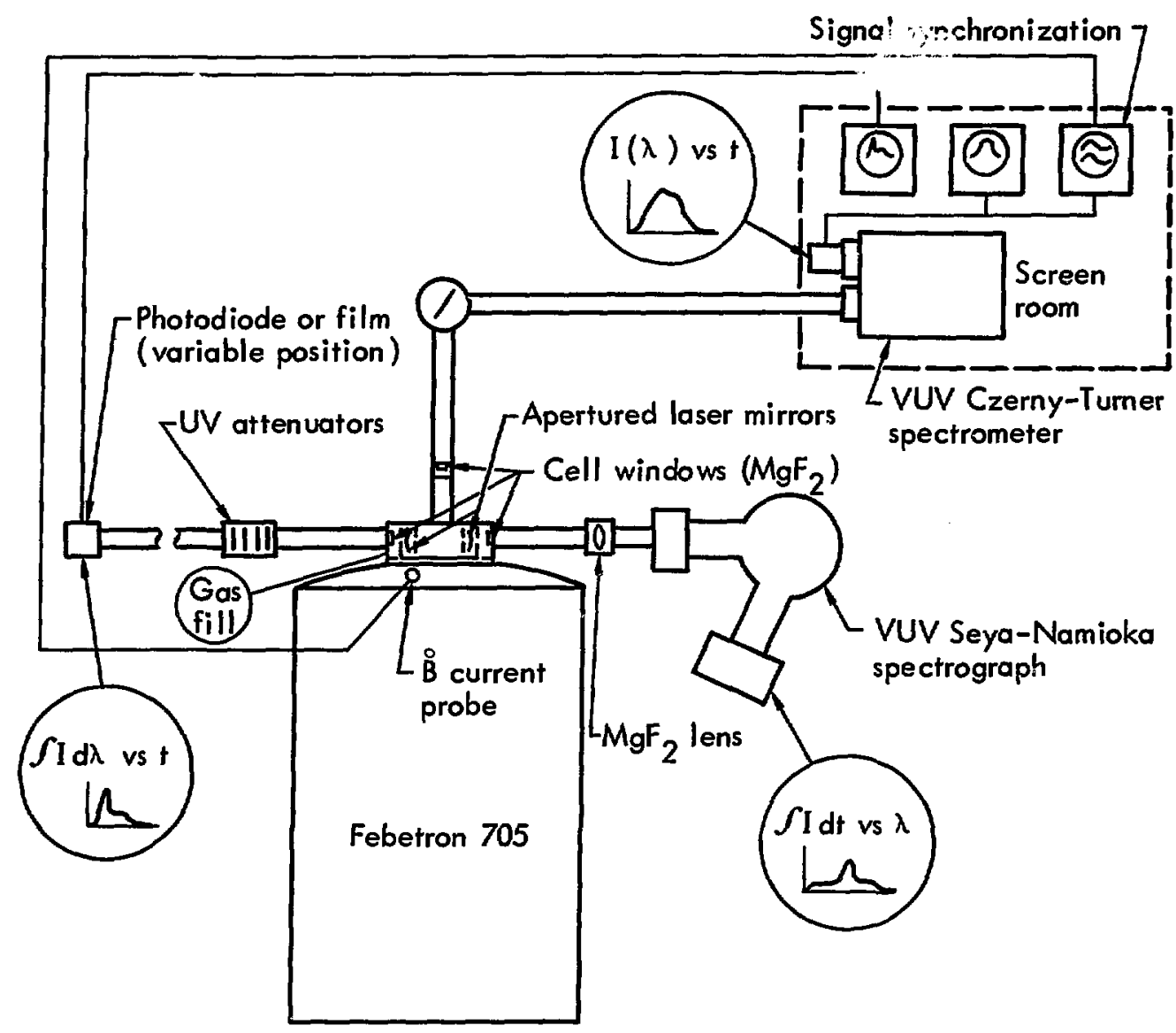

Fig. 119. Schematic of the experimental apparatus, illustrating the three diag : 4: systems: Seya-Namioka spectrograph, photodiode, wh Caerny-Turner qpectrometer viewing the sidelight.

ather by $5 \mathrm{~cm}$. The mirrors were fabricated from highly polished $\mathrm{MgF}_{2}$ substrates which were $\mathrm{Al}$ costed and $\mathrm{MgF}_{2}$-overcoated. The reflectance and iransmiltance of the mirrors at $1700 \AA$ were measured to be $80 \%$ and $8 \%$ respectively. The 2 -mm-diam intracavity agerlures limited the field of view of the diagnostic systems essentially to the volume contained in the cylinder defined by the apertures. Research gade xenon and high-vacuum turbomolecular pumps were used in the fill system. The spectral composition of the radiation along the optical axis was detected by $20.75 \cdot \mathrm{m}$ Sey2-Namioka spectrograph using Kodak SWR film and capable of spectral resolution of $0.1 \AA$.

The mirrors, rabricuted by Acton Research Corp., withstood over 50 ahot before deterionating. Some mirrors of poorer optical polish deterionted with one shot.
The temporal characteristics were observed with an ITT 4115 photodiode having a 2-nsec risetime. Spatial propagation studies were performed by replacing the photodiode with SWR film mounted on a variable-length line-of-sight pipe, and attenuation in this pipe was accomplished with calibrated sapphire attenuators. The spontaneous radiation emitted perpendicular to the cavity axis was monitored by a 0.82-m Czerny-Tumer spectrometer with a typical bandpass of $5 \AA$ about a center wavelength of 1715 \&.

Our conclusion of stimulated emission derives from the following five observations:

1. The spectral linewidth (measured photographically) narrowed from $\sim 160 \AA$ (as observed without mirrors) to a half-width of $\sim 15 \AA$ with the optical cavity. Figure 120a illustrates the typical 


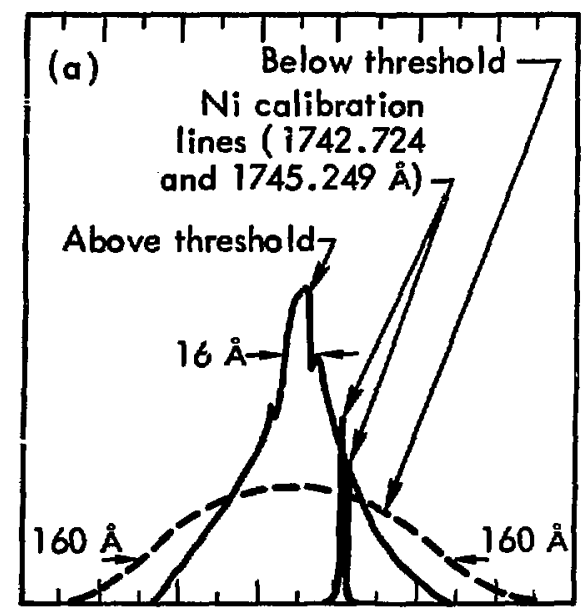

15801620166017001740178018201860

Wavelength $-\AA$

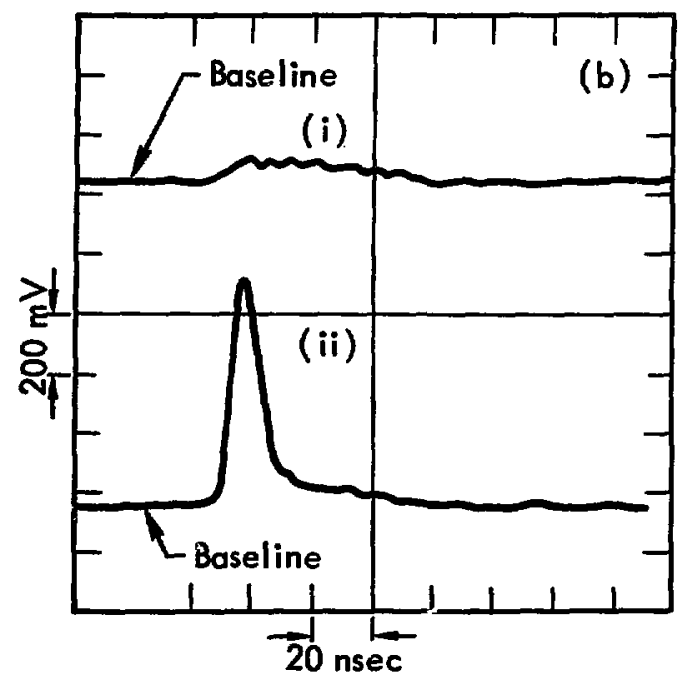

Fig. 120. (a) Time-integreted spectol output (logrithmic densitometer trece) from spectrotraph for conditions below and sbove threchold, showing $\mathrm{Ni}$ calibration lines. Two unidmtified aberption lines speat at 1708 and 1726 A. (b) Spectrilly iategated photodiode output as a function of time for (i) conditions below threshold, and (i) conditions sbove threitoif.

spectral output of the laser for conditions above and below threshold along with a calibration spectruin.

2. Marked changes in the time dependence of the directed output radiation were observed for conditions abr and below the oscillation threshold. This dramatic change in the temporal pulse shape is seen a an intense 10-nsec pulse as shown in Fig. 120b. Spectral line narrowing was observed only in conjunction with this fast pulse.

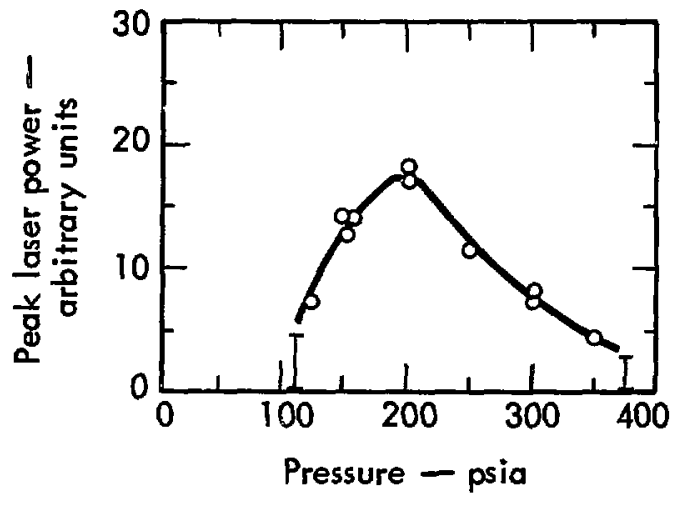

Fig. 121. Laser peak power vs xenon pressure. Note the sxistence of thresholds at about 110 and 375 psia. Maximum standard deviation of the data points was 0.612 .

3. The radiation was spatially coherent, as observed photographically by positioning film at various distances from the laser cavity. The half-angle of divergence so determined was $5 \mathrm{mrad}$, which is nine times the divergence of the fundamental mode $(0.6 \mathrm{mrad})$. Since the ratio of the diameter of a higher order mode to the fundamental mode is a constant, ${ }^{69}$ the divergence of the higher order mode is larger by this constant factor. In our case, the diameter of the fundamental mode at the $2-\mathrm{mm}$ aperture is $0.186 \mathrm{~mm}$. Assuming that the highest order mode is the size of the aperture, the diameter ratio is 10.7 , which agrees well with observation.

4. There was a definite oscillation threshold that depended on xenon gas pressure. Figure 121 illustrates the dependence of the peak laser output power on xenon pressure. Sharp threshold phenomena are oiserved at 125 and 375 psia.

5. A dip in the sidelight spontaneous emission at 1715 \& occurred simultaneously with the 10-nsec pulse observed on the photodiode.

The direct observation of spatial coherence and threshold effects reinforces the earlier reports of spectral narrowing ${ }^{65}$ and conclusively demonstrates the existence of optical gain on the $1700-\AA$ xenon dimer transition. Furthermore, we observed that the oscillation was concurrent with the pump pulse and that the laser behaved as a sin-switched oscillator. Temporally, three types of pulses were observed: a single pulse 10.20 nsec in duration, a double pulse, or a single pulse followed by a knee about 30 nsec in duration.

Because of the rapid energy-transfer processes known to occur in rare gas mixtures 70 in the gas, liquid, and solid phases, ${ }^{71}$ we have extended these 
studies to include binary argon/xenon and krypton/xenon mixtures. Under certain conditions substantially enhanced laser outputs were observed with the mixtures. In the case of argon/xenon mixtures, the stimulated emission spectrum arising from the xenon dimer transition shifted $\sim 8 \&$ to the blue side of the $1722 \pm 1 \AA$ value observed in pure xenon. A shift of this nature is expected on the basis of the collision-induced absorption 72 of xenon in this spectral region.

\section{OBSERVATIONS OF STIMULATED EMISSION FROM HIGH-PRESSURE KRYPTON}

AND ARGON/XENON MIXTURES

We describe here some initial experimintal findings concerning the observations of stimulated emission from high-pressure krypton and argon/xenon mixtures excited wih a pulsed relativistic electron beam. Kinetic analyses of these electron-beam-xcited plasmas appear in earlier publications. 67 The article preceding this one gives details of the experimental apparatus, which allows the simultaneous measurement of (1) the spectrally integrated time dependence of the spatialiy directed oscillator output, (2) the temporally integrated spectrum of the emitted radiation (photographed spectrum), and (3) the behavior of the spontaneolis emission of the excited material viewed perpendicular to the oscillator axis.

The molecular continuum of krypton arising from bound-free transitions of the excited krypton dimer $\mathrm{Kr}_{2}^{*}$ and centered at about $1500 \AA$ is analogous to the corresponding spectral feature in xenon at $1700 \AA$. As a first approximation, the level structure of the $\mathrm{Kr}_{2}$ system can be understood mutatis mutandis from the published data on $\mathrm{Xe}_{2}$. We have observed strong stimulated emission on this transition in krypton at $1457 \pm 1 \AA$ with a linewidth of $8 \AA$ as shown by the spectrum in Fig. 122a. This emission occurred in a 10-nsec pulse as observed on the photodiode. Due to a small but unavoidable impurity of xenon in the krypton sample, the xenon $1470-\hat{A}$ resonance line is a prominent absorptive feature in the spectrum. Moreover, our observations indicate that this absorptive loss dominates the optical losses of the system and easily prevents oscillation unless considerable care is taken to limit contamination from xenon. Furthermore, since the $1470-\AA$ xenon resonance line absorption has a strong wavelength dependence in the region which overlaps the wavelength of the observed stimulated emission, we expect that the precise values of the krypton stimulated-emission center frequency and bandwidth will be dependent on the xenon impurity level. The

\footnotetext{
'Soe Rer, 61. For thew Inreer diatomics, Hund's coupling case $c$ is appropriate.

the cavity wes bounded by curved 1-m-rudius mirrors with measured transmittences and ronoctences of about $5 \%$ and $80 \%$ reppectivoly.
}

pressure dependence of the peak power (detected with an ITT 4115 photodiode) is illustrated in Fig. 122b. A threshold ${ }^{\dagger}$ for oscillation was observed at 250 psia; pressure cell limitations prevented an examination of the region above 500 psia.
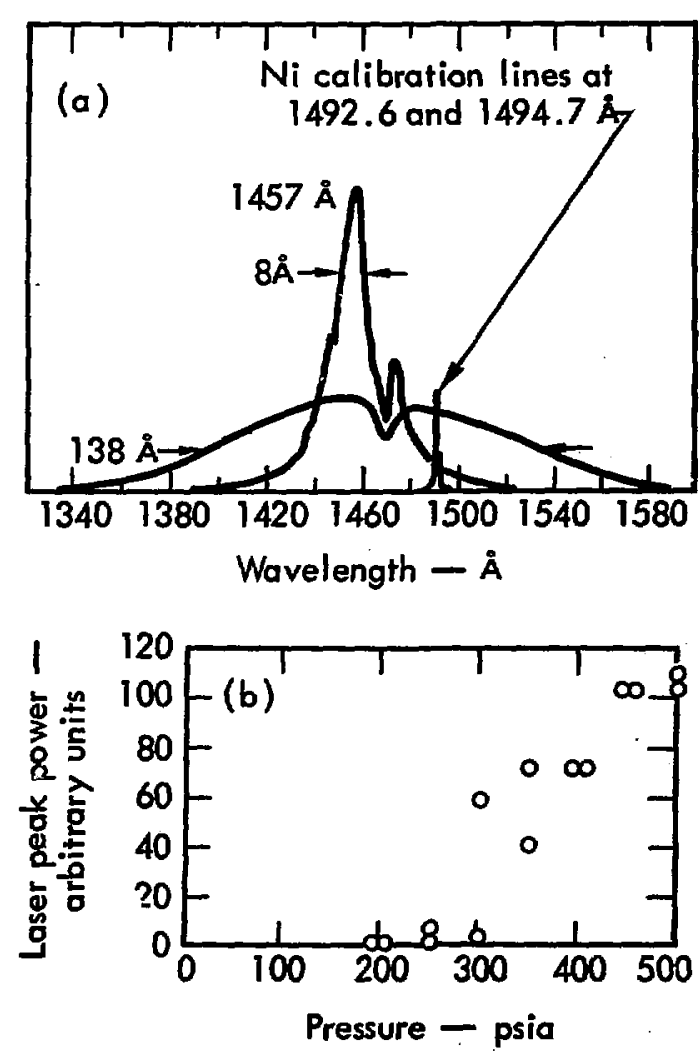

Fig. 122. (a) Timointegrated poctral output (logrithmic densitometer trace) showing Ni calbration lines. Minor absogption bands tentativaly attributed to the $X^{1} \Sigma_{g}^{+} \rightarrow a^{11} \Sigma_{\mu}^{-}(\Delta v=0,1)$ trancition of $N_{2}$ so observed, in addition to the Xenon resonicace storption. at 1.770 A. Sec M. O Jaw and Y. Tandke, J. Chem. Fhys. 32, 754 (1960). (b) Proswor dependence of the kypton oucillator output. Note the threinold at about 250 pin. 
Because of the rapid energy-transfer processes known to occur between certain rare-gas atoms and molecules in the gas, ${ }^{70}$ liquid, ${ }^{71}$ and solid ${ }^{71}$ phases, we have extended these studies to include rare gas mixtures. Of particular interest is the resonant energy transfer process $^{70}$

$$
\begin{aligned}
\operatorname{Ar}_{2}\left({ }^{1,3} \Sigma_{u}^{+}\right) & +\operatorname{Xe}\left({ }^{1} S_{0}\right) \rightarrow \operatorname{Ar}\left({ }^{1} S_{0}\right) \\
& +\operatorname{Ar}\left({ }^{1} S_{0}\right)+\operatorname{Xe}\left({ }^{1} P_{1}\right)
\end{aligned}
$$

which efficiently quenches the $1300-\AA$ argon continuum and leads to the generation of the xenon continuum at $1700 \AA$ through a strong coupling of the argon and xenon kinetic chains in argon/xenon mixtures. We report below a very substantial enhancement of the stimuiated emission from $\mathrm{Xe}_{2}$ at about $1720 \AA$ in argon/xenon mixtures along with the observation of a blue shift of the mixt:ie spectrum in comparison to the spectrum of pure xenon.

Figure 123a displays the dependence on xenon pressure of the peak oscillator output power (registered on an ITT 4115 photodiode) for argon/xenon mixtures (for a constant total pressure of 250 psia) and for pure xenon. In this case the enhancement, as measured by the relative peaks of the two curves, is a factor of $\sim 2$ and must be considered as evidence of considerably improved Frformance. In addition, the use of argon/xenon mixtures results in a reduction of the oscillation threshold in terms of xenon partial pressure. We note, however, that these data pertain to an oscillator configuration which contained no provision for control of the mode volume. Calibrated sapphire attenuators were used to prevent detector saturation over the full dynamic range of the measurements. Among the several factors which may contribute to the increased oscillator output are the following: (1) The possibility of lower excitedstate/excited-state collisional loss rates in the argon kinetic chain as compared to the corresyonding xenon kinetic processes. For fixed excimer density, the excited state losses will be the same; therefore for this process the differential losses would have to occur higher in the kinetic chain. (2) Lower collisional absorption ${ }^{\mathbf{7 2 , 7 3}}$ arising from xenon-xenon encounters (at fixed temperature and wavelength, this effect scales as the square of the xenon density). (3) Oscillation over a larger effective mode volume due to the decrease in the optical losses noted in (2) above, or due to a more beneficial electron-energy deposition in the gas. Internal apertures are necessary to limit the mode volume in order to separate these geometrical averaging effects from increases in localized inversion density. ${ }^{67}$
An indication that collisional absorption ${ }^{73}$ originating from xenon-xenon collisions ${ }^{*}$ is an important factor in the increased output is given by the observation of a blue shift in the stimulated

\footnotetext{
At $\sim 1700$ \& there is also the possibility of a component due to argon-xenon collisions which would scale like the product of the argon and xenon densities. The importance of this quality depends upon the details of the molecular potential curves for the ArXe system. Measurements are currently in progress for the evaluation of these losses.
}
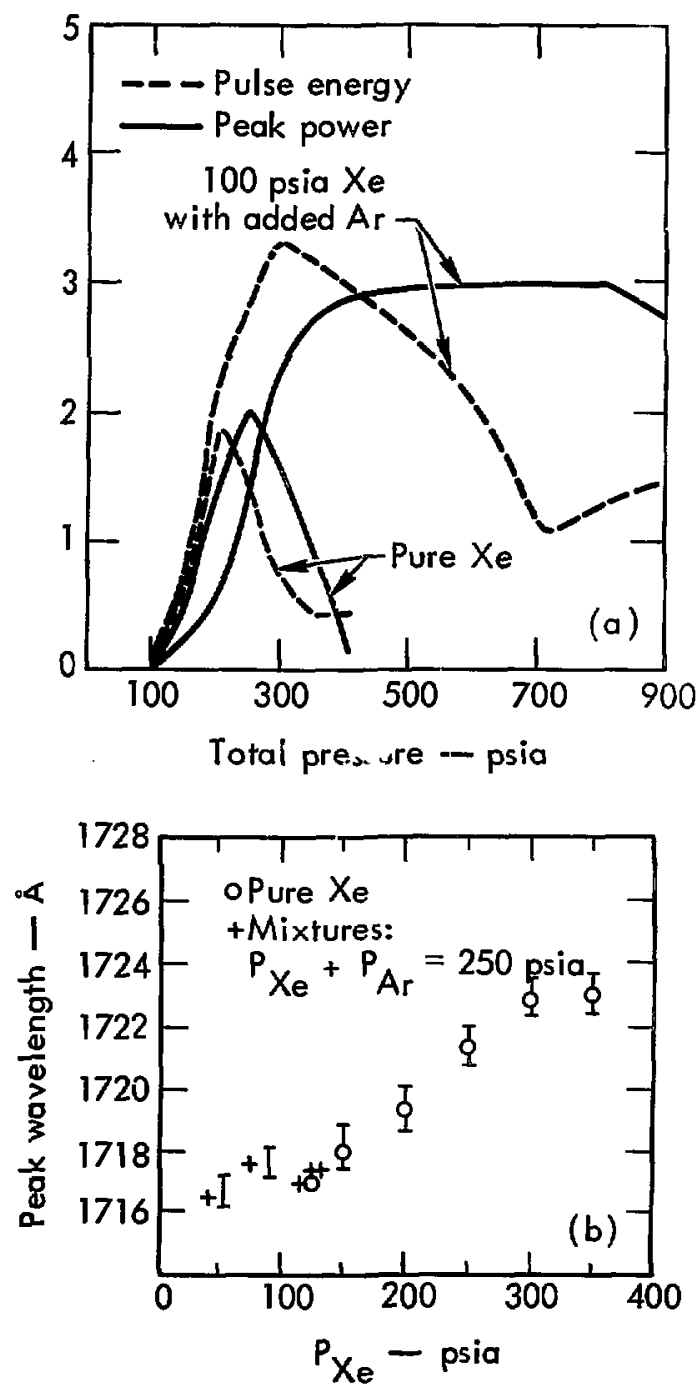

Fig. 123. (a) Pressure dependence of the laser pulse energy and peak power for argon/xenon mixtures and pure xenon. (b) Dependence of the laser peak wavelength on xenon partiai pressure. 
emission spectrum of the argon/xenon mixtures. Figure 123b illustrates the dependence of the laser peak wavelength on xenon partial pressure. Blue shifts as large as $7 \AA$ are observed, and the shift is independent of argon pressure. Since the collisional absorption $^{73}$ increases toward shorter wavelengths, a blue shift as observed in the experiment is anticipated when these losses are diminished. Refined experiments and calculations are presently in progress to more precisely determine the relative contributions to the enhanced output of points (1), (2), and (3) noted above.
Finally, we observe that the demonstration of efficient energy transfer has important implications for laser systems operating on molecular bound-free transitions. It reduces the required density of the active material, and thus lowers the collisional absorption, a quantity which represents a strong limitation on the extractable energy density. In addition, since we have some freedom in selecting the minority acceptor system, the output wavelength is at our disposal. Systems exhibiting efficient energy transfer to $\mathbf{H g}$ or $\mathbf{Z n}$ may enable the ge! aration of intense stimulated emission in the near ultraviolet and visible spectral regions.

\section{KINETIC MODEL OF ULTRAVIOLET INVERSIONS IN HIGH-PRESSURE RARE GaS PLASMAS *}

The ultraviolet continua resulting from bound-free transitions of excited rare-gas dimers have been known for decades. ${ }^{74}$ Recently, observations of stimulated emission at about $1760 \AA$ from rare gas excited with relativistic electron beams have been reported: by Basov et al. ${ }^{64}$ from exciton levels in condensed xenon, and by Koehler et al. ${ }^{65}$ from expanding to the $1,3 \Sigma_{u}^{+} \rightarrow 1 \Sigma_{g}^{+}$continuum ${ }^{61}$ of $\mathrm{Xe}_{2}$ in high-pressure xenon gas. The ${ }^{3} \Sigma_{u}$ state represents the lowest excited moiecular a te and arises from the correlation of ${ }^{3} P_{2}$ and ${ }^{1} S_{0} \times$ on atoms. ${ }^{61}$

In the fowing we show that stimulated ultraviolet emission should be possible in the afterglow of a plasma consisting of initially cool electrons and cold gas atoms and ions. Under these conditions dissociative recombination of $\mathrm{Xe}_{2}^{+}$copiously generates excited atom $c$ species. ${ }^{75}$ This process is illustrated with the potential curves shown in Fig. 124. Here it is assumed that some (or one) of the dissociative recombination paths (i.e., the neutral repulsive curves which cross the bound molecular ionic $A^{2} \Sigma_{u}^{+}$state) terminate on atomic states which lead to the production of ${ }^{3} P_{2}$ atoms either directly or through collisional and radiative cascade from higher levels. In the example of argon, Frommhold and Biondi ${ }^{76}$ have shown that one of the dissociative paths is linked radiatively to the ${ }^{3} P_{2}$ levels by a fast $(\sim 100-n s e c)$ transition at $6965 \AA$. Both electrons and atoms can participate in the collisional processes. Slow ci-trons can be very important particularly in processes involving magnetic transitions and transitions requiring a change in the electronic spin state. ${ }^{*}$ The confluence of these processes rapidly collapses the excited atomic manifold to the ${ }^{3} P_{2}$ state. For simplicity in this calculation we consider only two excited atomic states as illustrated in Fig. 124: the ${ }^{3} P_{2}$ denoted by $\mathrm{Xe}^{*}(L)$; and some higher state, represented by $\mathrm{Xe}^{*}(U)$, which is linked (here we use a rate of $2 \times 10^{7} / \mathrm{sec}$ ) to the ${ }^{3} P_{2}$ level.

The kinetic processes that are incorporated in the calculational model are given below. ${ }^{\mathbf{7 8}}$ For our present investigation, we restrict ourselves to an examination of the plasma afterglow. Here the electrons are sufficiently cool that the rates of direct excitation and ionization of ground state atoms by electron collisions are negligible compared to other processes and therefore have been neglected.

$$
\begin{array}{ll}
\mathrm{e}^{-}+\mathrm{Xe}^{*} & \rightarrow \mathrm{e}^{-}+\mathrm{e}^{-}+\mathrm{Xe}^{+} \\
\mathrm{Xe}_{2}^{*}+\mathrm{Xe}_{2}^{*} & \rightarrow \mathrm{Xe}_{2}^{+}+\mathrm{e}^{-}+\mathrm{Xe}+\mathrm{Xe} \\
\mathrm{Xe}^{* *}+\mathrm{Xe} & \rightarrow \mathrm{Xe}_{2}^{+}+\mathrm{e}^{-} \\
\mathrm{Xe}^{+}+\mathrm{Xe}+\mathrm{Xe} \stackrel{\mathrm{X} \mathrm{Xe}_{2}^{+}+\mathrm{Xe}}{ }
\end{array}
$$

\footnotetext{
For the example of neon, see Ref. 77. In this case Phelps determined the relaxation rates of ${ }^{3} P_{1} \rightarrow{ }^{3} P_{2}$ through collisions with neon atoms and electrons. For the atomic collision partners the cross section is $\sim 10^{-19} \mathrm{~cm}^{2}$ while for the electrons the cross section is $\sim 10^{-13} \mathrm{~cm}^{2}$, which is essentially the theoretical maximum imposed by unitarity for $S$-wave scattering of thermal electrons.
}

\footnotetext{
*This work was partially supported by the Joint Services Electronics Programs (U.S. Army, U.S. Navy, and U.S. Air Force) under Contract DAAB07-71-C-0300.
} 


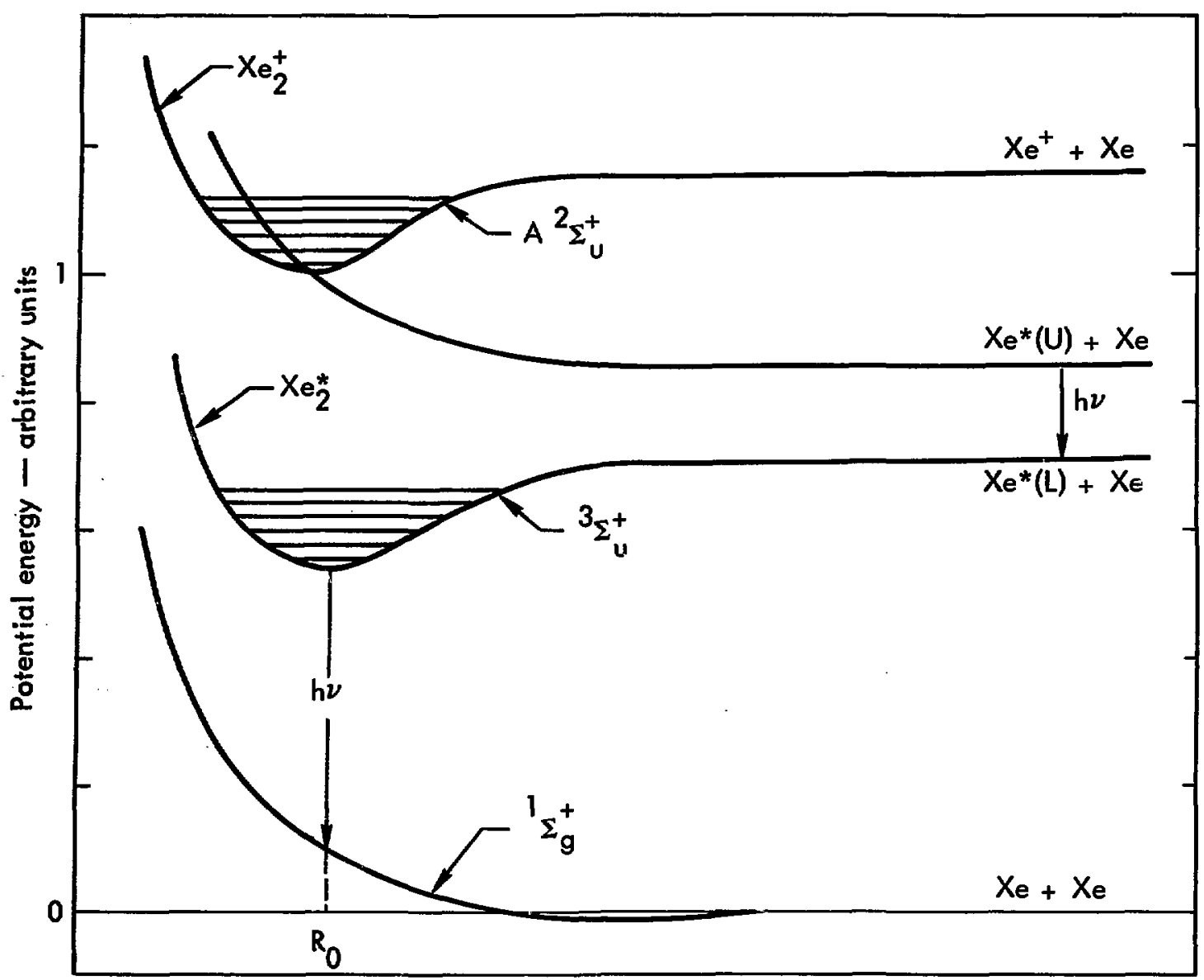

\section{Internuclear separation - R}

Fig. 124. Potential enengy curves as a function of internuclear separation $R$ of $\mathrm{Xe}_{2}$ and $\mathrm{Xe}_{2}^{+}$used in the colculational model reprewenting the $\mathrm{X}_{\mathrm{s}_{2}}$ molecular dimer ground state $\left({ }^{1} \Sigma_{g}^{+}\right)$, first excited state $\left({ }^{3} \Sigma_{u}^{+}\right)$, a representative excited state, and the lowest bound $\mathrm{Xe}_{2}^{+}$ionic level $\left(A^{2} \Sigma_{u}^{+}\right)$.

$$
\begin{array}{ll}
\mathrm{Xe}^{*}+\mathrm{Xe}+\mathrm{Xe} & \rightarrow \mathrm{Xe}_{2}^{*}+\mathrm{Xe} \\
\mathrm{Xe}_{2}^{+}+\mathrm{e}^{-} & \rightarrow \mathrm{Xe}+\mathrm{Xe} \\
\mathrm{Xe}_{2}^{*} & \rightarrow \gamma+\mathrm{Xe}+\mathrm{Xe} \\
\mathrm{Xe}^{* *}+\mathrm{Xe} & \rightarrow \mathrm{Xe}^{*}+\mathrm{Xe} \\
\mathrm{Xe}^{* *}+\mathrm{e}^{-} & \rightarrow \mathrm{Xe}^{*}+\mathrm{e}^{-} \\
\gamma+\mathrm{Xe}_{2}^{*} & \rightarrow \mathrm{Xe}_{2}^{+}+\mathrm{e}^{-}
\end{array}
$$

Reaction (1) illustrates the loss of excited xenon atoms (or dimers) through collisions with relatively low energy electrons. These low energy electrons can promote an excited xenon atom to either a higher level of excitation or to the ionized state. "Vojaczek ${ }^{79}$ has examined the electron collisional iouzation of the metastable ${ }^{3} P$ states of argon and obtained a cross section, using the classical method of Gryzinski, ${ }^{80}$ of $\sim 10^{-15} \mathrm{~cm}^{2}$ for this process. This large value is anticipated in light of the structural similarity between excited argon and the ground state of potassium. $t$ In evaluating the rate for reaction (1) we have convolved

\footnotetext{
Electrons with kinetic energies greater than about $3.81 \mathrm{eV}$ can lonize the lowest excited state of atomic xenon $\left({ }^{3} P_{2}\right)$.

$\dagger_{\text {Both systems are composed of a core and an outer } 4 s}$ electron. The peak cross section fo. onization of ground state potassium is $\sim 8.4 \times 10^{-16} \mathrm{~cm}^{2}$ as reported in Ref. 81 .
} 
this cross section with a Maxwellian electron-velocity distribution function $f(v)$ in the manner described by Kihara. ${ }^{82}$ The assumption of a Maxwellian distribution is justified since the rate of the dominant process tending to establish a Maxwellian distribution greatly exceeds the various inelastic rates. Notice that process (1) provides a significant cooling mechanism for the electron gas."

Reaction (2) illustrates Penning ionization of excited dimers (or corresponding atoms). These processes represent important loss mechanisms for excited ipecies and will clearly tend to depress the maximum density of $\mathrm{Xe}_{2}^{*}$ molecules. Although the cross section is not presently known for this reaction, the rate has been measured for the process 84

$$
\begin{aligned}
\mathrm{He}\left(2^{3} s_{1}\right)+\mathrm{He}\left(2^{3} s_{1}\right) & \rightarrow \mathrm{He}^{+}\left(1^{2} s_{1 / 2}\right) \\
& +\mathrm{He}\left(1^{1} s_{0}\right)+\mathrm{e}
\end{aligned}
$$

involving the helium metastable $\mathrm{He}\left(2^{3} S_{1}\right)$, giving a cross section of $\sim 10^{-14} \mathrm{~cm}^{2}$. We expect similarly large values to be operative $n$ the case of xenon and note that this process can provide a significant heating of the electron gas in the afterglow.

Ionic dimers can be formed through HornbeckMolnar processes 85 and three-body conversion of atomic ions as shown in reactions (3) and (4) respectively. Since the three-body mechanism dominates the molecular ion production for the gas densities under consideration, we have neglected the Hornbeck-Molnar process in our analysis. $f$ For xenon the rate for the three-body process (4) has been recently given ${ }^{87}$ as $(3.57 \pm 0.17) \times 10^{-31} \mathrm{~cm}^{6} / \mathrm{sec}$. Excited $\mathrm{Xe}_{2}^{*}$ dimer formation occurs by reaction (5) with a rate constant ${ }^{88} \beta=2.5 \times 10^{-32} \mathrm{~cm}^{6} / \mathrm{sec}$. At elevated temperatures the inverses of reactions (4) and (5) become important. These are incorporated in our model through the principle of detailed balance. The process of dissociative recombination (6) provides a rapid channel for the conversion of ions to excited atomic species. O'Malley ${ }^{89}$ has derived the temperature dependence of the dissociative recombination coefficient of the form

$$
\alpha\left(T_{e}, T_{\nu}\right)=C T_{e}^{-1 / 2}\left[1-\exp \left(-E_{v} / k T_{\nu}\right)\right],
$$

\footnotetext{
* It can be shown on the basis of sypical excitation cross sections and the considerations of detail balance (for example, see Ref. 83) that superelastic heating processes are small compared to this cooling process.

F For argon, this rate has been estimated as $\sim 10^{-11} \mathrm{~cm}^{3} / \mathrm{sec}$ (see Ref. 86). We further neglect the possible role of clustered ions such as $\mathrm{Xe}_{m}^{+}$, there $m>3$.
}

where $E_{v}$ is the vibrational energy level of the ion, $T_{e}$ and $T_{v}$ represent the electron and vibrational temperatures respectively, and $C$ is a constant evaluated to match the value ${ }^{75}$ at $T_{e}=T_{y}=300^{\circ} \mathrm{K}$.

Process (7) represents the bound-free ${ }^{3} \Sigma_{u}^{+} \rightarrow 1 \Sigma_{g}^{+}$ radiative transition shown in Fig. 124. Large spin-orbit coupling in high- $Z$ systems breaks the spin selection rule, and the radiative lifetime of $\mathrm{Xe}_{2}^{*}$ is believed to be $\sim 20 \times 10^{-9}$ sec. $^{65,78}$ The relaxation processes indicated by reactions (8) and (9) which collapse the manifold of atomic excited states to the ${ }^{3} \boldsymbol{P}_{\hat{\mathbf{Z}}}$ are desired, since this level correlates with the lowest bound molecular excited state ${ }^{61}\left({ }^{1} S_{0}+{ }^{3} F_{2} \leftrightarrow{ }^{3} \Sigma_{u}^{+}\right)$. Both electrons and atoms can effectively participate in these processes. ${ }^{77}$ Since the ${ }^{3} \Sigma_{i l}^{+} \rightarrow 1 \Sigma_{g}^{+}$continuum emission is sufficiently energetic to photoionize the excited dimers, reaction (10) represents the loss of $\mathrm{Xe}_{2}^{*}$ due to photoionization. Because of the Rydberg state structure of $\mathrm{Xe}_{2}^{*}$, we estimate the cross section by comparison with the value for ground state cesium 90 of $\sim 10^{-19} \mathrm{~cm}^{2}$.

Further basic assumptions involved in the calculation of this kinetic model are as follows. We assume only single ionization so that $n_{e}=\mathrm{Xe}_{2}^{+}+\mathrm{Xe}^{+}$, and we assume that gas kinetic, ion, and vibrational temperatures $\left(T_{g}, T_{+}, T_{v}\right)$ are equal because of the high gas density. We further assume that the time evolution of the excited plasma is sufficiently rapid that we may neglect the directed motion of the particles (spatial homogeneity is assumed). This assumption may not be valid in a laser-produced plasma subject to violent shock waves. We have also neglected bremsstrahlung cooling of the electron gas and heat diffusion. We include the three-body electron-ion recombination mechanism which becomes important as one considers sufficiently high electron densities. Here we take an effective $\alpha=10^{-10} \mathrm{~cm}^{3} / \mathrm{sec}$.

A set of equations was written to describe the time evolution of the electron, atomic ion, molecular ion, and excited state densities as well as the electron temperature $T_{e}$ and the gas temperature $T_{g}$. These resulting nonlinear differential equations were numerically integrated on a computer.

In Fig. 125 we illustrate the time dependence of the excited dimer density ( ${ }^{3} \Sigma_{u}^{+}$state) for various initial electron densities $n_{e}(r$. At time $t=0$, defined to occur at the end of the primary plasma production pulse, the excited state and molecular ion densities were chosen to be 0 and the initial electron and gas temperatures were set equal to $3 \mathrm{eV}$ and $0.04 \mathrm{eV}$ respectively. Notice that increasing $n_{e}(0)$ results initially in a larger excited dimer density (EDD) and that the peak EDD moves toward earlier times. One sees that there is an optimum value of $n_{e}(0)$ of 


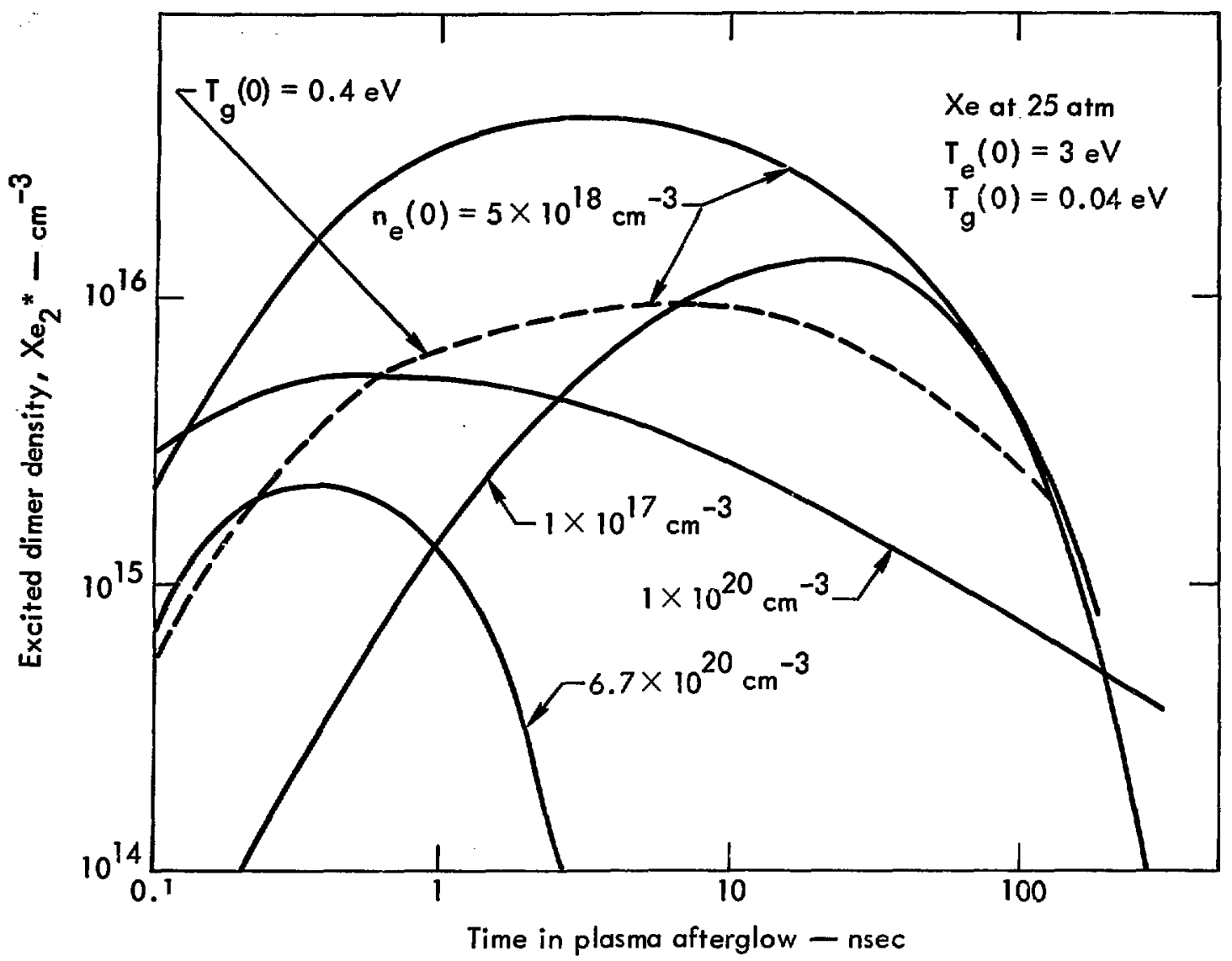

Fig. 125. Time evciution of the excited dimer density corresponding to the ${ }^{3} \Sigma_{u}^{+}$state for various values of initial electron density $n_{e}(\mathbf{0})$.

$\sim 5 \times 10^{18} \mathrm{~cm}^{-3}$ (corresponding to a fractional ionization of about $1 \%$ ). Further increase in $n_{e}(0)$ beyond the optimum results in a decreased peak EDD. This is easily explained since in the process of dissociative recombination, energy is added to the gas; as $n_{e}(0)$ is increased, a correspondingly greater amount of energy will be dsposited in the gas. The higher $T_{g}$ results in a decreased rate of production of the requisite excited atoms as well as the thermal dissociation of the excited dimers. The dashed line shows the effect of a higher initial gas temperature on the temporal characteristics of the EDD for $n_{e}(0)$ $=5 \times 10^{18} \mathrm{~cm}^{-3}$. We observe the expected result that a higher $T_{\mathrm{g}}$ gives a lower peak EDD. For the optimum $n_{e}(0)$ we fir $t_{t}$ that $0.4 \%$ of the initial energy is stored in the ${ }^{3} \Sigma_{u}^{+}$stare. To estimate the importance of the destructive processes (Eqs. (1) and (2)) the computer code was run with thest processes suppressid. For the case of $n_{e}(0)=5 \times 10^{18} \mathrm{~cm}^{-3}$ we find that the peak EDD shifts to later times and increzses by almost two orders of magnitude.
We note that since the stimulated-emission cross section 72 of $\mathrm{Xe}_{2}^{*}$ is $\sim 3.5 \times 10^{-18} \mathrm{~cm}^{2}$, an excited state density of $10^{17} \mathrm{~cm}^{-3}$ produces an optical gain coefficient of approximately $0.4 \mathrm{~cm}^{-1}$. For this excited-state density the loss due to photoionization is small; however, the optical loss due to ground state absorption ${ }^{91}$ could be quite large. This loss, which is strongly temperature-dependent, is presently being incorporated in our model.

Figure 126 illustrates a comparison of these calculations with the measured intensity of the $1700-\AA$ continuum emission from xenon. Experimentally, ${ }^{78}$ the plasma is created by means of the passage of a pulsed relativistic electron beam (of duration $50 \mathrm{nsec}$ ) through gaseous xenon at a pressure of $1.1 \mathrm{~atm}$. The experimental result (solid line) shows two distinct peaks. The first is due primarily to direci pumping processes, while the second peak we feel is the result of the recombinational process previously described. Here the initial excited-state densities (at $t=0$ ) are estive ?d using the ratio of the leights of the two 
experimental peaks. A lower bound for $n_{e}(0)=5$ $\times 10^{15} \mathrm{~cm}^{-3}$ is obtained by balancing the charged particle production, using tabu'. ited values 92 for the $d E / d x$ of our beam, with recombinational losses. Notice that for $n_{e}(0)=2 \times 10^{16} \mathrm{~cm}^{-3}$ the agreement between theory and experiment is good.
Similar agreement has also been obtained for $i \mathrm{ir}$ and $\mathrm{Kr}$ using the appropriate rate constants. In comparing all of these gases, we find that our results are consistent with a cross section for reaction (2) of $\sim 10^{-14} \mathrm{~cm}^{2}$. Any substantially different value for this cross section results in qualitative disagreement.

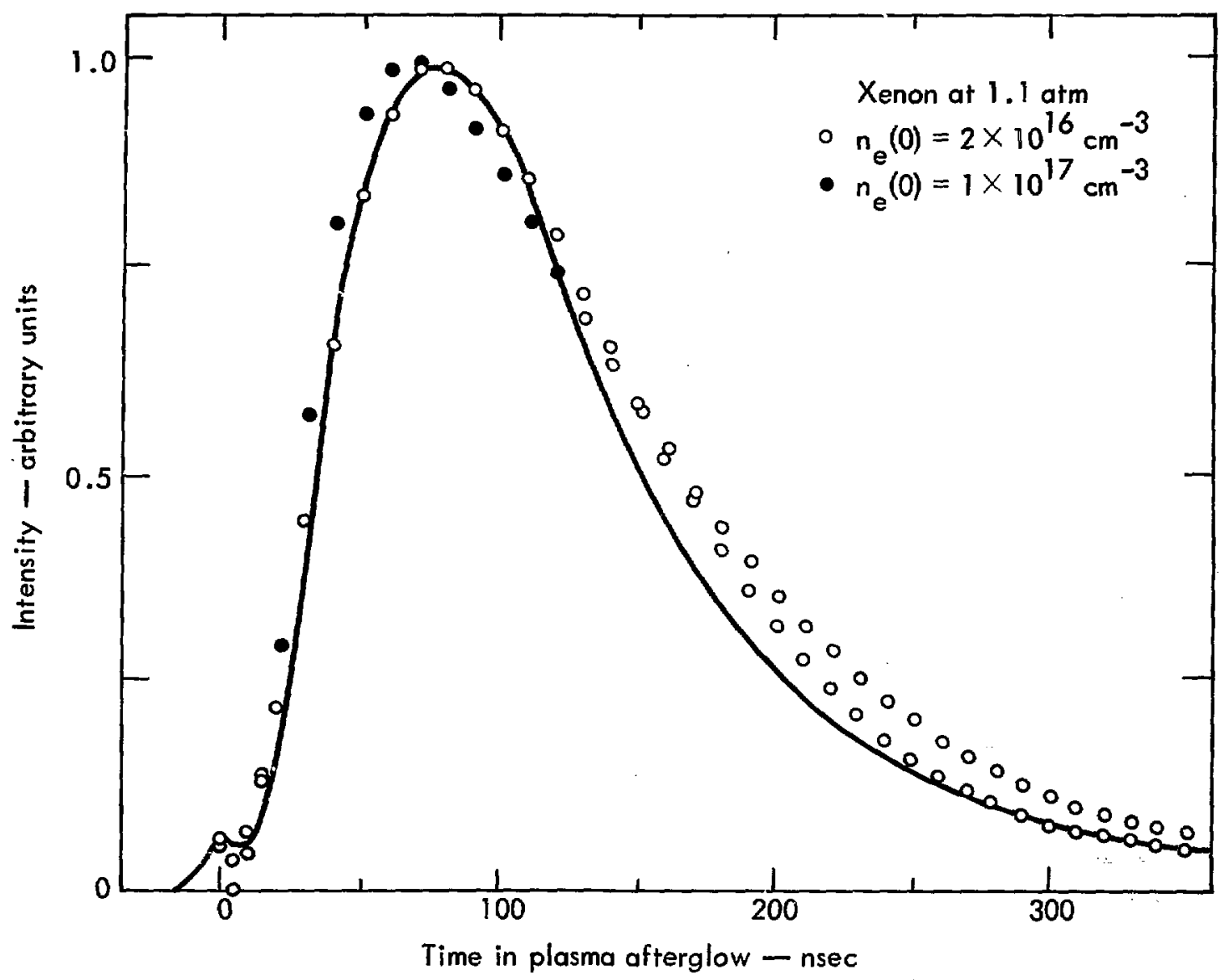

Fig. 126. Comparison between the temporal characteristics of the observed $1700-\AA$ continuum emission and our theoretical predictions. The data are normalized to the second peak.

\section{HIGH-RESOLUTION INFRARED SPECTROSCOPY}

The $\mathrm{CO}_{2}$ laser is of interest to the laser fusion effort because of its high power output, high efficiency, and simplicity. Short pulsewidths (subnanosecond) are desirable for laser fusion applications. Si:ce the limiting pulsewidth of a $\mathrm{CO}_{2}$ laser amplifier is inversely proportional to its bandwid'h for linear amplification, we have been studying the $\mathrm{CO}_{2}$ amplifier bandwidth by means of high-resolution infrared spectroscopy.

The primary contribution to the bandwidth of high-power, high-pressure $\left.\mathrm{Pl}_{1} \mathrm{~atm}\right) \mathrm{CO}_{2}$ amplifiers is pressure broadening due to phase-interrupting collisions between the $\mathrm{CO}_{2}$ molecules and other gases 


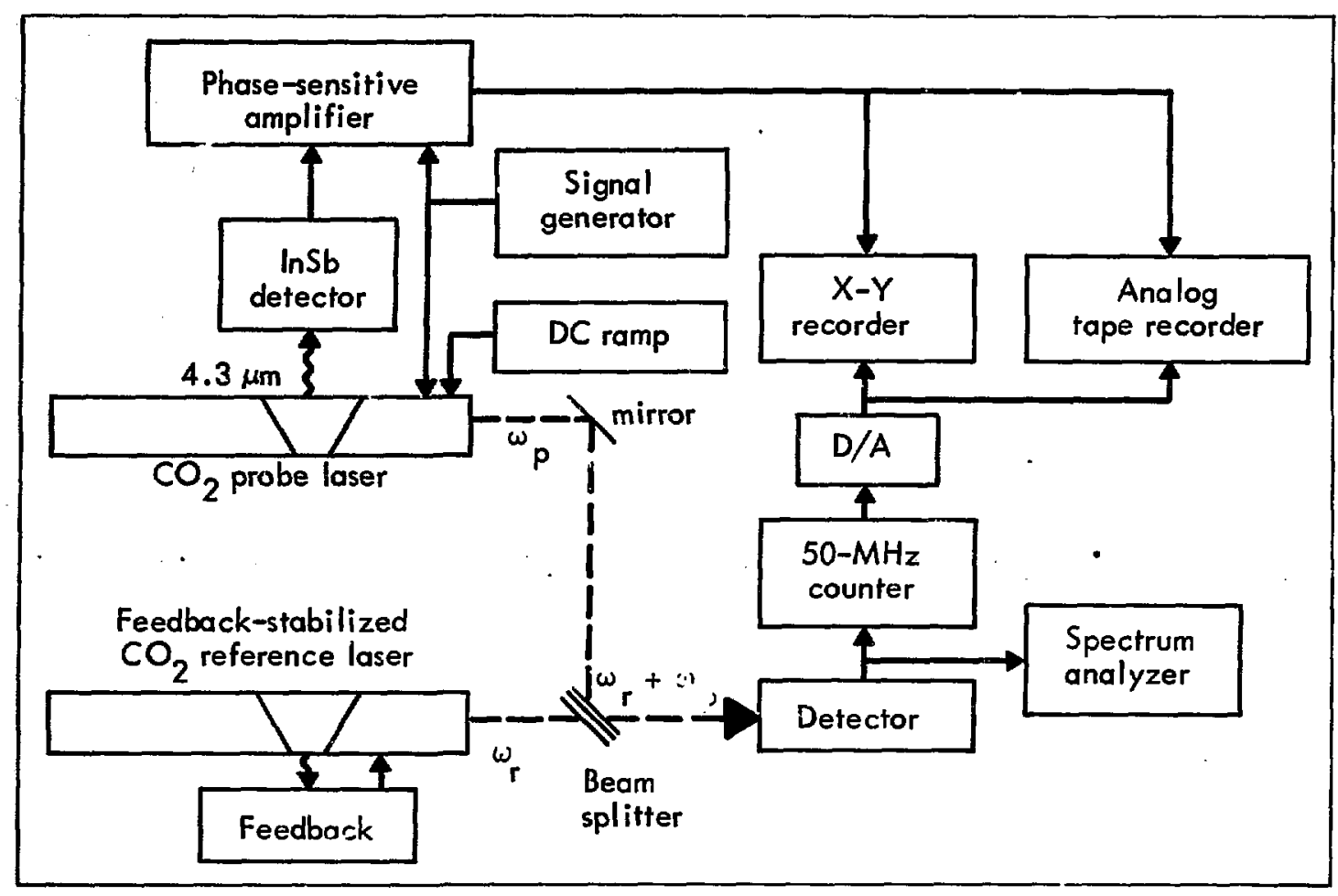

Fig. 127. Schematic of the high-resolution infrared spectrometer.

in the amplifier such as $\mathrm{He}, \mathrm{N}_{2}, \mathrm{H}_{2}$, and Xe. A detailed study of the collision dynamics not only provides the pressure broadening information necessary for determining the bandwidth of the $\mathrm{CO}_{2}$ amplifier, but can also provide an understanding of rotational relaxation processes in these amplifiers. This latter information is essential in determining the total energy that can be extracted from the amplifier in a single pulse. ${ }^{93}$ Since quantitative data on $\mathrm{CO}_{2}$ pressure broadening is scarce, ${ }^{93-95}$ we have been experimentally investigating the broadening coefficients for $\mathrm{CO}_{2}$ in collision with the perturbers $\mathrm{CO}_{2}, \mathrm{H}_{2}, \mathrm{D}_{2},{ }^{3} \mathrm{He}$, ${ }^{7} \mathrm{He}, \mathrm{N}_{2}, \mathrm{O}_{2}, \mathrm{CO}, \mathrm{NO}, \mathrm{Ar}, \mathrm{Ne}, \mathrm{Kr}, \mathrm{Xe}, \mathrm{NH}_{3}$, and $\mathrm{N}_{2} \mathrm{O}$.

All of these measurements have been made on the high-resolution infrared spectrometer described in previous reports. ${ }^{96} \mathrm{~A}$ schematic of the spectrometer is shown in Fig. 127. Two stable, single-line, single-mode $\mathrm{CO}_{2}$ lasers form the heart of the spectrometer. A precision grating on each laser allows individual selection of any of the $\mathrm{CO}_{2}$ 9. or $10-\mu \mathrm{m}$ rotational-vibrational transitions, and a piezoeleciric tuner (PZT) can be used to scan each line across its gain width. Both lasers are equipped with intracavity Brewster-window absorption cells (Fig. 128). In one laser (the reference), the absorption cell is filled with $\mathrm{CO}_{2}$, and the laser is used as a local oscillator stabilized to the $\mathrm{CO}_{2}$ line center. The absorption cell in the other laser (the probe) contains a mixture of $\mathrm{CO}_{2}$ and a perturbing gas. The $4.3-\mu \mathrm{m}$ fluorescence from the $\mathrm{CO}_{2}$ gas $^{97}$ subjected to the intense standing-wave electric field of the laser cavity is detected synchronously by applying a small sinusoidal modulation to the PZT upon which the laser output mirror is mounted.

Data from the probe laser is recorded on magnetic tape along with the heterodyne signal obtained from the probe and reference oscillators as the former is swept $\pm 10 \mathrm{MHz}$ with respect to the $\mathrm{CO}_{2}$ line center by applying a dc ramp voltage to its PZT mirror holder. After the data is recorded it is computationally analyzed by first integrating the iluorescence signal and then fitting the integral with a nonlinear least-squares routine. The fitting curve involves 1 narrow Lorenztian component, superimposed on a second-order background signal which arises from the convolution of the laser power variation and the Doppler profile cf :he absorbing transition.

Th: lineshape, containing the collision information, was found is be Lorentzian, within experimental error, in all cases. A typical result exhibiting the 


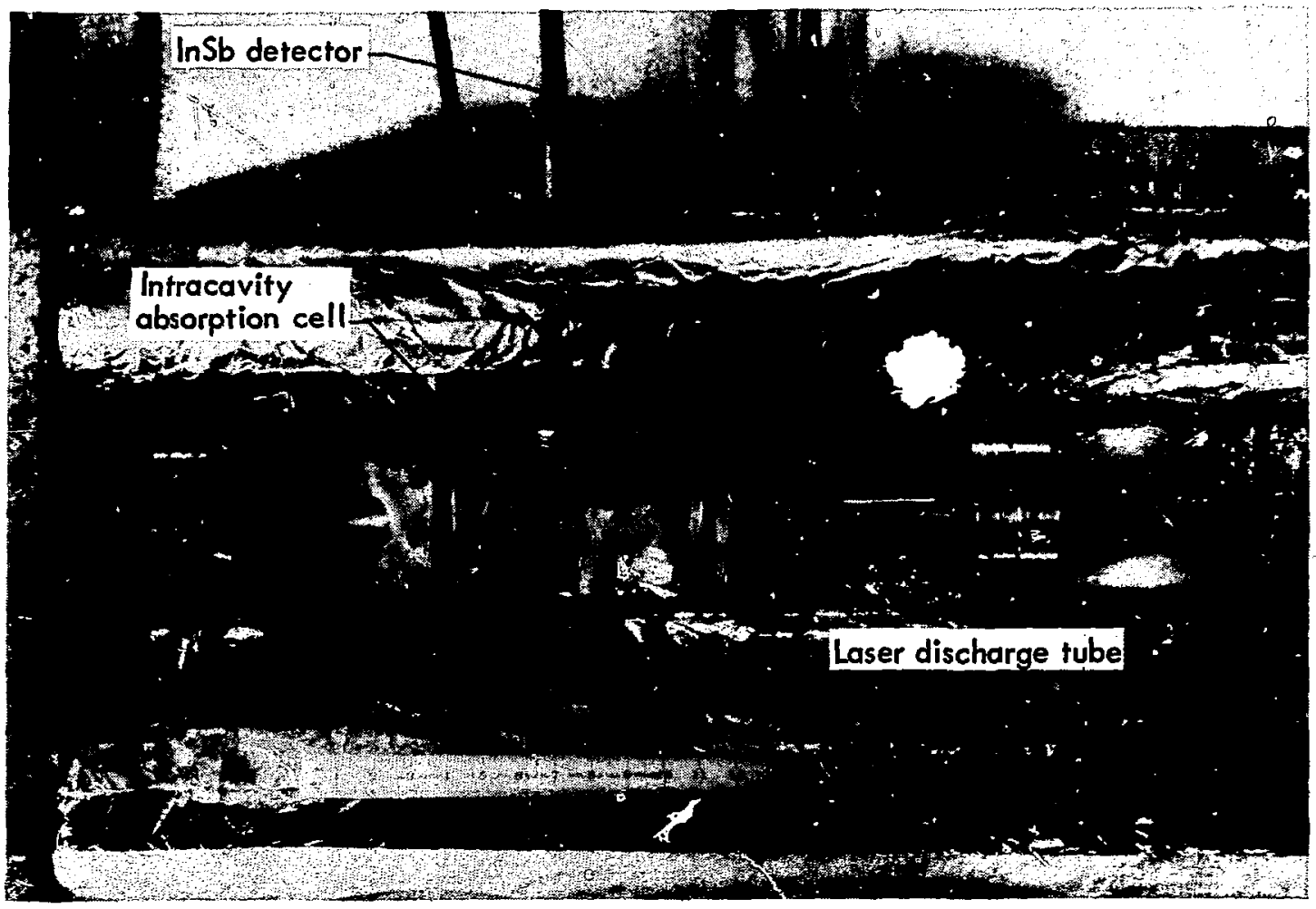

Fig. 128. The InSb detector, which is sensitive to the $4.3-\mu \mathrm{m} \mathrm{CO}$, fluorescence, is on top of the Brewster-window intracavity absorption cell. The inser discharge tube is to the right.

Table 15. Stummary of $\mathrm{CO}_{2}$ pressure-broadaning resulte. The measured pressurebroadening coefficients have been converted to cross sections by means of kinetic theory. For comparison, some previous cross-section results are presented-

\begin{tabular}{|c|c|c|c|c|}
\hline \multirow{2}{*}{$\begin{array}{l}\text { Collision } \\
\text { partmess }\end{array}$} & \multicolumn{2}{|c|}{ Pressure brosdening coefficient (MHz/Torr) } & \multicolumn{2}{|c|}{ Cross section $\left(10^{-14} \mathrm{~cm}^{2}\right)$} \\
\hline & This work & Freed and Jrvan ${ }^{97}$ & This work & Ducsit and Hoogs \\
\hline 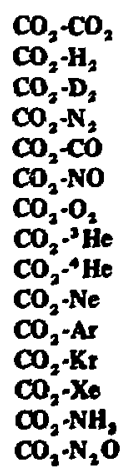 & $\begin{array}{r}7.1 \\
11.0 \\
7.8 \\
6.6 \\
6.1 \\
5.3 \\
5.5 \\
5 . \\
5.2 \\
5.0 \\
5.0 \\
5.1 \\
4.9 \\
8.1 \\
7.5\end{array}$ & $\begin{array}{l}7.6 \\
- \\
= \\
= \\
= \\
= \\
= \\
= \\
= \\
=\end{array}$ & $\begin{array}{l}1.3 \\
0.60 \\
0.59 \\
1.1 \\
0.99 \\
1.0 \\
0.92 \\
0.36 \\
0.40 \\
0.73 \\
0.90 \\
1.1 \\
1.1 \\
1.1 \\
1.4\end{array}$ & $\begin{array}{c}1.30 \\
- \\
\overline{0.87} \\
\overline{-} \\
\overline{0.84} \\
\overline{0.37} \\
= \\
= \\
= \\
= \\
=\end{array}$ \\
\hline
\end{tabular}




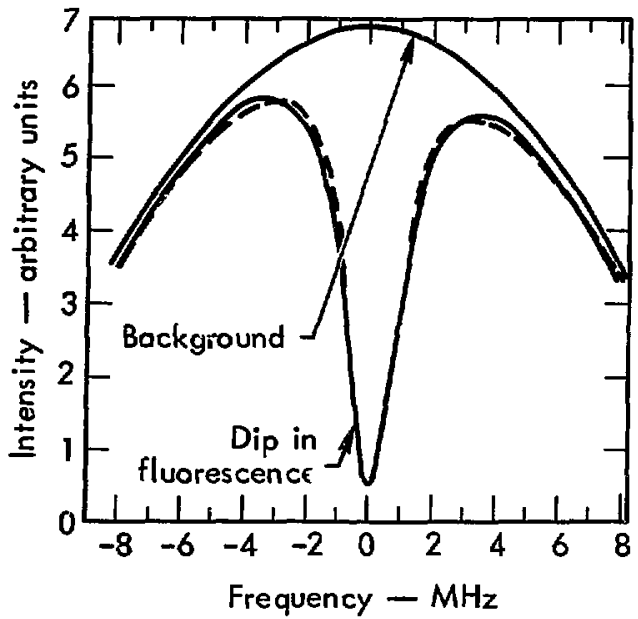

Fig. 129. Measured fluorescence dip in $\mathrm{CO}_{2}$ (lower solid curve) and a computer fit to the data (dashed curve). Upper curve is background.

characteristic dip in the fluorescence is depicted in Fig. 129 along with the computer fit to the data. The linewidth $\Delta \nu$ (FWHM) of the Lorentzian component was obtained at several different pressures for the various perturbers investigated while using a constant laser intensity. All measurements were made with the $\mathrm{CO}_{2} \quad$ 10.6- $\mu \mathrm{m} P(20)$ line.

The results of a typical investigation of selfbroadening in $\mathrm{CO}_{2}$ are plotted in Fig. 130. It is evident from the figure that the data points are fitted extremely well by a straight line whose slope is the

\section{Gas Laser Technology}

\section{Direct Electron-Beam Lasers}

Recont success with the $\mathrm{Xe}_{2}^{*}$ and $\mathrm{Kr}_{2}^{*}$ uv lasers indicate that excimer systems are prime candidates for new lasers which span the visible and near-uv spectral regions. To extend the scope of recent excimer laser experiments and to investigate the properties of lasers directly excited by relativistic beams, two high-current-density electron beam sources are being established as laser excitation facilities. These MEG (megavolt electron gun) diodes can provide rectangular beams over areas in excess of $80 \mathrm{~cm}^{2}$ at $1.2 \mathrm{MeV}$ with current densities of several $\mathbf{k A} / \mathrm{cm}^{2}$ in a 50-nsec pulse.

The first MEG with a $2-b y-10-\mathrm{cm} 1-\mathrm{MeV}$ beam will be used to study the scaling properties of the uv excimer lasers and the constraints imposed by optical pressure broadening coefficient. For $\mathrm{CO}_{2}$, the slope is 7.1 MHz/Torr. The linewidth and pressure for $\mathrm{CO}_{2}$ in collision with other gases are also found to be linearly related, and these results are tabulated in Table 15.

The primary source of error is in our data acquisition system, and this limits the accuracy of our results to $\pm 5 \%$.

Current work includes an extension of this investigation to other $\mathrm{CO}_{2}$ rotational lines, as well as an investigation of the power dependence of the broadening coefficient.

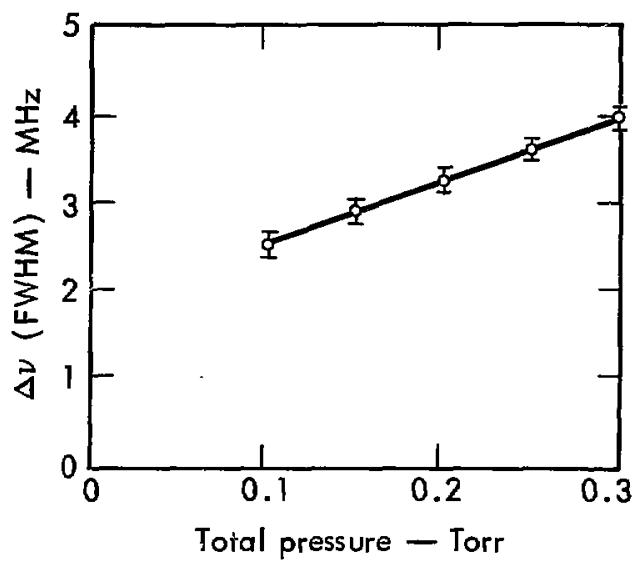

Fig. 130. Linewidth $(\Delta v)$ of the $10^{\circ} \mathrm{O} \rightarrow \mathrm{00^{ \circ }} 1 \mathrm{CO}_{2}$ tran sition vs pressure for $\mathrm{CO}_{2}$ self-broadening. The slope of the data points is $7.1 \mathrm{MHz} /$ Torr. materials, in order to determine the utility of vacuum-uv lasers and vacuum-uv spontaneous emission sources for laser excitation. The second MEG, which will provide a $2 \cdot$ by $-50-\mathrm{cm}$ beam, will be applied to investigate traveling-wave excitation techniques and visible excimer systems which appear promising.

An important aspect of schemes involving direct injection of relativistic beams is the need to control and characterize the beam properties in the medium of interest. Toward this end, experiments to generate, propagate, and extract uniform rectangular beams are in progress at LLL utilizing an existing diode electron gun. Uniform beams 2 by $10 \mathrm{~cm}$ with greater than $90 \%$ uniformity at $1 \mathrm{MeV}$ and $3 \mathrm{kA} / \mathrm{cm}^{2}$ have been generated by employing a new convex graphite cathode 
which cortrols and utilizes shank emission. With proper selection of anode material and thickness we have achieved arode lifetimes in excess of 20 shots at $3.5 \mathrm{kA} / \mathrm{cm}^{2}$. Wu are continuing efforts to transport the beam to a laser chamber using vacuum coax and neutral-gas drift tubes.

\section{Electron-Beam Sustainer Lasers}

Investigation of electron-beam sustainer laser systems will be accomplished by establishing two large-aperture electron gun facilities to encompass the e-beam sustainer parameter space. Both electron guns will be capable of a maximum emission area of 10 by $100 \mathrm{~cm}$. One system, consisting of a high-current-density $\left(\sim 10-\mathrm{A} / \mathrm{cm}^{2}, 300-\mathrm{keV}\right)$ cold cathode diode, is suitable for applications where a fast $(<1-\mu s e c)$ excitation pulse is required. The second system, consisting of a thermionic tetrode, will possess extreme flexibility in current and pulse length variations.

An obvious application of the above sustainer systems is to pump dipole-forbidden vibrational transitions in which energy extraction is accomplished by a nonlinear process. Of all the possible candidates, an anti-Stokes amplifier or dc-field-induced oscillator utilizing the $\mathrm{H}_{2}$ molecule is the most attractive from the standpoint of transition frequency. Preliminary estimates for the achievable inversion in an e-beam-pumped $\mathrm{H}_{2}$ medium are uncertain due to the

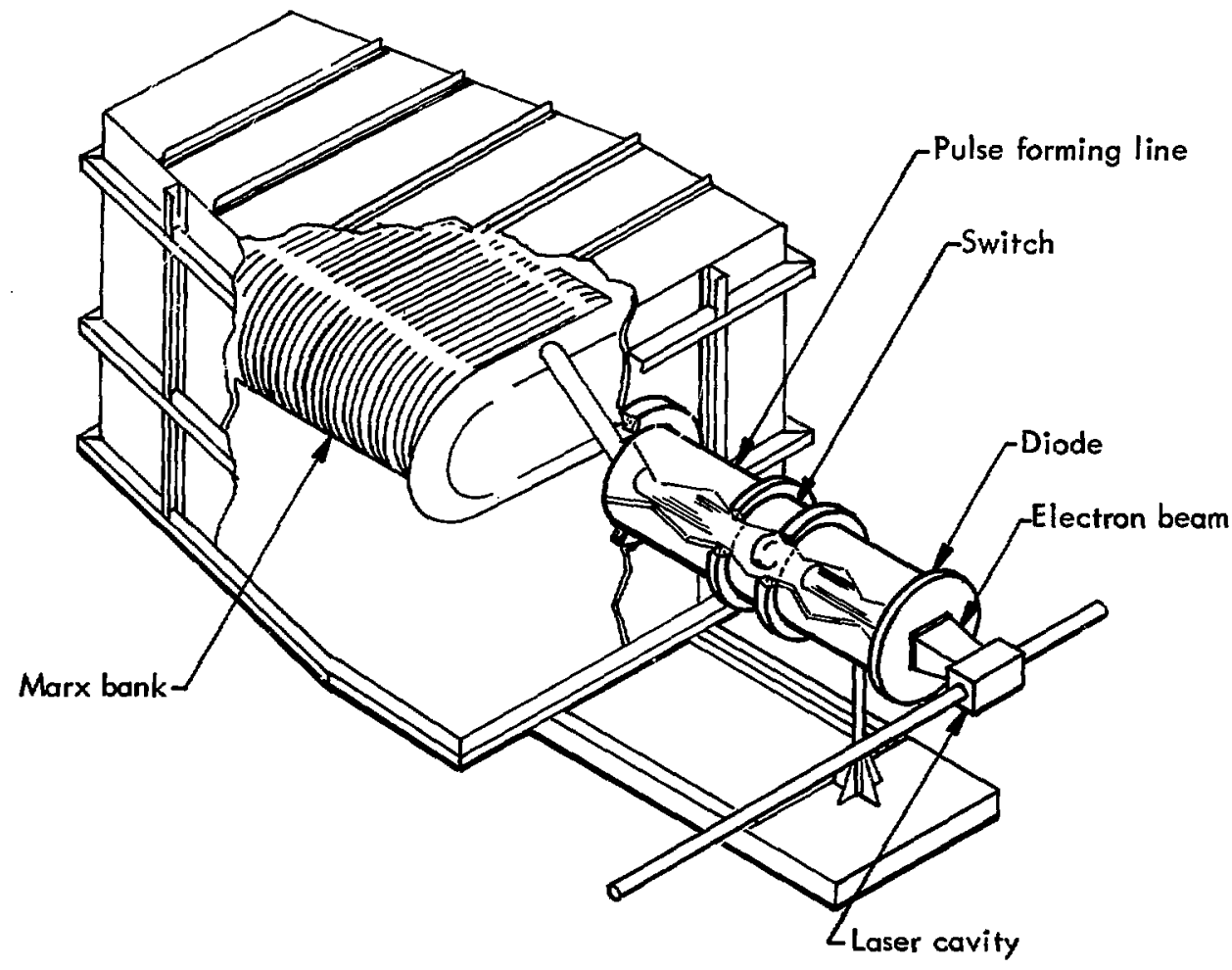

Fig. 131. Cutaway view of the MEG-I electron beam generator. 


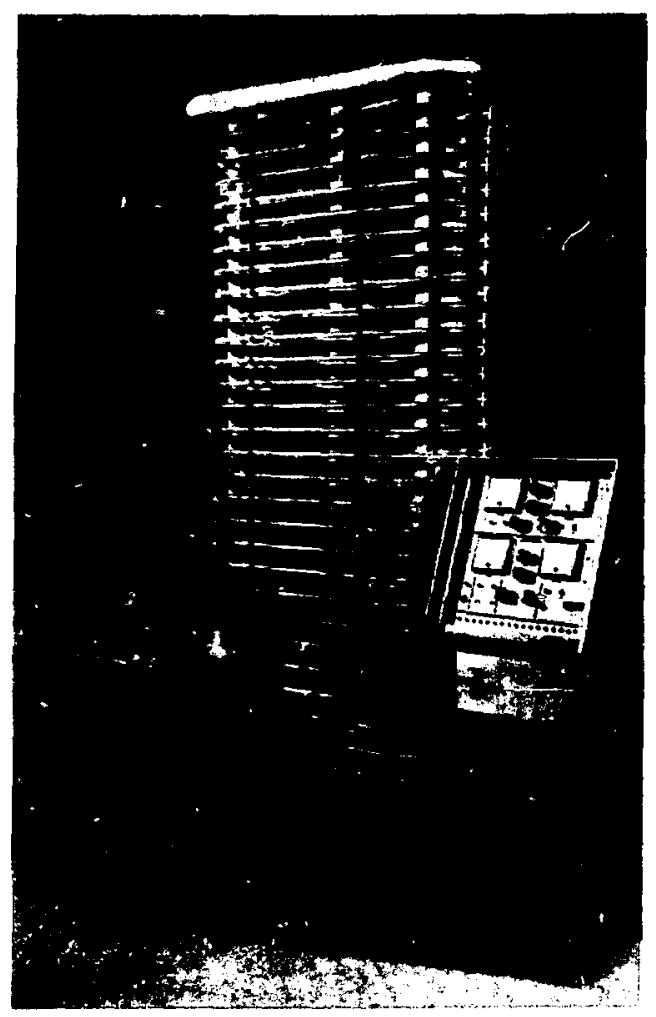

Fin. 132. Marx bask for the MSG-1 machine.

sbence of experimental information conceming the near-resonant $\boldsymbol{V}-\boldsymbol{V}$ relaxation rates. Experiments to determine the temperature dependence of these rates will allow the design of the optimum configuration to pump the largest inversion in $\mathbf{H}_{2}$.

\section{Eiectron-Beam Photolytic Sources}

Of the three lasers presently being developed for pellet irradiation, two (Nd:glas and iodine) are flahlamp-pumped systems and therefore show poor efficiencies. If an efficient photolytic source could be spectrally matched to the pump bands of these two systems, the resultant impact on the design of large lasers would be tremendous.

Potentially efficient photolytic sources are the various scintillators and fluorescent agents which produce narrow emission bands when irraliated by electron beams. To investigate their spplicability for Leer pumping, a Febetron 706 (0.6 MeV, 3 neec) electron gun will be used to determine the fluoressent yields of various geous, liquid, and solid fluors under electron irrediation.

\section{Excitation Source Development}

The technology of pulsed high-current relativistic electron beams has been developed primarily for simulation of weapons effects. Its application to the pumping of lasers requires extension of the technology in specific areas.

An electron beam generator, MEG-I, is currently being installed. Nominai characteristics are I MeV, $100 \mathrm{kA}$, and $50 \mathrm{nsec}$ pulse width (FW/M). Jitter from trigger to output is $<8$ nsec; reproducibility is 295\%. A cutaway view of the machine is shown in Fig. 131. The Manx bank is shown in Fig. 132.

The design of the diode of the MEG-I machine involves several factors. The cathode-anode gap spacing is determined to be $\sim 1 \mathrm{~cm}$ by Child's law (i.e., space-charge-limited operation). The impedance is then given by

$$
Z=\left(\frac{433}{V^{1 / 2}}\right)\left(\frac{d_{\text {eff }}^{2}}{A_{\text {enodt }}}\right),
$$

where $V$ is in $M V$ and $Z$ in $\Omega$. The factor $d_{e f f}$ includes the effects of gap closure due to platma motion generated by cathode whisker explosions (traveling with a velocity $\approx 2 \times 10^{6} \mathrm{~cm} / \mathrm{sec}$ ) and plasma motion emanating from the anode (velocity $\approx(2-6)$ $X 10^{6} \mathrm{~cm} / \mathrm{sec}$ ).

At a 1.cm cathode-anode gap both end and shank emission can be important. Figure 133 shows the regions of operation, which depend on the apect ratio of gap to cathode width. With the small apect ratio a planar zeometry is approximated, and end emission dominates, producing a uriform beam. With a large epect ratio a needle geometry is approximated, and shank emission dominates, producing a hollow beam. At intermediate spacings, both end and shank emission occur, producing a uniform centrd beam with a halo separated (as dictated by space charge at the comers) by a low density region; the energy is approximately equally distributed in the central region and the halo (Fig. 134). In all caxes, the J $\times$ B self-force causes curvature of the trajectories.

To generate a uniform beam, one can either attempt to suppress the shank emission by oiling and controlling ahank fields, or contour the cathode in order to fold the end and ahank emission together. This effectively reduces the space charge buildup at the comers, as shown in Fig. 135.

To get some operating data before the arrivd of the MEG-I, preliminary experiments were performed on an existing machine with a 2-by-10-cm cross rection and a current density of $3 \mathrm{kA} / \mathrm{cm}^{2}$. The results indicate that the nonuniformity at the mode plane was less than 8\%, and that a low eloctron transwerse enery was obtained. 


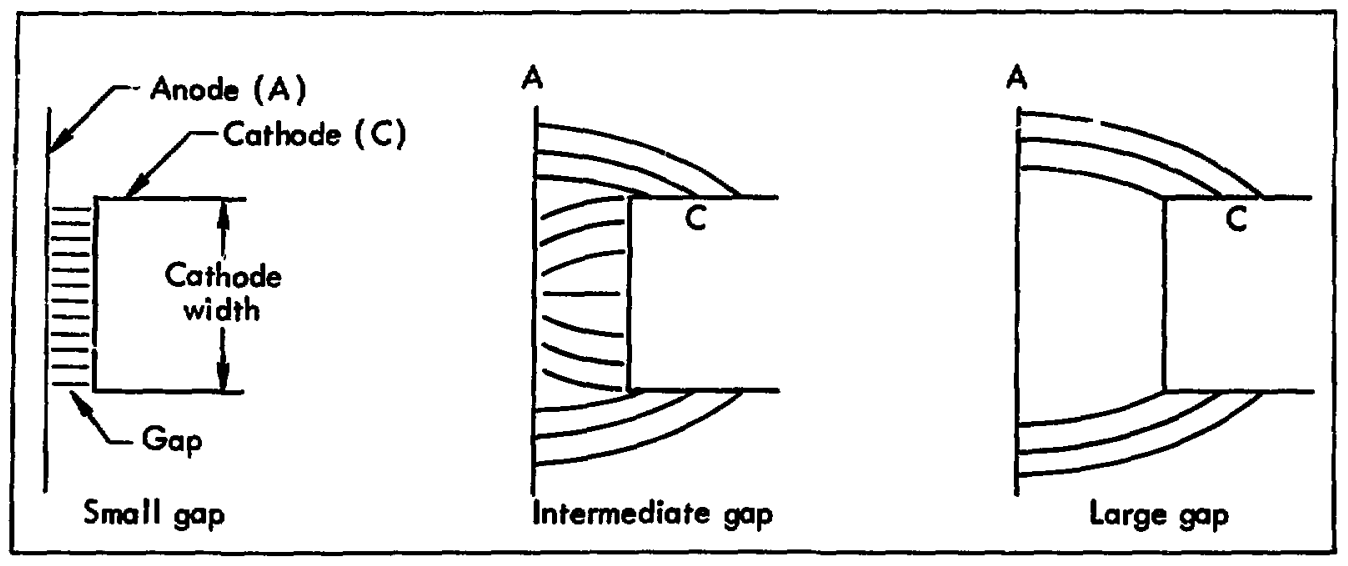

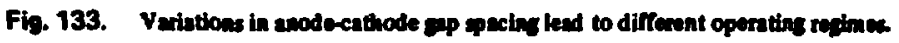

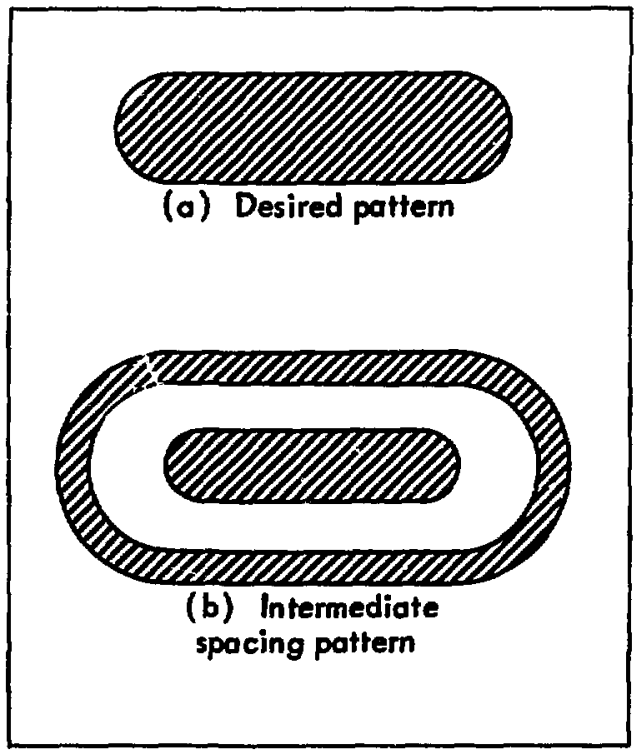

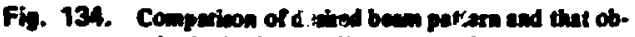

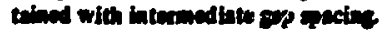

Anode survival is a critical factor in the performance of these machires since each anode frilure necessitates the moving of the maphine and cleaning of the diode. Anode failures are genersilly attributed to poor rise and fil times and postpulse ringing. Our preliminary experiments were encouraging since we were able to obtain at least 20 shots before mode rupture.

The next problem was how to efficiently deliver the beam some $30 \mathrm{~cm}$ from the machine to a lacer

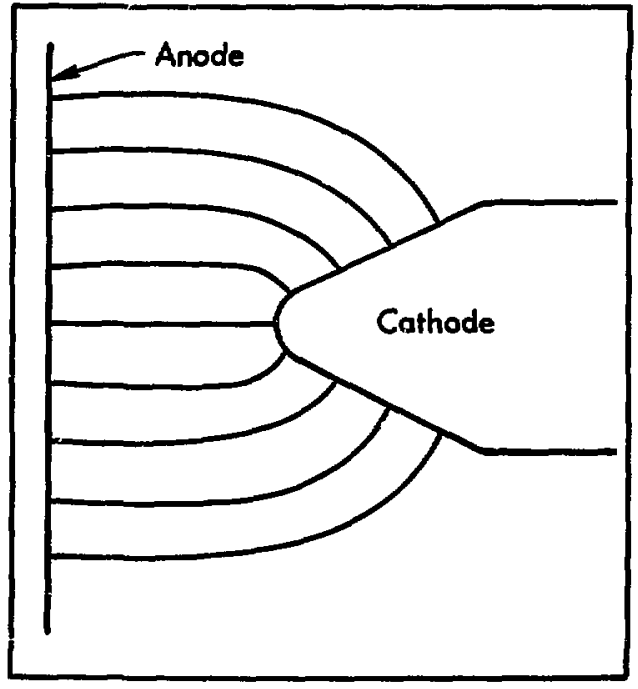

Fig. 135. Conlownd cathode wad to inprove electron tem pattin.

chamber. Two solutions were envisioned: transport in a vacuum coax by extending the cathode shank inside a "hat," or transporting the beam in neutrl ga, i.e., $\mathrm{N}_{2}$ at $\sim 1$ Torr. The former approach has the problem that ahank emission to the coax walls is a lose, and moreover no one had ever tried a rectangular geometry. The latter approach has the drawback that unless the electrons have low relative transverse energy in a rectengular guide, spiraling cnused by the electron 
angular momentum forces the beam to exit the guide as round beam having a diameter equal to the smallest guide dimension.

The vacuum coax configuration was tested by inserting a dummy conducting structure into the diode. The results confirmed that the shank emission could be minimized or eliminated.

The neutral-gas drift technique was also tested. A $3-\mathrm{kA} / \mathrm{cm}^{2}$ beam generated at the anode plane was drifted with $80-90 \%$ efficiency in a distance of $30 \mathrm{~cm}$ while maintaining the high degree of beam uniformity. This efficiency is adequate since MEG-1 has extra current capability. A final stage of drift through $2 \mathrm{~cm}$ of room air may be utilized to completely isolate the experiment from the machine.

Preliminary design is under way for a $2-\mathrm{m}$, traveling-wave, transversely pumped laser system which will utilize a 500-keV electron beam. A fast Marx bank, stripline, peaking switches, and a low-inductance diode are being developed.

\section{$\mathrm{CO}_{2}$ Laser System}

\section{INTRODUCTION}

The $\mathrm{CO}_{2}$ leser system program at $\mathrm{LLL}$ has two main objectives. The first is to establish $\mathrm{CO}_{2}$ system scaling parameters through analytical and computational modeling supported by experimental measurements. The second is to define and construct a small state-of-the-art $\mathrm{CO}_{2}$ system with which we can test design concepts and perform plasma physics experiments.

Since we are undertaking the constnuction of a research tool for the study of target plasmas, we feel that the quality of the optical pulse is as important *s the amount of energy in it. With high optical quality the pulse energy needed to carry out meaningful sesearch is only tens of joules. In view of the intended use of our experimental $\mathrm{CO}_{2}$ system, we have imposed several requirements on its design. (1) Reliability of the system must be high. (2) Pulse repetition rate should be at least 1 nulse/min, so that data can be acquired in a reasonable time span. (3) Preventive maintenance to maintain system reliability should not be required more often than every 1000 shots. (4) Electrical noise must be minimized within economic limits.

After considering several alternatives, we have concluded that we can minimize costs in money and time by narrowly specifying the devices we need and then purchasing them from vendors who sell similar units. The components we have chosen for the $\mathrm{CO}_{2}$ laser system meet all the above requirements.

\section{SYSTEM DESIGN}

The design of the system is simplified by the constraint that, insofar as possible, amplifiers and other components be purchased from vendors. After evaluating a number of amplifiers, two types were selected: (1) uv-preionized, and (2) cold-cathode, e-beam-sustained. The design problem is therefore reduced to choosing amplifier apertures, gains, amplifier positions, isolators, and beam guiding optics with the constraint that fluences be kept lower than the thresholds for medium breakdown and eptics damage. A computer program for simulating laser systems (BOBG) has been used extensively to calculate the spatial shupe of the optical pulse as it passes through hypothetical systems. BOBG uses a primitive rate equation model to describe the amplifying media; nevertheless, most of our work to date suggests that, within narrow limits, this simple theory of a saturating amplifier is a fair predictor of $\mathrm{CO}_{2}$ amplifier behavior and that consequently BOBG can provide crude but reliable system simulations. Among the inputs to BOBG are the transverse gain profiles of the amplifiers, their small-signal gains, and their effective saturation energies, $E_{s}$. The transverse small-signal profiles of our amplifiers have been measured and small-signal gains have been calculated using a family of four- and five-temperature models for $\mathrm{CO}_{2}-\mathrm{N}_{2}-\mathrm{He}$ media. Rheault et al. ${ }^{98}$ find that $E_{s}=155 \mathrm{~mJ} / \mathrm{cm}^{2}( \pm 10 \%)$ for 1-nsec pulses in TEA amplifiers, while our own preliminary measurements suggest that $E_{s}$ is between 120 and $175 \mathrm{~mJ} / \mathrm{cm}^{2}$ for 2-nsec pulses containing six rotational lines. In addition, we know that $E_{s}$ will increase with increasitig pressure, at least doubling at 2 atm. In predicting the performance of our system with BOBG we have used an effective saturation energy density of $160 \mathrm{~mJ} / \mathrm{cm}^{2}$-atm and rneasured small-signal gains. Figure 136 is a schematic diagram of the $\mathrm{CO}_{2}$ 

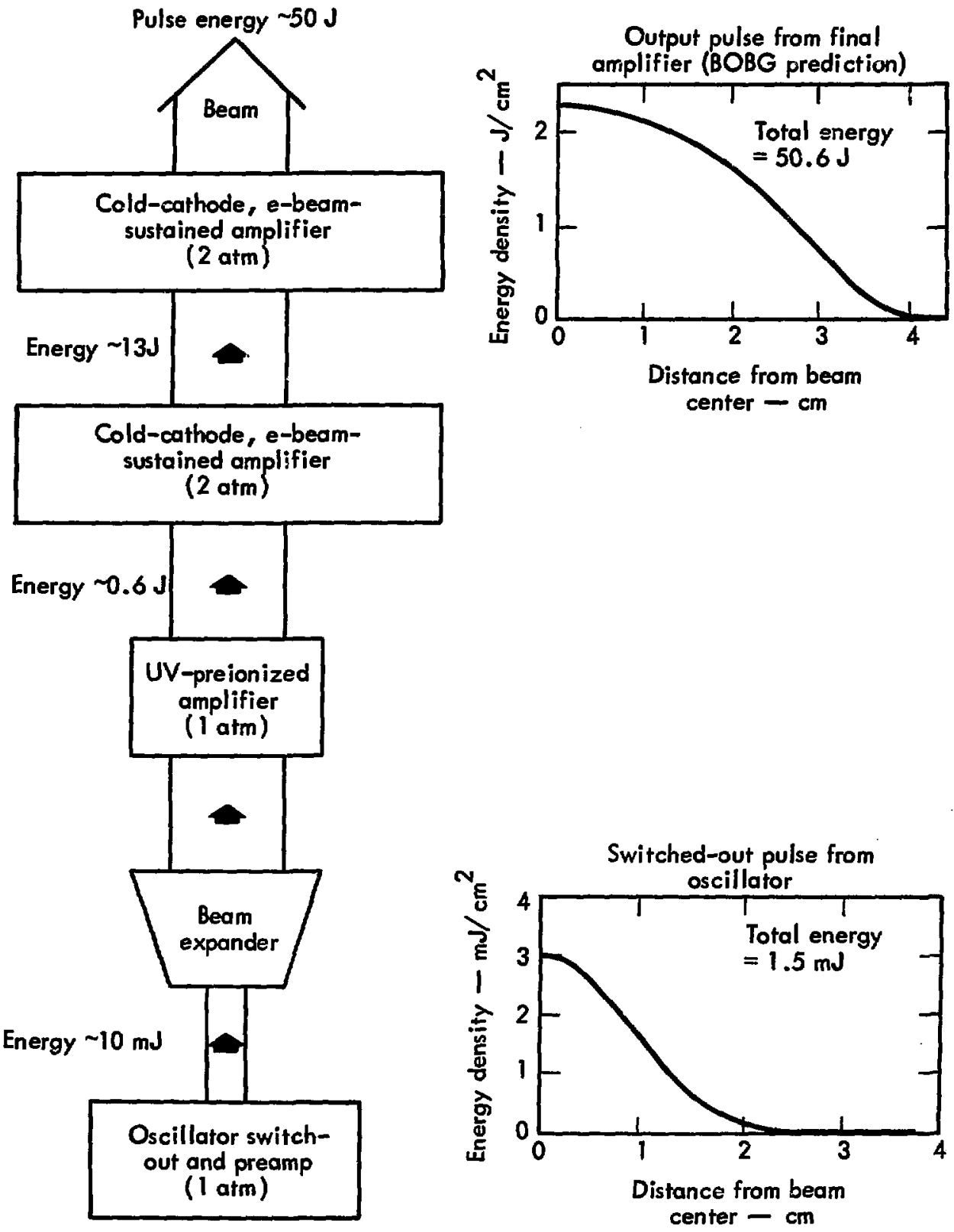


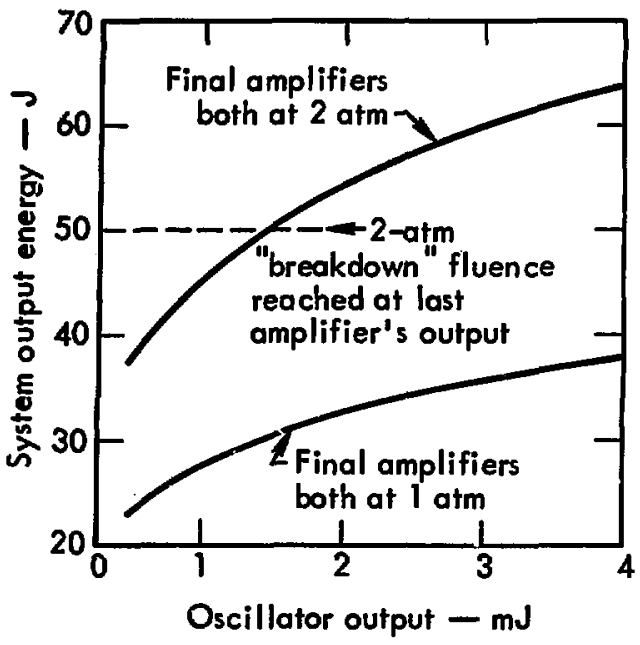

Fid. 137. False encery from the ayctem as a tunction of oncillator pulse energy, ss predicted by BOBG for 1-and 2-atm cperation of the two finel amplifers, which aro coli-cathode, obeen-arstained units.

system which shows the input and output pulses' spatial shapes, the latter as predicted by BOBG. Figure 136 also shows the pulse energy at the output of each amplifier stage. Note that the final amplifier is saturating and consequently the output pulse's spatial shape deviates substantially from the Gaussian spatial profile of the input pulse. The small-signal gain of the amplifier chain is $10^{6}$. Figure 137 shows the predicted pulse energy from the system as a function of the oscillator's output energy.

The energy density in the beam center of the output pulse is near or perhaps slightly above the breakdown threshold for the gain medium in the 2-atm amplifier. Detsiled breakdown measurements will be discussed below; however, from the studies performed so far, it appears that exceeding the "breakdown" fluence will lower the index of refraction of the medium near the beam center. This distortion of the index, if permitted to exist over too long a path, will refract energy out of the beam and distort the phase fronts of the propagating pulse so that it cannot be easily focused on a small target.

It is apparent that one of our most important needs i for reliable system simulation codes. More sophisticated computer models have been prepared which more realistically treat the multilevel nature of the $\mathrm{CO}_{2}$ molecule with finite rotational and intramode relexation rates. These codes are under evaluation and have not yet been used as an aid to system design. As reliable models for pulse propagation in $\mathrm{CO}_{2}$ amplifiers or in isolators become available, we intend to use them to better optimize gas mixtures, beam expansion ratios, and other system parameters.

The $\mathrm{CO}_{2}$ system is primarily intended to replicate target experiments performed with $1.06-\mu \mathrm{m}$ sources, changing only the wavelength, so that the effects of irradiating targets with $10.6-\mu \mathrm{m}$ pulses may be compared quantitatively to the effects obtained with 1.06- $\mu \mathrm{m}$ irradiation. Consequently, the system must deliver a single l-nsec-or-shorter pulse to a low$f$-number optical system at whose focus is a target. Two major concems are to prevent self-oscillation in the amplifier chain and to protect the chain from damage which can be caused by energy reflected from the target. To tackle these problems, we require knowledge of the reflectivities due to the target, windows, exposed metal, Rayleigh or Mie scattering, etc. A simple calculation illustrates the magnitude of the problem. Any rough scattering surface more than a few square wavelengths in area may be divided into a number of small surface elements, each of which radiates coherently but with random phase into a solid angle $\Omega$. If the strface is ideally rough, that is Lambertian, the effective scattering angle, $\Omega$, is $\pi$ steradians and each small surface element has an area $\lambda^{2} / \pi$. If we have provided no isolators, our amplifier chain will have an oscillator output mirror at one end and a number of these small coherent scatterers, called coherence areas, at the other. A very optimistic assumption would be to imagine that only one coherence area was important at the output end. In that case, the threshold gain for lasing of the amplifier chain is easily found to be given by $G_{t h}=(2 \pi L / \lambda) \approx 6$ $X 10^{6}$ for an amplifier chain length $L=10 \mathrm{~m}$ and $\lambda=10.6 \mu \mathrm{m}$. In practice a large number of coherence areas would be present and the threshold gain would be much lower. Mie scattering theory predicts that a single $0.2 \lambda$-radius dust particle has a scattering cross section given by $\pi \lambda^{2}$ and would, therefore, provide the sort of feedback which would be disastrous to a system with no isolators. It follows, then, that isolators are required to perform target experiments. We have already compiled some preliminary data on saturable absorber isolators. 99 In addition, an InSb Faradayrotator isolator has been designed and is being fabricated, and a focusing or Keplerian beam-expansion telescope has been designed which may protect delicate switchout optics from pulses reflected from target plasmas. In the coming months, besides assembling our system and target area and studying the dynarisics of the rexcited $\mathrm{CO}_{2}$ molecule, we will direct our efforts toward finding and modeling suitable isolators. 


\section{Oncillator}

The mode-locked switched-out $10.6-\mu \mathrm{m}$ pulse will be generated by an oscillator system very similar to that described previously. 100 Our recent efforts in this regard have been directed toward packaging the uv-preionized gain medium in an effort to reduce electrical noise.

\section{Amplifien}

System requirements dictate gain stages having the following characteristics:

1. Small-signal gain $\sim 4 \% / \mathrm{cm}$.

2. Gain uniformity within $\pm 10 \%$ over the full aperture.

3. Reliability $>0.9968$ at $50 \%$ confidence.

4. Full if shielding.

5. Commercial devices where feasible.

Fig. 138. Geth mulfomity curves for a commercially avail blo TEA $\mathrm{CO}_{2}$ enplifiet. Lacing on $P(20)$ line, fou mixtare $\mathrm{He} / \mathrm{CO}_{2} / \mathrm{N}_{2}=66.1 / 23.1 / 10.8$, totul pres. mive $=760$ Torr.

Fig. 139. Artiate conception of the finimed $\mathrm{CO}_{2}$ aydem.

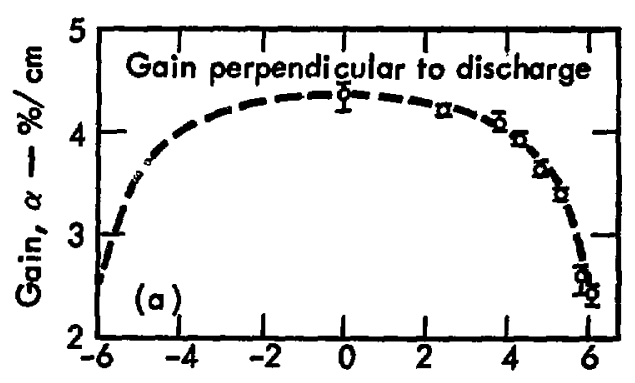

Distance from centerline $-\mathrm{cm}$

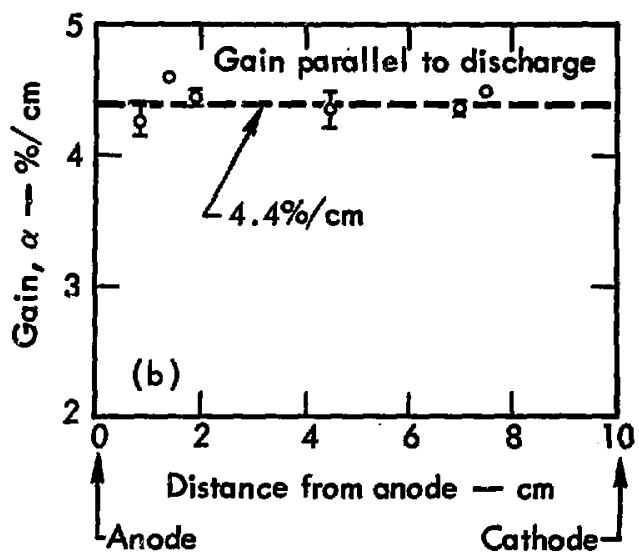

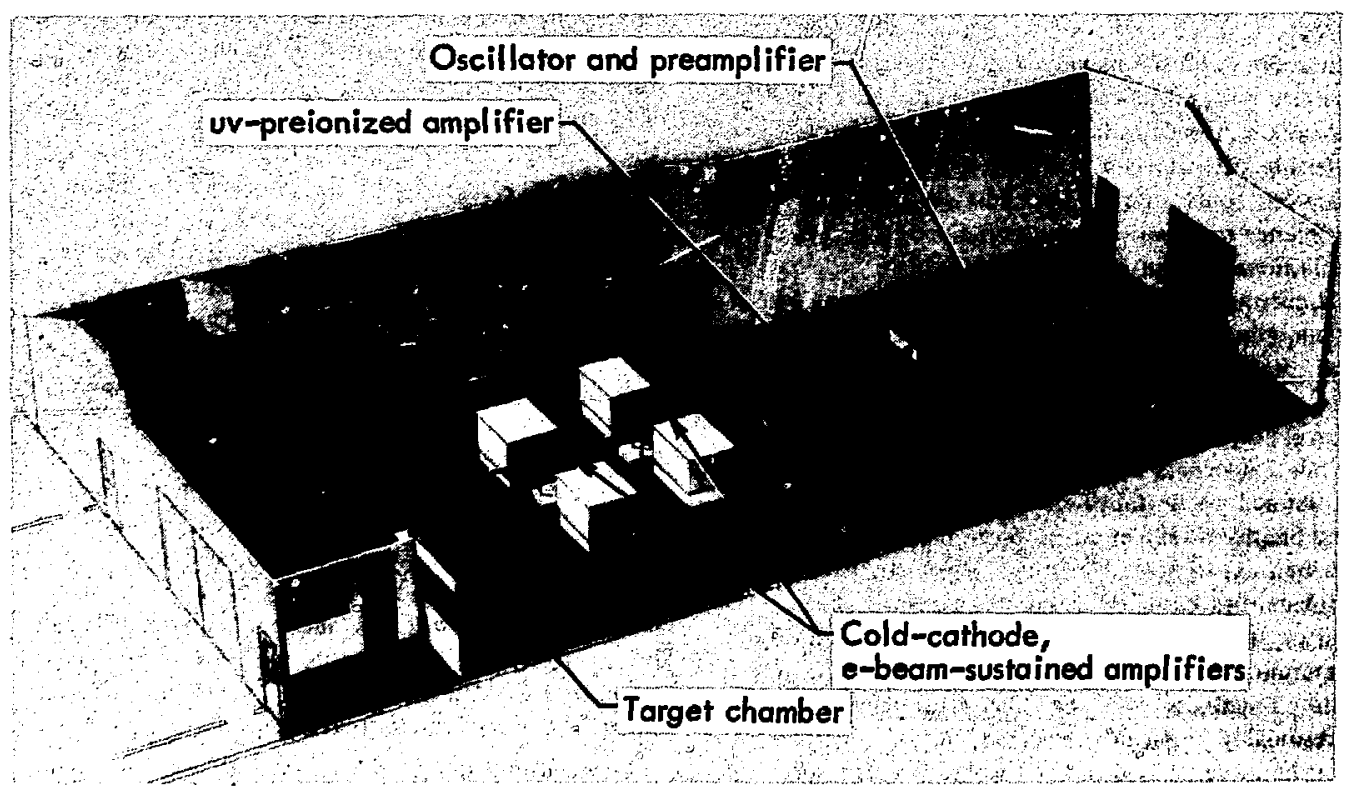


The specified dimensions and operating pressures of the various stages are as follows:

\begin{tabular}{lccc} 
& $\begin{array}{c}\text { Dimensions } \\
(\mathrm{cm})\end{array}$ & $\begin{array}{c}\text { Pressure } \\
\text { (atm) }\end{array}$ \\
\cline { 2 - 2 } Oacillator & $3 \times 3 \times 150$ & & 1 \\
Preamplifier & $4 \times 4 \times 150$ & 1 \\
1st amplifier stage & $10 \times 10 \times 100$ & 1 \\
2nd and 3rd stages & $10 \times 10 \times 100$ & 2
\end{tabular}

A program was initiated to determine what existing components would meet the system requirements. Typically a gain-producing component (i.e., amplifier) was characterized by scanning across the aperture with a probe $\mathrm{CO}_{2}$ beam and detector system, recording small-signal gains at several discrete locations." Repeatability data was accumulated by recording several data points for the same location, and reliability information was obtained by combining all data points. Several hundred data points were recorded for a given amplifier over a period of several days. A typical gain uniformity curve is shown in Fig. 138.

A critical path diagram for building the $\mathrm{CO}_{2}$ system has been devised, and at this writing the assembly is on schedule. Experiments employing the entire system are expected to begin in March 1974. Figure 139 is an artist's conception of the finished $\mathrm{CO}_{2}$ system.

"See the previous semiannual report, UCRL-50021-72-2 (pp. 28-29).
The articles in this section describe some of our current efforts in doing experimental measurements on and computational modeling of $\mathrm{CO}_{2}$ gain media.

\section{Energy Fuence Limitations for Namosecond}

\section{$\mathrm{CO}_{2}$ Pulse Ampitication}

The limitation to nanosecond $\mathrm{CO}_{2}$ pulse amplification for laser fusion research is generally thought to be the ionization of the gas mixture by the laser pulse - i.e., gas breakdown. However, there is no generally agreed-upon definition of breakdown threshold. Kroll and Watson define the breakdown threshold to be the maximum laser intensity at which the electron multiplication and loss rates are equal. 101 An altemative definition may be the minimum laser intensity at which the electron plasma frequency becomes equal to the laser radiation frequency. The difference between the values obtained from these two definitions can be as great as 1000.102

Experimentally, the breakdown thıeshold is commonly determined by focusing a laser beam into the mixture under study. A bright visible spark may be observed at or near the focal point of the mirror or lens. The laser intensity at which spark formation occurs once in 100 pulses, $1 \%$ probability, is defined to be the breakdown threshold by Berger and Smith. ${ }^{103}$ Hill et al., however, use $50 \%$ probability in their experiment. ${ }^{104}$ Others negiect to mention the probabilities used.105 Even if a single probability is agreed upon, additional ambiguities in the threshold determination are introduced by the variation in size and brightness of the observed sparks, which presumably indicates electron density differences. Furthermore, it is difficult to observe faint sparks in a TEA $\mathrm{CO}_{2}$ laser because of the background of visible radiation from the glow discharge. We have accordingly performed our experiments in such a way that the results do not necessarily depend on the visual observation of spark formation.

Figure 140 shows a diagram of our setup. A 10-cm-diam meniscus Ge lens is used to focus the narosecond, 0.7-cm-diam, Gaussian-shaped (temporally and spatially) $\mathrm{CO}_{2}$ laser beam near the center of the double-discharge test cell. The $1 / e$ beam diameter at the focus is approximately $0.05 \mathrm{~cm}$. Descriptions of the construction and operation of the test cell and the generation of the nanosecond $\mathrm{CO}_{2}$ laser pulses have been reported previously.106,107 The apertures in front of the two detectors are fixed at $4.2 \mathrm{~cm}$, a factor of 6 greater than the incoming laser beam diameter. All the laser radiation which passes through the apertures is focused onto the photon drag detectors by the focusing mirrors. The attenuation of the $\mathrm{CaF}_{2}$ attenuators and the linearity of the two photon-drag detectors in the range of our operation have been checked throughout the experiment. Our setup allows us to measure both the input and output laser intensities and temporal pulse shapes as a function of input intensity. From these measurements we can plot a graph of input versus output intensities. When the input laser beam is not perturbed, all the points should fall on a $45^{\circ}$ line. If breakdown occurs in the test call as the input energy is increased, not all the input laser radiation will be transmitted to the signal detector. As a result, the measured points will fall below the $45^{\circ}$ line mentioned before. We scek, therefore, the maximum input energy intensity at which the input and output intensities are equal.

Previous experiments have shown that the presence of impurities in the gas medium can reduce the 


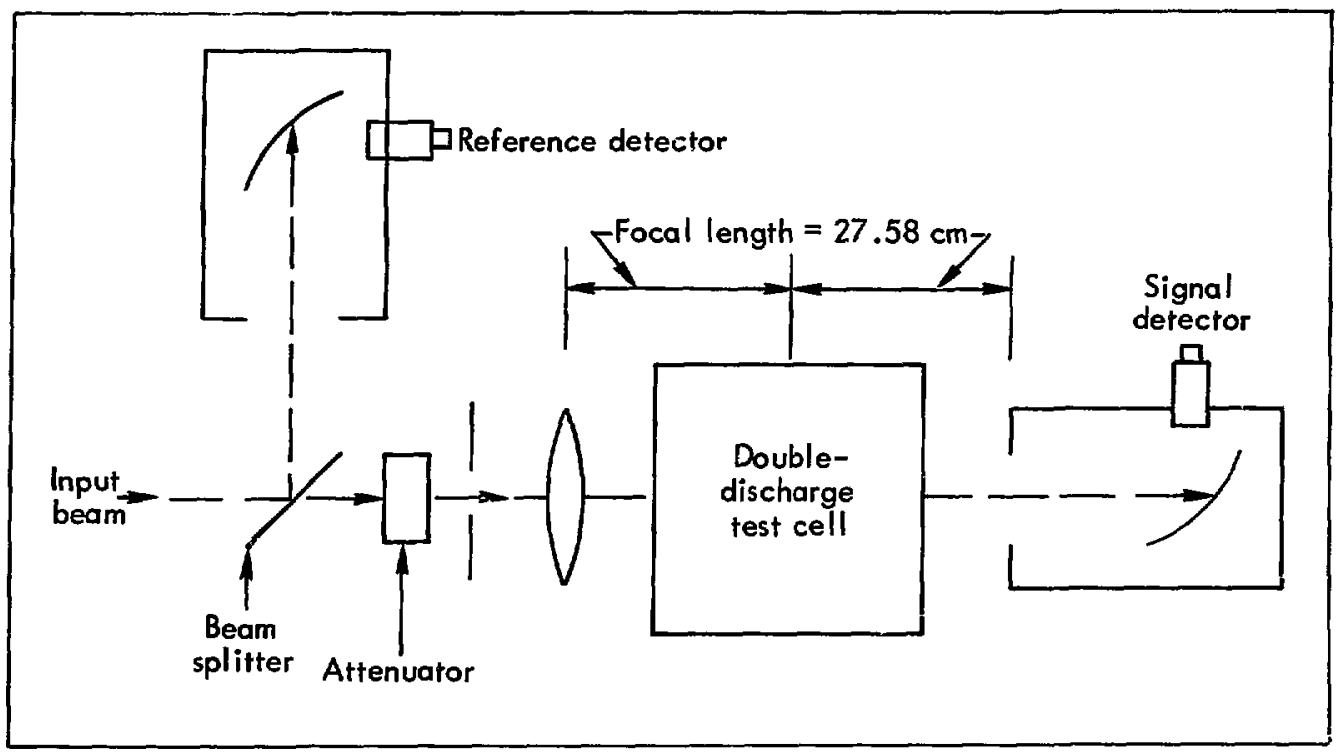

Fig. 140. Experimental setup for studying breakdown in $\mathrm{CO}_{2}$ amplification of nanosecond laser pulses.

breakdown threshold intensity by several orders of magnitude. $105,108,109$ Thus, at the start of each run, we flush the double-discharge test cell for at least 5 min to remove any contaminants that may have accumulated during the down period. During the flushing and normal operation, the flow rate is such that the gas in the cell is replaced 60 times/min; however, there are no in-line filters to remove any dust particles that may be in the gas cylinders. The system is fired at about 1 pulse/min during normal operation.

Figure 141 shows our data for a 1-atm, 5:1:1, $\mathrm{He}: \mathrm{CO}_{2}: \mathrm{N}_{2}$ gas mixture without preionization of the test medium. Each point on the graph represents the weighted average value of six groups of data obtained at different times. Each group of data contains about 15 pulses. A normal distribution is fitted to the pulse heights from each group to obtain the means and standard deviations of the distributions. The six means and standard deviations for each input energy (known to $\pm 6 \%$ ) are then combined to give the weighted average values according to the equation

$$
\begin{aligned}
& \bar{X} \pm \Delta \bar{X}=\frac{\sum_{i} x_{i}}{\sum_{i} w_{i}} \pm \frac{1}{\left(\sum w_{i}\right)^{1 / 2}}, \\
& w_{i}=\frac{1}{\left(\Delta X_{i}\right)^{2}},
\end{aligned}
$$

where $X_{i}$ and $\Delta X_{i}$ are the mean and the standard deviation of each group of data. A $45^{\circ}$ line is fitted

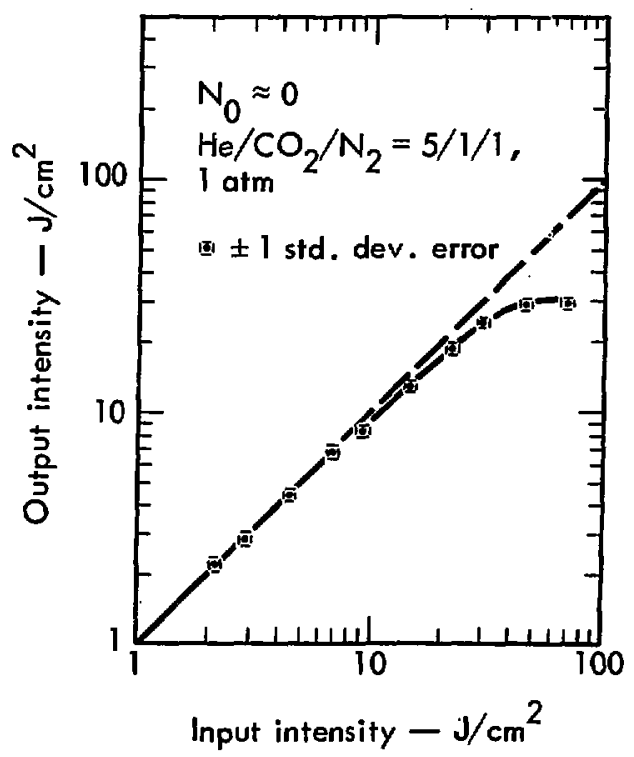

Fig. 141. Plot of output interdity vs input intensity with no preionization. Intensities are at the lens focus. 
through the four points with the lowest input laser energy. As the input energy is increased above $7 \mathrm{~J} / \mathrm{cm}^{2}$, the data points are observed to fall below the $45^{\circ}$ line.

In order to correlate our measurements with those obtained by the observation of spark formation, we note that the probabilities for spark formation are $100 \%, 50 \%$, $5 \%$, and $0 \%$ at about $20,14,9$, and $7 \mathrm{~J} / \mathrm{cm}^{2}$ respectively. These results may be compared to the value of $1 \%$ probability at $10 \mathrm{~J} / \mathrm{cm}^{2}$ found for 70 nsec pulses at the same pressure and gas mixture by Berger and Smith. 103 It is important, however, to observe that the absolute value in our energy measurement is uncertain because of the large differences found between various calorimeters for nanosecond $\mathrm{CO}_{2}$ laser pulses in this energy range. Our relative uncertainties, however, are small.

Figure 142 shows our data with the discharge on the test cell. The $45^{\circ}$ line is identical to the one obtained in Fig. 141. The change in slope, in this case, recurs at a laser input energy of about $3 \mathrm{~J} / \mathrm{cm}^{2}$. However, in contrast to the case of no preionization, we lind a distinct change in the visual character of the breakdown. Figure 143 shows the differences for an input energy of about $70 \mathrm{~J} / \mathrm{cm}^{2}$. As the input laser energy is reduced (when the discharge is present), the filament observed in Fig. 143b becomes narrower and fainter. No filament formation is observed at input energies below $20 \mathrm{~J} / \mathrm{cm}^{2}$.

Reflection, absorption, or refraction can cause the loss of the laser radiation. The fact that no spark or filament formation is observed below about $20 \mathrm{~J} / \mathrm{cm}^{2}$ when a discharge is present in the test cell indicates that the beam loss is not due to reflection and that the absorption is probably small. In addition, if absorption is the cause of the observed beam loss, the beam diameter would not change appreciabiy. Thus, a long as the aperture in front of the signal detector is substantially greater than the incoming laser beam diiameter $(0.7 \mathrm{~cm})$, the point at which the transmission curve slope changes from $45^{\circ}$ should not be affected. When the aperture size is reduced to $1 \mathrm{~cm}$, the break point is shifted lower. This suggests that the cause of the beam energy loss is refraction.

We note that the incoming laser beam is spatially Gaussian. Thus the electron generation rate is larger at the center of the beam than at its edges. This results in a lower index of refraction at the center than in the wings of the beam and causes the radiation to be refracted from the center. If we amplify a heavily saturating incoming laser pulse in a 4-to-5-m-long gain medium with small-signal gain of abost $5 \% / \mathrm{cm}$, a crude estimate indicates that the increase in the laser beam spot size due to beam refraction would equal that due to gain saturation, and considerable phase front distortion would be expected. Thus, beam refraction effects should be taken into account in the design of relatively long $\mathrm{CO}_{2}$ laser systems. Computer simulation of this effect is in progress. To date, preliminary runs have shown the refraction of light out of the beam center and the distortion of phase fronts, and have indicated that a $\sim 1-\mathrm{cm}$-long region of high electron density $\left(10^{16}\right.$ to $\left.10^{17} \mathrm{~cm}^{-3}\right)$ should exist just in front of the geometrical focus of the lens. This would have the appearance of a streak or filament.

\section{Rotational Relaxation Measurements}

The modeling of a short-pulse ( $<1-n$ sec), high-power $\mathrm{CO}_{2}$ laser system intended for laser fusion studies

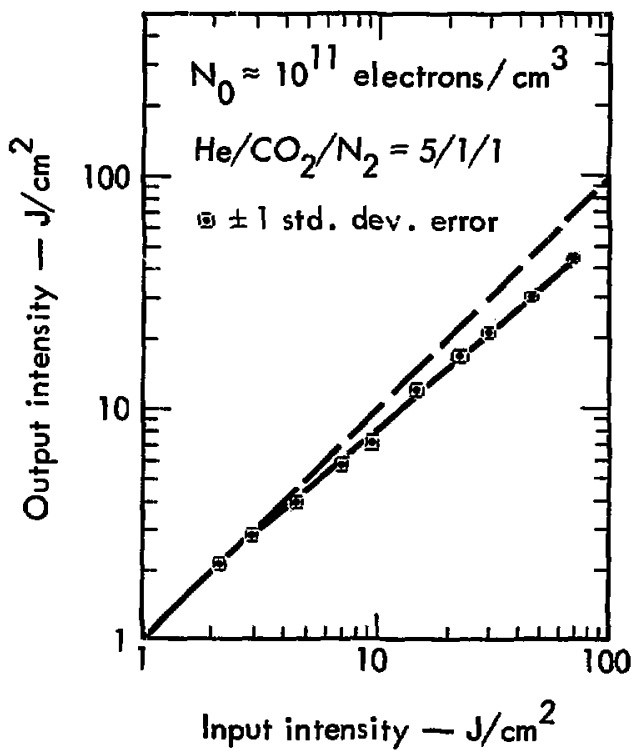

Fig. 142. Plot of ontput intensity vs input intensity with a preionization-producing dischange in the test cell. Intensities are at the lens focus.

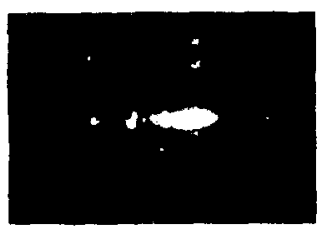

(a)

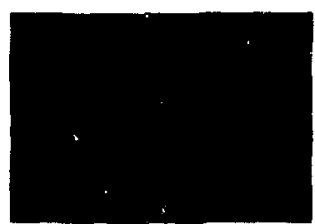

(b)
Fig. 143. Visual character of the breakdown at about $70 \mathrm{~J} / \mathrm{cm}^{2}$ at the focus of a nanosecond $\mathrm{CO}_{2}$ laser pulse in a mixture of $\mathrm{He} / \mathrm{CO}_{2} / \mathrm{N}_{2}=5 / 1 / 1$. (a) With no proionization of the gas mixture. (b) Whth prelonization. 
requires an accurate characterization of rotational relaxation processes in the upper and lower laser levels. Since these processes proceed at rates comparable to the laser pulse durations, they have a dominant influence on the energy extraction efficiencies of such lasers.

We have carried out an experimental study of rotational relaxation in the upper $\mathrm{CO}_{2}$ laser level $\left(00^{0} 1\right)$ and its dependence on the rotational quantum number $J$. The conclusion from these investigations is that, to a first approximation, we may model the $\mathrm{CO}_{2}$ upper vibrational laser level as a rotational bath in which all pertinent levels equilibrate with a similar time constant upon perturbation by a $\mathrm{CO}_{2}$ laser pulse.* Such a simplification permits us to ignore detailed stepwise energy-transfer processes among the rotational sublevels during the period of amplification of a short laser pulse. In addition, our results provide useful experimental input for molecular collision calculations in which we could analyze a binary encounter between an excited $\mathrm{CO}_{2}$ molecule and another partner in terms of nonvanishing multipole moments characterizing the interaction 111 ; in this application, the selection nules and predictions for the temporal behavior of the various $J$ sublevels would have to conform to the results of our study.

Our approach is very similar to earlier work of Cheo and Abrams, who primarily directed their attention to extracting the rotational rate constants for pure $\mathrm{CO}_{2}$ and for $\mathrm{CO}_{2}$ in combination with mixtures of $\mathrm{He}$ and $\mathrm{N}_{2} .112$ In addition they reported a qualitative $J$ independence for rotational relaxation in the upper $\mathrm{CO}_{2}$ laser level. Our work was aimed at a more thorough examination of the $J$ independence in the $00^{0} 1$ vibrational siate. At least three significant differences exist between the two efforts: (1) a more extensive scan of $J$ levels was made in our work, i.e., the 9.6- $\mu \mathrm{m}$ probe beam was varied from $P(10)$ through $P(34)$ as opposed to $P(22)$ through $P(38)$, and our results are presented in quantitative form; (2) our pulses were about an order of magnitude shorter, i.e., about 2 nsec vs 20 nsec, thus giving us a significant increase in temporal resolution; and (3) our study was made in a tripartite mixture of $\mathrm{CO}_{2}-\mathrm{He}-\mathrm{N}_{2}$ at two different pressures to complement the observations of Cheo and Abrams' work done in pure $\mathrm{CO}_{2}$.

In Fig. 144 we show the pertinent energy-level diagram of $\mathrm{CO}_{2}$. The experiment consisted of injecting two spatially and temporally overlapping laser beams, one in the 10.6- and the other in the 9.6- $\mu \mathrm{m}$ band, into a low-pressure $\mathrm{CO}_{2}$ amplifier. The temporal gain at a particular rotational transition in the $9.6-4 \mathrm{~m}$ band

" $J$ independence of rotational relaxation in molecular laser levels has been assumed in Ref3. 93 and 110 . was monitored with a low-powsr probe beam. This gain was altered by the passage of a $2 \cdot n s e c$ pulse at the $P(20)$ line in the $10.6 \cdot \mu \mathrm{m}$ band. In this manner the response of the rotational manifold in the $00^{0} 1$ vibrational state could be observed by successively selecting rotational transitions in the $9.6-\mu \mathrm{m}$ band and detecting the corresponding response of a particular rotational state $(J)$ in the upper laser level. A tacit assumption in this procedure is that the influence of lower state population rearrangements on the probe signal may be neglected. This is true if the Fermi resonance mixing the $10^{\circ} 0$ and $02^{\circ} 0$ levels operates on a longer time scale than the rotational relaxation among sublevels in the $00^{0} 1$ state. Furthermore, we can argue that the influence of the lower-level temporal effects on the $9.6-\mu \mathrm{m}$ signal will be mitigated because of mixing among the many rotational sublevels in the $10^{\circ} 0$ state. We also ignore any inter- or intra-mode $\boldsymbol{V} \cdot \boldsymbol{V}$ interactions in these studies. Recent investigations indicate that a certain intermode $V \cdot V$ collisional process is at least a factor of 2 slower than the rotational relaxation in the $00^{\circ} 1$ state."

In Fig. 145 the experimental arrangement used in the investigations is shown. The $P(20)$ pulse $(10.6 \mu \mathrm{m}$, $\sim 2$ nsec) originates in a three-stage Rogowski TEA oscillator whose output is mode-locked with an intracavity, acoustooptic germanium crystal. Details of this oscillator, the pulse switchout system, and the spectral content of the selected pulse have been reported in detail in a previous semiannual report. 114 After the pulse passes through a TEA amplifier, a 1-m grating spectrometer selects the $P(20)$ line. This line is then amplified by a second TEA amplifier and combined with a selected weak, orthogonally polarized transition in the $9.6-\mu \mathrm{m}$ band at a $1 / 2$-in.-thick germanium plate. Cain signals in the experiment were obtained only for the $P(10)$ through $P(34)$ lines in the 9.6- $\mu \mathrm{m}$ band because the grating-tuned probe laser did not proride sufficient output for detecting transitions outside this range. The probe $(9.6 \% \mathrm{~m})$ and saturating $(10.6-\mu \mathrm{m})$ beams enter and exit the low-pressure flowing $\mathrm{CO}_{2}$ medium through openings in two hollow cathodes. The two laser frequencies emanating from the amplifier are separated and individually detected by utilizing the indicated Brewster angle plates and a monochromator. The temporal response of the two detection schemes is a few nanoseconds or less. The triggering system for the experiment, which involves a $6328-\AA$ iaser beam passing through a rotating chopper wheel, has been described in detail in a previous semiannual report. 114

*The rate constant for the $V-V$ interband process $C O$, $\left(01^{\prime} 1\right)+\mathrm{CO}_{2}\left(00^{\circ} 0\right) \rightarrow \mathrm{CO}_{2}\left(00^{\circ} 1\right)+\mathrm{CO}_{2}\left(01^{2} 0\right)$ has been found to be $(5.3 \pm 1) \times 10^{5} \mathrm{sec}^{-1}$ Torr $^{-1}$ (Ref. 113). 
In Fig. 146 we show a typical response of the 9.6- $\mu \mathrm{m} P(20)$ gain signal to the perturbation which is due to the temporal buildup of the $J=19$ rotational state. This buildup comes from collisional interactions with other member states in the rotational manifold. The total amplifier pressure was 9.4 Torr. In Fig. 147 we show representative decay waveforms for the various gains in the $9.6-\mu \mathrm{m}$ band, depicting the corresponding time-dependent populations in the rotational states surrounding the perturbed $j=19$ level.

In the absence of a detailed theory describing the present experiment, we perform a three-segment linear least-squares computer analysis of the oscillograms

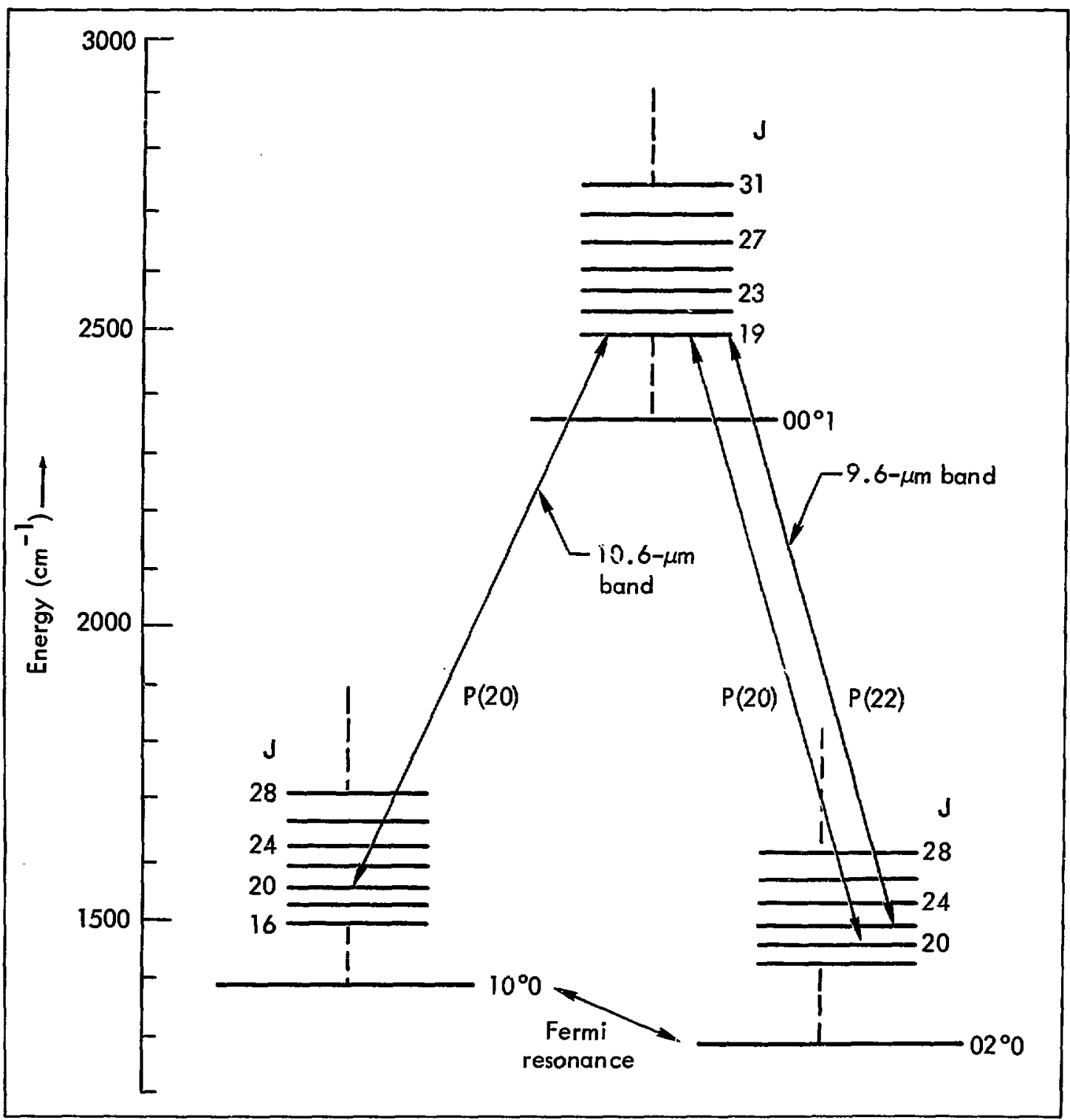

Fig. 144. Energy level diagram of certain low-lying vibrational levels in $\mathrm{CO}_{2}$ indicating the rotational substructure. Not shown are the lower !ving $02^{2} \mathrm{O}, 01^{1} \mathrm{O}$, and $00^{\circ} \mathrm{O}$ vibrational levels. 
shown in Fig. 147 to extract the various non- $P(20)$ 9.6- $\mu \mathrm{m}$ decay times. The resultant fits are fairly good even though this technique suffers on at least two counts: (1) the decays are not simple linear functions of time, and (2) in the signal traces for transitions originating near the $J=19$ level, i.e., $P(18)$ and $P(22)$, there is an initial rapid buildup following the main decay which made it difficult to establish a baseline

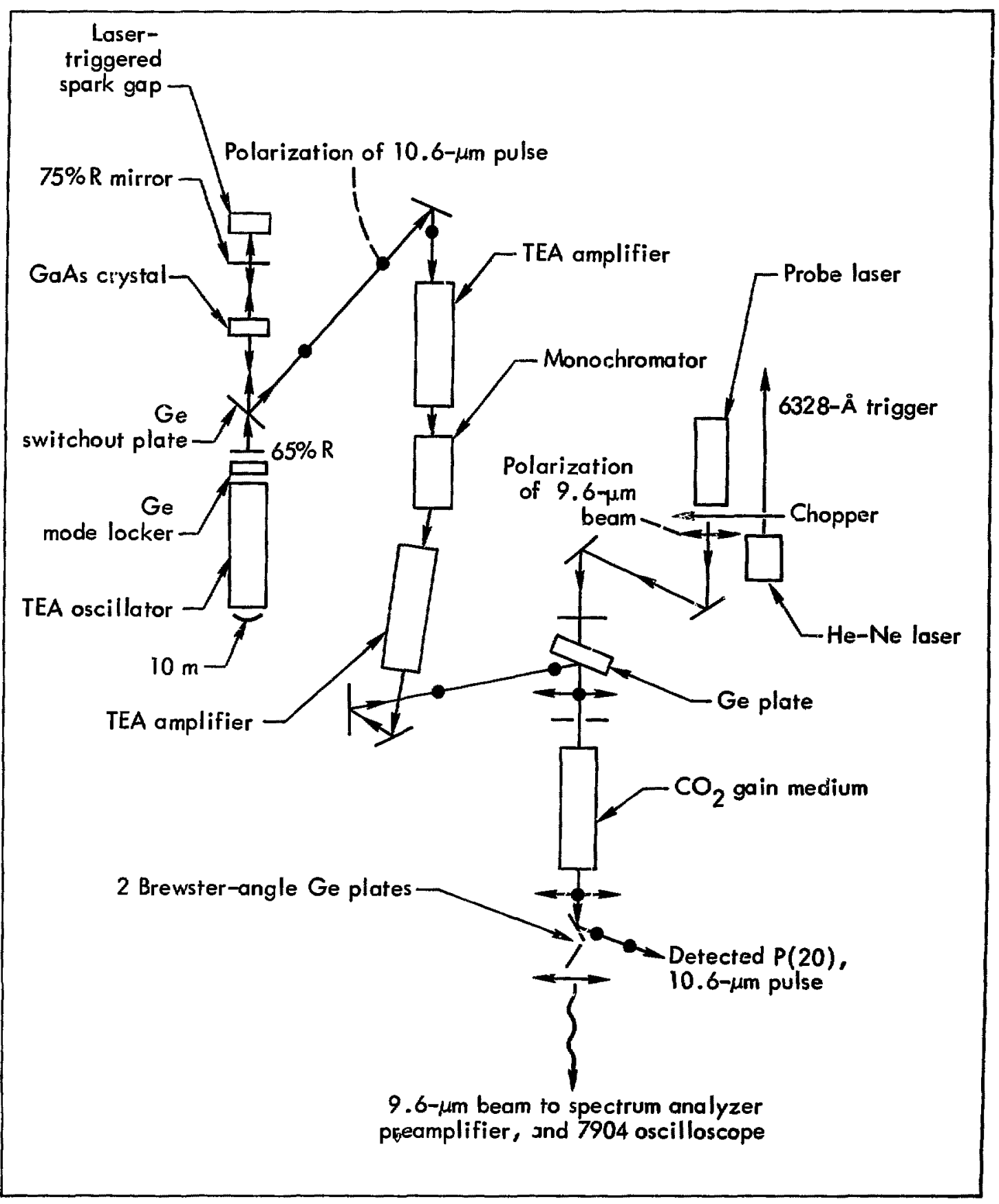

Fig. 145. Schematic diagnam of the experimental arrengement for studying rotational relexation. 


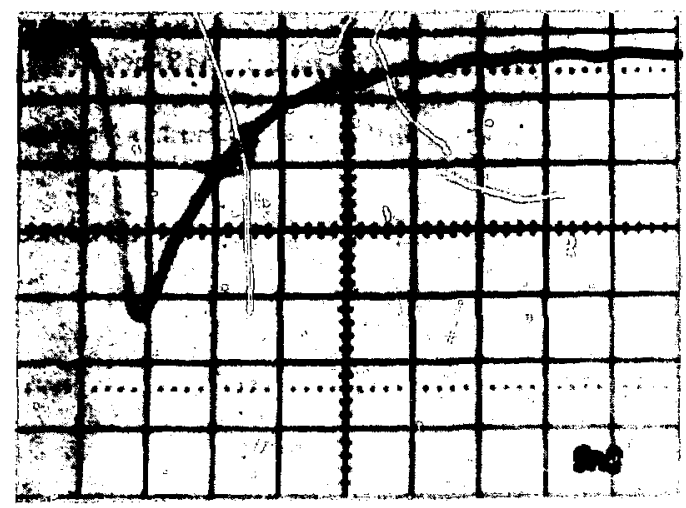

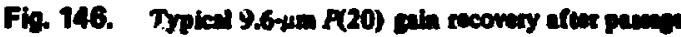

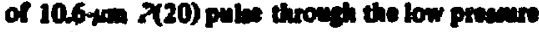
enptitio.

for these casen (in practice it is defined at the point of recovery from gain decay).

The reails on our preliminary data are shown in Fig. 148, in which we plot the total decay time vs the relevant rotational state in the $00^{0} 1$ level. The error bars encompass all the analyzed data centered about an avenge value for each $J$ level. The minirium in decay times existing sear the perturbed $J=19$ state is strongly suesestive of a quadrupole-quadrupole collisional interaction, i.e., $\Delta= \pm 2$ transitions. Another point of view can be obtained by drawing two horizontal lines centered about the decay lime of 19 nee $\pm 25 \%$, within whose bounduries the vast mujority of the data is concentrated, thus implying $J$ independence in the rotational relaxation to within this degree.

Anulytic work is now in progres to explain our observations. Such an andysis will then be used to further refine existing computer codes that describe the thort-pulse energy extrer isin from the $\mathrm{CO}_{2}$ laser system.

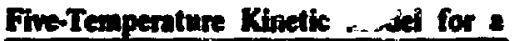

\section{$\mathrm{CO}_{2}$ Lewer Amplifier}

We have developed amity of four. and five-tempenture kinetic modeh for $\mathrm{CO}_{2}$ laser amplifiers. The five-temperature model for a TEA $\mathrm{CO}_{2}$ cacillator reported by Manes and Sequin has been extended to include rotational and intramode vibrational relexation procesces.lls This model unumes that the $\mathrm{CO}_{2} \cdot \mathrm{N}_{2}$ system may be dexcribed by four Landau-Teller energy-dexsily equations which specily the dynamics of the $\mathrm{CO}_{2}$ and $\mathrm{N}_{2}$ vibrational modes. The requirement that the vibntional modes and rotational manifolds be in dynamic equilibrtum has

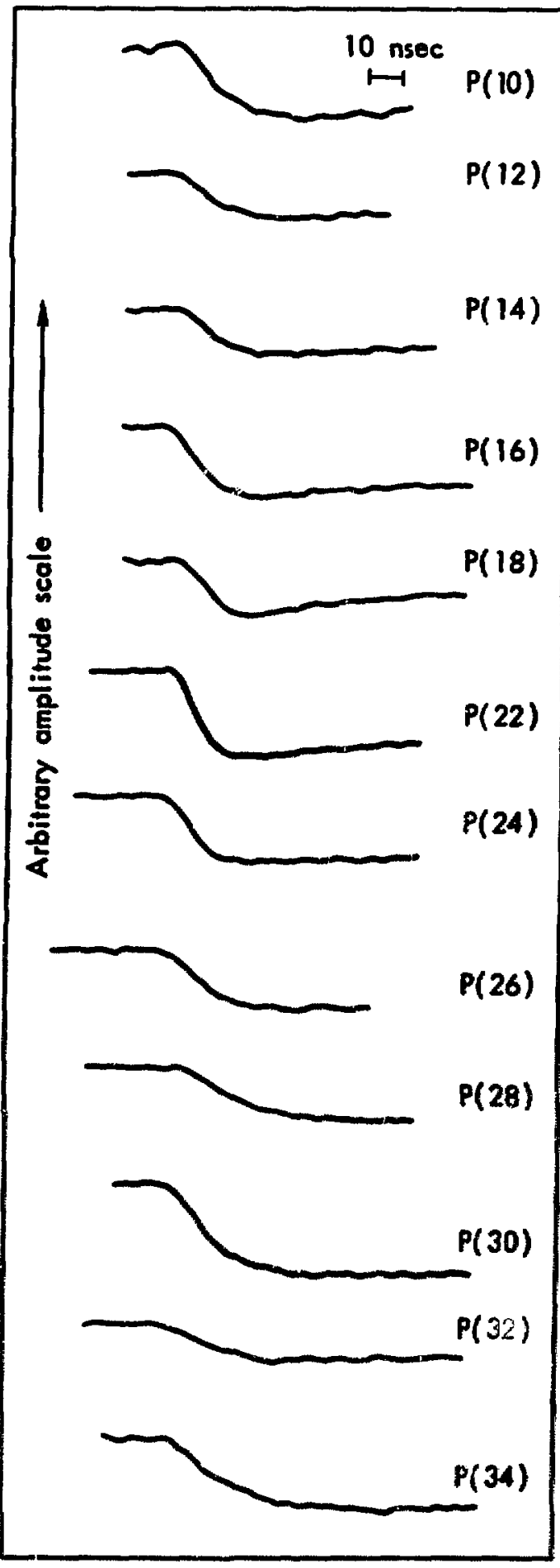

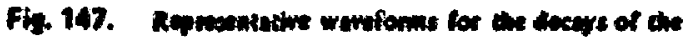

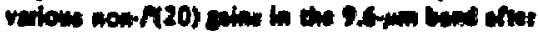

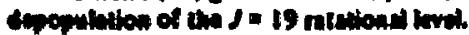




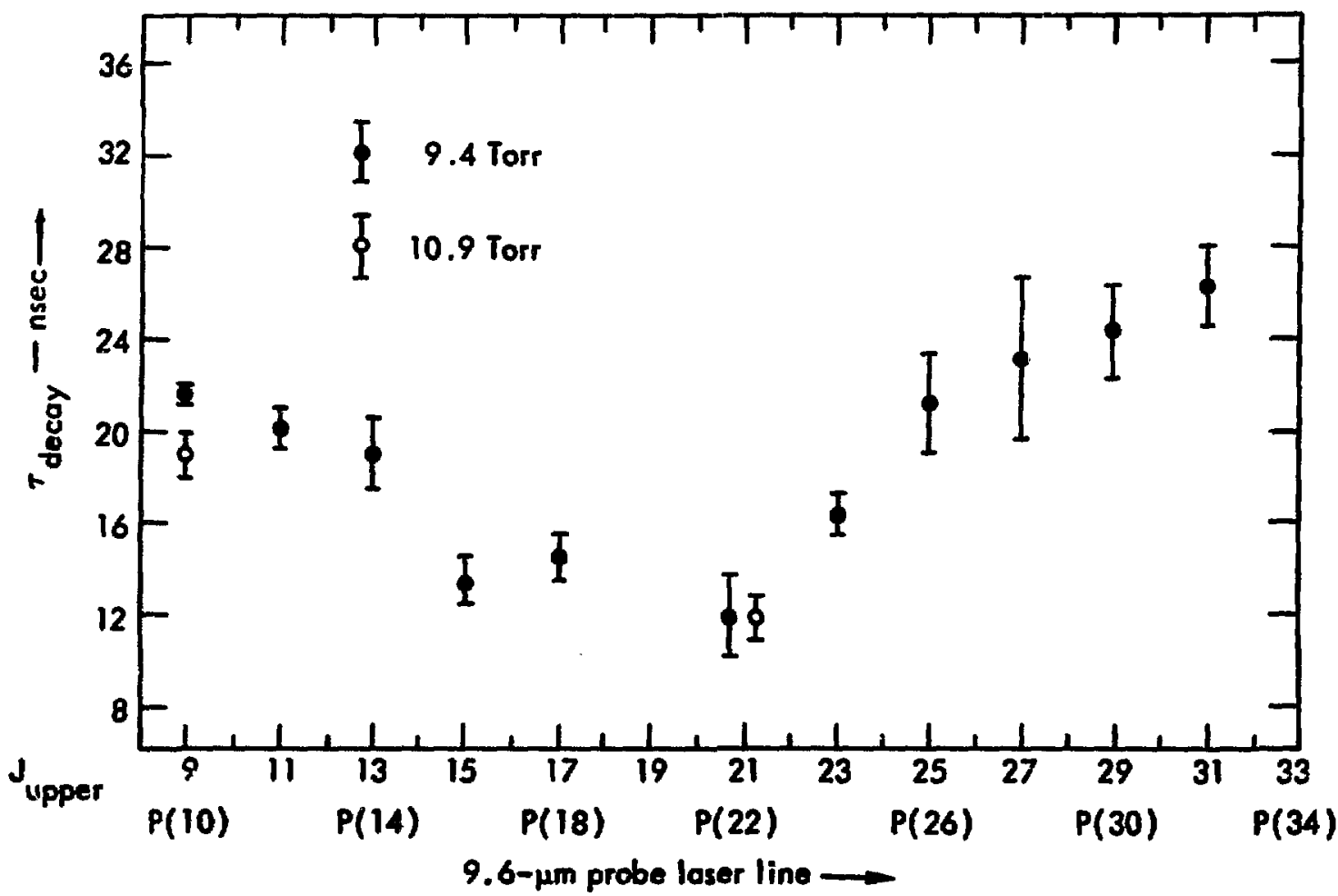

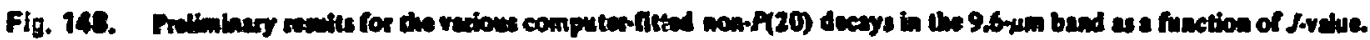

been lifted by the inclusion of several additional rate equations.

Fizure 149 is a schematic representation of this mode. 10.6. $\mathrm{wm}$ pulses interact with rotational levels which are coupled to their respective rotational manifolds with rotational relaxation time constants of aboul 0.2 neec at atmospheric presure. These rolational manifolds (or vibrational levels) are then coupled to their vibntionul modes with vibrational intrumode relaxation times which ate thought to be at leat twice as lone ss the rolational relaxation times. The vibrational modes relax logether and with the eround stale as decribed in Ref. IIS.

The incluation of the additional rapid rate equations has not altered the good sereement between experimentully observed and theoretically predicted ocillator pulie thapes.lis a pube propagation code using this modet has been written.

\section{Fon-Tenpenture Kantic Modet for a}

\section{CO. Len Amithex}

Vibrational temperature modals 16 axe now widely used to deacribe the production of population inversions in electrically and thermally pumped carbon-dioxide laser oxcillators and amplifiers.115,117 We have given 118 a detailed derivation and discussion of this type of model, specifically a four-tempenture (4-T) kinotic madel for as electron-beam-initiated $\mathrm{CO}_{2}$ Iuser emplifier. Our report inciudes some sumple calculations, a comparison of predictions of the model with experimental dat reported for a LASL ebeam device, ${ }^{119}$ and a procedure for running the associated computer code on the LLL time-sharing computer systern.

The distingulshing feature of this kind of model is its ability to exploit the wide disparity in the rates of the many elementary energy-transfer procesues that occur as the laser medium (continting of $\mathrm{CO}_{2}$ and additive gases) is pumped into an anplifying condition. Certain vibntional enery modes of the colliding molecules are identified which quickly equilibrate within themaelves. This allows the populations of all enery levels in one of these modes to be specified by a single vibrational (Boitumann) temperalure, which is different from the gas kinetic temperature and the temperatures of other vibntional modes. Further, it allows the firy of energy into and out of these levels to be characterized by one or a few collective excitation and relaxation rates. 
In the 4-T model, the effects of the additive gases $\mathrm{H}_{2}$ and $\mathrm{He}$, the radiation terms, and the temperature dependence of vibrational relaxation rates are included. The rate equations of the model govern the temporal behavior of the gas kinetic temperature $T_{g}$ and the populations of the carbon dioxide and nitrogen energy levels. These quantities in turn determine the time dependence of the amplifier gain and stored energy density. The four basic temperatures of the model are $T_{z}$, one vibrational temperature for nitrogen, and two separate vibrational temperatures for carbon dioxide. One of the $\mathrm{CO}_{2}$ temperatures refers to the asymmetric stretch mode, and the second to the bending and symmetric stretch modes, which are assumed to be in equilibrium.
To illustrate the predictions of the 4-T model, we briefly consider some effects of changing the operating pressure of the amplifier, and the effects of gain saturation that arise in amplifying short-duration light pulses.

The extent to which the gain and, especially, the stored energy density can be increased by operating the amplifier at higher initial pressure is shown for the 3/1/1 mixture in Fig. 150. In this sample calculation we keep constant the ratio $E / N$ of sustainer electric field to neutral particle density (thereby keeping the electron excitation rate coefficients constant). Figure 150 shows that for the $3 / 1 / 1$ mixture the peak values of both the gain, $\alpha_{0}$, and the stored energy density per unit pressure, $E / P_{0}$, change very little for

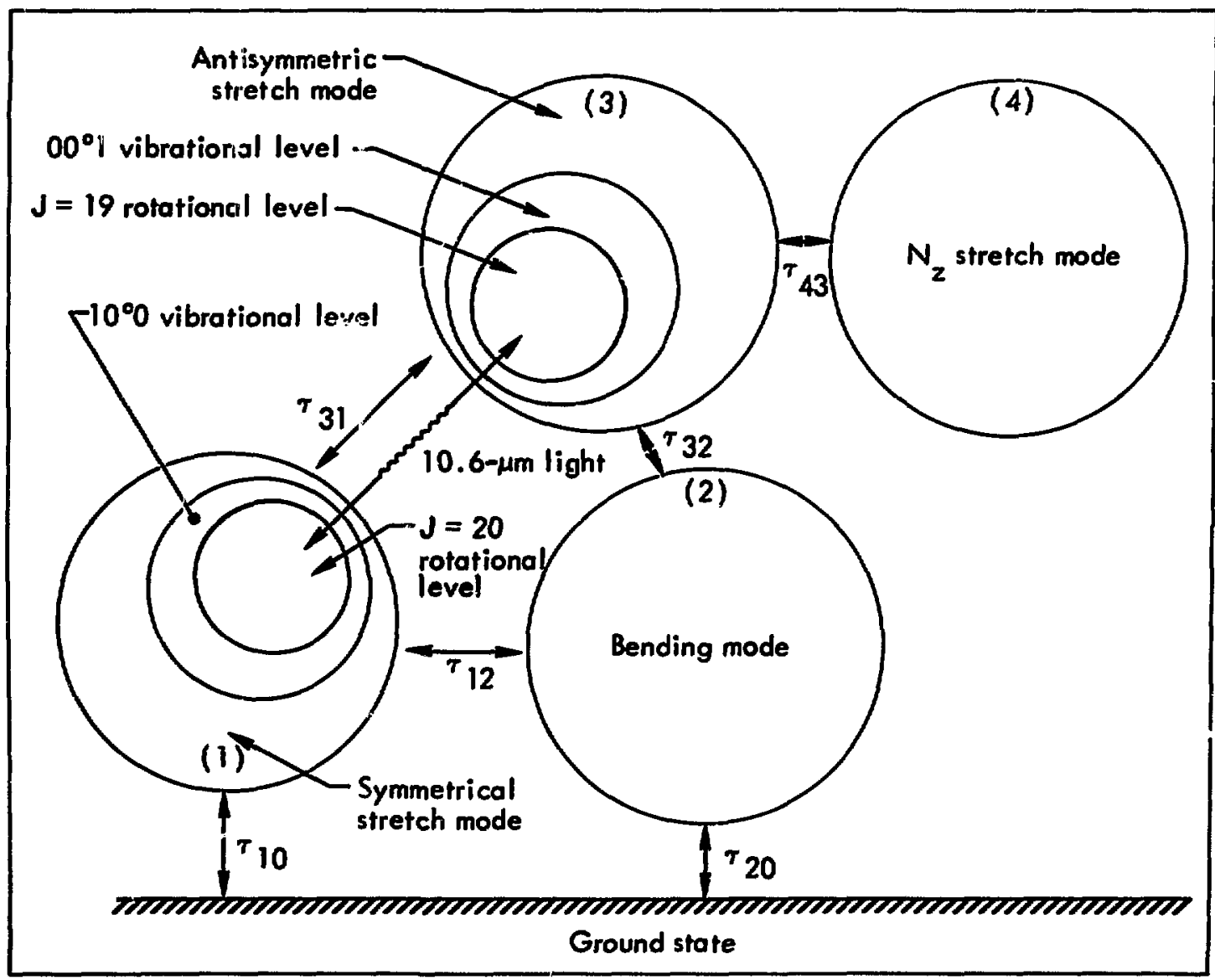

Fig. 149. Schemntic nepreantation of the 5-T kinetic model for $\mathrm{CO}_{2}$, indicating that the antisymmetric untch mode conlainin

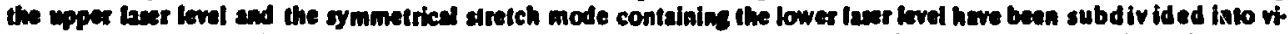
brationd lewels which in tum are further subdivided into rotaliond levets. In this way the axumption of thend equilibrivm of the modes has been remored. 


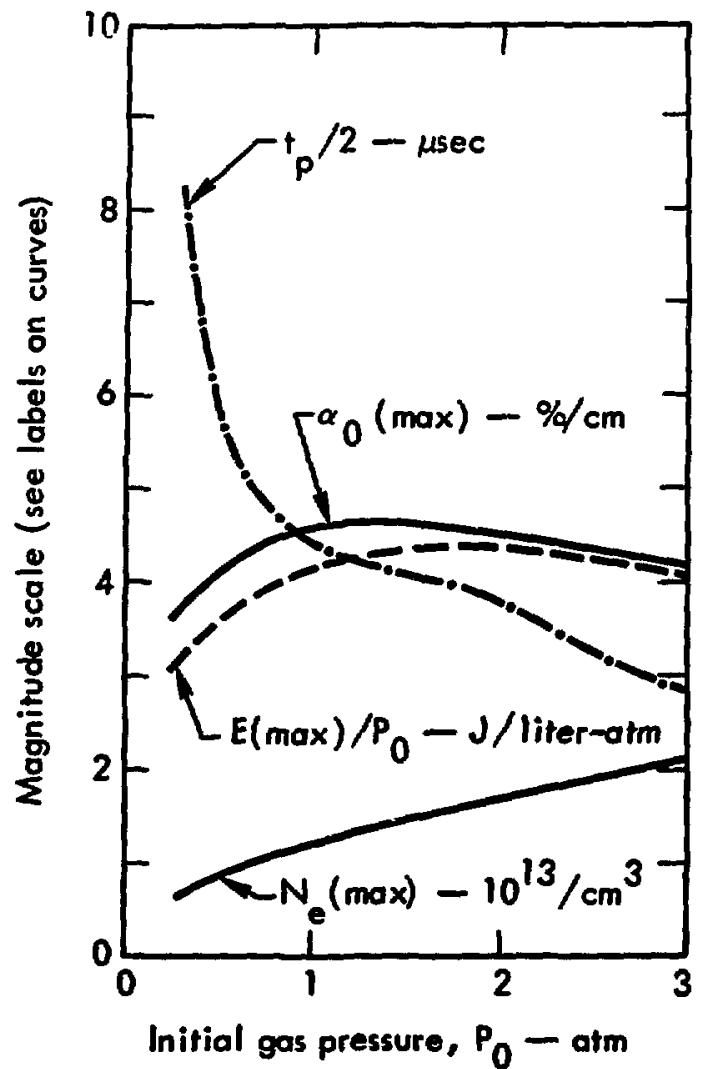

Fin. 150. Clicubtion with $4-\mathrm{T}$ modd of the eftect of hitily!

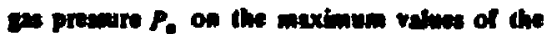

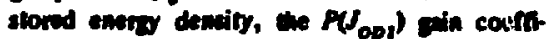
cient, the menter conity of cocondery electrons, ad the time $f_{p}$ at whish muxinimin gin ocenr. For fis celculation He/N, $\mathrm{CO}_{3}=3 / 1 / 1, E H Y=$ $2 \times 10^{-1} \cdot \mathrm{V} / \mathrm{cm}^{2}, T_{k}(0)=293^{\circ} \mathrm{K}$, and duntion

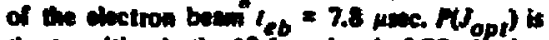

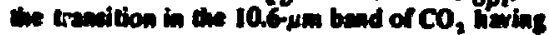
maxisum erin.

pressures in the range of $3 / 4 \mathrm{~atm}$ to $3 \mathrm{~atm}$. The shortening of the upper laser level's relaxation time and the increased rate of as heating at higher initial pressures cause the peak values of these quantities to occur earlier, tending to nullify any siznificant increase in their magnitudes. The stored energy density, $E(\max )$, ilself increases almost linearly with $\boldsymbol{P}_{\mathbf{0}}$.

The level of input radiation intensity required to saturate the amplifier on the optimum $F(n$ vibrational-rotational Inasition is illustrated in Fig. 15t. This figure shows the amplifier gain in the presence of a relatively long-duration (100-nsec) pulse

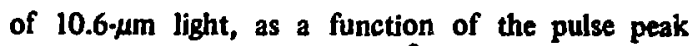
intensity $I_{0}$ (photons/sec-cm ${ }^{2}$ ). The "saturation intensity," defined as that magnitude of $I_{0}$ which reduces the gain to one-half its small-signal value, is seen to be $I_{\text {sat }} \approx 8.0 \times 10^{25}$ photons $/ \mathrm{sec}-\mathrm{cm}^{2}$ (corresponding to $1.5 \mathrm{MW} / \mathrm{cm}^{2}$ ) for this case of a 3/1/1 mixture at 580 Torr.

The computer code which solves the coupled, nonlinear rate equations of the 4-T model contains the following adjustable parameters: the initial temperature and pressure of the gas, the relative amounts of $\mathrm{CO}_{2}, \mathrm{~N}_{2}$, and $\mathrm{He}$ in the mixture, the duration and intensity of the electron beam, the electronic excitation sate coefficients and recombination and attachment coefficients, the vibrational relaxation rates, and the tum-on time, initial intensity, and duretion of an input light pulse. By varying these parameter values, the gain calculations can be closely tailored to the conditions of a given set of experiments.

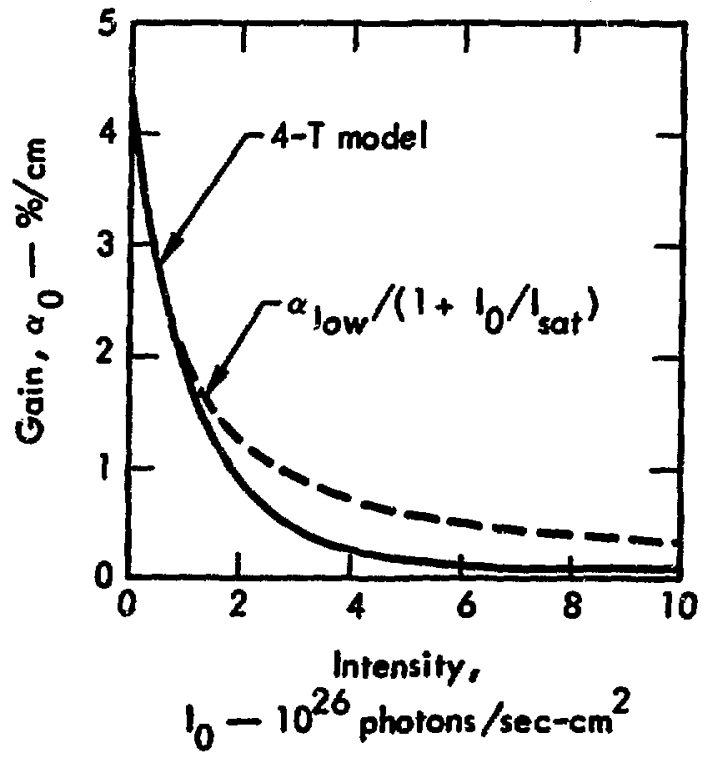

Fin 15t. Cuculation with 4-T modd of the wifoct on

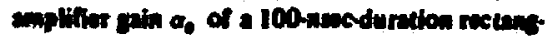

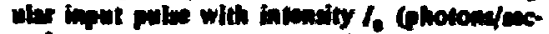
$\mathrm{cm}^{2}$ ). For thl celculation $\mathrm{He} / \mathrm{N}_{3} / \mathrm{CO}_{2}=3 / \mathrm{U}$, $E I N=2 \times 10^{-10} V / \mathrm{cm}^{2}, P_{0}=0.763 \mathrm{~atm}, \mathrm{ad}$ $T_{R}(0)=293^{\circ} \mathrm{K}$. The delved hime inclestes how

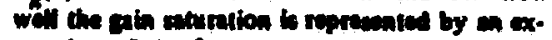

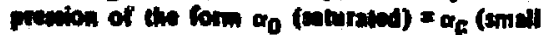

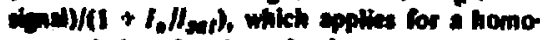

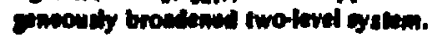




\section{INTRODUCTION}

In the previous semiannual report ${ }^{120}$ we gave the primary pumping and lasing processes for the lodine photodineosintion leser. We aiso indicated three items of concern:

- The primary photolytic quantum yield (the fraction of lodine atoms formed in the excited ${ }^{2} P_{1 / 2}$ state as opposed to the ground $2 P_{3 / 2}$ state).

- The energy storage capability as determined by the pressure-broadened lasing cross section.

- The lifeame of the excited state at high pressure as determined by the deactivation kinetics.

By extmpoiating our presure-broadening messurements on mixtures of gaseous iodine compounds with inert gas at less than atmospheric pressure, we indleated that at pressures of reveral atmospheres it should be possible to obtain (1) amplification of pulves shorter than 100 puec, (2) stored energy of several $J / \mathrm{cm}^{2}$ at reasonable gaine, and (3) an excited-state lifetime of tens of $\mu$ sec. Encouraged by then projectione, we have carried out both experi ental and theoretical investigations to better understand the lodine laser and to improve its performance.
Under superatmospheric conditions (i.e., pressures higher than atmospheric), the effects of three-body collisions, especially, must be carefully considered in relation to the primary $I^{*}$ quantum yield, lasing cross section, and deactivation lifetime. The pressure region up to about $3 \mathrm{~atm}$ can be explored experimentally without major fabrication difficulties for amplifier design purposes, but it is desirable to be able to reliably project performance to the considerably higher pressures required to obtain pulses ahorter than 100 psec from a mode-locked oscillator. Mode locking over one-fourth of the pressure-broadened bandwidth, $\Delta v$, requires a pressure $\geq 5$ atm for 100 -psec pulses, 120 and more sophisticated theories 121 indicate even higher pressures. Hence, our approach has involved (1) an experimental effort directed toward obtaining data for the cross section $\sigma$ and lifetime $r$ for pressures up to $\sim 3 \mathrm{~atm}$, and (2) a complementary theoretical effort aimed at explaining the data to 3 atm and projecting it to $\gtrsim 10 \mathrm{~atm}$.

In addition to characterizing superatmospheric behavior, we have also carried out experiments aimed at improving performance through optimization of photodiscociation and photolytic I* quantum yield.

\section{FHOTODISOCIATBON OUANTUM EFFICIENCY}

The quaritum efficiencies of $n-C_{3} F_{7} l$ and $\mid-C_{3} F_{7} l$ relative so that of $\mathrm{CF}_{3} \mathrm{I}$ were determined from their relative insing performance and pump-band abeorption crose sactions. A mall correction was aleo made for the difference in enerry absorption of the materials dive to the elitht epoctrol hift of their pump bands and the corresponding epectral charge in the output of the lamps. The materials were an nun in the some lawer system under identical operating conditions. Their pumpband crow soctions were measured accurately and are presented in Fiz. 152.

The quentum efficiency of $\mathrm{CF}_{3} \mathrm{I}$ was reported by Oenwre of al.122 to be 0.68 . This number was used to calculate the relative quuntum efficiencies of $n C_{3} F_{7} I$ and $i C_{3} F_{7} l$ presented in Table 16. The fact that come of the celculated quantum efficiencies exced the muximum value of unity indicates that the coumption for the quantum efficiency of $\mathrm{CF}_{3} \mathrm{I}_{3}$ as well at tha inforred axumption that the quenching resctions are identical, are both dightly in error.
Table 16. The quantum officiencies for forming oxcitudstat photodinocinted iodine for thren fluorinated atkyl lodides

\begin{tabular}{|c|c|c|c|}
\hline Mandel & $\begin{array}{c}\text { Pmomise } \\
\text { (Ton) }\end{array}$ & 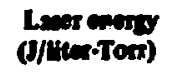 & Qumbing \\
\hline$C_{2}, 1$ & $\begin{array}{l}10 \\
20 \\
30\end{array}$ & $\begin{array}{r}0.029 \\
.031 \\
.035\end{array}$ & $\begin{array}{l}\text { ases } \\
- \\
-\end{array}$ \\
\hline$n \cdot C_{2} F_{q} I$ & $\begin{array}{l}10 \\
20 \\
30\end{array}$ & $\begin{array}{l}.055 \\
.051 \\
.059\end{array}$ & $\begin{array}{l}1.05 \\
0.93 \\
0.45\end{array}$ \\
\hline$\left|-C, F_{7}\right|$ & $\begin{array}{l}10 \\
20 \\
30\end{array}$ & $\begin{array}{c}.054 \\
.051 \\
0.050\end{array}$ & $\begin{array}{l}1.20 \\
1.04 \\
0.91\end{array}$ \\
\hline
\end{tabular}

Trom Oparn of al."129 


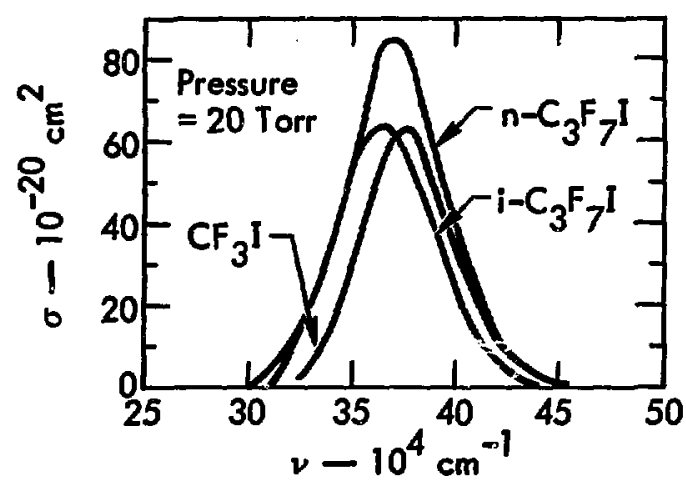

Fig. 562 Comparion of the mesared photon-absorption

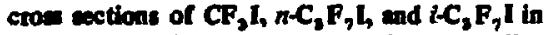
the vicinity of the pamp band for the lodine photodimocistion lacer.

\section{KINETIC MODELING}

The initial objecuive of our theoretical effort has been to develop a model describing the kinetics of CF $_{3}$ I photodissociation when diuted by noble gas at high presures (1 atm to $\geq 10 \mathrm{~atm}$ ) in order to obtain conservative estimates for the investion lifetime under a variety of experimental conditions. In this effort we can take advantage of considerable earlier basic researcin which has attempted to elicidate primary processes and determine their rates. Nevertheless, considcrable uncertainty about reactions and rates remains, and several rates have had to be extimated. Our initial extimates were deliberately convervative so that we could rapidly identify potential problem and determine if the lifetimes were adequate in the "worst case." Eventually, we will attempt to obtain reasonable sgreement with the experimental data at $1.3 \mathrm{~atm}$ by adjusting certain nte estimates. Wt hope this will permit extrapolation to hipher prescures ( $10 \mathrm{~atm})$ with fair reliability.

Previous attempts to model $\mathrm{CF}_{3} \mathrm{I}$ photolysis have ill considered low premure behavior. $123-130$ Although threebody procestes are then notigible to a good approximation, laree temperature rises $\left(>100^{\circ} \mathrm{K}\right) \mathrm{can}$ occur durins photolyais under some flachlamp pumping conditions. A lure $\Delta T$ considerably complicates the kineties, necescitates specification of the tempernture dependence of the rates, and can lead to pyrolysis under strons pumping. When we consider that several twobody rates have still not been determined even at room temperature, it is not ruppieing that low-preasure modeline attempts have not provided unambizuous azreement with experiment, even though they have apparendly been moderately succesuful at identifying the moat important reactions and providing lifetime estimates.

In contrast, photolysis under superatmospheric diluent conditions (for comparable partial pressures of CF $_{3} \mathbf{I}$ and pumping rates) according to our model produces only moderate-to-negligible temperature change $\left(\$ 50^{\circ} \mathrm{K}\right)$ as is hown below. This can considerably reduce the importance of several questionable two-body reactions and thus gubstantially increase the reliability of the two-body rate estimates. In addition, rapid thermalization of radicals created with excess transintional energy allows us to neglect other proposed reactions 131,132 having uncertain rates and/or validity. To do this, we need to specify the "three-borly" ree imbination nates for iodine atoms. Fortunately, ground-state iodine atom recombinaton is an exhanatively studied three-body proces. 13 .: 136

The approuch sdopted in our first-cut modeling effort was tos use the two-body reactions and rites used by Hohle and Kompa127,128 along with some considered by Zaleskiti, 129 Two-body deactivation of I* by either He or Ar is known to be so slow 137 thit it may be neylected even at $10 \mathrm{~atm}$. We then introduced a series of three-body seactions for jodine atom recombination in a conservative fachion.

In eeneral, we need rates at $\sim 300^{\circ} \mathrm{K}$ for three pouible types of threebody todine recombination, namely:

$$
\begin{aligned}
& I+I+M+I_{2}+M, \\
& I+I+M+I_{2}+M,
\end{aligned}
$$




$$
\mathrm{I}^{*}+\mathrm{I}^{*}+M \rightarrow \mathrm{I}_{2}+M,
$$

where $M$ is the third-body catalyst. The primary third bodies considered have been $\mathrm{He}$ or $\mathrm{Ar}_{2} \mathrm{CF}_{3} \mathrm{I}$, and $\mathrm{I}_{2}$. Several three-body tecombination rates for iodine atoms (including $M=\mathrm{He}, \mathrm{Ar}$, and $\mathrm{I}_{2}$ ) have recently been measured, 135,136 but these undoubtedly apply to the case of two ground-state $I$ atoms due to the presence of significant amounts of the very efficient quencher $I_{2}$ during the experiments.

Some insight into the estimation of three-body rates involving excited iodine atoms can be gained from the potential energy curves for $I_{2}$ shown in Fig. 153. Due to the repulsive nature of the excited $1 \Sigma_{0+}$ state, which correlates with dissociation into two $\mathrm{I}^{*}$ atoms, we would expect the rate for reaction (c) to be considerably slower than the rate for reaction (a). Likewise, the shallower well for the ${ }^{3} \Pi_{0}+u$ state, which correlates with $I^{*}+I$, might be expected to Iead to a slowet recombination rate for $I^{*}+I$.

We can also argue on physical grounds that recombination involving $I^{*}$ atoms should be slower than recombination of ground-state I atoms. To do this we use a more sophisticated analysis of three-body recombination, one that treats the recombination as a ieaction sequence involving an equilibrium

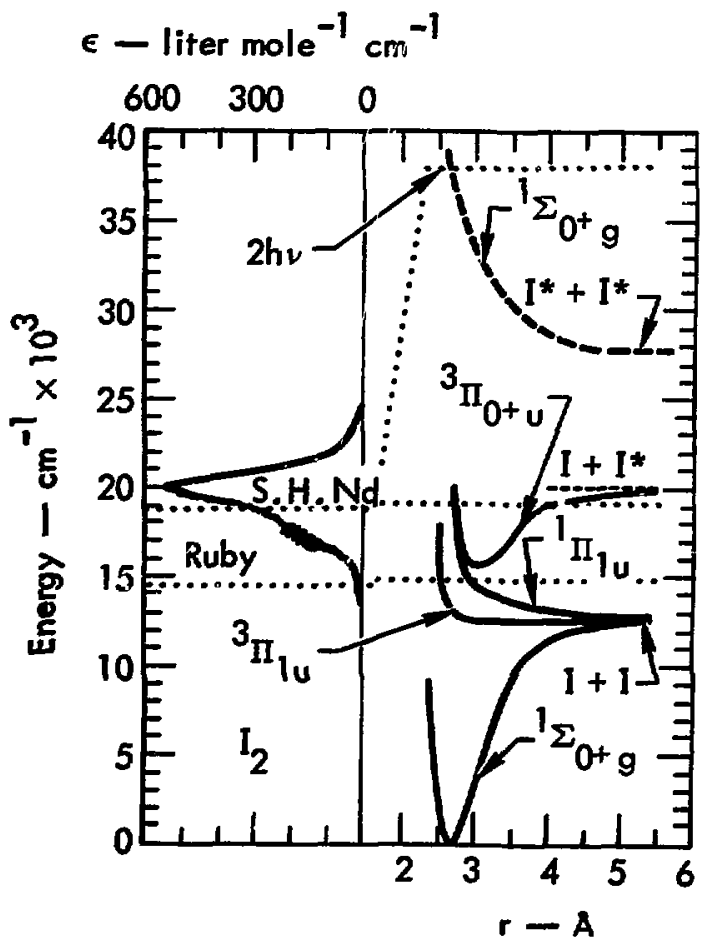

Fig. 153. Potentid curves and photon abeorption spectrum for $I_{2}$ (from Wileon' 1 "). concentration of some intermediate species. ${ }^{134}$ This follows from the physical expectation that the equilibrium concentration of intermediates involving $I^{*}$ should be lower than those involving only I. Or, putting it another way, the probability of forming weakly bound intermediates should be significantly lower for $I^{*}$ than for $I$. With these considerations in mind, it should be apparent that the estimation of three-body recombination rates in the three situations outlined below is expected to be basically conservative.

In the adoption of rates for reactions (a), (b), and (c) for different species $M$, three sitiations arise that require logically different treatment: (1) when no experimental rates are available, (2) when the experimental rate for reaction (a) has been deduced to be less than the estimated gas-kinetic three-body collision rate, and (3) when the experimental rate for (a) has been found to be greater than the estimated collision rate.

The course we followed in situation (1), where no experimentally deduced rates were available, was to set the reaction rate constants for all the reactions (a), (b), and (c) equal to a crude (order-of-magnitude) estimate for the gas-kinetic three-body collision rate. Due to the considerable uncertainty in other factors affecting the actual recombination rate constant, the simplest possible model for the gas-kinetic collision rate was employed. The model for triple collisions of $A+B+C$ which we adopted does nct proceed much further than the elementary statement that the three-body rate should simply be the two-body rate times the probability of finding a third body sufficiently close. That is,

$$
\begin{aligned}
R(A+B+C) \equiv \frac{\text { No. 3-body colls. }}{\mathrm{cm}^{3}-\mathrm{sec}} \\
=\left(\frac{\text { No. 2-body colls. }}{\mathrm{cm}^{3} \text {-sec }}\right)\left(\begin{array}{l}
\text { Probability of } \\
\text { finding a 3rd } \\
\text { body }(C) \text { nearby }
\end{array}\right) \\
=\left([A][B] v_{A B}{ }^{\sigma}{ }_{A B}\right)\left([C] \frac{4}{3} \pi\left(d_{A B}\right)^{3}\right)
\end{aligned}
$$

where

$$
\begin{aligned}
& v_{A B} \equiv \text { the relative velocity of the } A B \text { pair, } \\
& \sigma_{A B} \equiv \text { the collision cross section for } A+B, \\
& d_{A B} \equiv \text { the range of the } A B \text { interaction, }
\end{aligned}
$$

and [ ] indicates species concentration (molecules/ $\mathrm{cm}^{3}$ ). For comparison, the required three-body recombination rate, $k$, for the reaction

$$
A+B+C \rightarrow A B+C
$$


is defined by

$$
\frac{d}{d t}[A B] \equiv k[A][B][C],
$$

from which it is seen that the units of $k$ are $\mathrm{cm}^{6} /(\text { molecule })^{2}$-sec for concentrations in molecules/ $\mathrm{cm}^{3}$. From our previous conservative estimate that

$$
\frac{d}{d t}[A B]=R,
$$

we obtain

$$
k_{G K}=\frac{4}{3} \pi v_{A B} \sigma_{A B}\left(d_{A B}\right)^{3},
$$

wher 2 the subscript $G K$ indicates that we are calculating a gas-kinetic rate constant.

A considerable range of sophistication is available even in the preceding simple formula, depending on how we determine $\sigma_{A B}$ and $d_{A B}$. For our purposes, we have chosen a "hard-sphere" model to define

$$
\sigma_{A B}=\pi\left(\frac{d_{A}+d_{B}}{2}\right)^{2} \equiv \pi\left(d_{A B}\right)^{2},
$$

where $d_{A}$ and $d_{B}$ are tabulated collision diameters 139 obtainea from viscosity data. From kinetic theory it is known that $v_{A B}=\left(8 k_{B} T / \pi \mu\right)^{1 / 2}$, where $k_{B}$ is the Boltzmann constant, $\mu \equiv m_{A} m_{B} /\left(m_{A}+m_{B}\right)$, and $m_{X}$ is the mass of species $X$. Finally, our gas-kinetic formula for the recombination rate constant becomes

$$
k_{G K}=\frac{4}{3} \pi^{2}\left(d_{A B}\right)^{5}\left(\frac{8 k_{B} T}{\pi \mu}\right)^{1 / 2}
$$

For iodine atom recombination we have estimated $d_{I}$ by the tabulated collision diameter of $\mathrm{Xe}(\sim 4 \AA)$ since $X e$ is the closest atom to $I$ in the periodic table for which data is available. Then at $300^{\circ} \mathrm{K}$, with $m_{\mathrm{I}}=127 \mathrm{amu}$,

$$
k_{G K} \approx 4.26 \times 10^{-32} \frac{\mathrm{cm}^{6}}{(\text { molecule })^{2}-\mathrm{sec}}
$$

or

$$
k_{G K} \approx 1.54 \times 10^{+16} \frac{\mathrm{cm}^{6}}{(\mathrm{~mole})^{2} \cdot \mathrm{sec}}
$$

To illustrate the sigrificance of the above model, we make the following observations. The simplicity of the model is manifest in that it is independent of the nature (size, structure, etc.) of the third body. Due to its $T^{1 / 2}$ dependence, $k_{G K}$ is relatively insensitive to anticipated temperature rises of the order of $100^{\circ} \mathrm{K}$ above room temperature (which produces $\sim 15 \%$ increase in $k_{G K}$ ). However, the fifth-power dependence on $d_{A B}$ makes $k_{G K}$ rather sensitive to the details of the "collision diameter." For example, a change from $d=4 \AA$ to $d=5 \AA(25 \%$ increase in d) increases $k_{G K}$ by a factor of $(5 / 4)^{5} \approx 3$. srence to the $I_{2}$ potential energy diags am in Fig. 1 s 3 shows that our choice of $d_{1}=4 \AA$ is reasonable but that somewhat higher values could be envisioned. Even so, a factor-of-3 uncertainty is readily tolerable in our effort to get an order-of-magnitude, lower-limit estimate of inversion lifetime. In addition, it should be pointed out that for physical significance we have the requirement on the probability factor in Eq. (1) that $[C](4 / 3) \pi\left(d_{A B}\right)^{3} \ll 1$. The corresponding restriction on the diluent pressure $p_{C}$ at room temperature is:

$$
\begin{aligned}
& p_{C} \ll 76 \mathrm{~atm} \text { for } d_{A B}=5 \AA, \\
& p_{C} \ll 149 \mathrm{~atm} \text { for } d_{A B}=4 \AA .
\end{aligned}
$$

Both these limiting pressures are comfortably higher than the pressure region of interest to us (1-10 atm).

In situation (2), where the rate for $21+M$ (reaction (a)) has been exprarimentally deduced to be less than $k_{G K}$, the rates for reactions (b) and (c) were set equal to the experimental value for reaction (a).

In situation (3), where the rate for reaction (a) was experimentally deduced to be greater than $k_{G K}$ (as in the case of $M=I_{2}$ due to a relatively stable intermediate species, $I_{3}$ ), the rate for reaction (a) was set at the experimental value while the rates for reactions (b) and (c) were set equal to $k_{G K}$.

This conservative approach was intended to provide "worst case" lower limits for the inversion lifetime. To the extent that the calculated lifetimes might prove undesirably short, we can identify rates that need closer "crutiny. In addition, the sensitivity of the lietiarc to certain more questionable rate "estimates" (e.g., $I^{*}+I+I_{2} \rightarrow 2 I_{2}$ ) can be examined.

\section{The Kinsiac Model}

Any attempt to model the time dependence of the inversion in an "energy-storage" laser amplifier medium must take into account the important pumping and loss mechanisms. In our study of the $\mathrm{CF}_{3} \mathrm{I}$ photodissociation laser with substantial rare gas dilution, we have assumed that flash photolysis provides the primary pumping process via the reaction

$$
\begin{aligned}
\mathrm{CF}_{3} \mathrm{I} & +h v_{0.27 \mu \mathrm{m}} \rightarrow \mathrm{CF}_{3} \\
& +\alpha \mathrm{I}^{*}\left[{ }^{2} P_{1 / 2}\right]+(1-\alpha) \mathrm{I}\left[{ }^{2} P_{3 / 2}\right]
\end{aligned}
$$


Where $\alpha$ is the fraction of iodine atoms formed in the excited state (I* quantum yield). Laser action occurs on the spin-flip transition by the "reaction"

$$
\begin{aligned}
\mathrm{I} *\left[2 P_{1 / 2}\right]+h \nu_{1.315 \mu \mathrm{m}} & \rightarrow\left[{ }^{2} P_{3 / 2}\right] \\
& +2 h \nu_{1.315 \mu \mathrm{m}^{\circ}}
\end{aligned}
$$

We have neglected any direct chemical pumping of I* since present experimental evidence for it is at best weak and ambiguous. 140 Moreover, the highly dilute condition $\left(p_{\mathrm{CF}, \mathrm{I}} \ll p_{\mathrm{He}}\right)$ leads to rapid thermalization of hot radicals, minimizes thermal dissociation, and eliminates pyrolysis, thereby pracluding several potential sources of direct chemical pumping.

Radiative losses of $I^{*}$ have also been neglected in our model. Spontaneous emission has been ignored due to the long radiative lifetime $(\tau \approx 0.13 \mathrm{sec})$ of $I^{*} .141$ Stimulated emission has not been considered since our objective is to estimate the potential stored-energy density and inversion lifetime under pressure-broadened conditions, where the cross section for stimulated emission is sufficiently low that superfluorescence is suppressed in an amplifier having useful dimensions.

Thus losses are limited to collisional or reactive quenching. Heterogeneous loss of $I^{*}$ via wall collisions is certainly negligible in the interesting regions of diluent pressure $\left(>_{1} \mathrm{~atm}\right)$, tube diameter $\left(>_{1 \mathrm{~cm}}\right)$, and time $(<100 \mu \mathrm{sec})$ as can readily be verified from the diffusion lifetime formula and data by Husain and Donovan. ${ }^{137}$ Therefore, we consider only homogeneous collisional phenomena including chemical reactions as loss mechanisms, and radicals are assumed to be thermalized before reaction.

A list of collisional/reactive species which were considered in our model to be present during and after photolysis and which could influence the inversion is given in Table 17. These species were incorporated into a model consisting of the 22 reactions listed in Table 18. The values of the rate constants used in the simultaneous numerical solution of the associated set of differential equations (including the flashlamp

Table 17. List of collisional/reactive spocies treated by the kinetic iodine-laser model

\begin{tabular}{ll} 
Diluent & He or Ar \\
Active medium & $\mathrm{CF}_{3} \mathrm{I}$ \\
Lesing species & $\left.\mathrm{I}^{*}\left[{ }^{2} P_{1 / 2}\right], \mathrm{I}^{2} P_{3 / 2}\right]$ \\
Other radical & $\mathrm{CF}_{3}$ \\
Resction products & $\mathrm{C}_{2} \mathrm{~F}_{6}, \mathrm{I}_{2}$ \\
Impurity & $\mathrm{O}_{2}$ \\
\hline
\end{tabular}

pumping dynamics) are also listed with an indication of the data source. The reaction equations are grouped by type:

\begin{tabular}{cll}
$\begin{array}{c}\text { Reaction } \\
\text { number }\end{array}$ & & Type \\
\cline { 1 - 1 } $1-5$ & & Two-body deactivation \\
$6-8$ & & Two-body recombination \\
$9-10$ & & Radical-molecule reaction \\
$11-14$ & & Three-body recombination $\left(I^{*}+I^{*}\right)$ \\
$15-18$ & & Three-body recombination $\left(I^{*}+I^{\prime}\right.$ \\
$19-22$ & & Three-body recombination $\left(I^{*}+\right)$
\end{tabular}

Several possible reactions have been omitted for reasons indicated earlier.

\section{Calculational Tools}

The computer code NEST ${ }^{142}$ has been adapted to handle the kinetics of $\mathrm{CF}_{3} \mathrm{I}$ photolysis for various primary $I^{*}$ quantum yields $I^{*} /\left(I+I^{*}\right)$ in the presence of a variety of pressure-broadening diluent gases (e.g., He, Ar). The code has previously been used in studies of $\mathrm{H}_{2}+\mathrm{F}_{2}$ reaction kinetics with photolytic initiation at LLL. ${ }^{143}$ NEST formulates and numerically solves a set of simultaneous differential rate equations for a specified set of reactions and rate constants. Among other things, it provides the time dependence of the concentrations of the various species, effective reaction rates, temperature, pressure, etc. Such information was used to produce the plots presented below.

At present, lack of suitable thermodynamic and kinetic information precludes effective modeling of the photolysis of other fluorinated alkyl iodides that have been used in photodissociation lasers.

\section{Computational Results}

Within the context of the first-cut philosophy, calculational tools, and kinetic model described above, a wide range of experimental parameter space can be simulated. The initial results (presented below) were obtained for the following conditions.

The partial pressure of $\mathrm{CF}_{3} \mathrm{I}$ was held fixed at 100 Torr. The pressure of He diluent in the range $0 \leqslant p_{\mathrm{He}} \leqslant 10$ atm was the main parameter varied. The only other variation in initial conditions was the $\mathrm{O}_{2}$ impurity concentration, which was assumed to be directly proportional to the He concentration at a level of $0.8 \mathrm{ppm}$. This was intended to roughly simulate the deactivation from impurities (mainly $\mathrm{O}_{2}+\mathrm{H}_{2} \mathrm{O}$ ) in Matheson "Ultra High Purity (Gold Label)" He (99.999\%). 144

The initial temperature was set at $300^{\circ} \mathrm{K}$ in all cases.

The flashlamp profile used is shown in Fig. 154. It was intended to roughly simulate, in a convenient 


\begin{tabular}{|c|c|c|c|c|c|c|}
\hline \multirow{3}{*}{$\begin{array}{c}\text { senction, } \\
R\end{array}$} & \multirow{3}{*}{$\begin{array}{c}\text { Reactant } \\
\text { species }\end{array}$} & \multirow{3}{*}{$\begin{array}{c}\text { Product } \\
\text { species }\end{array}$} & \multicolumn{2}{|c|}{$k_{f t}$ values ${ }^{\mathrm{a}}$} & \multirow[b]{3}{*}{ References } & \multirow{3}{*}{ values $^{\mathrm{v}}$} \\
\hline & & & $\left.\left(\mathrm{cm}^{3} / \text { mole }-1 \mathrm{e}\right)^{n}\right]^{\mathrm{b}}$ & $\left(\mathrm{cm}^{3} / \mathrm{mole}^{n}\right]^{\mathrm{b}}$ & & \\
\hline & & & $\sec$ & $\sec$ & & \\
\hline 1 & $1^{*}+\mathrm{CF}_{3} \mathrm{I}$ & $\mathrm{I}+\mathrm{CF}_{3} \mathrm{I}$ & $4 \times 10^{-16}$ & $2.41 \times 10^{8}$ & 128 & $-3 / 2$ \\
\hline 2 & $\mathrm{I}^{*}+\mathrm{CF}_{3}$ & $1+\mathrm{CF}_{3}$ & $3.7 \times 10^{-12}$ & $2.23 \times 10^{12}$ & 128 & $-3 / 2$ \\
\hline 3 & $I^{*}+C_{2} F_{6}$ & $\mathbf{I}+\mathrm{C}_{2} \mathrm{~F}_{6}$ & $4.3 \times 10^{-16}$ & $2.59 \times 10^{8}$ & 128 & $-3 / 2$ \\
\hline 4 & $I^{*}+\mathbf{O}_{2}$ & $1+0_{2}$ & $8.6 \times 10^{-12}$ & $5.18 \times 10^{22}$ & 128 & $-3 / 2$ \\
\hline 5 & $I^{*}+I_{2}$ & $1+I_{2}$ & $5 \times 10^{-12}$ & $3.01 \times 10^{12}$ & 128 & $-3 / 2$ \\
\hline 6 & $2 \mathrm{CF}_{3}$ & $\mathbf{C}_{2} \mathbf{F}_{6}$ & $1 \times 10^{-11}$ & $6.02 \times 10^{12}$ & 128 & - \\
\hline 7 & $1+\mathrm{CF}_{3}$ & $\mathrm{CF}_{\mathbf{3}} \mathbf{I}$ & $5 \times 10^{-11}$ & $3.01 \times 10^{13}$ & 128 & $+1 / 2$ \\
\hline 8 & $\mathrm{I}^{*}+\mathrm{CF}_{\mathrm{s}}$ & $\mathrm{CF}_{3} \mathrm{I}$ & $5 \times 10^{-13}$ & $3.01 \times 10^{11}$ & 127 & -1 \\
\hline 9 & $\mathbf{I}+\mathrm{CF}_{3} \mathrm{I}$ & $\mathrm{I}_{2}+\mathrm{CF}_{3}$ & $\begin{array}{l}1.22 \times 10^{-10} \\
\times \exp [-17,800 / R T]\end{array}$ & $\begin{array}{l}7.34 \times 10^{13} \\
X \exp [-17,800 / R T]\end{array}$ & 129 & $+1 / 2$ \\
\hline 10 & $:=\mathbf{C F}_{\mathbf{3}} \mathbf{I}$ & $\mathrm{I}_{2}+\mathrm{CF}_{3}$ & $3 \times 10^{-18}$ & $1.8 \times 10^{6}$ & 129 & -1 \\
\hline 11 & $2 I^{*}+M_{6}^{d}$ & $I_{2}+M_{6}^{d}$ & $4.26 \times 10^{-31}$ & $1.54 \times 10^{16}$ & $-c$ & -2 \\
\hline 12 & $2 I^{*}+C F_{3} I$ & $\mathrm{I}_{2}+\mathrm{CF}_{3} \mathrm{I}$ & $<10^{-34}$ & $<3.62 \times 10^{13}$ & 128 & -2 \\
\hline 13 & $2 I^{*}+I_{2}$ & $2 \mathbf{I}_{3}$ & $4.26 \times 10^{-32}$ & $1.54 \times 10^{16}$ & $-e$ & -2 \\
\hline 14 & $2 \mathrm{I}^{*}+\mathrm{He}$ & $\mathrm{I}_{2}+\mathrm{He}$ & $<2.76 \times 10^{-33}$ & $<1 \times 10^{15}$ & 135 & -2 \\
\hline 15 & $2 \mathrm{I}+M_{6}^{\mathrm{d}}$ & $\mathrm{l}_{2}+M_{6}^{\mathrm{d}}$ & $4.26 \times 10^{-32}$ & $1.54 \times 10^{16}$ & $--^{e}$ & +1 \\
\hline 16 & $2 \mathrm{I}+\mathrm{CF}_{\mathbf{3}} \mathrm{I}$ & $\mathrm{I}_{2}+\mathrm{CF}_{3} \mathrm{I}$ & $<10^{-34}$ & $<3.62 \times 10^{13}$ & 128 & +1 \\
\hline 17 & $2 I+H e$ & $\mathrm{I}_{2}+\mathrm{He}$ & $2.76 \times 10^{-33}$ & $1 \times 10^{13}$ & 135 & +1 \\
\hline 18 & $2 I+I_{2}$ & $\mathbf{2 I}_{2}$ & $3.86 \times 10^{-30}$ & $1.40 \times 10^{18}$ & 136 & +1 \\
\hline 19 & $I^{*}+I+M_{6}^{d}$ & $I_{2}+M_{6}^{d}$ & $4.26 \times 10^{-32}$ & $1.54 \times 10^{16}$ & $-e$ & $-1 / 2$ \\
\hline 20 & $I^{*}+I+\mathrm{CF}_{3} I$ & $1_{2}+C F_{3} 1$ & $<10^{-34}$ & $<3.62 \times 10^{13}$ & 128 & $-1 / 2$ \\
\hline 21 & $I^{*}+1+\mathrm{He}$ & $I_{2}+H e$ & $<2.76 \times 10^{-33}$ & $<1 \times 10^{15}$ & 135 & $-1 / 2$ \\
\hline 22 & $1^{*}+1+1_{2}$ & $2 \mathrm{I}_{2}$ & $4.26 \times 10^{-32}$ & $1.54 \times 10^{16}$ & $-c$ & $-1 / 2$ \\
\hline
\end{tabular}

${ }^{2}$ The $k_{f r}$ value is the rate constant for the forward reaction (reactant species $\rightarrow$ product species).

${ }^{b}$ For two-body reactions $n=1$, for three-body reactions $n=2 . R=1.99 \mathrm{cal} / \mathrm{mole}^{\circ} \mathrm{K}$ (in reaction 9 ).

$c_{\text {The }} v_{f,}$ value is the weighting factor for the forward reaction describing its net effect on the inversion, $\Delta N \equiv I^{*}-$ th 1 . A negative value of $v_{f r}$ means that the forward reaction reduces the inversion.

${ }^{\mathrm{d} y} \mathrm{M}_{6}$ we mean any thing other than $\mathrm{He}, \mathrm{I}_{2}$, or $\mathrm{CF}_{3} I$ (i.e., $I^{*}, \mathrm{I}, \mathrm{CF}_{3}, \mathrm{C}_{2} \mathrm{~F}_{6}, \mathrm{O}_{2}$ ).

Maximum rate estimated from gas kinetics.

inathematical form, the rise and fall times of an experimental apparatus used at LLL. ${ }^{145}$ The simulated lamp rises linearly to peak output in $1 \mu \mathrm{sec}$, falls exponentially with a $2-\mu \mathrm{sec}$ decay constant, has a width (FWHM) of $\sim 2 \mu \mathrm{sec}$, and the area under the pulse is equal to that of a square pulse with unity height and a width of $2.5 \mu$ sec. The pump profile was nomalized so that a peak inversion corresponding to $\sim 1$ Torr at room temperature $\left(\sim 3.3 \times 10^{16}\right.$ molecules $)$ $\mathrm{cm}^{3}$ ) was produced. The normalization used results in photodissociation of $24 \%$ (independent of He pressure) of the initial $\mathrm{CF}_{3} 1$ concentration ( 100 Torr at $300^{\circ} \mathrm{K}$ ).
In the numerical calculations, the exponential tail was truncated to zero at $13 \mu \mathrm{sec}$.

For all the results shown below, the primary $I^{*}$ quantum yield on photodissociation was set equal to 0.67. This is based on the exper nental deduction of $\alpha=0.67$ by Ogawa et al. ${ }^{122}$ and is consistent with the relative primary quantum yields of other fluorinated alkyl iodides determined by Gregg and Huss. ${ }^{146}$

Figure 155 shows the time dependence of the inversion $\left(\Delta N \equiv I^{*}-I / 2\right)$ calculated for various He diluent pressures ranging from 0 to $10 \mathrm{~atm}$. The peak inversion for $10 \mathrm{~atm} \mathrm{He}$ is only $16 \%$ lower 
than that for no He. The peak inversion time lies between 4 and $7 \mu \mathrm{sec}$ in all cases and tends toward the earlier time as the He pressure is raised. The

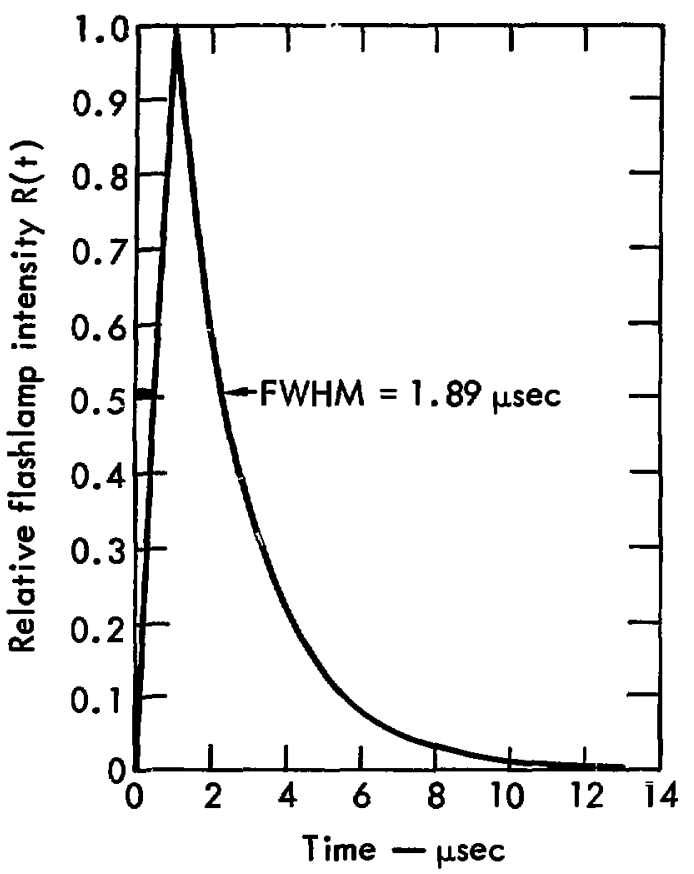

$R(t) \equiv\left\{\begin{array}{l}\text { at, } 0 \leq t \leq 1 \mu \text { sec } \\ -\left(t-t_{U}\right) \\ \exp , 1 \mu \text { sec } \leq t<\infty\end{array}\right.$

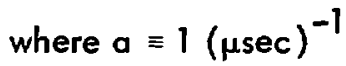

$t_{0} \equiv 1 \mu \mathrm{sec}$

$\tau \equiv 2 \mu \mathrm{sec}$

units of $\uparrow \equiv \mu \mathrm{sec}$

$\int_{0}^{\infty} R(t) d t=\frac{a_{0}{ }^{2}}{2}+\tau=2.5 \mu \sec$

Fig. 154. Relative flashlamp intensity profile, $R(t)$, used in the kinetic calculations. Note that $R(t)$ is dimensionless. For the calculations presented in the following figures, the dissociation rate is normalized as follows:

$P(t)=3.09 \times 10^{22} \times R(t)$ molecules $/ \mathrm{cm}^{3}$-sec.

This normalization provided an inversion of about 1 Torr at low He dilution and about 2.4\% dissociation of the initiol pressure of $\mathbb{C F}_{3} \mathbf{I}$ (100 Torr). The dissociation fraction does not take into account chemical recombination. width between $90 \%$ of maximum points varies from $\sim 17$ to $\sim 4 \mu \mathrm{sec}$ with increasing He concentration.

The time from peak inversion until the inversion has fallen to half its peak value, i.e., $t_{1 / 2} \equiv t_{p / 2}-t_{p}$, is plotted as a function of the partial pressure of $\mathrm{He}$ in Fig. 156. Note from the slope of the $\log -\log$ plot that $t_{1 / 2}$ is very nearly proportional to $\left(p_{\mathrm{He}}\right)^{-1 / 2}$ with only a very slight deviation even at $10 \mathrm{~atm}$. This relatively modest pressure dependence is very striking in its simplicity; its significance, if any, is not completely understood.

The calculated change in temperature $(\Delta T)$ and pressure $(\Delta p)$ as a result of the photolysis are plotted versus the He partial pressure in Fig. 157. The $\Delta T$ curve asymptotically approaches an inverse dependence on $p_{\mathrm{He}}$ at high $p_{\mathrm{He}}$. This is expected for constant energy input to a medium consisting essentially of one species and one constant heat capacity. As mentioned earlier, the progressively smaller $\Delta T$ achieved when $p_{\mathrm{He}}$ is increased favors the accuracy of many of the rates and the adequacy of the two-body reactions of the model.

The $\Delta p$ curve in Fig. 157 agrees quite well with what one would calculate using the $\Delta T$ curve and the ideal gas relationship, $p_{1} / p_{2}=T_{1} / T_{2}$. The result is

$$
\Delta p=\left(\frac{p_{1}}{T_{1}}\right) \Delta T=\frac{\left(p_{\mathrm{CF}_{3} \mathrm{I}}+p_{\mathrm{He}}\right)_{1}}{300^{\circ} \mathrm{K}} \Delta T .
$$

For high $p_{\mathrm{He}}$ (much higher than $p_{\mathrm{CF}_{3} \mathrm{I}}$ ) the $\Delta T$ curve shows that $\Delta T$ ci $1 / p_{\mathrm{He}}$ and we therefore expect $\Delta p$ to approach a constant value. It is comforting that this appears to be happening in Fig. 157. The modest values of $\Delta p / p_{\mathrm{He}}$ under suparatmospheric conditions obviously pose no structural threat, in contrast to the shock waves of some chemical lasers.

In order to provide a better understanding of the reactive pumping and quenching mechanisms for the inversion as a function of time for different regimes of diluent pressure, we present in Figs. 158-160 graphs of the net "effective" reaction rates $v_{f r}\left(L_{f r}-L_{b r}\right)$ for the main reactions affecting the inversion. The net reaction rate for a particular reaction $R$, taking into account the species concentrations as well as ooth the forward and backward rate constants, is represented by $\left(L_{f r}-L_{b r}\right)$. The factor $v_{f r}$ is a weighting factor that describes the effect of the forward reaction on the inversion $\Delta N=I^{*}-1 / 2$. For example, $\nu_{f r}=-3 / 2$ for reaction 1 in Table 18 since it decreases the $I^{*}$ concentration by one atom while increasing the I concentration by one atom. The result is equivalent to decreasing $\Delta N$ by $3 / 2$ atoms. The factors $\nu_{f r}$ are given in Table i8 for all the reactions. Note that $v_{f r}<0$ implies a net quenching effect, whereas $\nu_{f r}>0$ implies a net pumping of $\Delta N$. 


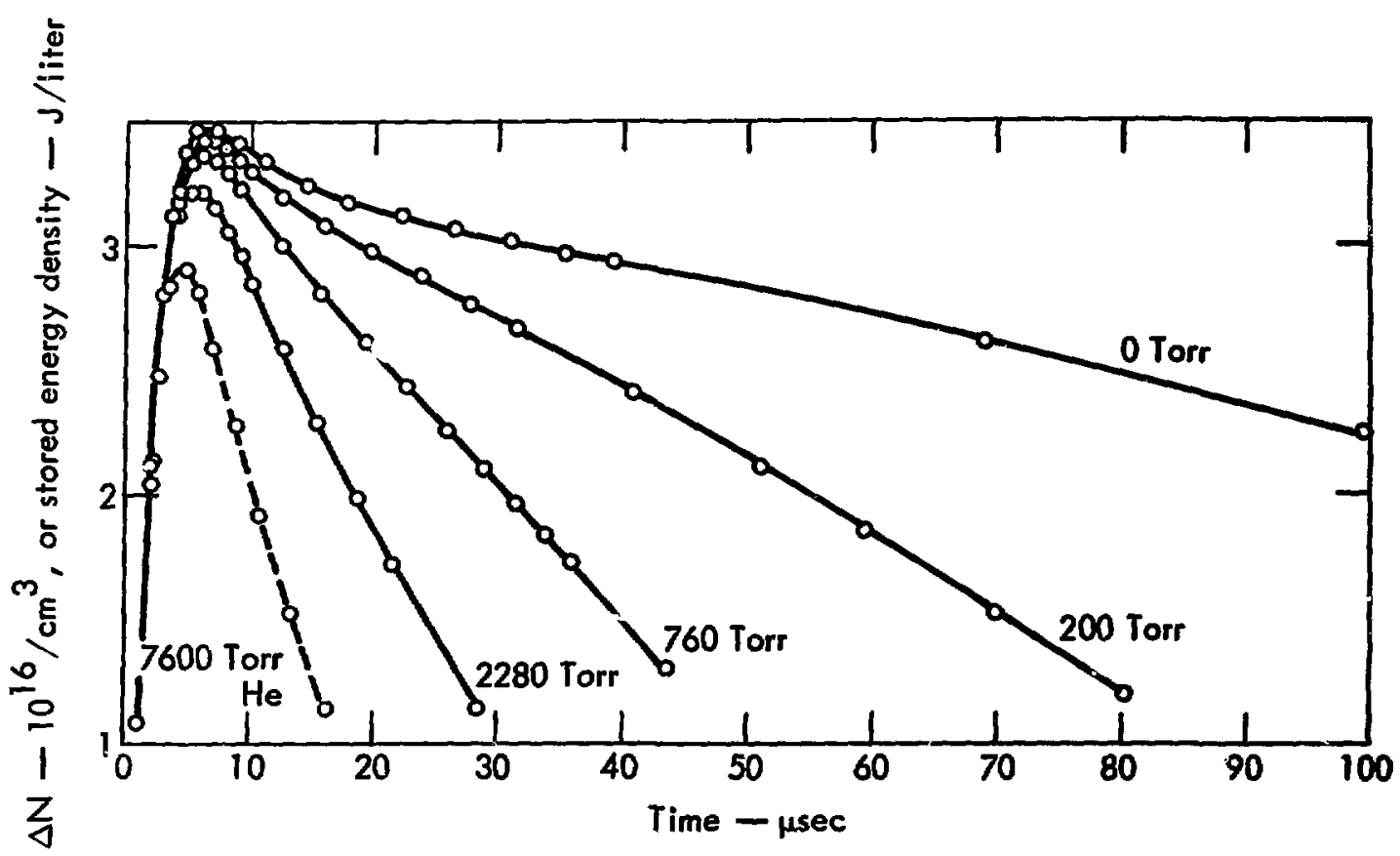

Fig. 155. Time dependence of the celculated inversion $\Delta W$ for a constant initid pertid presmre of CF, 1 (100 Ton) and

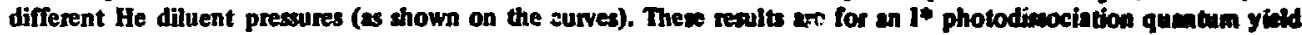
$\alpha=0.67$. Note that the ordinate provides either $\Delta N\left(10^{16}\right.$ moleculed $\left./ \mathrm{cm}^{3}\right)$ or, very nearly, the stosed enery deasthy (J/liter) since $(2 / 3) h \nu \approx 10^{-19} \mathrm{~J}$.

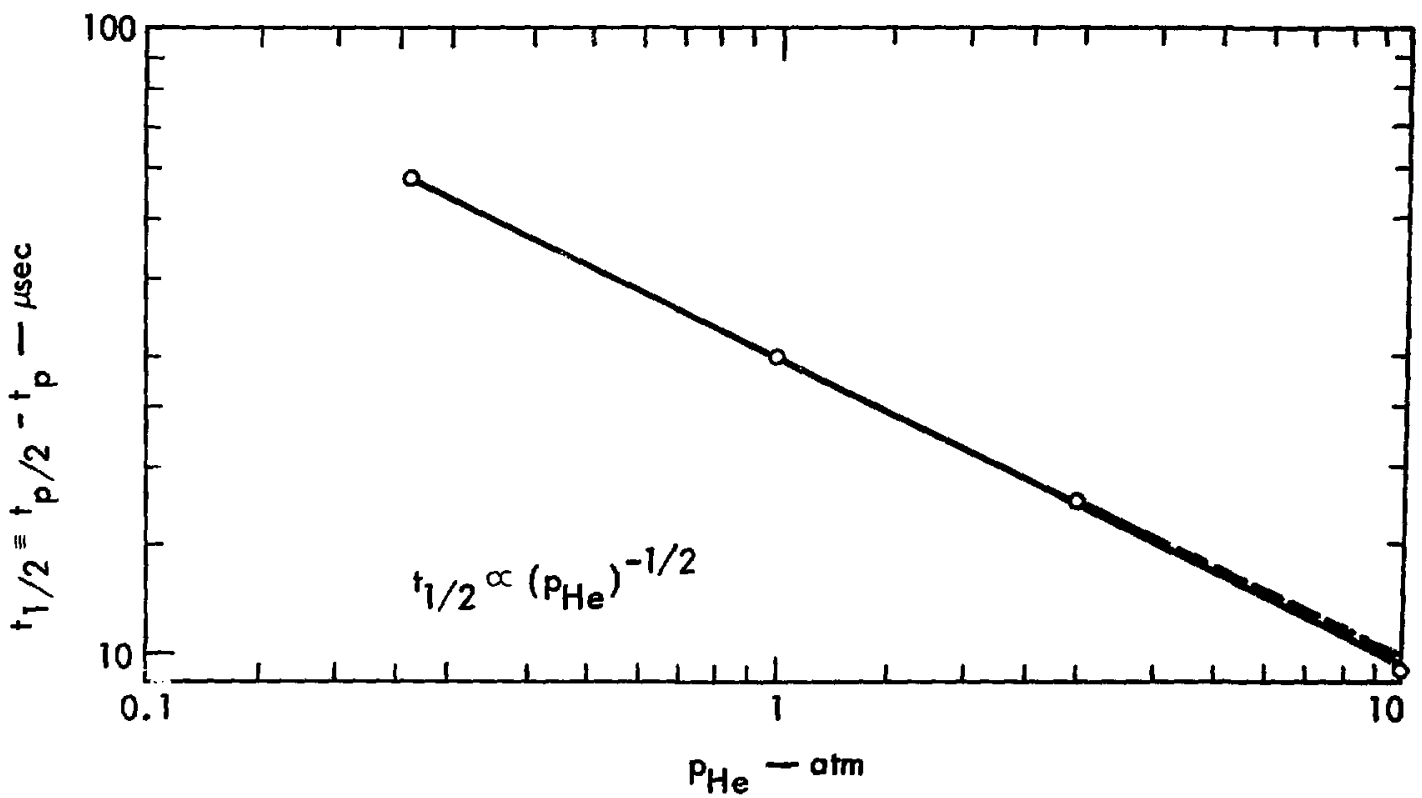

Fig. 150. Half-life of the inversion as a function of the He diluent presure. Solid line connects the culculated pointa amoothly. Dashed line indicates the extrapolation of the initial liner portion of the calculated curve thowing $t_{1 / 2} \propto\left(p_{H e}\right)^{-1 / \pi}$. 


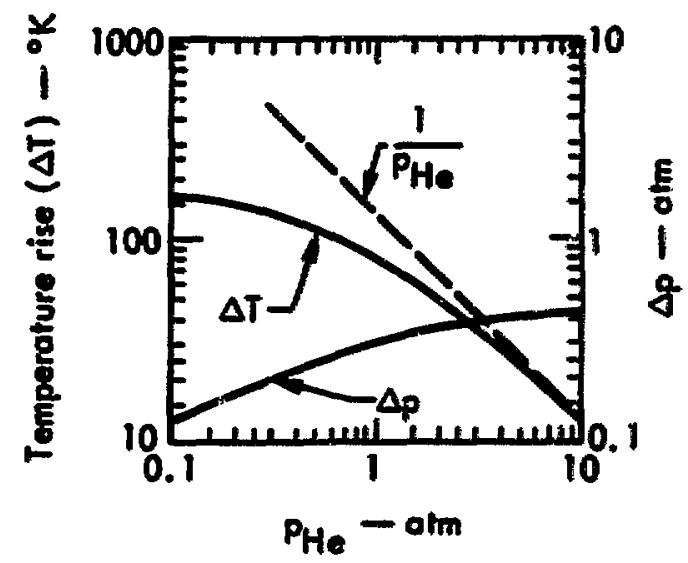

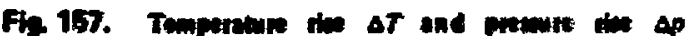

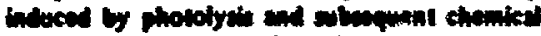

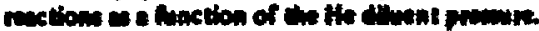

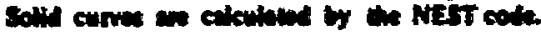

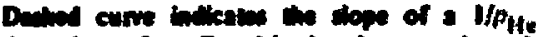

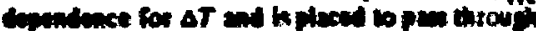
cre celculand vilus at phe $=10 \mathrm{ath}$.

The fashiamp contribution to the inversion does not fit conveniently on the scale of Fig. 158-160. Consequently, cnly a portion of its tell has been shown in these figures. The full curve may be obtuined from Fig. 160 by noting that the pumping rate is of $(1)$ while the quenching rate is $[(1 \cdot a) / 2 \mid P(t)$. Therefore,

$$
\frac{d}{d t}\left(\left.\Delta M\right|_{\text {Jleshtomp }}=\left(\frac{3}{2} a \cdot \frac{1}{2}\right) f(t)\right. \text {. }
$$

For $=2 / 3$ we see that the net effective pumping rate is only $P(t) / 2$. This points out dramatically the desirability of using a photolytic msserial having a quantum efficiency $\alpha=1$ ff pumping efficiency is concern.

One major point of interes for our morel is the effect of intirect chemicel pumpine such pumping is sen from Fie: 158-160 to be esentially confined to reaction 7 within the $\Delta N=\Delta N_{\text {peek }} / 2$ points. Nole. however, that the effective nate of rewctions 2 and 7 in esentidly identical (within the rule-constent uncertanty limits) in the region of interest $(t \geq 5$ unec) wher the fistlamp nte of contribution to quenching has allen below the contribution due to rexction 2. Thus, within the context of our model and the validity of the recontents und, indirest chemical pumping ts presuat but herdly able to keep up with the quenehing due to the deactivation by $\mathrm{CF}_{3}$. If the (uncertain) mate for cesction 2 were a little smalker, the indinet pumping resction 7 could provide a smali increas in $\Delta N$ and migh explein the weak "chemical oumpins" observed by Howl and Komps 127.120 after cermintion of the fexhemp pulse.

Some schemes hav been proposed147 for chemical pumping that involve exim CF, production. It is clear from the delicate bulmce between reactions 2 and 7 indicated here that these schemes must be curefully eriluased before a positive effect en be defended.

Another major point of interest is that in highpresure tie diluent cases (Fip. IS9 and 160) resction 14 involving awo t" atons is the muin threebody deactivation mechanism on the inversion tail. We should reatl that the rate for reaction 14 is probably significently overestimated and note that reaction 14 is a prime contritutor of the $t_{2}$ that is driving reaction 5 . Hence, hould expect to find considerably loneer decuy times experimentally, and very early results indiente this to be the case. If In

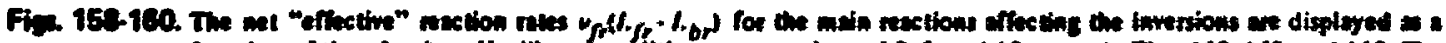

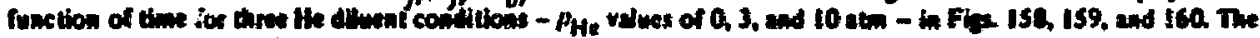

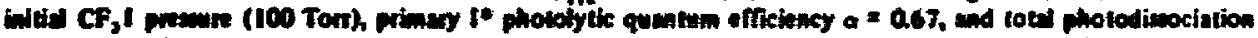

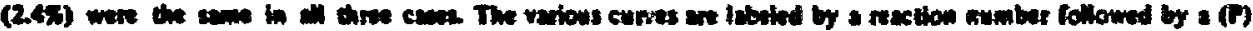

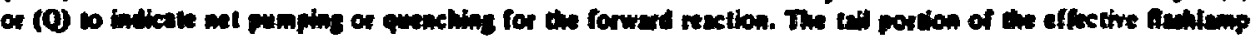

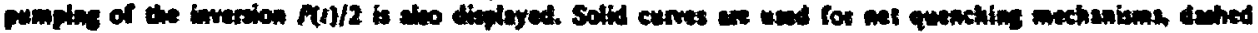

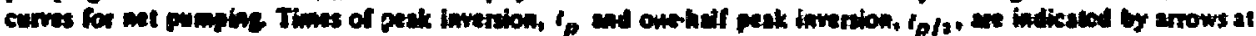

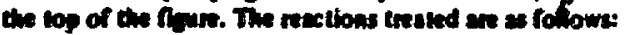

$$
\begin{aligned}
& \text { Rl: } \mathrm{I}+\mathrm{CF}_{3} \mathrm{i} \rightarrow \mathrm{I}+\mathrm{CF}_{\mathrm{a}} \mathrm{I} \\
& \text { 22: } 1 *+C F, \rightarrow 1+C F \text {, } \\
& \text { R4: } 1 *+0, \rightarrow 1+0 \text {, } \\
& \text { RS: } I^{*}+I_{2} \rightarrow 1+I_{2} \\
& \text { R7: } 1+\mathrm{CF}_{3} \rightarrow \mathrm{CF}_{2} \mathrm{I}
\end{aligned}
$$

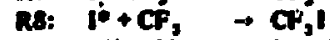

$$
\begin{aligned}
& \text { RII: } 2^{*}+M_{4} \rightarrow I_{2}+M_{0} \text {, where } A_{6}=10,1, C F_{2}, C_{2}, t_{0}, O_{2} \\
& \text { RI4: } 2^{*}+\mathrm{Hi} \rightarrow \mathrm{I}_{2}+\mathrm{He}
\end{aligned}
$$

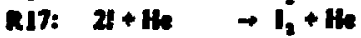

$$
\begin{aligned}
& \text { R21: } 10+i+i k \rightarrow I_{2}+H_{e}
\end{aligned}
$$




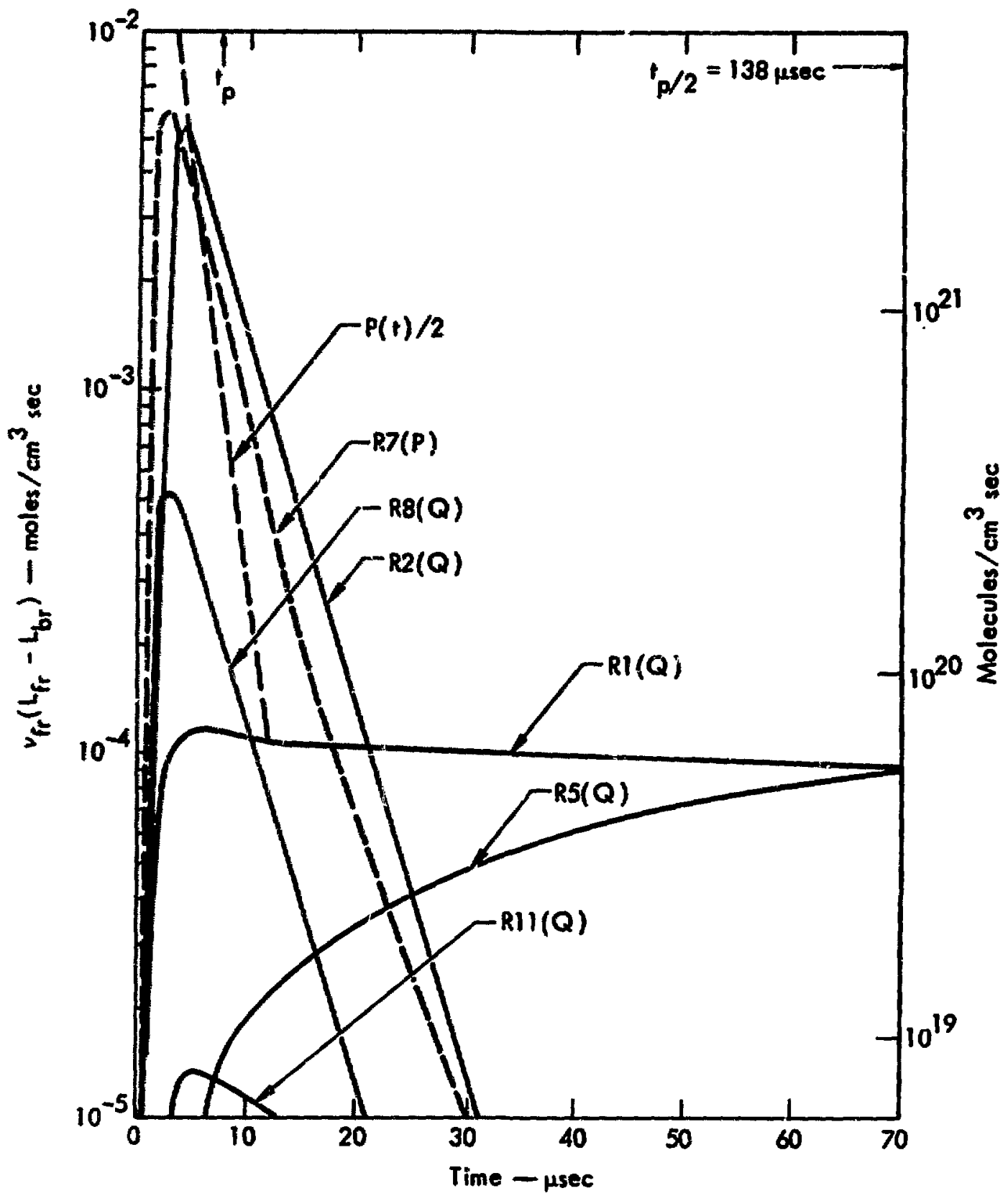

Fie. 150. Helium diment partiat prewne, 0 atm (ao helium).

all cases examined, $I_{2}$ has tumed out to be the dominant deactivator at long times, although reaction 14 is of considerable importance at $P_{\text {tle }}=10$ atm just from the standpoint of dedetivation.

Our first effor's at conservalively modeling $\mathbf{C F}_{\mathbf{3}}$ I photodisscciation kinetics when heavily difuted with
He for pressure-broadening purposes have indicated that even with a primary $1^{*}$ quantum yield of 0.67 , inversions of the order of 1 Torr can be produced and maintained for times $\gtrsim^{10} \mu$ sec even at $p_{\text {He }}=10 \mathrm{~atm}$. The inversion lifetimes calculated seem adequately long for short-pulse laser amplifiers. Aso, the lifetine at 10 atm is probably adequate for 


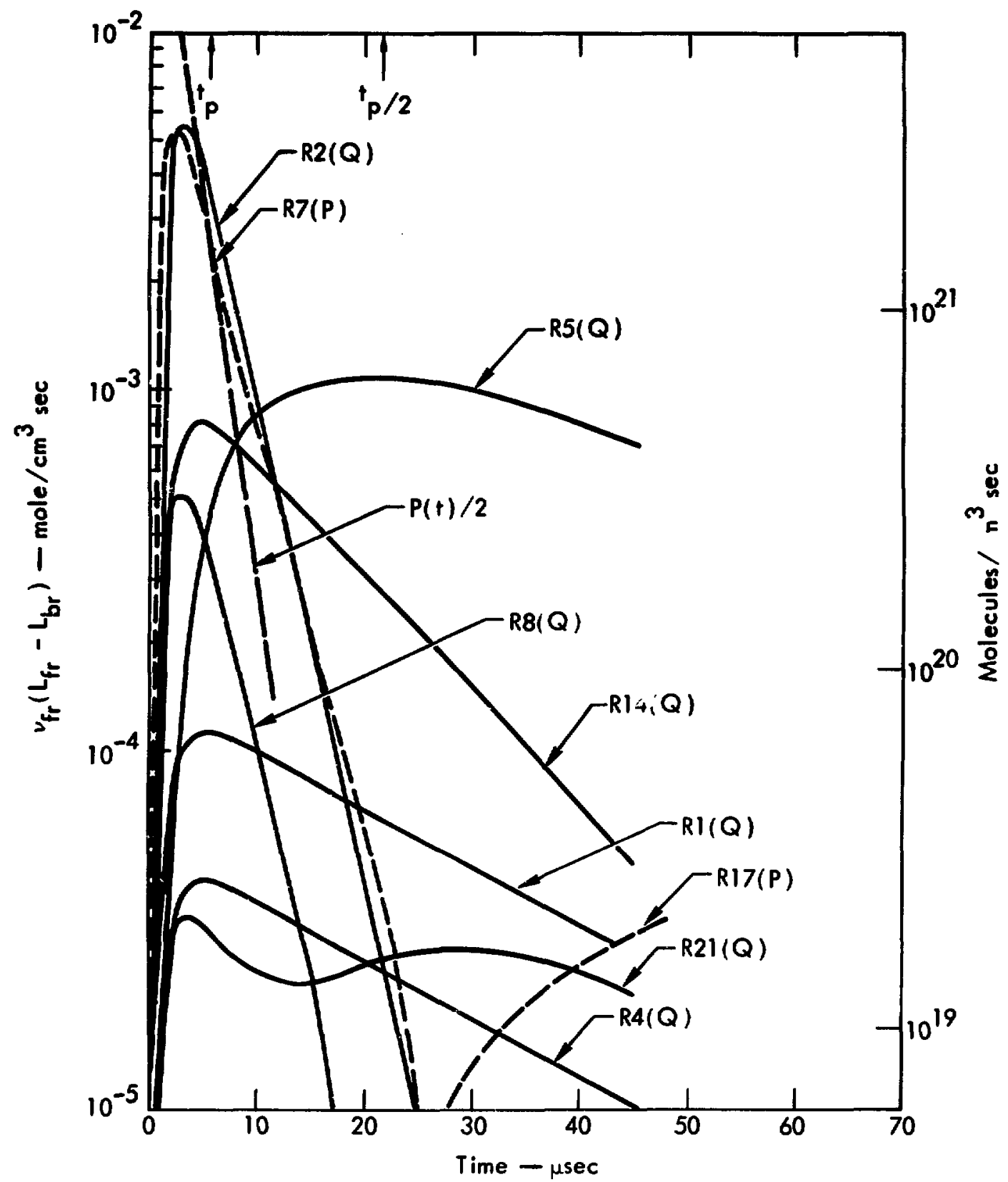

Fig. 159. Helihm diluent partial pressure, 3 atm.

short-pulse production using active mode-locking. However, this problem needs closer scrutiny in light of recent developments on the theory of transient mode-locking. 149

We still need to compare our calculations with suitable data for a closer evaluation of the projection capabilities of our model. However, it is encouraging that our attempt to simulate worst-case kinetics yields inversion lifetimes at high He-diluent pressures that do not preclude the possibility of exploiting pressure broadening for significant reduction of the stimulatedemission cross section in the iodine laser. The resultant increase in predicted energy-storage capability and the ability to amplify pulses shorter than 100 psec argue for the continued careful evaluation of the iodine laser for potential laser-fusion applications. 


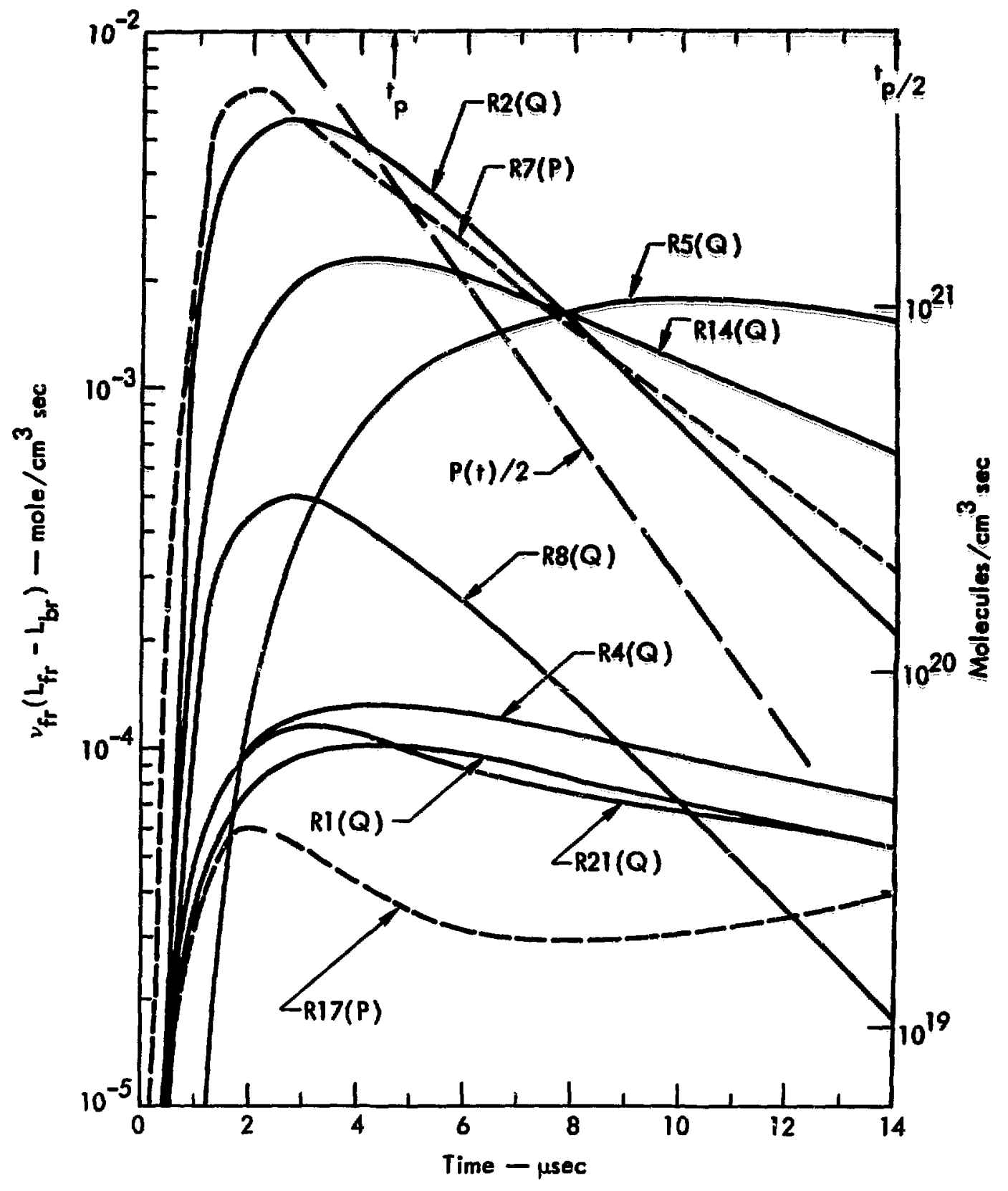

Fig 160. Hellum diluent partial prescure, 10 atm. 


\section{BASIC STUDIES AND ADVANCED CONCEPTS}

\section{MThosustion}

Durins the pat six monthe rexeych has provened in four broud ares of investiation - lifhi propan. tion celculations, experimental device deviopment, frequency compraion tochniques, and leser materials studies. In the following article the man points of propen in esch of thes anes ape reported.

The research deacribed here is beine carried out in apport of the lerer fusion effort. Since laer fusion drvices entell operation at the hidhest power densitios chieveble, monlines optical effects ap ungvoidably present. Detried celculations of litht propagtion in nonlines modia ure required for understendins

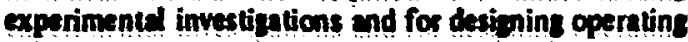
oysume. The empheis in the present research ho been pleced on developins fort and efricient technigues for "thetching" the lint propsation, 4 opposed to the very ecurate but timesconaming computationd mothod drendy in existence. Lorencien codes provide a suitsble technique for achievine this soil. The Lepranien method has been applied to the computation of steady atave and time-dependent cases fis stimulated Ruman scatering.

The rowth of smdl-scale selfofocusing is the principal limitation to the operation of dass issers in the short pule regime. $A$ brief analytical estimate of the threshold for smallecele selffocusing is siven, howing that small filaments con develop over a broad mee of spatial frequencies.

Thene small perturbations in phase and amplitude ariee, even for en ided beam, from innate inhomopentities in the laer material. It is shown that for an $\mathrm{ms}$ variation of $\lambda / 8$ in the optical path in the medium, intensity fluctuations of the order of 100\% will develop in a distance of approximately $2 R_{\text {min }}$, where $2_{\text {min }} i$ the e-folding length for the growth of the most unstable spatial frequency. Thus the amall-ecale instability imposes a very real limit in the design of any Imer system.

Another manifestation of the index nonlinearity in laser das is the spectral broadening associated wilh selfphase modulation. The combined effects of self-phase modulation and linear dispersion have been investigated for both $\mathrm{CS}_{2}$ and laser glass, over a range of pulce lengths of interest. Computed frequency spectra are given $a$ a function of the parameters of the pulse and the distance, traveled in the medium.

In the area of device development, the 20-psec mode-locked nuby lases system has been improved.
Preliminary studies have been carried out on the desien of a ruby disk system, although no sctual construction of this device is snticipated. The ruby system presently enerales $250 \mathrm{~m}$ from an aperture $1.5 \mathrm{~cm}^{2}$ in area. Extention to $1102 \mathrm{~J}$ with an area of $5 \mathrm{~cm}^{2}$ is discusyd. This device is being used for experimenls in frequency convession at high intensity via nonlinear procesist.

The possibility of constructing a small-diameter (1.3-cm) Nd:YAG disk laet he been invalizated. Design considerations and tat resulu are preatented. Serious problem have been encountered in obtain. ine lever mulerial of adequate quelity. Results are viven for optical ain and beam quality in a lest device.

As a prototype of uonlinear frequency conversion, - well $*$ device for storing enery without smallesignal gin, stimulated Raman scatlering (SRS) is of greal interest. Recent experimental results on SRS in molecular gases at high pressure are presented. A 1.06. $\mu \mathrm{m}$ laser delivering a I-nsec (FWHM) bandwidthtimited pulse of I.J energy has been consinucted for this research. The oulput is frequency-doubled with up to $30 \%$ conversion efficiency. Working at the shorter wavelength minimizes the problems of windew damage and reflective feedbock. At the high powers being used, many Stokes and anti-Stokes comprnents are observed. Investigation of the oulput with a 10-psec-resolution streak cannera revealed severe temporal modulation on all components. The origin of this modulation is not understood but is still under study.

In the area of laser materials, the spectrum of the $\mathrm{Nd}^{3+}$ ion in laser glass is being inveshigaled. By monitoring the absorption from the $4 / 11 / 2$ state of the Nd ion to the pump band after passage of an intense laser pulse in an amplifier, the lower level relaxation time can be obtained. As a preliminary 10 this measurement, the cross sections for absorption from the $t_{11 / 2}$ statc to both the $4 F_{3 / 2}$ and ${ }^{4} G_{5 / 2}$ states have been measured. The value of the stimulatedemission cross section obtained from these measurements is two-thirds of the accepted value. The reason for this discrepancy is presently unclear.

A tunable dyc-laser facility for spectroscopic investigation has been developed. Its capabilitics are described. 
The propapation of light in a nonlinear medium is described by the vector wave equation

$$
\nabla \times \nabla \times E+\frac{1}{c^{2}} \frac{\partial^{2}}{\partial t^{2}} E=-\frac{4 \pi}{c^{2}} \frac{\partial^{2} p}{\partial t^{2}},
$$

where $E$ is the electric field of the light weve and $P$ is the polarization vector induced in the medium by the preacence of the light wave. For a component of the lifht wave at optical frequency $\omega$, with a corresponding frequency component of the polariza. tion, we can express the constitutive relation for the medium as

$$
P_{\omega}=x(\omega) E_{\omega}=\left[x_{1}(\omega)+\not x_{2}(\omega)\right] E_{\omega},
$$

where the suacteptibility $x$ is written $s$ the sum of the red term, correspondins to refrective index offocts, and an imaginay torm, corresponding to absorption. Asaming that no dspolarization is present, so that sealar theory applies, Eq. (1) now soumes the form

$$
\begin{aligned}
& \nabla^{2} E_{\omega}+\frac{\omega^{2}}{c^{2}}\left[1+4 \pi x_{1}(\omega)\right. \\
& \left.+i 4 \pi x_{2}(\omega)\right] E_{\omega}=0 .
\end{aligned}
$$

In general, we are interested in light waves that are nearly plane waver. (A) equivilent formulation exists for nearly spherical wrves.) A nearly plane wave is one in which the light field $\mathbf{E}_{\omega}$ is given by the product of a slowly varying complex amplitude $\downarrow$ and a rapidly acillating plane wave term

$$
E_{\omega}=\psi e^{(k z-\omega) ;}
$$

the propagation vector $k$ eatisfies

$$
k^{2}=\frac{\omega^{2}}{c^{2}}\left[1+4 \pi x_{1}^{(0)}\right]=\frac{n_{0}^{2} \omega^{2}}{c^{2}},
$$

whese $\chi_{1}^{(0)}$ is the linear susceptibility of the medium, and $n_{0}$ is the ordinary, linear refractive index. Equation (3) now takes the form

$$
\left[\nabla^{2}+2 i k \frac{\partial}{\partial z}+4 \pi \frac{\omega^{2}}{c^{2}}\left(x_{1}-x_{1}^{(0)}+i x_{2}\right)\right] \psi=0 \text {. }
$$

We can drop the second derivative of $\downarrow$ with respect to 2 in comparison with the $\operatorname{term} 2 i k(\partial \psi / \partial z)$, since it is assumed that the complex amplitude does not vary significantly over a wavelength. (If this is not the case, $\omega$ in a focel region, this description may not apply.) Defining an absorption coefficient a as

$$
\alpha=4 \pi x_{2}\left(\omega / n_{0} c\right)
$$

and an index nonlinesrity $\mu$ as

$$
\mu=2 \pi\left(x_{1}-x_{1}^{(0)}\right) / m_{0}^{2}=\left(n-n_{0}\right) / n_{0},
$$

we arrive st the angittal equation,

$$
2 i k\left(\frac{\partial}{\partial z}+\frac{\alpha}{2}\right) \psi+\nabla^{2} \psi+2 k^{2} \mu \psi=0,
$$

whese the Laplaciun, divergence, and gradient operator are assumed to contain only components perpendicular to 2 . In the nonatationary case, the integration slong 2 must be replaced by integration along $[(\ell \cdot x / v)=$ constant $]$. The complex amplitude $\downarrow \mathrm{cm}$ be written in terms of a real amplitude and phese,

$$
\psi=A e^{i t} \text {. }
$$

If the quantities

$$
I=A^{2} / Z_{0}
$$

where $I$ is the intensity and $Z_{0}$ is the free-space impedance, and

$$
\mathbf{u}=\nabla \phi / k
$$

are defined, and are subatituted in Eq. (9), one obtains the set of equations 150

$$
\begin{aligned}
& \left(\frac{\partial}{\partial z}+u \cdot \nabla\right) I+I(\nabla \cdot u)=a I, \\
& \left(\frac{\partial}{\partial z}+u \cdot \nabla\right) u=\nabla[u+P(I)],
\end{aligned}
$$

where

$$
P(n)=\frac{\left[\frac{\nabla^{2} l}{I} \cdot \frac{1}{2}\left(\frac{\nabla}{I}\right)^{2}\right]}{4 k^{2}}
$$

Equations (13)-(15) represent the equations of light fow, corresponding to the equations of fluid flow. Equation (13) is just the expression of conservation of power in the light beam (equation of continuity), and Eq. (14) represents the bending of the light trajectories due to the effects of refraction $(\mu)$ and diffraction $P(I)$. In the correspondence to fluid flow, 
12 plays the role of the body force, and $P(I)$ the role malogous to prescure.

We recognize that the characteristics of both Eqs. (13) and (14) are given by

$$
\mathbf{u}=\frac{d \mathbf{r}}{d z} \text {. }
$$

Thus, along the curves given by Eq. (16) we can write the Lagrangian derivatives

$$
\frac{D I}{D z}=\alpha I \cdot I(\nabla \cdot u)
$$

and

$$
\frac{D u}{D z}=\nabla[\mu+P(n)]
$$

Equations (16)-(18) can be integrated simultaneously, as a system of equations, to yield a set of values (r, $u, I$ at each value of $z$. Each set will be characterized by the values of $r$ at $z=z_{0}$, called $r_{0}$. The family of curves $r\left(r_{0}\right)$ are called the "trajectories" of the light beam. They have the following useful property: in the case of cylindrical symmetry, we can integrate the intensity from $r_{1}$ to $r_{2}$, where $r_{1}(z)$ and $r_{2}(z)$ are two trajectories. Then we have, from Eq. (13),

$$
\begin{aligned}
\frac{\partial}{\partial z} \int_{r_{1}}^{r_{2}} r d r I & =\int_{r_{1}}^{r_{2}} r d r\left[-\frac{1}{r} \frac{\partial}{\partial r}(r I u)+\alpha I\right] \\
& +r_{2}\left(\frac{d r_{2}}{d z}\right) I\left(r_{2}\right) \cdot r_{1}\left(\frac{d r_{1}}{d z}\right) I\left(r_{1}\right) .
\end{aligned}
$$

From Eq. (16), the first term in brackets on the right side of Eq. (19) is canceled out by the last two terms, leaving

$$
\frac{\partial}{\partial z} \int_{r_{1}}^{r_{2}} r d r I=\int_{r_{1}}^{r_{2}} r d r \alpha I .
$$

In the absence of absorption, the trajectories are seen to be the boundaries of the regions of constant power. This theorem, Eq. (20), provides a useful check on the accuracy of the numerical integration, and has even been used in place of Eq. (17) to obtain the intensity. ${ }^{151}$

The method of trajectories has been used $t \leq$ study self-focusing and the propagation of light past the self-focus in a nonlinear medium. ${ }^{152}$ It piovides the advantage of an adaptive grid, which expands, contracts, and deflects, as dictated by the topography of the phase front. The method has proved to be faster than fixed grid calculations of comparable accuracy by factors of 5 to 10 .

\section{NUMERICAL CALCULATION OF STIMULATED RAMAN SCATTERING}

\section{IN A LASER BEAM}

The objective of this study is to calculate the structural and temporal characteristics of a laser beam undergoing stimulated Raman scattering and to describe the Stokes (frequency-downshifted) and anti Stokes (upshifted) light produced by the scattering. This will be done by means of a set of computer programs under development, known collectively by the name SAGITTARIUS, which solve numerically the equation of quasi-optics ior the laser, Stokes, and anti-Stokes beanis, using the method of trajectories. The programs will be used to help obtain a physical understanding of the nature of the Stokes and antiStokes beam produced under a variety of circumstances and to simulate processes occurring in actual and potential laboratory experipnents.

The SAGITTARIUS codes solve the equation for the slowly varying envelope of the electric field, $\psi(r, z, t)$, in an axially symmetric beam, as outlined in the preceding article. There are two classes of problems to be solved: steady-state problems in which the time derivative does not appear, and the time-dependent problems. Of these, the class of time-dependent problems is much richer $r$ and has more relevance to the calculation of Raman scattering in pulsed laser beams. The objective of the present work is to produce a fully time-dependent code in which the dependent variables are calculated as functions of the two spatial variables $r$ and $z$, in addition to the marching variable t. A one-dimensional time-independent code, in which quantities are calculated as functions of $r$ and a marching variable $z$, has been completed and is being tested. The purpose of the one-dimensional code is to gain experience with the numerical methods associated with the trajectory or ray-path approach ${ }^{153,154}$ and to provide a relatively compact, fast running program for calculating steady-state Stokes and anti-Stokes production. As of June 1973, the steady-state program had been completed and was being tested 
in the calculation of Stokes and anti-Stokes light production.

Finite difference forms of the trajectory equations are solved in the time-independent SAGITTARIUS code. This code features an implicit difference scheme which gives unconditional numerical stahility. In addition, the physical result of the conservation of power is satisfied identically even when the $\Delta r$ between trajectories is not made to approach zero. The program is well behaved numerically and includes all the relevant physics correctly. For a beam having a Gaussian radial profile, the code has given the known position of the beam waist and the intensity at the beam waist to about $1 \%$ accuracy using only 10 trajectories. Further testing of the performance of this code for the propagation of the field (in free space) from a uniformly illuminated aperture, which should give the Airy pattem, is now in progress. If it calculates the diffraction rings successfully, the code should be useful in helping experimenters set up optical systems whose diffraction radial profiles are presisely known.

The extension to the time-dependent case is fairly simple conceptua?ly, but introduces certain difficulties in the program implementation. The equations for the time-dependent case are obtained from the stationary case by replacing the operator $\partial / \partial z$ by the derivative along a characteristic in the $(z-v t)$ plane. We then have the trajectory slope defined by

$$
\frac{\partial r}{\partial z}=u \text {. }
$$

Conservation of power is expressed by

$$
\left(\frac{1}{\nu} \frac{\partial}{\partial t}+\frac{\partial}{\partial z}\right) \int_{r_{1}(z, t)}^{r_{2}(z, t)} I(r, z, t) 2 \pi r d r=0,
$$

where $I$ is the intensity, and the diffraction equation of motion is given as

$$
\frac{1}{v} \frac{\partial u}{\partial t}+\frac{\partial u}{\partial z}+u \frac{\partial u}{\partial r}=\frac{1}{4 k^{2}} P(I)
$$

If all the $z$-steps were of width $\Delta z=v \Delta t$, the time-dependent problem could be solved in principle by working with a sequence of static problems in a coordinate moving to the right with velocity equal to the group velocit: $v$. In practice this does not work for focused beams since it is necessiry for such beams to have the $\Delta z$ steps of unequal lengths. $A$ further difficulty arises when several beame have to be followed simultaneously, each with a different group velocity. One solution to both these problems is to perform interpolations along $z$ and allow a variable $\Delta z$; a code implementing this solution has been written, buti is not yet tested.

In order to calculate stimulated Raman scattering, several sets of trajectory equations are introduced, one set corresponding : $: 0$ the laser (pump) beam and the others corresponding to the various orders of Stokes and anti-Stokes light at appropriate frequencies. Both the time-independent and time-dependent codes are written to permit an arbitrary number of bearns, with the Raman interaction terms in the equations for the various beams handled by subroutines. Two sets of such subroutines jave been written for the time-independent coais: laser-Stokes interaction, and laser-Stokes-anti-Stokes interaction. The laser-Stokes interaction changes only the power conservation equation, but the inclusion of anti-Stokes production modifies the diffraction equation also.

In the case of the time-dependent code, additional equations are introduced to follow the time dependence of the slowly varying envelope of the normal coordinates of molecular ossillations, and also to determine the spatial and temporal variation of the difierence in probability between quantum states of the molecules of the scattering medium. The complete code will therefore have the ability to describe properties of the medium in addition to the properties of the light passing through the medium. Work on these aspects of the time-dependent code, in particular how they relate to the trajectory deseription and to changes in the phase anomalies, is now in progress.

\section{HOMOGENEITY REQUIREMENTS FOR MINIMIZING SELIF.FOCUSING DAMAGE-}

Bulk optical damage in solid dielectrics is known to occur at incident intensity levels much lower than the intrinsic dielectric breakdown value. Under controlled conditions at low powers, such premature breakdown has been shown to arise from the catastrophic intensity enhancement near a self-focus of the incident optical bearn (we are assuming that extrinsic damage mechanisms such as inclusions are inoperative). Under less controlled conditions, and at higher powers, the damage pattern is very complex. Much experimental and theoretical evidence suggests that, in this case too, self-focusing is responsible, but that the observed damage arises from self-focusing of fluctuations on the incident intensity profile, and not from the self-focusing of the beam as a whole. Since the self-focal length of small-scale fluctuations can be 
much shorter than that of large-scale intensity variations, the self-focusing of fluctuations determines the effective damage threshold of beams propagating in nonlinear dielectrics.

Theoretical studies of self-focusing fluctuations are available in which the fluctuations are already present in the incident beam, and are simply enhanced by self-focusing, or diminished by diffraction. We point out that even when the incident beam is "clean," intensity fluctuations can accumulate during propagation through an inhomogeneous medium. Since all media are inhomogeneous to some extent, it is imperative to determine the magnitude of the influence of weak random inhomogeneities on the self-focusing process. Fortunately, there are simple approximate theories of random propagation which are easily modified to include the initial stages of self-focusing. In this work, we have exploited one of these theories to provide information on the influence of random inhomogeneities on bulk damage thresholds.

Starting with the "slowly varying envelope equation" for the optical field amplitude,

$2 i k \frac{\partial E^{\prime}}{\partial z}+\nabla_{T}^{2} E^{\prime}+\frac{k^{2}}{\epsilon_{0}}\left(\epsilon_{1}+\frac{1}{2} \epsilon_{2}\left|E^{\prime}\right|^{2}\right) E^{*}=0,(1)$

we let

$E^{*}=e^{x+i \sigma}\left\{E_{0} \exp \left(i \frac{k \epsilon_{2}}{4 \epsilon_{0}}\left|E_{0}\right|^{2} z\right)\right\}$,

where the bracketed expression is a "zeroth order" solution of Eq. (1) when $\epsilon_{1}=0$. Here $\chi, \sigma$ are supposed to be small perturbations which depend on $x, y$, and $z$, and which are introduced by fluctuations in the initial field, or in the linear dielectric constant $\epsilon_{1}$.

Upon substituting Eq. (2) into Eq. (1) and discarding terms of second order in $\chi$ and $\sigma$, we find a coupled set of linear equations for $x(x, y, z)$ and $\sigma(x, y, z)$ which may be solved easily for the transverse Fourier transforms $\hat{\chi}\left(a_{x}, A_{y}, z\right)$ and $\hat{o}\left(a_{x}, q_{y}, z\right)$. The result is

$$
\begin{aligned}
\hat{\chi}(q, z) & =\hat{\chi}(q, 0) \cosh (z / l)+\hat{\sigma}(q, 0) \frac{q^{2} \ell}{2 k} \sinh (z / l) \\
& +\frac{\ell q^{2}}{4 \epsilon_{0}} \int_{0}^{z} \hat{\epsilon}_{1}\left(q, z^{\prime}\right) \sinh \left[\left(z-z^{\prime}\right) / \ell\right] d z^{\prime},(3) \\
\hat{\sigma}(q, z) & =\hat{o}(q, 0) \cosh (z / l)+\hat{\chi}(q, 0) \frac{2 k}{q^{2} \ell} \sinh (z / \ell) \\
& +\frac{k}{2 \epsilon_{0}} \int_{0}^{z} \hat{\epsilon}_{1}\left(q, z^{\prime}\right) \cosh [(z-z) / \ell] d z^{\prime} .(4)
\end{aligned}
$$

Here $\ell$ is a function of $q$ :

$$
\ell=\frac{2 k / g^{2}}{\sqrt{\left(I_{0} / I_{q}\right)-1}},
$$

where $I_{0}$ is the mean incident intensity and $I_{q}$ is a "critical intersity" for self-focusing a fluctuation of transverse wave number $q$ :

$$
I_{q}=\frac{n^{3} c g^{2}}{8 \pi \epsilon_{2} k^{2}}=P_{1} q^{2} / 2 \pi
$$

$P_{1}$ is the lower critical power for self-focusing of a smooth Gaussian beam of any radius, and $n^{2}=\epsilon_{0}$. We have assumed the beam is strong enough that $I_{0}>I_{q}$. Note that

$$
\frac{I_{0}}{I_{g}}=\frac{4 \delta k^{2}}{g^{2}},
$$

where $\delta$ is the fractional index change due to $I_{0}$ :

$$
\delta=\left(n-n_{0}\right) / n_{0} .
$$

From these solutions for $\hat{\chi}$ and $\hat{\sigma}$, we may compute a variety of important properties of the propagating field. Let us first find the effect of a medium without statistical fluctuations (i.e., with $\epsilon_{1}=0$ ) on initial fluctuations. We will consider a medium of thickness $b$ bounded by vacuum on both sides. In the vacuum, a ripple of transverse wave number $q$ oscillates as

$$
\exp \left(i \frac{q^{2} x}{2 k}\right)
$$

In the medium the fluctuations grow as

$$
\exp \left(\frac{z q \sqrt{4 \delta k^{2}-q^{2}}}{2 k}\right)
$$

if the background intensity $I_{0}$ is strong enough to focus ripples of transverse wave number $q$, or the fluctuations oscillate as

$$
\exp \left(\frac{i z q \sqrt{q^{2}-4 \delta k^{2}}}{2 k}\right)
$$

if it is not.

To find the amount by which the ripple amplitude is changed on passage through the slab, we match a vacuum solution to a medium solution at the beginning 


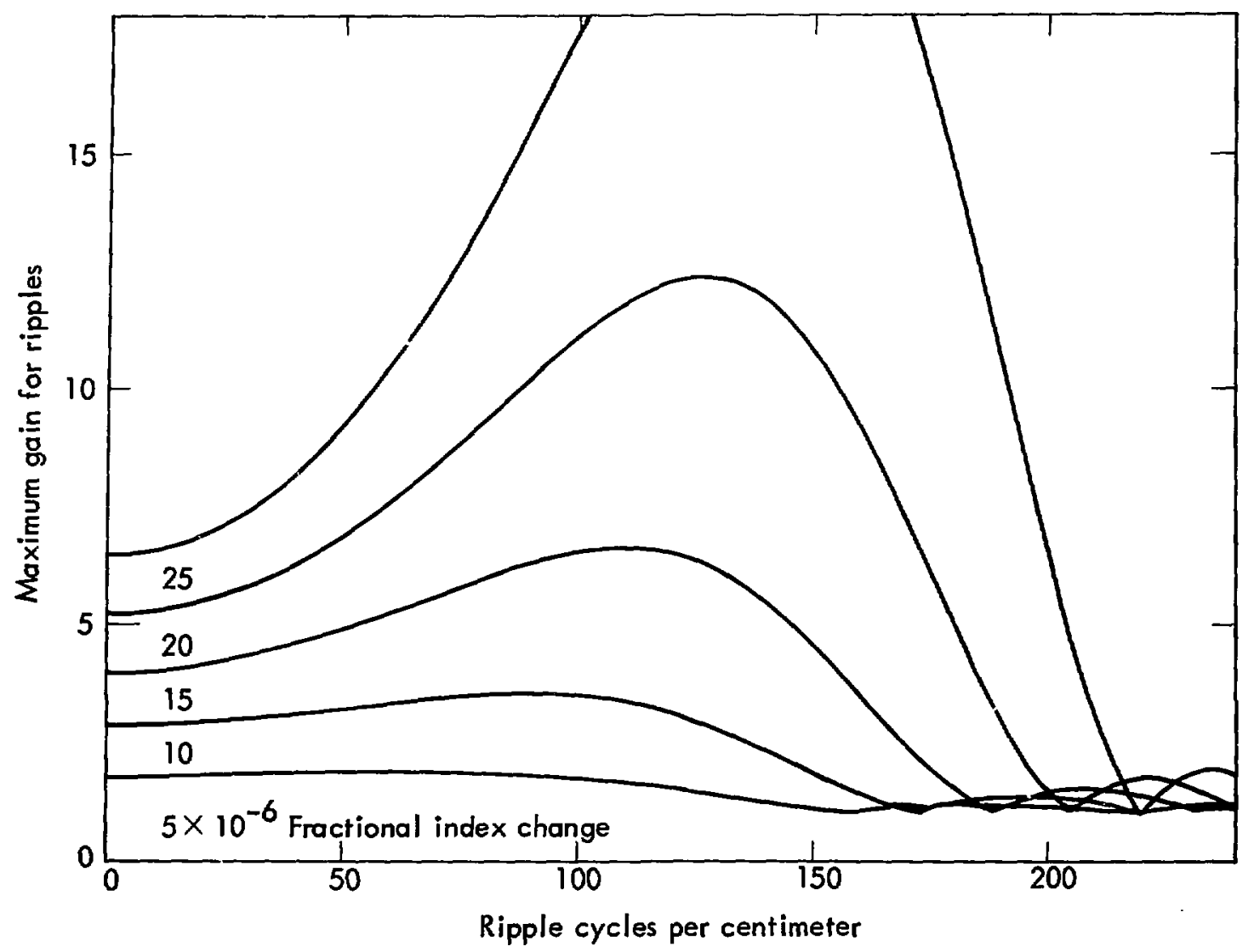

Fig. 161. Maximum gain of small transverse ripples on a uniform beam of $1-\mu m$ light in a $2-\mathrm{cm}$ slab of optical gain medium. Gains are shown as a function. If the crest-to-crest ripple spacing for various fractional refractive index changes $\delta=\left(n-n_{0}\right) / n_{0}$ due to the i-cid:nt beam. The gain depends on the phase with which the ripple strikes the slab; the quantity plotted is the maximum value. The gains first increase as the ripples become more closely spaced (because the spatial index gradients which amplify the ripples are larger), and then drop off as diffraction becomes stronger than self-focusing. Note the large gains available for small index variations, and the broad range of ripple sizes which can be amplified.

of the slab of material. The resulting ripple is then allowed to grow through the medium. The solution at the end of the slab is then matched to a vacuum solution again. The ripple gain through the slab is defined as the ratio of the amplitudes of the vacuum solutions on the two sides of the slab. This gain depends on the phase of the vacuum solution entering the slab, and we vary this phase to find the maximum-gain input wave. Figure 161 shows curves of such phase-maximized gains as a function of the transverse crest-to-crest ripple spacing. A number of curves are shown, one for each value of $\delta$ in the range from $5 \times 10^{-6}$ to $25 \times 10^{-6}$. The gains are for a $1-\mu \mathrm{m}$ wavelength and a $2-\mathrm{cm}$ slab, which are values relevant to glass disk lasers. The high per-disk gains shown for large $\delta$ would be very dangerous in a multidisk amplifier. Since amplification takes place for ripples with a large range of transverse scale (extending to ripples comparable to the beam size), suppression of initial fluctuations to very low levels over a broad range of wave numbers will be required if self-focusing damage is to be avoided.

In addition to the growth of initial fluctuations, variation in the medium itself can cause fluctuations to arise. These fluctuations will then be amplified and may lead to damage. We can gain insight into the influence of inhomogeneities by considering a localized fluctuation $\epsilon_{1}(q, 2)$ in the vicinity of $z_{0}$ which vanishes outside the small interval $\Delta z$. The last terms in Eqs. (3) and (4) then give

$x(q, z) \approx \frac{\ell q^{2} \Delta z \hat{\epsilon}_{1}}{4 \epsilon_{0}} \sinh \left[\left(z-z_{0}\right) / l\right]$, 
$\hat{\sigma}(q, z) \approx \frac{k \Delta z \hat{\epsilon}_{1}}{2 \epsilon_{0}} \cosh \left[\left(z \cdot z_{0}\right) / \ell\right]$.

This is exactly the solution we would have found if a phase fluctuation of magnitude

$$
\hat{\sigma}\left(q, z_{0}\right)=k \Delta z \frac{\hat{\epsilon}_{1}}{2 \epsilon_{0}}
$$

had been introduced at $z_{0}$ in a homogeneous medium. Since the effect of an index inhomogeneity is to retard the phase by precisely this amount, the result satisfies our intuition.

For an index fluctuation of order $1 \%$ confined to $\Delta z \approx 100 \mu \mathrm{m}$, Eq. (13) predicts a phase fluctuation of order $\pi$ for $1-\mu \mathrm{m}$ radiation. If the transverse scale of the fluctuation is such that $\ell=l_{\min }$, then the output ripple amplitude $R_{\text {out }}$ satisfies

$$
R_{\text {out }}^{2}=\left(\frac{k \Delta z \epsilon_{1}}{2 \epsilon_{0}}\right)^{2} \cosh \left[2\left(z-z_{0}\right) / \ell_{\min }\right]
$$

which grows exponentially with characteristic length $\ell_{\min }$.
Since index fluctuations are expected to occur randomly, it is necessary to develop a statistical theory of the growth of field fluctuations. Only one result of this theory will be reported here, since the derivation is rather complicated. The approximate mean-square field fluctuation at distance $z$ caused by random index fluctuation in a self-focusing medium is

$$
\left\langle\delta \mathrm{E}^{2}\right\rangle \approx \frac{1}{2}\left\langle\Lambda^{2}\right\rangle \mathrm{I}_{0} \mathrm{q}_{0}^{4} l_{0}^{2}\left[\frac{\sinh \left(z / l_{0}\right)}{\left(z / l_{0}\right)}-1\right] .
$$

Here $\left\langle\Lambda^{2}\right\rangle$ is the mean-square fluctuation in optical path length to $z$ (an observable quantity). The index fluctuation spectruin is assumed to be peaked at wave number $q_{0}$, and $\ell_{0}$ is simply $\ell$ (Eq. (5)) evaluated at $a_{0}$. For an $\mathrm{ms}$ path length fluctuation of $v$ wavelengths, Eq. (15) gives, in the worst case,

$\left\langle\delta E^{2}\right\rangle_{\max } \approx 8 \pi^{2} v^{2} I_{0}\left[\frac{\sinh \left(z / \ell_{\min }\right)}{\left(z / \ell_{\min }\right)}-1\right]$.

For an rms distortion of $\lambda / 8$, Eq. (16) implies a relative fluctuation of order unity at distance $z \approx 2 Q_{\text {min }}$.

\section{THE ROLE OF LINEAR DISPEASION IN PLANE-WAVE}

SELF.PHASE MODULATION

In the previous semiannual report, ${ }^{155}$ we described the results of a computer calculation which coupled the effects of self-phase modulation and material dispersion. We described an algorithm for solving the nonlinear differential equations, and we showed some preliminary results of that calcuiation. Shocks were shown to form on the leading edge of intense short pulses propagating in $\mathrm{CS}_{2}$, and the grating-pair compression recipe for $\mathrm{CS}_{2}$-chirped pulses ${ }^{156}$ was modified to take self-dispersion into account. It was shown that the parameter responsible for dispersively reshaping pulses is given by $\lambda^{3}\left(d^{2} n_{0} / d \lambda^{2}\right)$ where $\lambda$ is the free-space wavelength, and $n_{0}$ is the linear index of refraction.

In this report we present new developments since the last report period.

\section{Pulse Propagation in $\mathrm{CS}_{2}$}

We report here a more complete study of the shock instabilities encountered when an intense short pulse propagates through $\mathrm{CS}_{2}$. For the same conditions as previously discussed (22-GW/ $\mathrm{cm}^{2}$, 5-psec-duration, $1.06-\mu \mathrm{m}$ pulses), the deleterious effect of amplitude noise has been examined with initial peak-to-peak intensity ripples of $10 \%, 2 \%, 0.2 \%$, and $0.04 \%$. Results are shown in Fig. 162. Note that the $10 \%$ noise triples in $2 \mathrm{~cm}$. The shock formation previously predicted 155 can only be recognized in the case of $0.04 \%$ ripple.

We have aiso repeated the calculation of Shimizu 57 (as a check of our work) and we find excellent pulse shape agreement if we increase by $15 \%$ the propagation distance for our calculated pulse. At present we are unable to explain this minor discrepancy.

Pulse Propagation in $\mathrm{CS}_{2}$-Filled Waveguides

Optical waveguide propagation may serve as a technique for measuring nonlinear coefficients of materials, and may aid in the reshaping of relatively weak laser pulses. The structure allows long propagation distances at relatively high peak intensities 


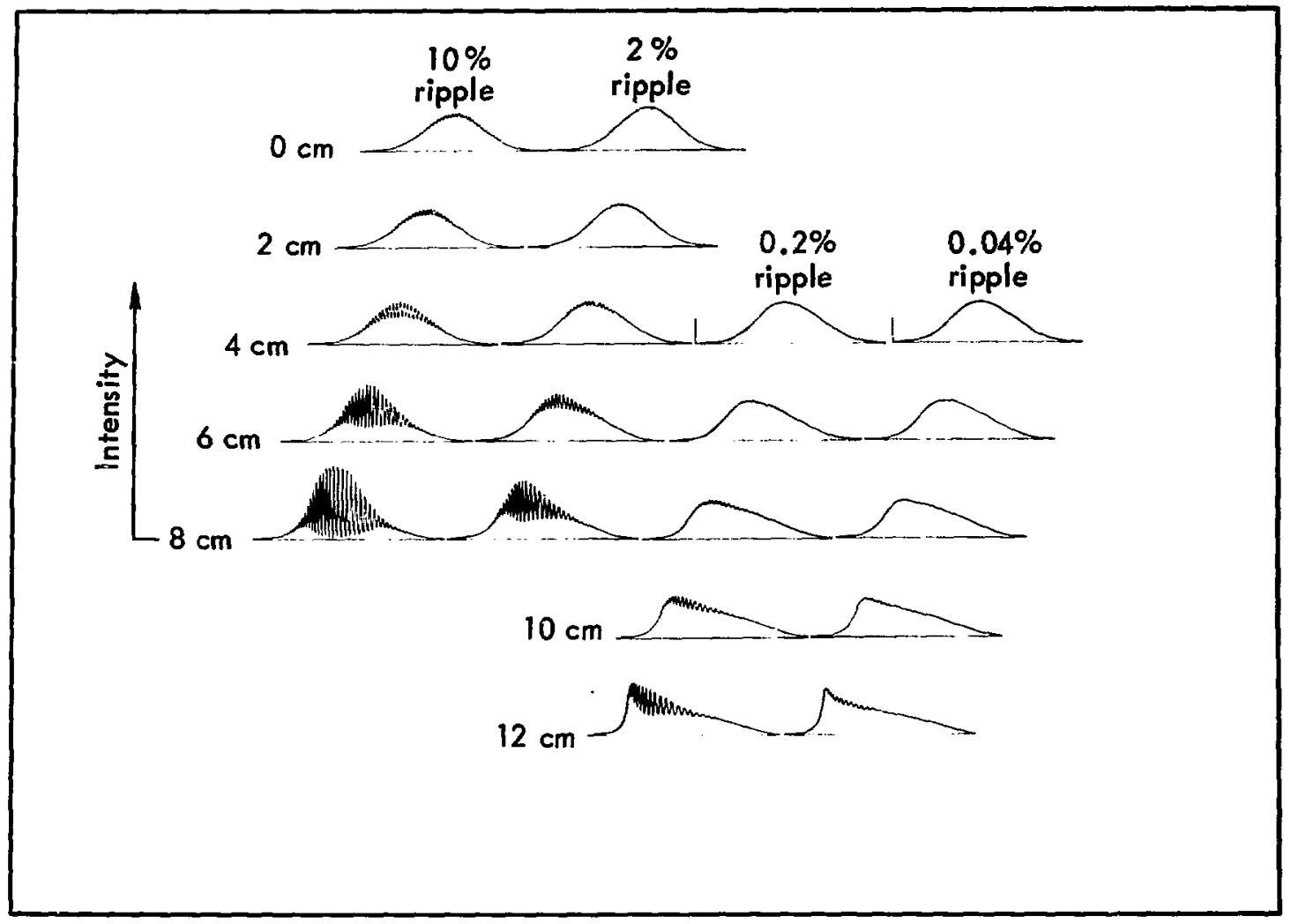

Fig. 162. Demonstration of isstability due to amplitude noise. Calculated results are shown for cases of 10\%, 2\%, $0.2 \%$, and $0.04 \%$ peak-to-peak intensity ripple on a laser beam propagating through $\mathrm{CS}_{2}$. Peak power is $22 \mathrm{GW} / \mathrm{cm}^{2}$, pulse duretion 5 psec, horizontal axis represents time.

without the added complication of self-focusing. The optical Kerr effect has recently been observed in glass fibers, 158 and spectral broadening in $\mathrm{CS}_{2}$-filled fibers has also been reported. ${ }^{159}$

We have calculated the dispersive effects that would be encountered in chirping $\mathrm{cw}$ dye-laser pulses via self-phase modulation in $\mathrm{CS}_{2}$-filled waveguides. In a 10- $\mu \mathrm{m}$-diam $\mathrm{CS}_{2}$-filled waveguide, for instance, material dispersion dominates the guide dispersion, but attenuation can be quite severe. Since $\lambda^{3}\left(d^{2} n_{0} / d \lambda^{2}\right)$ at $0.59 \mu \mathrm{m}$ is 2.67 times greater than it is at $1.06 \mu \mathrm{m}$, $\mathrm{CS}_{2}$ dispersion in the visible region will have more influence than it had in the infrared. We have calculated the reshaping for $0.59-\mu \mathrm{m}$ picosecond pulses (considering only material dispersion) with peak powers of 100,50 , and $10 \mathrm{MW} / \mathrm{cm}^{2}$. The attenuation $\alpha$ was chosen so that $\alpha^{-1}=200 \mathrm{~cm}$. The results are shown in Fig. 163. The 100-MW/ $\mathrm{cm}^{2}$ pulse travels less than a meter before shocking. As the $50-\mathrm{MW} / \mathrm{cm}^{2}$ pulse shocks, attenuation prevents catastrophic reshaping and the pulse shape is quite stable in the region of the shock (160-240 cm). (In comparison, for the case with no attenuation the pulse reshapes severely as it shocks.) As the shock forms the pulse "speeds up" because the transient conditions favor spectral broadening to the Stokes side $(\Delta \omega<0)$. The pulse spectrum his broadened to approximately eight times its original width by $240 \mathrm{~cm}$, and then does not broaden any more. At this point, dispersion and attenuation drop the peak intensity of the pulse, and the nonlinear effects go away. In the $10-\mathrm{MW} / \mathrm{cm}^{2}$ case, the pulse developed into a skewed shape by $170 \mathrm{~cm}$, similar to the $50-\mathrm{MW} / \mathrm{cm}^{2}$ case after $80 \mathrm{~cm}$. The $10-\mathrm{MW} / \mathrm{cm}^{2}$ case developed only twice the initial spectral broadening by $170 \mathrm{~cm}$, and then retained its shape and spectrum for the remaining $2.3 \mathrm{~m}$ of propagation.

Grating-Pair Compression of Pulses from CS $_{2}$-Filled Waveguides

We have calculated the grating-pair compressibility of the pulses emanating from $\mathrm{CS}_{\mathbf{2}}$-filled waveguides. 

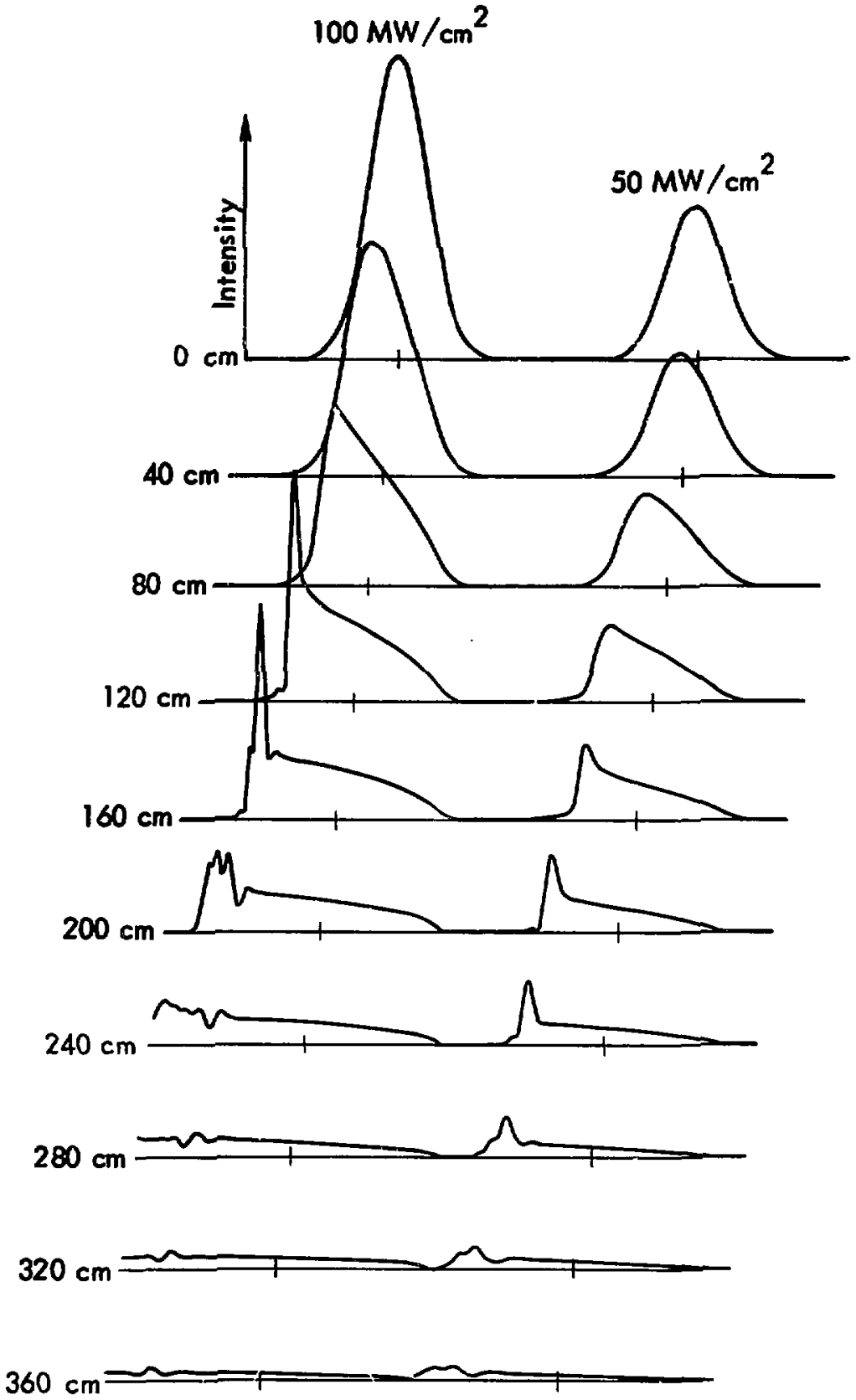
The $50-M W / \mathrm{cm}^{2}$ case in Fig. 163 has been studied after 2 and $4 \mathrm{~m}$ propagation and is shown in Fig. 164. In the case of $2-\mathrm{m}$ propagation the grating-pair compressible portion is everal picoseconds past the shock feature, and we find that the compression is not nearly as critically dependent upon grating-pair dispersive delay setting as was the case for the 1.06- $4 \mathrm{~m}$, $22 . \mathrm{GW} / \mathrm{cm}^{2}$ pulse after $10 \mathrm{~cm}$ propagation in $\mathrm{CS}_{2} \cdot 155$ Since the self-phase-modulated spectrum is far narrower for $50 \mathrm{MW} / \mathrm{cm}^{2}$ input intensity, the duration of the compressed pulse is reduced $t / 3$ only 0.5 psec. Although the "compressed" pulve does have approximately six times the peak power of the "uncompressed" pulse, the grating pair may not have been necessary, because in compressing the smooth portion of the pulse the grating pair had dispersed the already short shock.

We now turn ow attention to the $4 \mathrm{~m}$ case. We noted before that the nonlinearity became less important as linear attenuation and dispersion diminished the power. In Fig. 164 the 4-m case with relative compression setting of 2.3 is of interest. This value of compression setting was chosen because it would exactly cancel the dispersion due to linear $\mathrm{CS}_{2}$ propagation in the final $2 \mathrm{~m}$. The similurity to the uncompressed pulse after $2 \mathrm{~m}$ propagation supports our suggestion that virtually no sonlinearities arise in traversing the final $2 \mathrm{~m}$. Figure 164 also shows that the optimally compresued $4 \mathrm{~m}$ pulee correzponds to a grating-pair relative compresion setting of 5.0, which is very clos: to the sum of the optimally compressing grating-pair setting for the $2-\mathrm{m}$ case (3.0) and the setting to compensate for the last $2 \mathrm{~m}$ of nearly linear propagation (2.3). (A grating-pair relative compreacion of 1.0 corresponds to a groove spacing of $1200 / \mathrm{cm}$, an angle of incidence of $60^{\circ}$, and a epparation of $8 \mathrm{~cm}$.) Note that although the optimally compressed pulse in the $4-\mathrm{m}$ case resembles the optimally compressed $2 \cdot \mathrm{m}$ pulse, the compressed $4-\mathrm{m}$ pule has more intense secondary lobes. It is apparent that there was still some influence of the nonlinear effect during the last $2 \mathrm{~m}$ of propagation; otherwise the two curves would have been identical. Because the 2-m pulse can be compressed with less secondary structure than can the $4 \mathrm{~m}$ pulse, we conclude that no more than $2 \mathrm{~m}$ of $\mathrm{CS}_{2}$-filled waveguide should be used to prepare 5.psec, $50-\mathrm{MW} / \mathrm{cm}^{2}$ pulses for grating-pair compression.

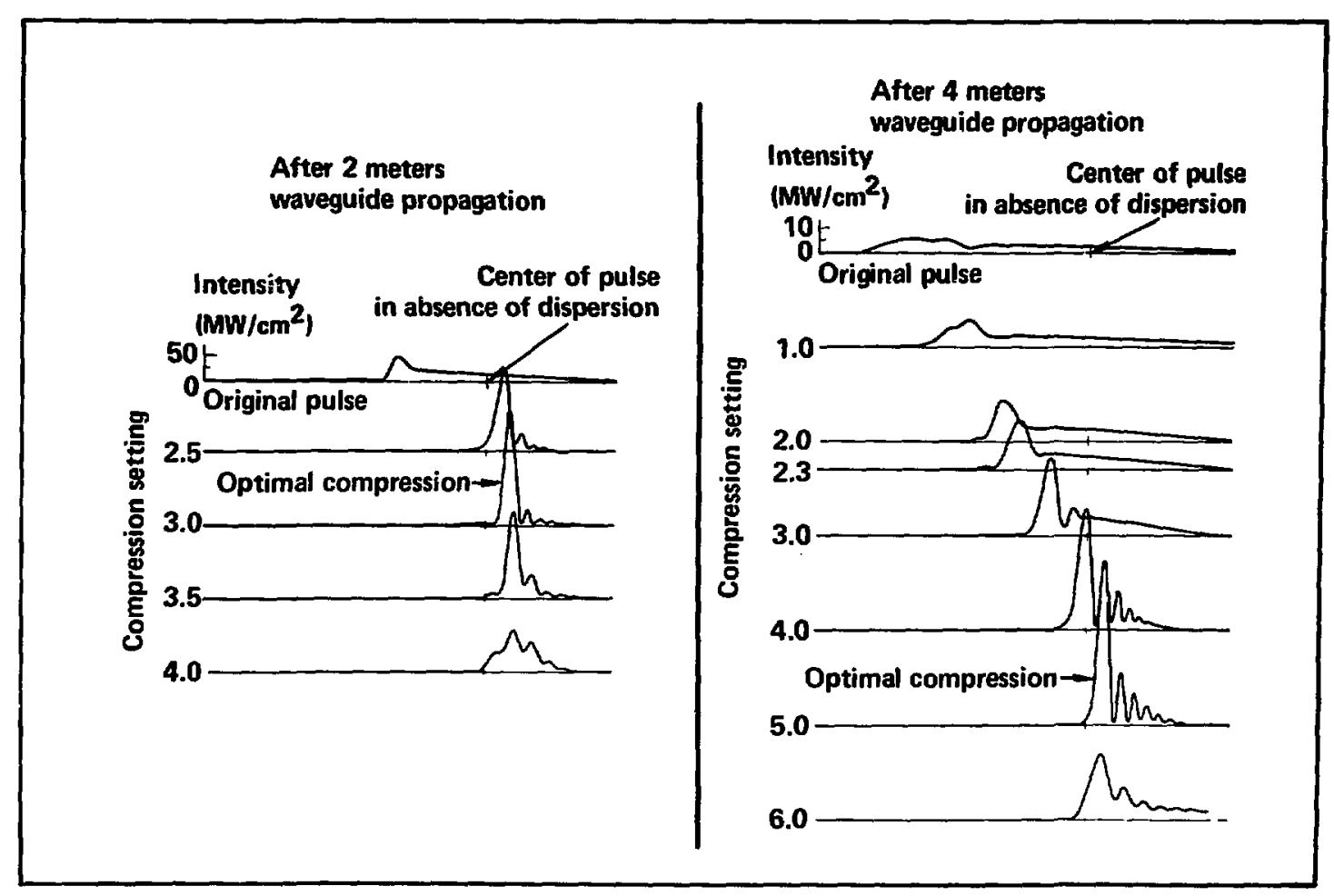

Fig. 164. Calculated grating-pair compressibility of pulses emanating from $\mathrm{CS}_{2}$-filled waveguides. The 0.59- $\mu \mathrm{m}$ pulves entering the guide were 5 psec in duration and had a peak power of $50 \mathrm{MW} / \mathrm{cm}^{2}$. Detailed comments on this figure are given in the text. 


\section{Pulse Propagation in Glass}

The nonlinear index of refraction $\left(n_{2}\right)$ in glass was first noted by Duguay, Hansen, and Shapiro, ${ }^{160}$ and was subsequently studied by Alfano and Shapiro. ${ }^{161}$ This nonlinearity is considered to be responsible for the $100-200 \mathrm{~cm}^{-1}$ spectral width of mode-locked neodymium-glass laser oscillators, and is responsible as well for the catastrophic self-focusing problems in high-power glass laser amplifiers. Thus knowledge of the values of $n_{2}$ and of the effects due to $n_{2}$ is of paramount importance in the design of high-power glass laser systems.

Although the resonance (gain) will also provide some linear dispersion, we will address first the problem of dispersive self-phase modulation in unpumped Owens-Illinois ED-2 glass. This glass was chosen for study because of its possible utility in large laser systems. We assume that $n_{2}$ for ED-2 is $1.3 \%$ that of $\mathrm{CS}_{2}{ }^{160,161}$ and that the response is nearly instantaneous. Although the relaxation time of the nonlinearity in glasses is unknown, spectral broadening experiments indicate that it is probably far faster than 1 psec. 160,161 We have arbitrarily chosen a relaxation time of $0.1 \mathrm{psec}$, which allows us to take longer step sizes than if we had chosen an instantaneous nonlinear response. The index of refraction for this glass is given by 162

$n=1.555067+\frac{0.004406}{\lambda^{2}-0.012773} \cdot 0.002963 \lambda^{2}$

$-0.000025 \lambda^{4}$

This equation was doubly differentiated to evaluate $\lambda^{3}\left(d^{2} n_{0} / d \lambda^{2}\right)$, which is compared to that of other relevant materials in Fig. 165. At $1.06 \mu \mathrm{m}$, for example, this glass has a dispersive effect which is one-third that of $\mathrm{CS}_{\mathbf{2}}$.

Figure 166 shows the results of our calculated evolution of a $10-G W / \mathrm{cm}^{2}, 5-\mathrm{psec}, 1.06-\mu \mathrm{m}$ pulse in unpumped ED-2 glass. The pulse flattens because the chirp which develops at the center is of the wrong sign for compression by the material dispersion. The small shock which forms on the leading edge (after $380 \mathrm{~cm}$ propagation) is absent if we set the Kerr relaxation time to zero.

Pulse Propagation in Pumped Neodymium-Doped Laser Amplifiers

In the simplest description of solid-state amplifiers, the nonlinear index changes come from the host (glass,

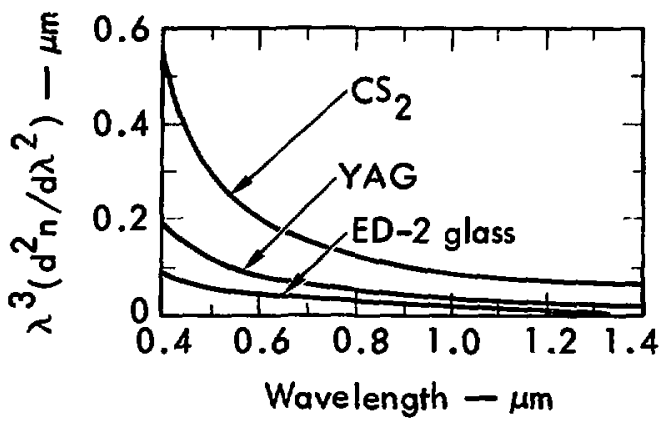

Fig. 165. Dispersive parameters for some important laser materials.

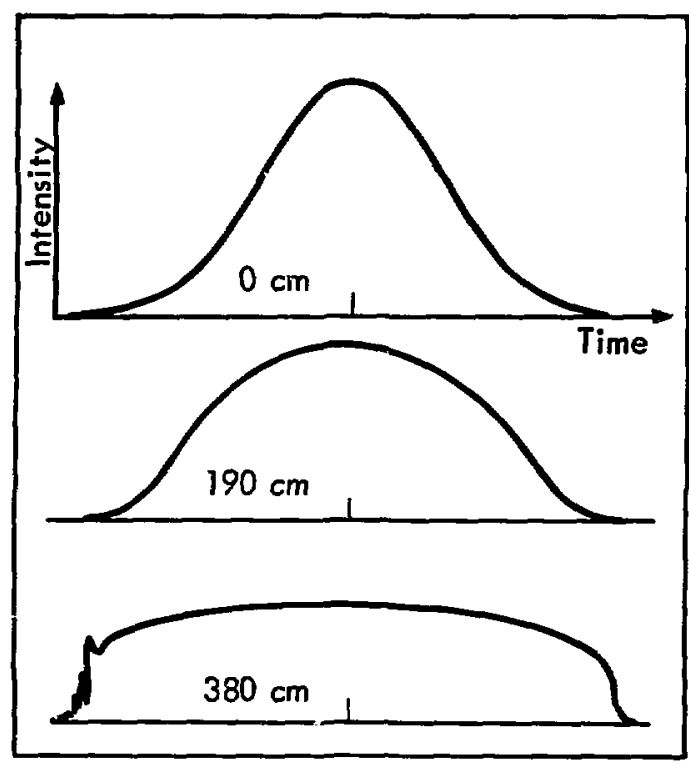

Fig. 166. Calculated evolution of a $10 \mathrm{GW} / \mathrm{cm}^{2}, 5-\mathrm{psec}$, 1.06- $\mu$ m pulse in urpumped ED-2 glass

YAG, or sapphire) while the dispersion is due to both the host and the resonance. The resonance contribution can be modeled in some cases by a nonsaturating homogeneously broadened resonance (for simplicity) of full spectral width $2 / T_{2}$. For $\Delta \omega \equiv \omega-\omega_{0}$, the ratio of the real part of the susceptibility to the imaginary part is given by $\Delta \omega T_{2}$. Thus one can write the linear complex index (near resonance) as the sum of host and resonance contributions: 


$$
\begin{aligned}
n_{c}(\Delta \omega)= & n_{h o s t}(\Delta \omega)+n_{\text {res }}(\Delta \omega) \\
= & n_{h o s t}(\Delta \omega)+\frac{\Delta \omega T_{2} g(0)}{2 k_{0}\left[1+\left(\Delta \omega T_{2}\right)^{2}\right]} \\
& -i\left\{\frac{g(0)}{2 k_{0}\left[1+\left(\Delta \omega T_{2}\right)^{2}\right]}\right\},
\end{aligned}
$$

where $n_{\text {host }}$ is the index of refraction of the host material, $g(0)$ is the line-center gain coefficient, and $k_{0}$ is t'le free-space wave vector. Both the imaginary (gairi) and real (dispersive) parts of this expression are important.

Fig. 167. Calculated evolution of a $14-G W / \mathrm{cm}^{2}, 5 . \mathrm{psec}$, 1.06- $\mu \mathrm{m}$ laser pulse in a pumped piece of ED-2 glass. Note the shock forming on the trailing edge.
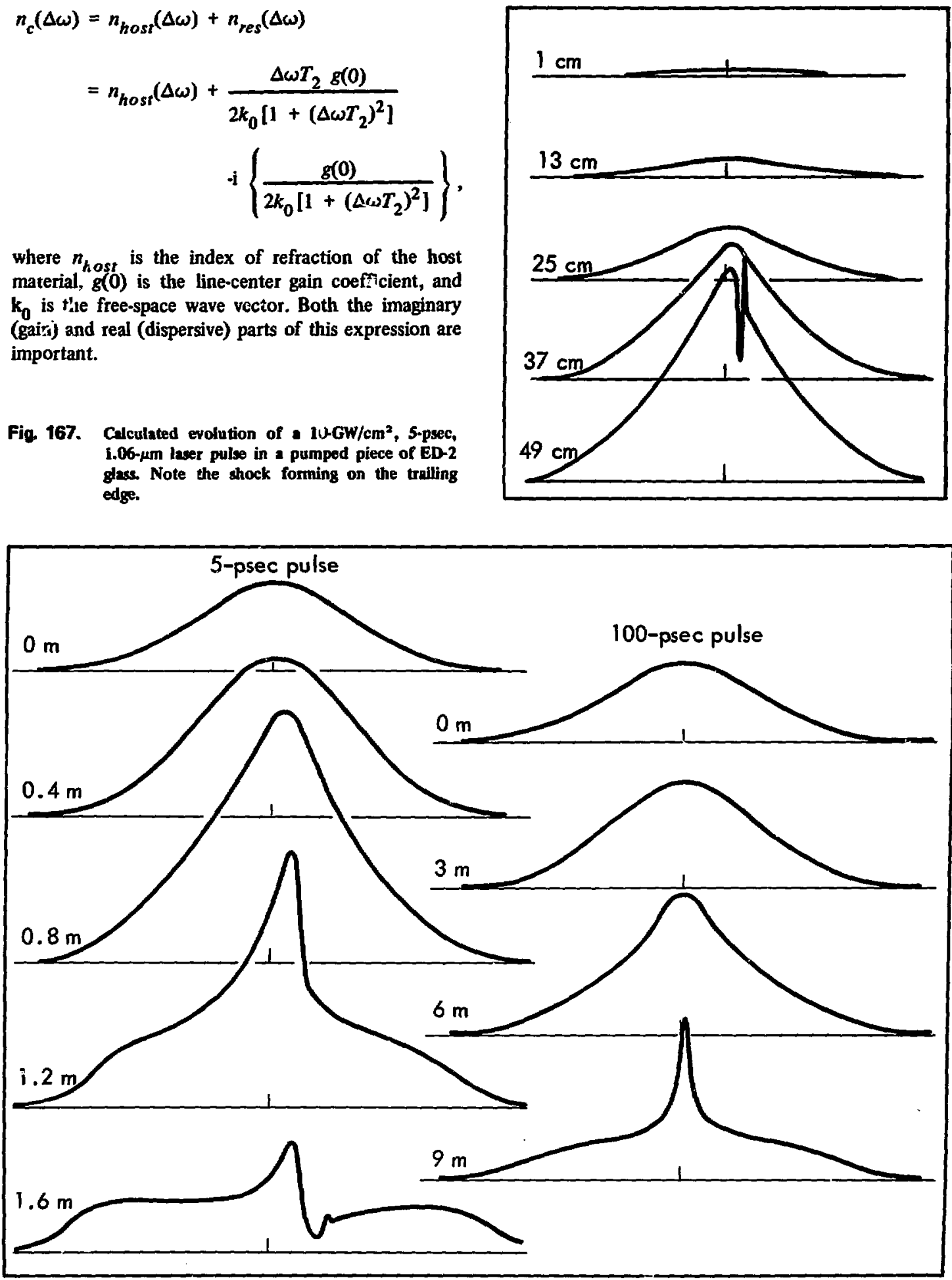

Fig. 168. Calculation of pulse evolution in a leser chain where the staging is represented by a distributed loss, $\alpha$ An a-value of $0.075 \mathrm{~cm}^{-1}$ was needed to stabilize the 5 -psec pulse, $0.089 \mathrm{~cm}^{-1}$ for the 100 psec pulse. 
like a "string of beads" (see Fig. 8-67).

"The periodic version of ZOHAR has also been used to study relutivistic filamentation. The growth rates found in these simulations, which are performed with fire? ions, are typically half the maximum value

$$
\gamma_{\max }=\left(\omega_{\mathrm{p}}^{2} / 4 \omega_{0}\right)\left(v_{0 s}^{2} / c^{2}\right) q .
$$

Again, sharp narrow concentrations of field intensity characterize the nonlinear stage.

For parameter ranges projected to be typical for laser fusion, the spatial growth rate in the steady state for relativistic filamentation is slower than that for filamenration driven by other mechanisms, such as the ponderomotive force. However, the relativistic mech. anism may play an important role in the initial transient response of the plasma. In simulations of the nonlinear stage of relativistic filamentation, electrostatic potentials as large as $100 \mathrm{keV}$ were seen in the filament. If the ions had not been held fixed in the simulations, they would have been expelled from the channel at high energies. Such a strong initial "kick" due to the relativistic effect should make the subsequent development of the ion-inertia-limited form of filamentation considerably faster.

The physical processes responsible for relativistic filamentation can also produce self-modulation of laser light in a plasma. 67 When the laser-light intensity is sufficiently high, relativistic nonlinearities 68 cause sidebands to develop around the incident-light frequency. Ultimately, as more and more sidebands de-

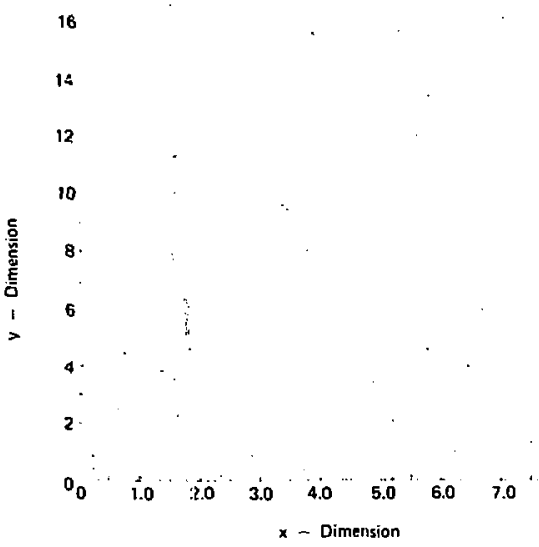

Fig. 8-67. Phot of ion positioning, $y$ versus $x$, in the plane of the simuiation for a system $4 \lambda_{0} \times 2 \gamma_{0}$ velop, the frequency of the light can be broadened considerably. We have sinula'ed this effect with the periodic version of ZC:IAR, using fixed ions since at high intensities the modulation affects only the electrons. We find that the modulations become quite deep and nonsinusoidal in their nonlinear stages. The initial exponentiation rate for the linearly most un. stable mode is typically half its theoretical maximum value. The latter is identical with the maximum growth rate for relativistic filamentation.

In these simulations, it is the incident wave that filaments. However, in simulations of Raman side. scattering in plasma slabs (using the open-sided version of ZOHAR), we have seen relativistic self-focusing of the Ranian decay electromagnetic wave. (With moving ions, we see indications of both Raman- and Brillouinscattered light self-focusing in density-depressed channels.) Therefore, in assessing processes that occur in laser-plasma interactions, we must consider the filamentation of both the incident and the decay electromagnetic waves.

\section{Self-focusing of an Initially Gaussian Beam}

Intense laser light is capable of pushing plasma aside when the field energy density $E_{0} 2 / 8 \pi$ becomes cumparable to the plasma thermal energy density $n_{0} T$. For time scales long compared to those needed for an ion acoustic wave to cross the dimensions of interest. the resulting lowering of the plasma density $n$ is described by $46 n=n_{0} \exp \left(-v_{o s}{ }^{2} / 4 v_{t h}{ }^{2}\right)$, where $v_{\text {os }} \equiv\left(\mathrm{eE}_{0} / \mathrm{m}_{\mathrm{e}} \omega_{0}\right)$ is the oscillation velocity of an electron in the light-wave electric field $E_{0}$ and $\left.v_{t h} \equiv[T e+T i) / m e\right]^{1 / 2}$ is a weighted electron thermal speed.

Self-focusing of laser light in plasmas is one consequence of this nonlinear dependence of plasma density on field strength. The self-focusing phenomenon has been studied previously 69 in the weak nonlinearity limit, $v_{\text {os }}{ }^{2} \ll v_{\mathrm{th}}{ }^{2}$. In this limit the plasma density is given by expanding the exponential: $n \cong n_{0}\left(1-v_{o s}{ }^{2} / 4 v_{t h}{ }^{2}\right)$. Self-focusing can then be described by the familiar "nonlinear Schroedinger equation" of nonlinear optics, with a cubic nonlinearity. 70 The solutions of the latter equation for a cylindrical beam indicate that the light intensity becomes infinite in a finite propagation distance, for all laser powers above a certain threshold.

This prediction of infinite intensity is of course nonphysical. Before such large intensities are reached, the weak-nonlinearity assumption $v_{o s}{ }^{2} \ll v_{t h}{ }^{2}$ breaks down, and the cubic nonlinearity in the Schroedinger equation is no longer an adequate description. To investigate the development of a self-focusing beam past the point where the weak-nonlinearity assump- 


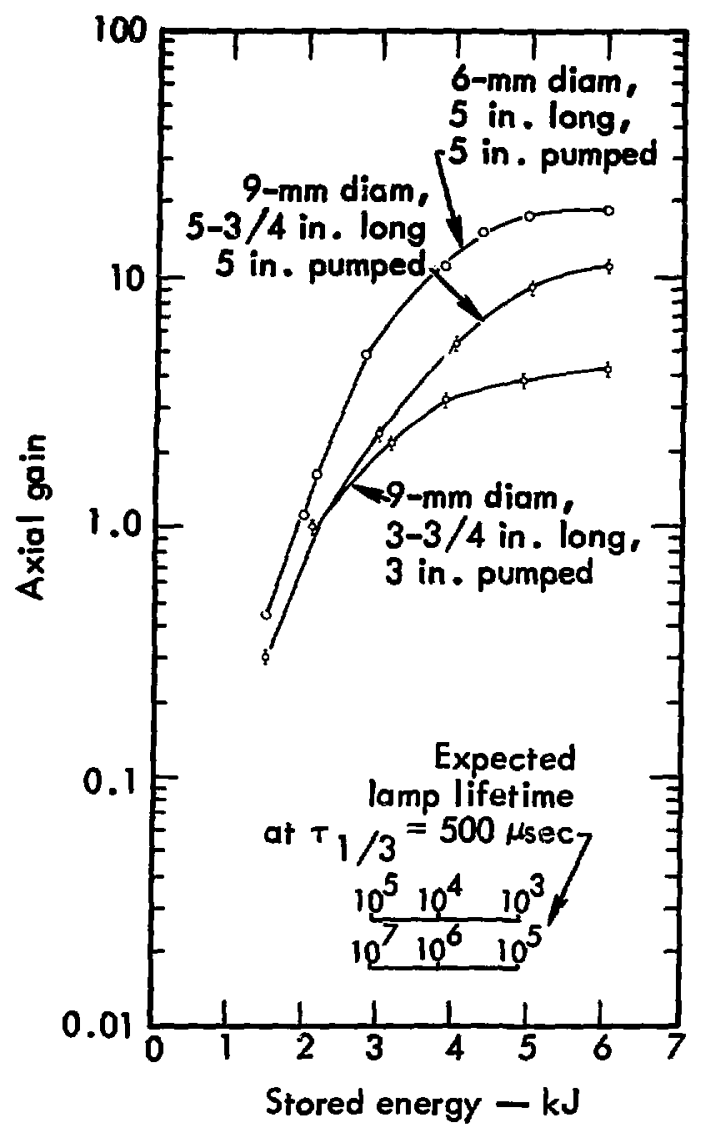

Fig 16\%. Optimiration of the premplifier stage containing 6- and 9-mm-dim ruby sods with 0.05\% doping in four-lamp clovedeaf hesd. The improvement in performance gined by eliminating the unpumped rod ends can be seen by comparing the two upper curves; the fact that the rod diameters are different is not significant for the uniform pumping asumed here.

By using the new pumped-end 6-mm amplifier preceding the current pair of $1 . \mathrm{cm}$-diam amplifiers (with unpumped rod ends), it was possible to observe self-focusing of the entire beam in the last $1-\mathrm{cm}$ amplifier due to the larger energy outputs when it was operated near maximum gain with a 5-mm input beam diameter. Figure 171 shows such a typical input and output beam spatial distribution. By telescoping the beam further, spatial nonlinear effects were eliminated. These results agree both with calculations and previous experimental expectations as to the nature and location of propagation problems. The final system design calls for a $7.5-\mathrm{mm}$ beam diameter propagating in $1-\mathrm{cm}$ rods with pumped ends, which is expected to totally eliminate this problem.

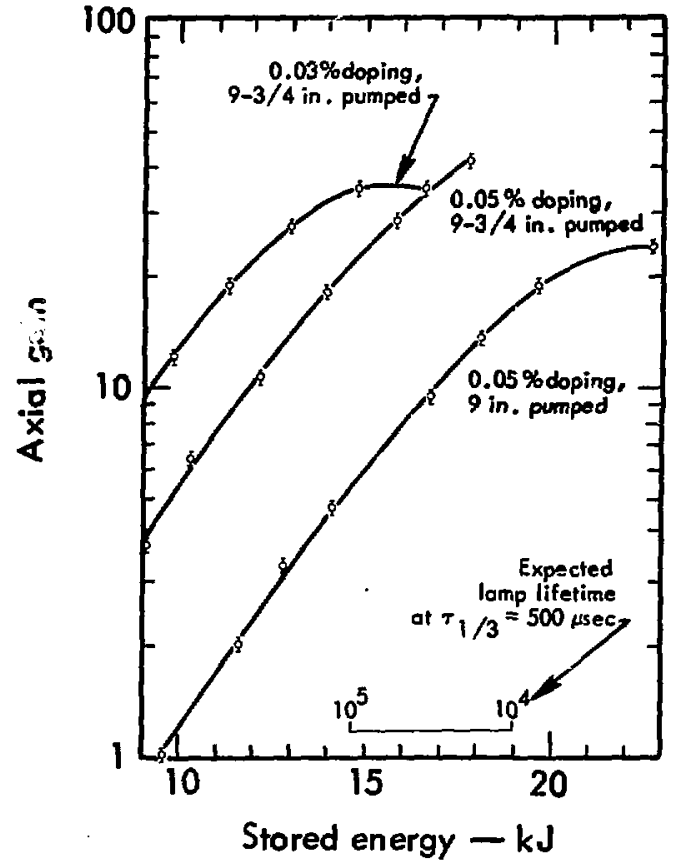

Fig 170. Optimization of the amplifier, containing a 14 mm-diam ruby rod $9-3 / 4$ in. long in a six-lamp clovedeaf hesd.

Finally, in connection with the ruby disk amplifiers, we have carefully studied the prospects for edge-coating the ruby disks to suppress parasitic oscillations. We have tested one very promising glass coating which has an index of refraction $n=1.8$ compared to $n 1.77$ for the ruby. A study of reflection versus angle for the coating was made using semicircular-cross-section disks with the coating on the perimeter, as has been done by NRL to evaluate glass disk edge-coatings. The data are shown in Fig. 172. Attenuation in a single pass of $>99 \%$ was typical, indicating that amplifier gain coefficients $>0.15 \mathrm{~cm}^{-1}$ are ot dinable without parasitic oscillation. The coated disks were placed in proximity to high-energy flashlamp light, with varying amounts of masking on the face and coated edge. We found that direct exposure of the coatings to flashlamp light led to irreversible damage to the coating, but a masking of only $1 \mathrm{~mm}$ of the disk face as well as the entire coated edge is sufficient to prevent any detectable short-term coating damage.

In order to assess the nonuniformity of the $\mathrm{Cr}_{\mathbf{r}}$ doping in the 35-by-70-mm elliptical-cross-section disks, we used an isodensitometer in which the light source was filtered to emit only in the ruby pump 


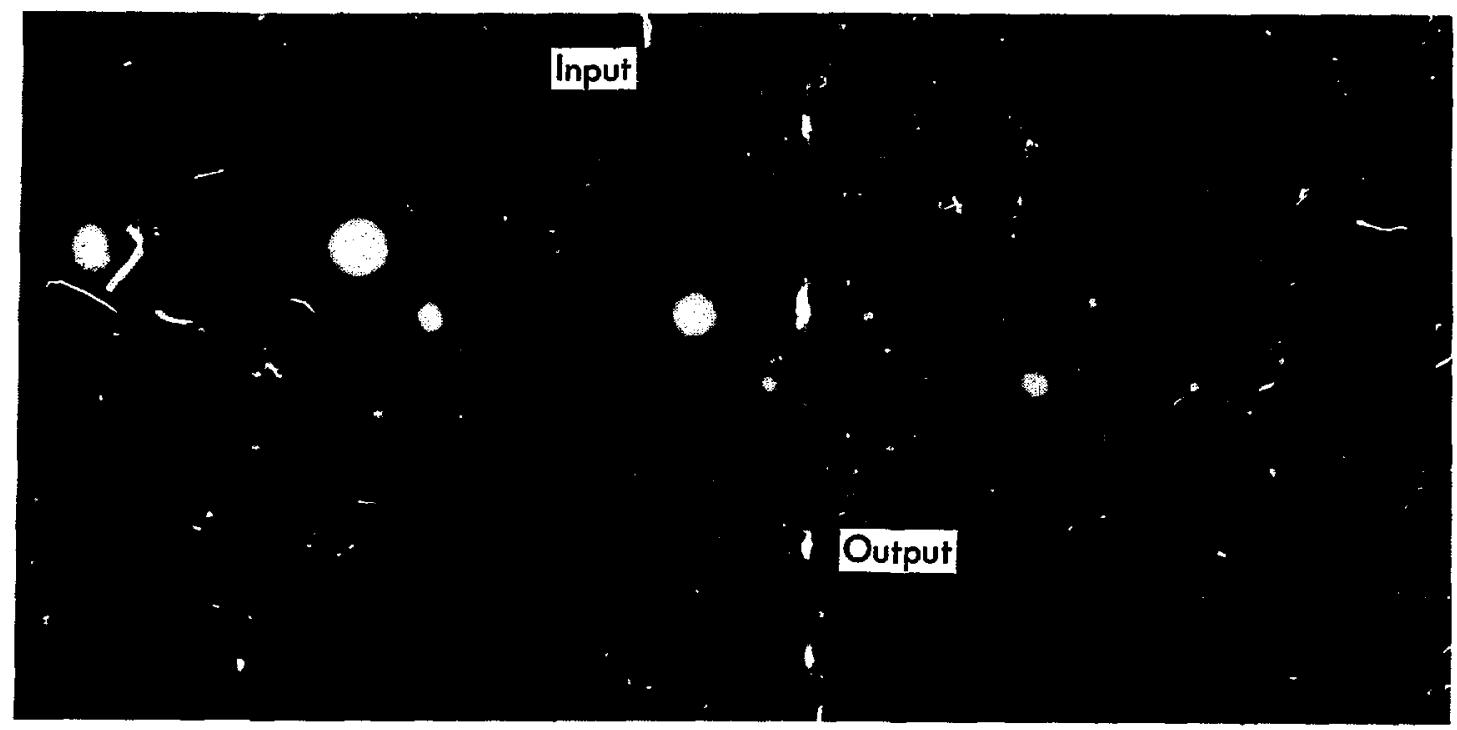

Fig. 171. Input and output near-field spatial patterns for the 1-cm-diam ruby amplifier, showing the partial focusing of the entire output beam due to intensity-dependent refractive index effects.

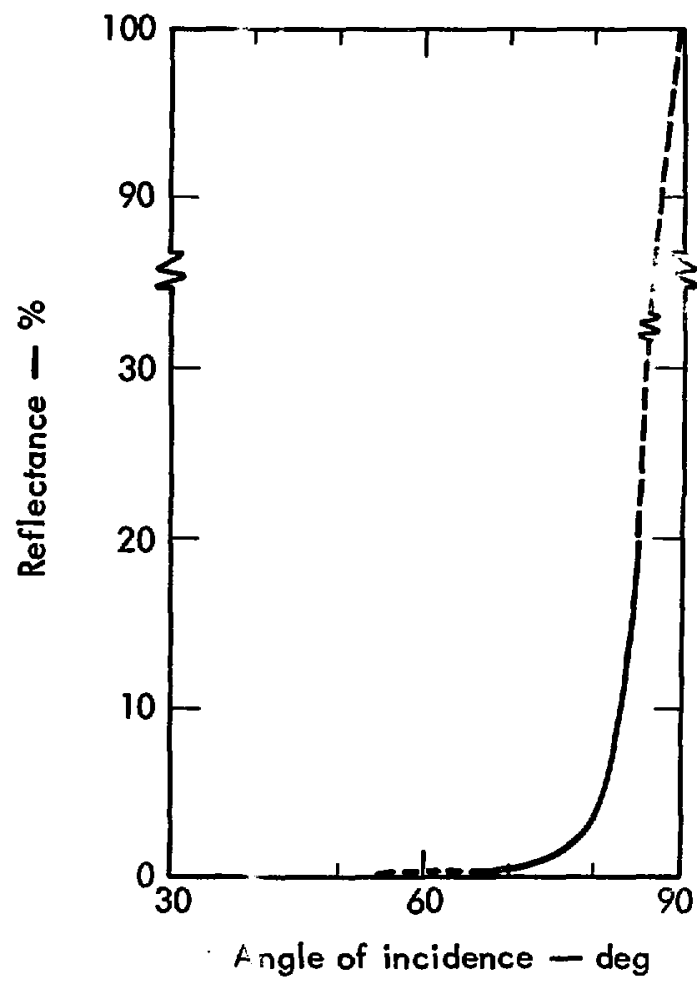

Fig. 172. Plot of measured reflectance vs angle of incidence at the ru'y-coating interface.

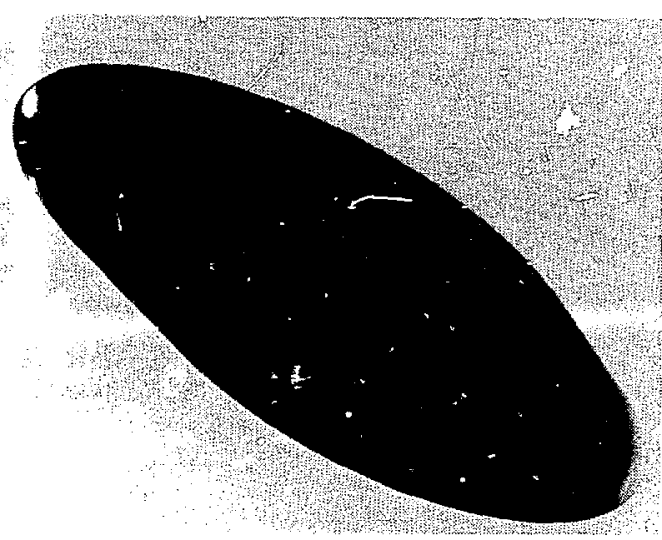

Fig. 173. One of the disks for the ruby amplifier. Elliptical cross section is 35 by $70 \mathrm{~mm}$.

bands. Figure 173 is a photograph of one of the disks. For nine disks of the same thickness, the average total variation in the doping of $0.05 \%$ nominal $\mathrm{Cr}$ was $1.5 \%$, which is well within the limits required for achieving sufficiently flat gain profiles over the active aperture.

With respect to the ruby-disk head design, preliminary data on the $\mathrm{Nd}$ :YAG disk amplifier (presented in the following article) seems to indicate that our present design is sound as far as being able to supply sufficient pump light to obtain the required 
gains. Further studies of the gain uniformity are required, however. We are no. planning to proceed with actual construction of the disk amplifiers for at least 9-12 months. The system is now being moved to Bldg. 174 and shortly after its optimization, several high-priority experiments will be undertaken.

\section{Nd:YAG DISK AMPLIFIER}

In the design of high-brightness, short-pulse amplifiers, Nd:YAG is an attractive candidate for the gain medium. Relative to Nd:glass, it has a high small-signal gain, $g$, and a comparable nonlinear index, $n_{2}$. A simple analysis indicates that for a specified output-beam intensity, self-focusing and self-phase modulation will be smallest for the material with the smallest value of the ratio $n_{2} / g$. This suggests investigating the performance of Nd:YAG amplifiers. We have encountere two probiems in this development. First, the quality of available YAG disks is poor. Second, suitable edge-coatings must be developed in order to take advantage of the high gain potential of the material.

In the previous semiannual report, ${ }^{164}$ a photograph was shown of an amplifier employing Nd:YAG disks. These disks are not edge-coated, which limits achievable gains, but nevertheless it is possible to make a preliminary evaluation of their performance. The system employs six disks having elliptical cross sections 13 by $25 \mathrm{~mm}$ and $6 \mathrm{~mm}$ thick, pumped in a 9-in.-long, six-lamp cloverleaf head. Selection of the disk size was limited by the technology for growing YAG material. The optical quality of YAG crystals is poor compared to that of other crystal materials such as ruby. A typical YAG boule has only limited regions of usable material. As indicated in Fig. 174, a number of striae "planes" radiate from a central core which runs the length of the boule. The 13-by-25-mm disks were cut from between these planes, in the region of "good" material quality. Since the present limit of boule size constrains rod dimensions to about $10 \mathrm{~mm}$ in diameter, the disk dimensions could not be much larger than this without sacrificing quality at the edges. Therefore we limited the disks to $13 \mathrm{~mm}$ along the minor axis, hoping to ensure good quality throughout the disk.

The appropriate head design and the position of the flashlamps within the head were determined from a study using the ZAP computer code. A comparison was made between the six-lamp cloverleaf arrangement, for which parts were available, and a six-lamp close-packed arrangement using a simple cylindrical reflector. It was initially decided to use water circulation within the head to improve head cooling. The maximum calculated pumping efficiency for both configurations was $0.7 \%$ for identical total flashlamp loadings (including flashlamp feedback) in both arrangemeits. On the basis of availability of parts, ease of filtering the flashlamp light, better cooling possibilities, and our past experience with the six-lamp cloverleaf head in obtaining goud gain profiles, we elected to use the cloverleaf head.

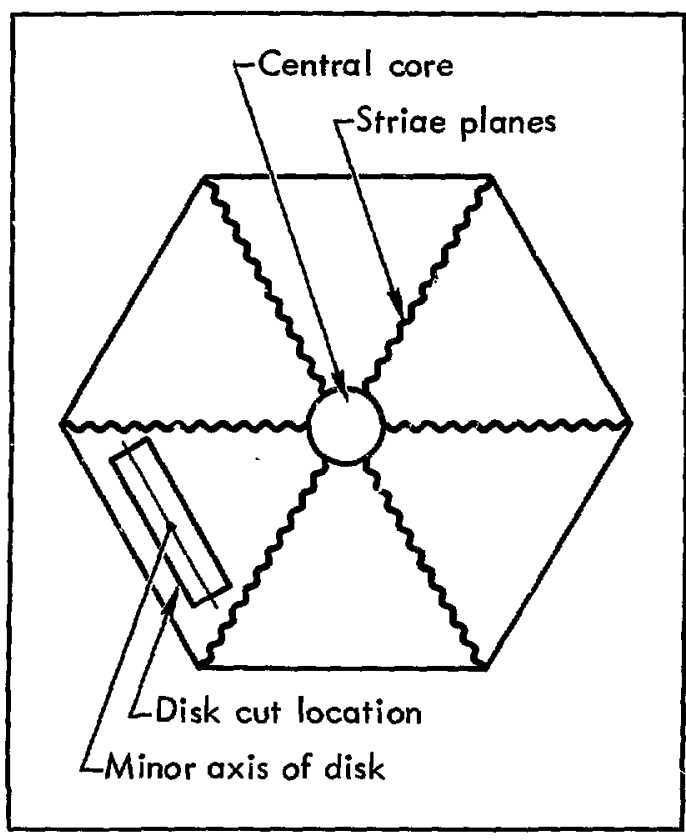

Fig. 174. End view of YAG boule showing central core, striae planes, and location of disk cut.
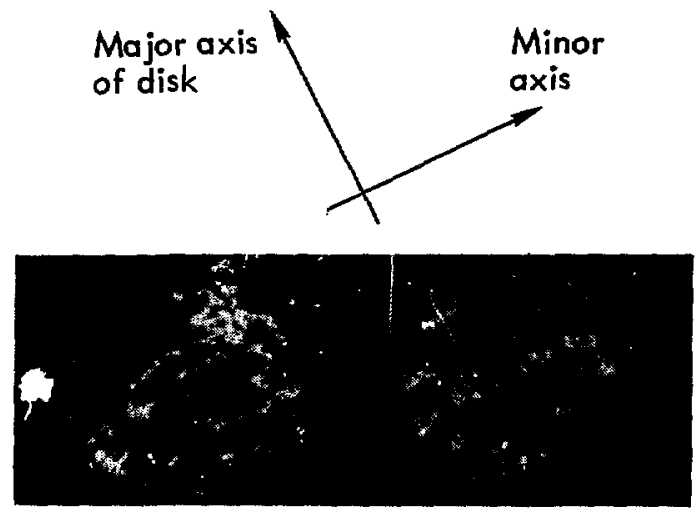

Fig. 175. Burn pattern frum an oscillator containing six highly striated YAG disks. 
Our calculations indicate that the position of the lamps within the cloverleaf huad has a pronounced effect on efficiency, which increases as the lamps are moved closer to the reflector, i.e., farther from the disks. Therefore all ampifier evaluation tests conducted thus far have been with the lamps in their outermost position. (The end plates were made to allow for adjusting the lamp pcsition.)

Disk quality, as expected, wis found to have a pronounced effect on amplifier performance. Even though individual disks were of acceptable quality, the additive effects of optically stacking six disks were disastrous to the overall optical quality. The presence of striae caused the most pronounced effects, as was determined by testing the amplifier head in an cocillator configuration. A typical "bum pattem" of the output of the oscillator with six highly striated disks is shown in Fig. 175. An interferogram of the six disks optically stacked in their holder is shown in Fig. 176. It is possible that most of the probiems are due to inhomogeneities caused by strain induced during polishing rather than poor crystal growth. If this is the case, annealing of the disks may improve the situation. We are investigating this possibility.

Amplifier gain was measured by observing the pulse trains before and after amplification, on the same cocilloscope. Initially a 3-mm probe beam was used to evaluate axial gain. Maximum gain occurred with no delay between amplifier and oscillator triggering,

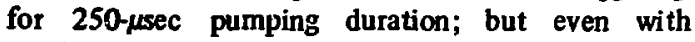
oscillator delays as great as $\mathbf{1 5 0} \mu \mathrm{sec}$, no pronounced effects were noted in the gain. Also, it was noted that increased bank voltage, i.e., increased stored energy, had little effect on the gain. This indicates that a gain saturation occurred, which we attributed to superfluorescence and/or parasitic oscillation effects within the individual disks, since all pulses within the oscillator train were amplified to the same extent. The maximum observed gain was 3.1, which corresponds to a gain coefficient of $0.27 \mathrm{~cm}^{-1}$. Further gain measurements were mide using a $12-\mathrm{mm}$ beam, which filled the entire disk aperture. Figure 177 is a plot of gain vs stored bank energy. The same gains were observed for the 12-mm-diam beam as for the $3-\mathrm{mm}$ beam.

At present we are attempting to improve the Nd:YAG disk amplifier quality by careful selection of disks after they have been annealed. With improved material quality, we will be able to evaluate gain

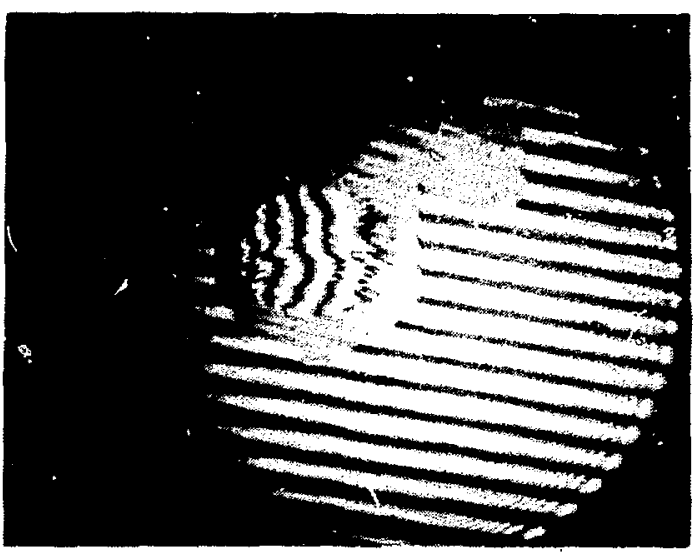

Fig. 176. Interferogram of the six highly striated YAG disks optically stacked in their holder.

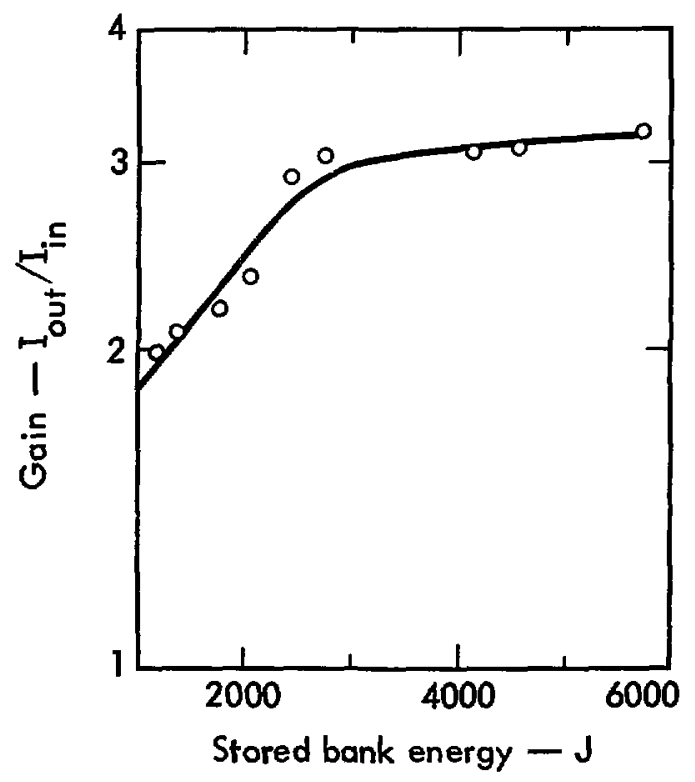

Fig 177. PLot of gain of YAG disk amplifier vs stored bank energy. Note saturation at higher bank energies.

profiles accurately across the amplifier aperture. Furthermore, if material quality merits it, we will begin to look into edge-coating possibilities in the near future.

\section{STIMULATED RAMAN SCATTERING IN GASES AT HIGH PRESSURE}

There are several interesting possibilities for the application of stimulated Raman scattering (SRS) to the problems of laser fusion. Perhaps the most obvious is the use of SRS as a method of isolating a highly reflecting plasma from the laser amplifier chain. The frequency of the laser beam could be shifted out of 
the amplifier gain bandwidth so that any reflected light reentering the amplifier chain would not experience gain and would thus be unlikely to cause damage to the amplifier. SRS might also be used to extract energy from an inverted medium as a type of two-photon amplifier which clearly has attractive properties. The rational implementation of these possible applications requires that we first have a detailed understanding of the properties of SRS. We have consequently undertaken a study of SRS in high pressure gases directed toward these and other applications.

The laser system being used for these experiments has been previously described. 165 We have experimentally verified that the 1-nsec (FWHM) output pulse is bandwidth-limited ( $\triangle V$ (FWHM) $=0.016 \mathrm{~cm}^{-1}$ ) for energies up to $1 \mathrm{~J}$, and that the system is capable of achieving a pulse energy of up to $3 \mathrm{~J}$. The beam divergence, which is approximately three times the diffraction limit, is due to the poor optical quality of the Nd:YAG amplifier rods and the nonuniform pumping geometry of the double-ellipse, Nd:glass amplifier heads which also produce nonuniformities in the nearfield intensity distribution. The system is currently being modified to eliminate these difficulties.

We have chosen to examine the details of SRS generated by a collimated beam in a $1 \cdot \mathrm{m}$-long gas cell. This geometry eliminates many of the ambiguities present in previous experimental investigations of SRS in which the laser beam was focused. With the collimated beam we avoid questions of the intensity distribution in the focal region and competition between forward and backward SRS for 1-nsec pulses. The spatial, angular, frequency, and temporal distributions for the various orders of the SRS now may be studied as functions of laser intensity and gas pressure. The results of these studies, while interesting in their own right, may further provide experimental checks on a two-dimensional Raman scattering code which is now under development ${ }^{154}$ and which may give direction for optimization of future energyextraction schemes utilizing SRS.

Our initial studies were made on $\mathrm{SF}_{6}$ gas at a pressure of $18 \mathrm{~atm}$. This gas was chosen since the broad linewidth of the vibrational transition $\left(10^{11} \mathrm{~Hz}\right)$ ensures that the stimulated scattering will be in the steady-state regime for 1-nsec pulses. In addition, this pulsewidth is short compared to the acoustic transit time in the scattering volume, thereby inhibiting the occurrence of stimulated Brillouiu scattering (SBS). The relatively small Raman shift $\left(775 \mathrm{~cm}^{-1}\right.$ ) makes it possible to study the conversion of energy to many Stokes and anti-Stokes orders using standard visible and infrared photographic techniques.

In $\mathrm{SF}_{6}$, SRS occurred at a threshold laser power of about $1 \mathrm{GW} / \mathrm{cm}^{2}$ with the incident laser wavelength of $1.06 \mu \mathrm{m}$. This power level was unfortunately very close to the damage threshold for the pressure cell windows, necessitating their frequent replacement and presenting serious danger of damage to the laser system due to amplification of light backscattered from a window damage site. Consequently, taking advantage of the high laser power to obtain second-harmonic-generation conversion efficiencies of $30 \%$, and of the fact that the Raman gain coefficient is proportional to the frequency of the exciting laser source, we have done all subsequent work using the second harmonic beam at power levels less than $500 \mathrm{MW} / \mathrm{cm}^{2}$. This eliminates the window damage and laser feedback problems.

Using $5300-\AA$ beam, SRS in $\mathrm{SF}_{6}$ has been generated at wavelengths from 4738 to $7076 \AA$ in steps of $775 \mathrm{~cm}^{-1}$ with conversion efficiencies of $80 \%$ into successive Stokes orders. The first objective was to study the time dependence of the conversion to the various Stokes and anti-Stokes orders. The time dependence was investigated using a streak camera with 10 psec resolution (sce article by Carman, Spracklen, and Thomas earlier in this repart in Diagnostics section, p. 89). the $5300-\AA$ beam and the SRS Stokes lines could be simultaneously displayed on the streak camera trace. When no SRS occurred, the 1-nsec, $5300-\AA$ pulse showed no detectable time structure; when SRS did occur, all of the Stokes orders exhibited severe temporal modulation with a period of about 100 psec as shown in Fig. 178. The initial reaction to these observations was that the temporal structure was due to strong coupling of the stimulated waves. Since this coupling would be strongest near the

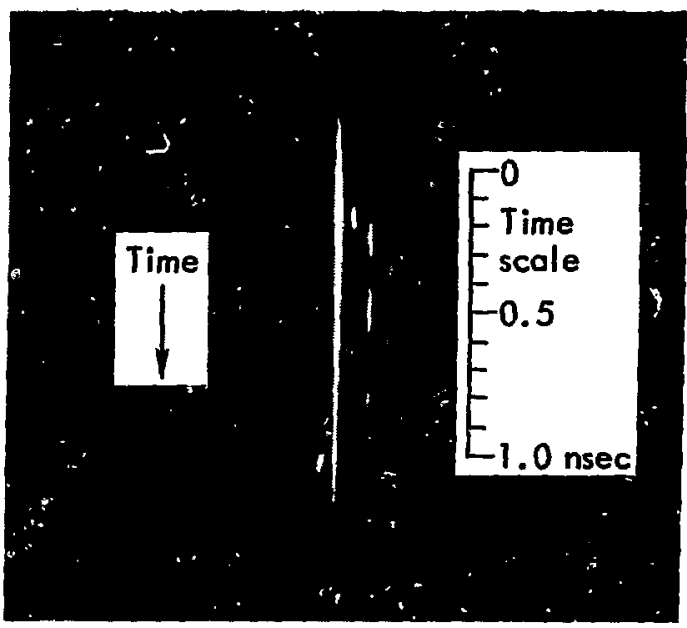

Fig. 178. Streak camera record of the time dependence of intensity in the laser and first four RamanStokes waves appearing in order from left to right. Time incresses moxing down the trece, with the scite as shown. 
Stokes/anti-Stokes phase matching angle, it was logical to assume that the time dependence in other, more dispersive, gases would be qualitatively different. Consequently, we have successfully generated SRS and examined its time dependence in the following gases: $\mathrm{CO}_{2}, \mathrm{~N}_{2}, \mathrm{~N}_{2} \mathrm{O}, \mathrm{CH}_{4}, \mathrm{H}_{2}$, and the liquid, $\mathrm{CS}_{2}$. Although the results for each gas examined have indeed been qualitatively different, no obvious pattern in the data has emerged, and no satisfactory explanation of the results can yet be given. The interpretation of the results from some of the gases is additionally complicated since the SRS occurs in the transient regime due to a long phonon lifetime. To further investigate the proposal that the time structure is due to mutual coupling of the stimulated waves, an existing computer code (RAMANS) is being modified to calculate the propagation properties for three coupled waves. Other possible explanations for our observations have also been examined experimentally, and some conclusions can be drawn from these investigations.

Since the existing laser has no detectable frequency chirp, the observed time dependence cannot be due to the laser light's phase changing rapidly compared to the phonon lifetime. ${ }^{166}$ The possibility of interference in the time domain caused by a small frequency shift of the Stokes wave - arising perhaps from anharmonicity of the molecular vibrational levels or simultaneous occurrence of SRS and SBS - has also been eliminated. We have ruled out, in addition, the possibility of coherent oscillation of the molecular vibrational population, the occurrence of relaxation oscillations due to competition between forward and backward SRS, ${ }^{167}$ and the possibility of gain modulation due to dispersion between the pump and Stokes waves. Another possible explanation for the time dependence of the SRS arises from a time-dependent spatial intensity distribution of the pump beam. To study this possibility, we are modifying the laser system to eliminate spatial nonuniformities in the pump beam.

Clearly these aspects of SRS must be well understood before its application to problems in other areas becomes practical. To this end, investigations along the lines discussed above are continuing.

\section{STUDIES OF THE ${ }^{4} I_{11 / 2}$ LEVEL IN ED-2 NEODYMIUM-DOPED GLASS}

An experiment is under way to measure the nonradiative lifetime of the $4 / 11 / 2$ level in Owens-Illinois ED-ż glass (henceforth called "ED-2 glass"). This is the lower level for the $1.06-\mu \mathrm{m}$ laser traissition. Published observations ${ }^{168}$ suggest that the $4_{I_{11 / 2}} \rightarrow 4_{I_{9 / 2}}$ lifetime in other laser glasses is in the range 50-100 nsec. A much shorter relaxation time in ED-2 glass could influence amplifier design parameters.

As a preliminary effort, we have observed and identified excited-state absorptions from the ${ }^{4} I_{11 / 2}$ energy level of the neodymium ion in ED-2 glass. An energy level scheme for the neodymium ion is shown in Fig. 179, using the notation taken from Mann and DeShazer. ${ }^{169}$ The splitting of the $F$ and $I$ levels is not depicted in the drawing. One excited-state absorption transition will be used to monitor the ${ }^{4} I_{11 / 2}$ dynanics. Preliminary excited-state absorption spectra indicate that the measured $0.66-\mu \mathrm{m}$ peak cross section is in good agreement with theory.

\section{Excited-State Absorption Observations}

All absorption measurements were performed on a Cary 14 spectrophotometer. Figure 180 shows a double-trace absorption spectrum for sample 1 (a 1/2-in.-thick polished disk of nominally $3 \%$ doped ED-2 glass). One trace was taken at room temperature and the other at $500^{\circ} \mathrm{C}$. The chart gives the optical density versus wavelength. Note that at elevated temperatures new features appear at $0.66 \mu \mathrm{m}$
$(6600 \AA)$ and $1.06 \mu \mathrm{m}$. These absorptions correspond to upward transitions which arise from the larger thermal population $\left(\sim 4 \%\right.$ of the ions at $\left.500^{\circ} \mathrm{C}\right)$ of the ${ }^{4} I_{11 / 2}$ level.

To measure the ${ }^{4} I_{11 / 2} \rightarrow{ }^{4} I_{9 / 2}$ life time, we require information about the ${ }^{4} I_{11 / 2}$ population; it appears that the $0.66-\mu \mathrm{m}$ transition is the only useful monitor of this population because it does not overlap normal absorption bands from the ${ }^{4} I_{9 / 2}$ ground state. The $1.06-\mu \mathrm{m}$ excited state absorption may not be easily monitored because of the difficulty in discriminating a probe from the intense $1.06-\mu \mathrm{m}$ pulse necessary to saturate a flashlamp-pumped Nd:glass amplifier.

\section{Estimates of Excited-State Absorption}

\section{Cross Sections}

In thermodynamic equilibrium, the transmission of light through a sample of length $\ell$ is given by $T(\lambda)=\exp \left[-n \sigma_{a b s}(\lambda) \ell\right]$, where $n$ is the density of ions in the lower level of the transition, and $\sigma_{a b s}(\lambda)$ is the absorption cross section. The transmission and the length can be accurately measured, while $n$ must be inferred in order to estimate excited-state absorption cross sections. To a good approximation, the probability of occupation of the ${ }^{4} I_{11 / 2}$ state is given by the sum of the Boltzmann factors over all Stark sublevels of the ${ }^{4} I_{11 / 2}$ state divided by the sum of Boltzmann factors over both the ${ }^{4} / g_{9 / 2}$ and ${ }^{4} / 1 / 2$ states. Line locations for ED-2 glass are taken from 
Ref. 170, from which we find that the probability of occupation in the ${ }^{4} / 1 / 2$ level (from sample 1 at $\therefore\left(0^{\circ} \mathrm{C}\right)$ is approximately 0.0377 . We assume 171 that the density of neodymium ions in a $3 \%$ doped rod is $2.89 \times 10^{20} / \mathrm{cm}^{3}$. (Although DeShazer in Ref. 170 reports $20 \%$ less neodymium density in the same glass, we will for the time being use the value reported in Ref. 171.) Let us first address our attention to the 0.66- $\mu \mathrm{m}$ absorption $\left({ }^{4} I_{11 / 2} \rightarrow{ }^{4} G_{5 / 2}\right)$. We estimate that one-third of the optical density shown in Fig. 180 at the peak of the new feature is due to the expanding tail of the ground state absorption (which appears to the right of the feature), and the remaining two-thirds is due to excited state absorption. With this assumption we find that the maximum of $\sigma_{a b s}\left({ }^{4} I_{11 / 2} \rightarrow{ }^{4} G_{5 / 2}\right)$ is given by $0.78 \times 10^{-20} \mathrm{~cm}^{2}$. Krupke has calculated ${ }^{172}$ that for a $290-\AA$-wide band, the peak should be $0.8 \times 10^{-20} \mathrm{~cm}^{2}$. Our measurement is in

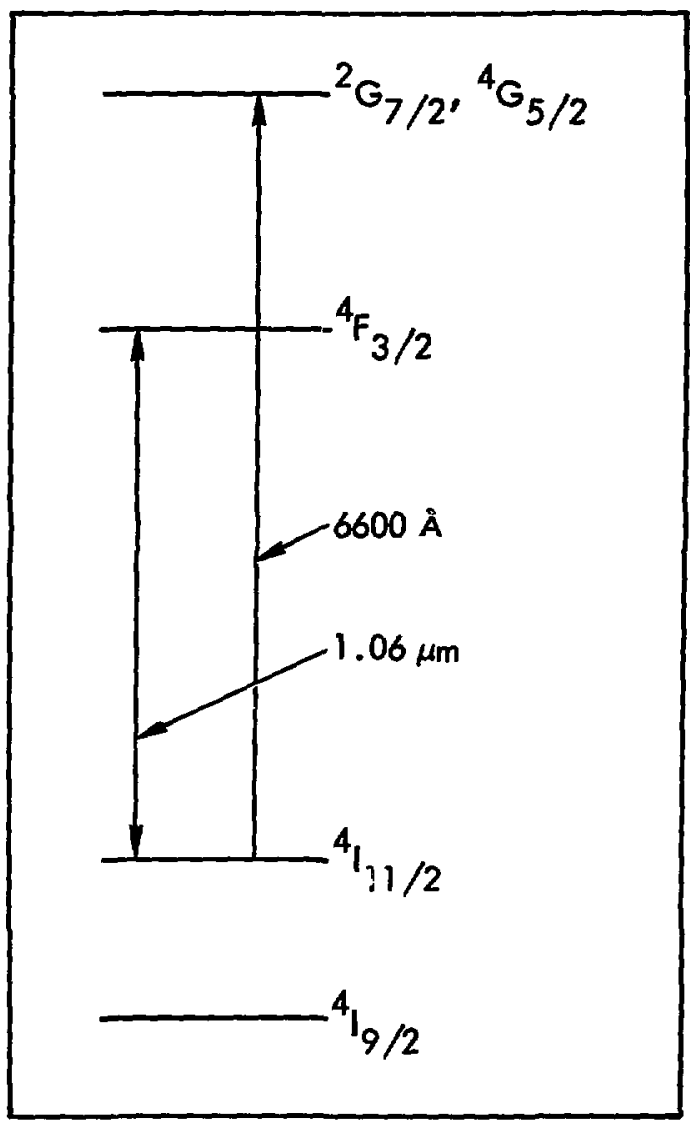

Fig. 179. Level scheme for the neodymium ion in glass. Other levels not involved in the dynemics studies in this article are not depicted, nor is the splitting of each of the four levels.
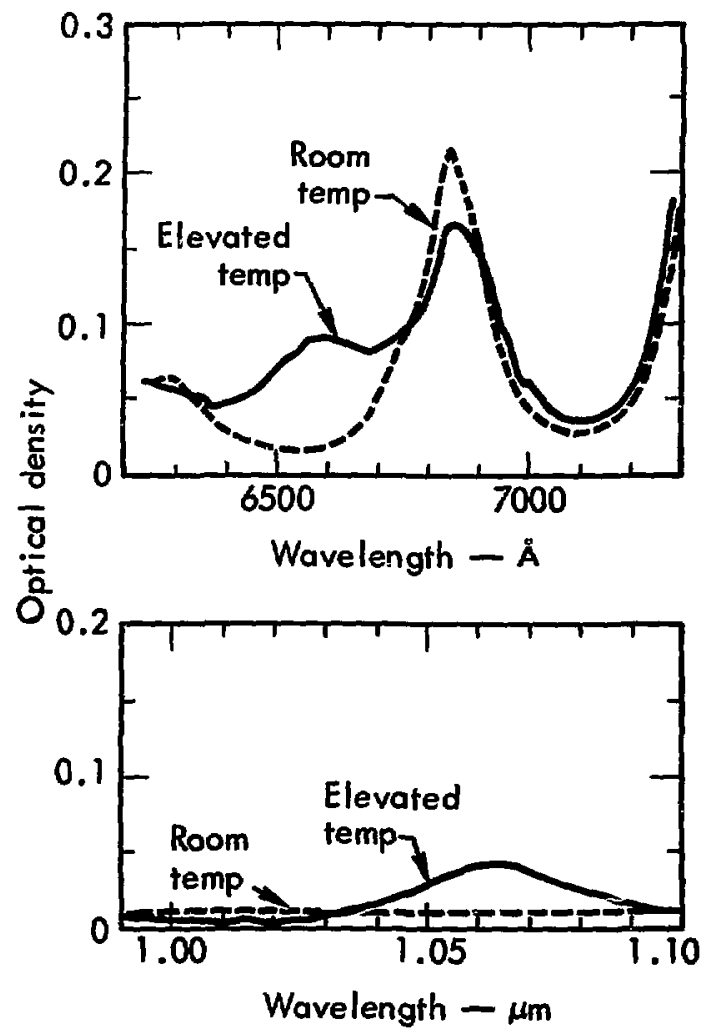

Fig. 180. Tracings of selected regions of the absorption spectrum for sample 1. The epectrometer was operated in the optical density mode. Note the appearance of the two excited-state absorption features.

close agreement, but our choice of the factor $2 / 3$ was quite arbitrary.

At $500^{\circ} \mathrm{C}$, we find that the maximum value of $\sigma_{a b s}\left({ }^{4} I_{11 / 2} \rightarrow{ }^{4} F_{3 / 2}\right)$ is $0.56 \times 10^{-20} \mathrm{~cm}^{2}$. There is no background due to nearby ground-state absorptions. At reduced temperatures, measurements on a second sample give values of $\sigma_{q b s}\left({ }^{4} I_{11 / 2} \rightarrow{ }^{4} F_{3 / 2}\right)$ of $0.6 \times 10^{-20}$ and $0.7 \times 10^{-20} \mathrm{~cm}^{2}$ measured at 350 and $300^{\circ} \mathrm{C}$ respectively. At present published data are insufficient to infer from our measurements the value of the pesi stimulated-emission cross section $\sigma_{\text {stim }}\left({ }^{4} F_{3 / 2} \rightarrow{ }^{4} I_{11 / 2}\right)$.

\section{${ }^{4} I_{11 / 2}$ Nonradiative Lifetime Measurements}

Experiments are presently under way to use the newly found $0.66-\mu \mathrm{m}$ excited state absorption to measure the ${ }^{4} I_{11 / 2}$ nonradiative lifetime. A flashlamp-pumped cresyl violet perchlorate dye-laser pulse (tunable from 6500 to $6800 \AA$ ) is passed 
through a 2-by-20-in. flashlamp-pumped Nd:glass laser head simultaneously with a nearly saturating $1.06-\mu \mathrm{m}$ light pulse. The $1.06-\mu \mathrm{m}$ pulse is about 1 nsec in duration, the dye laser pulse about 300 nsec. The monitored recovery of the $6600-\AA$ probe signal should give a direct readout of the $4 I_{11 / 2}$ nonradiative relaxation time. Since the dye laser takes approximately 900 nsec to tum on, large optical delays are useful in such experiments. For this reason, the experiment is set up on the Long Path Laser, where such delays are available.

Let us assume that a neodymium-glass laser amplifier can leave approximately $1 / 2 \%$ of its ions in the ${ }^{4} I_{11 / 2}$ level after amplifying a suitably bright $1.06-\mu \mathrm{m}$ pulse. With these $1.45 \times 10^{18}$ excited ions $/ \mathrm{cm}^{3}$, the excited-state absorption coefficient would be $0.0115 \mathrm{~cm}^{-1}$. Thus the transmission of a $0.66-\mu \mathrm{m}$ probe beam will be $57 \%$ for a $50-\mathrm{cm}$ laser amplifier rod. This is an acceptable signal to monitor.

It is important to show that the amplifier flashlamp light will not influence the proposed measurement.
This is easy to demonstrate if we assume that upward pumping probabilities are nearly equal for ions in the ${ }^{4} I_{9 / 2}$ and ${ }^{4} I_{11 / 2}$ state. We know that the flashlamp takes about $400 \mu \mathrm{sec}$ to remove $1 \%$ of the ${ }^{4} I_{9 / 2}$ ions. Our measurement is performed in $100 \mathrm{nsec}$; so if the flashlamp is on, it should reduce our ${ }^{4} I_{11 / 2}$ population by the multiplicative factor $0.01 \times(0.1 \mu \mathrm{sec})$ ) $(400 \mu \mathrm{sec})=2.5 \times 10^{-6}$. Since the flashlamp can be nearly off at the time of the experiment, this effect can be further reduced by another factor of 10 if necessary.

Two matched photomultipliers will be used to measure the attenuated and unattenuated pulse shapes. The dynamic linear range of these tubes was measured to be below $0.75 \mathrm{~V}$ output for a 200-nsec pulse into $50 \Omega$. Pulse intensities have been adjusted accordingly. At present a lifetime between 2 and 200 nsec could be measured. The upper limit is due to the dye-laser pulse duration, and the lower limit results from the fact that the saturating pulse and the probe pulse are passing in opposite directions in the 20-in. rod.

\section{TUMABLE LASER SPECTROSCOPY}

In order to address some of the spectroscopy-related questions in the laser fusion program, we have constructed and characterized a tunable $\mathrm{cw}$ dye-laser which is pumped by a cw argon ion laser at $5145 \AA$ and can operate from 5300 to $7000 \AA$ with linewidths less than $10^{-3} \AA$ throughout this range. ${ }^{173}$ In addition, we have nearly completed fabricating a pulsed dye laser pumped by the 3371- $\AA$ line of a pulsed nitrogen laser. The potential tuning range of this device utilizing a varriety of dyes is 3400 to 7400 \& with similar narrow linewidths. ${ }^{174}$ Harmonic generation can extend the lower wavelength region to about $2500 \AA$.

In Fig. 181 we show the spectral output of the $\mathrm{cw}$ dye laser operating at about $5900 \AA$ using the dye thodamine $6 \mathrm{G}$ and tuned by a single intracavity prism. The observed linewidth is $\leqslant 0.25 \AA$. Further narrowing to about $10^{-3} \AA$ or less is readily accomplished by use of additional (fine-tuning) intracavity etalons. A schematic diagram of the pulsed dye laser is shown in Fig. 182.

Our intention is to utilize these tunable lasers as sources to probe the excited state dynamics of novel atomic/molecular gas-laser candidates emitting in the

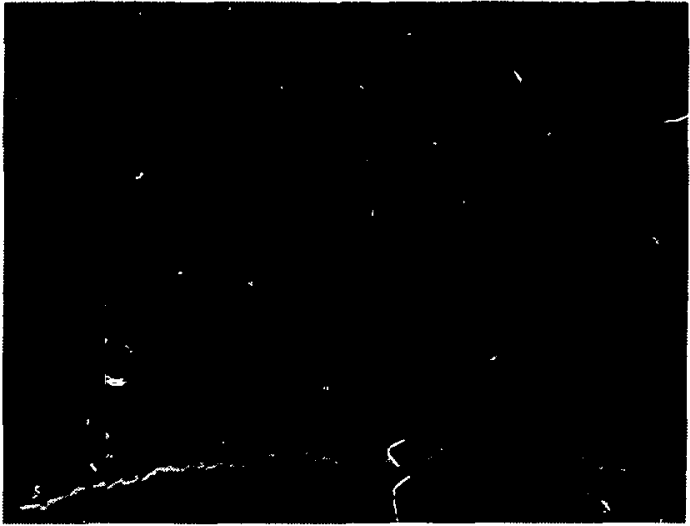

Fig. 181. Spe trum of a cw dye laser tuned by use of a single intracavity prism and operating at $\sim 5900$ A. Each dot corresponds to a wavelength resolution of 0.058 \& on the optical spectrum analyzer. Additional intracavity etalons can narrow the line to $\sim 0.001 \mathrm{~A}$.

visible spectral region, and to study the $\mathrm{Nd}$ :glass laser medium. 


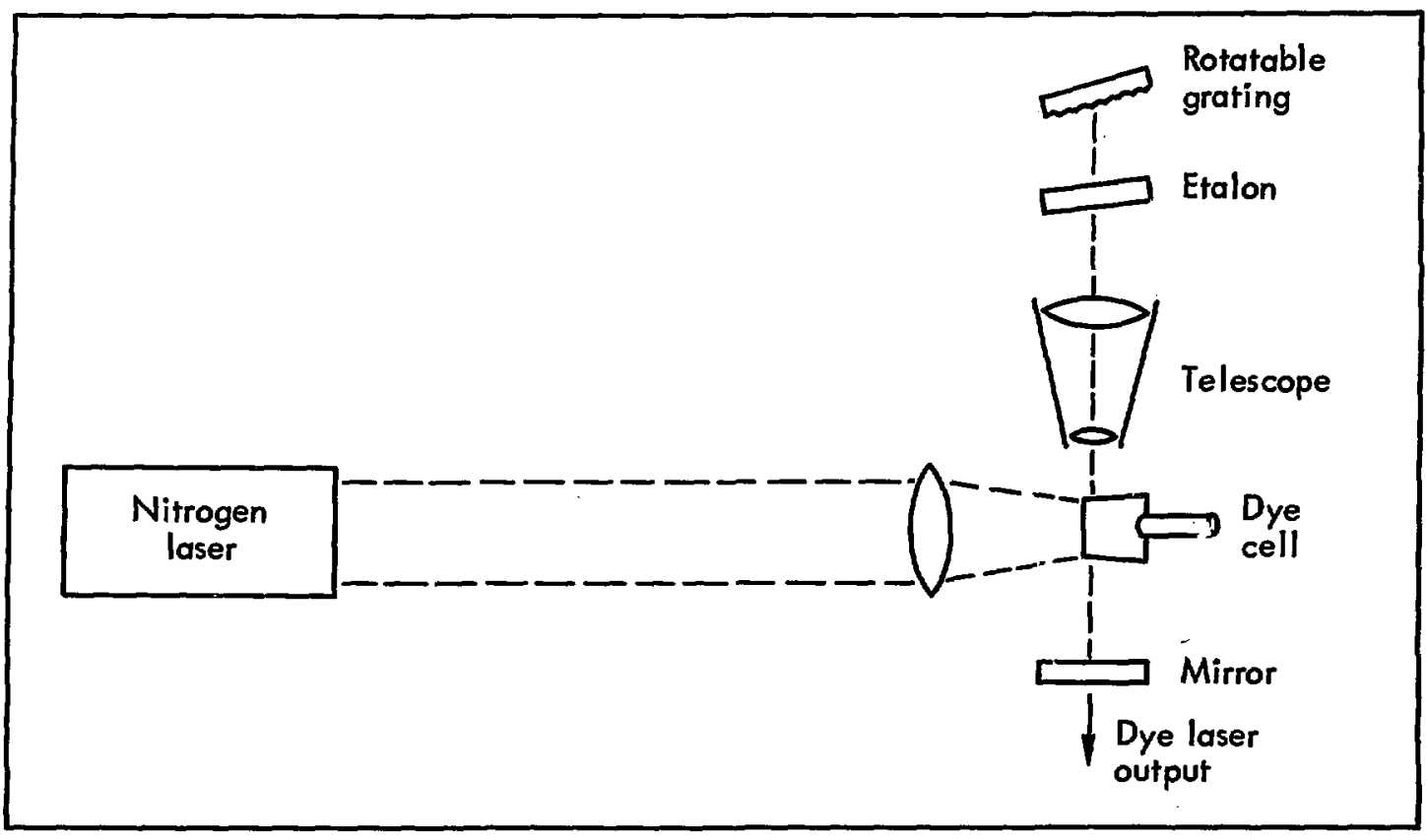

Fig. 182. Schematic diagram of a pulsed dye laser excited by the 3371-A line of a pulsed nitrogen laser. The rectangulnz-crosesection nitrogen beam is focused into the dye cell, and the subsequent absorption region in the liquid defines the dye leser axis. In order to increase the resolntion available from the tuning grating and thus decreuse the lnser linewidth, a telescope is used to expand the small excited dye region so that it fills up 2 lange fraction of the grating. The etalon is used to further reduce the laser linewidth. The tuning range of this device is about 2500 to $7400 \mathrm{~A}$, throughout which linewidths of $\sim 0.004 \mathrm{~A}$ are obtainable. 


\section{LASER PROPAGATION}

\section{TWO-DIMENSIONAL PROPAGATION AND SELF-FOR.USING CUDE}

The importance of assessing diffraction and self-focusing effects in the design of high-power glass laser systems is already well established. While axially symmetric codes yield many of the important aspects of self-focusing in amplifier chains, the use of disks leads to elliptical patterns in the beam within the pumped media which must also be examined. To this end, a time-dependent, two-transverse-dimensional propagation and self-focusing code has been developed, based on cubic spline approximations in $r \cdot \theta$ coordinates. The use of $r-\theta$ geometry allows the introduction of elliptical beam profiles in a natural way and also makes possible radial zoning in graded step sizes.

A fast, cylindrically symmetric, time-indeperdent code has also been developed to supplement the time-dependent LAMP code. The new code carries 1000 radial zones, uses only two-thirds of the CDC 7600 small core memory, and advances the solution at the rate of 40,000 zone cycles per second.
Like the LAMP code, it is fully implicit and is based on cubic spline interpolations.

Figure 183 shows two-dimensional isointensity profiles at two propagation distances for a propagating and self-focusing Gauss-Hermite $\mathrm{TEM}_{33}$ mode. The wavelength is $1.06 \mu \mathrm{m}$. The contours represent fractions of the maximum intensity in increments of 0.1. The loss of contours in certain portions of the pattem is due to the relatively stronger self-focusing in other portions of the pattem. Note that the overall symmetry of the mode structure is preserved despite the self-focusing.

Calculations have also been carried out to examine the self-focusing of elliptical beams. ${ }^{175}$ Preliminary results agree with Marburger's formula for self-focusing distance if the latter is multiplied by a factor of $1 / \sqrt{2}$. This factor is necessary to bring the Marburger formula into agreement with the well-known Kelley ${ }^{176}$ formula when the major and minor elliptical axes are equal. (a)

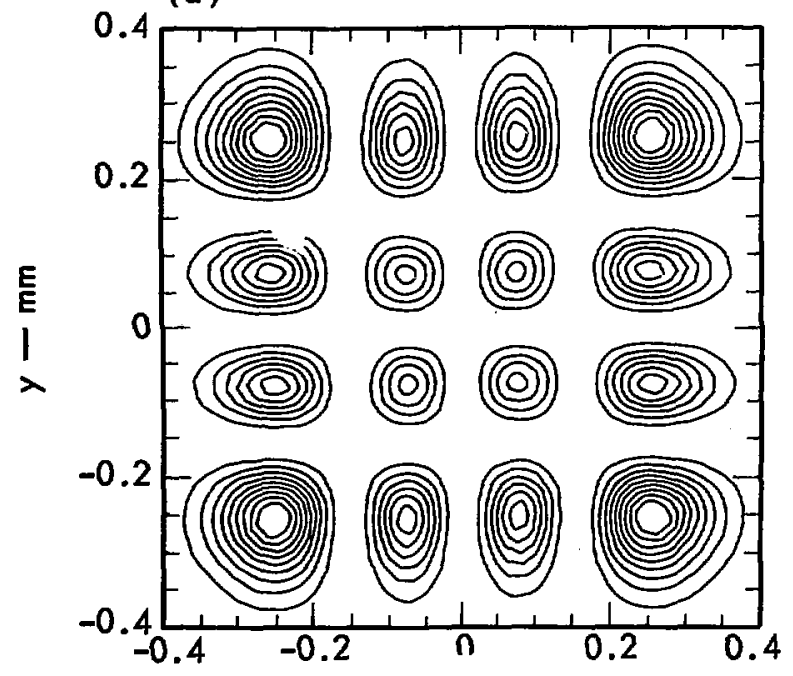

(b)

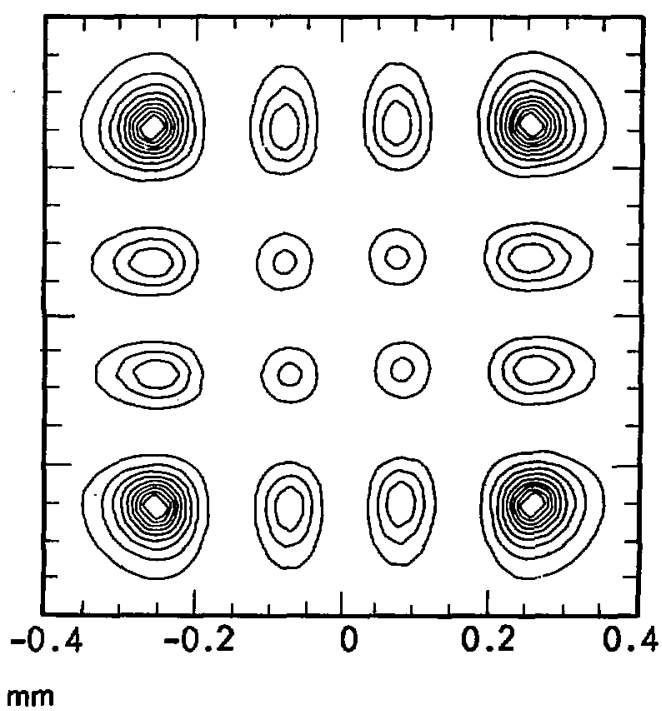

Fig. 183. Self-focusing of Gauss-Hemite $\mathrm{TEM}_{3 \mathrm{3}}$-mode isointensity contours. The weakest self-focusing is indicated by the smallest number of contours. (a) Propagation distance $z=10^{-5} \mathrm{~cm} ; I_{\max }=9.95 \times 10^{12} \mathrm{~W} / \mathrm{cm}^{2}$. (b) Propagation distance $z=3 \times 10^{-4} \mathrm{~cm} ; I_{\max }=21 \times 10^{12} \mathrm{~W} / \mathrm{cm}^{2}$. 
A recent measurement ${ }^{8}$ su $_{c}$ gests the large value of $4 \times 10^{-12} \mathrm{~cm} / \mathrm{W}$ for the nonlinear absorption coefficient in 3\%-doped Nd:glass. It so, nonlinear absorption will have a profound effect on the propagation of intense pulses, since it can prevent self-focusing by spatially and temporally flattening the pulse shape while limiting "te maximum intensity attainable.

We became interested in this problem while investigating the self-focusing behavior of a typical amplifier chain consisting of two YAG amplifiers followed by two glass amplifiers having known radially dependent gains. The results presented here are for a $1.5-\mathrm{mJ}$ pulse of $150 \mathrm{psec}$ duration and $0.06 \mathrm{~cm} e^{-1}$ intensity radius. The value used for the nonlinear dielectric coefficient is $\epsilon_{2}=4 \times 10^{-13}$ esu.

Without nonlinear absorption, self-focusing occurs in the last amplifier. The onset is shown in Fig. 184, in which we display consecutive radial intensity profiles starting at the time of maximum on-axis intensity. Note the formation of a filament on-axis. The modulations of intensity which form filaments are apparently related to fundamental propagation modes. ${ }^{177}$ The energy of this pulse is $23 \mathrm{~J}$. Figure 185 shows the catastrophic self-focusing that occurs at a distance $4 \mathrm{~cm}$ farther downstream and at an energy of $32 \mathrm{~J}$.

We find a much different result when nonlinear absorption is included (Fig. 186). With otherwise the same conditions as theje for Fig. 184, note that the maximum intensity has been decreased by a factor

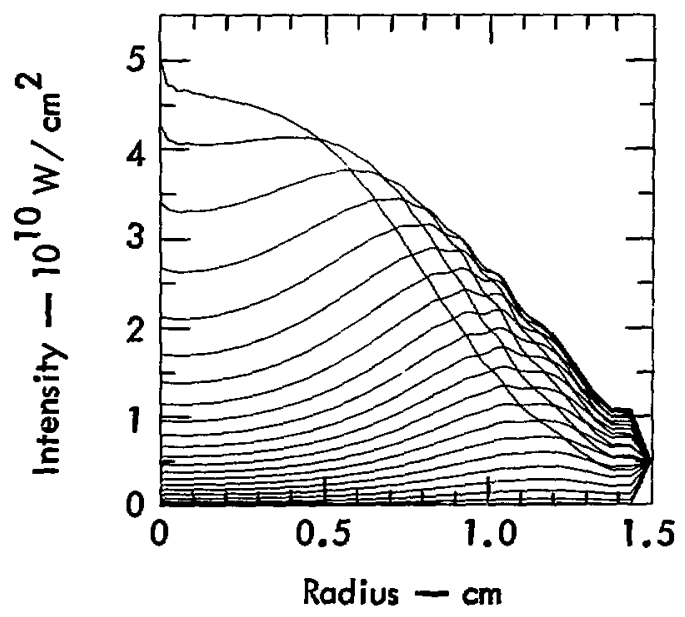

Fig. 184. Radial intensity profiles at successive times following the time of maximum on-axis intensity. The position of the pulse is near the end of the last glass amplifier.

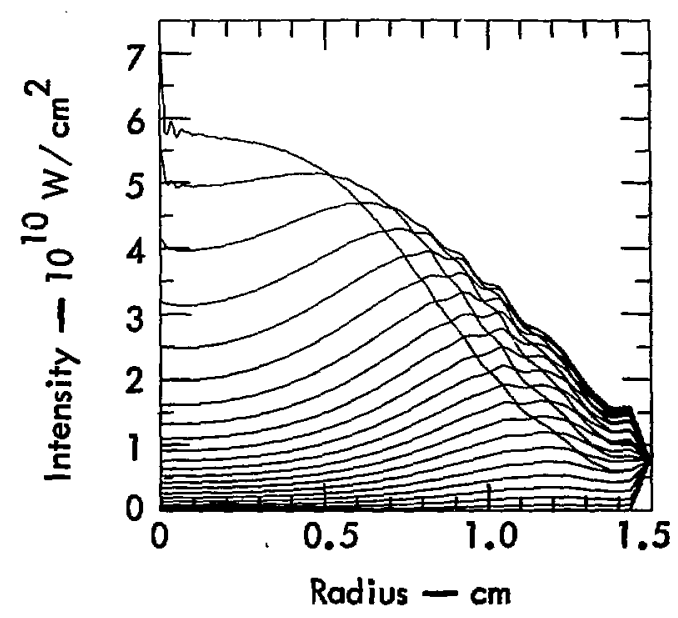

Fig. 185. Catastrophic self-focusing at a point $4 \mathrm{~cm}$ downstream from that of Fig 184. The pulse energy is $32.3 \mathrm{~J}$.

of 3; the pulse energy is only $15.8 \mathrm{~J}$, and the population inversion is far less saturated than before. The temporal profile is now much flatter than in the first case because of the intensity-limiting effect, and self-focusing does not occur. Finally, note that the difference between the two cases would be even greater if the small-signal gain were constant rather than increasing with radius in the two glass amplifiers.

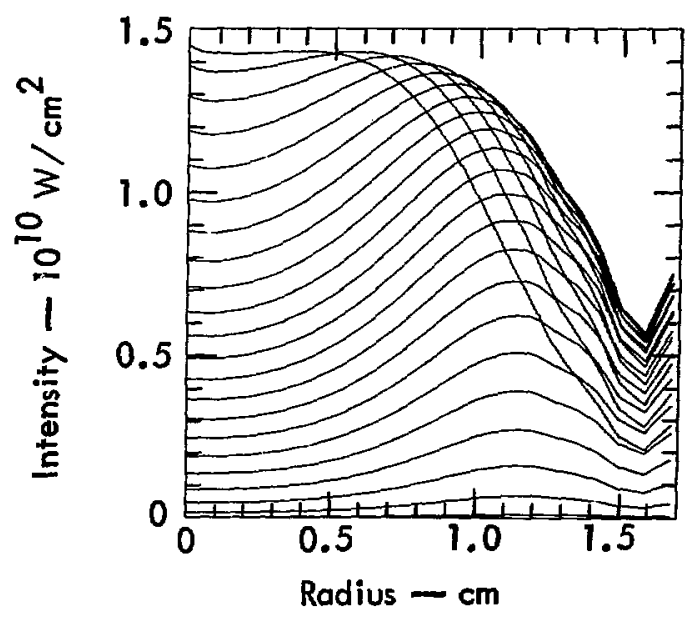

Fig. 186. Radial intensity profiles for the same conditions as in Fig 184, except that the effect of nonlinear absorption is included. 
Comparison of such calculations with the actual observation of self-focusing in such systems, and with more recent measurements, ${ }^{178}$ leads us to conclude that the nonlinear absorption cofficient, at least in ED-2 glass, must be considerably smaller than the value given by Penzkofer and Kaiser. ${ }^{8}$

\section{LASER-PULSE-INDUCED $\mathrm{CO}_{2}$ BREAKDOWN}

Gas breakdown is a fundamental limitation on the propagation of intense pulses through $\mathrm{CO}_{2}$ amplifiers, since it sets an upper limit on the permissible energy flux. The breakdown is caused by an electronavalanche process fed by the energy absorbed by the electrons via inverse bremsstrahlung. In the simplest model, the electron-production rate (ionization rate) is proportional to the energy-absorption rate, resulting in exponential growth. When this growth rate exceeds losses for a sufficient time, the medium breaks down light is absorbed and strongly refracted or reflected by the electron plasma. We are developing a version of the LAMP pulse-propagation code to aid in understanding current breakdown experiments. 179

In a typical experiment, the pulse is sharply focused by a lens, and the output information (burn pattern, energy content, etc.) is taken one focal length beyond the focus. Thus it is important that the code be able to treat the pure focusing problem accurately, since small discrepancies in the phase front near the focus result in amplitude fluctuations at greater distances. These, combined with nonlinear refractive index changes, can lead to completely spurious results. A careful analysis of the analytic solution for a focusing Gaussian beam, compared with the numerical scheme used, and practical experience have led to a number of rules of thumb regarding permissible mesh and step sizes in terms of the parameters of the problem.

The results presented here refer to a 1-nsec, 50-mJ Gaussian pulse focused by a lens of $27.6 \mathrm{~cm}$ focal length. Electrons are produced by the avalanche process and lost by radiative recombination. Their optical properties are treated by the Drude theory. As the light is focused, the local electron density increases from an initial value of $10^{6} \mathrm{~cm}^{-3}$, but no noticeable effect on the light is seen until densities on the order of $10^{15} \mathrm{~cm}^{-3}$ are reached. As the pulse passes a rixed position, the electron density builds up, and, therefore, the refractive index decreases. Thus there is a progressively stronger refraction of light away from the axis, as shown in Fig. 187. Here we see radial-intensity profiles at successive times starting from the time of maximum on-axis intensity. Note that the on-axis intensity drops rapidly to zero from $4 \times 10^{10} \mathrm{~W} / \mathrm{cm}^{2}$ (in about $0.1 \mathrm{nsec}$ in the present case). Because of the distribution of energy in the back of the pulse, it is possible for the final maximum electron density to also be off-axis as shown in Fig. 188, in which the radial dependence of the

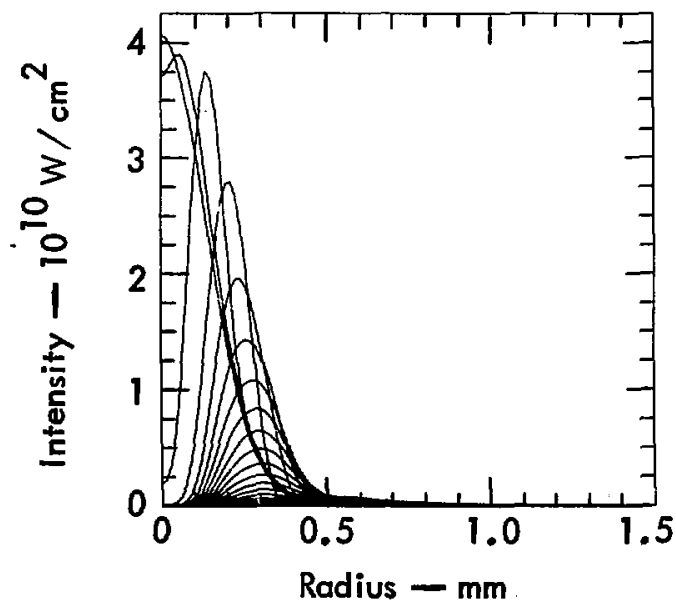

Fig. 187. Successive cross sections of the laser pulse starting $a$. the time of maximum on-axis intensity.

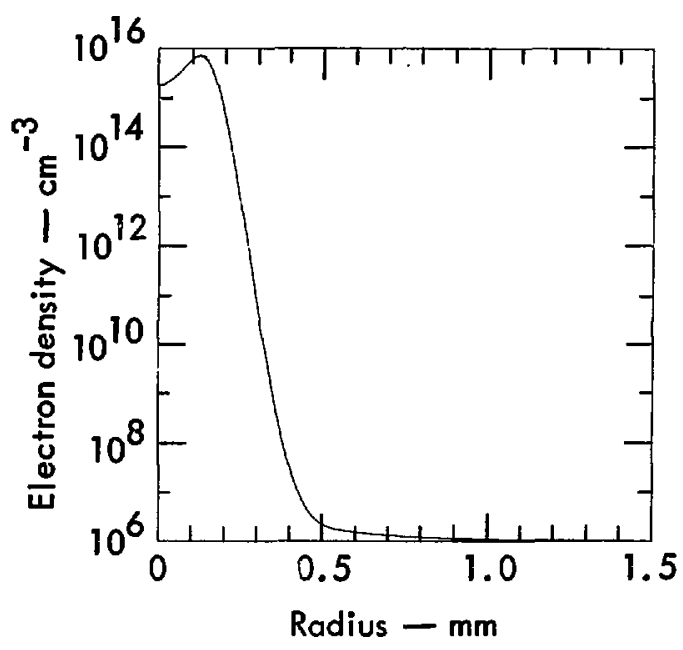

Fig. 188. Electron density as a function of radius just after passage of the pulse. The depth of the central depression depends on the loss rate.

electron concentration is shown just after passage of the pulse. This "smoke-ring" structure, which occurs when the on-axis concentration has started to decay while the sides are still building up, has been postulated previously to explain the long loss of transverse 
transmission in intracavity breakdowa. ${ }^{180}$ Finally, we show in Fig. 189 the time development of the on-axis electron concentration. Note that the maximum on-axis concentration (at the maximum on-axis intensity) is two orders of magnitude larger than its final value shown in Fig. 188. Figures 187-189 all refer to the position of maximum intensity, which is $1 \mathrm{~cm}$ in front of the focal point.

Two interesting features have been noted in the preliminary calculations carried out to date. First, the principal effect of the electrons on the light is reactive rather than dissipative; i.e., the pulse shape is severely distorted, but there is little absorption of energy (less than 10\%). Secondly, after leaving the focal region,

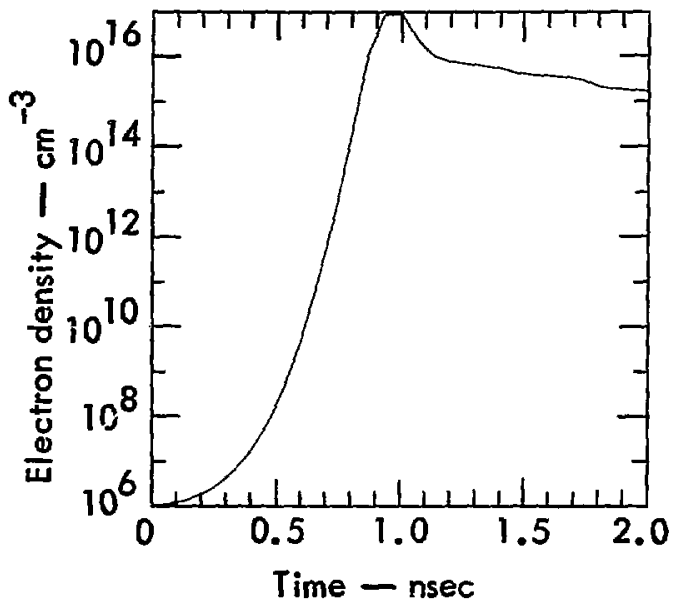

Fig. 189. Time development of the electron cascade. The electron density is maximum at the time of maximum on-axis intensity, after which it decays because of radiative recombination.

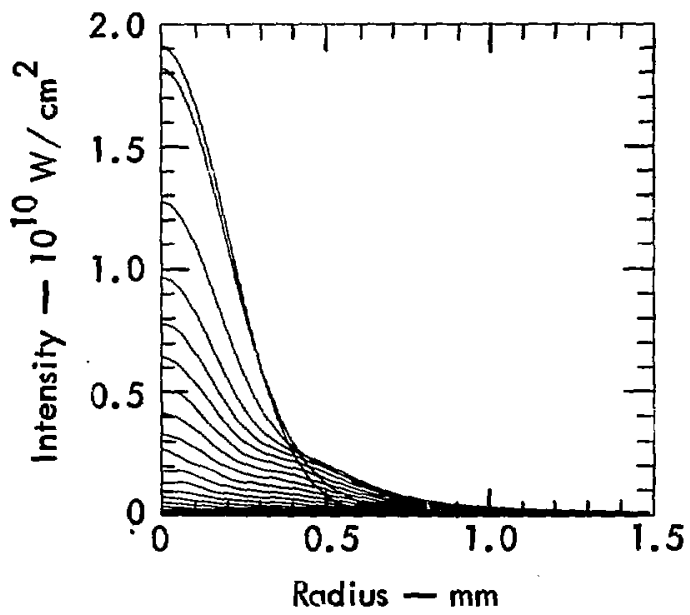

Fig. 190. Successive cross sections of the laser pulve at a position $2 \mathrm{em}$ beyond the focal point, starting at the time of maximum on-axis intensity. The puise regains a normal shape, but it has acquired a long tail.

the pulse tends to reestablish a Gaussian-like shape with a long tail, as shown in Fig. 190, which refers to a position $2 \mathrm{~cm}$ past the focal point.

Our hcpe is to normalize calculations such as these to experiment, leading to a model generally applicable to breakdown in a $\mathrm{CO}_{2}$ medium with gain. Recently, considerable interest has focused on the possibility that avalanche breakdown and the accompanying refractive effects are responsible for the stabilization of self-focused light filaments in solids. It may be possible to apply the same model with modifications to this important problem of dielectric breakdown of solid laser media. The main difference in this case will be the greater energy absorption than that in a gas. 


\section{Laser-Plasma X-Ray Studies*}

\section{INTRODUCTION}

Investigations of the radiation characteristics of laser-produced plasmas are among the most instructive methods for exploring these plasmas and their interaction with the incident laser pulse. Recent progress in our studies of laser-plasma radiation is discussed in the following articles.

A central requirement in these investigations is the ability to make reliable measurei...nts of the total $\mathrm{x}$-ray yield. Suitable diagnostic techniques are essential, and we $t$ ave therefore explored a variety of $x$-ray detection systems. The most promising have been used to measure $\mathrm{x}$-ray yields from plasmas produced with two different LLL laser systems and various target materials.

A second important aspect of laser-plasma radiation is the character of the emitted x-ray spectrum. Recent measurements have begun to clarify the nature of the high-energy spectrum - i.e., above about $10 \mathrm{keV}$. This portion of the spectrum could be of crucial importance for laser fusion because its characteristics may help to elucidate significant laser-plasma coupling mechanisms, and because the high-energy electrons that give rise to these $x$ rays may be inimical to efficient pellet compression.

\section{X-RA YIELDS FROM LASER-PRODUCED PLASMAS}

An important part of the study of laser-produced plasmas is the investigation of $\mathrm{x}$-ray yields as a function of laser anu target characteristics. In attempting to explore this dependerce, we have used a wide variety of target materials and two LLL laser systems with. significantly different pulse characteristics. Since ar. effective investigation of laser-plasma $x$ rays requ ires. the development of reliable diagnostic techniques, wi: have attempted to evaluate a variety of $x$-ray deteciion methods that are potentially suitable for curreni $o$ : future $\mathrm{x}$-ray measurements.

\section{Detector Investigations}

An experimental determination of the efficiency with which laser light is converted to $x$ rays through plasma production involves both $x$-ray and laser-pulst calorimetry. The calorimetry of laser pulses, at leas in the energy regimes of our current laser systens, can be carried out with commercially available calorimeters. However, x-ray calorimetry of $1-\mathrm{k}^{2} \mathrm{v}$ photons is a relatively undeveloped field involving it: own peculiar difficulties. There are no commercially available $x$-ray calorimeters for this energy region. I. has therefore been necessary to build suitable detector; or adapt detectors designed for other applications to our specific needs. The detectors we have investigated so far include ionization chambers, two types of silicon

\footnotetext{
Portions of this work were supported by the Defense Nuclear Agency.
}

detectors, x-ray film, MOSFET's (metal-oxide-siliconfield-effect transistor), and specially designed thermopiles. Some of these detectors are shown schematically in Figs. 191-193.

We have carried out a series of comparative measurements in a continuing effort to evaluate the reliability, accuracy, and convenience of the various $\mathrm{x}$-ray detectors. In all of the comparisons, laser-plasma $\mathrm{x}$ rays were measured simultaneously by two detectors located either adjacent to each other or in symmetrical positions relative to the laser beam and target. Absorbers of the same thickness, generally $1 / 3$ - or $1 / 2$-mil beryllium, were positioned in front of both detectors. Various targets and two laser systems - the Plasma X laser and the Long Path Laser - were used.

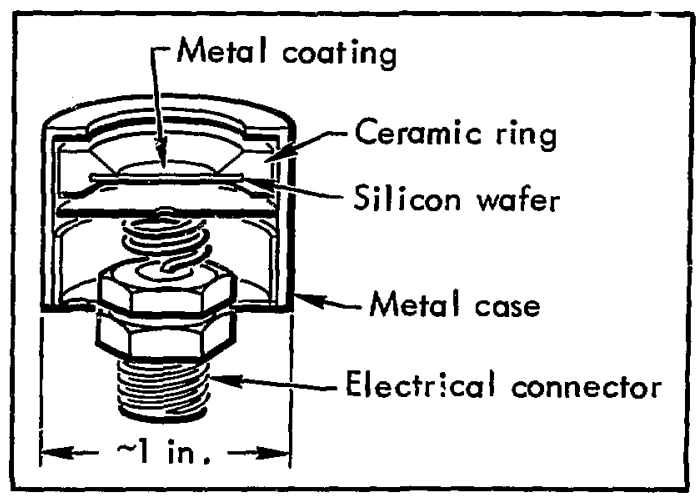

Fig. 191. Silicon surface-barrier (SiSB) detpctor. 


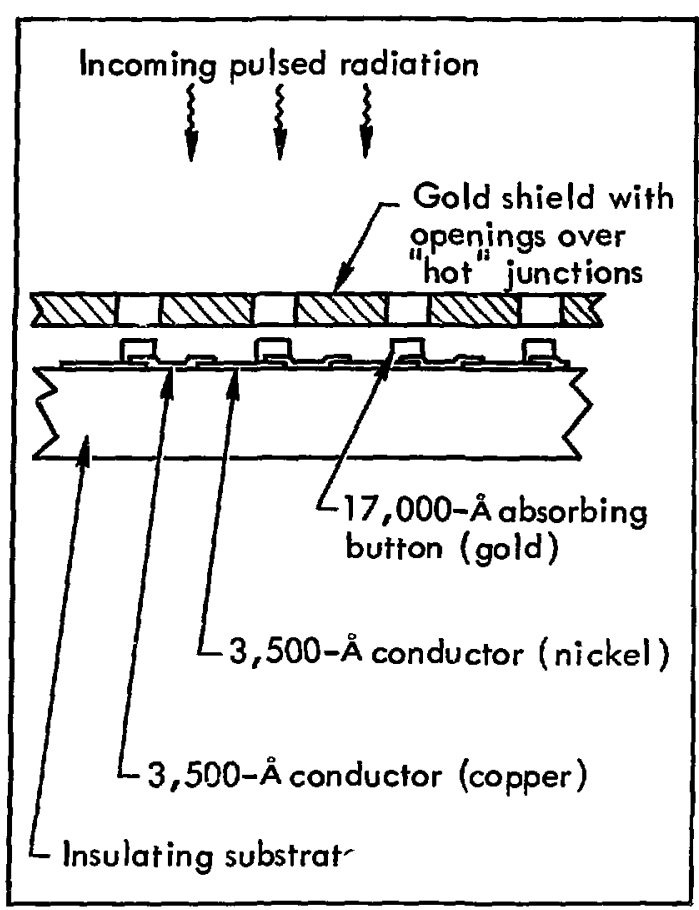

Fig. 192. Cross section of a thin-film thermopile matrix used for detecting pulsed $x$ rays (not to scale). Note that the reference junctions are shielded from incoming radiation.

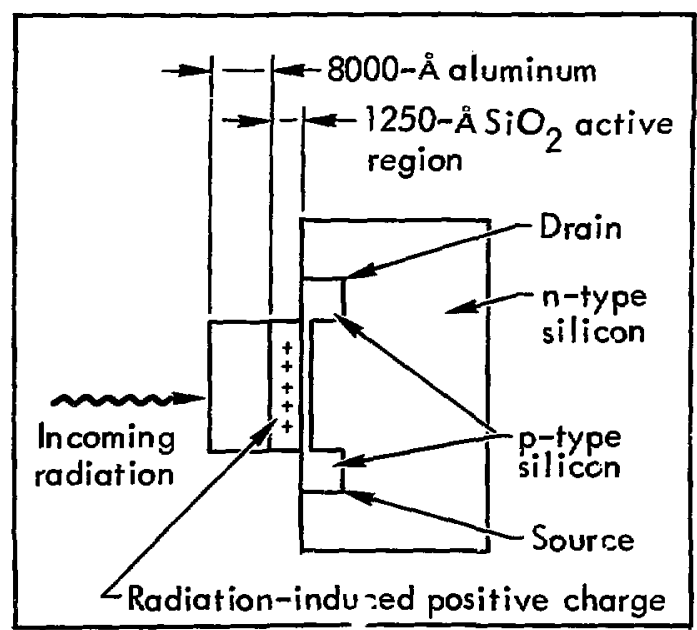

Fig. 193. Cross section of a MOSFET x-ray detector (not to scale). The radiation-induced positive charge results in an increased resistance between the drain and source terminals. The increased resistance remains after irradiation and can therefore be measured in a noise-free environment.
Measurements in which the silicon surface barrier (SiSB) detector is compared with other detectors are summarized in Table 19. In general, the detectors agree with each other - except for the ionization chamber. Additional investigations, discussed below, have helped to explain why the ionization chamber gave discrepant results."

The two types of silicon detector are evidently in good agreement, as might be expected. In fact the very convenient SiSB detector is in good agreement with nearly all of the other detectors so far explored. On the basis of this agreement, together with a consideration of the mechanism involved in the detection process, we have gained increasing confidence in the SiSB detector. It has been used for many of our recent measurements. However, we should no:e that additional comparison checks are required, and that some of the apparent agreement may be fortuitous. In particular, the MOSFET comparisons are uncertain since they involve the simplifying assumption that the detected spectrum has an effective photon energy near $2 \mathrm{keV}$. In view of such corsiderations, we conclude that our best current determinations of $x$-ray yields - based largely on SiSB measurements - have an accuracy which may be no better than a factor of two. Of less significance is the precision of the silicon detector measurements, which is approximately $\pm 10 \%$.

The low $x$-ray yields generally indicated by the jonization chamber have led us to a careful scrutiny of some of the reasons for detector malfunction. Table 20 is a partial list of mechanisms that can lead to detector errors. Investigations that included changes in operating voltage and effective source strength have provided convincing evidence that the ionization chamber suffers from incomplete charge collection, probably because of volume recombination or, possibly, electron capture resulting from impurities in the counting gas. It is evident that a comparatively high charge density is produced just inside the entrance window as a result of the predominantly low-energy spectrum.

It seems clear that additional $\mathrm{x}$-ray detector studies are essential. They will provide improvements in reliability and accuracy, and will permit the development of measuring techniques that will be appropriate for more intense sources. (For example, in its present form the SiSB detector is best suited to relatively low $\mathrm{x}$-ray fluxes.) Among the detectors soon to be investigated are an improved ionization chamber, nickel bolometers, and semiconducting temperature sinsors.

\section{X-Ray Measurennents with the Plasma X Laser}

An extensiv: series of $x$-ray yield measurements has been carried out with plasmas produced by the 


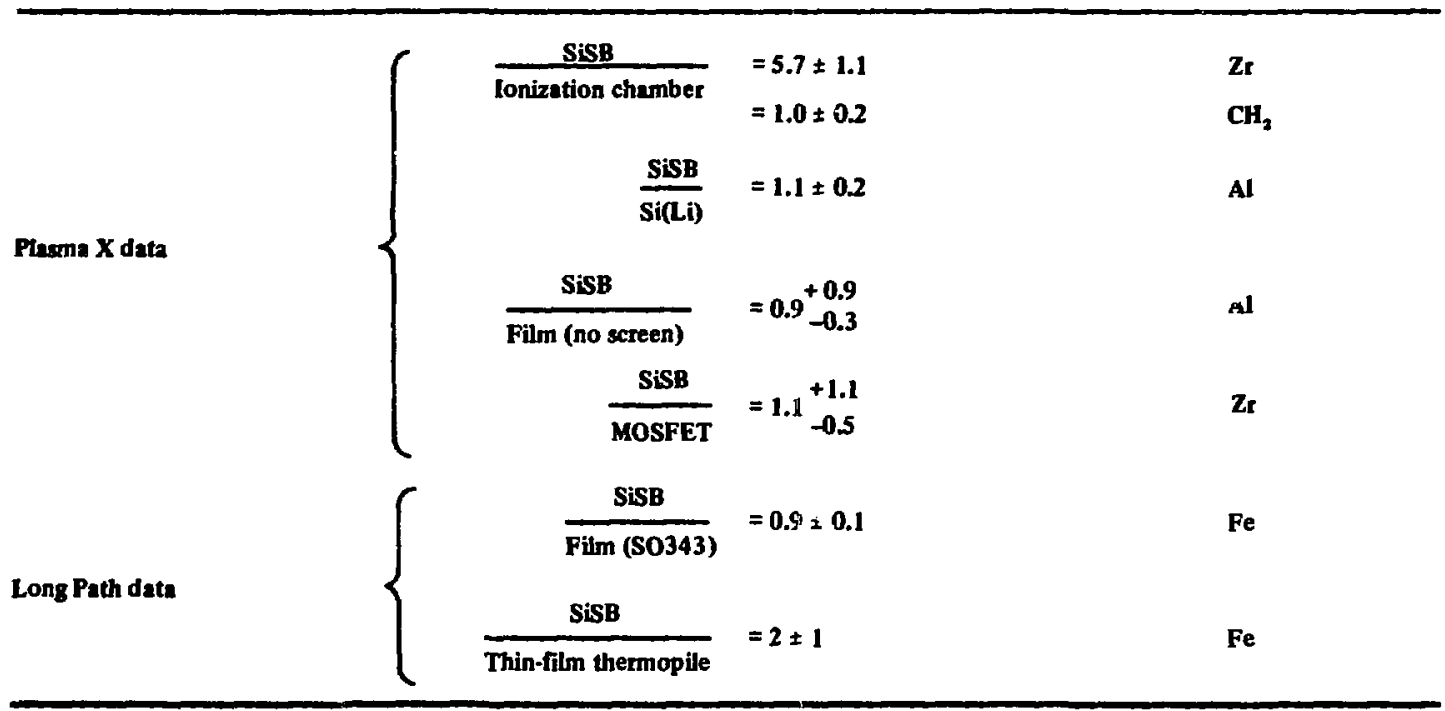

'SiSB - silicon surface barrier detector.

$\mathrm{Si}(\mathrm{Li})$ - lithium-drifted silicon de tector.

Table 20. Machanisms causing incorrect charge collection in ion thambers and silicon detectors

Charge loss

Charge amplification

\begin{tabular}{lll}
\hline Ion chamber & $\left\{\begin{array}{l}\text { Diffusion } \\
\text { Electro: capture } \\
\text { Volume recombination } \\
\text { Columnar recombination } \\
\text { Preferential recombination }\end{array}\right.$ & Gas multiplication \\
Si cerector & $\left\{\begin{array}{l}\text { Recoinbination } \\
\text { Plasma time effects }\end{array}\right.$ & Long-term trapping \\
\hline
\end{tabular}

Plasma X laser. ${ }^{181}$ In this system, 1-to-2-J, subnanosecond pulses of $1.06-\mu \mathrm{m}$ light are focused on planar targets located in an evacuated chamber. We have been primarily concerned with light-to-x-ray "conversion efficiencies," defined as the percentage of incident laser energy reemitted from the target in the form of $x$ rays above a specified cutoff energy. In the context of this report, we have assumed that $x$-ray emission from these planar targets is "isotropic," but confined to $2 \pi$ steradians. Although our measurements with curved film strips support the assumption of an isotropic distribution at medium photon energies $(\sim 5 \mathrm{keV}$ ) this assumption is unwarranted at very high and very low energies. Measurements of the laser light backscattered into the $f / 3$ focusing lens give a value of $4 \pm 2 \%$, which appears to be nearly independent of target material. Since the targets are tilted about $12^{\circ}$ from the vertical, specular reflections are not detected. Our recent investigations using the Plasma $X$ laser have included a group of $x$-ray conversionefficiency determinations with the SisB detector. A 1/2-mil beryllium window, corresponding to one mean free path for $1.2 \mathrm{keV}$ photons, established the low-energy cutoff of the detection system. In these measurements, we obtained efficiencies from as low as $0.005 \%$ and $0.02 \%$ for $\mathrm{CH}_{2}$ and carbon targets, respectively, to about $1.5 \%$ for iron targets. 


\section{Long-Path Laser Experiments}

The Long Path Laser ${ }^{182,183}$ produces pulses whose characteristics are very different from those obtained with the Plasma $X$ laser. During a recent series of Long Path experiments, the focused laser pulse delivered 20 to $50 \mathrm{~J}$ in $3 \mathrm{nsec}$. These experiments included a series of $\mathrm{x}$-ray conversion-efficiency measurements involving an iron target and a wide range of detector-window thicknesses. The data obtained with the SiSB x-ray detector are summarized in Table 21. Laser light backscattered into the $f / 7$ focusing lens, also measured during these experiments, was about $0.2 \%$ of the incident puise.

The Long Path $x$-ray conversion data are of special interest in connection with our efforts to examine the effects of a low-level precursor to the main laser pulse. Mallozzi ${ }^{184}$ has reported a striking increase in $x$-ray yields when the main pulse was preceded by a precursor or "foot" about 10 nsec long and having an intensity about $1 / 1000$ of the peak intensity of the main pulse. We used a similar precursor, which could be switched on or off at will, during the recent Long Path experiments. The precursors were 5 and $10 \mathrm{nsec}$ long, and the intensity was varied from 1/300 to $1 / 1800$ of the main-pulse intensity. X-ray conversionefficiency measurements with and without the precursor were carried out for the range of window thicknesses listed in Table 21. In these measurements we found no evidence of a pronounced increase in soft $x$ rays resulting from the precursor pulse; any changes in $x$-ray conversion efficiency due to the precursor were cortainly less than a factor of 2 .

In assessing the precursor experiments, two points need emphasis. First, although it is difficult to compare
Table 21. X-ray conversion efficiencies for the Long Path Laser and an iron targat

\begin{tabular}{lcc} 
Absorber & $\begin{array}{c}\text { Mean-freepath } \\
\text { cuteff energy } \\
\text { Al }(1500 \mathrm{~A})\end{array}$ & $\begin{array}{c}\text { Converion } \\
\text { efriency }(2 \pi) \\
(\%)\end{array}$ \\
Be $\{1 / 3 \mathrm{mil})$ & 0.30 & 10 \\
Be $(1 / 2 \mathrm{mil})$ & 0.98 & 3 \\
Be $(5.33 \mathrm{mil})$ & 1.2 & 1.5 \\
\hline
\end{tabular}

${ }^{\mathrm{a} X}$ rays were measured with an SiSB detector. The laser delivered 20 to $50 \mathrm{~J}$ in 3 -nsec pulses.

conversion efficiencies pr sperly because of differences in the methods of defining the low-energy cutoff, it appears that our $x$-ray conversion efficiencies with or without a precursor agree with those Mallozzi obtained using a precursor to within a factor of 2 or 3. The difference might represent inaccuracies in measurement techniques. A second important consideration is the significant difference between our Long Path Laser and the laser employed by Mallozzi. Our pulse length, $3 \mathrm{nsec}$, is twice that of the laser he employed, and therefore a precursor pulse may be of less significance. Furthermore, the frequency characteristics of our pulse - broad and complex - are believed to be considerably different from those of his laser. Finally, the detailed nature of the precursors themselves was certainly quite different in the two sets of experiments. Evidently, further experimentation, involving improved diagnostics and control of both laser and plasma, may be necessary to clarify the importance of laser-pulse precursors.

\section{PRELIMINARY MEASUREMENTS OF THE HIGH-ENERGY X-RAY SPECTRUM FROM LASER-PRODUCED PLASMAS}

Although the laser-plasma radiation above $10 \mathrm{keV}$ represents a very small fraction of the intal emission from current laser-produced plasmas, these high-energy $x$ rays may be of considerable significance, for two reasons.

First, they could provide a key to the nature of laser-target interactions that may play an important role in coupling laser energy into the evolving target plasma. Some of these anomalous absorption mechanisms are expected to produce a distorted electron spectrum involving a suprathermal electron distribution. These suprathermal electrons would give rise to an associated elevation of the high-energy $X$-ray spectrum. Consequently, an experimental investigation of the high-energy $x$ rays can lead to an improved understanding of potentially important laser-heating mechanisms.
A second motivation for exploring the high-energy $x$-ray spectrum is a concern that the associated high-energy electrons could interfere with a crucial aspect of the laser-fusion scheme. In particular, an excessive number of high-energy electrons might penetrate and preheat the core of the target pellet, thereby precluding efficient compression of the pellet.

We have completed a preliminary investigation of the intensity and shape of a laser-plasma $x$-ray spectrum from about 10 to $140 \mathrm{keV}$. The method involves several high-gain scintillation detectors used in conjunction with thick filters. Our experimental arrangement is shown schematically in Fig. 194.

The targets used for these experiments consisted of deuterated polyethylene $\left(\mathrm{CD}_{2}\right)$ disks, $0.31 \mathrm{~cm}$ thick. They were located at the center of an evacuated target chamber $(<0.03$ Torr). The plasmas to be studied were 
produced with the Long Path Laser. ${ }^{182,133}$ The spectral energy band to which each detector responded was determined by a thick $K$-edge filter located in front of each detector, as indicated in Fig. 194. These filtess transmitted $\mathrm{x}$ rays just below their $K$-edge and also $x$ rays of energies greater than about twice the $K$-edge energy (Fig. 195a). If the spectrum under investigation falls off rapidly with photon energy, then the resulting transmission will look typically like Fig. 195b. It was determined experimentally that our spectrum did indeed fall off rapidly, and that the $x$-ray energy in the tail was always $<20 \%$ of the total energy measured for each channel. Thus, the four $K$-edge filters, in conjunction with detectors 1 through 4 , provided a measurement of four $K$-edge notches of the spectrum, with the high-energy tails contributing small but accountable "backgrounds."

The detectors used in channels 2,3 , and 4 were Nal(Tl) crystals coupled to 56 AVP photomultiplier tubes. The crystal thickness for each channel was chosen so that the $K$-notch $\mathrm{x}$-rays were ictally absorbed, whereas the tail $x$ rays were about $50 \%$ transmitted. This further reduced the fraction of the measured signals that was due to the high-energy tails. For channel 1, a $1.9-\mathrm{cm}$-thick Pilot B fluor was used instead of $\mathrm{Nal}(\mathrm{TI})$. The notch-to-tail ratio for this channel was increased by a factor of 4 because of the $x$-ray response of the plastic fluor. Calibration for all detectors included measurements of the absolute dc sensitivities for $x$ rays from 8 to $98 \mathrm{keV}$, the detectoi gain as a function of photomultiplier voitage, and detector linearity in the pulsed mode.

The filter and detector thicknesses used in each channel are shown in Table 22. Since only four channels were available, channels $1 \mathrm{~A}$ and $4 \mathrm{~A}$ represent

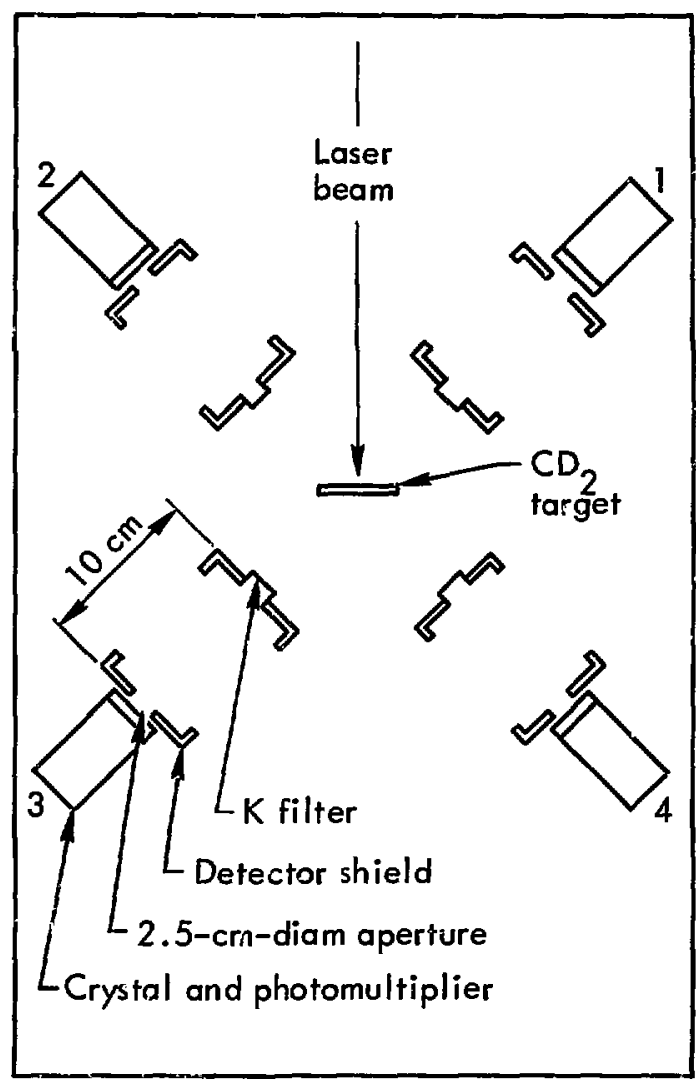

Fig. 194. Detector arrangement for high-enzrgy $x$-ray measurements. The laser beam is focused onto the target with an $f / 7$ lens. The detectors and their associated fiters are positioned with the aid of aluminum tubes inserted into the walls of the evacuated octagonal target chamber.

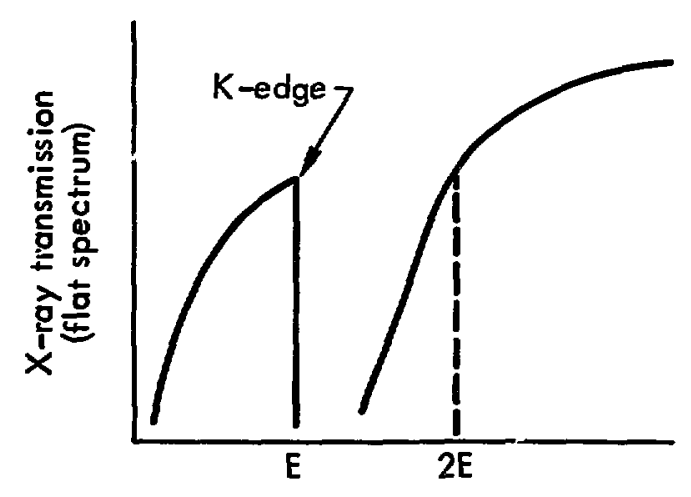

(a)
Photon energy

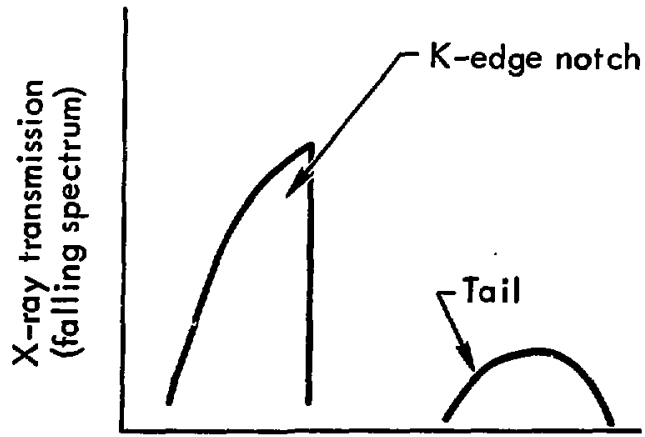

(b)
Photon energy

Fig. 195. Relative $x$-ray tranemiscion through a filer of the type used for the high-energy x-ray measurements (a) Transmission of a fut incident spectrum. (b) Tranamiscion of a rapidly falling spectrum (such as encountered in these measurements). 
Table 22. Filters and detectors used in measuring the high-energy $x$-ray spectrum from laser plasma

\begin{tabular}{clcc} 
Channel & \multicolumn{1}{c}{ Filter } & Kedge energy (keV) & Detector \\
\hline $1 \mathrm{~A}$ & $0.00095 \mathrm{~cm} \mathrm{Zn}$ & 9.66 & $1.9 \mathrm{~cm} \mathrm{Pilot} \mathrm{B}$ \\
1 & $0.0256 \mathrm{~cm} \mathrm{Sn}$ & 29.2 & $1.9 \mathrm{~cm} \mathrm{Pilot} \mathrm{B}$ \\
2 & $0.050 \mathrm{~cm} \mathrm{Er}$ & 57.5 & $1 \mathrm{~mm} \mathrm{Nal} \mathrm{(T)}$ \\
3 & $0.116 \mathrm{~cm} \mathrm{~Pb}$ & 88.0 & $3 \mathrm{~mm} \mathrm{NaI}(\mathrm{TI})$ \\
4 & $0.124 \mathrm{~cm} \mathrm{U}$ & 115 & $6.3 \mathrm{~mm} \mathrm{NaI}(\mathrm{Tr})$ \\
$4 A$ & $0.229 \mathrm{~cm} \mathrm{Er}$ & 142 & $3 \mathrm{~mm} \mathrm{NaI}(\mathrm{Tr})$ \\
\hline
\end{tabular}

a This filter was too thick to transmit $x$ rays below its $K$-edge. It was used as a broad $140-k e V$ channel.

auxiliary detector systems which replaced channels 1 and 4 for some of the shots. The results were normalized so as to produce a single spectrum including all six data points. This procedure is justified because the spectral shape did not change very much from shot to shot.

Since the four channels were looking at the source from different angles, an ancillary experiment was done to determine whether the source emitted the high-energy $x$ rays isotropically. The high- $Z$ filters listed in Table 21 were replaced by identical $0.15-\mathrm{cm}$ nickel filters. The nickel filters transmit $x$ rays with energies greater than about $50 \mathrm{keV}$, with most of the signal coming from the 60-to-100-keV $x$ rays. After accounting for differences in detector response, it was determined that the $x$-ray fluence at position 1 was comparable to the fluence at position 2 and that the fluence at position 3 was about the same as at position 4. However, the fluence in channel 3 or 4 appears to be nearly ten times higher than in 1 or 2 . This asymmetry is being investigated as a function of photon energy.

The $x$-ray signals were analyzed with the aid of a computer program designed to facilitate a correct interpretation of each measurement. The data points are plotted at the $K$-notch center point, defined as that energy for which half of the signal due to the $K$-notch $x$ rays is from $x$ rays above the center point and half from below. In order to permit a simple and consistent presentation of the results, we have normalized all data to a direction $135^{\circ}$ from that of the incident laser beam. The signals from channels 3,4 , and $4 \mathrm{~A}$ were divided by 10 to account for the asymmetry in the $x$-ray angular distribution. Thus the measured spectrum is essentially that emitted by the source in the direction corresponding to detector position 1 or 2 . A typical spectrum measurement, normalized in this way, is shown in Fig. 196. Its shape is noteworthy, since it is inconsistent with a single-temperature Maxwellian electron distribution.

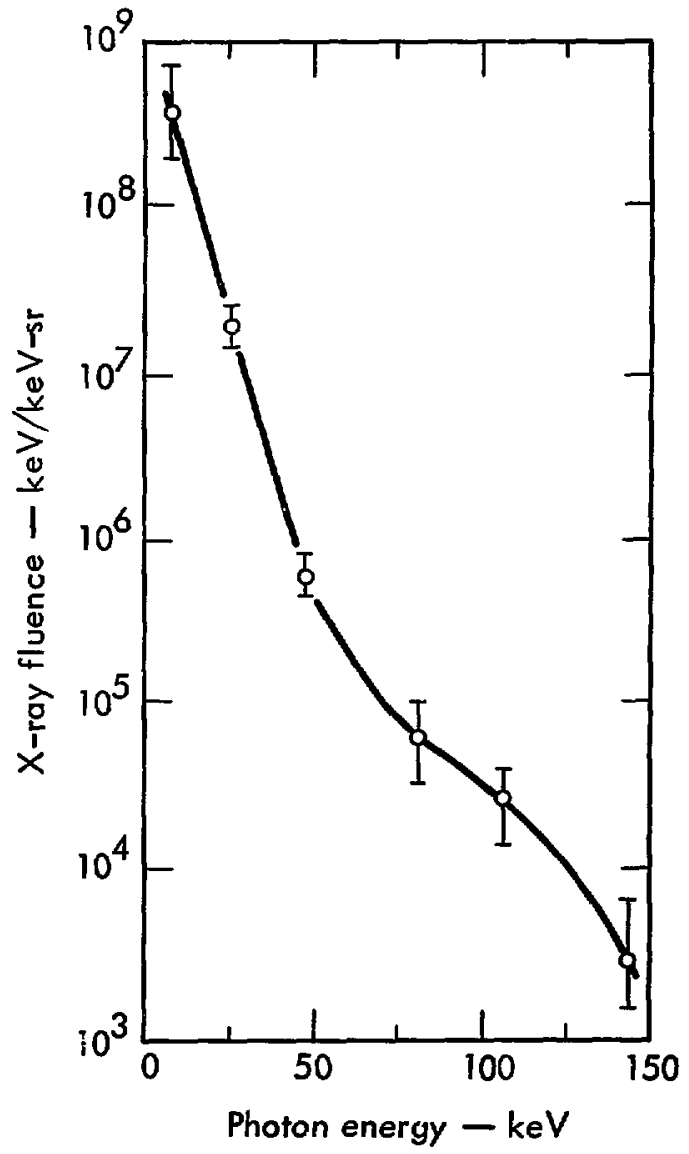

Fig. 196. High-enersy x-ray spectrum emitted from a plasma produced from a $\mathrm{CD}_{2}$ target irrediated with 55-J, 3.3-nsec pulses from the Long Path Laser. The spectrum has been normalized so as to represent the $x$-ray intensity as viewed at an angle of $135^{\circ}$ from the direction of the incident laser beam.

We estimate the error to be $\pm 25 \%$ for channels 1 and 2 and $\pm 50 \%$ fo: channels 3 and 4 . A factor- 
of-two error is assigned to channels $1 \mathrm{~A}$ and $4 \mathrm{~A}$, until better detector calibrations are done at those energies. However, channel 4A also suffers from background problems, and it is doubtful if the error stated here can be much reduced.
These results represent only a preliminary application of a very useful diagnostic technique. This technique can now be utilized to explore in detail the effects of laser and target characteristics on the anomalous high-energy $x$-ray spectrum.

\section{HAF:D-X-RAY EVIDENCE FOR RELATIVISTIC ELECTRONS}

There is current in $-\mathrm{t}$ in high-energy electron "tails" to the distribution function" 185,186 and also in the possibility of pair production by relativistic electrons produced at the laser focus. ${ }^{187}$ For this reason, we have more closely analyzed some earlier data on very hard $x$ rays that were taken in April 1972.188

The experiments were done with the Long Path Laser. A 6-nsec double pulse was used whose intensity peaks were separated by $3 \mathrm{nsec}$; the total output energy in the pulse was $80 \pm 10 \mathrm{~J}$. The light path made only five passes through the disk amplifier rather than nine passes as in the original design. Optical

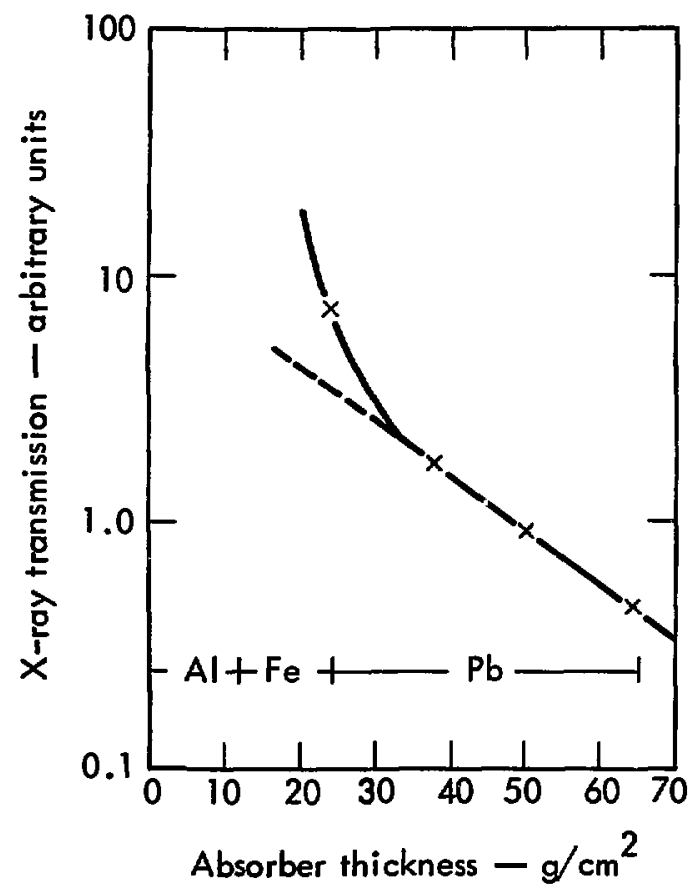

Fig. 197. Trananission curve of hand $x$ rays observed in experiments of April 1972. The aluminum was the vacuum-chamber cover plate and the "canning" container for the fuor. The iron was detector ahielding. Lead was added as shown. The absorption coefficient $\mu$ is determined from the slope of the straight-line portion of the curve. spectrometer measurements of this new pulse indicated a narrower overall spectral width $(\sim 60 \AA)$ than that of the previous pulse $(\sim 100 \AA) .^{183,188}$

Although the narrowing of the overall spectral width seemed to be a minor change, an unusually penetrating hard component of the $x$ rays was seen when this pulse was incident on our standard polyethylene $\left(\mathrm{CH}_{2}\right)$ target. The detector was a plastic fluor $(15 \mathrm{~cm}$ diam by $20 \mathrm{~cm}$ long) originally intended for neutron measurements with $\mathrm{CD}_{2}$ targets; its front face was located $22 \mathrm{~cm}$ from the target.

The $x$-ray transmission curve obtained from these measurements is plotted in Fig. 197. Since the measurements were not originally intended to be used for an absorption curve, the experimental accuracy $(\sim 10 \%)$ is not as good as might have been desirable. Nevertheless, a well-characterized curve is seen, with

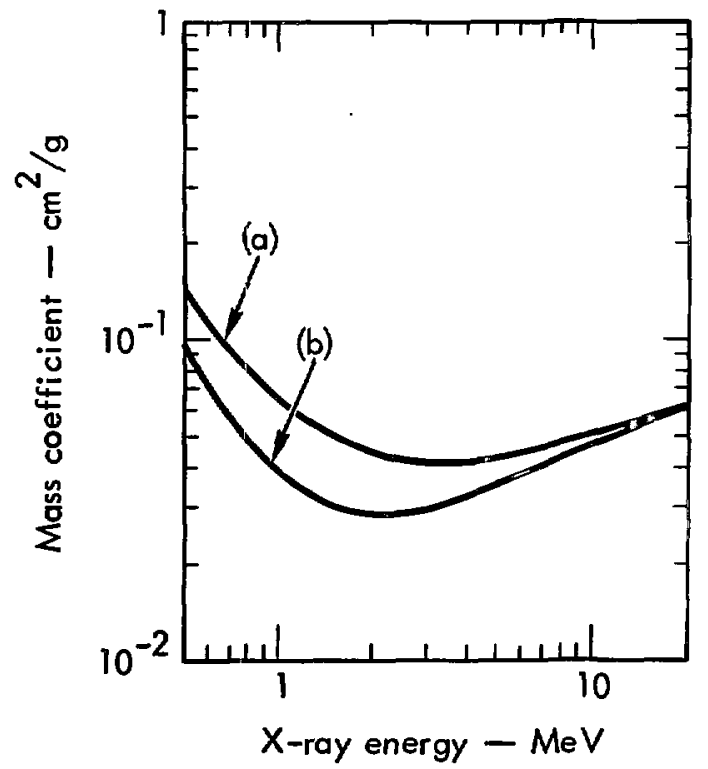

Fig. 198. Attenuation (a) and absorption (b) curves for lead. 109 Curve (a) assumes that all of the Compton-scattered photons miss the detector; curve (b) assumes that all of the Comptonscattered photons are collected by the detector with the same efficiency as the original photons. 
a hard component whose absorption coefficient $\mu$ is approximately $0.05 \mathrm{~cm}^{2} / \mathrm{g}$, as determined by the slope of the straight-line portion of the curve.

In Fig. 198 we show the theoretical $x$-ray attenuation and absorption curves for lead, as given by Evans. ${ }^{189}$ For our actual geometry, the effective absorption coefficient will fall between the two curves because only some fraction of the Compton-scattered photons will reach the detector. The measured value of $\mu\left(0.05 \mathrm{~cm}^{2} / \mathrm{g}\right)$ corresponds to an x-ray energy of either about $1 \mathrm{MeV}$ or $10 \mathrm{MeV}$; the former number is the only plausible one.

Bremsstrahlung $x$ rays having an average energy of about $1 \mathrm{MeV}$ must have been emitted by electrons of this energy or greater. Such electrons are essentially relativistic, since their total energy is greater than $3 m c^{2}$. Thus, in this set of experiments, the measurements definitely indicate the presence of at least a few relativistic electrons.

However, we are unable to estimate the absolute number of relativistic electrons produced, because we do not know where the $x$ rays were produced. In cold material, $1-\mathrm{MeV}$ electrons have a range of $0.4 \mathrm{~g} / \mathrm{cm}^{2}$ or more; the range in tot plasma would be higher, but even the cold-material range is already much greater than the dimensions of the laser focal spot. Thus, relativistic electrons created at the target would be expected to escape into the vacuum charnber, where they would travel to the walls, creating bremsstrahlung $x$ rays at many locations. Until further experimental work is done to isolate and measure these effects, it is not possible to estimate the absolute number of electrons produced.

Such extremeiy hard $\mathrm{x}$ rays were not seen in such abundance in earlier experiments ${ }^{188}$; the reasons for the difference are not known. Better reproducibility of the laser system and diagnostics is essential in order to improve these early measurements. The mechanisms of relativistic-electron production and the possibility of electron-positron pair production are discussed in the following article.

\section{PAIR PRODUCTION BY RELATIVISTIC ELECTRONS FROM AN}

INTENSE LASER FOCUS

The possibility of electron-positron pair production at the focus of a high-energy laser pulse has been considered for several years. It was found that the probabili of vacuum pair production by multiphoton absorptic is orders of magnitude too low for such producti $n$ to be detected even with presently available laser intensities. ${ }^{190,191}$ However, a different, two-step mechanism is also possible: production of relativistic electrons $\left(E>2 m c^{2}\right.$ ) by the laser light, followed by pair production by the scattering of these electrons in the Coulomb field of the nucleus. ${ }^{192}$ We have investigated this latter mechanism in greater detail, and have concluded that laser-driven plasma instabilities may be capable of producing relativistic electrons at lower intensities than previously estimated. ${ }^{187}$

From the viewpoint of laser fusion, probably the most significant outcome of this work is our greater understanding of the importance of very-high-energy electrons in many laser-produced plasmas. Relativistic treatment of the plasma electrons is probably required for an adequate calculation of anomalous absorption.

Pair production by relativistic electrons is often called the trident process (se Fig. 199). The cross section for this process is a quantum electrodynamic problem which was first calculated by Bhabha many years ago. 193 Bhabha was forced to make two approximations to obtain an analytic solution; we have done an exact calculation on the Livermore computers using a general lepton cross-section calculation program written by Brodsky and Ting. ${ }^{194}$ Our exact result is shown in Fig. 200, along with Bhabha's approximate solution.

Once the trident cross section is known, one must then try to estimate the energy distribution of the relativistic electrons. We investigated two cases of relativistic-electron production: first, a circularly polarized laser-light field in the absence of instabilities, and second, laser-pumped parametric instabilities.

For circularly polarized laser light, the analytic solution of Steiger and Woods for the coherent electron motion is available. ${ }^{195}$ We used their solution to calculate the electron energy as a function of laser intensity, and we found a threshold $I_{\pi}$ for pair production given by:

$$
I_{T}=\frac{2.6 \times 10^{19}}{\lambda_{\mu}^{2}} \mathrm{~W} / \mathrm{cm}^{2},
$$

where $\lambda_{\mu}$ is the wavelength of the laser light in microns. Such an intensity is at the upper limit of laser technology today. ${ }^{163}$ Furthermore, it is orders of magnitude above the thresholds for instabilities ${ }^{196}$; therefore, we were led to investigate the instabilities as possible sources of relativistic electrons.

A complete relativistic analytic investigation of laser-driven plasma instabilities was not possible, but some plausible and interesting results were obtained. 
If relativistic electrons are to be produced by electron trapping in the wave, one should look for waves whose phase velocity is approximately $c$, the velocity of light. Longitudinal plasma waves of small wave number $K$ do indeed have such a phase velocity. In fact, it was shown that the fastest growing wave number $K$ in the parametric ion-acoustic instability will have phase velocity $c$ at a plasma density $N_{e}$ given by:

$$
N_{e} \approx N_{c}\left(1-3 \frac{v_{t h}^{2}}{c^{2}}\right)
$$

where $N_{c}$ is the cutoff density $\left(N_{c}=\pi / r_{0} \lambda^{2}\right)$, and $\nu_{t h}$ is the electron thermal velocity $\left(v_{t h}^{2}=k T_{e} / m\right)$. For example, if the electron temperature $T_{e}$ is $1 \mathrm{keV}$, we have $N_{e}=0.994 N_{c}$. This is just the density regime where this instability has a low threshold; thus, the plasma waves with the required phase velocity probably do exist for accelerating electrons to relativistic energies.

We should also note that the nonrelativistic plasma-simulation calculations show hot-electron distributions out to velocity ratios $\left(v / v_{t h}\right)$ of 10 to $20 .{ }^{185}$ But for a $1-\mathrm{keV}$ electron temperature the velocity ratio $c / v_{t h}$ is approximately 23 ; therefore, the simulation calculations also suggest that the production of relativistic electrons is plausible.

The group velocity $V_{g}$ of a longitudinal plasma wave is given by

$$
V_{g}=3 V_{t h}^{2} / V_{p}
$$

Thus, when $V_{p} \rightarrow c, V_{g}$ is very small, and the energy of the waves remains localized. If relativistic electrons are captured by the wave near the center of the focal spot, we would expect them to be quickly propelled away frorn this center at the phase velocity and to

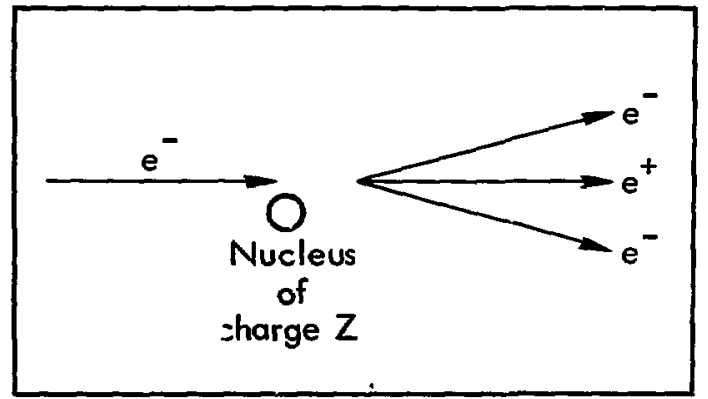

Fig. 199. Schematic drawing of the trident process of pair croduction by an incident electron of kinetic eviergy $E_{K}$, where $E_{K}>2 m_{0} c^{2}$, in the Coulomb field of a nucleus.

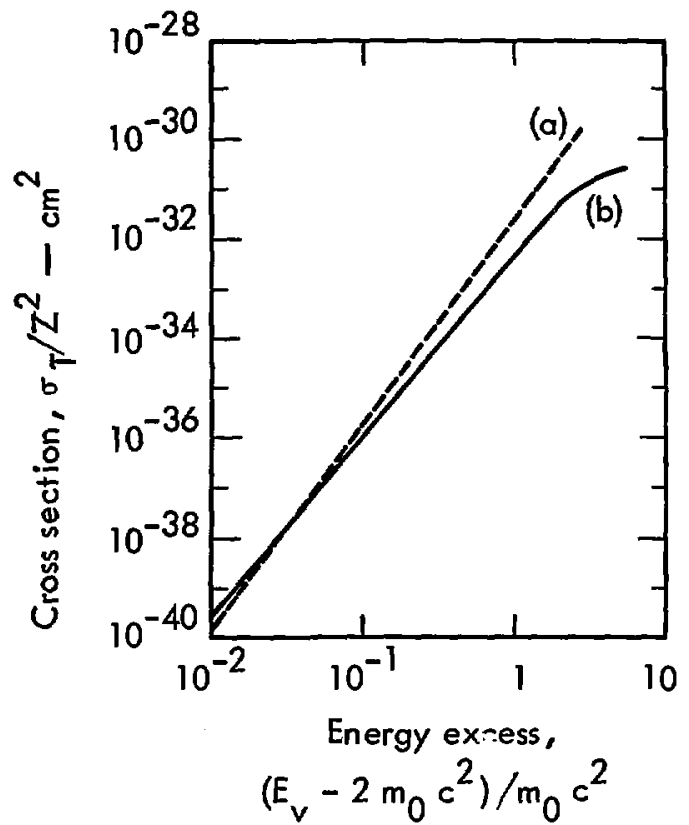

Fig. 200. Cross section of the trident process near threshold. Curve (a) is derived from Bhabha's approximate analytical solution ${ }^{193}$; curve (b) is our exact computer solution.

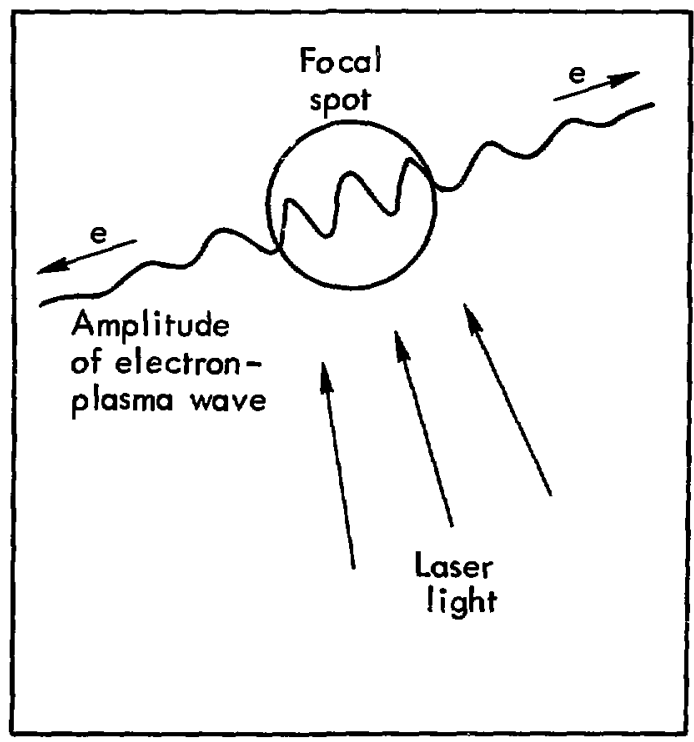

Fig. 201. Schematic of plasma waves in the vicinity of a focal spot. The wave velonity $v_{p} \approx c$, but the average amplitud: is almost stationary (see text). Thus, electrons trappod by the high amplitudes in the focal spot escape sut the sides, where the umplitude decreases. 
escape from the focal spot (see Fig. 201). Of course, in reality there are many plasma waves rather than just one, so this is admittedly a qualitative, oversimplified description.

The range of a relativistic electron in matter is well known; for an initial energy of $2 m c^{2}(\sim 1 \mathrm{MeV})$, it is about $400 \mathrm{mg} / \mathrm{cm}^{2}$ in cold material (greater in a plasma). This is larger than the usual dimensions of a focal spot; thus, we expect that the relativistic electrons will escape from the focal spot into the cold target, and into the vacuum chamber, where they can travel long distances. This is an additional complication for the experimentalist.
Finally, a comparison of the pair-production cross section with the bremsstrahlung cross section shows that most of the $x$-radiation emitted during the interaction of the relativistic electrons with matter is due to bremsstrahlung. Any experimental search for pair production will have to be done in the presence of these more copious amounts of $x$ rays, of course, no search need be attempted unless such $x$ rays are observed, indicating the presence of relativistic electrons.

Some $\mathrm{x}$-ray evidence for relativistic electrons in a laser-plasma experiment is described in the preceding article.

\section{LASNEX CALCULATIONS OF X-RAY EXPERIMENTS}

We are designing $x$-ray targets for use with the first two stages of the LLL glass laser with 100-psec pulses and energies of 50 to $250 \mathrm{~J}$. As a check on the design code LASNEX, we have compared its predictions with the results of two experiments. While the code calculations are reasonably successful in interpreting the data, this comparison has isc'ated several possible shortcomings in the code.

LASNEX ${ }^{197}$ is a two-dimensional Lagrangian hydrodynamics code. It describes the thermal motions of the electrons and ions by two temperatures, $T_{e}$ and $T_{i^{*}}$ The use of multigroup, flux-limited, diffusion radiation transport obviates the restrictive assumption of Planckian radiation spectra. The mean group opacities are computed with the supposition of local thermodynamic equilibrium (LTE) and a hydrogenic, average ion model. LASNEX calculates the depusition of laser energy via the mechanisms of both inverse bremsstrahlung and anomalous absorption. A flux-limited diffusion treatment compuses the transport and thermalization of the suprathermal electrons accelerated by laser heating. LASNEX computes the rate of thermonuclear burn and subsequent energy deposition by charged-particle reaction products. As demonstrated by Chase, LeBlanc, and Wilson, ${ }^{198}$ nonuniform laser illumination of a target can generate large magnetic fields, and LASNEX computes these fields and their impact upon the plasma hydrodynamics and thermal conduction.

We have checked the LASNEX code by comparing its predictions with the results of two slab experiments. In these experiments, performed at Battelle Memorial Institute (BMI) and LLL, pulses of $1-\mu \mathrm{m}$ light were focused to fluxes of $10^{14} \mathrm{~W} / \mathrm{cm}^{2}$ at the surface of thick iron slabs. The $x$ rays emitted from the resulting plasma were than detected. In the BMI experiments, 7 to $10 \%$ of the energy of a 1-nsec pulse of energy 14 to $20 \mathrm{~J}$ was reradiated as $x$ rays with energies greater than $1 \mathrm{keV} .^{183}$ In a similar experiment, performed with the LLL Plasma X laser, only $1.5 \%$ of the laser energy in a 50-psec pulse was reradiated as $\mathrm{X}$ rays with energies greater than $1.3 \mathrm{keV}$ (see above, p. 170). LASNEX simulations have indicated that, because of the difference in pulse lengths, the two results are not mutually contradictory.

In the BMI experiments, the target spot was conditioned before the arrival of the main laser pulse by a low-intensity $\left(10^{11} \mathrm{~W} / \mathrm{cm}^{2}\right)$ precursor pulse of known duration. The intensity of the main pulse is weak enough so that inverse bremsstrahlung, a fairly well understood process, may be the sole lightabsorption mechanism. With a complete knowledge of initial conditions, we would expect to be able to calculate the fraction of laser energy atsorbed by the plasma. Since the inversebremsstrahlung absorption coefficient varies as the square of the density, smaller density gradients in the vicinity of the critical density result in greater absorption. LASNEX simulations indicate that precursors of longer duration result in shallower density gradients and hence in greater absorption.

The reflectivity data from the Battelle experiments shows that for 20-nsec precursors only $10 \%$ of the main pulse was reflected, while for 1-nsec precursors 50 to $80 \%$ of the laser energy was reflected. Although LASNEX can correctly predict the variation of the absorption with precursor length, it greatly overestimates the fraction of light absorbed for short precursors. Even considering the effect of self-generated magnetic fields and the refraction of oblique rays, we predict a inflectivity of only $16 \%$ for a $1-n s e c$ precursor instead of the measured 50 to $80 \%$. At present, LASNEX does not treat self-focusing of the laser beam in the plasma or stimulated Raman or Brillouin backscattering. Perhaps more significantly, the local thermodynamic equilibrium (LTE) 
approximation must certainly exaggerate the average degree of ionization, $\bar{Z}$. The inverse-bremsstrahlung absorption coefficient varies roughly as $Z^{3}$; a mistake of $20 \%$ in calculating $Z$ results in an error of about $200 \%$ in the absorption of a typical pulse. We investigated the deficiency of the LTE approximation in an ancillary conputation which followed the time evolution of the ionic level populations with the rate equations for collisional and radiative processes. We conclude that calculations using the LTE approximation overestimate the degree of ionization in the region of the critical density. We plan to incorporate a non-LTE treatment in LASNEX in the near future.

Figure 202 is a plot of a typical LASNEX two-dimensional simulation showing the free expansion of the corona away from the target slab into vacuum. The laser beam, incident from the right, is focused on a spot $100 \mu \mathrm{m}$ in diameter. The light propagates through the rariffed plasma, being absorb ed until it reaches plasma of the critical density where it starts being reflected. In a highly ionized iron plasma, this critical density is $0.004 \mathrm{~g} / \mathrm{cc}$. Because the absorption coefficient varies as $\rho^{2}$, the electron temperature is highest near the critical density. The thermal conductivity of the hot electrons ( 0.5 to $0.8 \mathrm{keV}$ ) is so great that the corons is nearly isothermal for the electrons.

As shown in Fig. 203, the X-ray spectrum predicted by LASNEX for the BMI experiments successfully reproduces the observed data. The calculated and measured spectra are for a $1-\mathrm{nec}, 10^{14} \cdot \mathrm{W} / \mathrm{cm}^{2}$ pulse incident on an iron target. Both the calculations and the data show a cluster of strong line transitions near $950 \mathrm{eV}$.

Figure 204 shows the variation of the emission function with distance normal to the surface of the

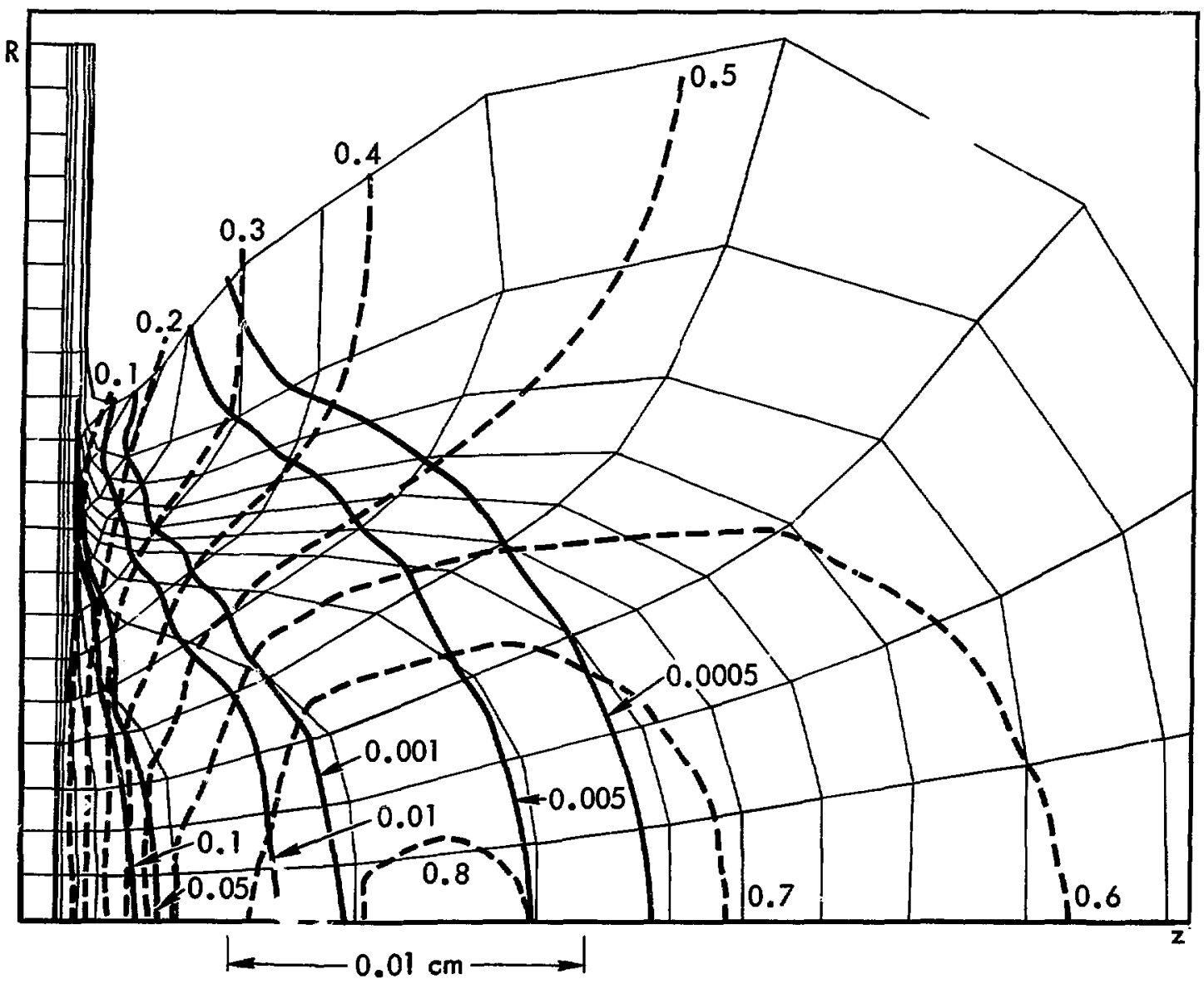

Fig. 202. Flasma blowoff from an iron dab illuminated from the right by a $1-$ nxec, $^{10^{14}}-\mathrm{W} / \mathrm{cm}^{2}$ pulse from an Nd:gass laser. The $z$ axis is an axis of revolution perpendicular to the slab. The thin lines represent the LASNEX Lagrangian grid, the solid lines are isodensity contours $\left(\mathrm{g} / \mathrm{cm}^{3}\right)$, and the dashed lines are electron isotherms (keV). This snapshot depicts the plasma 0.5 nsec after the arrivel of the main pulse. 


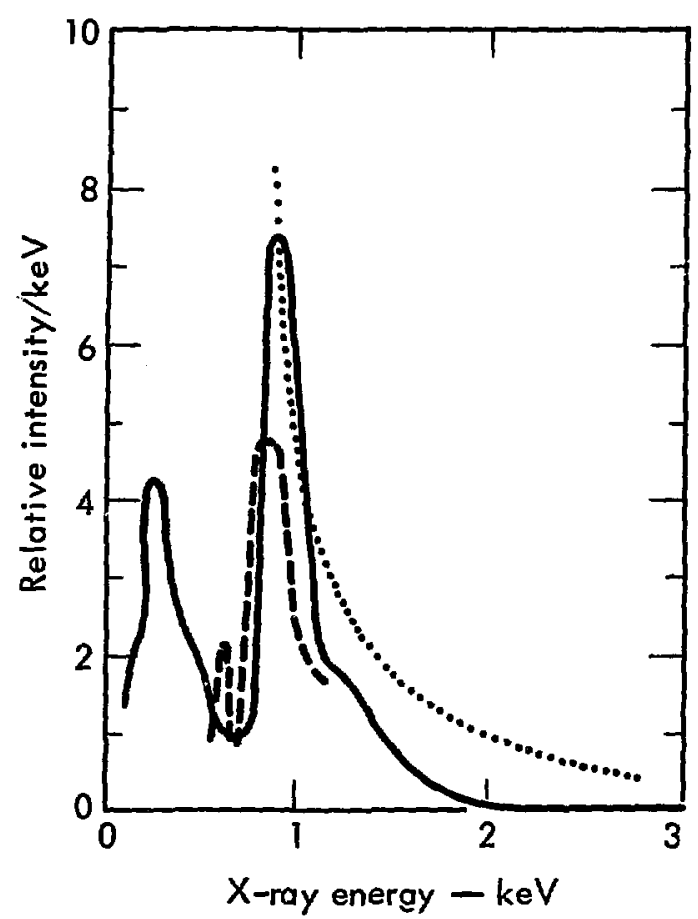

Fig. 203. Comparison of the $x$-ray spectrum predicted by LASNEX with those measured in the BMI experiment for a 1-nsec laser pulse incident on a flat iron slab. The solid line is the LASNEX prediction, the dashed line is a smooth curve drawn through the spectrum obtained from a highresolution bent-crystal spectrometer, and the dotted line gives the lower-resolution data obtained by a differential foil technique.

siab for the $950 \mathrm{eV}$ group. As before, the laser beam is incident from the right. The region emitting this radiation most strongly is beyond the critical density and has a lower electron temperature $(\sim 200 \mathrm{eV})$ than the corona $(\sim 2 \mathrm{keV})$.

Figure 205 shows the opacity of the plasma at the point of peak emission as a function of $x$-ray energy. The peak at $950 \mathrm{eV}$ arises from two $M \rightarrow L$ line transitions, $3 d \rightarrow 2 p$ and $3 p \rightarrow 2 s$. At the higher temperatures of the corona, the ions are so completely stripped of electrons that such bound-bound transitions are improbable and photorecombination is the dominant radiative process. As suggested by Fig. 205, most of the x-radiation between 1.5 and $9.5 \mathrm{keV}$ arises from photorecombination to the $L$-shell. During the course of the 1-nsec pulse, the narrow, relatively cool region between the critical density and solid density radiates line transitions at a fairly constant rate. By contrast, the radiating volume of the corona increases constantly during the pulse.

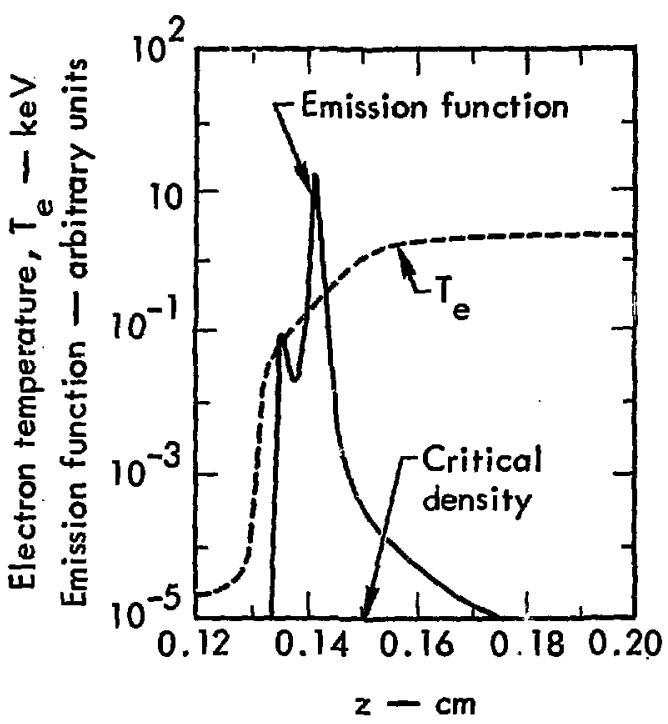

Fig. 204. Variation of the emission function (for 950-eV $x$ rays) and the electron temperature with distance along the $z$ axis $0.9 \mathrm{nsec}$ after the arrival of a I-nsec pulse (from the right). The emission function is $\rho K_{v} B_{v}\left[1-\exp \left(-h \nu / T_{e}\right)\right]$, where $\rho$ is the density, $\kappa_{v}$ is the LTE opacity, and $B_{p}$ is the Planck function. The critical density appears at $z=0.15 \mathrm{~cm}$.

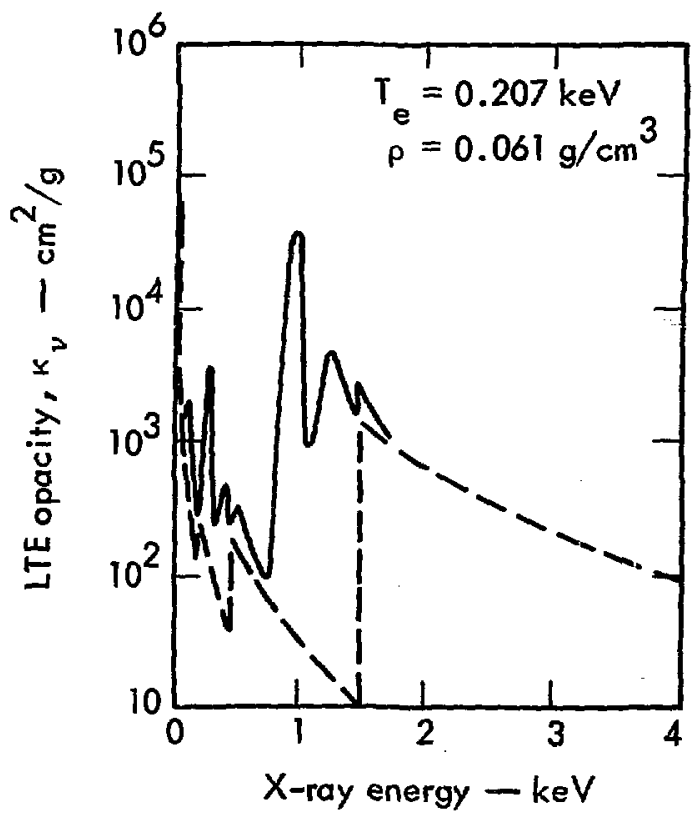

Fig. 205. LTE opacity $\kappa_{p}$ for the zone yielding the pesk in the emission function in Fig 204. The solid line is the opecity with contributions from line transitions; the dashed line is the opacity without line contributions. 
Table 23. X-ray conversion efficiencies for 1-11sec and 50-psec laser pulses on iron as measured in experiments at BMI and LLL and as predicted by the

LASNEX code

$\mathrm{X}$-ray conversion efficiency $-\%$

I-nsec pulse

\begin{tabular}{|c|c|c|c|}
\hline & $\begin{array}{l}\text { To x rays } \\
\text { of energy } \\
\geqslant 1 \mathrm{keV}\end{array}$ & $\begin{array}{l}\text { To } x \text { rays } \\
\text { of energy } \\
\geqslant 300 \mathrm{eV}\end{array}$ & Total \\
\hline BMI experiment & $\begin{array}{r}7-10 \\
-17\end{array}$ & 27 & $\overline{40}$ \\
\hline
\end{tabular}

50-psec pulse

\begin{tabular}{|c|c|c|}
\hline & $\begin{array}{l}\text { To } x \text { rays } \\
\text { of energy } \\
\geqslant 1.3 \mathrm{keV}\end{array}$ & Total \\
\hline LLL experiment & 1.5 & - \\
\hline LASNEX prediction & 0.9 & 2.7 \\
\hline
\end{tabular}

Table 23 summarizes the predicted and measured conversion efficiencies for 1-nsec and 50-psec pulses. For the 1-nsec pulse used in the Battelle experiment, LASNEX predicts a conversion of 11 to $17 \%$ for $x$ rays of energy greater than $1 \mathrm{keV}$, in reasonable agreement with the measured 7 to $10 \%$. The LASNEX calculation indicates that a substantial fraction of this radiation arises from recombination to the $L$-shell. For the 50-psec pulse used in the LLL experiment, LASNEX predicts a lower conversion efficiency $(0.9 \%$ for energies above $1.3 \mathrm{keV}$, a figure in fair agreement with the measured $1.5 \%$ ). Thus, the LASNEX simulations have faithfully reproduced the variation of conversion efficiency with pulse length.
I'his measure of success constitutes tentative certification of the use of LASNEX for the prediction of the emission of $x$ rays from laser-generated plasmas. Bearing in mind that LASNEX neglects non-LTE effects, self-focusing, and stimulated backscattering, we have commenced the design calculation for laser targets. While we have directed our attention primarily to these designs, we intend to further refine LASNEX - in particular, to include non-LTE opacities. Moreover, the experiments with 50- and 250-J iasers will serve as even more detailed checks of the approximations used in LASNEX. A complete understanding of these experiments will be crucial for the successful design of targets for use with higher power lasers.

\section{THE COMPUTER PROGRAM FOREST}

The computer program FOREST is designed to treat multiple-line $\mathrm{x}$-ray spectra which are emitted from laser-produced plasmas. It is a multiple-ion representation, in which targets composed of a single atomic element are considered, in a plane geometry. of one dimension. The plasmas are assumed to consist of ions in various states of ionization and excitation, and of free elections which obey a Maxwellian velocity distribution. Each bound electron of an ion is assigned three quantum numbers, $n, \ell, j$, which are the principal, azimuthal, and total quantum numbers, respectively. An ionic "configuration" is thereby specified by listing these numbers for ail bound electrons of the ion. Only singly excited and ground-state configurations are permitted. For practical reasons, each ion is restricted to at most ten shells of principal quantum number $n$. A total of 100 subshells or levels, also denoted by $n, \ell, i$, is therefore possible. Line transitions between these levels are reduced by the usual selection rules so that there can be at most 774 emission lines per ion with a fixed number of bound electrons. As the number of bound electrons increases, the number of lines is reduced somewhat by the filling in of the ower shells. Further reduction is permitted by the option of designating the uppermost shells as degenerate.

In general, each ion would be specified by $i$, the number of bound electrors, and $k$, the level to which the excited electron belongs. However, to reduce the number of variables, FOREST at present assumes 
"statistical equilibrium," which means that the relative distribution of electrons among the levels of a given shell $n$ is the same as for thermodynamic equilibrium. Thus each ion is specified by $i$ and $n$. The number of ions in the ionic chain is a variable with time, since ions at the ends of the chain can be created or destroyed as it is deemed reasonable.

Another computer program has been developed at LLL that calculates the energy level of each electron for any ionic configuration of $i$ bound electrons and nuclear charge $Z$ by solving the Dirac equation for the isolated ion with a self-consistent spherical potential. ${ }^{199}$ In addition, this program computes the $f$-values for single-electron line transitions. This information and the line frequencies for all the possible stages of ionization and single excitation are read into FOREST as input data.

For the present, the problem of radiative transfer is essentially ignored. All continuous radiation is assumed to escape without absorption. Once a line photon leaves its zone of origin, it is allowed to escape without absorption. However, the average probability of a line photon escaping the zone from the zone center is calculated for each line, assuming a Doppler profile, and is included in the reduction of the line radiative emission rate used in the rate equations for the ionic populations.

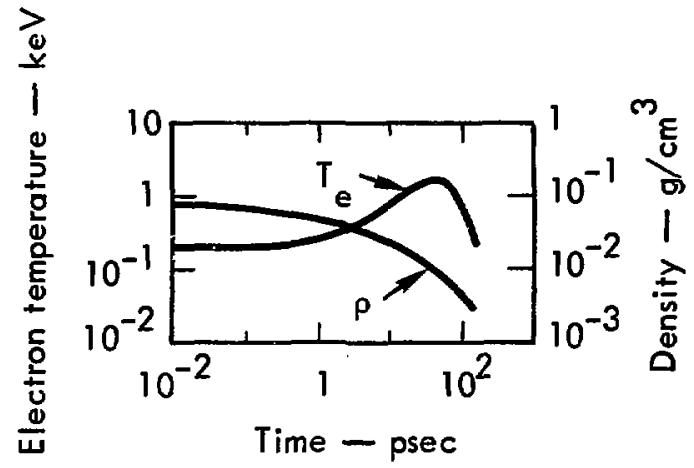

Fig. 206. The electron ature $T_{e}$ and density $\rho$ as functions of i st for a one-zone zirconium problem.

The rate equations for the ionic populations are solved simultaneously, where electron impact ionization and recombination, radiative recombination, electron impact excitation and de-excitation, and radiative line emission (reduced by self-absorption) are allowed. Bound-free absorption, dielectronic recombination, and autoionization are ignored. Conservation of particles is strictly observed to within computer round-off error.

Several one-zone problems with various assumptions about the electron temperature and density have been

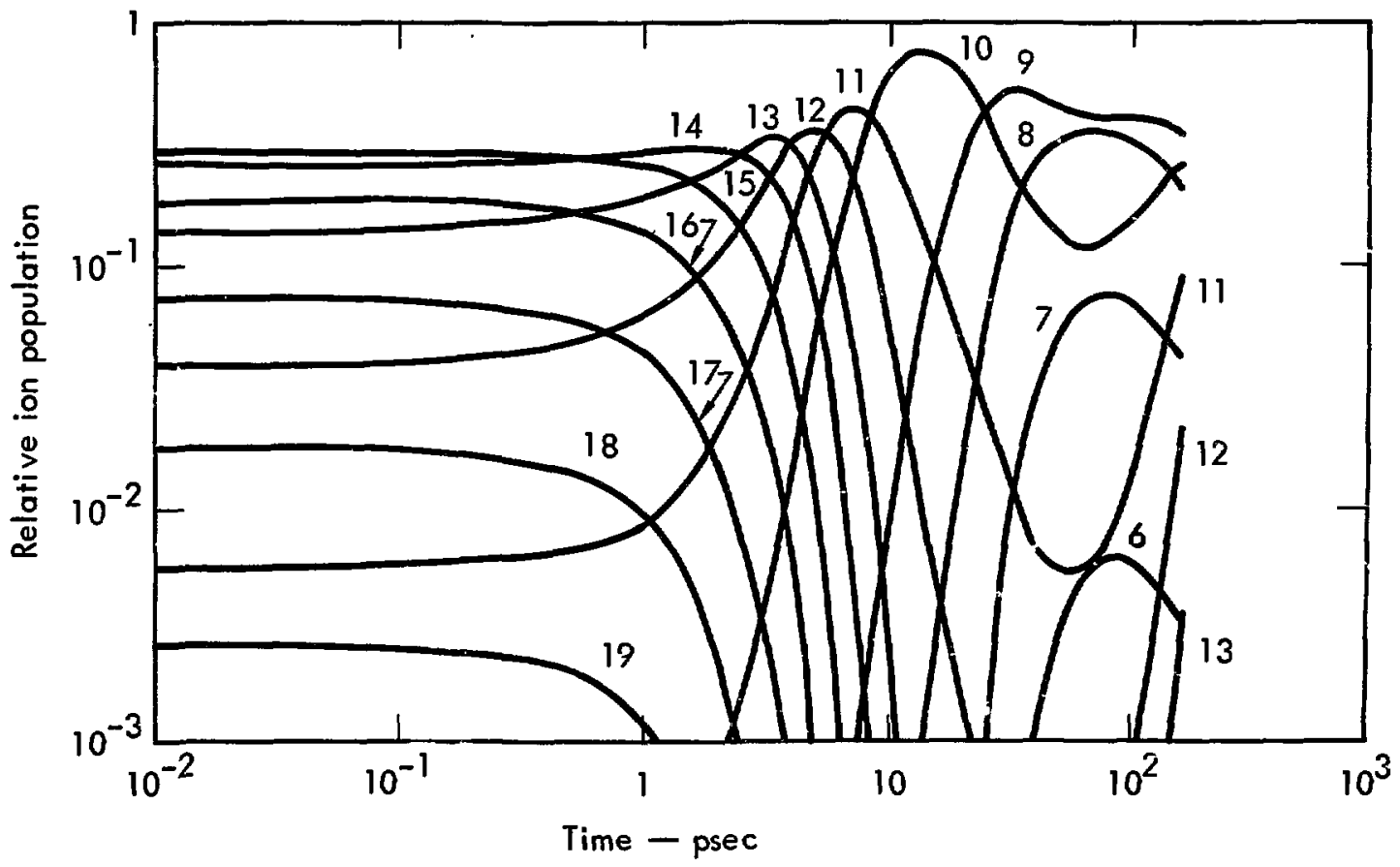

Fig. 207. The fraction of ions with a given number of bound electrons as a function of time for a one-zone zirconi im problem. The numb $a$ of bound electrons is indicated on each curve. 
run on FOREST for the purpose of testing the program. As an example of one such problem, consider a representative zone of a multiple-zone problem dealing with a zirconium foil heated by a typical pulse from the Plasma $X$ laser (duration of pulse, $\sim 100$ psec; total energy of pulse, $\sim 2 \mathrm{~J}$. Figure 206 gives possible variations of electron temperature $T_{e}$ and density $\rho$ with time for such a zone. (These variations were obtained from previously done representative calculations.) Using these values and starting the problem with the ions at their thermodynamic equilibrium populations of $T=200 \mathrm{eV}$ and $\rho=0.1 \mathrm{~g} / \mathrm{cm}^{3}$, FOREST computed the time variations of the populations of the ions up to the time of $200 \mathrm{psec}$, as shown in Fig. 207.

The $x$-ray spectrum of the emission lines and continuum is presently being programmed for graphical output display. The computer program WAZER will supply to FOREST the motion of the zone boundaries, the electron temperatures, and the densities as functions of time for multiple-zone problems. The calculated $x$-ray spectra will be compared with experimental spectra to facilitate understanding of the physics.

\section{Laser-Plasma Coupling - Plasma Instabilities}

\section{INTRODUCTION}

In order to design pellets for fusion we must understand the physics of laser-plasma coupling. A characteristic feature of this coupling is the excitation of plasma instabilities by the intense laser light. We are examining the effects of these instabilities on laser light absorption and reflection, and on energetic particle production. Particular attention has been given to understanding how energetic particles are produced by instability-heating near the critical density, with the object of finding regimes in which fewer energetic particles are produced. In the underdense plasma beyond the critical density, we have examined the turbulent heating due to the $2 \omega_{p e}$ decay of laser light, as well as the reflection due to the Raman and Brillouin instabilities. In addition, we have carried out an initial study of self-focusing of laser light.

\section{MODE-COUPLING SATURATION OF THE PARAMETRIC INSTABILITY}

AND ELECTRON HEATING

A problem of considerable interest in the pursuit of laser fusion is the possible formation of high energy tails on the electron distribution function due to parametric instabilities. These tails have been observed experimentally ${ }^{200,201}$ and in simulations. ${ }^{202}$ Energetic electrons so formed may lead to serious preheat and decoupling problems in the design of pellets. ${ }^{203}$ We report here a one-dimensional analysis of the saturation and heating mechanism for the parametric instability at the critical density (where the Inser frequency is equal to the plasma frequency). The analysis is based upon mode-coupling saturation of the linear instability and subsequent electron heating by quasi-linear diffusion due to the saturated plasma wave spectrum. The predictions are in good agreement with simulations and provide an upper bound for the suprathermal tail formation to be expected in pellet applications.

Previous authors have considered saturation of the decay instability in a plasma having comparable electron and ion temperatures. ${ }^{204}$ The dominant nonlinearity for the plasma waves is then their nonlinear Landau damping on the ions, a process which only spreads plasma wave energy to higher phase velocities. (When $\theta_{e} \gg \theta_{i}$, this process goes over into the resonant coupling of plasma waves by ion acoustic waves.) In these analyses electron-ion collisions play a central role as the process by which energy is coupled from the waves into the particles. In general, however, plasma wave energy spreads to both lower- and higher-phase-velocity waves. Coupling of energy to lower phase velocity can result in the transfer of energy from the waves into the particles by Landau damping. Hence a stationary nonlinear state is possible even when collisions are negligible. Since the energy transfer is via Landau damping, the particle heating will be characterized by the formation of high energy tails.

The nonlinear evolution of the parametric instabilities driven by an electric field oscillating near the plasma frequency has been extensively investigated in computer simulations. They show that for laser field intensities such that $\eta^{2} \equiv E_{0}^{2} / 4 \pi n \theta_{e} \gtreqless 1$, the instability saturation is simply determined by electron trapping in the unstable plasma waves. ( $E_{0}$ is the laser field amplitude, $n$ is the electron density, and $\theta_{e}$ is the electron temperature.) However, for $\eta^{2} \ll 1$, trapping in the electron plasma waves does not occur. Since $\eta^{2}$ is the ratio of the laser's light energy density 
to the plasma's thermal energy density, we expect the $\eta^{2} \ll 1$ regime to be the most relevant for laser pellet studies. Hence we have focused attention on both simulations and theory of the instabilities in this regime.

In order to discuss a specific instability, let us consider the case in which the laser frequency equals the electron plasma frequency. Then only the oscillating two-stream instability occurs. Typical simulation results for this case are shown in Figs. 208a, 209, and 210. The plasma wave energy exponentiates in agreement with the linear theory of the oscillating two-stream instability and saturates at a level several times the pump field energy (Fig. 208a). After saturation, the plasma wave spectrum $\left(\left.E_{k}\right|^{2}\right)$ assumes an approximate $k^{-2}$ shape for wave numbers greater than the linearly most unstable mode (Fig. 209). The heated electron distribution function
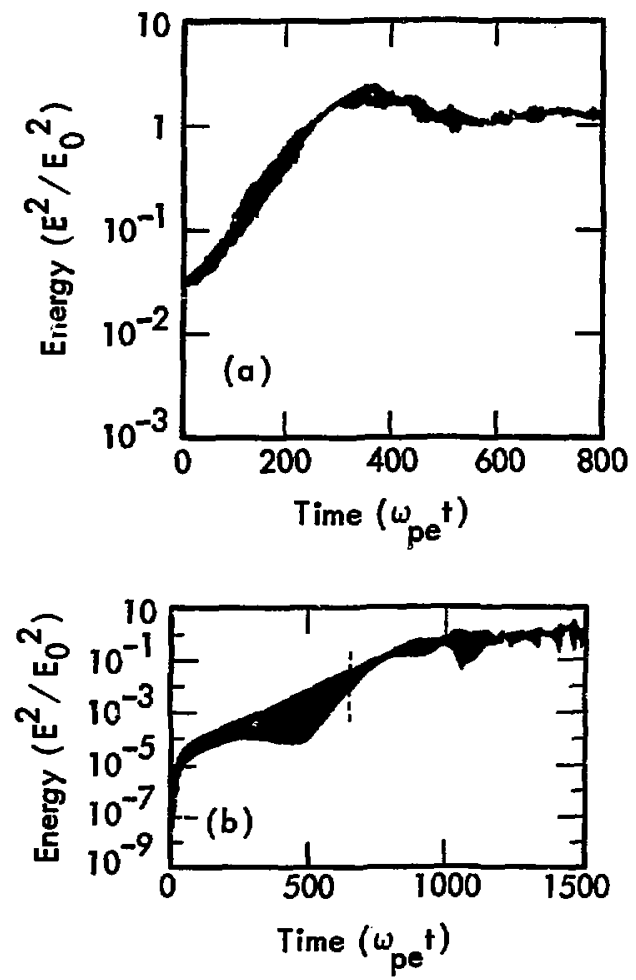

Fig. 208. Occillnting two-stream instability. Electric field energy is shown as a function of time (a) for a cimulation calculation with driver strength $\eta=$ $0.13, \omega_{0}=\omega_{p e}$, and $\theta_{e} / \theta_{t}=3.33$, and (b) for a hydrodynamic equation celculation with $\eta^{2}=$ 0.15, $\omega_{0}=\omega_{p e}$, and $\theta_{e} / \theta_{i}=3.00$. The dashed line indicates epproxinate starting conditions for the cimulation. has a central Maxwellian, an exponential tail, and then a sharp drop (Fig. 210). Our analysis will predict all these basic features observed in the simulations.

Since the fluctuating fields in the saturated state become as large as the pump field, this suggests that mode coupling may be the saturation mechanism. We apply Nishikawa's ordering scheme $e^{205}$ to the hydrodynamic equations for electrons and ions, but without neglecting the products of pairs of fluctuating quantities. The following equations are obtained for the evolution of the electron and ion density fluctuations.

$$
\begin{aligned}
& \frac{\partial^{2}}{\partial t^{2}} n_{e k}+{ }_{e k} \frac{\partial n_{e k}}{\partial t}+\omega_{e k}^{2} n_{e k}=\frac{i k e}{m_{e}} n_{i k} E_{0} \\
& +\frac{i k e}{m_{e}} \sum_{k^{\prime} \neq 0} n_{i k-k^{\prime}} E_{k^{\prime}}, \\
& \frac{\partial^{2}}{\partial t^{2}} n_{i k}+\nu_{i k} \frac{\partial n_{i k}}{\partial t}+\Omega_{k}^{2} n_{i k}=-\frac{i k e}{m_{i}} n_{e k} E_{0} \\
& -\frac{i k e}{m_{i}} \sum_{k^{\prime} \neq k} \frac{k^{\prime}}{k-k^{\prime}} n_{e k-k^{\prime}} E_{k^{\prime}} .
\end{aligned}
$$

We have followed Nishikawa's notation; $\omega_{e k}$ is the Bohm-Gross frequency, $\Omega_{k}$ the ion acoustic frequency, and $\nu_{k}$ is taken to be the electron or ion Landau damping. We numerically solve these coupled mode equations to predict the instability saturation.

Simultaneously the electron distribution function is evolved by solving the quasi-linear diffusion equation, 206

$$
\frac{\partial f}{\partial t}=\frac{\partial}{\partial v} D \frac{\partial f}{\partial v},
$$

where the diffusion coefficient $D$ is known in terms of the plasma wave amplitudes determined from the mode-coupling code, and is given by

$$
D(v)=\frac{e^{2}}{m^{2}} \int \frac{\gamma_{k} E_{k}^{2} d k}{\left(\omega_{e k}-k v\right)^{2}+\gamma_{k}^{2}} .
$$

Here $\gamma_{k}$ is the width of the resunance. As long as it is small, its detailed magnitude is not significant. For convenience we have taken $\gamma_{k}$ to be the magnitude of the Landau damping at $\nu=\omega_{k} / k$, which is probably an underestimate. From the updated distribution function we compute new values of $\nu_{k}$ and $\lambda_{D}$ for Eqs. (1) and (2). 
Figures $208 \mathrm{~b}$ and 210 show the calculated results for the typical case investigated in the computer simulations $\left(\eta^{2}=0.13, \theta_{e} / \theta_{i}=3.3\right.$ initially). As shown in Fig. 208b, the plasma wave energy exponentiates in agieement with linear theory and then saturates at a level of $\sim 2 E_{0}^{2}$, in agreement with the simulation results. The field spectrum is more coarsely represented in the mode-coupling program than in the simulations due to the larger mode spacing, but the $k^{-2}$ shape is evident. Figure 210 shows the good agreement between the calculated distribution function and the simulation result. After saturation, the heating rate is constant at about $0.032 m_{p e}$, whereas the simulation result is about $0.033 \omega_{p e}$. This close agreement is probably fortuitous, but this analysis does give generally correct heating rates.

From quasi-linear theory, we can show that the spectrum $E_{k}^{2} \propto k^{-2}$ leads to a diffusion coefficient, $D(v)$, which is linear in velocity. Since the spectrum depends on temperature (through terms like $\boldsymbol{\Omega}_{k}$ and $\left.\lambda_{p}\right), D(v)$ depends on the time-varying thermal velocity: $D(v) \propto v / v_{t h}(t)$. A simple calculation then gives the correct conclusion that the heating rate is constant and that exponential tails are formed. It should be noted that the $k^{-2}$ spectrum is not a sensitive function of particle damping but results from the mode-coupling dynamics. This was seen by varying the form of $v_{k}$ in the mode-coupling equations and observing that the $k^{-2}$ spectrum persists.

Our simple analysis then predicts the basic features of the oscillating two-stream instability and shows that the major effects are mode-coupling for saturation and diffusion for heating. We expect this simple model of turbulent heating at the critical density to provide an upper limit for the suprathermal tail formation in pellet applications. For example, we have also investigated cases in which the laser frequency is somewhat higher than the plasma frec vency, so that both the oscillating two-stream and ion acoustic instabilities are operative. The heated distribution function then has fewer high-energy electrons, basically because lower-phase-yelocity plasma waves are produced. In addition, we find that fewer very energetic particles are produced when one accounts for the finite spatial extent of the turbulence in pellet applications. This effect has been demonstrated in both theory and simulations. Additional improvements are discussed in the next article.

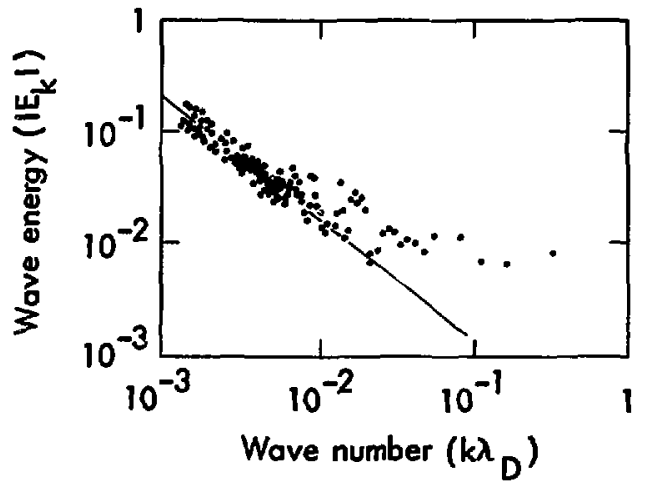

Fig- 209. Suturated turbulent-field epectrum from the timulation calculation at time $\omega_{p e^{l}}=703$.

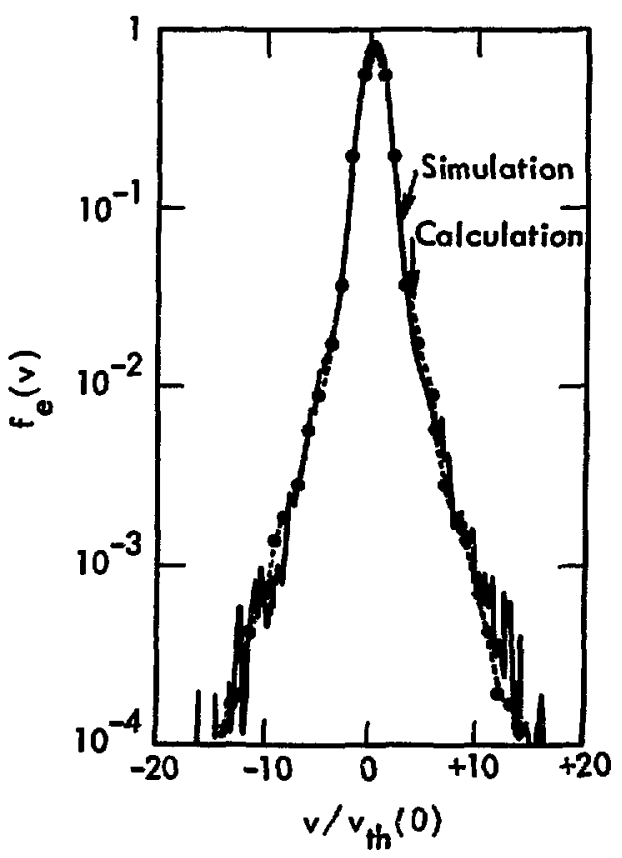

Fin. 210. Electron velocity distribution from the cinule. tion at ine $\omega_{p e} l=326$. Temperature at this tine is 25 times the ivitil clectron cemperr vire. Dulved line is the distribudion celculated from hydrodynanic equations for this tempere ture.

\section{IMPROVEMENTS IN TURBULENTLY HEATED DISTRIBUTION FUNCTIONS}

Since distribution functions play an important role in pellet design, we have given considerable attention to understanding them. Simple models of turbulent heating in a collisionless plasma predict distributions which are very rich in energetic particles. ${ }^{206}$ Although these ideal models give valuable upper limits for 
conservative pellet design, we expect that fewer energetic particles will be produced in practice. To explore this possibility we have been systematically improving our models of the turbulent heating. We discuss here two important mechaniams to reduce the energetic particle production: electron-ion collisions, and short-wavelength ion waves. There is a regime above the instability threshold in which electron-ion collisions balance the instability growth, leading to turbulent heating of electrons in the main body of the distribution. We examine this limit and estimate how far it extends above the instability threshold. In addition, when the electron/ion temperature ratio is large, unstable long-wavelength ion waves "shock-up" to produce ahort-wavelength ones. These latter ion waves couple fast-electron plasma waves into slower ones which produce less-energetic particles when they Landau-damp. Simulations show improved but still non-Maxweilian heated distributions in the limit of high electron/ion temperature ratios.

\section{Turbulent Heating Near}

the Intabifity livechold

Turbulent heating is simply the transfer of energy from a laser into particles via plasma waves. The character of the heated distributions depends on the mechanism by which the plasma waves damp their energy into the particles. Collisional damping leads to a heating of the main body of the distribution functions, because slower particles are deflected more frequently. In contrast, Landau damping of long-wavelength plasma waves produces energetic particles, since it is due to an acceleration of resonant particles (those with velocities near the phase velocity of the plasma wave).

We have previously concentrated on investigating turbulent heating in the collisionless limit, where Landau damping is the mechanism for wave damping. This is a realistic limit for many pellet calculations, particularly late in the laser pulse when the plasma temperature is many kilovolts. However, in some situations collisions play an important role; e.g., early in the pulse when the temperature is low, or for short-wavelength laeer light and pellets seeded with high- $Z$ material. Indeed, one can construct nonlinear solutions in which collisiounl damping solely stabilizes the instability, and hence very energetic particles are not produced. Let us first outline the general features of this "collisional" limit and then estimate when the collisionles behavior becomes dominant.

We consider the evolution of a plasma driven by a spalially homogeneous pump field oxcillating near the electron plasma frequency. This problem is theoretically tractable 207 if we make a number of assumptions: the instability is near threshold, only plasma waves which are collisionally damped are driven unstable, and the ion and electron temperatures are comparable. Standard weak-turbulence theory then predicts a wave kinetic equation which determines the nonlinear evolution of the electron plasma oscillations.

The general features of the nonlinesr solution can be obtained by simple energy-balance argunents. The energy flow into the waves driven unstable by the pump is balanced by the collisional damping of the total energy in all the plasma waves. Hence

$$
r_{\frac{1}{8 \pi}}^{2}=\nu_{e i} \frac{E_{T}^{2}}{8 \pi} \text {, }
$$

where $\gamma$ is the growth rate of the instability, $\nu_{e l}$ is the collisional damping rate, $E_{1}^{2} / 8 \pi$ is the energy density in the unstable waves, and $E_{T}^{2} / 8 \pi$ is the energy density in all the plasms waves. Since the plasma waves driven by the pump must be large enough to decay as efficiently into other plarma waves,

$$
E_{1}^{2} \approx \frac{E_{0}^{2}}{2}
$$

$E_{0}$ is the pump field amplitude, and the factor of 2 arises since sne usually defines $E_{0}$ as the peak amplitude of the pump field. The above equations yield

$$
\frac{E_{T}^{2}}{E_{0}^{2}} \approx \frac{\gamma}{2 p_{e i}} .
$$

Similarly the energy damped from the plasma waves must be supplied by the pump field. This defines an effective damping rate $\nu^{*}$,

$$
\nu^{*} \frac{E_{0}^{2}}{8 \pi}=\nu_{e l} \frac{E_{T}^{2}}{8 \pi} \text {. }
$$

Hence

$$
v^{*} \approx \gamma
$$

These estimates are borne out by the solutions of the wave kinetic equation. 208

The basic point is that one can construct nonlinear solutions in which the parametric instability is stabilized solely by collisional damping of the electron plasma waves. In this case anomalous absorption occurn, but with a heating of the slow electrons rather then a production of energetic tails. In the collisionless bimit, of course, the solution we oudlined is no longer 
passible. It is important to know when the "collisiond" solution gives way to the collisionless one. In other words, how far above the instability threshold (which is determined by collisions) is the turbulent heating dominmtly heating of slow electrons?

Although this question is still under investigation, it is worthwhile now to make some simple estimates. As a plausible first extimate, let us assume that the "collisional" solution holds as long as the total plasma wave energy predicted by it is less than the plasma wave energy predicted by our collisionless investigations. In the pump field intensity regime investigated in the simulations, one then estimates the two solutions to crose over when

$$
\frac{\gamma}{v_{e i}} \approx 4
$$

When $\gamma \lesssim 4 v_{\text {ei }}$ collisional damping can stabilize the instability, and heating of slow electrons is the dominant result. Clearly this estimate is not precise, but its magnitude is consistent with recent microwave experiments. 209

A study of the transition between the collisional and collisionles rolutions is now under way. The basic approach is to simulate the turbulent henting with a Vasov code, including a collision operator to realistically describe electron-ion collisions. These calcuiations will yield a mixture of main body heating and tail formation and show how this mixture depends on $\gamma / \nu_{e l}$, the laser frequency, and the electron/ion temperature ratio.

\section{The Role of Short-Wavelength lon Waves}

\section{in Turbulent Heating}

In the collisionless limit, Landau damping balances the instability growth. However, there is a pronising mechanism for producing improved distribution functions even in this limit. The basic notion is to couple the high-phase-velocity plasma waves generated by the instabilities into waves having much lower phase velocity. When these slower waves are Landau-damped, they producc less-energetic particles. Short-wavelength, finite-amplitude ion fluctuations $\left(k \lambda_{D} \approx 0.3\right.$, where $\lambda_{D}$ is the electron Debye length) efficiently couple fast and slow electron plasma waves. If the electron/ion temperature ratio is sufficiently large $(>10)$, these chort-wavelength ion waves are produced by a shockingup of the instability-generated long-wavelength ones.

Weak turbulence calculations assume that energy is transferred by ion waves from one plasma oscillation to mother only when the coupling is resonant; i.e., when the frequency- and wave-number matching conditions are satisfied. However, it has been pointed out and verified in computer simulations 210 that finite-amplitude ion waves efficiently couple electron plasma waves, even when the mode coupling is off-resonant. The condition for efficient energy transfer is that the ratio of the amplitude of the ion density fluctuation to the background density $\left(\delta n / n_{0}\right)$ be comparable to the frequency mismatch between the electron plasma oscillations; i.e. $\delta n / n_{0} \approx \Delta \omega / \omega_{p e}$. To give a specific example, an ion density fluctuation with an amplitude of $\sim 15 \%$ and a wave number $d \lambda_{D}=0.3$ rapidly couples energy from very fast electron plasma waves into one with a phase velocity of $\sim 3 v_{t h}$. This slower wave Landau-damps, producing energetic electrons with velocities $\sim 3 v_{\text {th }}$. Short-wavelength, finite-amplitude ion waves are then a natural way to reduce the energetic particle production by parametric instabilities. Fast plasma waves driven by the instability are coupled to rather slow ones, which then Landau-damp their energy into the particles.

These beneficial short-wavelength ion fluctuations can in fact be produced by a "shocking-up" of the instability-generated, longwavelength ion acoustic waves. To see this, let us consider turbulent heating in a finite region of a plasma in which the electrons are much hotter than the ions. The finite size of the turbulent region allows the plasma temperature to reach a quasi-steady level in which the heating is balanced by energy flow out of the region. (See the previous semiannual report ${ }^{186}$ for a discussion of other features of turbulent heating in a finite region.) The high electron/ion temperature ratio means that the instability-generated ion waves are very weakly damped. This allows them to shock-up (or generate harmonics) before they damp.

The shocking-up of ion waves in a plasma with $\theta_{e} \gg \theta_{i}$ can be readily shown. ${ }^{211}$ for $k \lambda_{D} \ll 1$, the dispersion relation for an ion wave is approximately $\omega=k v_{a c}$, where $v_{a c}$ is the ion acoustic velocity. Disturbances at different wave numbers travel at the same speed, as in ordinary sound waves. It can be shown that such waves shock-up in a time $\tau_{s} \approx\left[k v_{a c}\left(\delta n / n_{0}\right)\right]^{-1}$. For finite $k \lambda_{D}$, however, $\omega_{2 k} \neq 2 \omega_{k}$. The time for this frequency shift to become important is $\tau_{\omega}=1 / \Delta \omega \approx\left(3 k v_{c c} k^{2} \lambda_{D}^{2}\right)^{-1}$. Ion waves generate strong harmonics if $\tau_{s}<\tau_{\omega}$; that is, if the shock time is less than the frequency mismatch time. The condition for shocking is then $\delta n / n \gtrsim 3 k^{2} \lambda_{D}^{2}$. Hence finite-amplitude, long-wavelength ion waves efficiently generate harmonics.

We have carried out simulations to find the magnitude of the expected improvement in the electron distribution function. The one-dimensional electrostaiic code, modified for a finite laser-absorption region, was used. Laser-heated electrons that reach the boundaries of the simulated region are replaced by cold electrons. These cold electrons are the corona 
electrons flowing into the laser absorption region to maintain charge neutrality. This model is valid only as long as few laser-heated electrons can cross the corona without losing most of their energy. When a significant fraction of the laser-heated electrons cross the corona without energy loss, these hot electrons supply the neutralizing current. Then the infinite-medium results should apply.

In Fig. 211 we compare the heated electron distributions from simulations with an infinite interaction region and with a finite interaction region $1024 \lambda_{D}$ in width. Finite region results are shown for an electron/ion temperature ratio of 100 . These distributions have been normalized so that the mean energy is the same in both cases. There are fewer very high energy electrons and more main body heating in the finite region case, as expected. We have also performed finite region calculations with equal electron and ion temperatures. We find that the number of electrons in the tail is roughly independent of temperature ratio. In each case the ion wave growth was finally stopped by ion trapping. The ion waves were several times larger in the case with the high temperature ratio, and the short-wavelength components were many times larger. These shortwavelength ion waves resulted in the strong main body heating shown in Fig. 211. No such heating occurred in the equal-temperature case. Thus for a given average electron energy, a high electron/ion temperature ratio will result in far fewer high-energy electrons.

These results can be particularly important for longer wavelength lasers such as $\mathrm{CO}_{2}$, since the electron/ion temperature ratio is typically much larger than with $\mathrm{Nd}$ lasers. We are continuing this

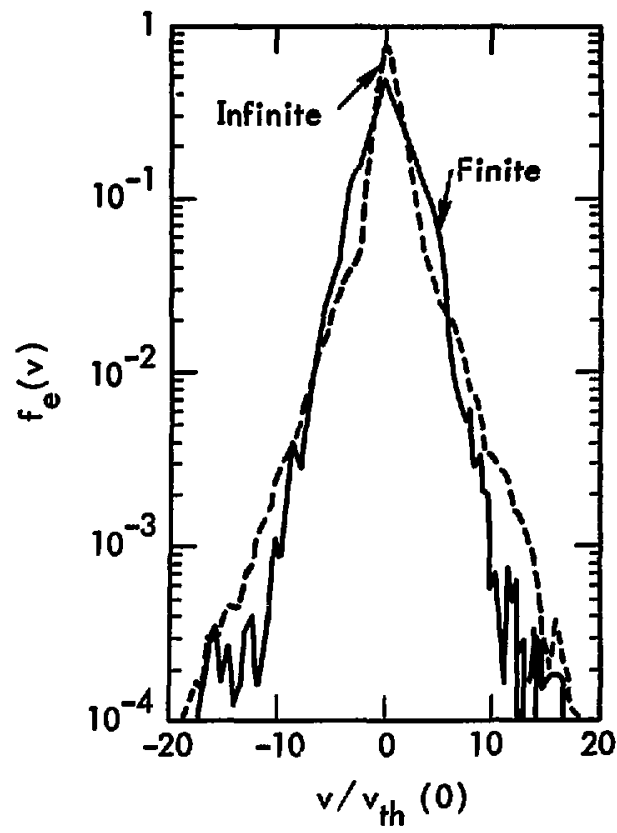

Fig. 211. Flectron velocity distributions as calculated from simulations with an infinite internction revion and with a finite interaction region. In the calculation for the finite region, width is $1024 \lambda_{D}$ and $\theta_{e} / \theta_{i}=100$.

investigation. More realistic mass ratios, better models of the finite region, and the time-dependent return current from LASNEX will be analyzed.

\section{PLASMA HEATING AT ONE-FOURTH THE CRITICAL DENSITY}

The mechanisms most considered for anomalous absorption of laser light occur at critical density, where the electron plasma frequency $\omega_{p e}$ is near the laser frequency $\omega_{0}$. But it is also important to understand heating and reflection processes which occur in the underdense region outside the critical density surface. Heating and reflection by the Raman instability have previously been considered 212,213 ; this instability occurs when the plasma dersity is less than one-fourth the critical density $\left(2 \omega_{p e}<\omega_{0}\right)$. When $2 \omega_{p e} \approx \omega_{0}$ a competitive heating mechanism appreciably increases absorption of laser energy. This mechanism is the decay of the laser light into two longitudinal plasma waves, 214 as compared to the Raman instability in which one of the decay waves is a transverse light wave. The $2 \omega_{p e}$ instability is particularly interesting because it is absorptive rather than reflective, and because it tums out to produce a softer spectrum of electron energies. Furthermore, because its decay waves carry energy away from the instability region more slowly than for the Raman process, the threshold for the $2 \omega_{p e}$ instability in an inhomogeneous plasma is expected to be lower.

\section{Theory}

We look first at the linear theory of the two instabilities. The decay-process wave vectors are shown in Fig. 212. Wave 2 is longitudinal for both instabilities, while wave 1 may be either longitudinal $\left(2 \omega_{p e}\right)$, or transverse (Raman), or a mixture. Since we deal with electron temperatures of the order of 1-2 keV, some modifications of existing theory are 
neeried. At thesc high temperatures the instability is suppresed by Landau damping of wave 2 unless $k_{2} \leqslant 3 k_{0}$, since $k_{0} \lambda_{D} \approx 2 v_{t h} / c \approx 0.1$, where $v_{t h}$ is the electron thermal velocity. Therefore we cannot use an expansion in powers of $k_{0}$ as in previous theories. Aso we find that the magnetic force term $\vee \times B$ makes an important contribution. Lastly we assume no particular polarization for decay wave 1 , because in the important regime where $k_{1} \ll k_{0} \approx k_{2}, \mathbf{E}_{1}$ is neither purely longitudinal nor transverse.

The result of the linear analysis is a vector equation for $E_{1}$,

$$
\begin{aligned}
\left(\omega_{2}^{2}\right. & \left.-\omega_{p e}^{2}\right)\left\{\left(\omega_{1}^{2} \cdot \omega_{p e} \cdot c^{2} k_{1}^{2}\right) \mathrm{E}_{1}+c^{2} \mathrm{k}_{1}\left(\mathrm{k}_{1} \cdot \mathrm{E}_{1}\right)\right] \\
& =\frac{\omega_{p e}^{2}}{4 k_{2}^{2}}\left[k_{2}^{2} \mathrm{v}_{0} \cdot \frac{\omega_{p e}}{\omega_{1}}\left(\mathrm{k}_{1} \cdot \mathrm{v}_{0}\right) \mathrm{k}_{1}\right] \\
& \cdot\left\{\left[k_{2}^{2} \mathrm{v}_{0} \cdot \frac{\omega_{p e}}{\omega_{1}}\left(\mathrm{k}_{1} \cdot \nabla_{0}\right) \mathrm{k}_{1}\right] \cdot \mathrm{E}_{1}\right\},
\end{aligned}
$$

where $v_{0}$ is the amplitude of oscillation in the laser field. The assumption that $\mathbf{E}_{1}$ is purely longitudinal or purely transverse will yield the dispersion relations for the $2 \omega_{p e}$ or Roman instability, respectively. Without making such an assumption, the condition for a nontrivial solution $\mathbf{E}_{1}$ to exist is the dispersion relation

$$
\begin{aligned}
\frac{4\left(\omega_{2}^{2} \cdot \omega_{p e}^{2}\right)}{\omega_{p e}^{2}}= & \frac{k_{2}^{2}}{k_{1}^{2}} \frac{\left|\mathrm{k}_{1} \times v_{0}\right|^{2}}{\left(\omega_{1}^{2}-\omega_{p e}^{2} \cdot c^{2} k_{1}^{2}\right)} \\
& +\frac{\left(k_{2}^{2}-k_{1}^{2}\right)^{2}}{k_{1}^{2} k_{2}^{2}} \frac{\left(\mathrm{k}_{1} \cdot v_{0}\right)^{2}}{\left(\omega_{1}^{2}-\omega_{p e}^{2}\right)} .
\end{aligned}
$$

When $k_{1}$ is not too small with respect to $k_{0}$, only one of the terms on the right-hand side can be important; which term (if either) depends on the frequencies. When

$\omega_{0} \cdot \omega_{2}=\omega_{1} \approx \omega_{t 1} \equiv\left(\omega_{p e}^{2}+c^{2} k_{1}^{2}\right)^{1 / 2}$,

the Raman instability is recovered, whose growth rate with zero frequency mismatch is

$\operatorname{Im}\left(\omega_{1}\right)=\frac{1}{4} \sqrt{\frac{\omega_{p e}}{\omega_{t 1}}} \frac{k_{2}}{k_{1}}\left|k_{1} \times v_{0}\right|$.

When $\omega_{1} \approx \omega_{p e}$ and the last term is retained, we find the dispersion relation for the $2 \omega_{p e}$ instability. Its growth rate with zero frequency mismatch is

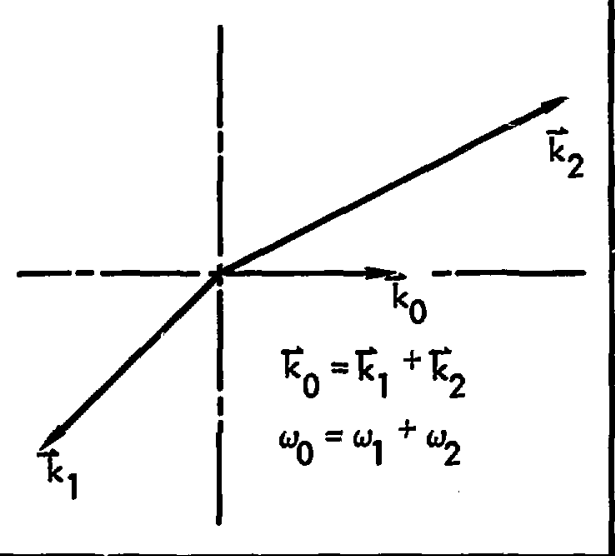

Fig. 212. Schematic diggram of the wave vectors for the $2 w_{\text {pe }}$ Raman decay instability. Wave $\left(k_{0}, w_{0}\right)$ is the laor light, waves 1 and 2 are the decay products.

$$
\operatorname{Im}\left(\omega_{1}\right)=\frac{1}{4 k_{1} k_{2}}\left|k_{1} \cdot v_{0}\right| i k_{2}^{2}-k_{1}^{2} \mid
$$

This is the first new result of the analysis. The second is that when $k_{1}$ is small with respect to $k_{0}$, Eq. (1) reduces to

$$
\left(\omega_{2}^{2} \cdot \omega_{p e}^{2}\right)\left(\omega_{1}^{2}-\omega_{p e}^{2}\right) \mathrm{E}_{1}=\frac{1}{4} \omega_{p e}^{2} k_{2}^{2} \mathrm{v}_{0}\left(\mathrm{v}_{0} \cdot \mathrm{E}_{1}\right) .
$$

This says that $E_{1}$ is parallel to $v_{0}$ regardless of the direction of $k_{1}$, and the maximum growth rate is $k_{0} v_{0} / 4$. Equation (3) or (4) correctly describes the case of small $k_{1}$ only when $k_{1}$ is parallel or perpendicular to $k_{0}$, respectively.

\section{Simulation Results}

Although many features of the Raman instability can be studied in one-dimensional simulations, t; $2 \omega_{p e}$ instability requires two space dimensions si: the plasma waves do not propagate parallel to the laser iight. In order to study the competition and nonlinear evolution of these two instabilities we have used the new plasma simulation code ZOHAR. ${ }^{215}$

A typical simulation result serves to demonstrate the basic linear and nonlinear phenomena. In particular, we simulate intense laser light propagating through a uniform underdense plasma in which the electron plasma frequency is half the frequency of the laser. In our system, decay processes such as those in Fig. 213a,b occur. All the expected modes, including those which are predicted only by the fully electromagnetic theory of $2 \omega_{p e}$ and Raman (such as 


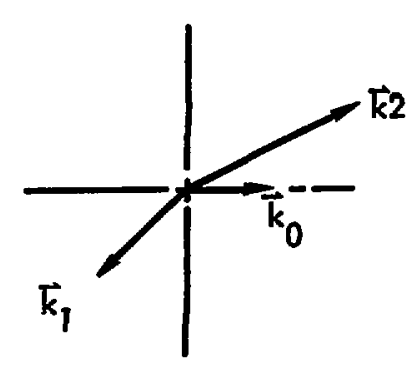

(a)

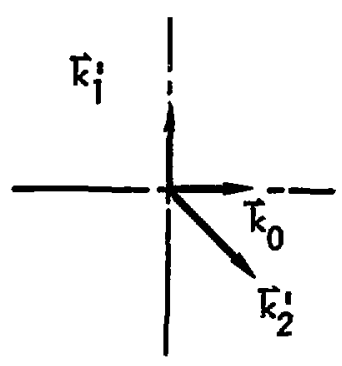

(b)

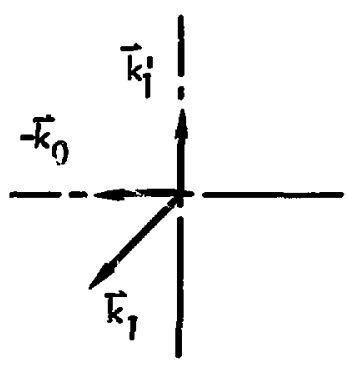

(c)

Fig. 213. Examples of $2 \omega_{p e}$ decays of an electromagnetic wwe, $k_{0}$, are shown in (a) and (b). The recombination of one wave from each docay into a lifht wave, $\cdot k_{0}$, is shown in (c).

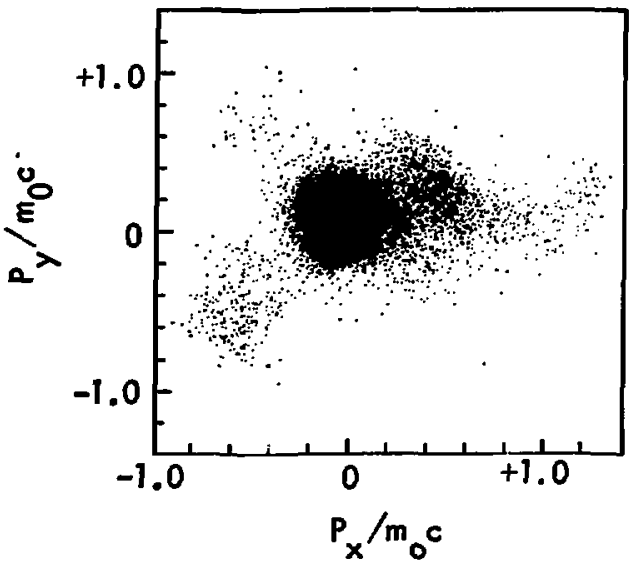

Fig. 214. Fhase space plot of electron momentum in the $y$-direction vs electron momentum in the $x$ direction, showing the trapping due to $2 \omega_{p e}$ decay and Ruman instabilities.

the one shown in Fig. 213b), have growth rates in agreement with the above theoretical calculations.

Figure 214 is a phase space plot of the electron distribution after the waves have grown and the main saturation mechanism, trapping of the electrons by the forward plasma waves, has set in. Very energetic particles are produced by this trapping. The $2 \omega_{p e}$ instability provides a spectrum of waves with phase velocities less than those of Raman, with the result that energy is given to a larger number of electrons and their energy spectrum is softer. In the example shown, the lower-phase-velocity $2 \omega_{p e}$ decay waves (such as $k_{2}$ in Fig. 213a) have reduced the Raman-decay-wave trapping width and therefore modified the electron distribution fren what it would be if the $2 \omega_{p e}$ instability had been ignored. When both processes are included, the Raman decay wave traps particles more efficiently. More particles are heated but the distribution function has a lower cutoff in energy.

The backward waves from $2 \omega_{p e}$ have higher phase velocities $\left(k_{2}>k_{1}\right.$ in Fig. 213a) and therefore trap relatively few particles. These waves are then only weakly damped and persist for a long time at high amplitudes. They can then recombine into other transverse waves. Specifically, the process shown in Fig. 213c occurs in which the higher-phase-velocity waves from two distinct $2 \omega_{p e}$ decays combine to produce a transverse wave with propagation vector $-\mathbf{k}_{0}$. Four-wave coupling also occurs. As an example, the second harmonic of $k_{1}$ (Fig. 213a) or $k_{1}^{\prime}$ (Fig. 213b) plus the laser produce a higher-frequency transverse wave. We emphasize that these recombination processes depend on the existence of a $2 \omega_{\text {pe }}$ decay of the type shown in Fig. 213b, and the magnetic force term must be included for this decay to be understood.

Later in time, the ion motion becomes important. The small $k_{1}$ decay products remaining at high amplitudes themselves become the pump waves for subsequent decay. Their frequencies are just above $\omega_{p e}$ and therefore they drive the oscillating two-stream and parametric instabilities, leading to further electron heating. Also occurring on the slower ion time scale are Brillouin scattering and filamentation. These are discussed in later articles in this section.

In addition to delineating the wave coupling processes and the nature of the heating which takes 
place near one-fourth the critical density, the simulations allow us to make some initial estimates of the efficiency of this heating. Basically we compute the energy flow to the plasma particles and from this define an effective damping rate, $\nu^{*}$, for the laser light. 216 We then estimate $\nu^{*} \approx 2 \operatorname{Im}(\omega)$, where $\operatorname{Im}(\omega)$ is the growth rate of the $2 \omega_{p e}$ decay instability. The absorption length for wave decay is then

$$
\lambda_{\mathrm{ab} z} \geq \frac{\lambda_{0}}{3} \frac{c}{v_{0}},
$$

where $\lambda_{0}$ is the laser wavelength and $v_{0}$ is the electron quivering velocity in the laser field. For efficient heating in a plasma with a density gradient, this absorption length must be less than the distance over which the plasma density changes by about $20 \%$, since the instability can only occur near the point where $\omega_{0}=2 \omega_{p e}$.

Future plans include the incorporation of a density gradient to make the simulations more realistic, and an examination of the effect of self-focusing on the instabilities.

\section{INSTABILITY-GENERATED LASER REFLECTIONS IN PLASMAS}

Although several plasma instabilities enhance Iaser-light absorption, the Raman and Brillouin instabilities increase the reflection of laser light. These instabilities represent the decay of the laser light into another electromagnetic wave plus either an electron plasma wave of an ion wave. To examine the importance of instability-generated reflection in laser-fusion schemes, we calculate the reflection of laser light propagating through an underdense plasma with a density gradient. A 1-1/2-dimensional electromagnetic, relativistic particle-simulation code is used, and both the Raman and Brillouin instabilities are studied. The instability-generated reflection is shown to decrease rapidly as the ratio of the laser light pressure to the plasma pressure becomes small. These nonlinear results scale in agreement with simple physical arguments. Although the model we use is rather ideal, the nonlinear results are consistent with experimental observations of small laser-light backscattering. Several techniques which further reduce these instability effects are demonstrated.

Since the linear theory of the Raman and Brillouin instabilities has been discussed in many papers, ${ }^{217}$ let us directly discuss the nonlinear results. In general one should allow for the possibility of beth backscatter and sidescatter ${ }^{218}$ of the laser light. To begin with the most tractable case for particle simulations, let us confine ourselves to examining the backscatter of the laser light. We then use a 1-1/2-dimensional electromagnetic particle simulation code. This code works directly with the electric and magnetic fields and is fully relativistic. The plasma density profile rises linearly from zero to 0.7 of the critical density $\left(10^{21} \mathrm{~cm}^{-3}\right.$ for Nd lasers) in a distance of 10 free-space laser wavelengths. This model is similar to the one used in valuable studies recently reported by Forslund et al.,219 with the exception that our code is relativistic. The critical density is not incluoed in this simple model in order to isolate the effects of the reflective instabilities from the usual parametric ones occurring near the critical density. Many free-space wavelengths of vacuum are included on either side of the plasma to allow for plasma blowoff. The laser light is introduced from the left, and electromagnetic waves are allowed to propagate freely into space from either simulation boundary. The simulation particles are charge-weighted to provide an optimum representation of the low- and high-density regions of the plasma.

It is instructive to study each instability individually. Since the Raman instability depends only on the electrons, it can be isolated by using immobile ions. Let us briefly describe the basic features of these simulations. In agreement with linear theory, the Raman instability maximizes where the plasma density $n \approx(1 / 4) n_{c r}$ (the critical density), where it drives an electron plasma wave with a wavelength nearly equal to the laser wavelength plus a long-wavelength backscattered transverse wave. For intense lasers the instability is simply saturated by electron trapping, whereas for less intense lasers other nonlinearities play a role (such as the resonant decay of plasma waves when ion motion is included). We will here discuss two important features of the nonlinear evolution: the laser reflection, and the generation of very energetic electrons.

As noted in Ref. 219, the reflection due to the Raman instability is not severe. Figure 215a shows the percentage of laser energy reflected as a function of $\eta^{2}\left(\eta^{2}=E_{0}^{2} / 4 \pi n_{c r} \theta_{e}\right.$, where $E_{0}$ is the laser-light electric field, $n_{c r}$ is the critical density, and $\theta_{e}$ is the electron temperature). Note that $\eta^{2}$ is simply the ratio of the laser light pressure to the plasma thermal pressure defined at the critical density. For this data the laser intensity was fixed, and the electron temperature was varied. The observed reflection is at most $9 \%$ (for $\eta^{2} \gg 1$ ) and decreases rapidly as the electron temperature is increased. For low electron temperatures, strong electron trapping occurs; this heats the electrons and brings the instability into 

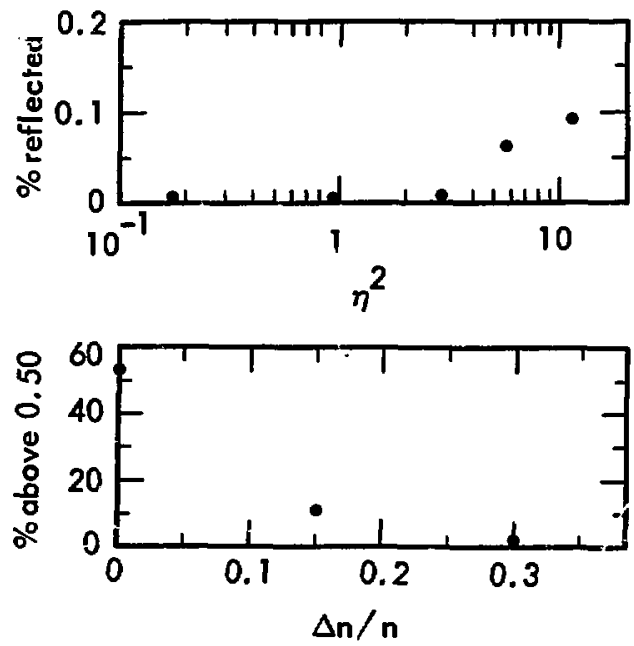

Fig. 215. Noalinear reaults for the Raman indebility with immobile ionk (a) Reflected laver energy (normalized to incident energy) vs $\eta^{2}$. (b) Percent of energy in electrons with velocities greater than $0.5 c$ ve ion wave amplitude. Laser intensity is $3 \times 10^{16} \mathrm{~W} / \mathrm{cm}^{2}$ (Nd), and the imposed ion ware has a wave number $k \lambda_{D}=0.2$, where $\lambda_{D}$ is the Debye length at $1 / 4$ the critical density.

the regime where the driven plasma waves are heavily damped. For higher electron temperatures $\left(\theta_{e} \gtrsim 5 \mathrm{keV}\right)$, the driven plasma waves are already appreciably damped and so are not. driven to large amplitudes.

A more serious feature of the Raman instability is the energetic electrons which are produced. Since the phase velocity of the plasma wave driven near $(1 / 4) n_{c r}$ is $\sim(1 / 2) c$, electrons acquire velocities nearly equal to $c$ when they are trapped by the wave. Hence we use a fully relativistic code. Since these electrons can preheat the core of a pellet, 220 it is important to explore means by which they can be suppressed. Finite-amplitude, short-wavelength ion fluctuations efficiently couple high-phase-velocity plasma oscillations into slow, damped ones which produce less-energetic electrons. ${ }^{210}$ Such ion waves can be produced in the underdense plasma surrounding a pellet by, for example, counterstreaming between different ion species in the blowoff or steepening of the ion waves produced by other instabilities. In order to demonstrate their beneficial effect, we have simply introduced a short-wavelength ion wave as an initial condition into our simulations of the Raman instability. Figure 215b shows the percentage of the kinetic energy in electrons with velocities greater than $0.5 c$ after the instability saturation as a function of ion wave amplitude. In this example the laser intensity is $3 \times 10^{16} \mathrm{~W} / \mathrm{cm}^{2}$. There is substantial reduction for ion wave amplitudes $\Delta n / n \approx 20 \%$. For less intense lasers, smaller-amplitude ion waves are equally effective.

One final point concerning the Raman instability should be noted. As Rosenbluth points out, ${ }^{221}$ the instability threshold depends on density gradient, because the resonance conditions for the laser decay can only be satisfied locally in the plasma. For a $10-\mu \mathrm{m}$ density gradient and an Nd laser, we find an abrupt shutoff of the Raman instability at an intensity of $\sim 10^{16} \mathrm{~W} / \mathrm{cm}^{2}$. This is in reasonable agreement with the theoretical estimates. 219,221

This suppression of the Raman instability by density gradient allows one to isolate and study the Brillouin instability (which is much less sensitive to density gradient). We now use an electron/ion mass ratio of 300 . This mass ratio sufficiently separates the time scales for iori and electron motion and also allows us to run many ion periods in the simulations. We have varied the mass ratio to spot-check our results.

Again let us briefly describe the basic features of these simulations. Ion waves, with half the wavelength of the laser, exponentiate as expected from linear theory. Correlated with the buildup of these ion waves, laser light reflection occurs. We measure this reflection by monitoring the Poynting vector on the left side of the system. This reflection exhibits relaxation oscillations which persist for a number of cycles, as is typical in coupled-mode interactions. We average the reflected power over the oscillations, since an average over these times scales is inherently carried out in experimental measurements.

The time-averaged reflection due to the Brillouin instability versus $\eta^{2}$ is shown in Fig. 216a. For $\eta^{2} \gtrsim 1$ the reflection is quite large ( $\sim 50 \%$ even for our $10-\mu \mathrm{m}$ plasma). However, it rapidly decreases as $\eta^{2}$ is made small. This is particularly important since laser fusion schemes operate in the $\eta^{2}<1$ regime. The data were obtained by varying the laser intensity and/or the thermal energy of the plasma, indicating the $\eta^{2}$ is a reasonable parameter. This decrease of the reflection with $\eta^{2}$ is consistent, for example, with results found by Forslund et al. ${ }^{219}$ that less reflection occurs when the electrons become heated due to either the Raman or other instabilities. The small reflection found when $\eta^{2} \ll<1$ is consistent with experimental observations of small laser-light backscattering.

A simple physical argument for the observations can be given. The energy density of ion waves is $\left(\Delta n / n_{0}\right)^{2} n_{0} k T_{e}$, where $\Delta n / n_{0}$ is the root-mean-square ion wave amplitude. The electron temperature er:ters since the electrons provide the pressure in an ion wave. A given laser intensity wili then produce smaller-amplitude ion waves when the electron thermal 

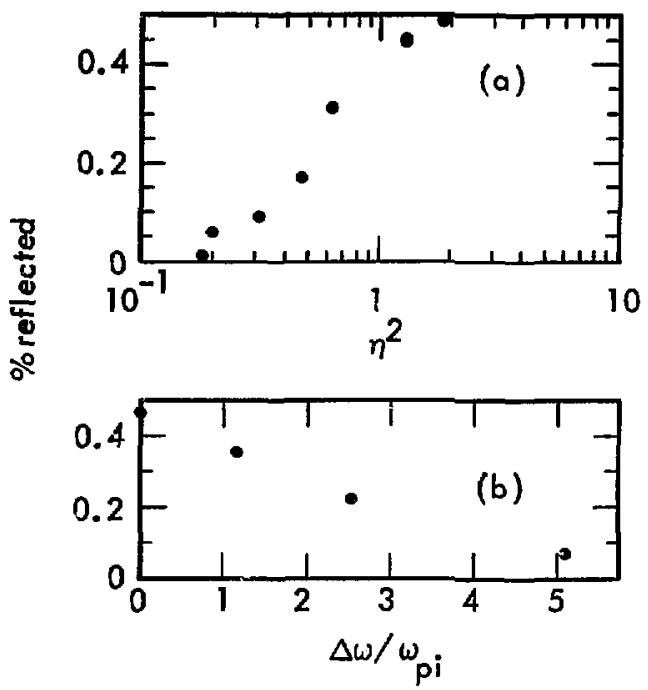

Fig. 216. Nonlinear results for the Brillouin instability. The electron/ion mass ratio is 300 and their temperature ratio is 5. (a) Reflected laser energy vs $\eta^{2}$. (b) Reflected laser energy ys laser bandwidth; $\omega_{p i}$ is the ion plasma frequency at the critical density.

energy is larger. Hence the reflection decreases, since it depends on the level to which the ion waves are driven.

In the small $\eta^{2}$ regime we have doubled the size of the plasma (to $20 \mu \mathrm{m}$ ), and found that the reflection approximately doubles. When we increase the electron/ion temperature ratio to 10 , the reflection increases severalfold. This again is as expected physically. A laser of given intensity produces larger ion waves if the damping of the waves is less. In this regard, it should be noted that the electron and ion temperatures remain closer together $\left(\theta_{e} / \theta_{i}\right.$ is $\sim$ 3-5) when one uses a short-wavelength laser (heating at higher plasma density) than when one uses a long-wavelength laser such as $\mathrm{CO}_{2}$.
A nonlinear theory is necessary to quantitatively predict the reflection. Several exact analytic solutions for the backscatter of laser light in a spatially homogeneous medium have been carried out, assuming the only nonlinearity is pump depletion. 222 These illustrate some characteristic features of the spatial backscatter in complementary limits in which the phonon is undamped and heavily damped. For the present problem these solutions need to be extended to allow both a density gradient and plasma expansion.

The instability-generated reflection is further reduced when the laser light has a finite bandwidth. Figure 216b shows the reflected power versus the laser bandwidth for a sample case in which the net laser intensity is $10^{16} \mathrm{~W} / \mathrm{cm}^{2}(\mathrm{Nd})$ and the electron temperature is $1 \mathrm{keV}$. The laser bandwidth is simply modeled by distributing the laser energy in many individual fields, each with a slightly different frequency and random phases. Similar results have been obtained by using a laser with random phase modulation. This decrease in the reflection agrees with simple estimates. Basically only that fraction of the power within a given frequency width (proportional to the linear growth rate of the instability) acts coherently. This is also found in linear instability calculations for a finite-bandwidth laser. ${ }^{196}$ For weaker intensities even smaller laser bandwidths are effective at suppressing the reflection. In another interesting limit we find that the reflection is also reduced when one uses two intense monochromatic lasers with different frequencies, due to destructive interference between the unstable plasma waves.

In summary, we have investigated the nonlinear evolution of the Raman and Brillouin instabilities in one dimension and demonstrated several techniques for suppressing their effects. The nonlinear results show small reflection when the plasma pressure is much less than the laser light pressure. Improved models of the reflective instabilities are being developed. In particular, we are investigating a 2-1/2-dimensional model which includes both sidescattering and self-focusing of the laser light.

\section{SELF-FOCUSING AND FILAMENTATION IN PLASMAS}

It has been predicted that the laser light in a plasma will focus itself into a narrower beam with higher intensity. 223,224 In a plasma this occurs because the intense light forces some of the plasma out of the beam, forming a channel with lower plasma density and therefore higher refractive index. The light intensity inside the channel then rises to achieve a balance between refraction into and diffraction out of the channel. Another possibility is that the beam will break up into many channels or filaments, ${ }^{225}$ perhaps initiated by diffraction pattems in the focused light. 226 It is important to examine the consequences oi such filamentation on laser heating of plasma.

We have begun a theoretical analysis of these effects. In order to estimate the rate at which filaments form, and the relation of this process to others involving ion motion, a dispersion relation has been derived 
describing the rate $\omega$ of formation of filaments with periodicity given by $k$ :

$$
\begin{aligned}
\frac{\omega^{2}-\omega_{i a}^{2}}{\omega_{p i}^{2}}=\frac{1}{4} k^{2} v_{0}^{2}\left[\left(\frac{\hat{\left(\mathbf{k}_{-} \cdot \hat{v}_{0}\right)^{2}}}{\omega_{-}^{2} \cdot \omega_{p e}^{2}\left(1+3 k_{-}^{2} v_{t e}^{2} / \omega_{0}^{2}\right)}\right)\right. \\
+\left(\frac{\left(\hat{\mathrm{k}}_{-} \times \mathrm{v}_{0}\right)^{2}}{\omega_{-}^{2} \cdot \omega_{p e}^{2}-c^{2} k_{-}^{2}}\right) \\
\left.+\left(\begin{array}{l}
\text { Repeat of above terms } \\
\text { with } k_{-} \rightarrow k_{+} \text {and } \\
\omega_{-} \rightarrow \omega_{+}
\end{array}\right)\right]
\end{aligned}
$$

where $\omega_{i a}$ is the ion-acoustic frequency, $v_{0}$ is the velocity of oscillation in the laser field of frequency $\omega_{0}$ and wave vector $k_{0}, k_{-}=k_{0}-k, \omega_{-}=\omega_{0}-\omega$, and $\hat{v}_{0}=v_{0} /\left|v_{0}\right|$.

The simplest case of filamentation is obtained when $k$ is perpendicular to both $v_{0}$ and $k_{0}$. The most rapid growth rate is

$$
\operatorname{Im}(\omega)=\frac{\omega_{p i} \nu_{0}}{\sqrt{2} c},
$$

which applies when

$$
\frac{v_{0}^{2}}{v_{t e}^{2}} \geq \frac{c^{2} k^{2}}{\omega_{p e}^{2}} \gtrsim \frac{\omega_{0} \omega_{p i}}{\omega_{p e}^{2}} \frac{v_{0}}{c} .
$$

Filamentation with $\mathrm{k}$ parallel to $\mathrm{v}_{0}$ is almost as rapid.

We have also begun a study of filamentation using the plasma simulation code ZOHAR. We find formation of filaments or channels a few wavelengths wide, with intensities inside the channels many times larger than the intensity outside, even at densities as low as $\sim 2 \%$ of the critical density. The channels appear to be stable structures.

Very similar physical processes underlie filamentation, Brillouin scattering, and the oscillating two-stream and ion-electron parametric instabilities. This is clear from Eq. (1), which includes all of these effects according to the relative magnitudes of the terms on the right-hand side. We are studying the interdependence of these phenomena.

\section{LARGE PLASMA-SIMULATION CODES USING VIRTUAL MEMORY}

This project aims at developing large plasmasimulation codes (electrostatic and electromagnetic) which utilize disk storage as virtual memory. These codes depart from the usual implementation (in which only the particles are kept on disk) in that the charge/current and field arrays are stored on disk and only brought to core in sections as they are needed. Thus the ultimate mesh size is not limited by memory size considerations. Even moderate-sized codes can benefit by requiring only a small fraction of the total memory, an advantage in a time-shared environment.

The code currently being implemented is of the electrostatic particle type with a two-dimensional potential mesh of $512 \times 513$ points. The north and south boundaries are periodic while the potential on the east and west boundaries is fixed. The particle mover is the usual central-difference type, with linear interpolation used in determining the force on each particle and in accumulating the charge array.

The particles are kept in 32 groups, each of which lies in a region coincident with a $16 \times 512$ section of field cells. As each section of field cells is brought into coie, the particles associated with that section are moved and their charge array section is created and sent to disk. The field array is stepped through in this manner until all the particles have been advanced through one time step and their charges accumulated into the charge array. The Poisson equation is then solved to convert the charges to potentials, and the whole process is repeated.

Particles which cross the outer boundaries of the $16 \times 512$-cell section and leave the group during the time step are sorted into separate arrays to be later merged with particles in adjacent groups. Those particles which have moved in the forward direction (into the section which will be processed next this time step) are kept in core to be merged immediately with their new group. Those particles moving into sections which have already been processed are put to disk, after having their charge accumulated, to be merged into their new group during the next time step. Most disk transfers are scheduled so as to overlap computation as much as possible, and double and triple buffering are used to facilitate this overlap.

The Poisson solver uses Fourier transforms in the periodic dimension (the long direction of the $16 \times 512$-point section) and Gauss elimination in the fixed-potential direction. The Fourier transform and first march of the Gauss elimination are done on the charge array sections before they are put to disk for the first time. The solution is completer by bringing the data back into core in the reverse order to be marched back and inverse-transformed. The whole Poisson solution is thus accomplished with only one extra round trip to disk. 
To date, the bookkeeping part of the particle-moving algorithm has been debugged and partially optimized for the LLL computer system. The Poisson solver has been debugged and tested with good results. Existing particle-moving, charge-accumulating, diagnostic, and initialization routines are being adapted to this code. A smaller version of this code (with
$16 \times$ 513-point mesh) is being implemented which fits entirely into core storage and will be used as a standard code. Results from both codes should match to the last bit (with the large code operating in the $16 \times 513$-point mode). It is expected that this code will run nearly as fast as existing codes with core-contained fields and particles kept on disk. 
For controlled thermonuclear reactor (CTR) applications, the fuel gain (ratio of fusion energy produced to fuel energy at ignition) must be greater than 1000-fold - ten times larger than can be achieved with uniformly ignited, nondegenerate fusion micropellets. Gains greater than 1000 -fold may be achieved if thermonuclear propagation and electron degeneracy are exploited. $220^{*}$

The 1000-fold gain required for CTR applications is determined by the combined efficiencies of the laser, the implosion, and the electrical generating equipment. Practical lasers suitable for laser implosion CTR may be $10 \%$ efficient. The implosion efficiency is only $5-10 \%$ because the blow off velocity is much larger than the implosion velocity, and because material already ablated coniinues to be heated in order to transport energy from the outer region of laser absorption inward to ablate the surface of the imploding pellet. $^{227}$ The thermal-electric generating efficiency is about $40 \%$, and in order to minimize capital costs, iess than $30 \%$ of the generated electrical energy can be allowed to circulate internally (to drive the laser).

The 100-fold gain that can be achieved with uniform heating is determined by the $10^{9} \mathrm{~J} / \mathrm{g}$ required to heat DT to a 10-keV ignition temperature and the $10^{11} \mathrm{~J} / \mathrm{g}$ produced by DT with $30 \%$ burnup. In a fusion microexplosion there is little or no advantage in igniting at less than $10 \mathrm{keV}$ because the time for the DT to self-izeat (via the $3.6-\mathrm{MeV}$ DT alpha particle) from initial temperatures significantly less than $10 \mathrm{keV}$ to optimum burn temperatures greater than $20 \mathrm{keV}^{\dagger}$ is comparable to the inertial confinement time. At $51 . \mathrm{eV}$, the "ideal" ignition temperature, the alpha heating rate equals the bremsstrahlung cooling rate - so that the net heating is zero. The DT burn efficiency, $\phi$, is ${ }^{228}$

$$
\phi \approx \frac{\rho R}{6+\rho R}
$$

\footnotetext{
"Other papers show gain relative to the laser light energy applied to the pellet. This gain is 20 times smaller than the gain defined here because we take into account the implosion efficiency which is only about $5 \%$.

$t_{\text {Recall the burn efficiency is proportional to the product }}$ of the burn rate $\rho \sigma v$ and the inertial confinement time $R / c$. The teinperature-dependent terms ov/c optimize at iemperatures of $20-40 \mathrm{keV}$.
}

or $33 \%$ at $\rho R \approx 3 \mathrm{~g} / \mathrm{cm}^{2}$. With uniform heating and a density of $1000 \mathrm{~g} / \mathrm{cm}^{3}$ a $2 \times 10^{6}-\mathrm{J}$ laser is required to achieve a $\rho R$ of $3 \mathrm{~g} / \mathrm{cm}^{2 *}$.

The gain that may be achieved if propagation and degeneracy are exploited is determined by the critical mass of DT that must be ignited to initiate propagation and by the minimum energy of the Fermi-degenerate eiectrons as a function of density:

The critical $p R$ which must be ignited to initiate propagation is about $0.3 \mathrm{~g} / \mathrm{cm}^{2}$. At this $\rho R$ the burn efficiency is $5 \%$, corresponding to $1.6 \times 10^{10} \mathrm{~J} / \mathrm{g}$, or about $3 \times 10^{9} \mathrm{~J} / \mathrm{g}$ in alpha particles. This is sufficient energy to heat three times more mass to $10 \mathrm{keV}$ temperatures. At $10-\mathrm{keV}$ electron temporatures, the 3.6-MeV alpha range is about $0.3 \mathrm{~g} / \mathrm{cm}^{2}$, so that a few times more mass will be heated by the alpha particles. Hence a pellet with $\rho R \gtrsim 1.5 \mathrm{~g} / \mathrm{cm}^{2}$ may be ignited by less than $1 \%$ of its mass in a hot central core with $0.3 \mathrm{~g} / \mathrm{cm}^{2}$ heated to $10 \mathrm{keV}$. Thus in principle the average ignition energy may be reduced from $10^{9} \mathrm{~J} / \mathrm{g}$ to less than $10^{7} \mathrm{~J} / \mathrm{g}$.

If propagation is utilized to reduce the ignition energy of a high-density pellet by more than one to two orders of magnitude - to less than $10^{7}-10^{8} \mathrm{~J} / \mathrm{g}$ - then electron degeneracy becomes important. If the DT is at a density of $1000 \mathrm{~g} / \mathrm{cm}^{3}$, the minimum energy of compression is $3 \times 10^{7} \mathrm{~J} / \mathrm{g}$. This minimum occurs if the DT electrons are highly Fermi-degenerate, i.e., if the thermal energy is much less than the Fermi energy:

$$
\mathscr{E}=\mathscr{G}_{F}\left[\frac{3}{5}+\frac{\pi^{2}}{4}\left(\frac{K T}{6}\right)^{2}+\ldots\right],
$$

where $\mathscr{E}$ is the internal energy, $\mathscr{E}_{F}$, the Fermi energy, is $\left(h^{2} / 8 m\right)\left(3 n_{e} / \pi\right)^{2 / 3}, h$ is Planck's constant, $m$ is the electron mass, and $n_{e}$ is the electron density. At $1000 \mathrm{~g} / \mathrm{cm}^{3}$ the Fermi energy is about $1 \mathrm{keV}$. In computer calculations of implosions with optimum pulse shapes most of the pellet mass is heated to temperatures of about $100 \mathrm{eV}$ during imp'ssion to densities of $1000 \mathrm{~g} / \mathrm{cm}^{3}$. If the electrons throughout most of the mass of DT are imploded to a highly

\footnotetext{
Using perfect gas heat capacity, $10^{5} \mathrm{~J}$ of energy is required to heat $10^{-4} \mathrm{~g}$ of DT to $10-\mathrm{keV}$ temperatures; with $2.20 \%$ implosion efficiency, this corresponds to a $2 \times 10^{6}$.J laser; a sphere of mass $10^{-4} \mathrm{~g}$ and density $10^{3} \mathrm{~g} / \mathrm{cm}^{3} \mathrm{has} a \rho \mathrm{\rho R}$. of $3 \mathrm{~g} / \mathrm{cm}^{2}$.
} 


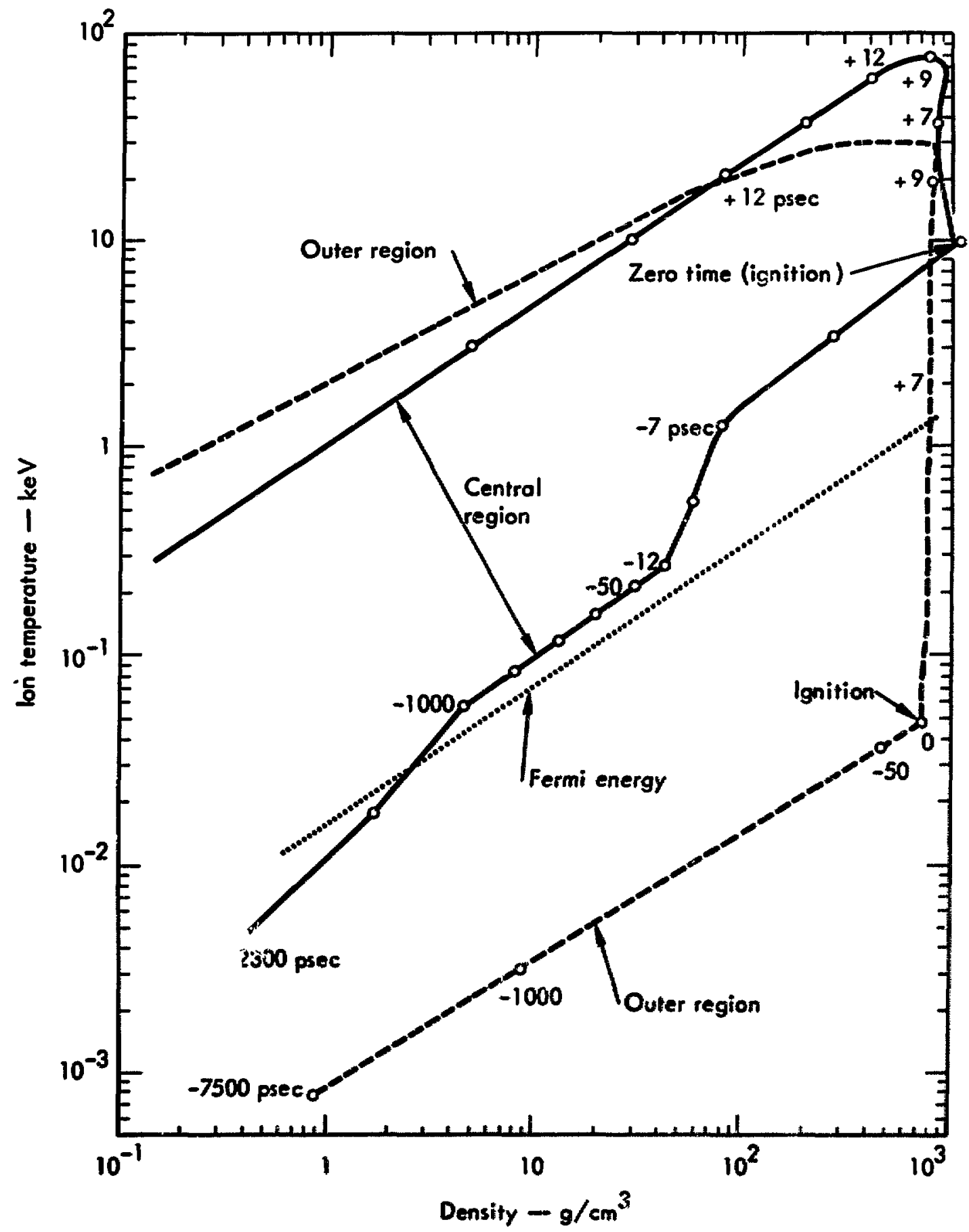

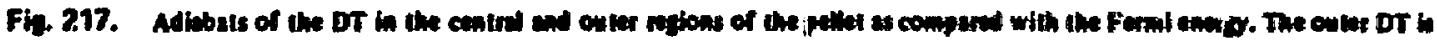

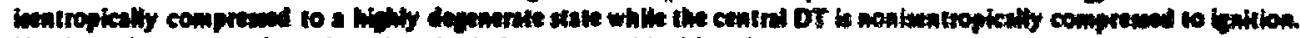

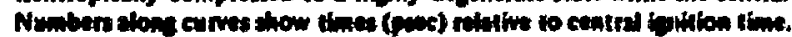


degenerate state, then the energy required to compress the pellet may be made as amall as the energy required to ignite a few percent of the DT mass in the central core. Otherwise the energy of compression may exceed the energy of ignition.

In a spherical implosion the central region is heated to higher temperatures than the outer region by convergence of the initial shock. Further heating of the central core relative to the outer region may be achieved by adjusting the lner pulse thape so that the characteristics of the hydrodymanic now in the imploding matter conlesce to form a strong shock in the matrer which compresses to form the $0.3-8 / \mathrm{cm}^{2}$ centril core. 227 Although oxiy $0.3 \mathrm{~g} / \mathrm{cm}^{2}$ in the central segion need be heated to $10-\mathrm{keV}$ temperatures, the average ignition enersy cannot be made arbitnrily small as the total aR of the pellet becomes much hager than $0.3 \mathrm{~d} / \mathrm{cm}^{2}$. This is dive to imperfect implosion symmetry and the fact that material just beyond the central $0.3 \mathrm{~g} / \mathrm{cm}^{2}$ will be heated by implosion to temperatures intermediate between $10 \mathrm{keV}$ and $100 \mathrm{eV}$ (characteristic of the outer region). In typical computer calculations of laser implosions the temperature incresses invernely with radius squared. Then the average imnition eneray is reduced by a factor proportional to $(0 R / 0.3)^{3}$, ft $>0.3$, or a factor of 30 when $\rho R=3 \mathrm{~g} / \mathrm{cm}^{2} .22 R$

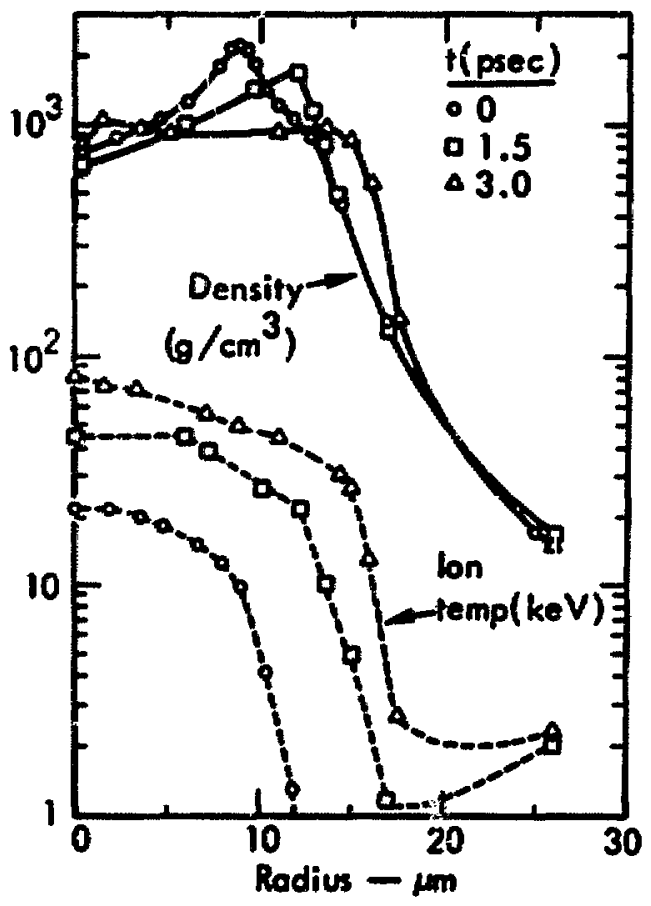

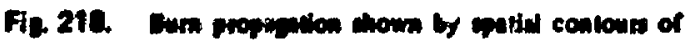

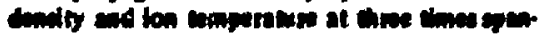
ning e s oune intered.
Figures 217.219 show the results of a LASNEX 229 computer calculation of a laser imploalon of $260 \mu g$ DT pellet. The pulse shape is optimized to fully exploit degeneracy and propagation. In Fig. 217 the solid and dashed lines show the thermndynamic paths (temperature vs denaity) of the fuel in the ceniral and outer regions of the pellet. The dotted line is the Fermi energy. The fuel in the outer region is near isentropically compressed to a state in which the electrons are highly Fermi.degenerate - density $800 \mathrm{~g} / \mathrm{cm}^{3}$ and thermal tempernture $<100 \mathrm{eV}$, kess then one-tenth the Fermi energy at this density. The fuel in the central region is compressed to $1000 \mathrm{~g} / \mathrm{cm}^{3}$ and 10.keV temperature. The numbers along the thermodynamic paths are times in picoseconds relative to zero time at ignition (when the central region resches $10 \mathrm{keV}$ ). The central region is thocked off its adiabal (parallel to the Fermi line) by shocks at 1000 and 12 peec prior to ignition. Seven picoseconds after tenition the central region has bootestrapped to $20 \mathrm{keV}$ and the outer region has been heated to $2 \mathrm{keV}$ by bum propagated out from the central region. At

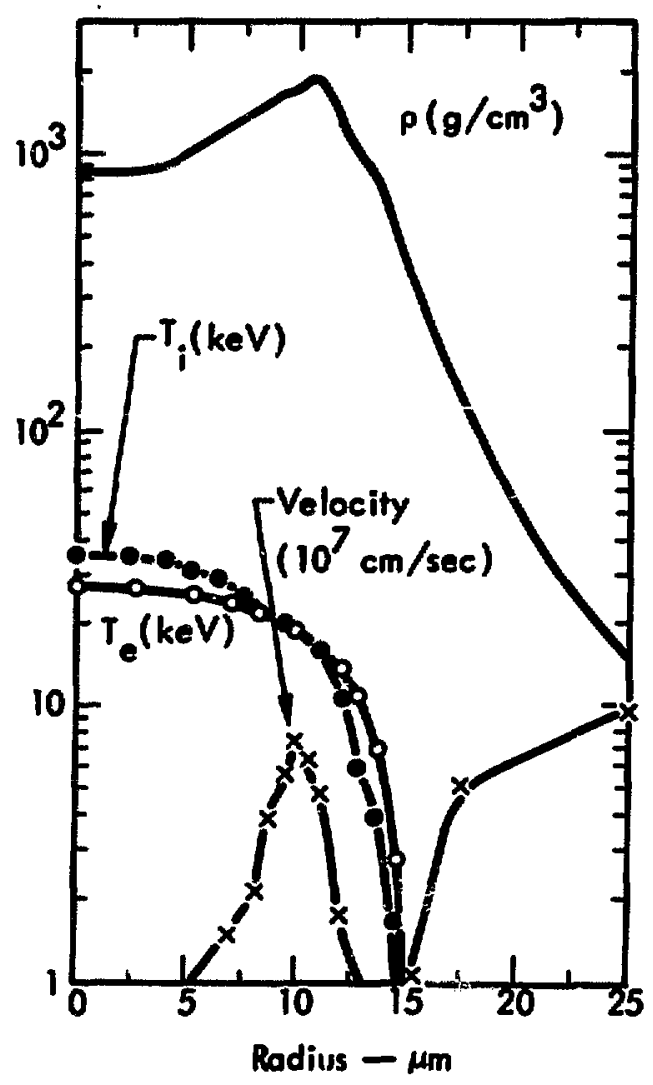

Fig. 219. Myoled strecture of the burn front thom by spatidit contoure of density, momperaturen and matise velocity. 
9 psec the central core has reached $80 \mathrm{keV}$ and the outer fuel is at $20 \mathrm{keV}$. At 12 psec the bum is $90 \%$ completed.

Figure 218 shows the propagation of the burn front outward from the central region. Ion temperature (dached lines) and density (solid lines) are plotted against radius at three times spanning 3 psec. During this time the burn front moves from $\sim 8$ to $\sim 15 \mu \mathrm{m}$, and the outer fuel is heated to $30-\mathrm{keV}$ ion temperatures.

Figure 219 shows the structure of the burn front. Matter velocity, electron temperature, icin temperature, and density are plotted against radius. There is an electron temperature precursor because at $10 \mathrm{keV}$ the DT alpha particles deposit about 75\% of their energy in electrons. However, the strong electron-ion coupling at density $1000 \mathrm{~g} / \mathrm{cm}^{3}$ heats the ions in a time short compared to the inertial time. In the central core the ion temperature exceeds the electron temperature (even though the alpha-particle heating of electrons and jons is equal at $30-40 \mathrm{keV}$ ) because the electrons are cooled by bremastrahlung and thermal conduction.
About twofold compression occurs in the burn front. The velocity profile outlines a shell of matter almost $4 \mu \mathrm{m}$ wide, $\rho R \approx 0.6 \mathrm{~g} / \mathrm{cm}^{2}$, about one alpha. particle range at the $20-\mathrm{keV}$ shell temperature. This shell is moving radially outward at a velocity of $\sim 2 \times 10^{8} \mathrm{~cm} / \mathrm{sec}$. The matter speed is $\sim 10^{8} \mathrm{~cm} / \mathrm{sec}$, and the sound speed is $1.4 \times 10^{8} \mathrm{~cm} / \mathrm{sec}$. The sum of the matter and sound speeds is approximately equal to the front speed. This is the Chapman-Jouguet detonation condition. ${ }^{230}$ However, bezause of divergence a steady-state condition is not achieved. The details shown in these figures are somewhat inaccurate because LASNEX uses a one-group flux-limited diffusion model to transport the DT alpha particles. ${ }^{229}$ Nevertheless, comparisons between these results and those with a multigroup model shorv only small differences.

In detailed computer calculations such as shown here the >1000-fold gains required for CTR applications are achieved when the pellet $\rho R$ is about $3 \mathrm{~g} / \mathrm{cm}^{2}$.

\section{A LASER-FUSION ROCKET PROPULSION SYSTEM}

Laser-initiated fusion is particularly attractive for smacecraft propulsion because the fusion energy release occurs in milliton rather than kiloton smounts, allowing a much lighter thrust chumber than ORION-like schemes. In our propulaion system a laser pulse strongly compreses a fusion fuel pellet to ignition; piasms debris from the resulting microexplosion is then magnetically redirected out the rear of the spacecraft to provide thrust. The 300-ton propulsion system shown in Fig. 220 would be capable of reaching any point of the solur system within one year with a 100-ton payload.

Vehicle mass is dominated by the mass of the heat rejection system and of the microexplosion

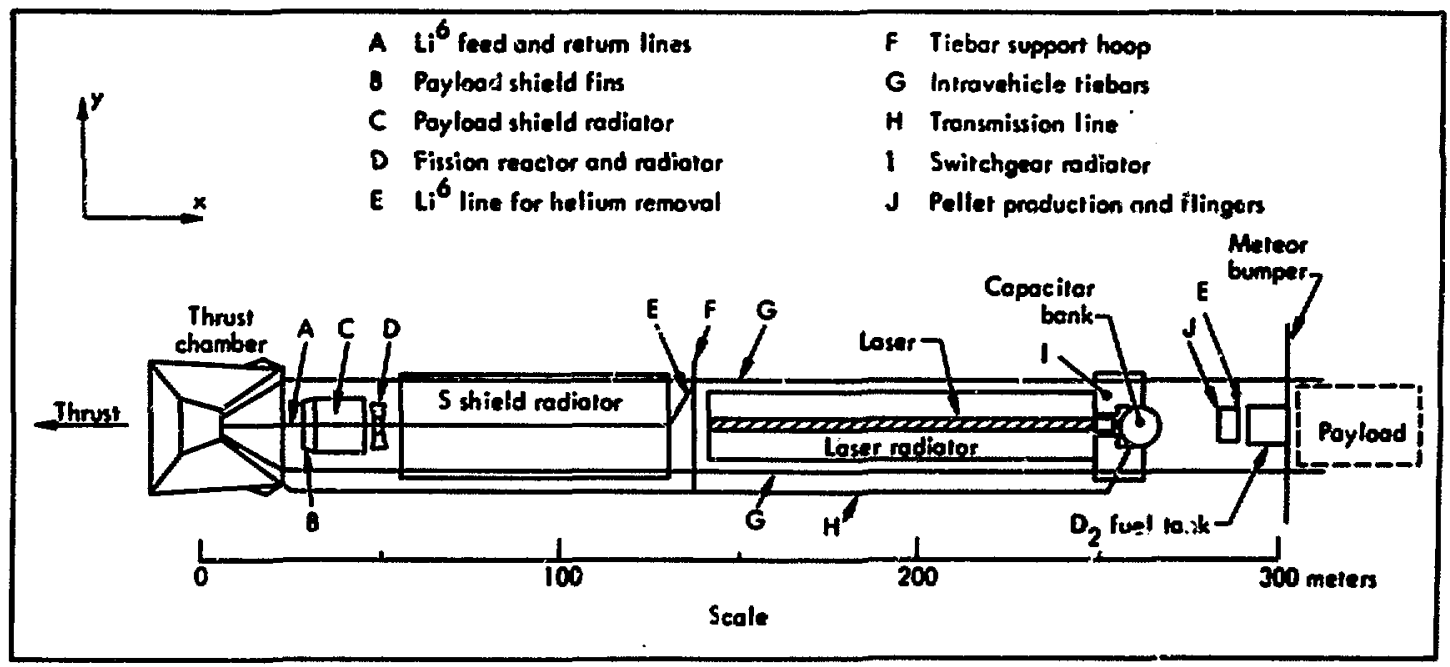

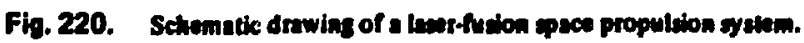


containment system. Since the latter mass is independent of the frequency of microexplosions, the specific thrust of the spacecraft is increased by exploding fuel pellets at a high frequency: 500 per second. The design of the pellets ${ }^{220}$ is dominated ky three constraints: the availability and cheapness of fuel, the need of a low ignition temperature to reduce laser mass, and release of as many ions in the microexplosion as possibie to increase specific thrust. The second constraint demands a DT fuel, while the first requires either tritium self-sufficiency for the spacecraft, or a preexisting fusion economy on earth to provide tritium. Unfortunately, the third constraint is generally in conflict with the first two due to severe radiative energy loses at high compression and the high ignition temperature of ionic product fuels. We chose to satisfy the first two constraints by using a pellet that is principally deuterium but also contains a small amount of tritium. Our chosen pellet optimizes the vehicle's specific impulse.

Three considerations dictate the design of the thrust chamber: it must magnetically redirect the plasma pellet debris to produce thrust, it must breed enough tritium for self-sufficiency, and it must emplace and explode 500 pellets per second. This thrust chamber is shown in Fig. 221. The microexplosion occurs inside an axially asymmetric magnetic mirror, generated by two current loops. ${ }^{231}$ The strongest field occurs at the small coil which turns the plasma flow, dumping it cat through the big coil to produce thrust. The magnetic-field-producing currents are large, requiring superconductors and extra structural support for the coils. The energetic neutrons and photons from the microexplosion must not be allowed to hit the superconductors; thus ${ }^{6} \mathbf{L i}$ neutron shields are placed between the coils and the microexplosion. The photons and newn. 'is that hit these shields deposit their energy, which must then be rejected from the spacecraft; therefore the solid angle subtended by the thrust chamber must be kept as low as possible. A minimum value is required, however, since it is in the ${ }^{6} \mathrm{Li}$ shield for the small magnet coil that tritium is bred from the $n^{6}{ }^{6} \mathrm{i}$ reaction. ${ }^{232}$ A radiation shicld is needed between the payload and the microexplosion to provide crew protection. X-ray spall in the walls closest to the microexplosion is a

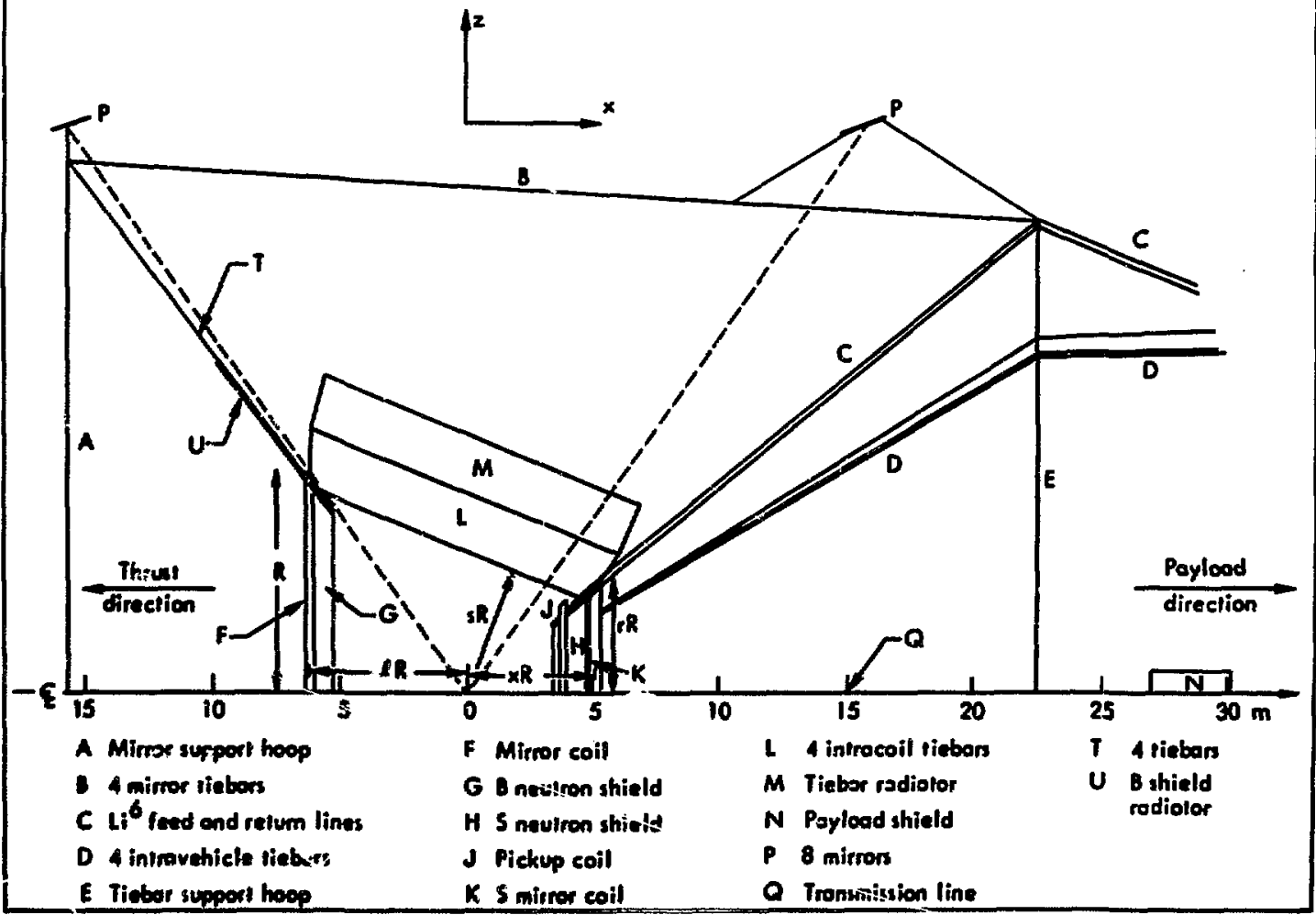

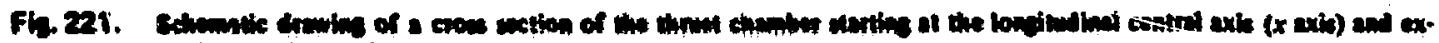

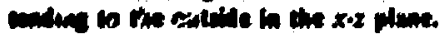


serious problem which we solve by a special carbon wall construction.

The need to emplace 500 pellets per second in the uhrust chamber limits its physical size. In our design a pellet moves $5 \mathrm{~m}$ from behind a protecting $B$-field to the microexplosion point at $2500 \mathrm{~m} / \mathrm{sec}$ where it is exploded while in motion by eight symmetrically converging laser beams. These beams originate from the same laser and are reflected into the thrust chamber by mirrors protected from microexplosion debris by the $B$-fields of the thrust chamber.

The laser used to compress and ignite the pellets differs from terrestrial CTR lasers due to the heat rejection problems in space. Like usual CTR lasers, it must have a high efficiency; but because all wasted energy must be radiatively rejected, it must also operate at a high iemperature. Thus, we use an $\mathrm{Hg}$ molecular laser operating at $1500^{\circ} \mathrm{K}$ and $3 \mathrm{~atm}$ with a $33 \%$ efficiency, producing repetitive $1 \cdot \mathrm{MJ}$ subnanosecond pulses. The laser is in the form of a long cylinder with two radiator fins extending along its length for inat removal.

The total heat rejection load of the spacecraft is large, over $2 \mathrm{GW}$, and accounts for more than 180 tons out of the 300-ton total for the vehicle. This mass is kept as low as it is by using heat-pipe radiators operating at $1500^{\circ} \mathrm{K},{ }^{233}$ depicted in Fig. 222. The fact that the laser, radiation shields, and radiators all operate at high temperatures requires extensive use of a superstrength nitrited $\mathrm{MoHf}$ alloy developed at LLL. ${ }^{234}$ The parallel operation of many independent heat pipes in each radiator provides built-in meteoroid protection while removing the need for hightemperature moving parts. Only the small magnet coil is unable to reject heat directly into a projecting heat-pipe radiator. Its heat is removed by pumping a flow of ${ }^{6} \mathrm{Li}$ through its neutron shield to an external heat-pipe radiator. This tritium-rich ${ }^{6} \mathrm{Li}$ flow is tapped and a portion is removed to the payload end of the spacecraft, where tritium is removed by diffusion through niobium windows. Pumping for the external radiator loop is performed by an MHD pump inside the small coil's neutron shield.

Power for excitation of the laser and for housekeeping needs is obtained from the microexplosion by a pickup coil wlich is exposed to the transiently expanding magnetic field in the thrust chamber. The current induced in the coil is dumped through a transmissicn line into an energy-storage capacitor bank located between the laser and payload. This capacitor bank stores sufficient energy for eight laser firings and periodically discharges into an electron beam generator which excites the laser. Auxiliary power is provided by a $1.5-\mathrm{MW}$ nuclear reactor.

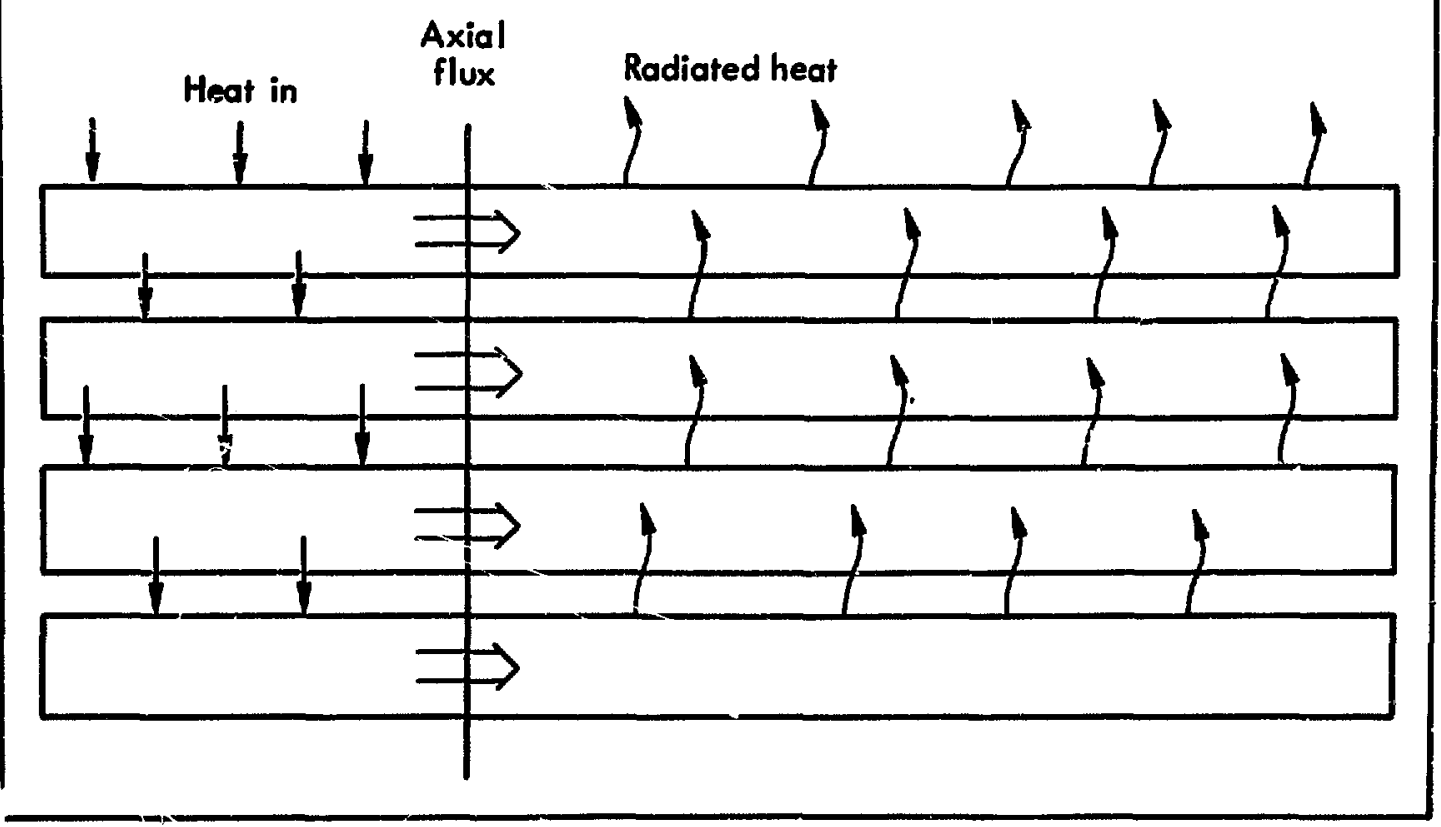

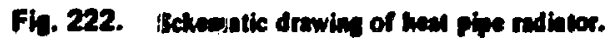




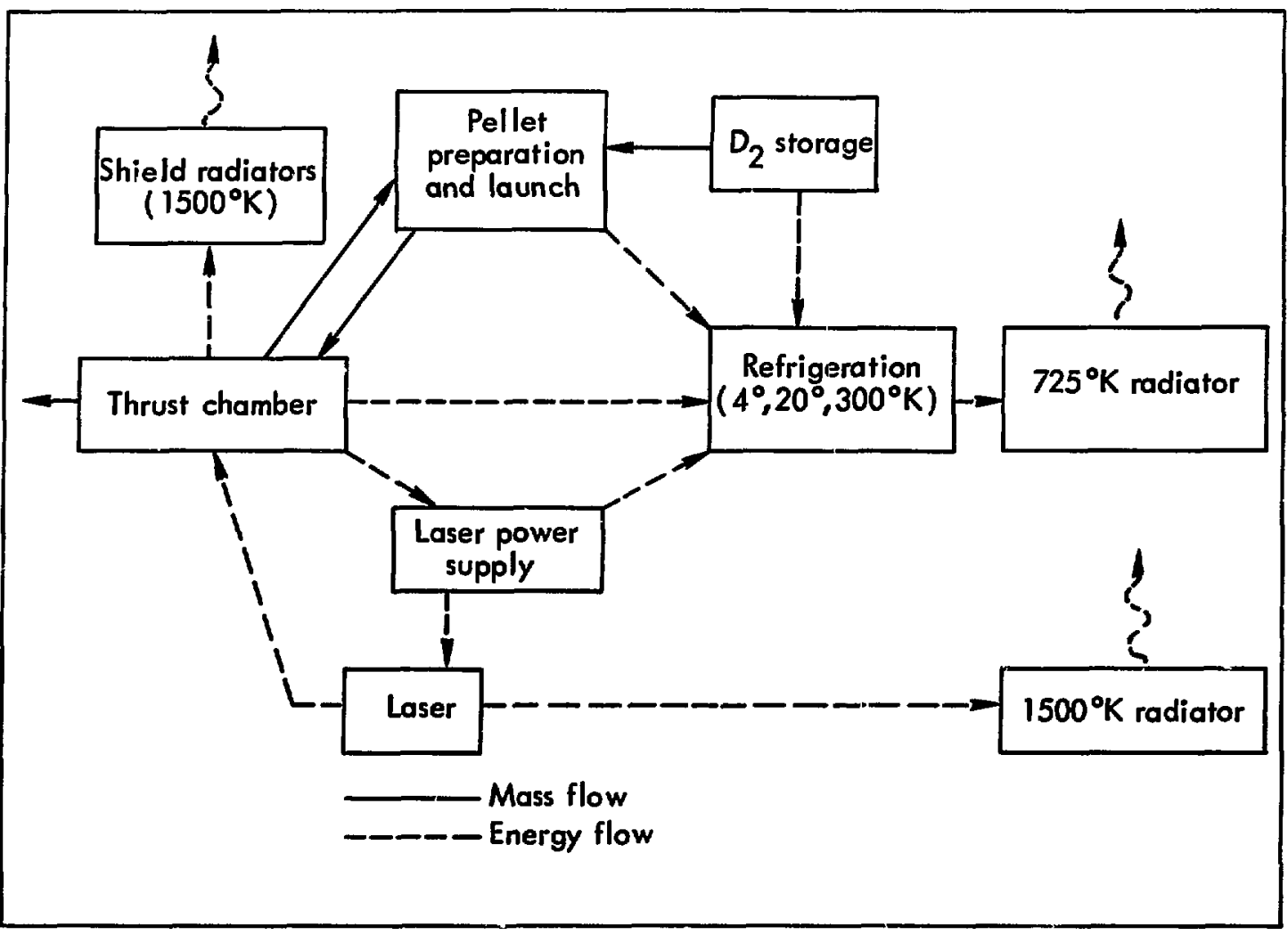

Fig. 223. Mas and enerty fow in the havifuion propulion gytem.

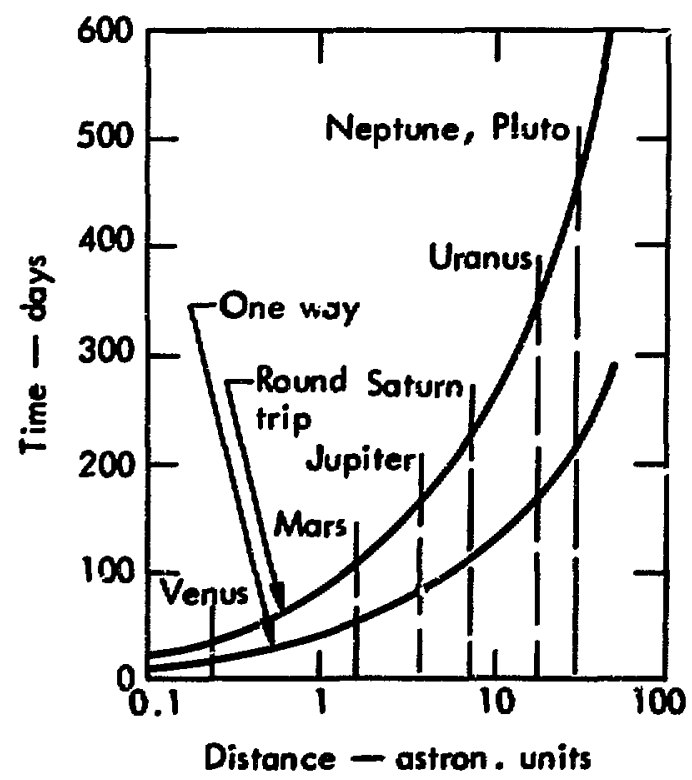

Fig. 224. Tondulued performence of the ber-fwion gropuinion ryenin with a 100 sou peylow.
Table 24. Vohich mass budopt for lawefusion rocket propulion system

\begin{tabular}{|c|c|}
\hline & Men (tom) \\
\hline 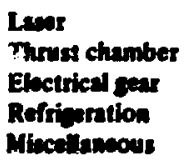 & $\begin{array}{r}84 \\
151 \\
14 \\
26 \\
25 \\
\end{array}$ \\
\hline Vealcie mas & 300 \\
\hline
\end{tabular}

Refrigeration for the superconductors, the electronic solid-atate switch gear, and the $D_{2}$ fucl tanks is provided by a heat engine durnping into a $725^{\circ} \mathrm{K}$ radiator.

All moving paris in the propulsion system are located in a crew-accestible region between the paylond and the laser. These are primarily the pellet accelerators, which are spinning diaks with $2500-\mathrm{m} / \mathrm{sec}$ 
rim speeds, constructed from an SiC-epoxy composite. This region of the spacecraft also includes the $D_{2}$ fuel tanks and a multilayer meteor bumper which provides shadow shielding for the vulnerable spacecraft components at high vehicle speeds.

The propulsion system's energy and mass flow are shown in Fig. 223, and its mass budget in Table 24. This design optimizes specific impulse at $550,000 \mathrm{sec}$ (pound-seconds of thrust per pound of fuel used), and provides a specific thrust of 7.5 milligees. Figure 224 illustrates the performance possible for this spacecraft with a 100-ton payload.

We expect that future laser-fusion rocket designs will lead to specific thrust increases of a factor of 2 or 3 with little specific impulse change, while possible specific impulse improvements will be much more modest. Nevertheless, the present laser-fusion propulsion system already offers performance superior by two orders of magnitude to previous propulsion-system designs.

\section{DIAECT CONVERSION POSSIBILITIES FOR LASER-FUSION POWER SYSTEMS}

We have examined some of the more readily apparent means for directly converting the fusion energy produced in laser-fusion 220 power plants to electricity. Such direct conversion is potentially of interest not only to reduce the waste heat engendered per unit of electrical power produced, but also because quite substantial savings in power-plant capital investment may be realized thereby. Furthermore, direct-conversion approaches admit of a potentially far fuller exploitation of the advantages fusion has over fission for electrical power generation. (See Table 25.)

In this survey we have been concerned with conversion systems which are seemingly well suited to all types of CTR systems, pulsed or quasi-cw, such as the neutron-scattering electrostatic converter, as well as conversion means best suited for pulsed-fusionenergy sources, such as ac plasma magnetohydrodynamic (MHD) means. Emphasis has been placed on conversiou systems that aptly exploit the characteristics of the exotic fuel systems, ${ }^{235}$ which output $99.9^{+} \%$ of their energy in $x$ rays and charged particles, i.e., are essentially neutron- and radionuclide-free. (See Table 26.)

\section{Pulsed-Fusion-Energy Conversion Means}

One obvious means of converting the energy produced from a pulsed-fusion-energy source (such as repeated thermonuclear microexplosions) is to expand the associated fireballs against an externally applied magnetic field. ${ }^{235-237}$ This is an especially attractive option when fusion fuels are used which do not engender significant amounts of penetrating radiation such as neutrons and gamma rays, so that the superconducting solenoid sources of magnetic flux can be located in close proximity to the fusion energy source, minimizing structural and superconductor mass and cost (Fig. 225). This is an example of how physics differences between fission and fusion may be advantageously exploited by proper choice of CTR power-plant parameters.

The fireball-compressed magnetic field may be coupled to an external electrical load in a variety of ways. It is worth noting, also, that this approach quite naturally frequency-shifts the microexplosion energy pulse from the picosecond time scales associated with fusion energy generation to the microsecond ones arising from fireball expansion to convenient (e.g., meter) scales, time scales which are much more technologically accessible.

Such means may also be employed for efficient direct conversion of the relatively large fraction of hard $x$-radiation which use of exotic fuels will inevitably entail. Pulsed injection of small quantities of intermediate- $Z$ gas (e.g., $\mathrm{Kr}, \mathrm{Xe}$ ) into the combustion

Table 25. Differencas between fission and fusion energy sources

\begin{tabular}{|c|c|c|c|c|c|c|}
\hline & \multicolumn{4}{|c|}{ Problems posed for } \\
\hline & & & & Fiscion & $\operatorname{Ean}_{j} \cdot \mathrm{CTR}$ & Advanced CTR \\
\hline 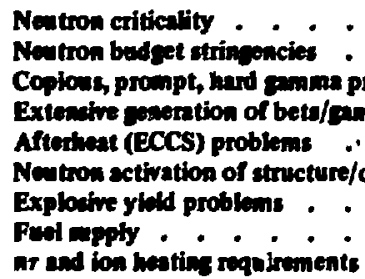 & 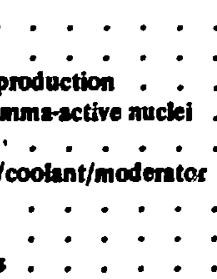 & & $:$ & $\begin{array}{l}\text { Yes } \\
\text { Yes } \\
\text { Yes } \\
\text { Yes } \\
\text { Yes } \\
\text { Yes } \\
\text { Yes } \\
\text { Yes } \\
\text { No }\end{array}$ & $\begin{array}{l}\text { No } \\
\text { Doubtful } \\
\text { Somewhat } \\
\text { Somewhat } \\
\text { Somewhat } \\
\text { Yea } \\
\text { No } \\
\text { No } \\
\text { Yes }\end{array}$ & $\begin{array}{l}\text { No } \\
\text { No } \\
\text { No } \\
\text { No } \\
\text { No } \\
\text { No } \\
\text { No } \\
\text { No } \\
\text { Yes }\end{array}$ \\
\hline
\end{tabular}




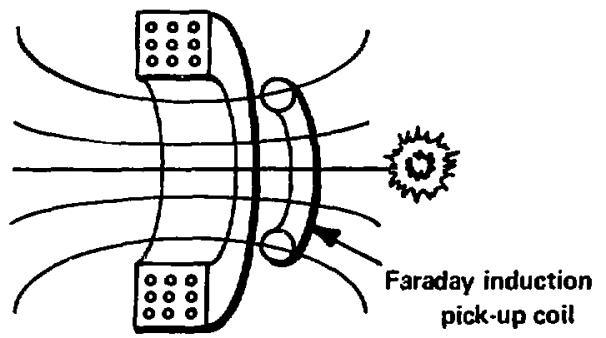

Features

- Basic feasibility demonstrated $-\geq 70 \%$ of keV fireball ingrnal energy converted to compressed $m$ ic field energy (Haught et al., 1970)

- Low magnetic field intensities suitable $-2-4$ Tesla for $10^{7}$ joule microexplosions

- $500-5000 \mathrm{kV}, \simeq 1 \mu$ sec time pulses available for direct transmission line excitation, or for transformation

DC field-generating

current system

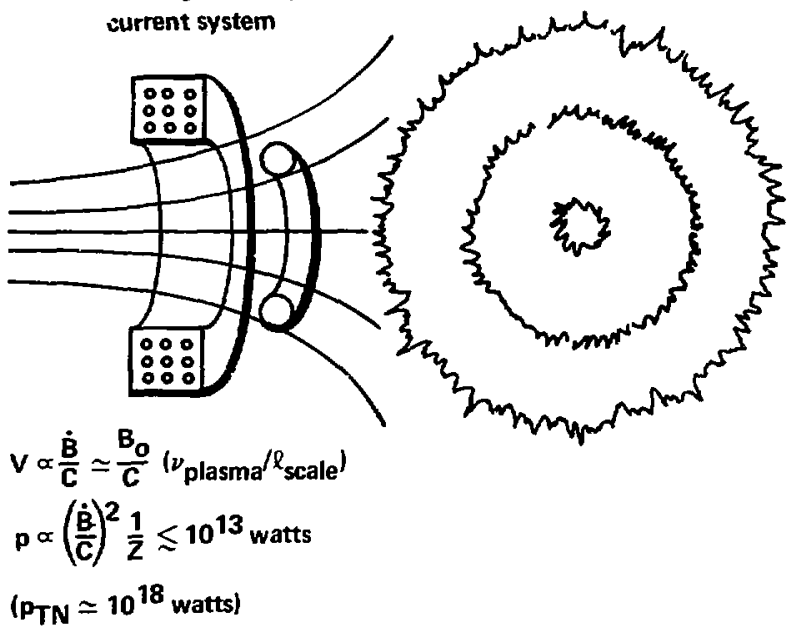

Fig. 225. Magnetohydrodynamic conversion of laser-fusion microexplosions to ac electrical power.

Table 26. Some non-heat-engine means of fusionbased electricity generation

Pulsed Systems (Laser-and Electron-Beam-Induced

Microexplosions):

MHD - fireball expansion against imposed magnetic field (Haught et al.)

MHD - exhausting (plasma + wall shielding layer) into MHD duct

CW or Pulsed Systems

MHD - exhausting plasmas (with buffer gas?) into
MHD duct
Compton generator - conversion of x-ray energy to
directed electron streams, with electrostatic
conversion
Neutronic Compton generntor - generation of
directed, high-energy proton streams by
neutron scattering, with electrostatic conversion

chamber just prior to microexplosion initiation will suffice to efficiently convert the fireball $x$-ray energy to low density plasma, which may be coexpanded with the fireball plasma itself, against the applied magnetic field. Such gas (or fog) injection may also be used to further lower the frequency (and thus also the magnitude) of the voltage pulse induced in pickup coils, by slowing fireball expansion to nearly any desired extent (e.g., one to two orders of magnitude). A sketch of one of the possible typos of laser-fusion CTR power plar: " in indicated in Fig. 226; this somewilat ancient concept, ${ }^{236}$ while employing ac MHD conversion, assumed use of neutronogenic fusion fuel.

MHD duct techniques ${ }^{238}$ may also be used to convert fireball energy directly to electricity. However, consideration of duct materials limitations will probably require the buffering of the multi-keV plasma with a much larger quantity of normal fluid before it is put into the duct. This in turn makes this approach more similar to a conventional heat engine, with its relatively high capital cost and low thermal efficiency. However, such MHD duct techniques may serva as total-efficiency-enhancing "topping" units for conventional steam-thermal converters, lowering 


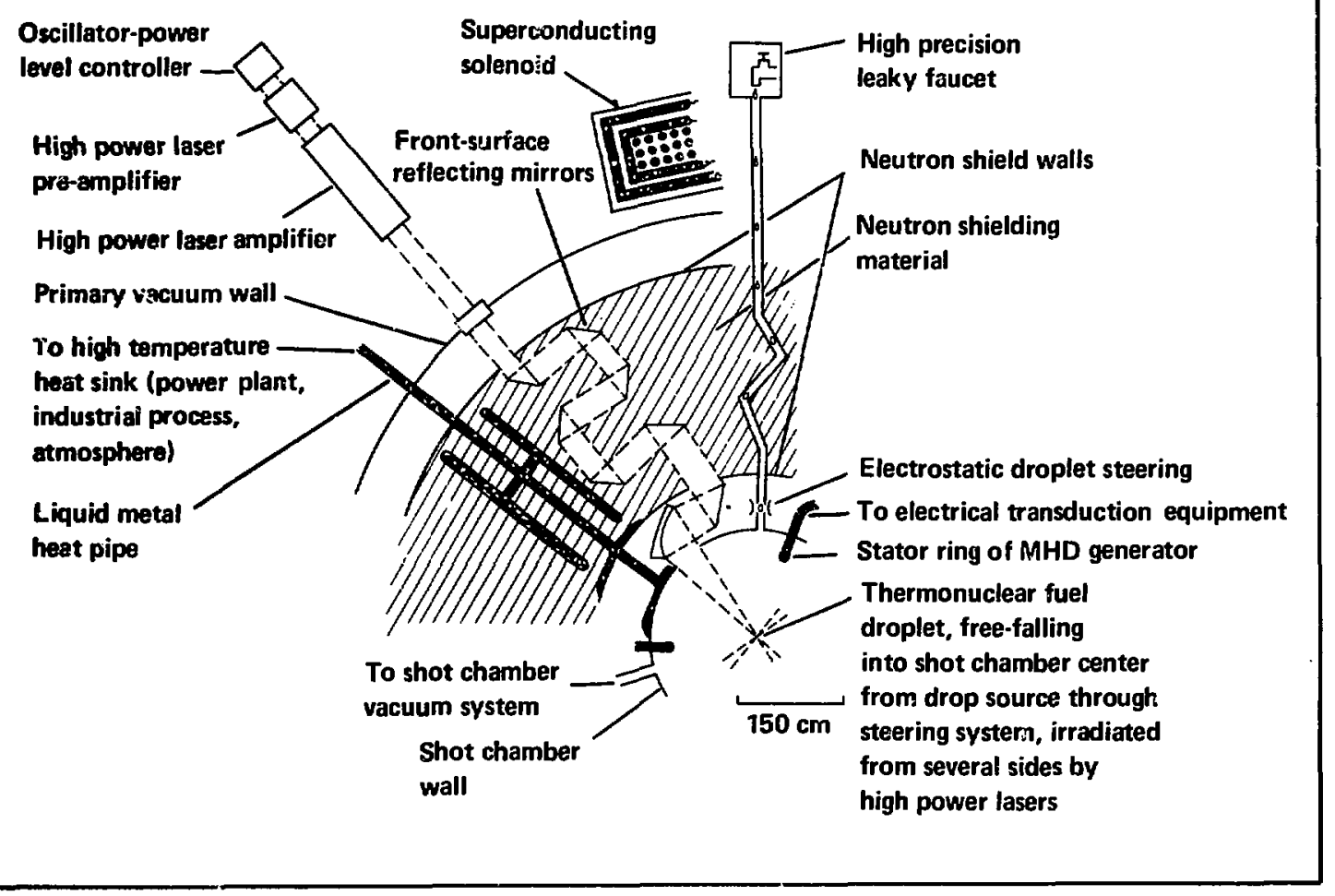

Fig. 226. Concept of a laser-fusion electrical powerplant with direct conversion.

rejected heat and capital cost per unit of power generated from fusion energy sources. The repetitive, pulsed nature of the fusion energy release may also markedly simplify the dc $\rightarrow$ ac conversion gear.

\section{Ceneral-Purpose Direct Conversion Means}

There are at least two other techniques of relatively low technological risk for directly converting fusion energy to electricity and which function about equally well for pulsed or quasi-cw systems. One of these employs the well-known Compton effect to convert a fraction of the $x$-ray energy emitted by a fusion plasma to quasi-directed (into the forward solid angle) electron beams (Fig. 227). There are two slight complications to this scheme, however - both of fairly fundamental origin. First, electrons have a nonnegligible mass, while photons do not; therefore, until photon energies become comparable to electron rest energies, it is kinematically impossibie for them to transfer large fractions of their energy to electrons. Second, hard $x$ rays have large Compton-scattering mean free paths in matter, while the electrons they scatter have much shorter ones. Therefore Compton generators have relatively little promise for converting "soft" photons (of energy $\leqslant 30-50 \mathrm{keV}$ ) to electrical energy in a technologically interesting fashion; they seemingly have relatively little interest for most DT-burning systems, in which both the electron and ion temperatures would be kept relatively low, and $\mathrm{x}$-radiation production is relatively small in any event.

However, Compton generators may be of considerable interest in exotic-fuel-buming CTR power plants, which are likely to be comparatively rich sources of hard $x$-radiation, due to the high electron and ion temperatures involved $\left(\gtrsim_{100 \mathrm{keV}}\right)$, and the large nuclear charges. In a $\mathrm{pB}^{11}$-burning system, ${ }^{*}$ for instance, early calculations indicate that a Compton-generator converter bank might be able to directly convert $\sim 30 \%$ of the emitted $x$-radiation to electrical encrgy. Such a generator would incidentally have to be a relatively complex, multilayer one, in

\footnotetext{
"One using the reaction $\mathrm{p}+\mathrm{B}^{11} \rightarrow 3 \mathrm{He}^{4}+8.68 \mathrm{MeV}$. See the article following this one.
} 
order to accommodate the greatly different electron and photon mean free paths. Such a device is indici d in Fig. 228, with the forward face section removed. This system contains the basic features needed in a $\mathrm{pB}^{11}$-buming pulsed-type CTR plant. Most of the microexplosion energy is directly converted to electricity via the ac MHD technique mentioned before; the $x$-ray fraction of it is partially converted in the surrounding Compton converter bank. $A$ foot of high density shielding outside the Compton bank reduces the external radiation level (due almost completely to $\gamma$ rays) to $\sim 10 \mathrm{mR} / \mathrm{hr}$. Present estimates indicate that such a system could produce electricity at $50-60 \%$ efficiency, with rejected heat at $\sim 500^{\circ} \mathrm{C}$.

Refer ing back to Fig. 227, a mechanism for converting multi-MeV neutron energy directly to electricity is sketched in the right-hand half of the figure. As far as direct conversion is concemed, this may be considered as a device for stripping the electron off a neutron, just as thin metal foils are used for removing electrons from atomic and ionic beams. The electron-stripped neutron - a proton - carrying on the average about half of the incident neutron's energy, is then made to do work against an imposed electric field, a now-familiar gambit.

This electrostatic generator differs from the type of Compton generator just mentioned, since multi-MeV protons have somewhat more respectable mean free paths than $\lesssim 100-\mathrm{keV}$ electrons. In particular, it appears technologically feasible to put intermediate foils between the anode and the cathode to "pick up" protons born with intermediate energies, which have done all the work possible against the imposed electrostatic field and are about to tum around and fall back into the anode layer from which they came; these foils exploit the relatively high opacity of most materials for low energy protons (see Table 27) and "pick them up" whenever their energy falls below 1.2 MeV. One thus has an automatic means of dropping the protons into the energetically correct "pocket."

Unfortunately, multi-MeV neutrons do have considerably longer mean free paths in cold matter than do multi-MeV protons, so that it is necessary to operate such a converter as a stacked bank of

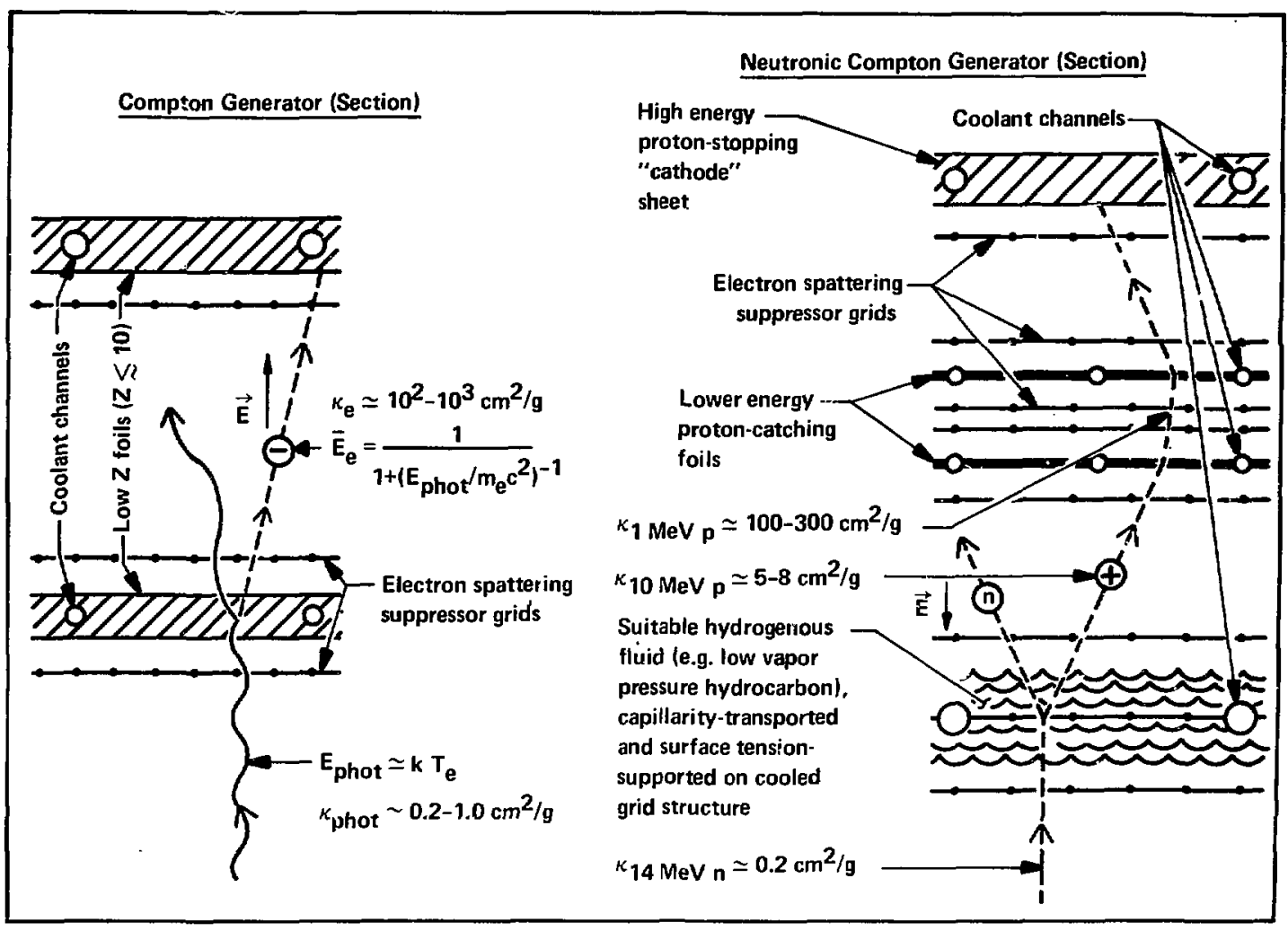

Fig. 227. Photon- and neutron-driven Compton generators. 


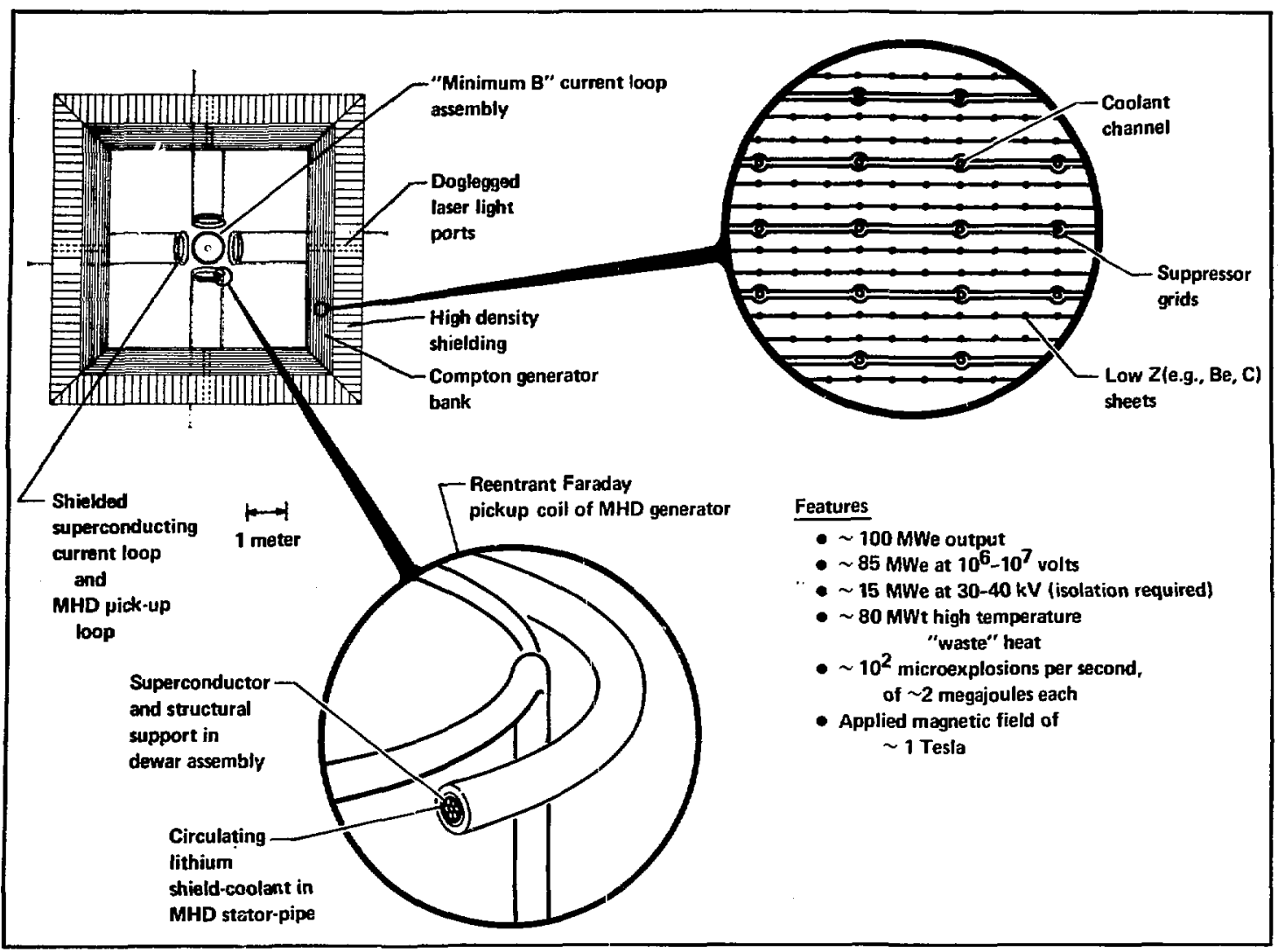

Fig. 228. Conceptual design of an MiD-converter/Compton-generator module (100-MWe) of a $\mathrm{pB}^{11}$-burning pulsed fusion powerplant.

individual sections, as in the Compton case. The conversion afficiency will be quite strongly dependent on the complexity of such a bank, increasing as the number of proton-catching foils in each section is increased, as the total thickness of each section is decreased, and as the number of sections in the bank is increased. Ultimate overall conversion efficiencies of $80-90 \%$ presently appear attainable.

Taken into account in estimating such efficiencies are the losses associated with removing the $\$ 100 \mathrm{~mW} / \mathrm{cm}^{2}$ from the layers of each section via grids of small coolant tubes. If neutronic Compton generator sections are operated with their layers largely parallel to an imposed magnetic field (as might be the case for plasma-energy direct conversion systems), total bank thicknesses of the order of $1 \mathrm{~m}$ might be feasible, due to the possibility of exploiting the "magnetic insulation" effect. 240 In the absence of external magnetic fields, severalfold greater thicknesses would be required by vacuum dielectric strength considerations.
Some of the salient features of such converters are noted in Table 28. A sketch of such a system in cross section is shown in Fig. 229.

\section{Conclusions}

One of the technologically most relevant differences between fission and fusion for electrical energy production is the very real possibility of efficiently and cheaply converting fusion energy directly to electricity. This is a particularly "live" option when the fusion energy is produced essentially free of gamma rays, neutrons, and radionuclides - when the fusion power reactor may be reasonably viewed simply as a source of billion-degree plasma, with virtually no radioactive "dirt" included. This seems to us to be a most valid reason for a serious second look at relatively exotic CTR fuels, which promise to deliver this type of performance.

This has been the basic rationale for the present general examination of low-technological-risk 
Table 27. Range and stopping-power tables for havy ions. (From L. C. Northeliffe and

A. F. Schilling.239)

iH roNs

\begin{tabular}{|c|c|c|c|c|c|c|c|c|c|c|c|c|c|}
\hline $\begin{array}{l}\text { DEA } \\
\text { SS un }\end{array}$ & & & & & manc & E & or . & $150 \mathrm{ch}$ & & & & & $\lim _{i=1}$ \\
\hline nEvramu & ot & c & al & II & MI & сE & $2 a$ & a6 & cu & IA & $\omega$ & $v$ & Av \\
\hline $\begin{array}{l}0.0125 \\
0.0160 \\
0.0200 \\
0.0250 \\
0.0320 \\
0.0460\end{array}$ & $\begin{array}{l}0.058 \\
0.060 \\
0.078 \\
0.089 \\
0.102 \\
0.116\end{array}$ & $\begin{array}{l}0.070 \\
0.002 \\
0.093 \\
0.106 \\
0.122 \\
0.130\end{array}$ & $\begin{array}{l}0.102 \\
0.111 \\
0.134 \\
0.151 \\
0.113 \\
0.196\end{array}$ & $\begin{array}{l}0.150 \\
0.190 \\
0.205 \\
0.231 \\
0.2364 \\
0.200\end{array}$ & $\begin{array}{l}0.1103 \\
8.222 \\
0.251 \\
0.2213 \\
0.323 \\
0.363\end{array}$ & $\begin{array}{l}0.216 \\
0.224 \\
8.279 \\
0.315 \\
0.3160 \\
0.406\end{array}$ & $\begin{array}{l}0.231 \\
0205 \\
0.300 \\
0.310 \\
0.316 \\
0.436\end{array}$ & $\begin{array}{l}0.240 \\
0.243 \\
0.320 \\
0.324 \\
0.014 \\
8.660\end{array}$ & $\begin{array}{l}8.913 \\
0.430 \\
0.404 \\
0.554 \\
0.635 \\
0.118\end{array}$ & 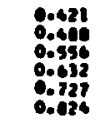 & 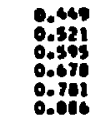 & 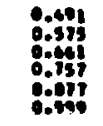 & $\begin{array}{l}0.0116 \\
0.0184 \\
0.0162 \\
60.0152 \\
0.0112 \\
0.0405\end{array}$ \\
\hline $\begin{array}{l}0.0500 \\
0.0600 \\
0.0700 \\
0.0800 \\
0.0900 \\
0.1000\end{array}$ & $\begin{array}{l}0.133 \\
0.167 \\
0.141 \\
0.175 \\
0.190 \\
0.204\end{array}$ & $\begin{array}{l}0.151 \\
0.175 \\
0.193 \\
0.209 \\
0.224 \\
0.202\end{array}$ & $\begin{array}{l}0.222 \\
0.246 \\
8.270 \\
0.293 \\
8.316 \\
0.330\end{array}$ & $\begin{array}{l}0.337 \\
0.336 \\
0.409 \\
0.443 \\
8.446 \\
0.916\end{array}$ & $\begin{array}{l}0.413 \\
0.455 \\
0.400 \\
0.941 \\
0.542 \\
0.022\end{array}$ & $\begin{array}{l}0.458 \\
0.358 \\
0.356 \\
0.060 \\
0.664 \\
0.060\end{array}$ & 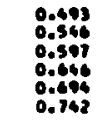 & $\begin{array}{l}0.528 \\
0.586 \\
0.841 \\
8.844 \\
8.940 \\
0.791\end{array}$ & $\begin{array}{l}0.013 \\
0.805 \\
0.800 \\
1.072 \\
1.152 \\
1.230\end{array}$ & 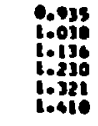 & 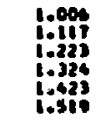 & 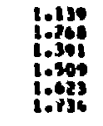 & $\begin{array}{l}6.0 \\
0.1\end{array}$ \\
\hline $\begin{array}{l}0.1230 \\
0.1800 \\
0.22000 \\
0.2560 \\
0.3200 \\
0.4600\end{array}$ & $\begin{array}{l}0.240 \\
0.293 \\
0.357 \\
0.444 \\
0.980 \\
0.756\end{array}$ & $\begin{array}{l}0.201 \\
0.337 \\
0.402 \\
8.406 \\
0.614 \\
0.174\end{array}$ & $\begin{array}{l}0.397 \\
80.460 \\
8.510 \\
8.814 \\
8.916 \\
1.179\end{array}$ & $\begin{array}{l}0.512 \\
80.909 \\
0.940 \\
1.031 \\
1.306 \\
1.049\end{array}$ & $\begin{array}{l}0.123 \\
00.86 \\
0.023 \\
1.023 \\
1.241 \\
1.364 \\
1.066\end{array}$ & $\begin{array}{l}0.198 \\
0.092 \\
1: 133 \\
1,171 \\
1.126 \\
2.146\end{array}$ & 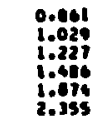 & $\begin{array}{l}0.024 \\
1.103 \\
1.316 \\
10501 \\
2.004 \\
2.320\end{array}$ & 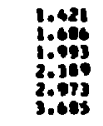 & 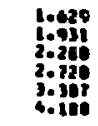 & 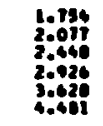 & 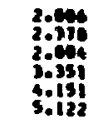 & \\
\hline $\begin{array}{l}0.5000 \\
0.6000 \\
0.7000 \\
0.0000 \\
0.9000 \\
1.0000\end{array}$ & $\begin{array}{l}1.001 \\
1.293 \\
1.615 \\
1.911 \\
2.359 \\
2.790\end{array}$ & $\begin{array}{l}0.999 \\
11.253 \\
1.539 \\
1.057 \\
2.207 \\
2.589\end{array}$ & $\begin{array}{l}1.542 \\
1.967 \\
2.994 \\
2.041 \\
3.605 \\
3.961\end{array}$ & $\begin{array}{l}2.128 \\
2.042 \\
3.210 \\
3.024 \\
6.910 \\
3.117\end{array}$ & $\begin{array}{l}2.511 \\
3.110 \\
3.140 \\
6.401 \\
5.207 \\
5.971\end{array}$ & $\begin{array}{l}2.161 \\
3.112 \\
6: 119 \\
9.9717 \\
9.0682 \\
6.533\end{array}$ & $\begin{array}{l}3.010 \\
3.127 \\
3.306 \\
3.344 \\
6.233 \\
1.116\end{array}$ & 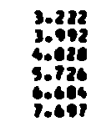 & $\begin{array}{r}4.042 \\
3.670 \\
6.769 \\
7.576 \\
9.810 \\
10.540\end{array}$ & $\begin{array}{r}3.262 \\
8.421 \\
7.466 \\
10.710 \\
11.040 \\
11.040\end{array}$ & $\begin{array}{l}3.026 \\
0.455 \\
0.116 \\
11.911 \\
11.054 \\
12.002\end{array}$ & 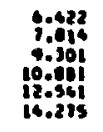 & $\begin{array}{l}0.1035 \\
8.0062 \\
0.707 \\
1.00076\end{array}$ \\
\hline $\begin{array}{l}1.2500 \\
1.6000 \\
2.0000 \\
2.5000 \\
3.2000 \\
4.0000\end{array}$ & $\begin{array}{r}3.967 \\
5.966 \\
8.643 \\
12.061 \\
19.456 \\
20.027\end{array}$ & $\begin{array}{r}3.453 \\
5.955 \\
0.112 \\
11.975 \\
10.433 \\
27.200\end{array}$ & $\begin{array}{r}5.524 \\
6.056 \\
11.435 \\
16.361 \\
26.510 \\
35.560\end{array}$ & $\begin{array}{l}7.040 \\
10.140 \\
16.145 \\
19.0918 \\
29.323 \\
62.006\end{array}$ & $\begin{array}{l}0.153 \\
81.536 \\
26.060 \\
22.471 \\
32.067 \\
46.704\end{array}$ & $\begin{array}{l}11.051 \\
18.524 \\
11.317 \\
26.131 \\
33.196 \\
99.1091\end{array}$ & 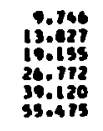 & $\begin{array}{l}10.461 \\
14.040 \\
20.629 \\
20.843 \\
49.169 \\
39.875\end{array}$ & $\begin{array}{l}14.095 \\
10.045 \\
20.706 \\
30.005 \\
32.946 \\
74.010\end{array}$ & 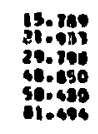 & 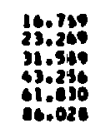 & 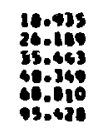 & 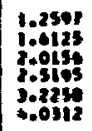 \\
\hline $\begin{array}{r}5.0000 \\
6.0000 \\
8.0000 \\
8.0000 \\
9.00000 \\
10.0000\end{array}$ & $\begin{array}{r}42.046 \\
59.307 \\
10.123 \\
99.236 \\
122.542 \\
167.989\end{array}$ & 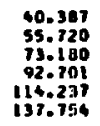 & $\begin{array}{r}31.053 \\
10.767 \\
70.149 \\
216.1962 \\
162.212 \\
116.2195 \\
110.195\end{array}$ & 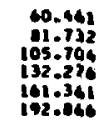 & 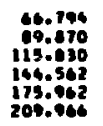 & $\begin{array}{l}71.210 \\
1752.09 \\
122.961 \\
133.169 \\
100.165 \\
221.170\end{array}$ & 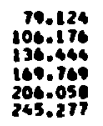 & 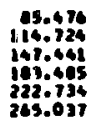 & 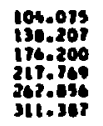 & 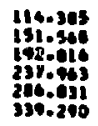 & $\begin{array}{l}120.447 \\
150.212 \\
2007.100 \\
260.315 \\
300.174 \\
3540.06\end{array}$ & 4 & 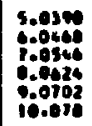 \\
\hline & $25+152$ & 1 & 63 & 2 & 0.406 & $\begin{array}{l}200.104 \\
300.975\end{array}$ & $313: 362$ & 14 & & & & & 12. \\
\hline
\end{tabular}

approaches for converting the various forms of pulsed-fusion-reactor energy output directly into electricity. It may well be that this review has overlooked some of the best approaches, and it is virtually certain that much better approaches will be forthcoming, as more people begin to think about these problems. It is certainly hoped that the present study will help to stimulate such work.

It would seem that fusion energy technologists should attempt to emulate the fabled butcher, who is reported to have so rendered and processed his pigs that profitable use was found for everything except the squeal. Figure 230 indicates how we see the fusion power pig being partitioned, for both early and more advanced pulsed CTR reactors. The electrical energy prcsuction advantages of early CTR over fission, and of advanced CTR over early fusion systems, are obvious.
Table 28. Advantegss and disedvanteres of direct convension of multiviny neutron energy to electricity

Potentially hith (5080\%) efficiency of comenion of 14. MeV neutron enery to elocticity

Ameñble to both poliad and ew sentron eanres converion

Requires relatively lure intrunieid volume (100-300 converter byen, hith electric ficids, eren when loyess are connected in parillel

Efriciency strondy complexity depondent

Cepable of operation in hiph megetic fietds (s 10T); "mapnetic insulation" exploitution

Substantially lower titiumbreding capebility

Probsbly les cepital-fintenatve than stean-thened sowrerton - bwet power cod

Potentially less rejected heat - kwer thems polintion

Subject to experimental evaluation in the near term 


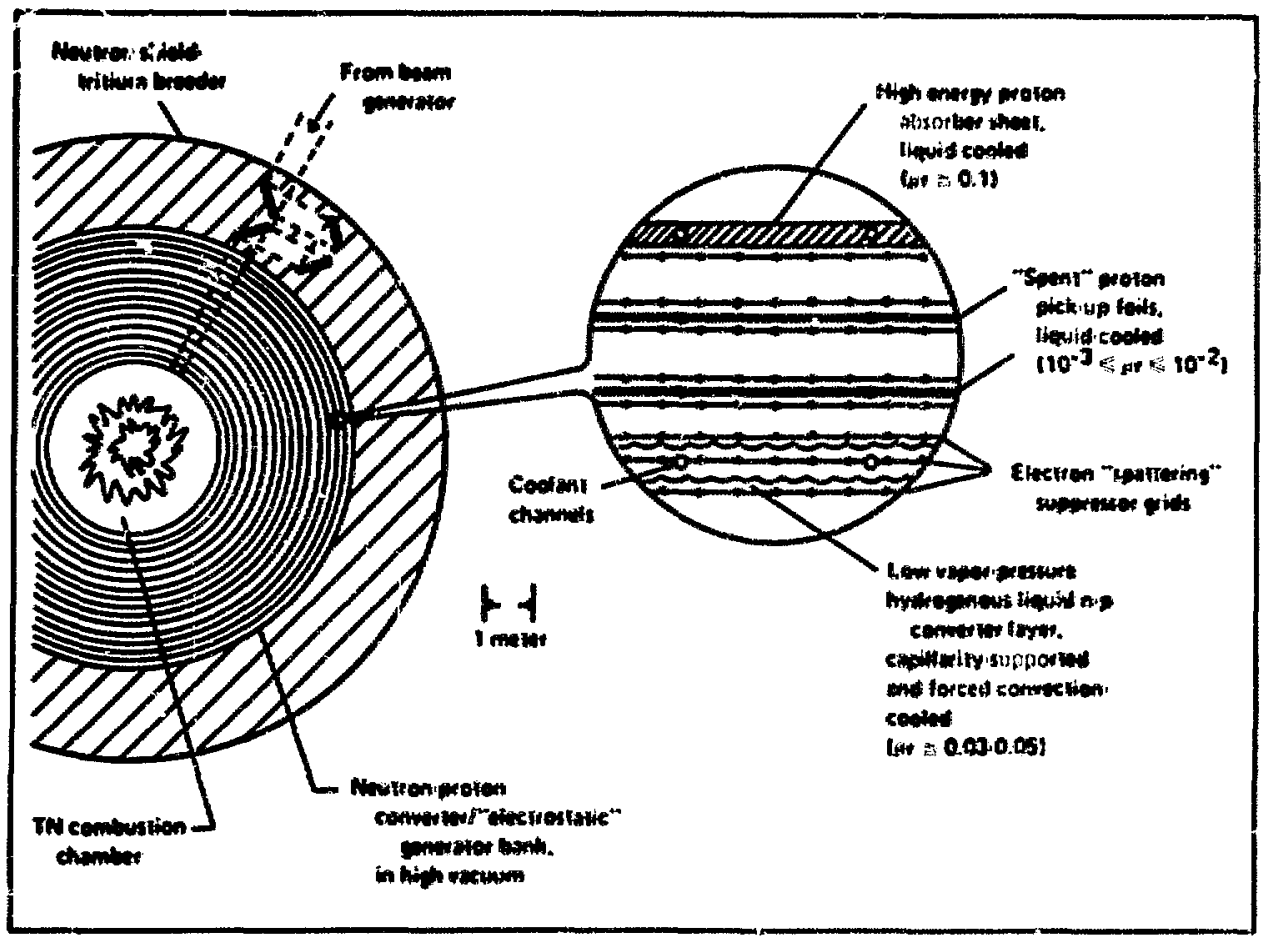

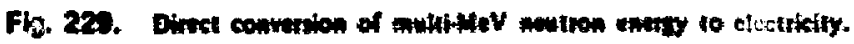

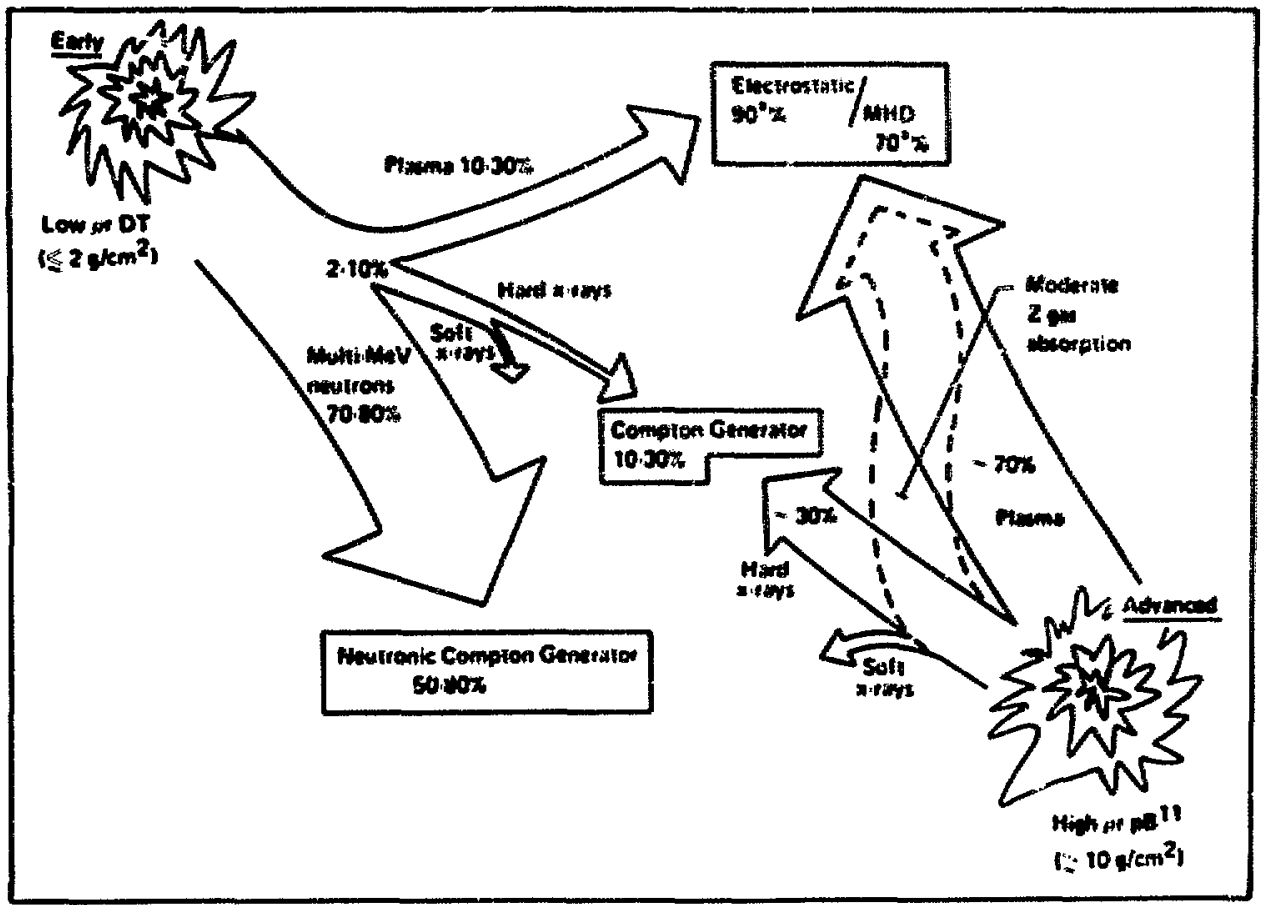

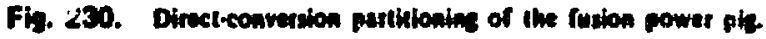


it the design of controlled-fusion resctor systems, virtually the only fuels considered in the past have been DT, DD, no DHe?." All three of these "standard" fuels suffer from significant shortcomings related primarily to their copious direct or indirect production of neutross, and secondiarily, in the case of DT and DHe ${ }^{3}$, to the necessity to breed fuel components. The attendent problems of reactor wall deterioration, of neutron-ectivated radiosctive waste disposs, and of afficiently converting neutron-bome fusion enery ( $-80 x$ for DT) to electricity have proved quite challenging and are presently being vigorously attucked. 241.243

An alternate approsch to these problems, however, is to seek "exotic" fusion-fuel systems that would not be subject to such difficulties. Ideally, such a fuel system would involw: no neutron- or adionuclide-producing reactions, would have cheap and incxhaustibly wailable reactants, would produce most of its energy as charsect panticles, and would have a sufficiently high energy-generation rate to be usable in projected laser fusion or other CTR systems. We have searched the light elements for such reactions 244 and have found one new and apparently very promising candidate in the reaction $\mathrm{p}+\mathrm{Bli}^{\mathrm{II}} \rightarrow 3 \mathrm{He}^{4}$ $+8.68 \mathrm{MeV}$ (denoted below as $\mathrm{pB}^{\prime \prime}$ ) that satisfies substantially all of the above criteria. Other possibly interesting fuel combinations include $\mathrm{pLi}^{6}, \mathrm{DLi}^{6}$, pise $^{9}, \mathrm{DBe}^{9}, \mathrm{pB}^{10}$, and $\mathrm{pN}^{15}$, though these suffer from refatively low reaction rates and in some cases substantid neutron and radionuclide production.

In the remainder of this article we describe in some detail the important charscteristics of the $\mathrm{pB}^{11}$ fuel system.

\section{Clea Bum}

The most salient feature of the $\mathrm{pB}^{11}$ fuel system is that $\mathbf{9 9 . 9 \%}$ of its reaction products are "citan" nonradioactive helium nuclei, with the remainder composed of $\leq 0.1 \%$ neutrons and $\leq 0.01 \%$ radioactive particles. The small residual contamination is caused by $(\alpha, n)$ and $(\alpha, p)$ reactions on $B^{11}$ that occur while the fusic: produced alpha particles (with energy wi-4 MeV) are slowing down in the buming plasma, and also by direct thermal $(p, y)$ and $(p, n)$ reactions. The characteristics of these side reactions, including their rales and contribstions to reactor radioisotope inventories, are detailed in Table 29 . The radiojsotope inventories are seen to be several orders of magnitude

\footnotetext{
"Edilor's nole: In this diccussion the authors have und the forms $\mathrm{He}^{3}, \mathrm{~B}^{1 / 2}$, etc. inatead of ${ }^{\prime} \mathrm{Hc},{ }^{\prime} \mathrm{B}$, elc. Sor notational convenience.
}

lower for $\mathrm{pB}^{\mathrm{J} 1}$ than those for fission and DT fusion reactors. 241

\section{Cost and Abundance}

Boron 11 comprises $80.22 \%$ of natural boron, and it is more abundant and cheaper to recover than either deuterium or $\mathrm{L}^{6}$. Table 30 detaiis the mean crustal abundances and costs of the various fusion fuels.

\section{Resction Rate and Imition Conditions}

Figure 231 shows the density-normalized thermonuclear reaction rates, ${ }^{245}\langle 0 v\rangle$, for $\mathrm{pB}^{11}$ and the standard fucls. The lower three $\mathrm{pB}^{11}$ curves show the $\pm 50 \%$ range of cross-section uncertainty, while the upper $\mathrm{pB}^{11}$ curve shows the possible amount of reaction rate augmentation due to the nonthemal effects discussed below. An analogous family of curves is employed in later figures to exhibit the results of these reaction rate uncertainties.

Figure 232 shows the electrical break-even Lawson numbers, $n_{T} \tau$, for $\mathrm{pB}^{11}$ and the standard fuels. (Here the total number of particles in the plasma, $n_{T}$. replaces the jon density, $n$, in calculating $n \tau$, to allow for the growing importance of the electron specific heat for fuels with $Z>1$.) The major implication of

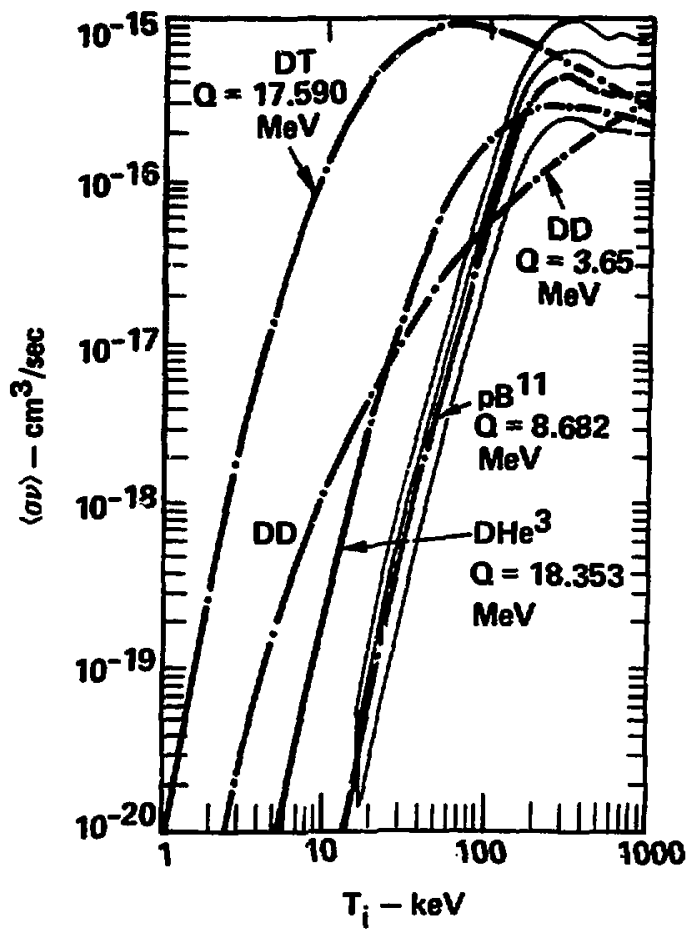

Fig. 231. Themally everaged nuclear resction ntes, $\langle 0 v\rangle$, for various fusion fuel. 
Thble 20. Side reactions for the $\mathrm{pB}^{11}$ fud wytam

\begin{tabular}{|c|c|c|c|c|}
\hline Reaction & $\frac{0}{(1 \mathrm{nV})}$ & 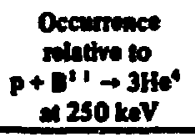 & 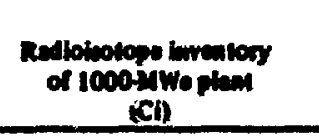 & andese \\
\hline$p+B^{\prime \prime}+C^{12}+\gamma$ & 16.0 & $5 \times 10^{-1}$ & 200 (axcenty & 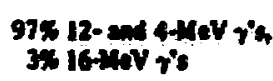 \\
\hline$p+D^{1} \rightarrow A+C^{21}$ & -28 & $15 \times 10^{-1}$ & $4: 10^{\circ}$ (andy stan) & Tiesmal neproam \\
\hline$a+B^{11} \rightarrow M+N^{14}$ & 0.2 & $510^{-3}$ & 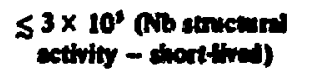 & Nontheral remation, \\
\hline$a+B^{11} \rightarrow p+C^{14}$ & 0.8 & $510^{-0}$ & $510^{\circ}$ (anund production) & $1, f\left(C^{14}\right)=\operatorname{cosen} r$ \\
\hline Dr fuslon reactor & - & - & $\sim 10^{\circ} \cdot 10^{\circ}$ (1):-ly atas) & \\
\hline Finion revettor & - & - & - $10^{10}$ (0.0idy stete) & \\
\hline
\end{tabular}

O'1 from activation of water shisiding $\left(c_{1 / 2}=2 \mathrm{~min}\right)$.

Teble 30. Fusion ful mesurces in the carth's cruse

\begin{tabular}{|c|c|c|}
\hline Reactunt & $\begin{array}{l}\text { Purt by } \\
\text { woingt of } \\
\text { carth's crust } \\
\text { (Ppa) }\end{array}$ & $\begin{array}{l}\text { Estimeted } \\
\text { mcovery or } \\
\text { production cost }\end{array}$ \\
\hline $\begin{array}{l}\text { B" }^{\prime \prime} \\
\mathrm{LH} \\
\mathrm{D} \\
\mathrm{He}^{3} / \mathrm{H}^{\prime}\end{array}$ & $\begin{array}{l}8 \\
4 \\
05 \\
\text { Neylioble (projected } \\
\text { production from D } \\
\text { and L5) }\end{array}$ & $\begin{array}{c}10-20 \mathrm{~d} / \mathrm{s} \\
20 \mathrm{~d} / \mathrm{s} \\
20-30 \mathrm{~d} / \mathrm{s} \\
\$ 10,000 / \mathrm{b} \\
(81 / \mathrm{g}(7)-\mathrm{CTR} \\
000 \mathrm{momg})\end{array}$ \\
\hline
\end{tabular}

Avallable technolosy applied to large scale (CTR) production.

bresent AEC official price.

these two figures is that if reactor ion temperatures of $150-250 \mathrm{keV}$ can be achieved, then $\mathrm{pB}^{11}$ can be bumed with the same order of difficulty (or ease) as DD and $\mathrm{DHe}^{3}$.

Figures 233 and 234 plot the density-normalized thermonuclear energy generation rates $\left(E_{T N} / n_{T}^{2}\right)$ and relativistically correct bremsstrahlung energy loss rates ${ }^{246}\left(E_{B r e m s} / n_{T}^{2}\right)$ for various ratios of the electron to the ion temperature, for $\mathrm{pB}^{11}, \mathrm{pLi}^{6}, \mathrm{DLi}^{6}, \mathrm{pBe}^{9}$. and the standard fuels. These graphs directly indicate the conditions under which the idealized ignition criterion for optically thin systems $\left(\tilde{E}_{T N}>E_{B r e m s}\right)$ is met. The indicated relative proportions of the fuel constituents have been chosen to maximize $\hat{E}_{T N}$ - E Erems. Also plotted in Figs. 233 and 234 are the bremsstrahlung loss rates for the steady-state case (believed typical of conditions in most feasible CTR reactors), where the heating of the electrons by the

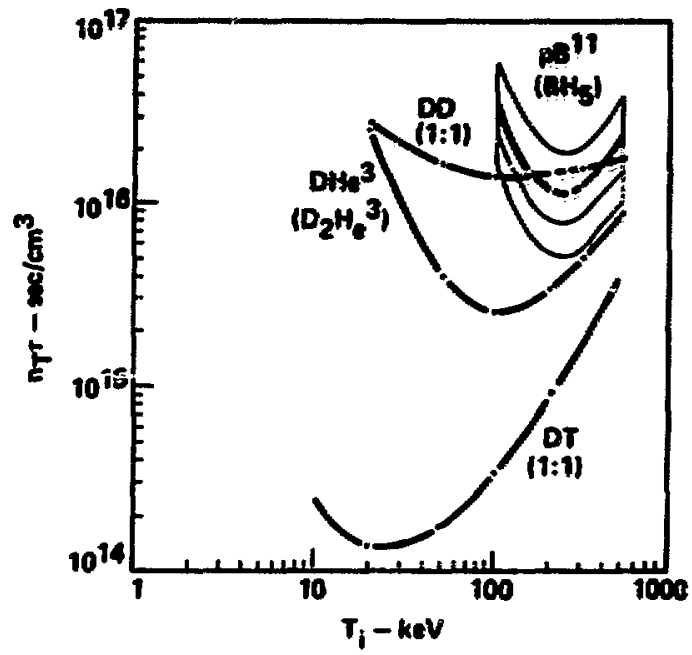

Fig. 292 Required Loweon sumbars $(n, f)$ for cocticel

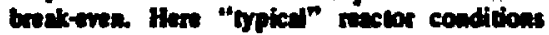

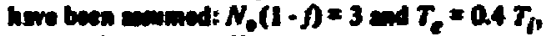

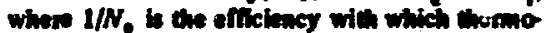

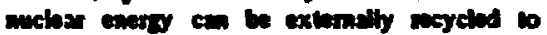
heat the plema derite the mat confimmenth and $f$ is the frection of hatond enesy proviled by themomclece selfheation.

fusion-heated ions is just balanced by their cooling by bremsstrahlung. The $\ln \Lambda=5$ and $\ln \Lambda=20$ cases are typical of fusion-microexplosion and mirrormachine systems, respectively, where In $\boldsymbol{\Lambda}$ is a density-dependent factor proportional to the dectron-ion coupling. It is seen under these conditions that DT can be readily igaited, $\mathrm{DHe}^{3}$ moderately well ignited, DD marginally ignited, and $\mathrm{pBe}^{9}, \mathrm{pLi}^{6}$, and 


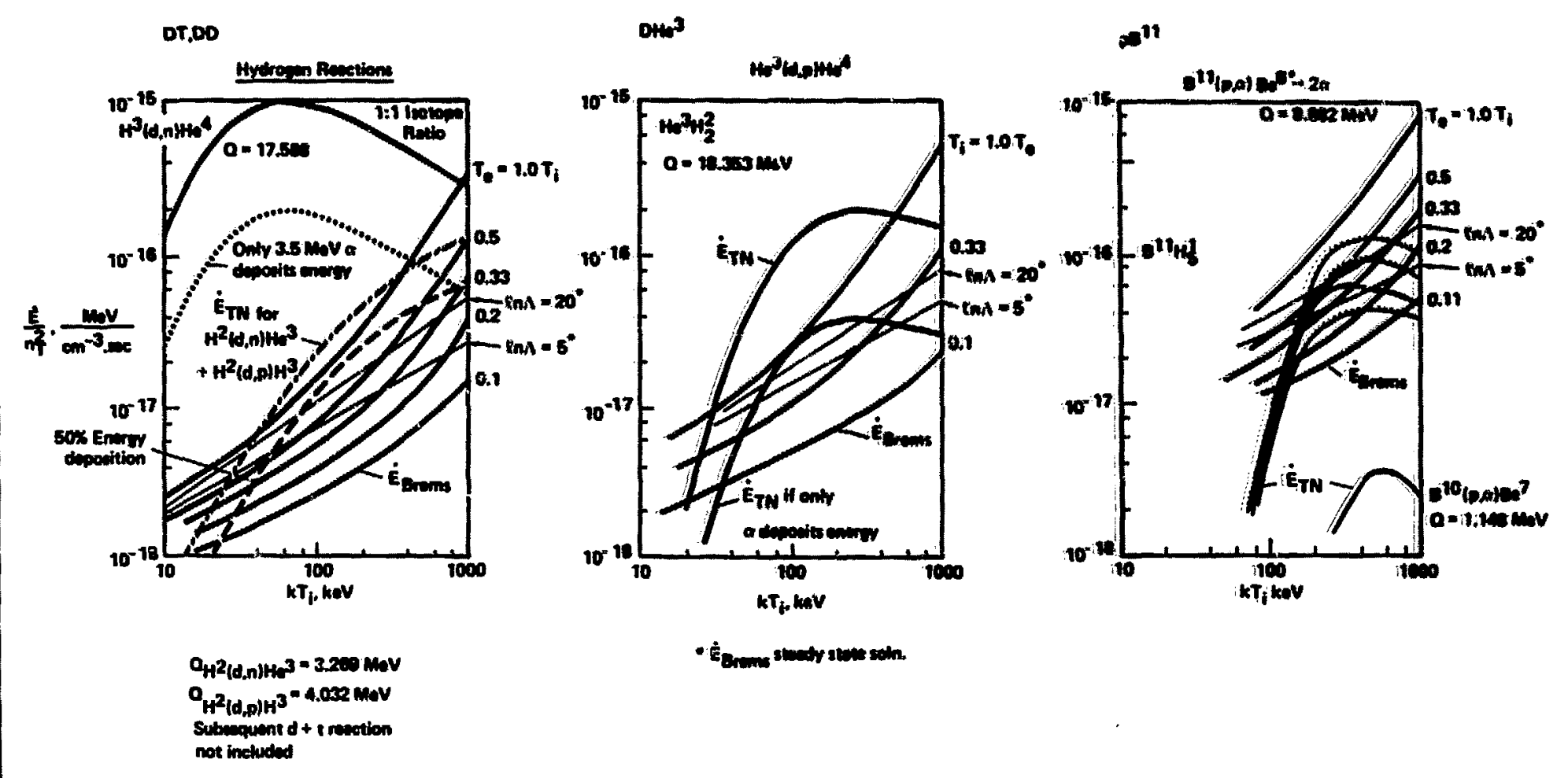

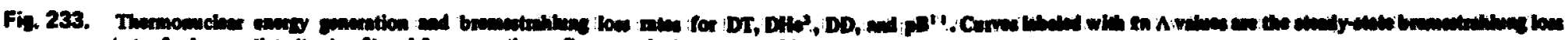

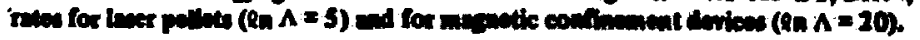




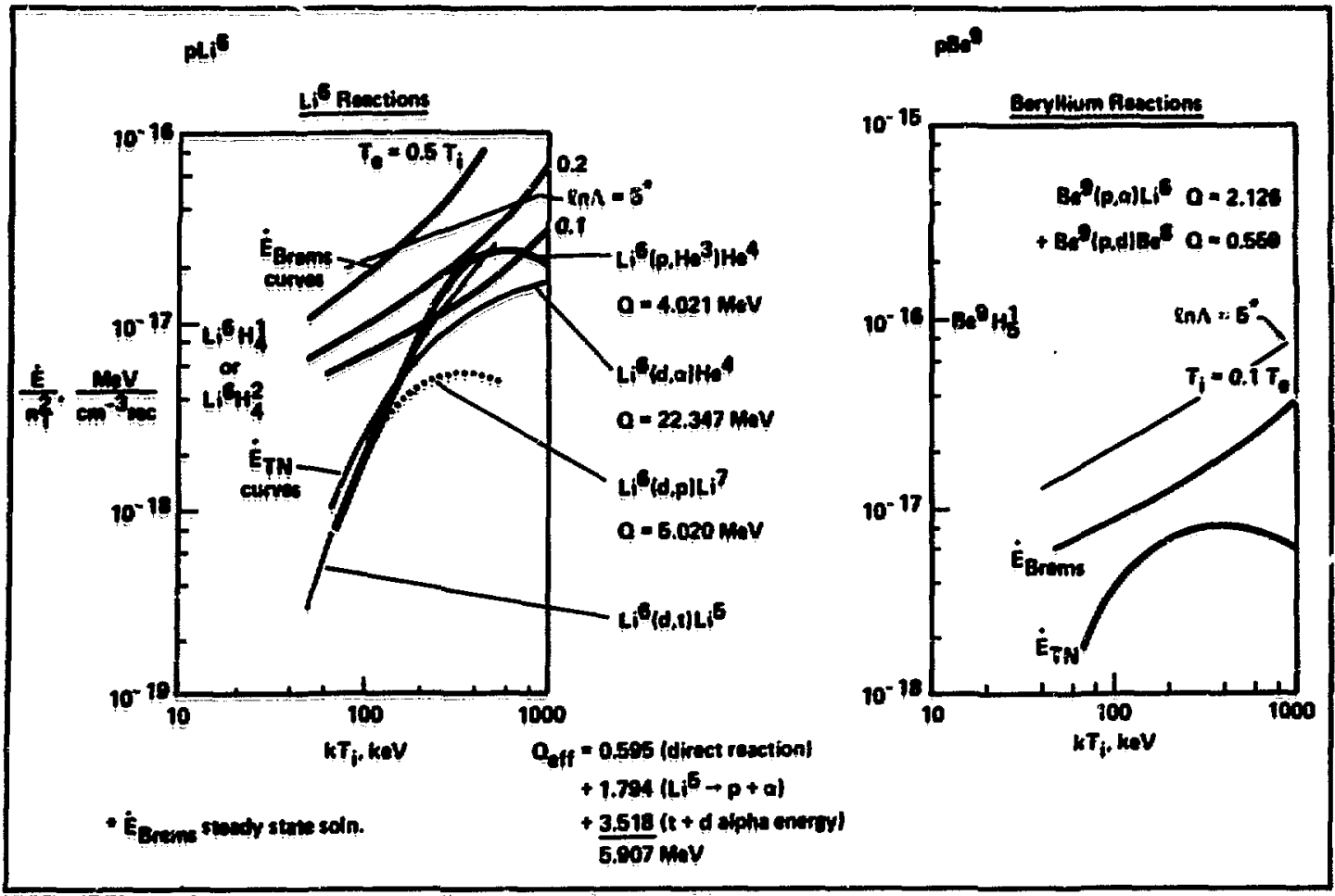

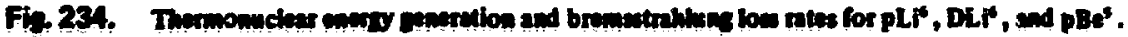

Ali $\mathrm{i}^{6}$ probably not ignited. It is evident that the degree of igition for $\mathrm{pB}^{11}$ is critically dependent on the crossection uncertainties. Within these uncertainties, it appeas that $\mathrm{pB}^{11}$ can probably be jgnited in later pellets and less probably in magnetic confinement reactors. It is important that laser pellets have more farorable ignition conditions to ensure that the fuel ignites, since the small energy-recycling efficiencies typical of lmer fusion require most of the plasma's internal energy to be provided by thermonuclear selfheating. Magnetic confinement schemes, by contrast, have high energy-recycling efficiencies, and could profit energetically from buming fuels even below igition.

\section{Nonthermal Effect}

Nonthermal effects that might significantly augment the $\mathrm{pB}^{11}$ reaction rate or reduce plasma radiation loses include:

1. An ion-ion fusion clain where at least one of the three alpha particles from each $\mathrm{pB}^{11}$ reaction upacatters a proton into the $\sim 0.8 \cdot \mathrm{barn} \cdot \mathrm{high}, 300$ keV-wide reaction resonance centered at $675 \mathrm{keV}$ proton energy so that it reacts before being rethermalized. In this case a diverging chuin reaction, exactly analogous to the fission case, would be extablished.244,247-251

2. A weak ion-ion fusion chain that did not diverge but still caused an augmentation of the population of the high-energy tail of the ion distribution, thus increasing the reaction rate above the themal value.

3. A depopulation of the low-energy, ion-coupled electrons due to upscattering by fusion-bom alpha particles, thus resulting in a lower electron temperature and less bremsstrahlung radiation Jos. 249,250

Preliminary analytic calculations indicate that effect 2 is probably the most significant, giving a bum-rate augmentation of $20-50 \%$ for laser fusion systems and 2-10\% for magnetic confinement reactors. (Here again the difference is the lower charged-particle coupling present in the higher-density laser-fusion systems.) Due to the relative closeness of the themonuclear energy generation and bremsstrahlung energy loss rates, such augmentations are important in attaining reasonable ignition. A computer code (FOKN) has been developed to definilely evaluate these nonthemal effects. ${ }^{249}$ It follows the energy distributions of the nuclear reactants and products in detail, calculating the 
low-ancle Rutherford centinging procens implicitly, utins the Fokker-Planck approximation, and celculating explicilly the lere-ente Coulomb and nuclear scatterines and nection procenas vis a Iranffer matrix. The definitive use of this cods, howerer, has been matly hampend by indequate exporimertal mesurements of the relevant nuchar revetion and salkering cross setions.

\section{Expetineingel Woik}

It is clest that before the eviluation of the polentiel of the pBil ful syoum can be completed, botter crosesection meauroments of the reactions involved must be mads. Such numurononts are preantly being

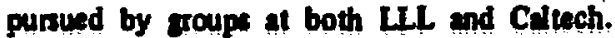

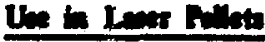

Studies utilizing the LASNEX197 cod to invertighte burning pBll pellets are presently in their initial stross. So far these studies refoct the crosenction uncertintien, with the peatimiatic crose wetions resulting in very poor bum and the optimistic vilues giving quite resconable fuel bumup. Nethod that are being employed to reach the ion temperature rme of 150-250 keV requipd for jprition include strone convenent hydrodynamic compremion and the homopreous inclusion of a small amount of a fuel with lower imition temperature, such $\approx$ DT or DD. Pollet postcomprequion vilues of $\mathrm{aR}-10 \mathrm{~J} / \mathrm{cm}^{2}$ and leer enerites of $\leqslant 1$ MJ are presently believed neceangy for efficient $\mathrm{pQ}^{11}$ bum. 
Photochemical procesaes utilizing tunable lasers may provide an alternattve to the gaseous diffusion, centrifupe, and electromagnetic procesaes for isotope sepantion. In a photochemical procest, use is made of the epectroscopic isotope effect in atomic or molecular vapor to excite one of several isotopes by means of a lawer. 3ince botope effects are generally mall a epectrally marrow source is required. The excited, or "prepared," isotope is then separated by a rubsequent physical or chemical process. The potential adyntages of the photochemical process are the relatively low energy required per separated atom, or molecule, and high eparation factor per epantion stage. Furthermore, the same general photochemical process should be applicable to isotopes of reveral elements.

The study of separation processes using tunable lasers is in a very preliminary phase. The tunable lasers upon which the photochemical isotope separation is based have been developed only recently. Many questions remain to be answered before the feasibility of eparation systems with a large throughput can be asessed.

The objective of the first phase of our isotope separation program is the assessment of technical feasibility of laser phoiochemistry for the separation of isotopes. In the later phases, assuming that feasibility is established, the separation of uranium isotopes will be of primary interest. The ultimate goal of the program is the development of a large-throughput process for the separation of uranium isotopes.

Photochemical processes for the separation of isotopes have been under consideration for many years. Early promise has not materialized, however, and photochemical processes are not in commercial use. This has, in general, been related to the lack of suitable excitation sources. For processes with a commercially significintit throughput, narrow line sources of high intensity are required. These requirements (narrow lines, high intensity) are mutually restrictive for thermal sources.

The careful work of Gunning and his collaborators 252,253 provides an example of the problems in translating a successful laboratory photochemical process into a commercial one. Gunining demonstrated the separation of ${ }^{202} \mathrm{Hg}$ from the six isotopes of natural mercury using a resonance lamp containing ${ }^{202} \mathrm{Hg}$ as the excitation source for the process. A product containing $80 \%{ }^{202} \mathrm{Hg}$ was obtained in a single step starting with natural mercury which contains $29.6 \%$ of ${ }^{202} \mathrm{Hg}$. Subsequent attempts to scale the process have failed, however. Resonance lamps that provide sufficient intensity for a commercial process must be operated at such high temperature and pressure that the emission lines are broadened to the degree that the isotope effect is lost.

It should be possible to overcorne the basic source problem with the use of lasers. The separation of light elements by lasur photochemistry has in fact recently been reported by several authors. Yeung and Moore 254 have separated $H$ and $D$ by the selective photodissociation of $\mathrm{CH}_{2} \mathrm{O}$ using a frequency-doubled ruby laser as the source. Separation of $H$ and $D$ by the photobromination of methanol in a mixture of methanol and deuteromethanol by the $2.7-\mu \mathrm{m}$ radiation from an HF laser has also been claimed by Mayer et al. ${ }^{255}$ Letukhov and co-workers 256,257 have described a two-step laser photochemical process for the separation of $14 \mathrm{~N}$ and $15 \mathrm{~N}$. In their process, ${ }^{15} \mathrm{NH}_{3}$ is excited preferentially with respe $\mathrm{it}$ to ${ }^{14} \mathrm{NH}_{3}$ by a selected line from the $\mathrm{CO}_{2}$ laser spectrum and then dissociated with a second photon from an intense ultraviolet thermal source. Letokhov has considered processes of this type in some detail.

There are no published reports on the use of laser photochemistry for the separation of heavy isotopes. However, several patents have been issued covering laser processes for the separatiun of uranium isotopes. ${ }^{258-260}$ Processes which have been considered

I $\quad$ I I

\begin{tabular}{|c|c|c|}
\hline $\begin{array}{l}\text { Introduction } \\
\text { of isotopically } \\
\text { mixed vapor }\end{array}$ & $\begin{array}{l}\text { Laser photo- } \\
\text { excitation of } \\
\text { desired isotope }\end{array}$ & $\begin{array}{l}\text { Extraction } \\
\text { of "prepared" } \\
\text { isotope }\end{array}$ \\
\hline
\end{tabular}

Fig. 235. Flow diagram showing the steps in a photochemical isotope-separation process using lasers. 


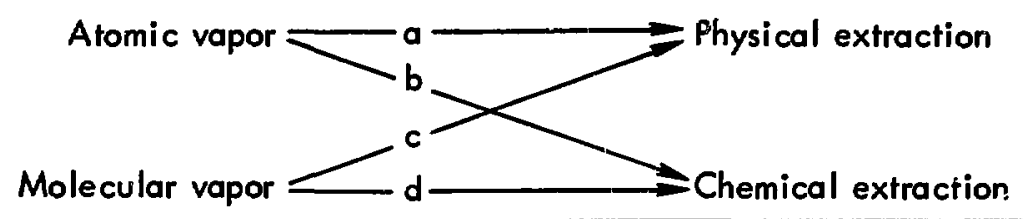

Fig. 236. The four basic processes being considered for photochemical isotope separation with lasers, labeled $a$ through $d$ in order of increasing complexity.

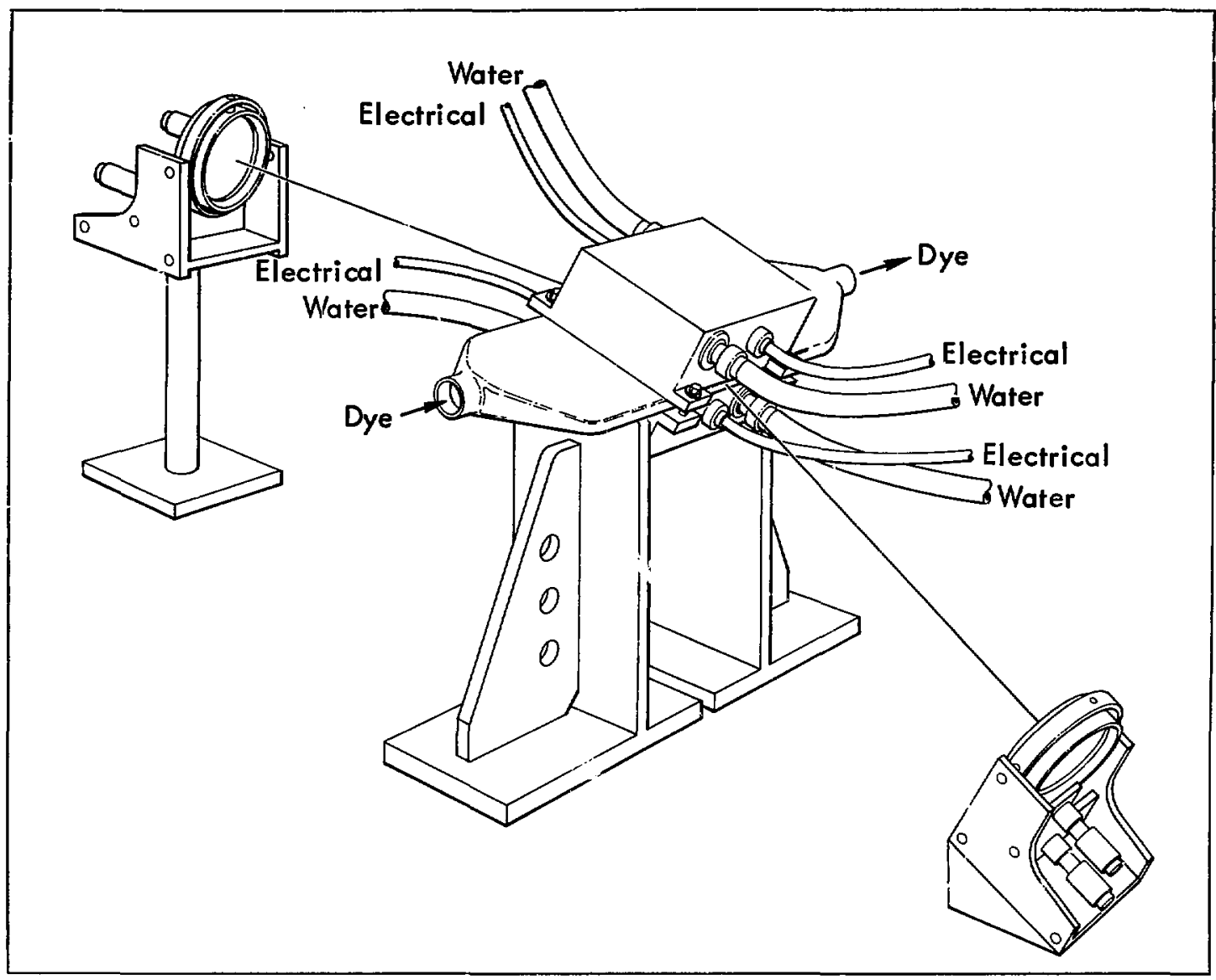

Fig. 237. The dye laser that will be used in the demonstra:un isotope-separation experiment. The laser is designed for an output of several watts and a pulse repetition rate of $300-500 \mathrm{~Hz}$. Dye flow is transverse to the output beam, tuning and line narrowing are done with a birefringent filter (nzt shown).

conform basically to the flow diagram of Fig. 235 . An atomic or molecular vapor is chosen as the starting material since a cleanly resolved isotope effect can be obtained in gases at high temperatures. Solids or liquids must generally be cooled to cryogenic temperatures to obtain the narrow spectral lines necessary for an efficient isotope separation process and are, therefore, less attractive as starting materials.
There are essentially four basic processes to be considered as indicated in Fig. 236. These are the combinations of an atomic or molecular vapor starting-material with physical or chemical extraction. The processes are listed from $a$ to $d$ in order of increasing complexity. In concept, an atomic vapor system with the physical extraction of ions by a combination of electric and magnetic fields ${ }^{257}$ is the 
simplest. The drawback of this system, for heavy elements, is that it must operate at such high temperatures that serious materials problems are encountered. System $d$, based upon the extraction of an excited or dissociated molecule by a chemical reaction, is perhaps the most attractive system. Its operating temperature could be much lower than that of a. Chemical extraction could also result in simplifications of the apparatus since magnets and electrodes, and the energy sources to run them, would not be required. However, a suitable photochemical scheme for uranium must be developed. To do this, we need to study the spectroscopy and photochemistry of volatile uranium compounds.

In the initial phase of the isotope separation program, we will conduct experiments which are necessary to establish feasibility. The spectroscopy and photochemistry of uranium and its volatile compounds will be studied to determine isotope effects, absorption and photoionization cross sections, excited-state lifetimes, cross sections for chemical reactions, energy exchange, and charge exchange. The measured spectroscopic parameters will then be used to model various isotope separation schemes.

To gain experience with the problems of isotope separation using laser photochemistry, we plan to employ the chemical process developed by Gunning et al., 252,253 but with a laser source instead of a resonance lamp, to separate mercury isotopes. To do this we will have to develop a $2537-\AA$ laser with an average power of aboet $0.1 \mathrm{~W}$. Accordingly, a highrepetition-rate, flashlampexcited dye laser is being built to operate at $5074 \AA$ (Fig. 237). The laser frequency will be doubled to obtain the desired wavelength. 
The LLL laser fusion program had about 270 people directly assigned to it at the end of this fiscal year, resulting in an FY 1973 yearly average of 250 full-time-equivalent employees. Of the total, $30 \%$ are scientific staff and the remaining $70 \%$ are engineers, technicians, and other support personnel. Following is a breakdown of the FY 1973 manpower by major

Solid state laser $\mathbf{R} \& \mathbf{D}$

Target development 110

Gas and chemical lasers

Isotope separation
39

100

2 task:

\section{OPERATING COSTS}

Operating costs for the January-June 1973 period totaled $\$ 6.9$ million, bringing the total for FY 1973 to $\$ 13.5$ million. Basic costs (which include wage expenses, overhead, and minor procurements) totaled $\$ 10.4$ million. Major procurements (generally orders over $\$ 10,000$ ) accounted for $\$ 2.3$ million, and computer time recharges amounted to $\$ 0.8$ million. Following is the FY 1973 operating cost breakdown by major task:

Solid state laser R \& D

Gas and chemica! lasers Isotope separation

\section{CAPITAL EQUIPMENT}

The total FY 1973 allocatiun tor capital equipment was approximately $\$ 1.1$ million, $\$ 0.8$ million of which was committed in the January-June time period. Following is the distribution of capital equipment expenditures by major task:

Solid state laser $\mathbf{R}$ \& D Target development 630

Gas and chemical lasers Isotope separation
10

350

140

Total 1,130

\section{FACILITIES}

The following general plant projects funded in FY 1972 were completed:

- Bldg. 174 (Solid State Research): Increment III was completed in March. This is a 4000-sq-ft addition consisting of a main laser bay, an oscillator development laboratory, a control room, and a mechanical equipment room. The 60 -ft-long main laser bay will allow continuation of the $1-\mathrm{kJ}$ glass laser through the stage of the $C$ modules - i.e., a designed energy output of $250 \mathrm{~J}$.

- Bldg. 166 ( $\mathrm{CO}_{2}$ Systems): A 4900-sq-ft addition to this building was completed in March.

- Bldg. 175 (Laser Diagnostics): The modification to this building was completed in March. The facility consists of four small laboratories used for development of special laser diagnostic techniques and equipment.

During the first half of FY 1973, design of a 4700-sq-ft addition to the chemical laser facility was completed. Construction bids averaging $40 \%$ above the Architećt/Engineer's estimated cost have forced a revision of the design which is currently in progress.

A High-Energy Laser Facitity with a total project cost of $\$ 20$ million was requested for funding approval in FY 1974. This 42,000-sq-ft facility will provide the basic structure and supporting equipment needed for developing and testing a glass laser system capable of delivering $10 \mathrm{~kJ}$ to a laser-fusion target. It will be built as an irradiation facility where the prime 
experimentation will be directed at understanding the interaction of high-energy, short-pulse laser beams with a wide range of target geometries. During May and June 1973, the AEC solicited and interviewed candidates for the Architect/Engineer services associated with the design of the building. Preliminary design studies to be done by the selected A/E firm are schetuled to begin in August 1973. Views of this facility are shown in Figs. 238 and 239.

On May 4, 1973, ground was broken for the start of construction of the LLL Laser Fusion Laboratory (Fig. 240). The total project cost of $\$ 6.8$ million is funded by the AEC through the San Francisco Operations Office. Norman Engineering Co. of Los Angeles was selected for the dual role of Architect/Engineer and Construction Manager for the construction portion of the project. The facility consists of three separate but interconnected buildings: a laboratory building of 38,000 sq $\mathrm{ft}$ (including a 10,000 -sq-ft basement), an office complex of $42,000 \mathrm{sq} f t$ to house 200 people, and a $9,000-$ sq-ft equipment building to house special heating, air conditioning, and electrical distribution equipment.

The laboratory building is designed to provide the close environmental control required for laser research. It will accommodate a variety of basic research activities and prototype laser systems aimed at investigating concepts which might eventually be utilized in designing one or more high-energy laser "tools." The office complex will consolidate scientific and support personnel into a common area designed for easy intercommunication.

Start of construction came almost a month ahead of schedule and only six months after funding approval. Two factors contributed significantly to this

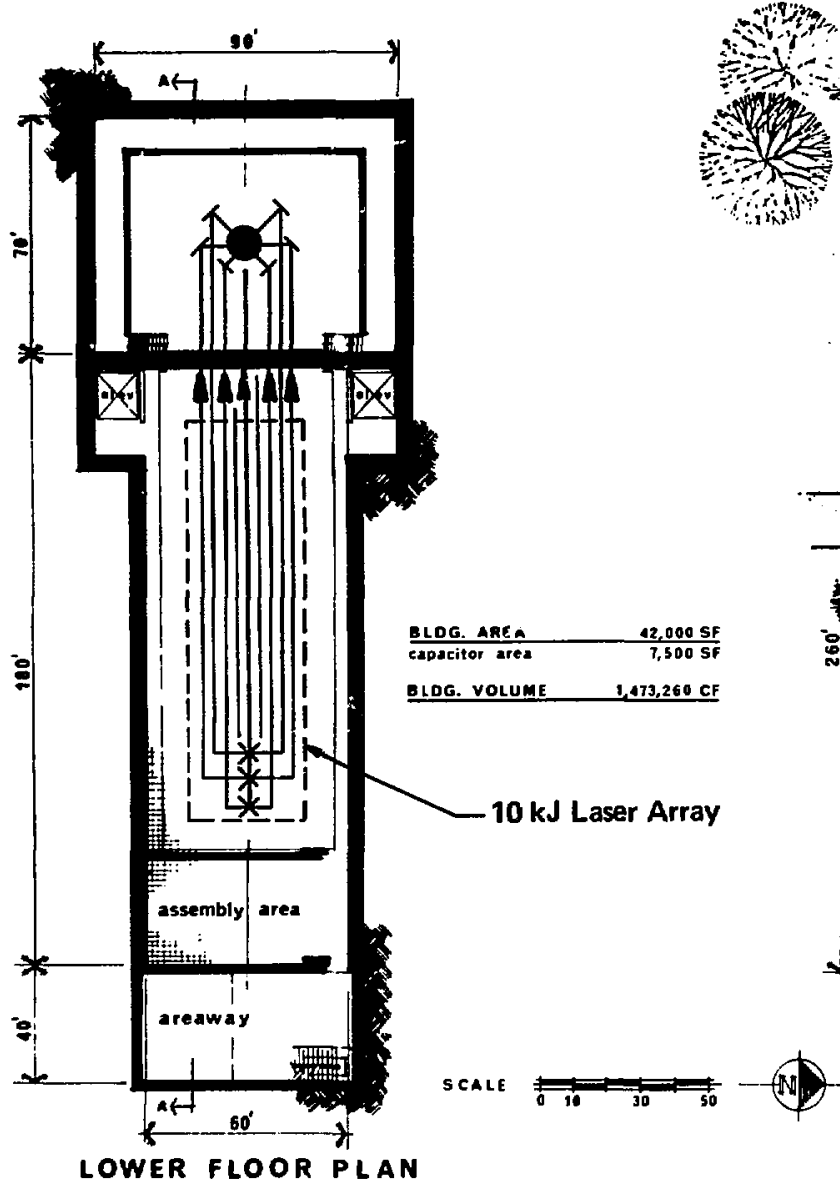

LOWER FLOOR PLAN

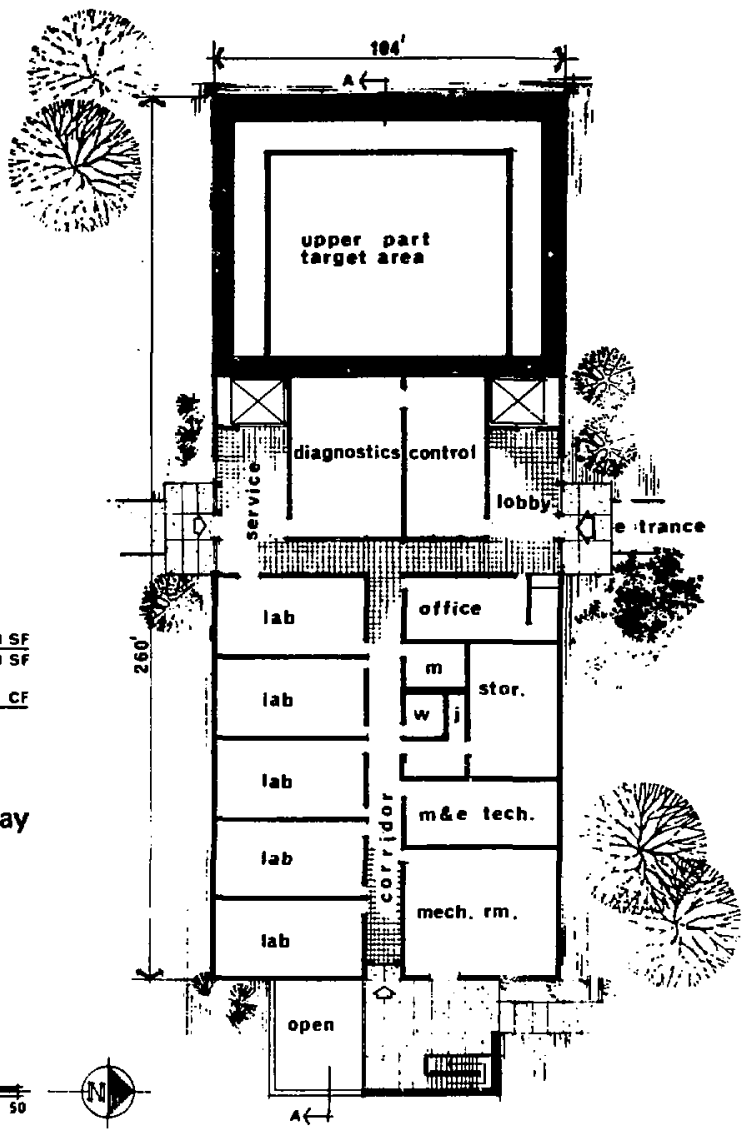

UPPER FLOOR PLAN

Fig. 238. Plan view of the High-Energy Laser Facility to be built at LLL. 


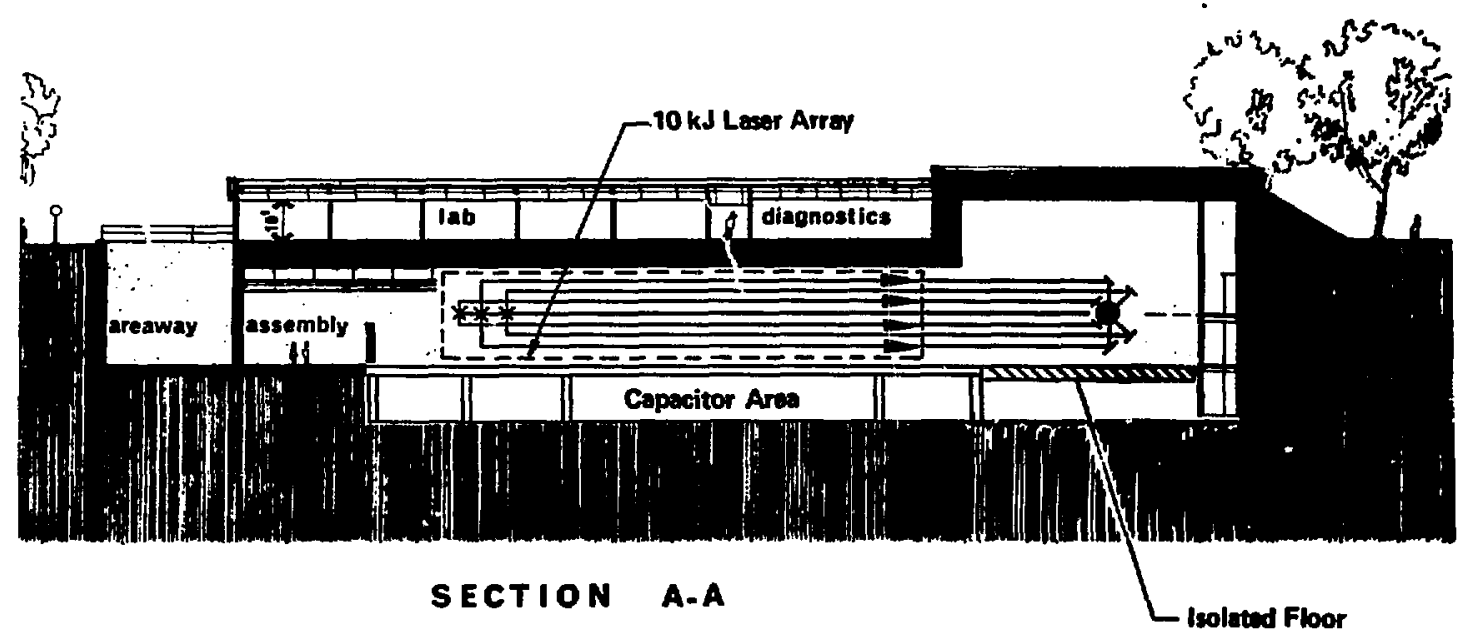

Fig. 239. Sectional view of the High-Energy Laser Fecility.

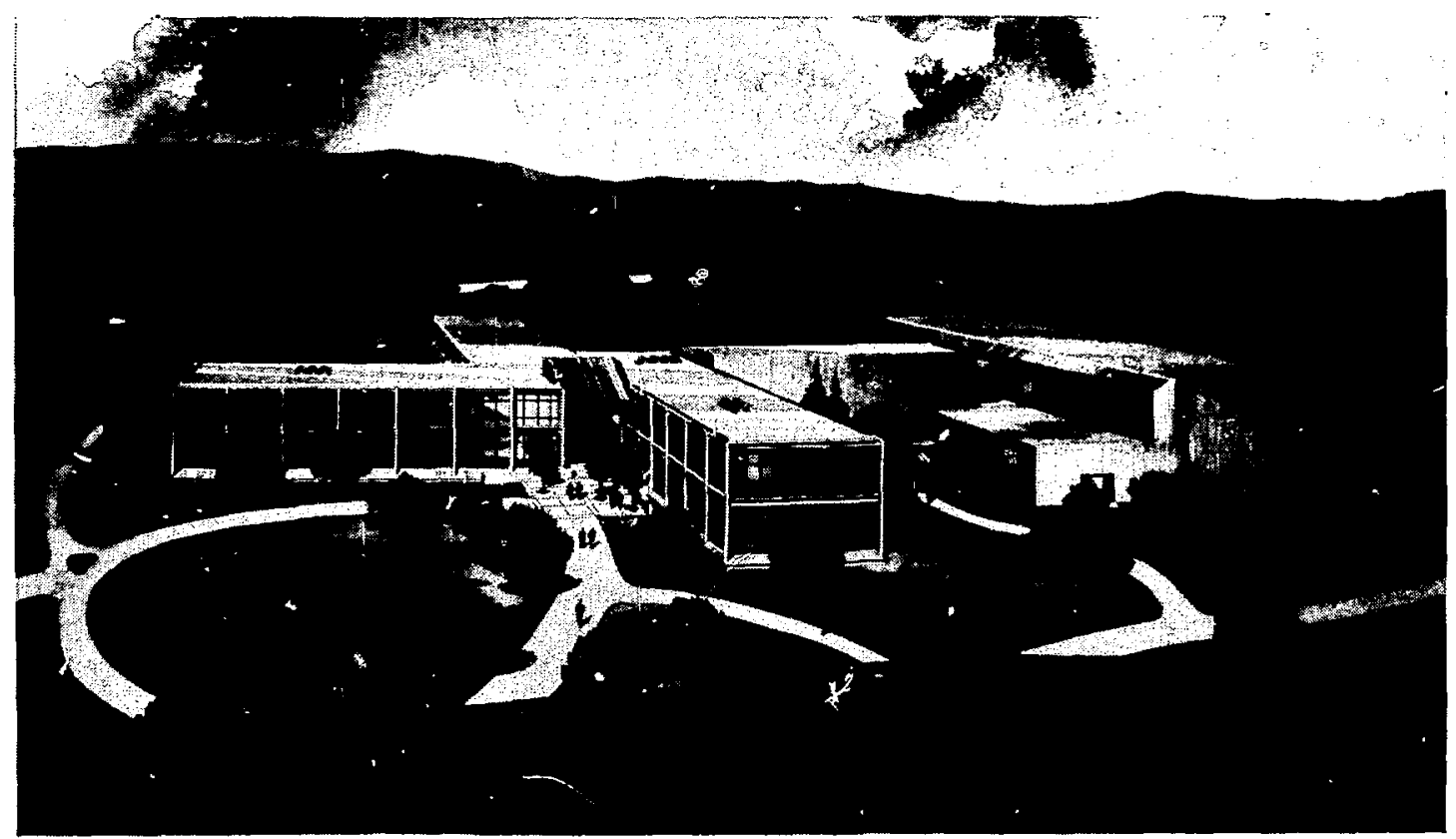

Fig. 240. The Laser Fusion Laboratory is now under construction.

accelerated schedule. First, preliminary design studies were done on vibrations, noise, temperature control, air distribution, humidity, and dust control. Results of these studies provided timely detailed criteria for the facility design. Second, a construction management concept coupled with a phased construction program meant that site and foundation construction could proceed before completion of the final design of the remainder of the building. Remaining construction "packages" will be released to phase with the work already under way; the targeted completion date is May 1974. 
1. Lasen-Fusion Program Semiannual Report - Jubs-December 1972, UCRL-S1021-72-2, PP. 7.8.

2. Ibid., pp. 16.19.

3. Jbid., pp. 11-16.

4. J. M. Mcllahon, J. L. Emmett, J. F. Holzrichter, and J. B. Trenholme, "A Glass Disk Laser Amplifier," IEEE J. Quent. Electron. (to be published Oet. 1973).

5. J. B. Trenholme, Fuonescence Amplification ind Parastic Osclllation Limitations in Diec Lasers, Naval Rexearch Labontory, Washington, D.C., NRL Memorandum Report 2480 (Jily 1972).

6. J. H. Gonz, "New Developments in Electronic Fiachtubes," Instrum Soc. Amer. S(1), ! (1966); see also ILC Technical Bulletin No. 1, LC, Inc., Sunnyvale, Calif.

7. I. M. Winex, "A Self-Calibnting Technique for Measuring Laser Beam Intensity Distributions," Appl. Opt. 5, 1437 (1966).

8. A. Penzkofer and W. Kaiser, "Nonlinear Loss in Nd-Doped Laser Glass," Appl. Phys. Lett. 21, 427 (1972).

9. P. Jacquinot and B. Roizen-Dossier, "Apodization," in Progress in Optics $I I$, edited by E. Wolf (North-Holland Pub. Co., Amsterdan, 1964). See alao Ref. 2.

10. S. Sackett and R. Healey, A Digital Computer Progran for the Numerical Solution of the Linear Poisson Equation, Lawrence Berkeley Laboratory Rept. UCRL18721 (1969).

11. Laser-Fusion Program Semiannual Report - Jamuary-June 1972, UCRL-50021-72-1, p. 104.

12. Ibid., pp. 9-13.

13. J. M. McMahon, Naval Research Laboratory, Washington, D. C., persoral communication (1973); the small-signal transmission of the saturable absorber changes by about $1 \%$ per ${ }^{\circ} \mathrm{C}$.

14. V. Met, "Working with Etalons," Microwaves (Sept. 1967), pp. 45-54.

15. E. Pierce, A Flowing Dye Cell and Pump System for Mode-Locked Oscillators, Lawrence Livermore Laboratory Engineering Note ENT-72-4 (May 2, 1972)(LLL internal report not circulated outside the Laboratory).

16. Laser-Fusion Program Semiannual Report - July-December 1971, UCRL-50021-71 Rev. 1, p. 9 ff.

17. S. B. Sutton, Lawrence Livermore Laboratory, unpublished data.

18. Laser-Fusion Program Semiannual Report - July-December 1971, UCRL-50021-71 Rev. 1, pp. 4-9.

19. L. Noble et al., Optical Pumps for Lasers, Final Report, U. S. Army Electronics Command R \& D Technical Report ECOM-0035-F (May 1971)(Contract DAAB07-70-C-0035; ILC, Inc.).

20. L. Noble and C. B. Kretschmer, Optical Pumps for Lasers, Triannual Report No. 1, U.S. Army Electronics Command R \& D Technical Report ECOM-0239-1 (March 1972) (Contract DAAB07-71-C-0239; ILC, Inc.).

21. J. Steffen et al., "Fundamental Mode Radiation with Solid-State Lasers," IEEE J. Quant.Electron. QE-8, 239 (1972).

22. A. L. Bloom, Properties of Laser Resonators Giving Uniphase Wave Fronts, Spectra-Physics Laser Technical Bulletin No. 2 (Aug. 1963).

23. Laser-Fusion Program Semiannual Report - Jamuary-June 1972, UCRL-50021-72-1, pp. 6-9.

24. Laser-Fusion Program Semiannual Report - July-December 1972, UCRL-50021-72-2, pp. 8-9.

25. R. L. Carman, B. C. Johnson, and L. L. Steinmetz, Opt. Commun. 7, 169 (1973).

26. Additional details on the theory of bandwidth-limited pulses are available in R. A. Saroyan, Lawrence Livermore Laboratory Rept. UCID-16247 (March 1973).

27. L. L. Steinmetz, T. W. Pouliot, and B. C. Johnson, Appl. Opt. 12, 1468 (1973).

28. G. I. Kachen and G. Shaw (editors), LLL Laser Projects Report No. 1, Lawrence Livermore Laboratory Rept. UCRL-50022-1 (May 1972).

29. J. Trenholme and K. Manes, A Simple Approach to Laser Amplifiers, Lawrence Livermore Laboratory Rept. UCRL-51413 (1973).

30. Laser-Fusion Program Semiannual Report - July-December 1972, UCRL-50021-72-2, pp. 63-65.

31. H. B. Dwight, Tables of Integrals and Other Mathematical Data (Macmillan, New York, 1961), 4th Ed.

32. J. B. Trenholme, Lawrence Livermore Laboratory, to be published.

33. J. Campbell and L. DeShazer, J. Opt. Soc. Amer. 59, 1427 (1969).

34. J. B. Trenholme, Lawrence Livermore Laboratory, private communication (1973). 

Laboratory report in preparation).

36. J. W. Goodman, An Introduction to Fourier Optics (McGraw-Hill, New York, 1968).

37. H. J. Weaver, Effect of Optical Systems on Diffrcicion (Lawrence Livermore Laboratory report in preparation).

38. J. W. Trenholme, J. Holzrichter, and W. F. Hagen, Lawrence Livermore Laboratory, private communication (1973).

39. W. F. Hagen, Lawrence Livermore Laboratory, private communication (1973).

40. R. A. Fisher, Picosecond Opticul Pulse Nonlinear Propagation Effects, Ph.D. thesis, University of California, Berkeley (1971).

41. P. Mace, Los Alamos Scientific Laboratory, private communication (1973).

42. R. E. Bellman and S. E. Dreyfus, Applied Dynamic Programming (Princeton University Press, Princeton, N. J., 1962).

43. J. P. Markiewicz and J. L. Emmett, IEEE J. Quant. Electron. QE-2, 707 (1966).

44. D. L. Mumaugh, COILS - Magnetic Projection of Current Systems Using Eltiptic Integrals, Lawrence Livermore Laboratory Rept. UCID-2465 (Apr. 1963).

45. Laser-Fusion Program Semiannual Report - July-December 1972, UCRL-50021-72-2, p. 19.

46. Ibid., pp. 48.50.

47. Laser-Fusion Program Semiannual Report - January-June 1972, UCRL-50021-72-1, pp. 95-98.

48. Laser-Fusion Program Semiannual Report - July-December 1972, UCRL-S0021-72-2, pp. 36-40.

49. L. L. Steinmetz, T. W. Pouliot, and B. C. Johnson, Lawrence Livermore Laboratory, paper presented at the IEEE/Opt. Soc. Amer. Conference on Laser Engineering and Applications, Washington, D. C., 1973.

50. Laser-Fusion Program Semiannual Report - July-December 1972, UCRL-50021-72-2, pp. 51-52.

51. S. W. Thomas ard L. W. Coleman, "Laser-Triggered Avalanche-Transistor Voltage Generator for a Picosecond Streak Camera," Appl. Phys. Lett. 20, 83 (1972).

52. Laser-Fusion Program Semiannual Report - July-December 1972, UCRL-50021-72-2, pp. 52-55.

53. Laser-Fusion Program Semiannual Report - July-December 1971, UCRL-50021-71 Rev. 1, pp. 18-19.

54. L. O. Heflinger, R. F. Wuerker, and R. E. Brooks, J. Appl. Phys. 37, 642 (1966).

55. R. E. Brooks, L. O. Heflinger, and R. F. Wuerker, IEEE J. Quant. Electron. QE-2, 275 (1966).

56. Laser-Fusion Program Semiannual Report - July-December 1972, UCRL-50021-72-2, p. 59.

57. R. F. Van Ligten, Proceedings of thc Society of Photo-Optical Instrumentation Engineers (SPIE) Seminar, May 1968, Vol. 15, p. 75.

58. Laser-Fusion Program Semiannual Report - July-December 1972, UCRL-50021-72-2, pp. 55-59.

59. T. C. Owen, L. W. Coleman, and T. J. Burgess, Ultrafast Optical Kerr Effects in CS, at $10.6 \mu \mathrm{m}$, Lawrence Livermore Laboratory Rept. UCRL-74131 Preprint (1973); also Appl. Phys. Lett. 22(6), 272 (1973).

60. Laser-Fusion Program Semianunal Report - January-June 1972, UCRL-50021-72-1, p. 34.

61. For details of the molecular energy levels, see R. S. Mulliken, J. Chem. Phys. 52, 5170 (1970).

62. Efficiencies between 10 and $100 \%$ have been reported for xenon excited by alpha particles: J. Fortner, L. Meyer, S. A. Rice, and E. G. Wilson, J. Chem. Phys. 42, 4250 (1965).

63. For a discussion of this and related topics, see R. E. Kidder in Physics of High Energy Density, edited by P. Caldirola and H. Knoepfel (Academic Press, New York, 1972), p. 306.

64. N. G. Basov, V. A. Danilychev, and Yu. M. Popov, Sov. J. Quant. Electron. 1, 18 (1971).

65. H. A. Koehler, L. J. Ferderber, D. L. Redhead, and P. J. Ebert, Appl. Phys. Lett. 21,198 (1972).

66. J. B. Gerardo and A. W. Johnson, IEEE J. Quant. Electron. QE-9, 748 (1973).

67. D. C. Lorents and R. E. Olson, Excimer Formation and Decay Processes in Rare Gases, Stanford Research Institute Report (Dec. 1972); E. V. George and C. K. Rhodes; Kinetic Model of Ultraviolet Inversion in High Pressure Rare Gas Plasmas, Lawrence Livermore Laboratory Rept. UCRL-74516 Preprint (1973), to be published.

68. This problem was alluded to very early in the development of optical lasers. See the section entitled "Summary and High-Frequency Limits," in L. A. Schawlow and C. H. Townes, Phys. Rev. 112, 1940 (1958).

69. A description of mode patterns is found in A. Kogelnik and T. Li, Proc. IEEE 54, 1312 (1966).

70. A. Gedanken, J. Jortner, B. Raz, and A. Szöke, J. Chem. Phys. 57, 3456 (1972). 
71. A. Cheshnovsky, B. Rax, and J. Fortner, Proc. Third Intemational Vocuum Ultraviolet Conference, Tokyo, 1971.

72. A. V. Phelps, Tunable Gas Lasers Utilizing Ground State Dissociation, University of Coloredo, Boulder, Cc'o., JILA Report No. 110 (Sept. 1972).

73. I. V. Kosinskaya and L. P. Polozova, Opt. Spectrosc. 30, 458 (1971).

74. Early work on continuous spectra is contained in Wolfgang Finkelnburg, Kontinuieriiche Spektren (Springer-Verlag, Berlin, 1938).

75. For the rates of dissociative recombination of the homonuclear rare grs ions at $T \approx 300^{\circ} \mathrm{K}$ see $\mathrm{H}$. J. Oakam and V. R. Mittelstadt, Phys. Rev. 132, 1445 (1963). The value for $\mathrm{Xe}_{2}^{+}$is $(1.4 \pm 0.1) \times 10^{-6} \mathrm{~cm}^{3} / \mathrm{sec}$. A further discussion of dissociative recombination is given by $\mathrm{J}$. $N$. Bardsley and M. B. Biondi, in Advances in Atomic and Molecular Physics, edited by D. R. Bates (Academic Press, New York, 1970), Vol. 6.

76. L. Frommhold and M. A. Biondi, Phys. Rev. 185, 244 (1969).

77. A. V. Phelps; Phys. Rev. 114, 1011 (1959).

78. A preliminary desctiption of these processes is given by B. Krawetz and C. Rhodes, Vacuum Ultraviolet Studies of Rare Gas Mixtures Excited with Pulsed High Energy Electron Beams, Lawrence Livermore Laboratory Rept. UCRL-73777 Preprint (1972); to be publiened in the Proceedings of the Symposium on High Power Molecular Lasers, Quebec City, Canada, May 15-17, 1972. See also E. V. George and C. K. Rhodes, MIT Quarterly Progress Report No. 108 (Jan 1973).

79. K. Wojaczek, Beitr. Plasmaphys. 5, 307 (1965).

80. M. Gryzinski, Phys. Rev. 115, 374 (1959); M. Gryzinskj, Phys. Rev. 138, A336 (1965).

81. H. S. W. Massey and E. H. S. Burhop, Electronic and Ionic Impact Phenomena (Oxford University Press, London, 1969), Vol. 1, p. 128.

82. T. Kivara, Rev. Mod. Phys. 24, 45 (1952).

83. A. C. G. Mitchell and M. W. Zemansky, Resonance Radiation and Excited Atoms (Cambridge University Press, 1934 and 1961).

84. A. V. Phelps and J. P. Molnar, Phys Rey. 89, 1202 (1953). See also H. S. W. Massey, Electronic and Ionic Impact Phenomena (Oxford University Press, London, 1971), Vol. 3.

85. J. A. Hornbeck and J. P. Molnar, Phys. Rev. 84, 621 (1951).

86. J. S. Dahler, J. L. Franklin, M. S. B. Munson, and F. H. Field, J. Chem. Phys. 36, 3332 (1962).

87. D. Smith, A. G. Dean, and I. C. Plumb, J. Phys. B5, 2134 (1972).

88. R. Boucique and P. Mortier, J. Phys. D: Appl. Phys. 3, 1905 (1970).

89. T. F. OMalley, Phys Rev. 185, 101 (1969).

90. G. V. Marr, Photoionization Processes in Gases, Vol. 28 of Pure and Applied Physics Series (Academic Press, New York, 1967), p. 108.

91. R. E. M. Hedges, D. L. Drummond, and A. Gallagher, Phys. Rev. A6, 1519 (1972).

92. M. J. Berger and S. M. Seltzer, Tables of Energy Losses and Ranges of Electrons and Positrons, N65-12506 (NASA, Washington, D. C., 1964).

93. F. A. Hopf and C. K. Rhodes, "Influence of Vibrational, Rotational, and Reorientational Relaxation on Pulse Amplification in Molecular Amplifiers," Phys. Rev. A 8, 912 (1973).

94. E. T. Gerry and D. A. Leonard, Appl. Phys. Lett. 8, 227 (1966).

95. D. W. Ducsik, An Experimental Determination of the Radiative Lifetime for $\mathrm{CO}_{2}$, B. Sc. thesis, Massachusetts Institute of Technology (August 1968), and E. Hoag, Avco Corp., Everett, Mass., private communication.

96. Laser-Fusion Program Semiannual Report - July-December 1972, UCRL-5C021-72-2, pp. 98-101.

97. C. Freed and A. Javan, Appl Phys. Lett. 17, 53 (1970).

98. F. Rheault, J. L. Lachambre, J. Gilbert, R. Fortin, and M. Blanchard, paper given at Seventh International Quantum Electrcinics Conference, Montreal, Canada, May 1972. See also Can. J. Phys. 50, 1876 (1972).

99. Laser-Fusion Program Semiannual Report - Jamuary-June 1972, UCRL-50021-72-1, pp. 49-50.

100. Laser-Fusion Program Semiannual Report - July-December 1972, UCRL-50021-72-2, pp. 20-24.

101. N. Kroll and K. M. Watson, Phys. Rev. 5A, 1883 (1972).

102. C. S. Naiman, M. 'Y. DeWolf, I. Goldbatt, and J. Schwart2, Phys. Rev. 146, 133 (1966); V. Chalmeton and R. Papoular, Phys. Lett. 26A, 579 (1968).

103. P. J. Berger and D. C. Smith, Appl. Phys. Lett. 21, 167 (1972).

104. G. A. Hill, D. J. James, and S. A. Ramsden, J. Phys. D: Appl. Phys. 5, 197 (1972). 
105. D. C. Smith, J. Appl. Phys. 41, 4501 (1970); D. C. Smith, Appl. Phys. Lett. 19, 405 (1971); M. C. Richardson, A. J. Alcock, K. Leoprold, and P. Burtyn, IEEE J. Quant. Electron. QE-9, 236 (1973).

106. Y. L. Pan, A. F. Bernhardt, and. J. R. Simpson, Rev. Sci. Instr. 43, 662 (1972).

107. Y. L. Pan, J. R. Simpeon, A. F. Bernhardt, and S. E. Kiergan, Lawrence Livermore Laboratory Rept. UCRL74604 Preprint (March 19:3).

108. D. E. Lencioni, Appl Phys. Lett. 23, 12 (1973).

109. G. H. Canavan and P. E. Nielson, Appl. Phys Lett. 22, 409 (1973).

110. E. E. Stark, W. H. Reichelt, G. T. Schrippert, and T. F. Stratton, Appl Phys Lett. 23, 322 (1973).

11. Such expansions have been handleil in the theoretical work on $V-V$ collisions by: $R$. D. Sharma and C. A. Brau, J. Chem Phys 50, 924 (1969); R. D. Sharma and C. W. Kem, J. Chem. Phys 55, 1171 (1971); R. D. Sharma, Phys. Rv. 177, 102 (1969); R. D. Sharma and C. A. Brau, Phjs. Rev. Lett. 19, 1273 (1967); F. T. Chan and C. L. Tang, J. Chem. Phys 40, 2806 (1969).

112. P. K. Cheo and R. L. Abrams, Appl. Phys. Lett. 14, 47 (1969): R. L. Abrams and P. K. Cheo, Appl. Phys Lett. 15, 177 (1969).

113. 1. Burak, Y. Noter, and A. Szöke, IEEE J. Quant. Electron. QE-9, 541 (1973).

114. . Laser-Fusion Program Semiannual Report - July-December 1972, UCRL-70021-72-2, pp. $20,28$.

115. J. Tulip, IEEE J. Quant. Electron. QE-6, 206 (1970); Y. Sato and S. Tsuchiya, J. Phys. Soc. (Japan) 30, 1467 (1971); A. L. Hoffman and G. C. Vlases, IEEE J. Quant. Electron. QE-8, 46 (1972); K. R. Manes and H. J. Seguin, J. Appl Phys 43, 5073 (1972); D. L. Lyon, IEEE J. Quant. Electron. QE-9, 139 (1973).

116. R. N. Schwartz, 2. I. Slawsky, and K. F. Herzfeld, J. Chem. Phys. 20, 1591 (1952); W. J. Witteman, Philips Res Rept., Suppl. 2 (1963); B. F. Gordictz, N. N. Sobolev, V. V. Sokovikov, and L. A. Shelepin, Phys Leat. 25A, 173 (1967).

117. J. Hester et al., Final Report on Program for Higñ Power Laser Techniques, Tech. Rept. AFAL-TR-67-114 (Apr. 1967); see also Quarterly Technical Status Reports on High Energy Pulsed Electric Laser, Avco Everett Research Lab., Repts. AERL-70-1393 (Sept. 1970), AERL-71-307 (Dec. 1970), and AERL71-1712 (Oct. 1971).

118. R. J. Harrach and T. H. Einwohner, Four-Temperature Kinetic Model for a $\mathrm{CO}_{2}$ Laser Amplifier, Lawrence Livermore Laboratory Rept. UCRL-51399 (June 1973). Our earlier work on this subject is described in the Laser-Fusion Program Semiannual Report - July-December 1971, UCRL-50021-71 Rev. 1, p. 36.

119. C. B. Mills, $\mathrm{CO}_{2}$ Gas Laser Studies, Los Alamos Scientific Laboratory Rept. LA-5151-MS (Jan. 1973); C. A. Fenstermacher, M. J. Nutter, W. T. Leland, and K. Boyer, Appl. Phys. Lett. 20, 56 (1972).

120. Laser-Fusion Program Semiannual Report - July-December 1972, UCRL-50021-72-2, pp. 86-88.

121. D. J. Kuizenga and A. E. Siegman, IEEE J. Quant. Electron. QE-6, 694 (1970).

122. T. Ogawa, G. A. Carlson, and G. C. Pimentel, J. Phys. Chem. 74, 2090 (1970).

123. M. A. Pollack, Appl, Phys. Lett. 8, 36 (1966).

124. D. E. O'Brien and J. R. Bowen, J. Appl. Phys. 40, 4767 (1969).

125. V. Yu. Zalesskii and A. A. Venediktov, Sov. Phys. $=$ JETP 28, 1104 (1969).

126. T. L. Andreeva et al., JETP Lett. 13, 449 (1971).

137. K. Hohla, Max-Planck-Institut für Plasmaphysik, Garching bei München, Rept. IPP-IV/33 (Dec. 1971)(in German). English translation, document number SC-T.722560.

128. K. Hohla and K. L. Kompa, Z. Naturforsch. 27a, 938 (1972)(in German). English translation, document number UCRL-Trans-10692.

129. V. Yu. Zalesskii, JETP 34, 474 (1972).

130. T. L. Andreeva et ai., High Energy Chem. 6(5), 368 (1973).

131. T. L. Andreeva et al., JETP Lett. 10, 271 (1969).

132.

133.

P. Gensel, K. Hohla, and K. L. Kompa, Appl. Phys. Lett. 18, 48 (1971).

A. D. Stepukhovich and V. M. Umanskii, Russ. Chem. Rev. 38, 590 (1969).

134. H. S. Johnston, Gas Phase Reaction Rate Theory (Ronald Press, New York, 1966), Ch. 14.

135. G. Burns et al., Proc. Roy. Soc. (London) A315, 81 (1970).

136. J. A. Blake and G. Burns, J. Chem. Phys. 54, 1480 (1971).

137. D. Husain and R. J. Donovan, "Electronically Excited Halogen Atoms," in Advances in Photochemistry, edited by J. N. Pitts, Jr., G. S. Hammond, and W. A. Noyes, Jr. (Wiley-Interscience, New York, 1971), Vol. 8, pp. 1-76. (See especially pp. 45-46.) 
138. K. R. Wilson, "Photofragment Spectroscopy of Dissociative Excited States," in Excited State Chemistry, edited by J. N. Pitts, Jr. (Gordon and Breach, New York, 1970), p. 44.

139. J. O. Hirschfelder, C. F. Curtis, and R. B. Bird, Molecular Theory of Gases and Liquids (Wiley, New York, 1954), p. $1110 \mathrm{ff}$.

140. K. Hohla and K. L. Kompa, Chem Phys. Lett. 14, 445 (1972).

141. R. H. Garstang, J. Res. Nat. Bur. Stand. Sect. A Ph. Ch. 68, 61 (1964).

142. E. B. Turner, G. Emanuel, and R. L. Wilkins, The NEST Chemistry Computer Program, The Aerospace Corp., El Segundo, Calif., Rept. TR-0059(6240-20)-1 (July 1970).

143. N. L. Rapagnani, The Effects of Various Chain-Branching Rates on the Pulsed HF Chemical Laser, Lawrence Livermore Laboratory Rept. UCID-16006 (Mar. 1972).

144. Matheson Gas Data Book (1971), 5th Edition.

145. F. T. Aldridge, Apph. Phys. Lett. 22, 180 (1973).

146. D. W. Gregg and E. B. Huss, Lawrence Livermore Laboratory, unpublished data.

147. R. K. Pearson, Lawrence Livermore Laboratory, private communication (1973).

148. F. T. Aldridge, Lawrence Livermore Laboratory, private communication (1973).

149. D. J. Kuizenga, Stanford University, Stanford, Calif., private communication (1973).

150. S. A. Akhunanov, D. P. Krindach, A. V. Migulin, A. P. Sukhorokov, aı, R. V. Khokhlov, "Thermal Self-Action of Laser Beams," IEEE J. Quant. Electron. QE-4, 568 (1968).

151. J. H. Weiner and Y. Partum, "Quantum Rate Theory for Solids. Il. One-Dimensional Tunneling Effects," Phys. Rev: 187, 1134 (1969).

152. A. J. Glass, Lawrence Livermore Laboia:ory, report in preparation.

153. A. J. Glass, paper TH-P14 presented at the Optical Society of America meeting in San Francisco, Calif., October 1972.

154. Laser-Fusion Program Semiannual Report - July-December 1972, UCRL-50021-72-2, p. 81.

155. Ibid., pp. 82-86.

156. R. A. Fisher, P. L. Kelley, and T. K. Gustafson, Appl. Rhys. Lett. 14, 140 (1969).

157. F. Shimizu, IEEE J. Quant. Electron. QE-8, 851 (1972).

158. R. H. Stolen and A. Ashkin, Appl. Phys. Lett. 22, 294 (1973).

159. C. V. Shank, E. P. Ippen, and T. K. Gustafson, paper 12.6 presented at the 1973 IEEE/OSA Conference on Laser Engineering and Applications (CLEA Conference), Washington, D. C., 1973.

160. M. A. Duguay, J. W. Hansen, and S. L. Shapiro, IEEE J. Quant. Electron. QE-5, 725 (1970).

161. R. R. Alfano and S. L. Shapiro, Phys. Rev. Lett. 24, 592 (1970).

162. R. M. Waxler, G. W. Cleek, I. H. Malitson, M. J. Dodge, and T. A. Hahn, J. Res. Nat. Bur. Stand. Sect. A Ph. Ch. 75, 163 (1971).

163. Laser-Fusion Program Semiannual Report -- July-December 1972, UCRL-50021-72-2, pp. 72-73.

164. Ibid., p. 99.

165. Ibici., pp. 70-72.

166. R. L. Carman, F. Shimizu, C. S. Wang, and N. Bloembergen, Phys. Rev. 2, 60 (1970).

167. R. V. Johnson and J. H. Marburger, Phys. Rev. 4, 1175 (1971).

168. P. C. Magnante, IEEE J. Quant. Electron. QE-8, 440 (1972), and Refs. 24 therein.

169. M. M. Mann and L. G. DeShazer, J. Appl. Phys. 41, 2951 (1970).

170. L. G. DeShazer, Informal Report No. 1, USAEC Subcontract No. 1434705 (June 1, 1973). Certain entries in this report have been slightly modified by $\mathrm{L}$. DeShazer by private communication.

171. J. D. Myers, Owens-Illinois Technical Brochure (undated).

172. W. F. Krupke, Lawrence Livermore Laboratory, private communication (1973).

173. R. R. Jacobs, H. Samelson, and A. Lempicki, J. Appl. Phys. 44, 263 (1973), and J. Appl. Phys. 44, 2775 (1973).

174. T. W. Hanson, Appl. Opt. 11, 895 (1972).

175. J. Marburger, "Self-Focusing with Elliptical Beams," in Damage in Laser Materials, NBS Special Publication No. 356 (U. S. Government Printing Office, Washington, D. C., 1972), p. 84.

176. P. L. Kelley, Phys. Rev. Lett. 15, 1005 (1965).

177. V. I. Bespalov and V. 1. Talanov, JETP Lett. 3, 307 (1966).

178. R. Speck, Lawrence Livermore Laboratory, private communication (1973).

179. Y. L. Pan, J. R. Simpson, and A. F. Bernhardt, IEEE J. Quant. Electron. QE-9, 714 (1973).

180. J. Tulip, K. Manes, and H. J. Seguin, Opto-electronics 3, 131 (1971). 
181. Laser-Fusion Program Semiannual Report - July-December 1971, UCRL-50021-71 Rev. 1, pp. 58-60.

182. S. W. Mead, R. E. Kidder, J. E. Swain, F. Rainer, and J. Petruzzi, "Preliminary Measurements of X-Ray and Neutron Emission from Laser-Produced Plasmas," Appl Opt. 11, 345 (1972).

183. L. W. Coleman, J. E. Swain, F. Rainer, and R. A. Saroyan, Pulse Characteristic of the Long Path Laser at $L L L$, Lawrence Livermore Laboratory Rept. UCRL-74626 Preprint (1973), submitted to IEEE J. Quant. Electron.

184. P. J. Mallozzi, H. M. Epstein, R. G. Jung, D. C. Applebaum, B. P. Fairand, and W. J. Gallagher, X-Ray Emission from Laser Generated Plasmas, report tu Advance Research Projects Agency, Vols. I and II (Jan. and Feb. 1972).

185. Laser-Fusion Program Semiannual Report - July-December 1972, UCRL-50021-72-2, pp. 118-121.

186. J. J. Thomson, R. J. Faehl, and W. L. Kruer, Mode Coupling Saturation of the Parametric Instability and Electron Heating, Lawrence Livermore Laboratory Rept. UCRL-74686 Preprint (Apr. 1973), submitted to Phys. Rev. Lett.

187. J. W. Shearer, J. Garrison, J. Wong, and J. E. Swain, Pair Production by Relativistic Electrons from an Intense Laser Focus, Lawrence Livermore Laboratory Rept. UCRL-74423 Rev. 1 Preprint (Apr. 1973), to be published in Phys. Rev. A.

188. Laser-Fusion Program Semiannual Report - January-June 1972, UCRL-50021-72-1, pp. 8486.

189. R. D. Evans, The Atomic Nucleus (McGraw-Hill, New York, 1955), p. 716.

190. F. W. Bunkin and I. I. Tugov, Dokl. Akad. Nouk SSSR 187, 541 (1969). English translation: Sov. Phys. - Dokl 14, 678 (1970).

191. E. Brezin and C. Itzykson, Phys. Rev. D 2, 1191 (1970).

192. F. V. Bunkin and A. E. Kazakov, Dokl. Akad. Nauk SSSR 193, 1274 (1970). English translation: Sov. Phys. - Dokl 15, 758 (1971).

193. H. J. Bhabha, Proc. Roy. Soc. London 152, 559 (1935).

194. S. J. Brodsky and S. C. C. Ting, Phys. Rev. 145, 1018 (1966).

195. A. D. Steiger and C. H. Woods, Phys. Rev. A 5, 1467 (1972).

196. Laser-Fusion Program Semiannual Report - July-December 1972, UCRL-50021-72-2, pp. $115-117$.

197. Laser-Fusion Program Semiannual Report - July-December 1971, UCRL-50021-71 Rev. 1, pp. 74-77, and January-June 1972, UCRL-50021-72-1, pp. 107-109.

198. J. B. Chase, J. L. LeBlanc, and J. R. Wilson, Lawrence Livermore Laboratory Rept. UCRL-73914 Preprint (May 1972), submitted to Phys. Fluids.

199. James Scofield, Lawrence Livermore Laboratory, private communication (1973).

200. W. F. Utlaut and R. Cohen, Science 174, 245 (1971).

201. H. Dreicer, J. C. Ingraham, and D. B. Henderson, Phys. Rev. Lett. 26, 1616 (1971).

202. W. L. Kruer, P. K. Kaw, J. M. Dawson, and C. Oberman, Phys. Rev. Lett. 24, 987 (1970); J. Katz, J. DeGrooî, and R. J. Faehl, Bull. Amer. Phys. Soc. 17, 1045 (1972).

203. Laser-Fusion Program Semiannual Report - Jamuary-June 1972, UCRL-50021-72-1, pp. 109-111.

204. E. Valeo, C. Oberman, and F. W. Perkins, Phys. Rev. Lett. 28, 340 (1972); D. F. Dubois and M. V. Goldman, Phys. Rev. Lett. 28, 218 (1972); W. L. Kruer and E. Valeo, Phys. Fluids 16, 675 (1973); Y. Kuo and J. A. Fejer, Phys. Rev. Lett. 29, 1667 (1972).

205. K. Nishikawa, J. Phys. Soc. Japen 24, 916 and 1152 (1968).

206. J. Katz, J. Weinstock, W. L. Kruer, J. S. DeGroot, and R. J. Faehl, Lawrence Livermore Laboratory Rept. UCRL-74334 Preprint (1973), to be published in Phys. Fluids, Oct. 1973.

207. See first three citations under Ref. 204.

208. W. L. Kruer and E. J. Valeo, Phys. Fluids 16, 675 (1973).

209. J. S. DeGroot and A. L. Pavel, to be published; see also H. Dreicer, R. F. Ellis, and J. C. Ingraham, Los Alamos Scientific Laboratory preprint LA-UR-73-481 (1973).

210. W. L. Kruer, Phys. Fluids 15, 2423 (1972).

211. J. M. Dawson, W. L. Kruer, and B. Rosen, Princeton University Rept. MATT-883 (1972).

212. D. W. Forslund, J. M. Kindel, and E. L. Lindman, Phys. Rev. Lett. 30, 739 (1973).

213. W. L. Kruer, K. G. Estabrook, and K. H. Sinz, Instability-Generated Laser Reflection in Plasmas, Lawrence Livermore Laboratory Rept. UCRi-74676 Preprint (1973), to be published.

214. E. A. Jackson, Phys. Rev. 153, 235 (1967); Martin V. Goldman, Ann. Phys. 38, 117 (19ú6).

215. Laser-Fusion Program Semiannual Report - July-December 1972, UCRL-50021-72-2, pp. 125-126.

216. W. L. Kruer and J. M. Dawson, Phys. Fluids 14, 1003 (1971). 
217. R. E. Kidder, in Proceedings of the International School of Physics (Enrico Fermi), Course XLV (Academic Press, New York, 1971), p $343 \mathrm{ff}$. , and references therein.

218. A. Galeev, G. Laval, T. O'Neil, M. Rosensluth, and R. Sagdeev, JETP Lett. 17, 35 (1973).

219. D. W. Forslund, J. M. Kindel, and E. L. Lindman, Phys. Rev. Lett. 30, 739 (1973).

220. J. Nuckolls, L. Wood, A. Thiessen, and ' . Zimmerman, Nature 239, 139 (1972).

221. M. N. Rosenbluth, Phys. Rev. Lett. 28, 565 (1972).

222. J. G. Meadors, J. Appl Phys. 40, 2510 (1969); C. L. Tang, J. Appl. Phys. 37, 2945 (1965).

223. J. W. Shearer and J. L. Eddleman, Laser Light Forces and Self-Focusing in Fully Ionized Plasmas, Lawrence Livermore Laboratory Rept. UCRL 73969 Preprint (1972), submitted to Phys. Fluids.

224. R. E. Kidder, Interaction of Intense Photon Beams with Plasmas (II), Lawrence Livermore Laboratory Rept. UCRL-74040 Preprint (1972). Preser ed at Japan-U.S. Seminar on Laser Interaction with Matter, Kyoto, Japan, Sept. 25-29, 1972).

225. P. Kaw, G. Schmidt, and T. Wilcox, University of California at Los Angeles, Plasma Physics Group preprint PPG-140 (1972).

226. Laser-Fusion Program Semiannual Report - July-December 1972, UCRL-50021-72-2, pp. 111-113.

227. J. Nuckolls, Lawrence Livermore Laboratory Rept. UCRL-74345 Preprint (1972).

228. J. Nuckolls, L. Wood, A. Thiessen, and G. Zimmerman, Lawrence Livermore Laboratory Rept. UCRL-74116 Preprint (1972).

229. Laser-Fusion Program Semiannual Report - Jamuary-June 1972, UCRL-50021-72-1, p. 107.

230. D. L. Chapman, Phil. Mag. 213, Series 5, 47, 90 (1899); E. Jouguet, J. Pure Appl. Mat. 70, Series 5, 1, 347 (1905); 2, 1 (1906).

231. R. W. Moir and C. E. Taylor, Magnets for Open-Ended Fusion Reactors, Lawrence Livermore Laboratory Rept. UCRL 72399 Preprint (Sept. 1970)

232. J. D. Lee, Some Neutronic Aspects of a DT Fusion Reactor, Lawrence Livermore Laboratory Rept. UCRL-72493 Preprint (May 1970).

233. R. W. Wemer and G. A. Carlson, Heat Fipe Radiator for a $50 \mathrm{MWt}$ Space Power Plant, Lawrence Livermore Laboratory Rept. UCRL-50293 (June 1967).

234. J. B. Mitchell, "Mechanisms of Growth and Loss of Coherency of HfN Particles in Molybdenum," Acta Met. 19, 1063 (1971).

235. T. Weaver and L. Wood, Exotic CTR Fuel ; for Direct Conversion Itilizing Fusion Reactors, Lawrence Livermore Laboratory Rept. UCID-16230 (1973).

236. B. Freeman, L. Wood, and J. Nuckolls, Some General Design Considerations Regarding Laser-Fusion CTR Power Plants, Lawrence Ljvermore Laboratory Rept. UCRL-74486 Preprint (1971).

237. A. F. Haught, D. H. Polk, and W. J. Fadar, Phys. Fluids 13, 2842 (1970) and private communication.

238. Richard J. Rosa, Magnetohydrodynamic Energy Conversion (McGraw-Hill, New York, 1968).

239. L. C. Northcliffe and R. F. Schilling, Nucl. Data 7A, 233 (1970).

240. F. Winterberg, Phys. Rev. 174, 212 (1968); Rev. Sci. Instr. 41, 1756 (1970).

241. D. Steiner, "A Review of the ORNL Fusion Reactor Feasibility Studies," Paper 709005, Energy 70, Las Vegas, Nevada (Sept. 1970).

242. L. Wood and T. Weaver, Some Direct Comversion Possibilities for Advanced CTR Systems, Lawrence Livermore Laboratory Rept. UCID-16229 (1973).

243. T. Weaver, J. Nuckolls, and L. Wood, Fusion Microexplosions, Exotic Fusion Fuels, Direct Conversion: Advanced Technology Options for CTR, Lawrence Livermore Laboratory Rept. UCID-16309 (1973).

244. T. Weaver, G. Zimmerman, and L. Wood, Prc pects for Exotic Fuel Usage in CTR Systems I. B ${ }^{I I}$ $(p, 2 \alpha) H e^{4}:$ A Clean High Performance CTR Fuel, Lawrence Livermore Laboratory Repts. UCRL-74191 and $\mathbf{- 7 4 3 5 2}$ Preprints (1972). See also Ref. 235.

245. W. A. Fowler, G. Caughlan, and B. Zimmerman, Ann. Rev. Astron. Astrophys. 5, 525 (1967); W. A. Fowler, G. Caughlan, and B. Zimmerman, to be published. See also N. Jarmie and R. Seagrave (editors), Los Alamos Scientific Laboratory Rept. LA-2014 (1957), and references cited therein, and F. Ajzenberg-Selov, Nucl. Phys. A152, 1 (1970), and references cited therein, for cross-section data.

246. S. Maxon, Phys: Rev. 5A, 1630 (1972).

247. M. Gryzinski, Phys. Rev. 115, 1087 (1959).

248. J. R. McNally, Jr., Nucl. Fusion 11, 187 (1971), et seq. 
249. G. Lee, G. Zimmerman, and L. Wood, Bull. Amer. Phys. Soc. 17, 989 (1972); also Concerning Electron-Ion Coupling and Charged Particle Energy Deposition During Vigorous Thermonuclear Bum, Lawrence Livermore Laboratory Rept. UCRL-74192 Preprint (1972).

250. G. Zimmerman and $L$. Wood, Lawrence Livermore Laboratory, to be published.

251. J. Wyatt and J. Luce, Lawrence Livermore Laboratory, private communication (1973).

252. K. R. Osborn, C. C. McDonald, and H. E. Gunning, J. Chem. Phys. 26, 125 (1957).

253. J. R. McDowell, C. C. McDonald, and H. E. Gunning, Can. J. Chem. 37, 1432 (1959).

254. E. S. Yeung and C. B. Moore, Appl. Phys. Lett. 21, 109 (1972).

255. S. W. Mayer, M. A. Kwok, R. W. F. Gross, and D. J. Spencer, Appl. Phys. Lett. 17, 516 (1970).

256. R. U. Ambartzumian, V. S. Letokhov, G. N. Markarso, and A. A. Paretzki, JETP Lett. 18, 91 (1973).

257. V. S. Letokhov, Science 180, 451 (1973).

258. J. Robieux and J. M. Auchair, French Patent No. 1,391,738 (March 12, 1965).

259. Avco Corporation, French Patent No. 2,094,967 (April 20, 1971).

260. K. Gürs, German Patent No. 1,959,767 (June 3, 1971). 
Listed here are laser-related publications produced by LLL authors during the report period. Brief abstracts accompany some of the listings. Papers presented at the 1973 IEEE/OSA Conference on Laser Engineering and Applications (May 30-June 1, 1973, Washington, D.C.) are listed separately, under the heading CLEA Conference Papers.

Carman, R. L., L. L. Steinmetz, and B. C. Johnson, "A Self-Driven Laser Oscillator for Directly Producing Bandwidth Limited Pulses of About One Nanosecond," Opt. Comm. 7, 169 (1973).

Coleman, L. W., J. E. Swain, F. Rainer, and R. A. Saroyan, Pulse Characterization for the Long Path Laser at $L L L$, Lawrence Livermore Laboratory Rept. UCRL-74626 Preprint (April 2, 1973). Submitted to IEEE J. Quant. Electron.

Characteristics of the pulse produced by the Laboratory's Long Path Laser are reported.

Emmett, J. L., "Physical Limitations on Lasers for Laser-Fusion," presented at Symposium of the Division of Plasma Physics, APS Annual Meeting, New York (1973).

Emmett, J. L., "A Review of Current Problems in High Power Laser Development," presented at Topical Conference on High Power and Tunable Lasers, sponsored by the Joint Services Electronics Program, University of Southern Calif̂́ninia, Los Angeles (1973).

Emmett, J. L., and W. F. Krupke, "High Power Lasers for Fusion Applications," presented at Spring Meeting of Optical Society of America, Denver (1973).

Fisher, R. A., "Dispersive Shcck Formation in Self-Phase Modulation," presented at the April meeting of the American Physical Society (1973).

This paper presents the results of a calculation which combines the effects of nonlinear self-phase modulation and host dispersion. Numerous cases are shown for propagation in $\mathbf{C S}_{2}$, glass, and YAG. It is shown that optical shocks will form under a large variety of circumstances.

Fisher, R. A., and W. Bischel, "The Role of Linear Dispersion in Plane-Wave Self-Phase Modulation," submitted to Appl. Phys. Lett. (1973).

It is shown by computer calculation that linear dispersion and self-phase modulation can strongly couple to influence pulse propagation phenomena.

Fleck, J. A., Jr., and R. L. Carman, "Laser Pulse Shaping Due to Self-Phase Modulation in Ampiifying Media," Appl. Phys Lett. 22, 546 (1973).

George, E. V., and C. K. Rhodes, "Kinetic Model of Ultraviolet Inversions in High Pressure Rare Gas Plasmas, Appl. Phys. Lett. 23, 139 (1973).

Gunn, S. R., “A Review of Calorimetric Measurements of Laser Energy and Power," J. Phys. E: Sci. Instrum. 6, 105 (1973).

Gunn, S. R., "Calorimeters for Measurement of the Energy of High-Power Laser Pulses," presented at the Calorimetry Conference, Worcester, Mass. (1973).

Hoff, P. W., J. C. Swingle, and C. K. Rhodes, "Demonstration of Temporal Coherence, Spatial Coherence, and Threshold Effects in the Molecular Xenon Laser," Opt. Comm. 8, 128 (1973).

Hoff, P. W., J. C. Swingle, and C. K. Rhcdes, “Observation of Stimulated Emission from High-Pressure Krypton and Argon/Xenon Mixtures," Appl. Phys. Lett. 23, 5 (1973).

Hopf, F. A., and C. K. Rhodes, "The Influence of Reorientational Collisions on Coherent Optical Processes," Opt. Comm. 8, 88 (1973). 
Hopf, F. A., and C. K. Rhodes, "Influence of Vibrational, Rotational, and Reorientation Relaxation of Pulse. Amplification in Molecular Amplifiers," Phys. Rev. A 8 (2) 912 (1973).

Hyde, R., L. Wood, and J. Nuckolls, Prospects for Rocket Propulsion with Laser-Induced Fusion Microexplosions, Lawrence Livermore Laboratory Report UCRL-74218 Rev. 2 Preprint (1972). This paper was presented at the 8th Annual AIAA/SAE Joint Propulsion Specialist Conference, New Orleans (27.29 Nov. 1972), AlAA Paper No. 72-1063.

We discuss the basic features and performance potential of the class of specific-impulse-maximizing exoatmospheric propulsion systems based on laser-induced fusion microexplosions. Considerations in and results of the integrated optimized design of the major components of such a propulsion system are presented, with emphasis on novel features. Technical uncertainties to which system performance is sensitive and present rates of resolution are treated, as are cost, launch and assembly considerations. The potential applications of such a $~ 300-$ ton propulsion system, capable of applying velocity increments in excess of 1000 miles/sec to $\sim 100-t$ ton payloads, are briefly noted.

Jacobs, R. R., "Technique for Gain Determinations in Pulsed $\mathrm{CO}_{2}$ TEA Lasers," Rev. Sci. Instrum. 44, 1146 (1973).

Karan, J., and J. J. Thomson, Non-Markovian Green's Function for Plasma Turbulence, Lawrence Livermore Laboratory Rept. UCRL-74800 Preprint (1973). Submitted to Phys. Lett. A.

Kruer, W. L., K. G. Estabrook, and K. H. Sinz, Instability-Generated Laser Reflection in Plasmas, Lawrence Livermore Laboratory Rept. UCRL-74676 Preprint (1973). Submitted to Nucl Fusion.

Owen, T. C., L. W. Coleman, and T. J. Burgess, "Ultrafast Optical Kerr Effect in $\mathrm{CS}_{2}$ at $10.6 \mu \mathrm{m}$," Appl. Phys. Lett. 22 (6), 272 (1973).

Pan, Yu-Li, "Generation of a Multigigawatt Nanosecond $\mathrm{CO}_{2}$ Pulse," IEEE J. Quant. Electron. (in press, 1973).

Rentjes, J., R. L. Carman, and F. Shimizu, “A Study of Self-Focusing and Self-Phase Modulation in the Picosecond Time Regime," Phys. Rev. (in press, 1973).

Rhodes, C. K., and P. W. Hoff, "Oscillation on the Ultraviolet Bound-Free Continua of Diatomic Molecular Xenon and Molecular Krypton," presented at the Conference on Laser Spectroscopy, Vail, Colo. (1973).

Saroyan, A., Notes on the Mathematical Expression for Bandwidth-Limited Pulses, Lawrence Livermore Laboratory Rept. UCID-16247 (1973).

Shay, H. D., LASNEX Simulation of X-Ray Generation, Lawrence Livermore Laboratory D.pt. UCID-16297 (1973).

Shearer, J. W., Comparison of Laser-Heated Fusion Plasma Proposals, Lawrence Livermore Laboratory Rept. UCRL-74628 Preprint (1973), submitted to 6th European Conference on Controlled Fusion and Plasma Physics, Moscow.

Shearer, J. W., "Problems of the Laser-Augmented Pinch Proposal," presented at 6th European Conference on Controlled Fusion and Plasma Physics, Moscow (1973).

Shearer, J. W., J. Garrison, J. Wong, and J. E. Swain, Pair Production by Relativistic Electrons from an Iritense Laser Focus, Lawrence Livermore Laboratory Rept. UCRL-74423 (1973). Submitted to Phys. Rev. A.

A discussion is given of electron-positron pair production by means of electrons accelerated to relativistic velocities at the focus of a laser beam.

Steinmetz, L. L., T. W. Pouliot, and B. C. Johnson, "Cylindrical, Ring-Electrode KD*P Electrooptic Modulator," Appl. Cpt. 12 (7), 1468 (1973).

Thomson, J. J., and G. Benford, "Green's Function for Markovian Systems," J. Math. Phys. 14, 531 (1973). 
Thomson, J. J., R. J. Faehl, and W. L. Kruer, Mode Coupling Saturation of the Parametric Instability and Electron Heating, Lawrence Livermore Laboratory Rept. UCRL-74686 Preprint (1973). Submitted to Phys. Rev. Lett.

Thomson, J. J., R. J. Faehl, and W. L. Knuer, "Electron Heating Due to Parametric Instability Turbulence," presented at the Anomalous Absorption Conference, Los Alamos, N. Mex. (1973).

Thomson, J. J., W. L. Kruer, S. Bodner, and J. DeGroot, Parametric Instability Thresholds and Their Control, Lawrence Livermore Laboratory Rept. UCRL-74637 Preprint (1973). Submitted to Phys. Fluids.

Trenholme, J. B., and K. R. Manes, A Simple Approach to Laser Amplifiers, Lawrence Livermore Laboratory Rept. UCRL-51413 (1972).

A mathematical description of a laser amplifier is presented in a simplified way. This is a consolidation and review of known results, and a study of their implications for laser amplifier design.

Tumer, C. E., Jr., and Yu-Li Pan, "Effects of Intense $\mathrm{CO}_{2}$ Radiation on Pulsed HF Chemical Laser Systems," IEEE J. Quant. Electron QE-9, 193 (1973).

Turner, C. E., Jr., Yu-Li Pan, and D. J. Blasberg, A New Approach for Demonstrating the Feasibility of Efficient Pulsed Chemical Lasers, Lawrence Livermore Laboratory Rept. UCRL-50022-4 (1973).

We are attempting to demonstrate that efficient pulsed chemical lasers are feasible by a unique experimental technique that uses a high-power $\mathrm{CO}_{2}$ laser to rapidly preheat chemical-laser gas mixtures before they are subjected to the electric discharge that produces the pulse of radicals for initiating the fast chemical reactions leading to lasing

Weaver, T., J. Nuckolls, and L. Wood, Fusion Microexplosions, Exotic Fusion Fuels, Direct Conversion: Advanced Technology Options for CTR, Lawrence Livermore Laboratory Rept. UCID-16309 (1973). Documentation of a briefing presented to the Committee on Advance Development and the Fusion Task Force of the Edison Electric Institute at the Los Alamos Scientific Laboratory, 1 May 1973.

In the past few years, several new technological options related to controlled fusion have been developed that promise to dramatically improve the long term prospects for the production of inexpensive electrical energy with minimal environmental impact. This paper very briefly describes three of these options: laser-initiated fusion microexplosions, exotic fuel usage, and new forms of conversion of fusion energy directly to electricity. While these technologies do not depend on each other for their useful realization, they rather naturally combine to form an extremely attractive fusion reactor system.

Weaver, T., and L. Wood, Exotic CTR Fuels for Direct-Conversion-Utilizing Fusion Reactors, Lawrence Livermore Laboratory Rept. UCID-16230 (March 16, 1973). Documentation of an invited talk before the AEC CTR Staff, AEC/Germantown, Md. (19 March 1973).

The prospects for the use of exotic CTR fuel systems to enhance the efficiency and economy of electrical energy production and to reduce neutron-induced structural damage and radioactivation relative to the standard fuels (DT, DD, and $\mathrm{DHe}^{3}$ ), are surveyed. The $\mathrm{pB}^{11}$ fuel system is found to be especially attractive in these respects, and it has an optimal $n \tau$ and burn temperature range $\left(T_{i}>100 \mathrm{keV}\right)$ comparable to those of $\mathrm{DHe}^{3}$. The importance of nonthermal effects in augmenting the performance of $\mathrm{pB}^{11}$ and exotic fuel systems (especially fusion chains) in general is emphasized, and calculational methods are outlined. The large gaps that exist in the knowledge of the nuclear cross sections needed to do these calculations accurately are pointed out.

Weaver, T., G. Zimmerman, and L. Wood, Prospects for Exotic Fuel Usage in CTR Systems I. $B^{11}(p, 2 \alpha) H e^{4}$ : A Clean, High Performance CTR Fuel, Lawrence Livermore Laboratory Rept. UCRL-74191/UCRL-74352 Preprint (Nov. 10, 1972). Paper 2D12 at APS Plasma Physics Division Meeting, Monterey, Calif. (November 1972).

The necessary and sufficient conditions for a given pair of nuclei to be of interest as fuels for various types of CTR systems are reviewed, with emphasis on those thermonuclear fuel combinations in which at least one nucleus of the pair has $Z$ such that $2<Z \lesssim 10$. Thermonuclear reaction rates are employed to develop relative figures of merit for all such fuel systems of interest, with emphasis on fuels developing most of their energy in charged particles. Nonthermal contributions to total nuclear fusion energy production rates are also treated, for representative systems. 
Wood, L., and T. Weaver, Some Direct Conversion Possibilities for Advanced CTR Systems, Jawrence Livermore Laboratory Rept. UCID-16229 (March 16, 1973). Documentation of an invited talk before the AEC CTR Staff, AEC/Germantown, Md. (19 March 1973).

Some of the means for directly converting fusion energy into electricity in various types of CTR systems are surveyed. It is concluded that relatively simple and efficient means exist for so converting essentially all forms of energy emitted from a thermonuclear reaction zone. It is noted that CTR power plants buming exotic fuels - neutron- and radionuclide-free nuclei combinations - maximally exploit the profound asymmetries between fission and fusion power sources, asymmetries that potentially strongly favor fusion-based means of electricity generation.

\section{CleA Conference Papers}

Following is a list of papers presented at the 1973 IEEE/OSA Conference on Laser Engineering and Applications, May 30-June 1, 1973, Washington, D.C.

Carman, R. L., W. H. Lowdermilk, J. Kysilka, M. Moran, and G. Kachen, "A 20-psec Mode-Locked Ruby Laser System of Brightness Greater Than $10^{18} \mathrm{~W} / \mathrm{cm}^{2}$-sr."

Coleman, L. W., and S. W. Thomas, "New 10 psec Streak Camera for Laser Pulse Diagnostics."

Emmett, J. L., "Review of Laser Devices for Fusion."

Fisher, R. A., and W. Bischel, "Pulse Self-Dispersion in Self-Phase Modulation."

Hoff, P. W., J. C. Swingle, and C. K. Rhodes, "Demonstration of Coherent Laser Oscillation in Molecular Xenci and Krypton."

Johnson, B. C., L. L. Steinmetz, R. L. Carman, and W. H. Lowdermilk, "A Laser Oscillator that Directly Produces Bandwidth-Limited Pulses in the 1-nsec Region and an Amplifier Chain that Maintains the Single Pulse Coherence."

Meyer, T. W., and C. K. Rhodes, "High-Resolution Pressure-Broadening Studies in $\mathrm{CO}_{2}$."

Owen, T. C., L. W. Coleman, and T. J. Burgess, "Ultrafast $10 \mu \mathrm{m}$ Detector Using the Optical Kerr Effect and a High-Speed Streak Camera."

Pan, Yu-Li, J. R. Simpson, and A. F. Bemhardt, "Breakdown Threshold for Nanosecond $\mathrm{CO}_{2}$ Laser Pulses."

Pearson, R. K., G. L. Hermann, and E. B. Huss, "Experimental Survey of Reactant Systems for HF Chemical Lasers."

Rhodes, C. K., "Review of Ultraviolet Lasers" (also submitted to IEEE J. Quant. Electron.).

Smith, D. L., and D. T. Davis, "Generation of Single 1-nsec Pulses at $10.6 \mu \mathrm{m}$ Without Mode Locking."

Speck, D. R., and E. S. Bliss, "Characteristics of a Subnanosecond Nd Amplifier System."

Steinmetz, L. L., T. W. Pouliot, and B. C. Johnson, "Cylindrical Ring Electrode KD*P Electrooptic Modulator."

Trenholme, J. B., "The Design of a Large Laser System for Fusion."

Tuccio, S. A., "CW Dye Laser Operation in the Blue."

Violet, C. E., J. Petruzzi, S. W. Mead, J. E. Swain, and R. A. Saroyan, "X Rays and Reflected Light from Laser Produced Plasmas." 